\title{
IR-Untersuchung von schwach gebundenen Molekülaggregaten im Überschallstrahl
}

\author{
Dissertation \\ zur Erlangung des mathematisch-naturwissenschaftlichen Doktorgrades \\ „Doctor rerum naturalium“ \\ der Georg-August-Universität Göttingen
}

\author{
im Promotionsprogramm Chemie \\ der Georg-August University School of Science (GAUSS)
}

vorgelegt von

Hannes Christian Gottschalk

aus Göttingen

Göttingen, 2020 


\section{Betreuungsausschuss:}

Prof. Dr. Martin A. Suhm, Institut für Physikalische Chemie

Prof. Dr. Ricardo A. Mata, Institut für Physikalische Chemie

\section{Referent:}

Prof. Dr. Martin A. Suhm

\section{Korreferent:}

Prof. Dr. Ricardo A. Mata

\section{Weitere Mitglieder der Prüfungskommission:}

Prof. Dr. Burkhard Geil, Institut für Physikalische Chemie

Dr. Sebastian Kruss, Institut für Physikalische Chemie

Dr. Tim Schäfer, Institut für Physikalische Chemie

Prof. Dr. Dirk Schwarzer, Max-Planck-Institut für Biophysikalische Chemie

Tag der mündlichen Prüfung: 29.09.2020 


\section{Danksagung}

Die Entstehung dieser Arbeit wäre nicht ohne die Hilfe zahlreicher Menschen möglich gewesen. An erster Stelle möchte ich mich deshalb bei Annika Iber für ihre fortwährende Unterstützung bedanken. Mein besonderer Dank gilt auch meiner Familie und meinen Freunden für ihre unschätzbare Hilfe über die Jahre hinweg.

Prof. Dr. Martin Suhm danke ich für die hervorragende Betreuung, seine Hilfsbereitschaft bei jeglicher Art von Problemen, sein entgegengebrachtes Vertrauen und nicht zuletzt die Möglichkeit, Teil von sehr unterschiedlichen und vielseitigen Forschungsprojekten wie dem Göbench oder dem Aufbau einer neuen Forschungsapparatur zu sein. Mein besonderer Dank gilt auch Prof. Dr. Ricardo Mata, der nicht nur ein engagierter Zweitbetreuer meiner Arbeit war, sondern der mein Interesse an der Computerchemie überhaupt erst geweckt hat. Außerdem danke ich Prof. Dr. Burkhard Geil, Dr. Sebastian Kruss, Dr. Tim Schäfer und Prof. Dr. Dirk Schwarzer für die Bereitschaft, Teil der Prüfungskommission zu sein.

Die Planung, der Aufbau und die Inbetriebnahme der Gratin-Jet-Apparatur wären ohne die Chemie-Werkstätten nicht möglich gewesen. Stellvertretend möchte ich hier Reinhard Hildebrandt, Mike Zippert, Andreas Knorr, Annika von Roden und insbesondere Volker Meyer meinen Dank aussprechen, die gemeinsam mit ihren Abteilungen jeden Abschnitt der Entstehung der neuen Apparatur mit außergewöhnlichem Engagement begleitet haben. Außerdem hatte ich das Vergnügen, Manuel Lange und Julia Stachowiak im Rahmen von Bachelorarbeiten sowie Charlotte Zimmermann und Taija Fischer im Rahmen von Masterarbeiten zu betreuen. Für ihr Engagement, ihre Ideen und die wertvollen Beiträge zu den Forschungsvorhaben - auch über die Abschlussarbeiten hinaus - möchte ich mich ebenfalls herzlich bedanken. Das gilt auch für die weiteren derzeitigen und ehemaligen Mitglieder der Suhm-Gruppe, wobei ich hier Dr. Jonas Altnöder, Thomas Forsting, Dr. Matthias Heger, Dr. Franz Kollipost, Dr. Sönke Oswald und Dr. Anja Poblotzki hervorheben möchte. Besonders danken möchte ich auch Katharina Meyer, mit der ich nicht nur das Büro, sondern auch die unweigerlichen Höhen und Tiefen eines Promotionsvorhabens teilen konnte, und Petra Lawecki, die mir nicht nur bei administrativen Problemen stets mit Rat und Tat zur Seite stand. Katharina Meyer, Sven Meyer und Lara Riemann danke ich für zahlreiche entspannende Mittagspausen, die mir sogar die Nordmensa schmackhaft gemacht haben. Dem BENCh-Graduiertenkolleg danke ich für das wissenschaftliche Umfeld, das unter anderem den Göbench ermöglicht hat.

Ohne Förderung wären viele Forschungsvorhaben nicht möglich - so auch in meinem 
Fall. Ich danke der Deutschen Forschungsgemeinschaft (DFG) für ihre Förderung im Rahmen des Schwerpunktprogramms (SPP) 1807 „Control of London Dispersion Interactions in Molecular Chemistry“ (Projekt-Nr. 271107160) und im Rahmen des Projekts „Diagonale und außerdiagonale Anharmonizität in Wasserstoffbrücken: Direkter infrarotspektroskopischer Nachweis in der Gasphase" (Projekt-Nr. 235616985). Gemeinsam mit dem Niedersächsischen Ministerium für Wissenschaft und Kultur (AZ 76211-10-4/16 (05) (74ZN1583)) danke ich der DFG (Projekt-Nr. 388444472) zudem für die Förderung im Rahmen des Großgeräteprojekts „FTIR-Clusterspektrometer mit Gasrückführung“.

Zu guter Letzt danke ich Katharina Meyer, Dr. Sönke Oswald, Arman Nejad und Annika Iber für das Korrekturlesen von Teilen dieser Arbeit und ihre äußerst hilfreichen Anmerkungen. 
Hiermit versichere ich, Hannes Christian Gottschalk, an Eides statt, dass ich die vorliegende Arbeit selbstständig verfasst und keine anderen als die angegebenen Quellen und Hilfsmittel verwendet habe.

Ort, Datum

Unterschrift 



\section{Inhaltsverzeichnis}

1 Einleitung 1

2 Theoretische und experimentelle Methoden 5

2.1 Theoretische Methoden . . . . . . . . . . . . . . 6

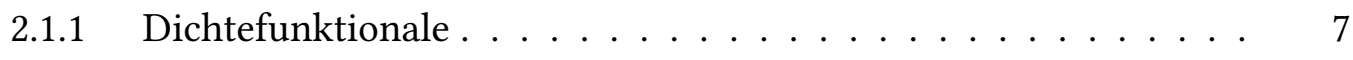

$2.1 .2 \quad$ DLPNO-CCSD $(\mathrm{T}) \ldots \ldots \ldots \ldots \ldots \ldots$

$2.2 \quad$ Filet-Jet-FTIR-Spektroskopie . . . . . . . . . . . . . . . . . . 12

2.3 Methoden zum Vergleich experimenteller und theoretischer Daten

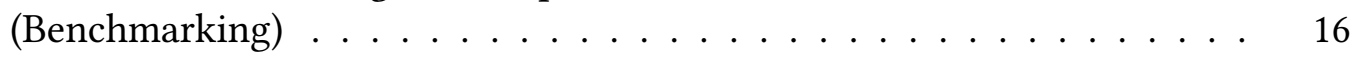

2.3.1 Benchmarking IR-spektroskopischer Parameter . . . . . . . . . 17

2.3.2 Benchmarking relativer Energien auf Basis eines Konformerengleichgewichts ................... 19

2.3.3 Fehlerbestimmung der experimentellen Intensitätsverhältnisse 30

3 Gratin-Jet-FTIR-Spektroskopie 33

3.1 Das Recycling-Konzept . . . . . . . . . . . . . . . . . . . . . 34

3.2 Gas- und Substanzbefüllung . . . . . . . . . . . . . . . . . . . . . . . 41

3.3 Evakuieren und Heizen . . . . . . . . . . . . . . . . . . . . . 44

3.3.1 Feinevakuieren . . . . . . . . . . . . . . . . 44

3.3.2 (Aus-)Heizen . . . . . . . . . . . . . . . . . . . . 45

3.4 Schlitzdüse und Expansionszone . . . . . . . . . . . . . . . . . . . 49

3.5 Spektrometer, Optik und Synchronisation . . . . . . . . . . . . . . 53

3.5.1 Spektrometer und Detektorkammer . . . . . . . . . . . 53

3.5.2 Optimierung von Synchronisation und Messparametern . . . . . 59

3.6 Untersuchung der im Laufe der Messzeit steigenden Wasserkonzentration 70

3.7 Leistungsfähigkeit der Gratin- im Vergleich zur Filet-Jet-Spektroskopie . 82

3.7.1 Vergleich der erreichten Kühlung . . . . . . . . . . . . . . . . 82

$3.7 .2 \quad$ Vergleich der Spektrenqualität . . . . . . . . . . . . . 83

3.7 .3 Fazit . . . . . . . . . . . . . . 93

4 O/C-Waagensysteme $\quad 97$

4.1 Furan-Mikrosolvatations-Leistungsüberprüfung (Göbench) . . . . . . . . . . 99

4.1 .1 Experimentelle Ergebnisse . . . . . . . . . . . . . . . 104

4.1 .2 Quantenchemische Rechnungen . . . . . . . . . . . 110 
4.1.3 Auswertung des O/C-Konformerengleichgewichts . . . . . . . 114

4.1.4 Auswertung IR-spektroskopischer Parameter . . . . . . . . . . . 123

4.1.5 Zusammenfassung . . . . . . . . . . . . . . . . 128

4.2 Alkohol-Anisol . . . . . . . . . . . . . . . . . . . 130

4.2.1 Experimentelle Ergebnisse . . . . . . . . . . . . . . 133

4.2.2 Quantenchemische Rechnungen . . . . . . . . . . . . 141

4.2.3 Auswertung des O/C-Konformerengleichgewichts . . . . . . . 154

4.2.4 Auswertung IR-spektroskopischer Parameter . . . . . . . . . . . 161

4.2.5 Analyse des Einflusses von Dispersionswechselwirkungen auf das

O/C-Konformerengleichgewicht . . . . . . . . . . . 167

4.2 .6 Zusammenfassung . . . . . . . . . . . . . . . . 169

5 Carbonylwaagensysteme $\quad 173$

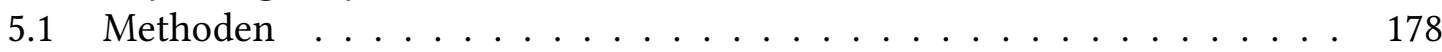

5.1.1 Experimentelles . . . . . . . . . . . . . . . 178

5.1 Quantenchemische Rechnungen . . . . . . . . . . . . . . 179

5.2 Experimentelle Ergebnisse . . . . . . . . . . . . . . . . . . . . 181

5.3 Auswertung des Me/Ph-Konformerengleichgewichts . . . . . . . . . 187

5.3.1 Ergebnisse der quantenchemischen Rechnungen . . . . . . . . . 187

5.3.2 Symmetrie der Dimerstrukturen _... . . . . . . . . . . . . 193

5.3.3 Benchmarks auf Basis des Me/Ph-Konformerengleichgewichts . . 199

5.4 Auswertung IR-spektroskopischer Parameter . . . . . . . . . . . . . . . 207

5.5 Analyse des Einflusses von Dispersionswechselwirkungen auf das Konformerengleichgewicht . . . . . . . . . . . . . 217

5.6 Zusammenfassung . . . . . . . . . . . . . . . . . . . . . 219

6 Zusammenfassung und Ausblick 225

$\begin{array}{ll}\text { Literatur } & 231\end{array}$

$\begin{array}{ll}\text { Lebenslauf } & 265\end{array}$ 


\section{Kapitel 1}

\section{Einleitung}

Die Computerchemie ${ }^{[1]}$ nimmt mit steigender Rechenleistung von Computersystemen ${ }^{[2]}$ und stetig verbesserten Algorithmen* eine immer wichtigere Rolle für die Erforschung chemischer Fragestellungen ein. ${ }^{[7,8]}$ So kann auf Basis quantenchemischer Rechnungen z. B. in der pharmazeutischen Industrie eine Vorauswahl potentieller Wirkstoffkandidaten erfolgen, bevor kostspielige experimentelle Versuchsreihen durchgeführt werden. ${ }^{[9-11]}$ Ähnliche Vorteile können geeignete Rechenmodelle auch in zahlreichen weiteren Forschungszweigen wie Katalyseforschung ${ }^{[12]}$ oder Materialwissenschaften ${ }^{[13]}$ bringen. Zudem können ${ }^{[14]}$ quantenchemische Modelle auch ein tieferes Verständnis grundlegender chemischer Prozesse ermöglichen, ${ }^{[12,15]}$ da nicht-observable Größen wie bspw. die Stärke einzelner Beiträge zur Wechselwirkungsenergie zugänglich sind. ${ }^{[16-23]}$ Voraussetzung für die erfolgreiche Anwendung solcher quantenchemischen Verfahren ist jedoch stets die Wahl einer geeigneten Methode, die für die untersuchte Fragestellung zuverlässige Ergebnisse bei vertretbarem Rechenaufwand liefert.

Um die Zuverlässigkeit und Genauigkeit einer quantenchemischen Methode einschätzen zu können, ist die Überprüfung ihrer Leistungsfähigkeit anhand von Referenzdaten, das sogenannte Benchmarking, von zentraler Bedeutung. Erst wenn eine Methode für eine Vielzahl möglichst diverser Systeme und zu berechnender Größen die Referenzdaten erfolgreich reproduziert, kann sie als geeignetes Werkzeug zur Vorhersage praxisrelevanter Daten angesehen werden. Ein Problem des Benchmarkings ist dabei die Gewinnung exakter Referenzdaten. Hierzu werden oftmals andere quantenchemische Rechnungen herangezogen. ${ }^{[7]}$ Äußerst beliebt sind dabei Ergebnisse, die mit dem „Gold-Standard“ der Quantenchemie, ${ }^{[24,25]} \operatorname{CCSD}(\mathrm{T}),{ }^{[26]}$ gewonnen wurden - Theorie benchmarkt also Theorie. ${ }^{[27,28]}$ Wenn möglich sollten jedoch experimentelle Daten als Referenz ${ }^{[29-35]}$ verwendet werden. ${ }^{[7]}$ Diese haben eine direktere Aussagekraft, da alle für praktische Anwendungen zur Verfügung stehenden Modelle auf diversen Näherungen basieren und somit nicht vollständig exakt sein können.

Das Ziel der vorliegenden Arbeit in diesem Kontext ist, akkurate experimentelle Referenzdaten zu gewinnen und deren Nutzbarkeit für das Benchmarking quantenchemischer

*Für eine interessante Diskussion über die Weiterentwicklung von Dichtefunktionalen s. Refs. [3-6]. 
Methoden zu überprüfen. Dabei werden sogenannte intermolekulare Waagensysteme ${ }^{\text {[31] }}$ untersucht. Bei diesen handelt es sich um Gleichgewichte aus zwei Konformeren des gleichen Molekülaggregats, ${ }^{[36]}$ die sich über chemische Variation der betrachteten Analyten hinsichtlich der Konformationspräferenz beeinflussen lassen. ${ }^{[37}$ Der inhaltliche Fokus liegt auf der Betrachtung der Regioselektivität bei der Ausbildung von Wasserstoffbrückenbindungen im Zusammenspiel mit sekundären Wechselwirkungen, insbesondere Dispersion. Ein weiterer Schwerpunkt der vorliegenden Arbeit ist die Optimierung der Rahmenbedingungen für die Aufnahme experimenteller Referenzdaten durch die Planung, den Aufbau, die Inbetriebnahme und eine erste Überprüfung der Leistungsfähigkeit einer neuen Forschungsapparatur mit Gasrecyclingsystem.

Um den grundlegenden Bedingungen quantenchemischer Rechnungen (isolierte Moleküle in der Gasphase bei $0 \mathrm{~K}$ ) möglichst nahezukommen, werden dazu Überschallexpansionen ${ }^{[38-45]}$ in Helium genutzt. In der adiabatischen Expansion wird die innere Energie der zu untersuchenden Moleküle bzw. Aggregate durch zahlreiche Stöße mit Trägergasmolekülen in Translationsenergie entlang der Ausbreitungsrichtung umgewandelt und es erfolgt eine Kühlung der Freiheitsgrade von bspw. Rotation und Vibration. ${ }^{[38,43]}$ Durch Mehrkörperstöße von mehreren Analyt- und mindestens einem Trägergasmolekül werden zudem schwach gebundene Analyt-Aggregate gebildet und durch die Aufnahme der Kondensationswärme durch das Trägergasmolekül stabilisiert. ${ }^{[43]}$ Bei ausreichend starker Verdünnung der Analyten (in dieser Arbeit etwa $<1 \%$ ) liegen bei größeren Abständen von der Düse in der Ruhezone der Expansion, in der kaum noch Stöße stattfinden, dann kalte, voneinander isolierte Analytmoleküle bzw. -aggregate vor. ${ }^{[40]}$

Die meisten praktischen Anwendungen chemischer Prozesse finden in flüssiger Phase bzw. in Lösung statt. ${ }^{[46]}$ Werden für letzteres protische Lösungsmittel wie Alkohole eingesetzt, spielen Wasserstoffbrückenbindungen für die Solvatation eine entscheidende Rolle. In der vorliegenden Arbeit sollen deshalb kleine wasserstoffbrückengebundene Aggregate aus einem Alkoholmolekül als Wasserstoffbrückenbindungsdonor und einem kleinen ( $\leq 12$ Schweratome) organischen Molekül als Wasserstoffbrückenbindungsakzeptor untersucht werden. Um die grundlegenden Lösungsmittel-Gelöstes-Wechselwirkungen an möglichst einfachen Modellsystemen zu untersuchen, ${ }^{[15]}$ wird nur der erste Schritt der (Mikro-)Solvatation ${ }^{[47,48]}$ - die Dimerbildung - betrachtet. Für die Ausbildung eines intermolekularen Waagensystems muss der organische Akzeptor zwei kompetitive Andockstellen für Wasserstoffbrückenbindungen bereitstellen. Hierzu können sich z. B. Moleküle eignen, die sowohl ein (C-basiertes) $\pi$-System als auch elektronegative Atome mit freien Elektronenpaaren $(\mathrm{O} / \mathrm{N} / \mathrm{F} / \mathrm{Cl} / \ldots)$ aufweisen. Kompakte Beispielverbindungen mit solchen vergleichbar attraktiven, eher lokalisierten $\mathrm{O}$ - und stärker delokalisierten C-zentrierten Bindungsstellen sind Furane ${ }^{[49]}$ oder Anisole ${ }^{[37,50]}$ (s. Abb. 1.1), die deshalb als sog. O/CWaagensysteme im Rahmen dieser Arbeit untersucht werden sollen. Während hier die chemisch unterschiedlichen (primären) $\mathrm{OH} \cdots \mathrm{O}-$ und $\mathrm{OH} \cdots \mathrm{C}$-Wasserstoffbrückenbindungen entscheidenden Einfluss auf die Konformation des Donor-Akzeptor-Dimers haben, spielen bei Waagensystemen mit gleichartigen (OH...O-)Wasserstoffbrückenbindungen Unterschiede in der Schwingungsnullpunktsenergie potentiell eine geringere und sekun- 
<smiles>[R]c1ccc([R])o1</smiles>

Furane<smiles>[R]c1c([R9])c([R])c(OC)c([R])c1[R]</smiles>

Anisole<smiles>[R]c1ccc(C(C)=O)c([R])c1</smiles>

Acetophenone

Abbildung 1.1: Lewis-Formeln der im Rahmen dieser Arbeit untersuchten Akzeptor-Stoffklassen der Furane $\left(\mathrm{R}^{1,2}=\mathrm{H}, \mathrm{Me}\right)$, Anisole $\left(\mathrm{R}^{1}=\mathrm{H}, \mathrm{Me}, \mathrm{Cl} ; \mathrm{R}^{2,4,5}=\mathrm{H}, \mathrm{Me} ; \mathrm{R}^{3}=\mathrm{H}, \mathrm{Me}, \mathrm{tBu}, \mathrm{F}, \mathrm{Cl}, \mathrm{Br}\right)$ und Acetophenone $\left(\mathrm{R}^{1,2}=\mathrm{H}, \mathrm{F}\right)$.

däre Wechselwirkungen wie Dispersion potentiell eine größere Rolle. Aus diesem Grund wird mit dem auf Acetophenon ${ }^{[31,51,52]}$ (s. Abb. 1.1) als Akzeptor basierenden Carbonylwaagensystem auch eine solche Klasse intermolekularer Waagensysteme betrachtet. Am Beispiel von Dispersionswechselwirkungen soll der Einfluss sekundärer Wechselwirkungen auf die Bindungsstellenpräferenz für das Carbonyl- und das Anisol-Waagensystem auf Basis des Local-Energy-Decomposition-Konzepts ${ }^{[20]}$ analysiert werden.

Für die Ermittlung der experimentellen Daten werden die verschiedenen, in einer Überschallexpansion erzeugten, wasserstoffbrückengebundenen Dimere an der sog. FiletJet-Apparatur ${ }^{[53-55]}$ mit FouRIER-Transformations-Infrarot-(FTIR-)Spektroskopie ${ }^{\text {[55-57] }}$ schwingungsspektroskopisch untersucht. Bei Ausbildung einer Wasserstoffbrückenbindung verschieben sich Schwingungsbanden der beteiligten Moleküle ausgehend von den Bandenpositionen der freien Monomere entlang der Wellenzahlachse, sodass sich die Aggregatbildung im IR-Spektrum nachweisen lässt. Im Rahmen der vorliegenden Arbeit wird dabei die $\mathrm{OH}$-Streckschwingungsbande betrachtet, die abhängig von der Stärke dieser Wasserstoffbrückenbindung signifikant (hier bis zu $160 \mathrm{~cm}^{-1}$ in Richtung niedrigerer Wellenzahlen) verschoben werden kann. ${ }^{[58,59]}$ Bei ausreichend unterschiedlichen Verschiebungen ermöglicht dies eine schwingungsspektroskopische Unterscheidung verschiedener Konformere eines Aggregats. Sollen so gewonnene experimentelle Daten als Referenz zum Benchmarking quantenchemischer Methoden herangezogen werden, ergeben sich allerdings einige Schwierigkeiten, ${ }^{[7]}$ z. B. durch Nullpunktsenergieeffekte. ${ }^{[60-62]}$ Auch die Auswahl geeigneter Größen für den Benchmark ${ }^{[35,60,63,64]}$ ist nicht trivial. ${ }^{[7]}$ So sind IR-spektroskopische Parameter wie die Stärke der Rotverschiebung der $\mathrm{OH}$-Streckschwingungsbanden verschiedener Dimer-Konformere oder deren Aufspaltung experimentell direkt aus den Schwingungsspektren zugänglich. Um dieselben Größen in quantenchemischen Rechnungen zu erhalten, müssen neben elektronischen Energien jedoch auch der Gradient des elektronischen Potentials (Optimierung zur Struktur niedrigster Energie) und die Hesse-Matrix (Berechnung der Schwingungsnull- 
punktsenergie und der Schwingungsfrequenzen der Normalmoden) berechnet werden, was angesichts der Rechenanforderungen nicht für alle quantenchemischen Methoden möglich ist. Zudem erfolgen die Schwingungsrechnungen bei der grundlegendsten Betrachtung zunächst nur in der doppelt-harmonischen Näherung, wohingegen die Berücksichtigung anharmonischer Korrekturen mit einem deutlich erhöhten Rechenaufwand einhergeht. Hier kann das Experiment der Theorie jedoch durch geschickte Systemwahl entgegenkommen. Die im Experiment naturgemäß vorhandenen anharmonischen Beiträge kompensieren sich zwischen verschiedenen Konformeren desselben wasserstoffbrückengebundenen Systems oftmals, wenn relative Größen wie Rotverschiebungen oder Bandenaufspaltungen herangezogen werden. ${ }^{[65-67]}$ So wird ein Vergleich mit harmonischen Rechendaten möglich. ${ }^{[3]}$ Aufseiten der Theorie leichter zugänglich sind die relativen Energien verschiedener Konformere. Aus diesem Grund liegt in der vorliegenden Arbeit ein besonderer Fokus auf der Bestimmung relativer Stabilitäten der beobachteten Konformere der gleichen Dimere. Informationen hierzu lassen sich aus den Schwingungsspektren auf Basis der relativen Intensitäten konformerspezifischer Banden gewinnen. Wie in Kapitel 2.3.2 erläutert wird, ist eine exakte Bestimmung des experimentellen Energieunterschieds aus diesen Daten allein jedoch nicht möglich.

Die im Rahmen dieser Arbeit für die Benchmarks beispielhaft herangezogenen quantenchemischen Methoden werden in Kap. 2.1 vorgestellt, der verwendete experimentelle Aufbau für die schwingungsspektroskopischen Untersuchungen (Filet-Jet) in Kap. 2.2. Das Vorgehen für die Benchmarks und die Ermittlung experimenteller Energieunterschiede aus den Jet-FTIR-spektroskopischen Daten wird im Anschluss in Kapitel 2.3 erläutert. Potentielle Verbesserungen gegenüber der hierfür genutzten Filet-Jet-Apparatur für die zukünftige Gewinnung experimenteller Referenzdaten bietet die im Rahmen dieser Arbeit geplante, aufgebaute und in Betrieb genommene Gratin-Jet-Apparatur, die in Kapitel 3 vorgestellt und ersten Leistungsüberprüfungen unterzogen wird. Die Regioselektivität bei der Ausbildung von Wasserstoffbrückenbindungen zu Akzeptoren mit O- und C-basierten Andockstellen wird anschließend in Kapitel 4 beleuchtet. In einer (Doppelblind-)Studie auf Basis des Furan-Mikrosolvatations-O/C-Waagensystems werden experimentelle und quantenchemische Daten (zumindest in einem ersten Schritt) ohne gegenseitige Kenntnis ermittelt. Anhand dieser Studie soll in Kapitel 4.1 gezeigt werden, dass sich mittels Jet-FTIR-Spektroskopie gewonnene Daten zu relativen Energien und IRspektroskopischen Parametern für das Benchmarking quantenchemischer Rechnungen eignen. Für die weiteren im Rahmen dieser Arbeit betrachteten Waagensysteme werden hingegen nur beispielhafte Benchmarks einiger populärer Rechenmethoden durchgeführt, um die Eignung der gewonnen experimentellen Daten als Benchmark-Referenz zu überprüfen. Mit dem Anisol-O/C-Waagensystem wird dafür in Kapitel 4.2 ein deutlich umfangreicherer Datensatz betrachtet, der gegenüber einer früheren Studie ${ }^{[37]}$ erweitert und neu ausgewertet wird und der sowohl Fälle eindeutiger $\mathrm{O}$ - als auch eindeutiger $\mathrm{C}$ Bindungsstellenpräferenz umfasst. Den Abschluss bildet in Kapitel 5 die Untersuchung des auf verschiedenen Konformeren gleichartig $\mathrm{OH} \cdots \mathrm{O}$-gebundener Methanol-Acetophenon(derivat)-Dimere beruhenden Carbonylwaagensystems. 


\section{Kapitel 2}

\section{Theoretische und experimentelle Methoden}

Quantenchemische Rechenmethoden wie die Dichtefunktionaltheorie (DFT, Kap. 2.1.1) oder Coupled-Cluster-Ansätze (Kap. 2.1.2) liefern zunächst Informationen zur elektronischen Struktur der vom Benutzer vorgegebenen Moleküle bzw. Aggregate im Vakuum bei 0 K. Sollen Wechselwirkungen mit benachbarten Molekülen, z. B. eines Lösungsmittels, in die Betrachtung aufgenommen werden, müssen diese explizit (durch Hinzufügen der Lösungsmittelmoleküle zur betrachteten Struktur) ${ }^{[68]}$ oder implizit (durch Betrachtung des Lösungsmittels als Kontinuum mit einer stoffspezifischen Permittivität) ${ }^{[69-72]}$ einbezogen werden. Hierdurch steigt der Rechenaufwand stark an (explizite Berücksichtigung) oder es werden zusätzliche Näherungen nötig (implizite Berücksichtigung). Ähnliches gilt für die Betrachtung von thermodynamischen Effekten wie Enthalpie- oder Entropiebeiträgen von z. B. Rotations- oder Schwingungsfreiheitsgraden, die bei größer werdenden Temperaturen eine immer wichtigere Rolle einnehmen. ${ }^{[73,74]}$

Je mehr Effekte in einer quantenmechanischen Rechnung berücksichtigt werden müssen und je mehr Näherungen dazu notwendig sind, desto schwieriger wird es, in einem Benchmark die Leistungsfähigkeit der zugrundeliegenden Methode bezogen auf die Berechnung der grundlegendsten Größe - der elektronischen Energie - zu beurteilen. Besonders anspruchsvolle Methoden wie $\operatorname{CCSD}(\mathrm{T})$ sind für viele Moleküle und Aggregate angesichts der zur Verfügung stehenden Rechenressourcen nur im Rahmen von Einzelpunktrechnungen zugänglich. Diese Methoden können also nur zur Berechnung elektronischer Eigenschaften einer vorgegebenen Geometrie, z. B. der elektronischen Energie oder des Dipolmoments, verwendet werden. Die wichtigsten in dieser Arbeit verwendeten quantenchemischen Methoden werden in Kapitel 2.1 kurz erläutert.

Sollen experimentelle Daten für den Benchmark einer quantenchemischen Rechenmethode genutzt werden, ${ }^{[7]}$ ist es essentiell, sich den inhärenten Bedingungen der quantenchemischen Rechnung so weit wie möglich anzunähern. Es sollten also möglichst isolierte Moleküle oder Aggregate möglichst nah am Temperaturnullpunkt untersucht werden. Solchen Bedingungen kann im Experiment, wie in Kapitel 1 bereits erwähnt, z. B. in Überschallexpansionen (oft als „Jet“, der Kurzform des englischen Begriffs „supersonic 
jet expansion“, bezeichnet) nahegekommen werden, ${ }^{[38-45]}$ in denen eine Kühlung der $\mathrm{zu}$ untersuchenden Moleküle und eine Stabilisierung schwach gebundener Aggregate erreicht wird. Bei Verwendung eines großen Überschusses eines geeigneten Stoßgases wird außerdem die Isolierung einzelner Aggregate voneinander gewährleistet, sofern das Stoßgas nicht selbst mit den Analyten aggregiert. Die Kombination einer solchen Überschallexpansion mit FouRIER-transformierter Infrarot-(FTIR-)Spektroskopie ${ }^{[55-57]}$ (JetFTIR-Spektroskopie) erlaubt die Untersuchung dieser Aggregate beispielsweise hinsichtlich ihrer relativen Konzentrationen und damit das Benchmarking quantenchemischer Methoden in Bezug auf den Energieunterschied. [31,37,50,75]

In der SuHM-Forschungsgruppe existieren mehrere Apparaturen, an denen Jet-FTIRExperimente durchgeführt werden können. Die in dieser Arbeit verwendeten Spektren wurden überwiegend am sogenannten Filet-Jet ${ }^{[53-55]}$ aufgenommen. Das Akronym Filet (vom englischen "fine, but lengthy") beschreibt dabei einerseits die Geometrie der langen $(600 \mathrm{~mm})$, aber schmalen $(0.2 \mathrm{~mm})$ Schlitzdüse, bezieht sich andererseits aber auch auf die Form der Expansionszone, die einem Filetstrang ähnelt. ${ }^{[76]}$ Der Filet-Jet wird in Kapitel 2.2 im Detail vorgestellt.

Ziel der im Rahmen dieser Arbeit durchgeführten Jet-FTIR-Experimente ist es, anhand der gewonnen experimentellen Referenzdaten die Leistungsfähigkeit quantenchemischer Methoden zu überprüfen, also Benchmarks dieser Methoden durchzuführen. Dazu müssen die aus dem Experiment gewonnenen Informationen, etwa über die Aufspaltung oder das Intensitätsverhältnis zweier charakteristischer Dimersignale in der Überschallexpansion, mit den berechneten Daten, beispielsweise zur Bandenaufspaltung oder zum Energieunterschied, verknüpft werden. Die hierzu verwendeten Methoden werden in Kapitel 2.3 erläutert.

\subsection{Theoretische Methoden}

Für die Berechnung verschiedener Moleküleigenschaften, wie Geometrie oder Schwingungsfrequenzen, steht eine ganze Bandbreite von Methoden zur Verfügung. Diese lassen sich in zahlreiche Kategorien unterteilen, z. B. Quantenmechanik gegenüber klassischer Mechanik (klassisches Kraftfeld) oder empirisch gegenüber $a b$ initio. Für die Rechnungen im Rahmen dieser Arbeit werden ausschließlich quantenmechanische Methoden verwendet. Dabei wird für die Geometrieoptimierung und harmonische Schwingungsrechnung der betrachteten Monomere und Dimere auf verschiedene Methoden aus dem Bereich der Dichtefunktionaltheorie (DFT) zurückgegriffen. Für einzelne Strukturen werden zudem Einzelpunktrechnungen mit einer wellenfunktionsbasierten Coupled-Cluster-Methode durchgeführt. Die verwendeten DFT- (Kap. 2.1.1), die im Rahmen dieser Arbeit eingesetzte Coupled-Cluster-Methode, DLPNO-CCSD(T) (Kap. 2.1.2), und die Einstellungen, die für die Rechnungen mit diesen Methoden verwendet werden, sollen im Folgenden kurz dargestellt werden. 


\subsubsection{Dichtefunktionale}

Die Dichtefunktionaltheorie basiert auf der Erkenntnis, dass die elektronischen Eigenschaften des Grundzustands eines Moleküls eindeutig aus seiner Elektronendichteverteilung $\rho(\boldsymbol{r})$ ableitbar sind. ${ }^{[7]}$ Die elektronische Energie ist über das Funktional $E[\rho]$ mit der Elektronendichte verknüpft und lässt sich somit bei Kenntnis des Funktionals und der Elektronendichte berechnen. Dabei wird bei der Betrachtung von Molekülen in der Regel der auf KoнN und SнAм ${ }^{[78]}$ zurückgehende Orbitalansatz verwendet. Bei einer ausreichend guten Modellierung des Dichtefunktionals lassen sich damit oft gute Ergebnisse bei vergleichsweise geringem Rechenaufwand erzielen, insbesondere in Geometrieoptimierungen. ${ }^{[75,79,80]}$

Ein Problem der klassischen KoHN-SHAM-DFT ist, dass Dispersionswechselwirkungen in der Regel nicht korrekt erfasst werden können. ${ }^{[81]}$ Durch hohe Parametrisierung (z. B. beim M06-2X-Funktional ${ }^{[82]}$ ) oder eine empirische Dispersionskorrektur, die dem Funktional hinzugefügt wird (z. B. die äußerst erfolgreiche D3-Korrektur ${ }^{[83]}$ ), können Dispersionswechselwirkungen jedoch ohne großen rechnerischen Mehraufwand berücksichtigt werden. Insbesondere letzterer Ansatz führt oftmals zu akkuraten Strukturen, auch in schwach gebundenen Situationen wie wasserstoffverbrückten Dimeren, ${ }^{[84]}$ die auch im Rahmen der vorliegenden Arbeit betrachtet werden.

Als zuverlässiges Funktional für die Untersuchung von intermolekularen Waagensystemen, die aus wasserstoffverbrückten Dimeren bestehen, hat sich in der Vergangenheit das B3LYP-Hybridfunktional, ${ }^{[85,86]}$ das neben HARTREE-Fock-Austauschenergie auch Beiträge vom B88-Austausch-[87] und LYP-Korrelationsfunktional ${ }^{[88]}$ aufweist, in Verbindung mit der D3-Dispersionskorrektur bewährt ${ }^{[37,50,89]}$ und soll deshalb auch hier wieder eingesetzt werden. In diesem und allen weiteren Fällen, in denen die D3-Korrektur genutzt wird, wird auf das BECKE-Johnson-Damping (BJ) ${ }^{[90-93]}$ zurückgegriffen. Weiterhin wird stets der Dreikörperterm $(\mathrm{ABC})^{[83]}$ der Dispersionskorrektur mit berücksichtigt.

In einzelnen Benchmarks wird darüber hinaus das M06-2X-Funktional untersucht. M06-2X konnte bereits erfolgreich dafür genutzt werden, nicht-kovalente Wechselwirkungen zwischen Basenpaaren in Biomolekülen ${ }^{[94]} \mathrm{zu}$ beschreiben oder das UV/IR-JetSpektrum von Propofol-Isopropanol-Clustern aufzuklären. ${ }^{[95]}$ In einer in der SuHMGruppe durchgeführten Jet-FTIR-Benchmarkstudie eines intermolekularen $\mathrm{O} / \pi$-Waagensystems zeigte das Funktional dagegen beispielsweise eine systematische Überschätzung der relativen Stärke der $\mathrm{OH} \cdots \pi$-Wechselwirkung. ${ }^{[37]}$ Mit neuen M06-2X-Rechnungen und der Betrachtung weiterer Waagensysteme soll der Datensatz in der vorliegenden Arbeit erweitert werden, um die Leistungsfähigkeit des hoch parametrisierten Funktionals detaillierter beurteilen zu können.

Vor allem Hybridfunktionale, die die Elektronen-Austauschenergie zum Teil über das HARTREE-FocK-Verfahren berechnen, weisen Rechenanforderungen auf, die bei Nutzung

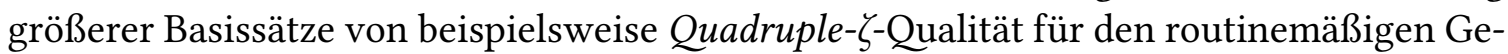
brauch zu hoch liegen können. Bei Verwendung kleiner Basissätze sinken zwar die Rechenanforderungen drastisch, die erzielbare Genauigkeit nimmt jedoch in der Regel eben- 
falls ab. Zur Lösung dieses Problems wurden von GrImme und Mitarbeitern mehrere Kompositmethoden vorgeschlagen, die HARTrEe-Fock- (HF-3c ${ }^{[96]}$, bzw. Hybrid-DFT$\left(\right.$ PBEh-3c ${ }^{[97]}$ ) oder HARTREE-FocK-freie DFT-Rechnungen (B97-3c ${ }^{[98]}$ ) mit der D3-Dispersionskorrektur und kleinen Basissätzen kombinieren. Die 3c-Methodenfamilie wurde dabei mit Korrekturen für unvollständige Basissätze (geometrische Counterpoise- $(\mathrm{gCP})^{[99]}$ und/oder kurzreichweitige Basissatzkorrekturen („short-ranged basis“ SRB) ${ }^{[96]}$ ) versehen und so parametrisiert, dass sich Basissatz- und Methodenfehler möglichst kompensieren. In dieser Arbeit werden die beiden DFT-Kompositmethoden B97-3c und PBEh-3c im Rahmen der betrachteten Carbonylwaagensysteme untersucht.

Sollen nicht die Rechenanforderungen durch kleine Basissätze gesenkt, sondern soll die Genauigkeit einer DFT-Rechnung durch ein höheres Theorieniveau erhöht werden, können sogenannte Doppel-Hybrid-Funktionale ${ }^{[68,100]}$ verwendet werden. Bei diesen wird nicht nur wellenfunktionsbasierte (HARTREE-Fock-)Austauschenergie, sondern zusätzlich auch wellenfunktionsbasierte Korrelationsenergie, z. B. aus der MøLLER-Plesset-Störungstheorie 2. Ordnung (MP2), ${ }^{[101]}$ berücksichtigt. Mit dem von GRIMME entwickelten B2PLYP-Funktional ${ }^{[102]}$ wird eine solche Methode auch im Rahmen dieser Arbeit verwendet. Zur Berücksichtigung von Dispersionswechselwirkungen wird auch dieses Funktional mit der D3-Korrektur inklusive BECKE-JoHnson-Damping und Dreikörperterm kombiniert (im Folgenden als B2PLYP-D3(BJ,ABC) bezeichnet).

In der vorliegenden Arbeit wird im Allgemeinen auf Basissätze von Triple- oder Quadruple-ร-Qualität zurückgegriffen. Für die Hybrid-DFT-Methoden B3LYP-D3(BJ, $\mathrm{ABC}$ ) und M06-2X werden dabei die AhLrichs-Basissätze def2-nZVP ${ }^{[103]}$ (mit $n=\mathrm{T}, \mathrm{Q}$ ) verwendet. Das Doppel-Hybrid-Funktional B2PLYP-D3(BJ,ABC) wird aufgrund des MP2Anteils mit den korrelations-konsistenten DunNing-Basissätzen cc-pVTZ ${ }^{[104]}$ für $\mathrm{H}$ bzw. aug-cc-pVTZ ${ }^{[105]}$ (aVTZ) für C, O und F genutzt. Die Kombination der Basissätze cc-pVTZ an H- und aug-cc-pVTZ an allen weiteren Atomen wird im Folgenden mit a'VTZ abgekürzt. Da für die B2PLYP-Rechnungen die sogenannte „resolution of the identity“(RI-)Näherung ${ }^{[68]}$ eingesetzt wird, ist hier außerdem die Verwendung der Hilfsbasissätze cc-pVTZ/C bzw. aug-cc-pVTZ/C ${ }^{[106]}(\mathrm{aVTZ/C})$ notwendig. Für die 3c-Kompositmethoden ist keine manuelle Basissatzauswahl nötig, da diese Methoden jeweils einen Basissatz beinhalten.

Die Rechnungen der meisten Methoden (B2PLYP-D3(BJ,ABC), B3LYP-D3(BJ,ABC), PBEh-3c, B97-3c) werden mit dem OrCA-Programmpaket ${ }^{[107-109]}$ in den Versionen 4.0.1 und 4.1.2 durchgeführt. Dabei werden die Konvergenzkriterien von SCF-Energie und Geometrieoptimierung gegenüber den Standardeinstellungen verschärft (VERYTIGHTSCF TIGHTOPT) und für die Nicht-Kompositmethoden (B2PLYP-D3(BJ,ABC), B3LYP-D3(BJ, $A B C)$ ) wird zusätzlich ein feineres Integrationsgitter verwendet (GRID5 NOFINALGRID). Wenn möglich (B3LYP-D3(BJ,ABC), PBEh-3c, B97-3c) wird die Frequenzrechnung mit einer analytischen Hesse-Matrix ${ }^{[110]}$ durchgeführt. Lediglich für B2PLYP-D3(BJ,ABC) muss auf eine numerische Rechnung zurückgegriffen werden.

Für die Berechnungen auf M06-2X-Niveau wird die Gaussian16-Software in der Revision A.03 ${ }^{[111]}$ eingesetzt, da in ORCA keine analytischen Frequenzrechnungen auf diesem 
Niveau durchgeführt werden können. Aufgrund der ausgeprägten Abhängigkeit des M062X-Funktionals von der Größe des Integrationsgitters ${ }^{[34,112,113]}$ wird dabei ein sehr feines Gitter genutzt (grid=SuperFineGrid). Auch in Gaussian16 werden die Konvergenzkriterien für SCF-Energie und Geometrieoptimierung verschärft (opt=tight scf=verytight).

\subsubsection{DLPNO-CCSD(T)}

$\operatorname{Das} \mathrm{CCSD}(\mathrm{T})$-Verfahren ${ }^{[26]}$ („,coupled clusters with singles, doubles and perturbative triples“, Coupled-Cluster-Verfahren mit Einfach-, Zweifach- und störungstheoretischen Dreifachanregungen) wird gern als „Goldstandard“ der Quantenchemie bezeichnet. ${ }^{[24,25,114]}$ Mit einer Skalierung $\mathcal{O}\left(N^{7}\right)$ zur siebten Potenz der Zahl der Basisfunktionen $N$ ist die $\operatorname{CCSD}(\mathrm{T})$-Methode etwas weniger rechenintensiv als CCSDT mit voller iterativer Berechnung der Dreifachanregungen, das mit $\mathcal{O}\left(N^{8}\right)$ skaliert. ${ }^{[68]}$ Dennoch wird aufgrund von Fehlerkompensation oftmals eine vergleichbare Genauigkeit erreicht. ${ }^{\text {115-117] }}$

Die Coupled-Cluster-(CC-)Methoden nutzen einen auf den Arbeiten von Coester und KüMMEL ${ }^{[118,119]}$ basierenden exponentiellen Ansatz, um die Elektronenkorrelation im Mehrelektronensystem zu beschreiben:

$$
\Psi_{\mathrm{CC}}=e^{T} \Phi_{0}
$$

$\Psi_{\mathrm{CC}}$ bezeichnet darin die CC-Wellenfunktion, $T$ einen Anregungsoperator und $\Phi_{0}$ die Referenzwellenfunktion, z. B. aus einer Hartree-Fock-Rechnung. Der Exponentialterm $e^{T}$ kann über eine Taylorreihe folgendermaßen ausgedrückt werden:

$$
e^{T}=\mathbf{1}+\boldsymbol{T}+\frac{1}{2} T^{2}+\cdots=\sum_{k=0}^{\infty} \frac{1}{k !} T^{k}
$$

Die Summierung bis $\infty$ verdeutlicht, dass die Ordnung der berücksichtigten Korrekturterme in der CC-Methodik im Gegensatz zum störungstheoretischen Ansatz nicht begrenzt ist. Während in der Störungstheorie allerdings alle Arten von Anregungen (Einfach-, Zweifach-, Dreifach-, usw. -anregungen) bis zur betrachteten Ordnung einbezogen werden, werden in der CC-Theorie nur bestimmte Anregungen berücksichtigt. Dies soll im Folgenden erläutert werden.

Der Anregungsoperator $T$ ist eine Summe aus Operatoren $T_{i}$, die jeweils alle $i$-fach angeregten Slater-Determinanten erzeugen:

$$
T=T_{1}+T_{2}+\cdots+T_{N_{\text {elec }}}
$$

Werden alle Anregungen einbezogen, ergibt sich das vollständige CC (Full CC), das äquivalent zur vollständigen „configuration interaction“ (Full CI) ist. Aufgrund der immensen Rechenanforderungen wird für alle bis auf die kleinsten Systeme jedoch nur eine begrenzte Zahl von Anregungsarten betrachtet. In der CCSD-Methode ${ }^{[120]}$ werden z. B. nur die 
Einfach- („Singles“) und Zweifach-Anregungen („Doubles“) berücksichtigt. Wenn die Terme gleicher Ordnung jeweils in Klammern zusammengefasst werden, ergibt sich damit:

$$
e^{T}=\mathbf{1}+T_{1}+\left(T_{2}+\frac{1}{2} T_{1}^{2}\right)+\left(T_{2} T_{1}+\frac{1}{6} T_{1}^{3}\right)+\left(\frac{1}{2} T_{2}^{2}+\frac{1}{2} T_{2} T_{1}^{2}+\frac{1}{24} T_{1}^{4}\right)+\cdots
$$

Hier kann in verknüpfte (gleichzeitig interagierende Elektronen, hier $T_{2}$ ) und nichtverknüpfte Mehrfachanregungen (mehrere nicht-interagierende Gruppen interagierender Elektronen, z.B. $T_{2}^{2}$ ) unterschieden werden. ${ }^{[68]}$ Durch die Berücksichtigung der nicht-verknüpften Terme sind die CC- im Gegensatz zu den CI-Methoden, die auch zur Berechnung der Elektronenkorrelation eingesetzt werden können, größenextensiv. Dies kann an einem einfachen Beispiel, dem Dimer aus zwei $\mathrm{H}_{2}$-Molekülen mit minimalem Basissatz, anhand des Vergleichs zwischen CID („CI with doubles“) und CCD („CC with doubles") verdeutlicht werden. In zwei voneinander isolierten $\mathrm{H}_{2}$-Molekülen erlauben beide Methoden jeweils die Zweifachanregung beider Elektronen in das antibindende Orbital. Im interagierenden Dimer aus zwei Molekülen entspricht diese Anregung nun aber einer Anregung von vier Elektronen und ist deshalb im Rahmen der CID nicht mehr möglich. Bei CCD bleibt die Anregung durch den Term $T_{2}^{2}$ hingegen erlaubt. CCD ist damit größenextensiv, CID nicht. Gleiches gilt für die weiteren Varianten (CISD, CISDT, CCSD, $\operatorname{CCSD}(\mathrm{T})$ usw.) dieser Methoden. ${ }^{[121]}$

Für die beliebte $\operatorname{CCSD}(\mathrm{T})-$ Methode wird „coupled clusters with singles and doubles" (CCSD) mit Dreifachanregungen kombiniert, die störungstheoretisch berechnet werden. Dazu wird auf die Formeln der Møller-Plesset-Störungstheorie vierter und fünfter Ordnung zurückgegriffen. ${ }^{[26]}$ Für die im Rahmen dieser Arbeit betrachteten Systeme wird jedoch die oben beschriebene Skalierung der Rechenanforderungen des kanonischen $\operatorname{CCSD}(\mathrm{T})$ von $\mathcal{O}\left(N^{7}\right)$ zum Problem, da solche Rechnungen mit den zur Verfügung stehenden Ressourcen nicht durchführbar sind.

Ein Ansatz zur Lösung dieses grundlegenden Problems basiert auf der Annahme, dass die zu erfassende dynamische Elektronenkorrelation prinzipbedingt lokal ist. Je weiter zwei Elektronen voneinander entfernt sind, desto weniger wechselwirken sie miteinander. Der für die Coupled-Cluster-Methodik nötige Rechenaufwand kann also verringert werden, wenn die aus der Referenz-Rechnung (z. B. HARTREE-FocK) erhaltenen kanonischen Molekülorbitale lokalisiert werden. Hierdurch muss nur die Wechselwirkung räumlich naher Elektronen betrachtet werden.

Die DLPNO-CCSD(T)-Methode ${ }^{[122-125]}$ stellt einen solchen lokalen CCSD(T)-Ansatz dar. In der Göbench-Leistungsüberprüfung (s. Kap. 4.1) wurde DLPNO-CCSD(T) in mehreren Beiträgen zur Berechnung der elektronischen Energie eingesetzt und konnte im Vergleich mit den experimentellen Referenzdaten gute Ergebnisse erzielen, ${ }^{[75,80]}$ weswegen es für die im Rahmen dieser Arbeit durchgeführten Einzelpunktrechnungen in der 2016 vorgestellten Implementierung ${ }^{[125]}$ verwendet werden soll.

Die Abkürzung „DLPNO“ steht für „domain based local pair natural orbitals“ und beschreibt das Lokalisierungskonzept der Methode. PNOs ${ }^{[126,127]}$ werden mit lokalen Korrelationsdomänen ${ }^{[128-131]}$ kombiniert, ${ }^{[108]}$ um eine lineare Skalierung der DLPNO- 
$\operatorname{CCSD}(\mathrm{T})$-Methode mit der Systemgröße zu erreichen. ${ }^{[125]}$ Dazu werden zunächst die besetzten Molekülorbitale lokalisiert, wofür standardmäßig das FosTER-Boys- ${ }^{[132]}$ oder alternativ das PiPEK-MEzey-Verfahren ${ }^{[133]}$ verwendet werden kann. Für jedes lokalisierte Elektronenpaar wird anschließend eine Korrelationsdomäne aus virtuellen Orbitalen gebildet. Die Größe des dazu verwendeten virtuellen Raums kann durch den PNO-Ansatz relativ klein gehalten werden, sodass die Rechenanforderungen moderat bleiben.

DLPNO-CCSD(T) wird von den Autoren als „Black-box“-Methode beschrieben, ${ }^{[125]}$ also als Methode, die ohne tiefergehende Kenntnisse der dahinterliegenden Theorie vom Anwender einfach genutzt werden kann. Das ORCA-Programmpaket stellt dabei drei Schlüsselwörter zur Verfügung, mit denen diverse Grenzwerte und Näherungsverfahren auf von den Autoren gewählte Voreinstellungen angepasst werden können. In der Reihenfolge LoosePNO, NormalPNO (Standard), TightPNO steigt dabei die Genauigkeit, aber auch die Rechenanforderungen nehmen zu. Für die Betrachtung schwach gebundener Systeme empfehlen die Autoren die Verwendung der strengsten Grenzwerte (TightPNO), ${ }^{[134]}$ die deshalb auch für die hier durchgeführten Rechnungen eingesetzt werden sollen. Die Auswirkungen der Verwendung der DLPNO-Näherung bezogen auf das kanonische $\operatorname{CCSD}(\mathrm{T})$ werden in den Referenzen [124] und [135] ausführlich diskutiert. Ein Vergleich der Ergebnisse von DLPNO- mit kanonischem CCSD(T) für den GMTKN55-Datensatz ${ }^{[136-138]}$ findet sich in Referenz [139]. Die Konvergenzkriterien für das SCF-Verfahren werden für alle DLPNO-CCSD(T)-Rechnungen mit dem Schlüsselwort TIGHTSCF gegenüber dem Standard etwas verschärft.

Auch für die Art der für die Korrelationsrechnung zu berücksichtigenden Elektronen gibt es vordefinierte Einstellungen. Bei den Standardeinstellungen werden nur die Valenzelektronen korreliert, während die kernnahen Elektronen „eingefroren“ werden. Über das NoFrozenCore-Schlüsselwort können die Kernelektronen jedoch in der Korrelationsrechnung mit berücksichtigt werden. Mit letzterer Einstellung kann die DLPNO-CCSD(T)-Methode in der zweiten Runde der Göbench-Leistungsüberprüfung besonders gut abschneiden (s. Kap. 4.1), weswegen diese Einstellung für einige der in Kapitel 5 dieser Arbeit durchgeführten Rechnungen testweise ebenfalls angewandt wird. Hierzu ist die Verwendung spezieller Basissätze nötig, die für die Berücksichtigung der Kernelektronenkorrelation ausgelegt sind. Im Falle der in Kapitel 5 durchgeführten Rechnungen kommen der DunNING-Basissatz aug-cc-pwCVQZ ${ }^{[105,140]}$ (kurz: awCVQZ) und der zugehörige Hilfsbasissatz für die RI-Näherung, aug-cc-pwCVQZ/C, ${ }^{[106,141]}$ zum Einsatz. Für die H-Atome, bei denen es nur Valenz- aber keine Kernelektronen gibt, werden stattdessen die entsprechenden „herkömmlichen“ DunNING-Basissätze aug-cc-pVQZ ${ }^{[105,142,143]}$ und aug-ccpVQZ/C ${ }^{[106]}$ verwendet. Letztere Basissätze werden bei DLPNO-CCSD(T)-Rechnungen mit eingefrorenen Kernelektronen für alle Atome genutzt. Die unterschiedliche Basissatzverwendung zwischen $\mathrm{H}$-Atomen und schwereren Atomsorten $(\mathrm{C}, \mathrm{O}, \mathrm{F})$ bei Verwendung der awCVQZ-Basis und die Verwendung der Hilfsbasissätze für die RI-Näherung werden im Folgenden nicht mehr gesondert gekennzeichnet. Rechnungen unter Berücksichtigung der Kernelektronenkorrelation werden also nur mit DLPNO-CCSD(T)/awCVQZ bezeichnet, Rechnungen mit eingefrorenen Kernelektronen mit DLPNO-CCSD(T)/aVQZ. 
Ebenso wie die Art der für die Korrelation zu berücksichtigenden Elektronen wird auch die für die DLPNO-Methodik genutzte Lokalisierungsmethode für einige Rechnungen testweise geändert. Dazu wird das standardmäßige Foster-Boys- durch das PipeKMEzEY-Verfahren ersetzt (\%MDCI LocMet PM end, s. Kap. 5). Alle DLPNO-CCSD(T)-Rechnungen werden mit ORCA in den Versionen 4.0.1 (DLPNO-CCSD(T)/awCVQZ) bzw. 4.1.2 (DLPNO-CCSD(T)/awCVQZ, DLPNO-CCSD(T)/aVQZ) durchgeführt.

Im Rahmen des DLPNO-CCSD(T)-Konzepts steht mit der "local energy decomposition“ (LED, lokale Energiedekomposition) ${ }^{[20]}$ ein Werkzeug zur Verfügung, mit dem die Wechselwirkung zwischen zwei Fragmenten in verschiedene Komponenten (Elektrostatik, Austausch, Dispersion) zerlegt werden kann. ${ }^{[22,23]}$ Die von dieser oder anderen Methoden ${ }^{[16-19,21]}$ bestimmten Werte stellen keine Observablen dar und können somit nicht experimentell verifiziert oder widerlegt werden. Dennoch wird erwartet, dass die LED-Methode zumindest Hinweise darauf liefern kann, welche Komponenten für die Gesamtwechselwirkungsenergie bedeutsam sind. Dies gilt insbesondere, wenn relative Effekte zwischen zwei ähnlichen Konformeren eines Waagensystems (s. Kap. 4 und 5) miteinander verglichen werden. Die LED-Analysen werden im Zusammenhang mit den DLPNO-CCSD(T)-Rechnungen mit ORCA durchgeführt. Dazu werden Standardeinstellungen verwendet (Schlüsselwort LED). Lediglich die maximale Zahl der für die LEDRechnung durchzuführenden Iterationen wird gegenüber dem Standard erhöht (\%mdci LocMaxIterLed 999 end). Die Dispersionsbeiträge $E^{\text {disp }}$ zur Gesamtwechselwirkungsenergie, die im Rahmen dieser Arbeit betrachtet werden sollen, werden dann durch Addition der in der LED-Analyse erhaltenen Werte für „Dispersion (strong pairs) “ und „Dispersion (weak pairs)“ berechnet.

\subsection{Filet-Jet-FTIR-Spektroskopie}

Der Filet-Jet ist seit seiner Inbetriebnahme $2004^{[53,76]}$ eine der erfolgreichsten Apparaturen der SuHM-Forschungsgruppe. $65^{[31-33,37,45,49,50,53-55,75,144-197]}$ der von 2005 bis einschließlich 2019 insgesamt 111 [7,31-34,37,44,45,49,50,53-55,67,75,144-239] veröffentlichten Forschungspublikationen unter Beteiligung von Martin Suhm (alle in Ref. [240] aufgelisteten „Suhm-Publikationen“ der Jahre 2005-2019, unter Nichtberücksichtigung der zwei Kommentare [241] und [242]) beinhalten Daten, die am Filet-Jet aufgenommen wurden. Dies entspricht einem Gesamtanteil von 58.6\%, wobei in jüngerer Zeit höhere Anteile vorlagen $(80 \%$ in $2016,67 \%$ in $2017,100 \%$ in 2018 und $63 \%$ in 2019).

Ursprünglich im Rahmen ihrer Promotion von Nicole BorHo geplant und in Betrieb genommen, ${ }^{[76]}$ hat der Filet-Jet seitdem einige Änderungen und Erweiterungen erfahren. ${ }^{[55]}$ So wurde z. B. der Messbereich ins ferne (FIR) ${ }^{[165]}$ und nahe Infrarot (NIR) ${ }^{[156]}$ erweitert und es wurde ein leistungsfähigeres Spektrometer (BRUKER IFS 66v/S) installiert. Unter Matthias Heger wurde das Signal-zu-Rausch-Verhältnis durch den Einbau einer potenteren Lichtquelle (150 W Wolframlampe), Optimierungen bei der Datenaufnahme (Einführung eines $5 \mathrm{~s}$ dauernden „pre-scan delays“ zur Stabilisierung der Spiegelbewe- 


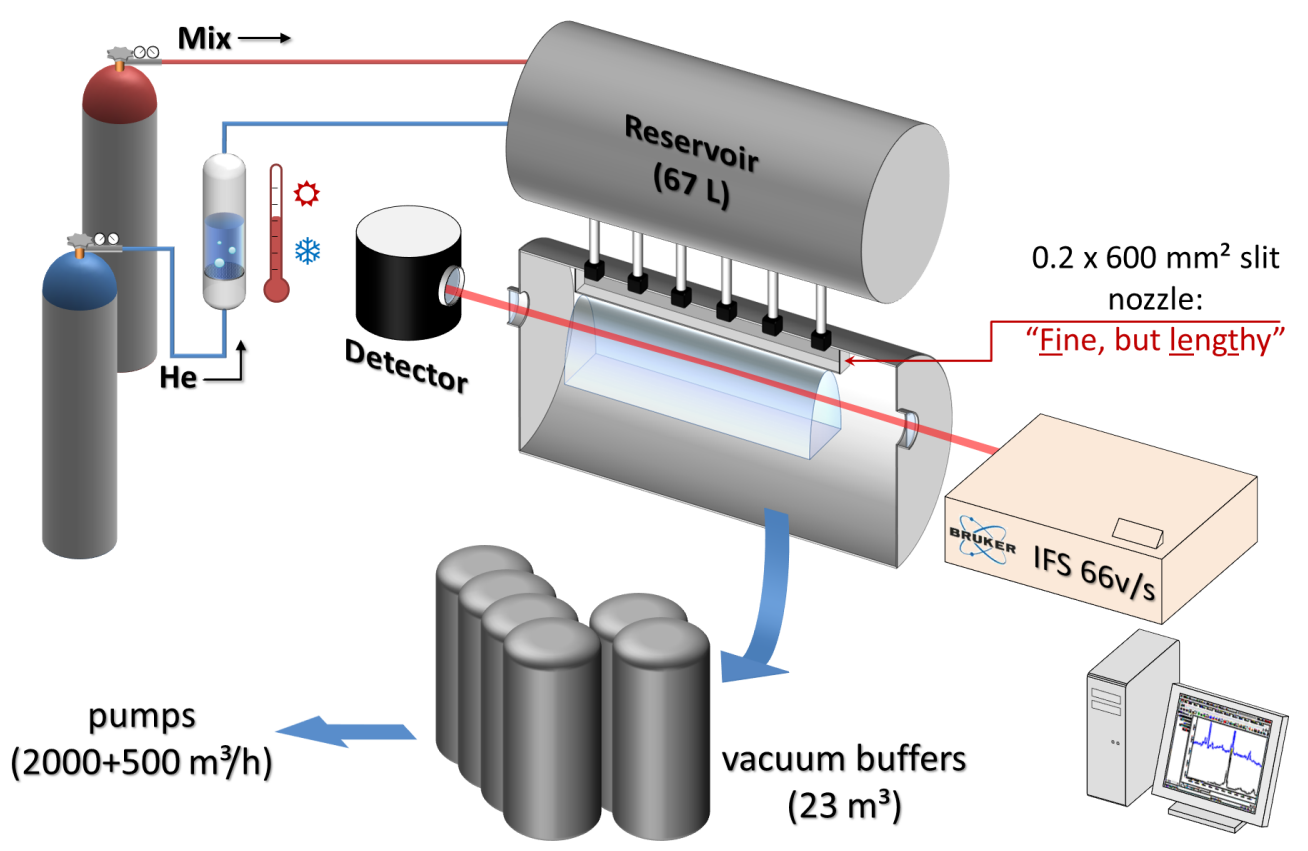

Abbildung 2.1: Schema der Filet-Jet-FTIR-Spektroskopie (wiedergegeben aus Ref. [243], lizenziert unter CC BY-NC-ND 4.0).

gung) und Veränderungen an der IR-Strahlgeometrie zur Verhinderung von Reflexion an der Düse weiter maximiert. ${ }^{[243]}$ Im Folgenden sollen die wichtigsten Aspekte der aus diesen Arbeiten resultierenden aktuellen Konfiguration, die für die Messungen im Rahmen der vorliegenden Arbeit genutzt wurde, kurz vorgestellt werden.

Für die FTIR-Spektroskopie leicht flüchtiger Moleküle (hier definiert als Sättigungsdampfdruck $p_{\mathrm{s}}>1$ mbar bei Raumtemperatur) und ihrer wasserstoffbrückengebundenen Aggregate im Überschallstrahl ist der Filet-Jet mit einer $(600 \cdot 0.2) \mathrm{mm}^{2}$ großen Schlitzdüse ausgestattet (s. Abb. 2.1). Durch diese wird die Gasmischung bestehend aus dem Trägergas (i. d. R. Helium) mit einem kleinen Anteil (je ca. 0.01-1\%) von einem oder zwei Analyten aus einem $67 \mathrm{~L}$ großen Reservoir gepulst in ein etwa $23 \mathrm{~m}^{3}$ großes Puffervolumen expandiert. Die Gasmischung kann über zwei temperierte Sättiger und einen dritten Gasflaschenanschluss erzeugt werden. Bei Raumtemperatur feste oder flüssige Analyten können dazu auf den Glasfilter in einem der Sättiger aufgebracht werden. Das Innere der Sättiger ist vollständig von einer Kühlflüssigkeit umschlossen, die jeweils mittels eines Kryostaten im Bereich zwischen etwa $-30{ }^{\circ} \mathrm{C}$ und Raumtemperatur temperiert werden kann. Zur Erzeugung der Gasmischung wird dann das Trägergas von unten durch den Glasfilter und die darauf befindliche Analytsubstanz geleitet, sodass die Analytsubstanz prinzipiell bei ihrem (temperaturabhängigen) Sättigungsdampfdruck aufgenommen wird. Bei längeren oder schnell aufeinander folgenden Nachfüllvorgängen wird der Sättigungsdampfdruck allerdings nicht dauerhaft erreicht, sodass sich in der Praxis geringere, nicht 
genau spezifizierbare Analytkonzentrationen im Gasgemisch einstellen. Über ein individuell schaltbares Magnetventil (Asco E262K220S1N00H1) in der jeweiligen Zuleitung (blau in Abb. 2.1, dort stellvertretend nur eine der Sättiger-Zuleitungen gezeigt) werden die so entstandenen Mischungen von Trägergas und Analyt ins Reservoir geleitet und vermengen sich dort. Der dritte, mit einem baugleichen Asco-Magnetventil ausgestattete Anschluss (rot) für eine Mischgas- oder eine weitere Trägergasflasche ermöglicht z. B. die Einbringung bei Raumtemperatur gasförmiger Analyten oder Trägergas/AnalytMischungen definierter Konzentration. Durch Änderung der Sättigertemperaturen und Anpassung der individuellen Öffnungs- und Schließzeiten der Asco-Magnetventile kann das Mischungsverhältnis zwischen den Analytsubstanzen variiert bzw. durch Trägergasbeimischung über den dritten Anschluss gezielt verdünnt werden. Die Befüllung des Reservoirs erfolgt über eine druckgeregelte Steuerung, sodass nur bis zum Erreichen des gewünschten Stagnationsdrucks Gas eingeleitet wird. Nach jedem Auslösen eines Gaspulses durch die Schlitzdüse wird das Reservoir durch diese Steuerung über die Sättiger und/oder den dritten Gasanschluss wieder bis zum Stagnationsdruck gefüllt.

Das Reservoir ist über sechs schnell schaltende Magnetventile (PARKer LUCIFER 221J3301E-299560-483816C2) mit der Düse verbunden. Eine Präexpansionskammer mit einem Volumen von etwa $0.5 \mathrm{~L}$ unmittelbar vor der Düse sorgt dafür, dass das Gas sich hinter den sechs Magnetventilen vor dem Durchströmen der Düse wieder gleichmäßig entlang der gesamten Schlitzlänge von $600 \mathrm{~mm}$ verteilt. Nach der Expansion durch die Düse strömt das Gas durch eine etwa 200 L große Jetkammer in das Puffervolumen. Aus diesem wird die Gasmischung anschließend mit bis zu zwei je dreistufigen Pumpständen, jeweils bestehend aus drei Wälzkolbenpumpen (Pumpstand I, im Laborjargon „großer“ Pumpstand: Pfeiffer WKP 2000 AD, Pfeiffer WKP 250 A, Pfeiffer DBP 050; Pumpstand II, im Laborjargon „kleiner“ Pumpstand: Pfeiffer WKP 500 A, Pfeiffer WKP 250 A, Pfeiffer DBP 050-3), abgepumpt.

Da der Filet-Jet sich das Puffervolumen mit dem Muesli- ${ }^{[244]}$ bzw. Chilli-Jet ${ }^{[45,226]}$ teilt, stehen beim Parallelbetrieb des Filet-Jets mit einer dieser Apparaturen von den $23 \mathrm{~m}^{3}$ Gesamtvolumen lediglich $12 \mathrm{~m}^{3}$ zur Verfügung. Darüber hinaus kann in diesem Fall nur der „kleine“ Pumpstand mit einer Nenn-Pumpleistung von $500 \mathrm{~m}^{3} / \mathrm{h}$ genutzt werden. Bei exklusivem Zugriff auf das Puffer- und Pumpsystem erhöht sich die Pumpleistung durch Hinzunahme des ,großen“ Pumpstands um $2000 \mathrm{~m}^{3} / \mathrm{h}$.

Unter anderem angesichts des hohen Gasdurchsatzes von bis zu 1 mol pro Puls ${ }^{[55]}$ durch die Düse wird der Filet-Jet in einem gepulsten Verfahren betrieben. Dadurch kann sich das durch die Düse geströmte Gasgemisch im Puffervolumen verteilen und solange durch die Pumpen abgepumpt werden, bis der Hintergrunddruck in der Jetkammer niedrig genug (d.h. <0.1 mbar) für die Auslösung des nächsten Pulses ist. Der benötigte zeitliche Abstand zwischen zwei etwa 100-200 ms langen Gaspulsen hängt dabei vom zur Verfügung stehenden Puffervolumen und der nutzbaren Pumpkapazität ab. Im exklusiven Betrieb beträgt der Abstand zwischen zwei 150 ms langen Gaspulsen gut 30 s, im Parallelbetrieb mit Muesli- oder Chilli-Jet liegt dieser bei etwa 90 s. Der Einschaltanteil der Düse an einem Puls-Abpump-Zyklus liegt also zwischen 0.2\% im Parallel- und 0.5\% im Exklusivbetrieb. 
Ein weiterer Vorteil des gepulsten Betriebes ist die Möglichkeit, mehrere Hintergrundspektren unmittelbar vor den jeweiligen Probespektren aufzuzeichnen ${ }^{[55]}$ und Störeffekte z. B. durch Intensitätsschwankungen der Signale atmospärischer Gase so möglichst gering zu halten.

Für die spektroskopische Untersuchung der Moleküle und Aggregate im Überschallstrahl steht ein FTIR-Spektrometer (BRUkER IFS 66 v/S) zur Verfügung. Der aus diesem Spektrometer ausgekoppelte IR-Strahl verläuft entlang der Schlitzdüse durch die Ruhezone der Expansion und wird auf den in einer evakuierbaren Kammer platzierten Detektor (z. B. HgCdTe- oder InSb-Detektor) fokussiert (s. Abb. 2.1). Der Strahl wird durch zwei in den Wänden der Jetkammer im Abstand von $776 \mathrm{~mm}$ angebrachte Linsen (Brennweiten $250 \mathrm{~mm}$ auf Detektor- und $500 \mathrm{~mm}$ auf Spektrometerseite) aus Kaliumbromid (KBr) oder Calciumfluorid $\left(\mathrm{CaF}_{2}\right)$ fokussiert. In das Spektrometer können Strahlteiler aus $\mathrm{KBr}, \mathrm{CaF}_{2}$ oder Mylar eingesetzt werden. Als Lichtquellen stehen ein Globar sowie zwei Wolframlampen mit 50 bzw. $150 \mathrm{~W}$ Leistung zur Verfügung. Spektrometer und Detektorkammer werden mit einer Scrollpumpe (EDwARDS XDS 35i) evakuiert und die wenige Zentimeter großen Spalte zwischen Spektrometer und Jetkammer sowie Jetkammer und Detektorkammer (s. Abb. 2.1) sind mit schwarzer Pappe ummantelt und werden mit Trockenluft gespült. Für die in dieser Arbeit gezeigten Spektren wurden in allen Fällen die Wolframlampe mit $150 \mathrm{~W}, \mathrm{CaF}_{2}$-Optiken und das InSb-Detektorlement eines in Sandwichbauweise gefertigten InSb/MCT-Detektors (INFRARED AssociATES) verwendet.

Bei Spiegelgeschwindigkeiten bis einschließlich $80 \mathrm{kHz}$ kann das sogenannte Gain Ranging ${ }^{[245,246]}$ genutzt werden. Hierbei werden im Interferogramm Bereiche abseits des zentralen Intensitätsmaximums (center burst) analog verstärkt, sodass bei der AnalogDigitalwandlung eine feinere Abtastung der Intensitätswerte möglich ist. Dies führt zu einer Minimierung des durch die begrenzte Zahl an zur Verfügung stehenden digitalen Intensitätsabstufungen hervorgerufenen Rauschens. Für Spiegelgeschwindigkeiten oberhalb von $80 \mathrm{kHz}$ kann das Gain Ranging am IFS-Gerät nicht genutzt werden, wodurch die für die Jet-Spektroskopie nutzbare Spiegelgeschwindigkeit effektiv auf diesen Wert begrenzt wird.

Alle im Rahmen dieser Arbeit am Filet-Jet aufgenommenen Spektren wurden im Akquisitionsmodus "Single Sided Fast Return“ gemessen. In diesem Modus wird das Interferogramm asymmetrisch um die Nullstellung des beweglichen Spiegels im Interferometer aufgezeichnet. Die Datenaufnahme beginnt kurz vor Durchfahren der Nullposition (und damit kurz vorm Center Burst im Interferogramm). Nach Durchfahren der Nullposition werden dann Daten bis zum Erreichen der gewünschten Auflösung (hier in allen Fällen $2 \mathrm{~cm}^{-1}$ ) aufgezeichnet („Single Sided“). Anschließend fährt der Spiegel zügig zur Ausgangsposition zurück, ohne dass Daten aufgenommen werden („Fast Return“). Dies führt zu kleinstmöglichen Datenaufnahmezeiten (auch Scanzeiten genannt), die bei $80 \mathrm{kHz}$ Spiegelgeschwindigkeit und $2 \mathrm{~cm}^{-1}$ Auflösung etwa $100 \mathrm{~ms}$ betragen. Für die Probemessungen, in denen die Überschallexpansion untersucht werden soll, sind Düsenpuls und Spektrometerscan synchronisiert. Die Überschallexpansion muss dabei über die gesamte Scanzeit aufrecht erhalten werden. Zusätzlich sind Vor- (hier $40 \mathrm{~ms}$ ) und Nachlaufzeiten 
(hier $7 \mathrm{~ms}$ ) für die Düsenöffnung nötig, damit während der Datenaufnahme näherungsweise gleichmäßige Expansionsbedingungen vorliegen. Durch möglichst kurze Scanzeiten kann die Länge des Gaspulses und damit auch der Druckanstieg in der Jetkammer begrenzt werden. Bei zu hohem lokalen Hintergrunddruck würde andernfalls die Stoßfront in den Messbereich des IR-Strahls rücken. ${ }^{\text {[9, }}$ (3,226]

Der lange Absorptionspfad entlang der Schlitzdüse führt zu guten Signal-zu-RauschVerhältnissen $(\mathrm{S} / \mathrm{N})$ selbst für schwach konzentrierte Substanzen (bis hinunter zu ca. $0.01 \%$ Anteil an der expandierten Mischung, s. Kap. 5.2) oder erlaubt den Nachweis von Banden mit äußerst geringen IR-Absorptionsquerschnitten, z. B. OH-Streckschwingungs-Obertönen. ${ }^{[50,181]}$ Solche Möglichkeiten haben den Filet-Jet, wie zu Beginn des Kapitels bereits erwähnt, zu einer der erfolgreichsten Jet-FTIR-Apparaturen in der Forschungsgruppe SuHm werden lassen. Der Nachteil des langen Absorptionspfads ist allerdings die große Düsenöffnungsfläche und damit der hohe Gasdurchsatz. Für den standardmäßig verwendeten Stagnationsdruck von 0.75 bar und eine Pulslänge von $150 \mathrm{~ms}$, wie sie für Messungen bei $80 \mathrm{kHz}$ Spiegelgeschwindigkeit und $2 \mathrm{~cm}^{-1}$ Auflösung genutzt wird, führt dies zu einem Heliumdurchsatz von etwa $0.3 \mathrm{~mol}$ pro Puls. Für ein einzelnes IR-Spektrum werden jedoch in der Regel je nach Signalintensität und gewünschtem S/N 100-1000 Pulse aufgezeichnet und gemittelt, sodass etwa 30-300 mol Helium verbraucht werden. Bei üblichen Konzentrationen von jeweils $0.01-1 \%$ werden zusätzlich noch Analyt-Stoffmengen von je bis zu 3 mol benötigt. Für eine typische Messreihe, bei der Mischungen zweier Analyten bei verschiedenen Konzentrationen untersucht werden, werden so schnell 5-50 g Substanz pro Analyt und 1-2 Druckgasflaschen (50 L, 200 bar) Trägergas benötigt. Aus finanziellen Gründen scheidet daher die Verwendung teurerer, aber besser kühlender Trägergase wie Neon ebenso wie die Untersuchung vieler Analytsubstanzen, die etwa im Rahmen einer Kooperation im Labormaßstab synthetisiert werden müssten, aus.

Diese Kostenfaktoren zusammen mit der bereits langen Laufzeit der Pumpen ( $>15$ Jahre) und des Spektrometers ( $>20$ Jahre) und der damit steigenden Ausfallwahrscheinlichkeit führten zur im Rahmen dieser Arbeit erfolgten Planung und Inbetriebnahme einer neuen Jet-FTIR-Forschungsapparatur. Die neue Gratin-Jet-FTIR-Apparatur basiert dabei auf dem bewährten und erfolgreichen Konzept des Filet-Jets, implementiert aber einige Detailverbesserungen und verbindet diese mit der Möglichkeit einer Rückführung des Gasgemisches vom Puffervolumen in das Reservoir. Hierdurch kann der Substanzverbrauch im Idealfall um mehr als eine Größenordnung reduziert werden. In Kapitel 3 soll der Gratin-Jet im Detail vorgestellt werden.

\subsection{Methoden zum Vergleich experimenteller und theoretischer Daten (Benchmarking)}

Aus Jet-FTIR-spektroskopischen Messungen können direkt (anharmonische) Wellenzahlen bestimmter Schwingungen verschiedener Aggregate ermittelt werden, sofern eine 
Zuordnung der Banden zu den untersuchten Aggregaten möglich ist. Solche anharmonischen Schwingungswellenzahlen sind damit wohl die in der Jet-FTIR-Spektroskopie am direktesten zugänglichen Größen. Stehen berechnete anharmonische Werte zur Verfügung, können die experimentellen Schwingungswellenzahlen prinzipiell unmittelbar für das Benchmarking quantenchemischer Methoden verwendet werden. Anharmonische Schwingungsrechnungen von Systemen, die aus mehr als nur ein paar Atomen bestehen, sind allerdings nicht routinemäßig mit ausreichender Genauigkeit möglich, wie z. B. die Göbench-Leistungsüberprüfung in Kap. 4.1 zeigt. Abhilfe schafft die Betrachtung relativer Unterschiede, also von Wellenzahldifferenzen. In der Verschiebung der OH-Streckschwingungsbande eines Dimers gegenüber der entsprechenden Bande des freien Monomers oder in der Bandenaufspaltung der OH-Streckschwingungsbanden zweier verschiedener gemischter Dimere einer bestimmten Donor-Akzeptor-Kombination kompensieren sich anharmonische Effekte aufgrund der ähnlichen chemischen Umgebung bei geschickter Wahl des betrachteten Systems zu einem großen Teil. ${ }^{[31,65-67]}$ Dies ermöglicht das Benchmarking harmonischer berechneter Werte anhand des Experiments, wenn entsprechende Sicherheitsmargen für die Rückkorrektur der anharmonischen experimentellen auf harmonische Werte ${ }^{[33]}$ berücksichtigt werden (Kap. 2.3.1).

Im Rahmen der vorliegenden Arbeit sollen Benchmarks quantenchemischer Methoden jedoch nicht nur aufgrund von Schwingungswellenzahlen bzw. Bandenverschiebungen oder -aufspaltungen erfolgen. Zusätzlich sollen berechnete Energieunterschiede zwischen verschiedenen Konformeren anhand experimenteller Daten überprüft werden. In der JetFTIR-Spektroskopie sind relative Energien allerdings nicht direkt zugänglich, sondern es können lediglich die Bandenintensitäten der für bestimmte Konformere charakteristischen Signale im Spektrum verglichen werden. In Kapitel 2.3.2 soll gezeigt werden, wie der berechnete Energieunterschied über die BoltzmanN-Verteilung mit dem beobachteten Intensitätsverhältnis verknüpft ist, sodass Jet-FTIR-Spektroskopie für das Benchmarking des berechneten Energieunterschieds genutzt werden kann. Für aussagekräftiges Benchmarking sind dabei realistische Fehlerbereiche für die ermittelten experimentellen Werte wichtig. Die Bestimmung dieser Fehler wird in Kapitel 2.3.3 erläutert.

\subsubsection{Benchmarking IR-spektroskopischer Parameter}

Da die im Rahmen dieser Arbeit durchgeführten Schwingungsrechnungen lediglich in der doppelt harmonischen Näherung erfolgen, ist ein Vergleich der darin ermittelten absoluten Bandenpositionen mit anharmonischen experimentellen Werten, wie bereits erwähnt, nicht sinnvoll. Für die hier untersuchten wasserstoffbrückengebundenen Dimere interessanter ist die Betrachtung von Rotverschiebungen, also des Wellenzahlunterschieds zwischen der Donor-Monomer- und der jeweils betrachteten Dimer-OH-Streckschwingungsbande. Anharmonische Beiträge zu diesen kompensieren sich durch die Differenzbildung zwischen Monomer- und Komplex-Bandenposition und durch gegenläufige Effekte der unterschiedlichen Anharmonizitätsbeiträge in der Regel zu einem hohen Grad, wie das Beispiel der einfachsten organischen Wasserstoffbrückenbindung im Methanol- 
Dimer zeigt. Für dieses reduziert sich der Netto-Beitrag der Anharmonizität zur Rotverschiebung auf etwa 1/3 der individuellen gegenläufigen diagonalen und außerdiagonalen Beiträge. ${ }^{[67]}$ Aufgrund der Größe dieser individuellen Beiträge und der potentiellen Außenseiterstellung des Methanols durch das kleine Trägheitsmoment des an die OH-Gruppe gebundenen Rests sollte für den Vergleich der absoluten Größen berechneter harmonischer und experimenteller anharmonischer Rotverschiebungen trotz der beobachteten Teilkompensation eine ausreichend hohe Sicherheitsmarge berücksichtigt werden. ${ }^{\text {[33] }}$

Um einen solchen direkten Vergleich zu ermöglichen, wurde von OswALD und SUHM eine Rückkorrektur der anharmonischen experimentellen Werte auf ihre harmonischen Anteile vorgeschlagen. ${ }^{[33]}$ Für Rotverschiebungen wird angesichts der erwarteten Kompensation anharmonischer Terme direkt der experimentelle anharmonische als Schätzung für den harmonischen Wert herangezogen, angesichts der Größe der gegenläufigen diagonalen und außerdiagonalen Beiträge zur Anharmonizität aber mit einer Sicherheitsmarge von $\pm 50 \%$. Aus der Rückkorrektur ergibt sich für den harmonischen Wert somit $\Delta \tilde{v}_{\mathrm{OH}, \exp } \pm 50 \%$. Zudem wird für den Vergleich mit den berechneten Werten die experimentelle Unsicherheit der Rotverschiebung berücksichtigt. Diese Unsicherheit wird im Rahmen dieser Arbeit über den Größtfehler der Fehler der individuellen Bandenpositionen berechnet. Die Fehler der Bandenpositionen werden jeweils mit $1 \mathrm{~cm}^{-1}$ (halbe Auflösung) abgeschätzt, sodass sich für die Rotverschiebung eine Unsicherheit von $2 \mathrm{~cm}^{-1}$ ergibt. Die Summe aus dieser Unsicherheit und dem Rückkorrektur-Fehlerintervall wird dann als experimenteller Fehlerbereich für den Vergleich mit den in doppelt-harmonischer Näherung berechneten Rotverschiebungen verwendet.

Ein deutlich kleinerer Fehlerbereich ergibt sich, wenn die Bandenaufspaltung $\Delta \Delta(\tilde{v}, \omega)_{\mathrm{OH}}(\mathrm{A}-\mathrm{B})$ zwischen den $\mathrm{OH}-$ Streckschwingungsbanden unterschiedlich wasserstoffbrückengebundener Dimere A und B herangezogen wird, da sich anharmonische Beiträge zwischen den unterschiedlichen Konformeren desselben Dimers nochmals stärker kompensieren als für die zuvor beschriebenen Rotverschiebungen. Damit ist eine genauere Rückkorrektur des anharmonischen experimentellen auf den harmonischen Wert möglich. Oswald und SunM ${ }^{[33]}$ schlagen hier als Fehler $10 \%$ des Mittelwerts der Rotverschiebungen der beiden betrachteten Dimerbanden vor, zu dem dann noch die Messungenauigkeit der Aufspaltung (hier wieder $2 \mathrm{~cm}^{-1}$ als Größtfehler der Kombination beider Einzelbeiträge) addiert wird.

Als quantitatives Maß für das Abschneiden der untersuchten quantenchemischen Methoden in Bezug auf die betrachteten experimentellen, schwingungsspektroskopischen Größen kann der ebenfalls von OswALD und SuHM vorgestellte $\delta^{2}$-Wert genutzt werden. ${ }^{[33]}$ Bei diesem handelt es sich um die mit dem inversen experimentellen Fehler gewichtete quadratische Abweichung zwischen berechnetem und (rückkorrigiertem) experimentellem Wert:

$$
\delta_{\mathrm{Xn}}^{2}(\mathrm{C}, \mathrm{Q})=\left(\frac{x_{\mathrm{Q}}-x_{\mathrm{exp}}}{\Delta x_{\exp }}\right)^{2}
$$

Darin bezeichnet „Xn“ die rückkorrigierte Observablenklasse (hier Dimer-OH-Streck- 
schwingungsbanden-Rotverschiebung und -Aufspaltung), „Q $Q$ " eine quantenchemische Methode (hier DFT-Methoden wie B3LYP-D3(BJ,ABC)/def2-TZVP oder B97-3c) und „C“ die betrachtete chemische Verbindung (hier verschiedene wasserstoffbrückengebundene Dimere). $x_{Q}$ kennzeichnet den berechneten, $x_{\text {exp }}$ den experimentellen Wert und $\Delta x_{\exp }$ den sich aus Rückkorrektur und Messungenauigkeit ergebenden experimentellen Fehlerbereich. Wird $\delta_{\mathrm{Xn}}^{2}(\mathrm{C}, \mathrm{Q})$ für eine bestimmte Observablenklasse Xn und eine bestimmte quantenchemische Methode $\mathrm{Q}$ über alle betrachteten Verbindungen $\mathrm{C}$ arithmetisch gemittelt, ergibt sich der Mittelwert $\overline{\delta_{\mathrm{Xn}}^{2}}(\mathrm{Q})$.

\subsubsection{Benchmarking relativer Energien auf Basis eines Konformerengleichgewichts}

Um Informationen über die Energieunterschiede zwischen zwei Konformeren aus einem Jet-FTIR-Spektrum zu erhalten, wird, wie bereits erwähnt, das Konzentrationsverhältnis der beiden betrachteten Konformere herangezogen. Die Beschreibung des dazu nötigen Vorgehens wurde in Ref. [31] publiziert und soll in Teilen dieses Kapitels in Ausschnitten wiedergegeben werden. Aus Ref. [31] übernommene Gleichungen und paraphrasierte Textpassagen werden dabei im Folgenden nicht gesondert gekennzeichnet oder mit einzelnen Quellenverweisen versehen. Eine Genehmigung für den Abdruck liegt vor.*

Das Konzentrationsverhältnis $c_{\mathrm{B}} / c_{\mathrm{A}}$ zweier Konformere A und B ist im thermodynamischen Gleichgewicht über die statistische Thermodynamik mit deren Energieunterschied $\Delta E_{\mathrm{B}-\mathrm{A}}^{0}$ verknüpft:

$$
\frac{c_{\mathrm{B}}}{c_{\mathrm{A}}}=\frac{g_{\mathrm{B}}}{g_{\mathrm{A}}} \frac{z_{\mathrm{B}}^{\mathrm{rot}}\left(T^{\mathrm{rot}}\right) z_{\mathrm{B}}^{\mathrm{vib}}\left(T^{\mathrm{vib}}\right)}{z_{\mathrm{A}}^{\mathrm{rot}}\left(T^{\mathrm{rot}}\right) z_{\mathrm{A}}^{\mathrm{vib}}\left(T^{\mathrm{vib}}\right)} \exp \left(-\frac{\Delta E_{\mathrm{B}-\mathrm{A}}^{0}}{R T_{\mathrm{c}}}\right)
$$

Hierin bezeichnet $g$ den Symmetrie-Entartungsfaktor, $z$ die Zustandssumme, $T$ die Temperatur und $R$ die universelle Gaskonstante. Die Superskripte „rot“ und „vib“ kennzeichnen Rotations- und Vibrationsfreiheitsgrade. Für wasserstoffbrückengebundene Dimere zweier kleinerer ( $<15$ Nicht-Wasserstoffatome), rigider Moleküle, wie sie im Rahmen dieser Arbeit betrachtet werden sollen, kann Gleichung 2.6 deutlich vereinfacht werden, da sich die Dimere chemisch gleichen. So liegt die Rotationstemperatur $T^{\text {rot }}$ in der HeliumÜberschallexpansion in der Regel bei 5-20 K und ist für die beiden Dimere A und B sehr ähnlich. Zudem weisen alle im Rahmen dieser Arbeit betrachteten Dimere $C_{1}$ - oder $C_{s^{-}}$ Symmetrie auf, sodass die in die Rotationszustandssumme eingehende Symmetriezahl $\sigma$ in allen Fällen 1 beträgt. Die Rotationszustandssummen $z_{\mathrm{A}, \mathrm{B}}^{\text {rot }}$ kürzen sich somit näherungsweise heraus. Die Vibrationstemperaturen $T^{\mathrm{vib}}$ liegen dagegen zwischen $T^{\text {rot }}$ für weiche und der Düsentemperatur $T^{\text {noz }}$ für starre Moden. Aufgrund der Rigidität der hier betrachteten Moleküle stimmen die starren Moden in den Dimeren A und B weitgehend überein

*Direkter Link zum Artikel: https: //pubs . acs.org/doi/10.1021/acs . jpclett.7b02337. Hinweis des Rechteinhabers: „Further permissions related to the material excerpted should be directed to the ACS.“ 
und sind zudem nur gering populiert. Die in Abhängigkeit von der Dimerkonformation unterschiedlichen, neugebildeten sechs intermolekularen Moden sind hingegen sehr weich und weisen damit wieder niedrige Vibrationstemperaturen auf. Hierdurch kürzen sich auch die Vibrationszustandssummen $z_{\mathrm{A}, \mathrm{B}}^{\mathrm{vib}}$ näherungsweise heraus. Mit diesen Vereinfachungen ergibt sich somit eine effektive Zwei-Niveau-BoltzmanN-Verteilung mit einer einzigen, effektiven Konformationsumwandlungstemperatur, die alle Unsicherheiten hinsichtlich der partiellen Gleichgewichtseinstellung im Jet und der abweichenden Zustandssummen in sich vereint:

$$
\frac{c_{\mathrm{B}}}{c_{\mathrm{A}}}=\frac{g_{\mathrm{B}}}{g_{\mathrm{A}}} \exp \left(-\frac{\Delta E_{\mathrm{B}-\mathrm{A}}^{0}}{R T_{\mathrm{c}}}\right)
$$

Die so gewonnene BolTZMANN-Formel in Gleichung 2.7 entspricht einer Betrachtung unter Vernachlässigung aller Entropieeffekte abseits der Konformersymmetrie, wenn sich die Symmetriezahl $\sigma$ zwischen den Konformeren A und B nicht ändert (s. oben). Da die Schwingungsnullpunktsenergie (ZPVE, vom englischen „zero-point vibrational energy“) auch am absoluten Temperaturnullpunkt noch vorliegt, kann im Allgemeinen nicht direkt der Energieunterschied der berechneten elektronischen Energien, $\Delta E_{\mathrm{B}-\mathrm{A}}^{\mathrm{l}}$, für das Benchmarking genutzt werden. Stattdessen muss - wie in Gleichung 2.7 auch dargestellt - der schwingungsnullpunktskorrigierte Energieunterschied $\Delta E_{\mathrm{B}-\mathrm{A}}^{0}$ verwendet werden. Trotz der Anharmonizität der neugebildeten intermolekularen Moden reicht hierbei in der Regel eine harmonische Berechnung der ZPVE aus, da für die Dimere A und B vergleichbare Anharmonizitäten erwartet werden. ${ }^{[50]}$ Dieser Umstand erweitert den Kreis der für Benchmarks zugänglichen Methoden immens, da längst nicht für alle Methoden anharmonische Schwingungsrechnungen verfügbar bzw. möglich sind. Doch auch harmonische Schwingungsrechnungen sind nicht für alle Methoden durchführbar (s. oben). Durch die Wahl eines Systems mit möglichst ähnlichen Schwingungsnullpunktsenergien in den Dimeren A und B kann jedoch versucht werden, den Einfluss der ZPVE zu minimieren, um einen direkteren Benchmark der elektronischen Energie zu erlauben. Dieser Ansatz wird mit den Carbonylwaagen verfolgt, die in Kapitel 5 vorgestellt werden.

Ein Problem beim Benchmarking über die Boltzmann-Verteilung ist, dass das Konzentrationsverhältnis $c_{\mathrm{B}} / c_{\mathrm{A}}$ in der Jet-FTIR-Spektroskopie nicht direkt experimentell zugänglich ist. Nach Zuordnung spezifischer Banden zu den Konformeren A und B, z. B. unterschiedlich rotverschobener $\mathrm{OH}$-Streckschwingungsbanden des Wasserstoffbrückendonors im betrachteten Dimer, kann lediglich das Verhältnis deren Intensitäten $I, I_{\mathrm{B}} / I_{\mathrm{A}}$, aus dem Spektrum ermittelt werden. Um aus diesem das Konzentrationsverhältnis zu berechnen, muss der integrierte IR-Absorptionsquerschnitt der jeweils zugrundeliegenden Schwingung berücksichtigt werden. Da dieser für die betrachteten Clusterbanden nahezu immer nicht bekannt ist, wird auf berechnete IR-Bandenstärken $(A)$, in der Regel aus doppelt harmonischen Schwingungsrechnungen, zurückgegriffen. Es ergibt sich:

$$
\frac{c_{\mathrm{B}}}{c_{\mathrm{A}}}=\frac{I_{\mathrm{B}}}{I_{\mathrm{A}}} \cdot \frac{A_{\mathrm{A}}}{A_{\mathrm{B}}}
$$


Für die Verknüpfung zwischen beobachtetem Intensitätsverhältnis und dem Energieunterschied folgt aus den Gleichungen 2.7 und 2.8:

$$
\frac{I_{\mathrm{B}}}{I_{\mathrm{A}}}=\frac{A_{\mathrm{B}}}{A_{\mathrm{A}}} \frac{g_{\mathrm{B}}}{g_{\mathrm{A}}} \exp \left(-\frac{\Delta E_{\mathrm{B}-\mathrm{A}}^{0}}{R T_{\mathrm{c}}}\right)
$$

Experimentell können somit nicht direkt der Energieunterschied $\Delta E_{\mathrm{B}-\mathrm{A}}^{0}$ oder die Konformationstemperatur $T_{\mathrm{c}}$, bei der die Verteilung der Konformere A und B in der Überschallexpansion ausgefroren wird, bestimmt werden. Aus dem beobachteten Intensitätsverhältnis ergibt sich lediglich der Quotient dieser beiden Größen, der zusätzlich noch von den Verhältnissen der berechneten Bandenstärken $A_{\mathrm{A}, \mathrm{B}}$ und der Entartungsfaktoren $g_{\mathrm{A}, \mathrm{B}}$ überlagert wird. Um das Ziel, den berechneten Energieunterschied anhand der experimentellen Daten zu benchmarken, zu erreichen, müssen also zunächst einmal die Verhältnisse $A_{\mathrm{B}} / A_{\mathrm{A}}$ und $g_{\mathrm{B}} / g_{\mathrm{A}}$ berücksichtigt werden. Hinsichtlich des letzteren kann für die im Rahmen dieser Arbeit betrachteten Dimere oftmals $C_{1}$-Symmetrie angenommen werden, sodass die Entartungsfaktoren keine Rolle spielen. Für eine genauere Betrachtung muss im Allgemeinen jedoch auf die Symmetrien der in quantenchemischen Rechnungen optimierten Strukturen für A und B zurückgegriffen werden, es fließen hier also bereits Rechenergebnisse ein. Ähnliches gilt für die Bandenstärken. Eine Option ist, grobe Annahmen für das Bandenstärkenverhältnis zu treffen, beispielsweise dass die Banden-

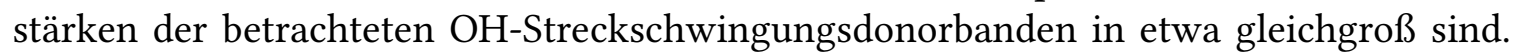
Diese Annahme kann dann mit einem großzügigen Fehlerbereich kombiniert werden $\left(A_{\mathrm{B}} / A_{\mathrm{A}}=1.0 \pm 0.5\right) .{ }^{[75]}$ Alternativ kann als zweite Option erneut auf Daten aus quantenchemischen Rechnungen zurückgegriffen werden. Wie auch die Symmetrie sind die berechneten Bandenstärken nur schwach mit dem berechneten Energieunterschied korreliert, ${ }^{[37]}$ sodass trotzdem noch sinnvolle Benchmarks des Energieunterschieds durchgeführt werden können. Darüber hinaus sind die berechneten Bandenstärken insbesondere für hohe Oszillatorstärken - wie sie vorliegen müssen, damit Banden im Jet-FTIR-Spektrum überhaupt sichtbar sind - in der Regel robust. Das gilt umso mehr für das Verhältnis der berechneten Bandenstärken, da sich Fehler durch Schwächen der genutzten Methode darin oftmals kompensieren. Aus diesem Grund liefern auch in der harmonischen Näherung berechnete Bandenstärkenverhältnisse im Allgemeinen eine ausreichende Genauigkeit für das Benchmarking des Energieunterschieds.

Unter der Annahme, dass die wie im letzten Absatz beschrieben ermittelten Werte für $A_{\mathrm{B}} / A_{\mathrm{A}}$ und $g_{\mathrm{B}} / g_{\mathrm{A}}$ ausreichend zuverlässig sind, kann nun also aus dem gemessenen Intensitätsverhältnis der Quotient $\Delta E_{\mathrm{B}-\mathrm{A}}^{0} / T_{\mathrm{c}}$ ermittelt werden. Um den berechneten Energieunterschied $\Delta E_{\mathrm{B}-\mathrm{A}}^{0}$ anhand experimenteller Daten zu benchmarken, muss somit $T_{\mathrm{c}}$ noch bestimmt werden. Die Höhe der Konformationstemperatur ist zum einen von den Expansionsbedingungen (z. B. Masse des Trägergases), zum anderen aber auch von der Höhe und Breite der Umwandlungsbarriere zwischen beiden Konformeren abhängig. Während für niedrige und schmale Barrieren Temperaturen bis fast hinab zur Rotationstemperatur $T^{\text {rot }}$ erreicht werden können, wird die Konformerenverteilung für hohe, 
breite Barrieren bei der Düsentemperatur $T^{\text {noz }}$ eingefroren. Letzterer Umstand kann genutzt werden, um mit einer erhöhten Düsentemperatur höherenergetische Konformere zu erzeugen und deren Population in der Überschallexpansion zu erhalten, gleichzeitig aber die Rotations-, Vibrations- und (senkrecht zur Ausbreitungsrichtung) Translationsfreiheitsgrade zu kühlen. Mit diesem Verfahren konnte kürzlich in einer RAMAN-spektroskopischen Messung beispielsweise das cis-Konformer der Ameisensäure untersucht werden. ${ }^{[34]}$ Auch am Gratin-Jet wurde die Möglichkeit, die Düse zu heizen, implementiert (s. Kap. 3). In der vorliegenden Arbeit wird dies jedoch noch nicht zu Forschungszwecken eingesetzt.

Da $T_{\mathrm{c}}$ nicht direkt experimentell bestimmt werden kann, muss diese Temperatur abgeschätzt werden. Strikte physikalische Grenzen werden durch die Thermodynamik gesetzt: Die Konformationstemperatur kann nicht unterhalb der Rotationstemperatur von minimal etwa $10 \mathrm{~K}$ und nicht oberhalb der Düsentemperatur $T^{\text {noz }}$ liegen. Da im Rahmen der vorliegenden Arbeit keine geheizten Düsen eingesetzt werden, liegt die obere Grenze bei Raumtemperatur, also bei etwa $300 \mathrm{~K}$. Weichere Grenzen ergeben sich aus dem Grad der Kühlung der Umwandlungskoordinate in der Überschallexpansion und damit aus der jeweiligen Barrierenhöhe und -breite der Konformerenumwandlung und können daher nur abgeschätzt werden. Für die O/C-Waagensysteme aus Alkoholen und Furanen bzw. Anisolen (Kap. 4) werden eher geringe Barrierenhöhen und somit Konformationstemperaturen von etwa $60 \pm 40 \mathrm{~K}$ für das $\mathrm{O} / \mathrm{C}$-Konformerengleichgewicht in den betrachteten Heliumexpansionen angenommen, da die Alkohole sich nur wenig oberhalb der Anisol- bzw. Furanebene bewegen müssen, um von einer Bindungsstelle zur anderen zu gelangen. ${ }^{[37,75]}$ Für die Carbonylwaagensysteme (Kap. 5) werden für die Konformerenumwandlung etwas größere Barrieren erwartet, da das Donormolekül sich für den Wechsel vom einen zum anderen freien Elektronenpaar am Carbonylsauerstoffatom deutlich stärker und um die Carbonylgruppe herum bewegen muss. Die Konformationstemperaturen in Heliumexpansionen werden deshalb auf $90 \pm 60 \mathrm{~K}$ geschätzt. Die nur auf einen relativ großen Bereich mögliche Eingrenzung der experimentell vorliegenden Konformationstemperatur ist damit eins der größten Hindernisse für das diskriminierende Benchmarking der Leistungsfähigkeit verschiedener Methoden. Drei Möglichkeiten, berechnete Energieunterschiede über die BoLTZMANN-Verteilung anhand gemessener Intensitätsverhältnisse zu überprüfen, sollen im Folgenden vorgestellt werden.

\section{Boltzmann-Plots}

Eine anschauliche Möglichkeit eines solchen Benchmarks ist eine Auftragung des Anteils $x_{\mathrm{A}}$ eines Konformers A an der Gesamtzahl der Konformere A und B gegen den berechneten Energieunterschied $\Delta E_{\mathrm{B}-\mathrm{A}}^{0}$, wie sie in Abbildung 2.2 dargestellt ist. Auf Grundlage der BoltzmanN-Verteilung ergibt sich für den Zusammenhang zwischen $x_{\mathrm{A}}$ und $\Delta E_{\mathrm{B}-\mathrm{A}}^{0}$ 


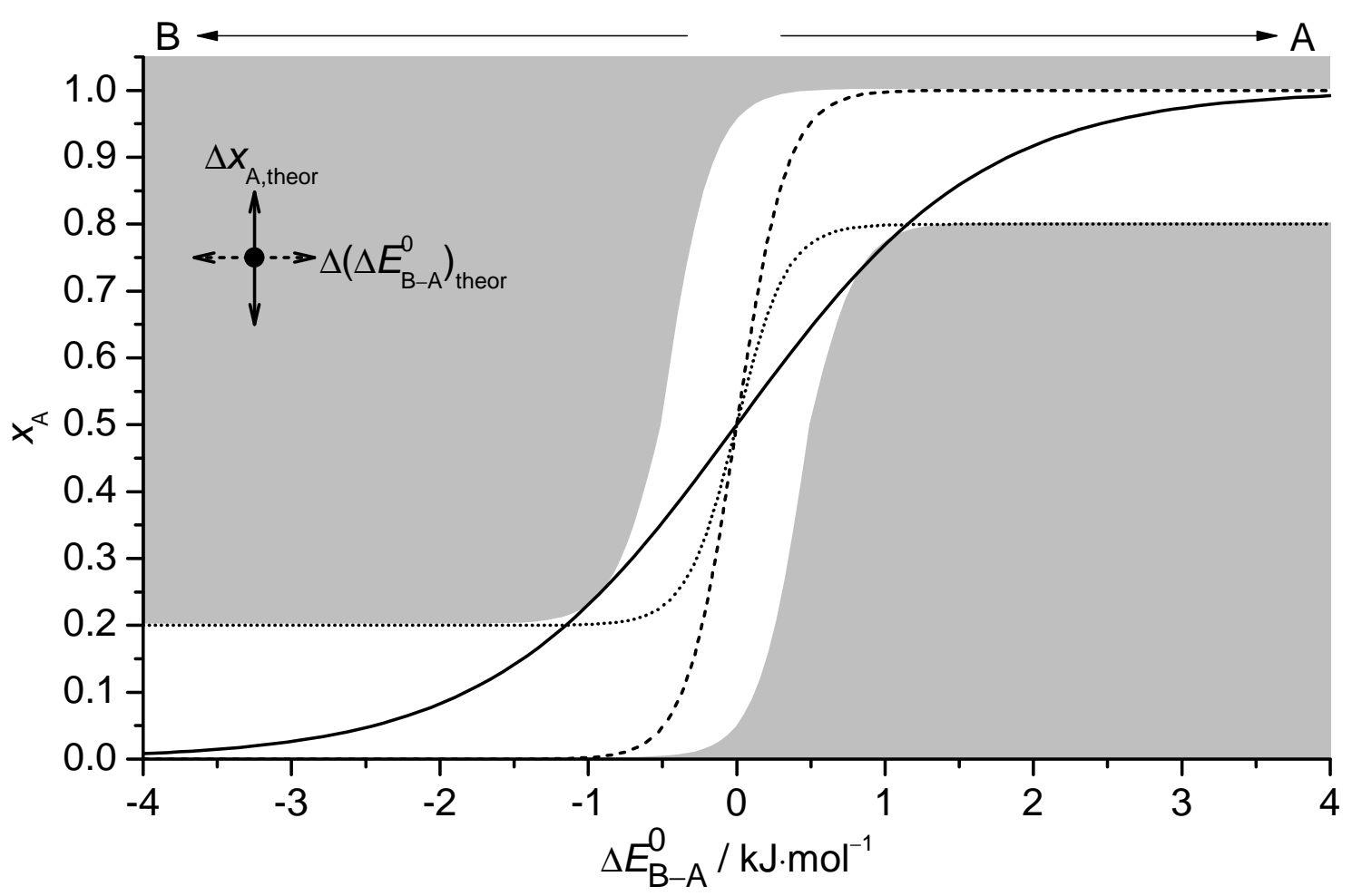

Abbildung 2.2: Darstellung der Konstruktion des Zielbereichs für Auftragungen des Anteils der A-Dimere $\left(x_{\mathrm{A}}\right)$ gegen den berechneten, schwingungsnullpunktskorrigierten Energieunterschied $\triangle E_{\mathrm{B}-\mathrm{A}}^{0}$. Konformationstemperaturen von $20 \mathrm{~K}$ (gestrichelte Kurve) bis $100 \mathrm{~K}$ (durchgezogene Kurve), sowie die Berücksichtigung von bis zu 20\% kinetischem Trapping (gepunktete Kurve) und erlaubte Fehler von bis zu $\pm 0.5 \mathrm{~kJ} / \mathrm{mol}$ in $\Delta E_{\mathrm{B}-\mathrm{A}}^{0}$ (gestrichelter Pfeil) bzw. \pm 0.1 in $x_{\mathrm{A}}$ (durchgezogener Pfeil) führen zum dargestellten weiß hervorgehobenen Bereich, in dem Datenpunkte liegen müssen, um mit den experimentellen Ergebnissen übereinzustimmen.

eine sigmoide Kurve:

$$
x_{\mathrm{A}}=\frac{c_{\mathrm{A}}}{c_{\mathrm{A}}+c_{\mathrm{B}}}=\frac{1}{1+\frac{I_{\mathrm{B}}}{I_{\mathrm{A}}} \cdot \frac{A_{\mathrm{A}}}{A_{\mathrm{B}}}}=\frac{1}{1+\frac{g_{\mathrm{B}}}{g_{\mathrm{A}}} \exp \left(-\frac{\Delta E_{\mathrm{B}-\mathrm{A}}^{0}}{R T_{\mathrm{c}}}\right)}
$$

Wie Gleichung 2.10 verdeutlicht, ist der Verlauf der Kurve dabei von der Konformationstemperatur $T_{\mathrm{c}}$ und vom Verhältnis der Entartungsfaktoren, $g_{\mathrm{B}} / g_{\mathrm{A}}$, abhängig. Unterschiedliche Verhältnisse $g_{B} / g_{A}$ für verschiedene betrachtete Konformerengleichgewichte führen also zu verschiedenen Kurvenverläufen und damit Zielbereichen. Je nach Symmetrie der Konformere sind somit gegebenenfalls einzelne Betrachtungen für verschiedene Entartungsfaktoren-Verhältnisse nötig. Alternativ kann das Verhältnis der Entartungsfaktoren in den Konformerenanteil $x_{\mathrm{A}}$ gezogen werden, sodass sich ein korrigierter Konformerenanteil $x_{\mathrm{A}, \text { eff }}$ ergibt. Dieser gibt dann allerdings nicht mehr den tatsächlich in der Expansion vorliegenden Anteil $x_{\mathrm{A}}$ wieder. Im Rahmen der vorliegenden Arbeit sollen die 
Boltzmann-Plots nur für die Auswertung von Konformerengleichgewichten mit Entartungsfaktoren-Verhältnissen von $g_{B} / g_{A}=1$ verwendet werden. Dadurch sind weder getrennte Betrachtungen für verschiedene Konformerensymmetrien noch die Verwendung des korrigierten Konformerenanteils $x_{\mathrm{A} \text {,eff }}$ nötig. Gleichung 2.10 vereinfacht sich damit $\mathrm{zu}$ :

$$
x_{\mathrm{A}}=\frac{c_{\mathrm{A}}}{c_{\mathrm{A}}+c_{\mathrm{B}}}=\frac{1}{1+\frac{I_{\mathrm{B}}}{I_{\mathrm{A}}} \cdot \frac{A_{\mathrm{A}}}{A_{\mathrm{B}}}}=\frac{1}{1+\exp \left(-\frac{\Delta E_{\mathrm{B}-\mathrm{A}}^{0}}{R T_{\mathrm{c}}}\right)}
$$

Eine höhere Konformationstemperatur führt zu einem flacheren Verlauf der sigmoiden Kurve (durchgezogene Kurve in Abb. 2.2), während die Kurve sich im Tieftemperaturlimit einer Stufenfunktion annähert (gestrichelte Kurve). Wie bereits erwähnt, kann der genaue Wert der Konformationstemperatur nur geschätzt werden. Weiterhin muss für die Bestimmung des auf den Konformerkonzentrationen beruhenden A-Anteils $x_{\mathrm{A}}$ neben den gemessenen Bandenintensitäten $I_{\mathrm{A}}$ und $I_{\mathrm{B}}$ auch das Verhältnis der berechneten Bandenstärken, $A_{\mathrm{A}} / A_{\mathrm{B}}$, berücksichtigt werden. Zudem kann keine unendliche Genauigkeit hinsichtlich der berechneten Größen Energieunterschied und Bandenstärkenverhältnis von den zu benchmarkenden Methoden erwartet werden. Anhand der verschiedenen fehlerbehafteten Größen wird somit anstelle einer unendlich schmalen Kurve ein verbreiterter Zielbereich definiert, wie im Folgenden erläutert wird.

Die in Abbildung 2.2 dargestellten, bereits genannten sigmoiden Kurven entsprechen Konformationstemperaturen von $20 \mathrm{~K}$ (gestrichelte Kurve) und $100 \mathrm{~K}$ (durchgezogene Kurve). Die genannten Temperaturen sollen hier als Beispiele für Abschätzungen des unteren bzw. oberen $T_{\mathrm{c}}$-Grenzwerts dienen. Für einzelne Konformerenpaare mit besonders großen Umwandlungsbarrieren kann zudem ein zusätzlicher Effekt in der Expansion auftreten: kinetisches Trapping. Dabei wird das Konformerenverhältnis, wie weiter oben beschrieben, effektiv bei der Düsentemperatur eingefroren, d. h. es findet keine Relaxation ins stabilere Konformer in der Expansion statt. Bei sorgfältiger Wahl der betrachteten Systeme, also z. B. bei Dimergeometrien, die kleine und flache Barrieren für die Konformerenumwandlung begünstigen, sollte dies nur bei Ausreißern der Fall sein. Somit wird nicht der gesamte Kurvenverlauf für eine Konformationstemperatur von $300 \mathrm{~K}$ berücksichtigt, sondern es wird lediglich maximal 20\% Trapping des metastabilen Konformers in der Expansion erlaubt. Eine entsprechend modifizierte Kurve für eine Konformationstemperatur von $20 \mathrm{~K}$ ist in der Abbildung gepunktet dargestellt. Der sich zwischen der unmodifizierten und der modifizierten 20-K-Kurve aufspannende Bereich erlaubt gleichzeitig einen Fehler $\Delta x_{\mathrm{A} \text {,theor }}$ in $x_{\mathrm{A}}$, der auf dem Fehler des berechneten Bandenstärkenverhältnisses $A_{\mathrm{A}} / A_{\mathrm{B}}$ beruht und dessen annehmbares Maß hier auf \pm 0.1 (durchgezogener Pfeil) festgelegt wird. Werden die drei dargestellten Kurven nun noch mit dem für die berechneten Energieunterschiede $\Delta E_{\mathrm{B}-\mathrm{A}}^{0}$ als akzeptabel eingestuften Fehlerbereich von $\Delta\left(\Delta E_{\mathrm{B}-\mathrm{A}}^{0}\right)_{\text {theor }}= \pm 0.5 \mathrm{~kJ} / \mathrm{mol}$ (gestrichelter Pfeil) kombiniert, ergibt sich der dargestellte weiß hervorgehobene Bereich, in dem die Datenpunkte aus $\Delta E_{\mathrm{B}-\mathrm{A}}^{0}$ ( $x$-Koordinate) und $x_{\mathrm{A}}(y$-Koordinate) liegen sollten, um mit dem Experiment vereinbar zu sein. Da in die Berechnung des A-Konformerenanteils $x_{\mathrm{A}}$ außerdem das experimentell bestimmte Inten- 
sitätsverhältnis $I_{\mathrm{B}} / I_{\mathrm{A}}$ eingeht, das mit einem Messfehler behaftet ist (s. Kap. 2.3.3), ergeben sich zusätzlich individuelle experimentelle Fehlerbalken für $x_{\mathrm{A}}$. Diese werden über eine GAUß'sche Fehlerfortpflanzung aus dem Fehler $\Delta\left(\frac{I_{\mathrm{B}}}{I_{\mathrm{A}}}\right)$ des gemessenen Intensitätsverhältnisses bestimmt:

$$
\Delta x_{\mathrm{A}, \exp }=\sqrt{\left(-\frac{\frac{A_{\mathrm{B}}}{A_{\mathrm{A}}} \cdot \Delta\left(\frac{I_{\mathrm{B}}}{I_{\mathrm{A}}}\right)}{\left(\frac{I_{\mathrm{B}}}{I_{\mathrm{A}}}+\frac{A_{\mathrm{B}}}{A_{\mathrm{A}}}\right)^{2}}\right)^{2}}
$$

Ein Vorteil dieser Form des Benchmarkings ist, dass Fehler in der quantenchemischen Vorhersage für den Energieunterschied auf der Abszisse, Fehler für das berechnete Bandenstärkenverhältnis dagegen auf der Ordinate auftreten. Je nach Lage des resultierenden Datenpunkts kann somit unter Umständen ein nicht mit den experimentellen Daten zu vereinbarendes Ergebnis Schwächen in einer der beiden vorherzusagenden Größen zugeordnet werden. Liegt ein Datenpunkt beispielsweise nur leicht unterhalb, aber weit rechts vom Zielbereich, könnte dies durch einen vergleichsweise kleinen Fehler im Bandenstärkenverhältnis verursacht sein, wohingegen der Fehler in der Energiedifferenz groß sein müsste. Ein Nachteil an dieser Form des Benchmarkings ist hingegen, dass pro Datenpunkt immer ein Wertepaar $\left(\Delta E_{\mathrm{B}-\mathrm{A}}^{0}, x_{\mathrm{A}}\right)$ nötig ist, um die Leistungsfähigkeit der untersuchten quantenchemischen Methode zu beurteilen. Schneller erfassbar sind Ansätze, bei denen anhand eines einzelnen Wertes pro Datenpunkt beurteilt werden kann, ob die Vorhersage aus der quantenchemischen Rechnung zu den experimentellen Ergebnissen passt. In der vorliegenden Arbeit sollen zwei solcher Ansätze, bei denen nur eine Zielgröße für den Benchmark benötigt wird, genutzt werden. Der erste Ansatz bringt das Experiment möglichst nah an die Theorie, indem ein experimenteller Energieunterschied $\Delta E_{\mathrm{B}-\mathrm{A} \text {,exp }}^{0}$ berechnet wird. ${ }^{[75,80]}$ Der zweite Ansatz bringt hingegen die Theorie möglichst nah an das Experiment, indem unter Einbeziehung der berechneten Daten eine Konformationstemperatur ermittelt wird. ${ }^{[80]}$

\section{Experimenteller Energieunterschied}

Für die Berechnung des experimentellen Energieunterschieds im ersten Ansatz kann Gleichung 2.9 (s. S. 21) nach $\Delta E_{\mathrm{B}-\mathrm{A}}^{0}$ umgeformt werden:

$$
\Delta E_{\mathrm{B}-\mathrm{A}}^{0}=-R T_{\mathrm{c}} \ln \left(\frac{I_{\mathrm{B}}}{I_{\mathrm{A}}} \frac{A_{\mathrm{A}}}{A_{\mathrm{B}}} \frac{g_{\mathrm{A}}}{g_{\mathrm{B}}}\right)
$$

Der Fehler des Energieunterschieds kann dann im Sinne einer konservativen Fehlerabschätzung als Größtfehler berechnet werden: ${ }^{[75]}$

$$
\Delta\left(\Delta E_{\mathrm{B}-\mathrm{A}}^{0}\right)=\left|-R \ln \left(\frac{I_{\mathrm{B}}}{I_{\mathrm{A}}} \frac{A_{\mathrm{A}}}{A_{\mathrm{B}}} \frac{g_{\mathrm{A}}}{g_{\mathrm{B}}}\right) \Delta T_{\mathrm{c}}\right|+\left|-R T_{\mathrm{c}} \frac{\Delta\left(\frac{I_{\mathrm{B}}}{I_{\mathrm{A}}}\right)}{\frac{I_{\mathrm{B}}}{I_{\mathrm{A}}}}\right|+\left|R T_{\mathrm{c}} \frac{\Delta\left(\frac{A_{\mathrm{B}}}{A_{\mathrm{A}}}\right)}{\frac{A_{\mathrm{B}}}{A_{\mathrm{A}}}}\right|
$$


Sollen zur Berechnung von $\Delta E_{\mathrm{B}-\mathrm{A}}^{0}$ dabei ausschließlich experimentelle Daten genutzt werden, müssen für die Verhältnisse der IR-Bandenstärken $\left(A_{\mathrm{B}} / A_{\mathrm{A}}\right)$ sowie der Entartungsfaktoren $\left(g_{\mathrm{B}} / g_{\mathrm{A}}\right)$, wie oben erläutert, Annahmen getroffen werden, z. B. vergleichbar große Bandenstärken mit 50\% Fehlertoleranz $\left(A_{\mathrm{B}} / A_{\mathrm{A}}=1.0 \pm 0.5\right)$. Für das Verhältnis der Entartungsfaktoren sind unter Umständen auch hier einzelne Betrachtungen für Kombinationen verschiedener Symmetrien erforderlich (s. Kap. 4.1). Der Fehler des gemessenen Intensitätsverhältnisses, $\Delta\left(\frac{I_{B}}{I_{A}}\right)$, wird, wie in Kapitel 2.3.3 beschrieben, aus dem Jet-FTIRSpektrum bestimmt und kann prinzipiell durch Erhöhung der Zahl der durchgeführten Scans verkleinert werden. Der Fehler des Energieunterschieds wird in der Regel jedoch durch den großen Fehler für $T_{\mathrm{c}}$ dominiert. Der Umstand, dass $T_{\mathrm{c}}$ nicht genau bestimmt werden kann, sorgt hier also für einen großen Fehlerbereich des rein experimentell bestimmten Energieunterschieds.

\section{Berechnete Konformationstemperatur}

Eine in dieser Hinsicht elegante Lösung stellt damit der zweite oben genannte Ansatz dar. Für die Berechnung der Konformationstemperatur $T_{\mathrm{c}}$ ergibt sich aus Gleichung 2.9:

$$
T_{\mathrm{c}, \mathrm{QM}}=-\frac{\Delta E_{\mathrm{B}-\mathrm{A}}^{0}}{R \ln \left(\frac{I_{\mathrm{B}}}{I_{\mathrm{A}}} \frac{A_{\mathrm{A}}}{A_{\mathrm{B}}} \frac{g_{\mathrm{A}}}{g_{\mathrm{B}}}\right)}
$$

In diesem Ansatz kann also eine Konformationstemperatur $T_{\mathrm{c}, \mathrm{QM}}$ aus dem experimentellen Intensitätsverhältnis $I_{\mathrm{B}} / I_{\mathrm{A}}$ und den theoretisch berechneten Werten für das Bandenstärkenverhältnis $A_{\mathrm{B}} / A_{\mathrm{A}}$, das Verhältnis der Entartungsfaktoren $g_{\mathrm{B}} / g_{\mathrm{A}}$ und den schwingungsnullpunktskorrigierten Energieunterschied $\Delta E_{\mathrm{B}-\mathrm{A}}^{0}$ errechnet und mit dem im Experiment erwarteten Wert $T_{\mathrm{c}, \exp }$ verglichen werden. Zum einen wird mit $T_{\mathrm{c}}$ hier die am stärksten fehlerbehaftete Größe als Zielgröße verwendet und fließt damit nicht in die Berechnung des für den Benchmark verwendeten Wertes ein. Zum anderen müssen abseits der Festlegung eines realistischen Erwartungsbereichs für die Konformationstemperatur $T_{\text {c,exp }}$ (s. oben) keine Annahmen getroffen werden. Alle Werte, die experimentell nicht ermittelt werden können, werden aus den quantenchemischen Rechnungen herangezogen. Damit liegt die Last auf Seiten der Theorie und es werden gleichzeitig mehrere Aspekte der betrachteten Rechnung untersucht: die elektronischen Energien im Energieunterschied, die Schwingungsrechnung in der Nullpunktskorrektur des Energieunterschieds sowie im Bandenstärkenverhältnis und die Geometrieoptimierung in Form der symmetrieabhängigen Entartungsfaktoren. ${ }^{[80]}$ Fehler in der Berechnung der verschiedenen Größen können sich dabei sowohl aufaddieren als auch kompensieren. Um eine zufällige Fehlerkompensation möglichst ausschließen zu können, sollte also z. B. die Robustheit der eingehenden Bandenstärkenverhältnisse durch Berechnung auf verschiedenen Theorieniveaus überprüft oder die Zahl der betrachteten Datenpunkte durch systematische Variation der untersuchten Konformere weit genug erhöht werden. ${ }^{[31]}$ 


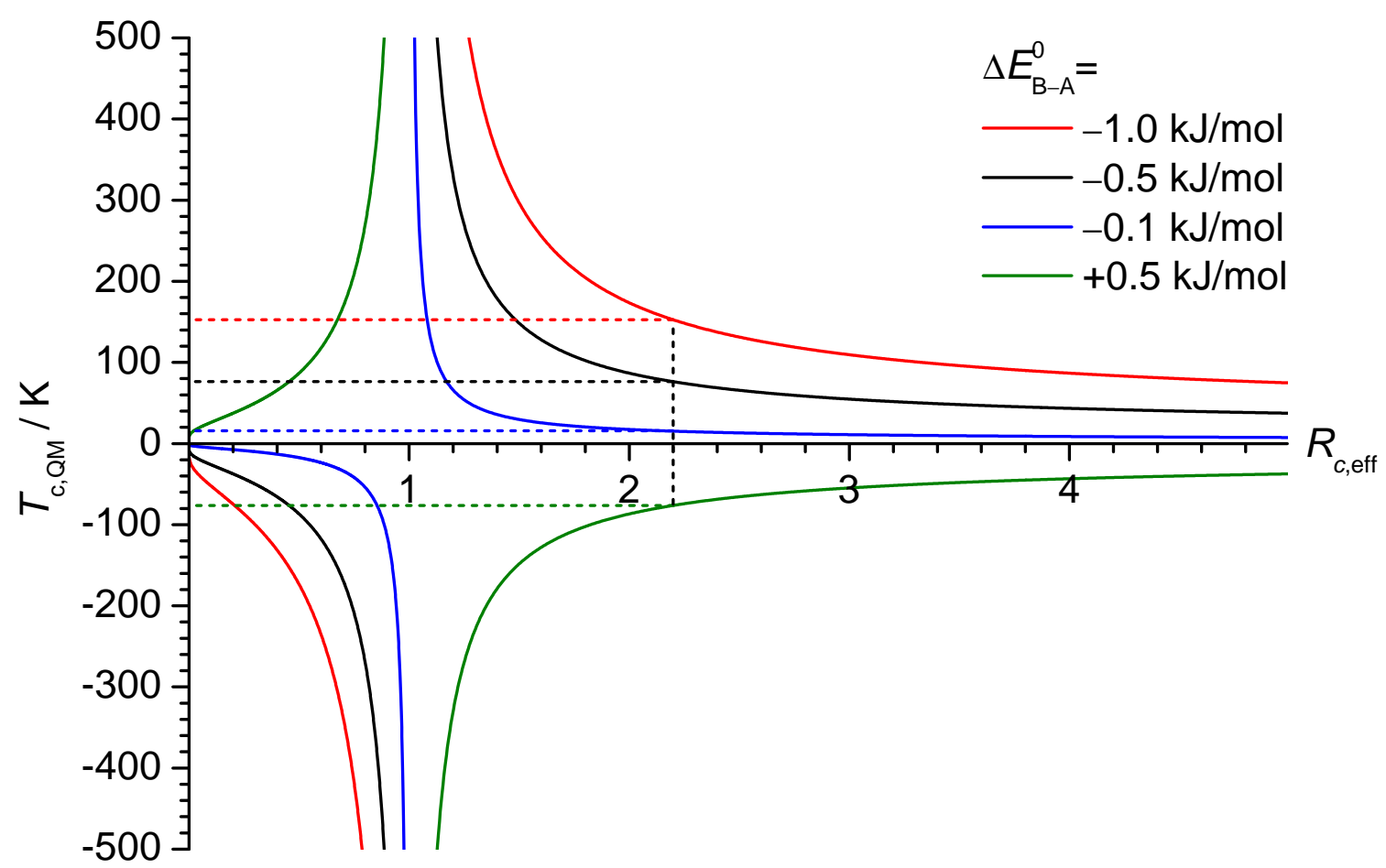

Abbildung 2.3: Verlauf der Konformationstemperatur $T_{\mathrm{c}, \mathrm{QM}}$ in Abhängigkeit vom effektiven Konzentrationsverhältnis $R_{c, \text { eff }}$ für verschiedene Energieunterschiede $\Delta E_{\mathrm{B}-\mathrm{A}}^{0}$.

Zum besseren Verständnis soll der Zusammenhang zwischen der Konformationstemperatur und den darin eingehenden Größen im Folgenden veranschaulicht werden. Dazu wird das Produkt der Intensitäts-, IR-Bandenstärken- und Entartungsfaktorenverhältnisse zunächst zu einem effektiven Konzentrationsverhältnis $R_{c \text {,eff }}$ zusammengefasst:

$$
R_{c, \text { eff }}=\frac{I_{\mathrm{B}}}{I_{\mathrm{A}}} \frac{A_{\mathrm{A}}}{A_{\mathrm{B}}} \frac{g_{\mathrm{A}}}{g_{\mathrm{B}}}
$$

Der Verlauf der Konformationstemperatur $T_{\mathrm{c}, \mathrm{QM}}$ in Abhängigkeit vom Verhältnis $R_{c, \text { eff }}$ ist in Abbildung 2.3 für verschiedene Energieunterschiede $\Delta E_{\mathrm{B}-\mathrm{A}}^{0}$ dargestellt. Deutlich sichtbar ist die Polstelle bei $R_{c \text {,eff }}=1$. Für Energieunterschiede $\Delta E_{\mathrm{B}-\mathrm{A}}^{0}<0$ (Konformer B stabiler) verlaufen die Kurven für $R_{c, \text { eff }}<1$ (weniger Konformer B) dazu zunächst im negativen Konformationstemperaturbereich, liegen auf der anderen Seite der Polstelle für $R_{c, \text { eff }}>1$ (mehr Konformer B) dann jedoch im positiven Temperaturbereich (rote, schwarze und blaue Kurve). Für einen Energieunterschied $\Delta E_{\mathrm{B}-\mathrm{A}}^{0}>0$ drehen sich die Vorzeichen für die Konformationstemperatur entsprechend um (grüne Kurve). Verschiedene Energieunterschiede gleichen Vorzeichens führen zu unterschiedlichen vorhergesagten Konformationstemperaturen $T_{\mathrm{c}, \mathrm{QM}}$ beim gleichen effektiven Konzentrationsverhältnis. Dies kann am Beispiel eines effektiven Konzentrationsverhältnisses von 2.2 (senkrechte schwarze gestrichelte Linie) verdeutlicht werden. Bei einem vorhergesagten Energieunterschied von 
$-1.0 \mathrm{~kJ} \cdot \mathrm{mol}^{-1}$ würde dies zu einer Konformationstemperatur von etwa $152 \mathrm{~K}$ führen und damit für die O/C-Waagensysteme beispielsweise oberhalb des erwarteten Bereichs von 20-100 K liegen (rot). Ein Energieunterschied von $-0.5 \mathrm{~kJ} \cdot \mathrm{mol}^{-1}$ liefert eine Konformationstemperatur von $76 \mathrm{~K}$ und läge damit mitten im erwarteten Bereich (schwarz), wohingegen ein Energieunterschied von $-0.1 \mathrm{~kJ} \cdot \mathrm{mol}^{-1}$ mit einer Konformationstemperatur von $15 \mathrm{~K}$ unterhalb des erwarteten Bereichs angesiedelt wäre (blau). Obwohl alle drei Energieunterschiede also das richtige Vorzeichen aufweisen, kann hier quantitativ zwischen zum Experiment passenden $\Delta E_{\mathrm{B}-\mathrm{A}}^{0}$ und zu kleinen bzw. zu großen Werten unterschieden werden. Ein falsches Vorzeichen wie im Fall von $+0.5 \mathrm{~kJ} \cdot \mathrm{mol}^{-1}$ führt, wie bereits beschrieben, zu einer negativen Konformationstemperatur (grün) und damit zu einem unphysikalischen Ergebnis.

Anhand des Kurvenverlaufs können jedoch auch einige Probleme der Auswertung über die Konformationstemperatur verdeutlicht werden. So liegt für ein effektives Konzentrationsverhältnis von 1 die bereits erwähnte Polstelle vor. Für untersuchte Konformerengleichgewichte, bei denen nahezu gleich viele Konformere A und B vorliegen, wird die Methode aufgrund des steilen Kurvenverlaufs also unzuverlässig. Liegen unter Berücksichtigung der Entartungsfaktoren exakt gleich viele Konformere vor, kann keine Konformationstemperatur bestimmt werden, da die Konformerenverteilung dann temperaturunabhängig immer gleich ist. Ähnliches gilt für einen berechneten Energieunterschied von $0 \mathrm{~kJ} \cdot \mathrm{mol}^{-1}$. In diesem Fall ist die über Gleichung 2.15 berechnete Konformationstemperatur ebenfalls 0. Sehr kleine berechnete Energieunterschiede (z. B. $0.01 \mathrm{~kJ} \cdot \mathrm{mol}^{-1}$ ) führen dadurch oft zu unrealistisch kleinen Konformationstemperaturen. Ein weiteres Problem tritt auf, wenn im Experiment nur ein Konformer beobachtet wird. In diesem Fall geht das Konzentrationsverhältnis gegen 0 bzw. $\infty$, sodass die Konformationstemperatur nicht mehr sauber definiert ist.

Darüber hinaus flacht die Kurve mit zunehmendem Abstand von der Polstelle zunehmend ab. Ausgehend von einem symmetrischen Fehler in $R_{c \text {,eff }}$ führt dies zu sehr asymmetrischen Fehlerbalken für $T_{\mathrm{c}, \mathrm{QM}}$. Eine Berechnung des Fehlers für $T_{\mathrm{c}, \mathrm{QM}}$ über eine Fehlerfortpflanzung nach GAUß oder nach dem Größtfehler ist also nicht sinnvoll. Stattdessen werden für die Fehlerbetrachtung in $T_{\mathrm{c}, \mathrm{QM}}$ Grenzwerte verwendet. Dazu werden mit dem für $R_{c, \text { eff }}$ ermittelten Fehler ein minimales und ein maximales effektives Konzentrationsverhältnis berechnet. Mit diesen minimalen und maximalen Verhältnissen werden dann die Grenzwerte für $T_{\mathrm{c}, \mathrm{QM}}$ bestimmt. In die Fehlerbetrachtung fließt dabei nur der experimentelle Fehler für das beobachtete Intensitätsverhältnis ein (s. Kap. 2.3.3). Mögliche Fehler in der berechneten Energie, den berechneten Bandenstärken und den sich aus der Symmetrie ergebenden Entartungsfaktoren gehen zulasten der Theorie und sorgen unter Umständen für einen $T_{\mathrm{c}, \mathrm{QM}}$-Wert außerhalb des Erwartungsbereichs. Dies verdeutlicht noch einmal, dass in diesem Benchmarking-Ansatz die Last möglichst weit auf die Seite der Theorie gelegt wird. ${ }^{[80]}$

Eine Besonderheit bei den $T_{\mathrm{c}, \mathrm{QM}}$-Grenzwerten ergibt sich durch die Polstelle im Kurvenverlauf. Liegt die Polstelle im Fehlerbereich von $R_{c, \text { eff }}$, ergibt sich für einen $\operatorname{der} T_{\mathrm{c}, \mathrm{QM}^{-}}$ 


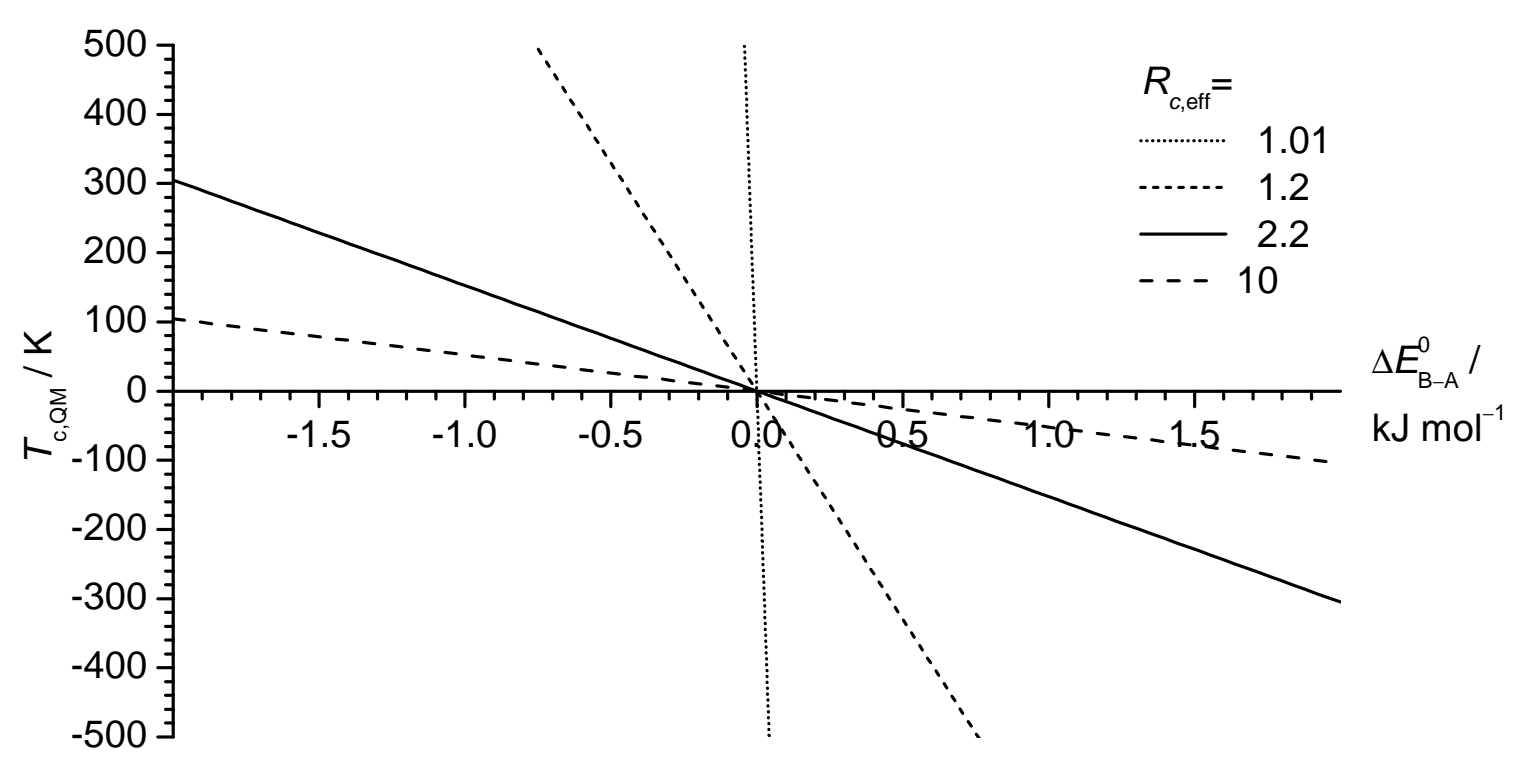

Abbildung 2.4: Verlauf der Konformationstemperatur $T_{\mathrm{c}, \mathrm{QM}}$ in Abhängigkeit vom berechneten Energieunterschied $\Delta E_{\mathrm{B}-\mathrm{A}}^{0}$ für verschiedene effektive Konzentrationsverhältnisse $R_{c, \text { eff. }}$.

Grenzwerte ein Vorzeichenwechsel. Eine maximale Konformationstemperatur kann dann also z. B. einen negativen Wert annehmen. Da der Grenzwert zuvor aber die Polstelle und damit $\pm \infty$ durchlaufen hat, wird in diesem Fall $+\infty$ als Grenzwert verwendet, bei Durchlaufen der Polstelle in anderer Richtung entsprechend dann $-\infty$.

Wie der steile Kurvenverlauf für $R_{c, \text { eff }}$-Werte nah an 1 und der flache Verlauf für Werte, die weiter von 1 entfernt sind, in Abbildung 2.3 bereits zeigt, eignen sich Konformerengleichgewichte mit eher ausgewogenen Verhältnissen besser für ein diskriminierendes Benchmarking als sehr einseitige Verhältnisse. Dies wird noch einmal deutlich, wenn $T_{\mathrm{c}, \mathrm{QM}}$ gegen den berechneten Energieunterschied $\Delta E_{\mathrm{B}-\mathrm{A}}^{0}$ aufgetragen wird (s. Abb. 2.4). Während die Konformationstemperatur sich bei einem Verhältnis nahe an 1, also z. B. von 1.01 (gepunktete Linie), äußerst schnell mit dem Energieunterschied ändert und nur für einen sehr schmalen $\Delta E_{\mathrm{B}-\mathrm{A}}^{0}$-Bereich realistische $T_{\mathrm{c}, \mathrm{QM}}$-Werte annimmt, nimmt der Betrag der Steigung der dargestellten Geraden mit zunehmendem Abstand zu $R_{c \text {,eff }}=1$ immer weiter ab. Für das bereits zuvor betrachtete Konzentrationsverhältnis von 2.2 (durchgezogene Linie) ergibt sich damit ein Bereich von $-0.66 \mathrm{bis}-0.13 \mathrm{~kJ} \cdot \mathrm{mol}^{-1}$, in dem die Konformationstemperatur zwischen 20 und $100 \mathrm{~K}$ liegt. In diesem Fall sind also berechnete Energieunterschiede in einem Intervall von $0.5 \mathrm{~kJ} \cdot \mathrm{mol}^{-1}$ mit den experimentellen Ergebnissen vereinbar. Liegt hingegen ein effektives Konzentrationsverhältnis von 10 vor (lange Striche), wird dieses Intervall mit $1.5 \mathrm{~kJ} \cdot \mathrm{mol}^{-1}$ in etwa dreimal so breit $(-1.91$ bis $\left.-0.38 \mathrm{~kJ} \cdot \mathrm{mol}^{-1}\right)$. So kann weniger gut zwischen erfolgreichen und nicht-erfolgreichen Rechenmethoden unterschieden werden. Auch in Abbildung 2.4 wird noch einmal deutlich, dass eine falsche Energiesequenz zu negativen Konformationstemperaturen führt.

Zusammenfassend wurden drei Ansätze vorgestellt, mit denen berechnete relative 
Energien zweier Konformere $\mathrm{A}$ und $\mathrm{B}$ anhand der Intensitätsverhältnisse konformerspezifischer Schwingungsbanden einem Benchmark unterzogen werden können. Jeder dieser Ansätze bringt spezifische Vor- und Nachteile mit sich. So ermöglicht das Benchmarking über Boltzmann-Plots $\mathrm{zu}$ einem gewissen Grad die getrennte Beurteilung berechneter Energieunterschiede und Bandenstärkenverhältnisse, benötigt jedoch immer eine Kombination zweier Werte (berechneter Energieunterschied $\Delta E_{\mathrm{B}-\mathrm{A}}^{0}$ und A-Konformerenanteil $x_{\mathrm{A}}$ ), um die Leistungsfähigkeit der Rechnung anhand der experimentellen Daten zu referenzieren. Die Bestimmung eines experimentellen Energieunterschieds liefert dagegen eine einzelne Größe, auf Basis derer das Benchmarking des entsprechenden quantenchemisch berechneten Energieunterschieds erfolgen kann. In die Ermittlung des experimentellen Energieunterschieds gehen keinerlei berechnete Größen ein, sodass ein rein experimenteller Referenzwert zur Verfügung steht. Die Kehrseite dieses Vorgehens ist jedoch, dass relativ grobe Schätzungen für die vorliegende Konformationstemperatur und das Bandenstärkenverhältnis der betrachteten Konformerbanden erfolgen müssen. Hierdurch ergibt sich ein relativ großer experimenteller Zielbereich. Zudem sind unter Umständen sowohl für die BolTZMANN-Plots als auch für die Bestimmung eines experimentellen Energieunterschieds getrennte Betrachtungen für verschiedene Konformerensymmetrien nötig. Einen gegenläufigen Ansatz verfolgt das Benchmarking über die Konformationstemperatur $T_{\mathrm{c}, \mathrm{QM}}$. Hier werden möglichst viele Daten aus der zu benchmarkenden quantenchemischen Rechnung herangezogen (schwingungsnullpunktskorrigierter Energieunterschied, Bandenstärkenverhältnisse, Entartungsfaktoren) und die experimentell am ungenauesten bestimmbare Größe, $T_{\mathrm{c}}$, wird als Zielbereich verwendet. Dies ermöglicht prinzipiell eine stärkere Diskrimination der Leistungsfähigkeit quantenchemischer Methoden als die Ermittlung des experimentellen Energieunterschieds, kann aber zu unbemerkter Fehlerkompensation zwischen den verschiedenen in die Auswertung eingehenden berechneten Größen führen. Zudem versagt die Methode bei experimentellen (effektives Konzentrationsverhältnis $R_{c, \text { eff }}=1$ ) oder berechneten Energieunterschieden von 0 und in Fällen, in denen nur ein Konformer im Experiment nachgewiesen werden konnte. Die Auswahl des jeweils genutzten Benchmark-Ansatzes sollte somit aufgrund der individuellen Vorteile, Nachteile und Grenzen an die Gegebenheiten des jeweiligen Systems und die Ziele der betreffenden Untersuchung angepasst werden.

\subsubsection{Fehlerbestimmung der experimentellen Intensitätsverhältnisse}

Sollen, wie im vorangegangenen Kapitel 2.3.2 beschrieben, quantenchemische Methoden unter Verwendung der BoLTZMANN-Verteilung mit experimentellen Daten verglichen werden, ist die entscheidende experimentelle Größe das gemessene Intensitätsverhältnis $I_{\mathrm{B}} / I_{\mathrm{A}}$ zweier Banden der betrachteten Konformere A und B. Im Rahmen der vorliegenden Arbeit werden dafür wasserstoffverbrückte Dimere betrachtet und es werden die Inten- 
sitäten der jeweiligen Donor-OH-Streckschwingungsbande verwendet. Für einen aussagekräftigen Benchmark ist es dabei wichtig, eine realistische Fehlerabschätzung für diese Messgrößen durchzuführen. Das Vorgehen zur Ermittlung des experimentellen Fehlers im Intensitätsverhältnis, $\Delta\left(\frac{I_{B}}{I_{A}}\right)$, soll im Folgenden erläutert werden.

Der Inhalt des vorliegenden Kapitels wurde in weiten Teilen im Rahmen des elektronischen Zusatzmaterials (Supplementary Material (IR)) von Ref. [80] in englischer Sprache veröffentlicht und wird im Folgenden teils als wörtliche deutsche Übersetzung wiedergegeben (Hinweis zum Urheberrecht: „Reprinted from f. Chem. Phys. 2020, 152, 164303, ${ }^{[80]}$ with the permission of AIP Publishing.").

Für jede Kombination aus Donor- und Akzeptormolekülen werden in der Regel mehrere Spektren bei verschiedenen Donor- und Akzeptorkonzentrationen aufgenommen. Jedes einzelne Spektrum $j$ besteht wiederum aus zahlreichen (meist 100-1000) zusammengemittelten Einzelscans, die bei identischen experimentellen Bedingungen aufgezeichnet wurden. Für die Auswertung wird zunächst in jedem Spektrum $j$ das Intensitätsverhältnis der beiden betrachteten Donor-OH-Streckschwingungsbanden der Konformere A und B bestimmt. Dazu werden das Verhältnis der Bandenintegrale (Integrationsmethode B in der Bruker Opus Software) sowie das Verhältnis der Peakhöhen (Differenz zwischen maximaler Absorbanz und Absorbanz der umgebenden Grundlinie) gebildet. Beide Verhältnisse werden je zweimal gebildet, z. B. durch zwei unabhängige Forschende oder durch Verwendung unterschiedlicher Integralgrenzen (bspw. engere Grenzen für Verhältnis 1, weitere Grenzen für Verhältnis 2) bzw. Grundlinienabsorbanzen (bspw. Absorbanzwert bei Rauschmaximum für Verhältnis 3, Absorbanzwert bei Rauschminimum für Verhältnis 4). Somit ergeben sich vier Intensitätsverhältnisse pro Spektrum. Das arithmetische Mittel dieser vier Verhältnisse, $\overline{\left(I_{\mathrm{B}} / I_{\mathrm{A}}\right)_{j}}$, wird als Intensitätsverhältnis des Spektrums $j$ verwendet. Um den Fehler dieses Intensitätsverhältnisses zu bestimmen, werden zunächst der statistische Fehler $s_{\text {stat }, j}$ und der Signal-zu-Rauschfehler $s_{\text {rausch }, j}$ berechnet. Für $s_{\text {stat }, j}$ wird die Standardabweichung des Mittelwerts inklusive des STUDENT'schen Faktors $s_{n, j}$ für das 95\%-Konfidenzintervall einer zweiseitigen Verteilung verwendet:

$$
s_{\text {stat }, j}=\frac{\sigma_{j}}{\sqrt{n_{j}}} \cdot s_{n, j}=\sqrt{\frac{\sum_{i=1}^{n}\left(\left(\frac{I_{\mathrm{B}}}{I_{\mathrm{A}}}\right)_{i, j}-\overline{\left(\frac{I_{\mathrm{B}}}{I_{\mathrm{A}}}\right)_{j}}\right)^{2}}{n_{j}\left(n_{j}-1\right)}} \cdot s_{n, j}
$$

In der Gleichung bezeichnet $n_{j}$ die Zahl der individuellen Intensitätsverhältnisse $\left(I_{\mathrm{B}} / I_{\mathrm{A}}\right)_{i, j}$ dieses Spektrums und $\sigma_{j}$ ist die Standardabweichung einer Stichprobe der Grundgesamtheit.

Für den Rauschfehler $s_{\text {rausch,j }}$ werden nur die Peakhöhen $S_{\mathrm{A}, k, j}$ und $S_{\mathrm{B}, k, j}$, wie sie in den beiden Durchgängen $k=1,2$ (durch zwei unabhängige Forschende oder durch Verwendung zwei unterschiedlicher Grundlinienabsorbanzen, s. oben) bestimmt wurden, berücksichtigt. Um den Fehler zu berechnen, werden die arithmetischen Mittel der jeweils zwei Peakhöhen der beiden betrachteten Banden, $\overline{S_{\mathrm{A}, j}}$ und $\overline{S_{\mathrm{B}, j}}$, genutzt. Das Rauschlevel 
$N_{j}$ des Spektrums (Wurzel der mittleren quadratischen Abweichung, engl. „root meansquare error", RMS-Fehler, mit der Opus-Software in einem angrenzenden analytfreien Spektralbereich bestimmt) dient als Fehler der Peakhöhen. Der Rauschfehler $s_{\text {rausch, } j}$ wird dann über eine GAUß'sche Fehlerfortpflanzung berechnet:

$$
\begin{aligned}
s_{\text {rausch }, j} & =\sqrt{\left(\frac{\partial}{\partial \overline{S_{\mathrm{B}, j}}}\left(\frac{\overline{S_{\mathrm{B}, j}}}{\overline{S_{\mathrm{A}, j}}}\right) \cdot N_{j}\right)^{2}+\left(\frac{\partial}{\partial \overline{S_{\mathrm{A}, j}}}\left(\frac{\overline{S_{\mathrm{B}, j}}}{\overline{S_{\mathrm{A}, j}}}\right) \cdot N_{j}\right)^{2}} \\
& =\sqrt{\left(\frac{N_{j}}{\overline{S_{\mathrm{A}, j}}}\right)^{2}+\left(\frac{\overline{S_{\mathrm{B}, j}} \cdot N_{j}}{\overline{S_{\mathrm{A}, j}}}\right)^{2}}
\end{aligned}
$$

Als Fehler $s_{j}$ des Intensitätsverhältnisses $\left(I_{\mathrm{B}} / I_{\mathrm{A}}\right)_{j}$ im Spektrum $j$ wird dann der größere der beiden Fehler $s_{\text {stat }, j}$ und $s_{\text {rausch, } j}$ verwendet.

Um möglichst genaue experimentelle Werte für das Intensitätsverhältnis zu erhalten, wurden teilweise mehrere Spektren derselben Donor-Akzeptor-Kombination bei gleichen oder ähnlichen Bedingungen gemessen. Zur Bestimmung der besten experimentellen Schätzung für das Intensitätsverhältnis $I_{\mathrm{B}} / I_{\mathrm{A}}$ kann dann der mit der inversen Varianz gewichtete Mittelwert der aus den einzelnen Spektren $j$ ermittelten Werte $\overline{\left(I_{\mathrm{B}} / I_{\mathrm{A}}\right)_{j}}$ verwendet werden. Mit $m$ als Zahl der zum finalen Wert beitragenden Spektren ergibt sich:

$$
\frac{I_{\mathrm{B}}}{I_{\mathrm{A}}}=\frac{\sum_{j=1}^{m} \overline{\left(\frac{I_{\mathrm{B}}}{I_{\mathrm{A}}}\right)_{j}} \cdot \frac{1}{s_{j}^{2}}}{\sum_{j=1}^{m} \frac{1}{s_{j}^{2}}}
$$

Der Fehler $s$ des finalen Intensitätsverhältnisses kann dann über die folgende Gleichung berechnet werden:

$$
s=\sqrt{\frac{1}{\sum_{j=1}^{m} \frac{1}{s_{j}^{2}}}}
$$

Für die Darstellung im Text und in den Tabellen der Auswertung wird der Fehler im Allgemeinen auf eine signifikante Stelle aufgerundet. 


\section{Kapitel 3}

\section{Gratin-Jet-FTIR-Spektroskopie}

Die Gratin-Jet-Spektroskopie (vom englischen Ausdruck "gas-recycling atom-economic infrared jet spectroscopy“) trägt eine der Hauptneuerungen gegenüber dem Filet-Jet (Kap. 2.2) im Namen: die Möglichkeit, die Gasmischung nach der Expansion ins Reservoir zurückzuleiten und wiederzuverwenden (gas-recycling), die zu einer deutlicheren Verringerung des Substanzverbrauchs (atom-economic) und damit zu einem nachhaltigeren Messkonzept führt. Dieses Messkonzept und der generelle Aufbau des neuen Gratin-Jets sollen im vorliegenden Kapitel erstmals vorgestellt und charakterisiert werden (Kapitel 3.1-3.6). Ein Vergleich der Eigenschaften und der Leistungsfähigkeit des Gratin- mit dem Filet-Jet erfolgt im Kapitel 3.7.

Das Grundprinzip der Gratin- (s. Abb. 3.1) entspricht dem der Filet-Jet-Spektroskopie. Das Gasgemisch, bestehend aus einem geringen Anteil von 1-2 Analytsubstanzen (Konzentrationen jeweils ca. $0.01-1 \%$ ) in einem Trägergas wird im Reservoir vorgehalten und durch die Schlitzdüse gepulst ins Puffervolumen expandiert. Die dabei entstehende Überschallexpansion wird von einem schwach fokussierten IR-Strahl gekreuzt und schwingungsspektroskopisch untersucht. Beim darauf folgenden Abpumpen des Gases aus dem Puffervolumen wird jedoch der konzeptionelle Hauptunterschied zum Filet-Jet deutlich: Das Gasgemisch wird nach der Verdichtung durch den Wälzkolbenpumpstand nicht verworfen, sondern zurück ins Reservoir geleitet und kann mehrfach neu expandiert werden (Kap. 3.1). Durch die Gasrückführung und den damit verbundenen Verlust der Notwendigkeit, kontinuierlich Gasmischungen im richtigen Konzentrationsverhältnis nachzuliefern, konnte das Verfahren zur Substanzbefüllung vereinfacht werden (Kap. 3.2). Auch abseits des Gas-Recycling-Konzepts wurden mehrere weitere Detailverbesserungen implementiert. So stehen zum Evakuieren des Vakuumsystems eine Turbomolekularpumpe und zum Ausheizen bzw. für den Messbetrieb bei erhöhter Temperatur eine Heizanlage zur Verfügung (Kap 3.3). Darüber hinaus wurde der Absorptionspfad durch die Erhöhung der Düsenlänge auf $700 \mathrm{~mm}$ gesteigert und die Expansionsgeometrie wurde optimiert, sodass geringere lokale Drücke auf der Niederdruckseite der Düse erreicht werden und die Verwendung eines kleineren Puffervolumens $\left(4 \mathrm{~m}^{3}\right.$ am Gratin- gegenüber 12-23 $\mathrm{m}^{3}$ am Filet-Jet) möglich wird (Kap 3.4). Außerdem kommt ein neues Spektrometer zum Einsatz, bei dem u. a. eine Parabolspiegeloptik zur IR-Strahlfokussierung verwendet werden 


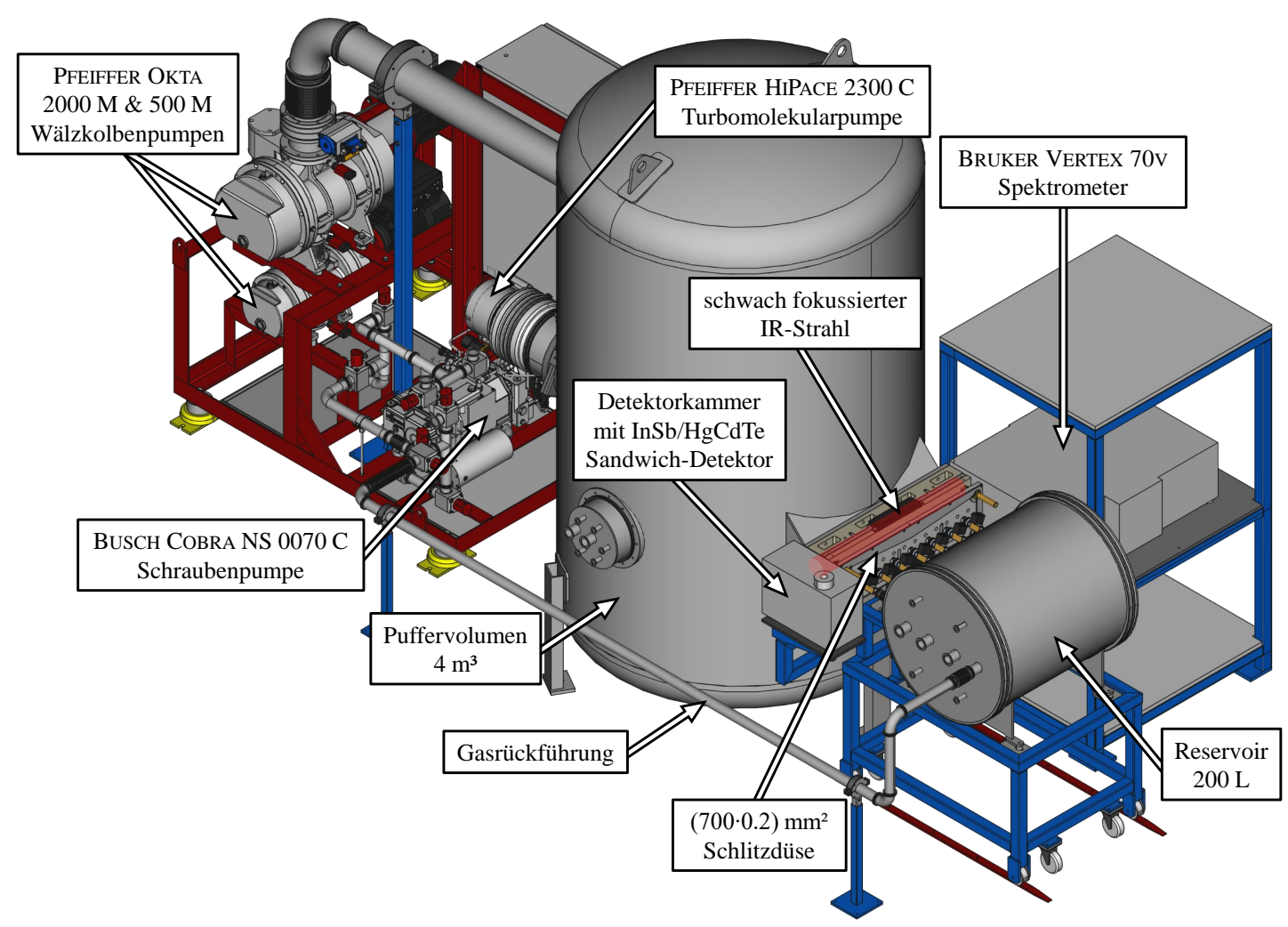

Abbildung 3.1: Gesamtansicht des Gratin-Jets. Das Gasgemisch wird in das Reservoir gefüllt und durch die $(700 \cdot 0.2) \mathrm{mm}^{2}$ große Schlitzdüse ins Puffervolumen expandiert. Die Überschallexpansion wird mit einem BRUKer Vertex 70v FTIR-Spektrometer untersucht. Anschließend wird das Gas durch zwei Wälzkolben- und eine Schraubenpumpe rekomprimiert und ins Reservoir zurückgeleitet. Bearbeitung von Ref. [247] (lizenziert unter CC BY 4.0).

kann und das einen Sandwich-Messbetrieb mit einem $\mathrm{HgCdTe/InSb-Detektor} \mathrm{ermöglicht}$ (Kap. 3.5). Zur Zeit wird der Kreislaufbetrieb allerdings noch durch sich im Laufe der Messzeit anreichernde Verunreinigungen wie Wasser beeinträchtigt (Kap. 3.6).

\subsection{Das Recycling-Konzept}

Das Grundprinzip eines Gasrecycling-Konzepts für die Jet-FTIR-Spektroskopie ist denkbar simpel: Der Auslass des Pumpstandes, der das Gas aus dem Puffervolumen abpumpt und wieder verdichtet, wird mit dem Reservoir verbunden, sodass das Gasgemisch nach der Expansion durch die Düse und der Kompression durch die Pumpen wieder zurück ins Reservoir geleitet wird. Bei ausreichend häufiger Wiederholung dieses Zyklus aus Expansion und Rekompression ergeben sich dann deutliche Verringerungen des Gasverbrauchs im Vergleich zur Neubefüllung des Reservoirs nach jedem Düsenpuls. Ein solcher Kreis- 
laufbetrieb bringt jedoch einige Anforderungen an Leckraten und Pumpenspezifikationen mit sich, die bewirken, dass die Umsetzung nicht trivial ist:

- Das Gas darf bei der Rekompression durch die Pumpen nicht verunreinigt werden, d. h. es dürfen keine Betriebsmittel sowie Schlepp-, Sperr- oder Umgebungsgase in den Schöpfraum der Pumpen gelangen.

- Aufgrund der direkten Verbindung zwischen Pumpstand und Reservoir entspricht der Druck am Auslass der letzten Pumpstufe dem im Reservoir. Bei Änderung des Stagnationsdrucks ändert sich also auch immer der Auslassdruck an der entsprechenden Pumpe. Ein Betrieb dieser Pumpe bei Auslassdrücken von idealerweise 0-1000 mbar muss also möglich sein, damit bei verschiedenen Stagnationsdrücken unterhalb des normalerweise am Auslass anliegenden atmosphärischen Drucks gearbeitet werden kann.

- Alle Stoffe, die sich zu Beginn des Kreislaufbetriebs im Vakuumsystem befinden, verbleiben während der gesamten Messdauer darin. Ein „Destillieren“ und damit eine fortwährende Aufreinigung zumindest von schwerflüchtigen Chemikalien im Messbetrieb, wie es beim Produzieren der zu untersuchenden Gasmischung über Sättiger möglich ist und die zu schnell „sauberer“ werdenden Spektren führt, kann im Kreislaufbetrieb nicht erfolgen. Die Anforderungen an die Chemikalienreinheit sind beim Gasrecycling also höher.

- Die gleiche Problematik gilt auch für die Vakuumbehälter sowie die vakuumführenden Teile der Pumpen und der Düse: Hier möglicherweise adsorbierte Stoffe, z. B. aus vorangegangenen Messkampagnen, müssen vor Beginn des Kreislaufbetriebs möglichst gründlich entfernt werden. Ein Evakuieren der Behälter auf $\sim 10^{-2}$ mbar, wie es am Filet-Jet üblich ist, reicht für eine Apparatur mit Gasrecycling-System also nicht aus.

- Alle durch Lecks in das Vakuumsystem eindringenden Stoffe (Luft, Pumpenöl, Kühlmittel) reichern sich im Laufe der Messzeit im System an. Die Anforderungen an die Leckraten der verbauten Komponenten sind also wesentlich höher als im in dieser Hinsicht äußerst unempfindlichen „Einweg“-Betrieb.

- Es darf im System keine kalten Stellen (z. B. durch ungleichmäßige Heizleistung oder Kühlmittelkontakt) geben, an denen im Gasgemisch vorhandene (Analyt-)Substanzen auskondensieren, da ansonsten die gewünschte Konzentration während der Messung nicht erreicht wird. Dies gilt insbesondere für den Betrieb des Systems bei erhöhter Temperatur mit schwerflüchtigen Substanzen. Auch für ein möglichst effektives Ausheizen (s. Kap. 3.3) sind kalte Stellen zu vermeiden.

Unter Berücksichtigung dieser Anforderungen wurde der Gratin-Jet für den Kreislauf(oder auch Recycling-)Messbetrieb des einmal eingefüllten Gasgemisches konzipiert. Ein 
zentraler Parameter ist hierbei die Leckrate für von außen ins System eindringende Verunreinigungen. Diese muss klein genug sein, um einen lohnenswerten Kreislaufbetrieb, d. h. mindestens über die Dauer eines Messtages von $8 \mathrm{~h}$, zu ermöglichen. Zur Bestimmung der Obergrenze für eine noch hinnehmbare Leckrate wurden einige Vorabschätzungen durchgeführt (basierend auf dem Vorgehen in Ref. [248]). Dazu wurde angenommen, dass Verunreinigungen tolerierbar sind, bis ihre Konzentration auf den Wert der Analytkonzentration steigt. Als typische untere Grenze für die Analytkonzentration wurden $0.1 \%$ gesetzt. Die tolerierbare Stoffmenge hängt nun vom betrachteten Volumen und Gesamtdruck ab. Da im Kreislaufbetrieb stets das gesamte Gasgemisch ins Reservoir gepumpt wird, wurden das Volumen des Reservoirs und der beabsichtigte Stagnationsdruck verwendet. Die kleinste Stoffmenge ergibt sich mit dem kleinsten geplanten Stagnationsdruck von 400 mbar. Das benötigte Volumen des Reservoirs hängt wiederum vom Düsendurchsatz ab, da maximal 10\% des im Reservoir vorhandenen Gasgemischs bei einem Puls durch die Düse strömen sollten. Andernfalls sind die Expansionsbedingungen über die Dauer des Pulses durch das Absinken des Stagnationsdruckes nicht mehr ausreichend konstant. Da die Schlitzdüse des Gratin-Jets auf der des Filet-Jets basiert, wurde der Durchsatz $D_{\mathrm{f}}$ der Düse am Filet-Jet in Abhängigkeit von Öffnungszeit $t_{\mathrm{on}}$ und Stagnationsdruck $p_{\mathrm{s}}$ für Öffnungszeiten $t_{\text {on }}$ von 75 bis $200 \mathrm{~ms}$ und Stagnationsdrücke $p_{\mathrm{s}}$ von 0.30 bis $0.60 \mathrm{bar}$ bestimmt. Dabei wurden näherungsweise lineare Abhängigkeiten des Durchsatzes von der Düsenöffnungszeit und vom Stagnationsdruck beobachtet, sodass Gleichung 3.1 für die Anpassung verwendet wurde:

$$
D_{\mathrm{f}}=\left(a \cdot t_{\mathrm{on}}+b \cdot p_{\mathrm{s}} \cdot t_{\mathrm{on}}+c \cdot p_{\mathrm{s}}+d\right)
$$

Aus den Messdaten konnten die Parameter $a-d$ durch die Anpassung $\mathrm{zu} a=$ $-2.23 \cdot 10^{3} \mathrm{mbar} \cdot \mathrm{L} \cdot \mathrm{s}^{-1}, b=54.4 \mathrm{~L} \cdot \mathrm{s}^{-1}, c=2.07 \mathrm{~L}$ und $d=-166 \mathrm{mbar} \cdot \mathrm{L}$ bestimmt werden. Um der Erhöhung der Schlitzlänge von $600 \mathrm{~mm}$ auf $700 \mathrm{~mm}$ (s. Kap. 3.4) Rechnung zu tragen, muss Gleichung 3.1 für den Gratin-Jet um den Faktor 7/6 korrigiert werden, sodass sich für den Durchsatz $D_{\mathrm{g}}$ am Gratin-Jet Gleichung 3.2 ergibt:

$$
D_{\mathrm{g}}=\frac{7}{6}\left(a \cdot t_{\mathrm{on}}+b \cdot p_{\mathrm{s}} \cdot t_{\mathrm{on}}+c \cdot p_{\mathrm{s}}+d\right)
$$

Der maximale Durchsatz wird bei maximaler Öffnungszeit und maximalem Stagnationsdruck erreicht. Für die größten vorgesehenen Werte von $200 \mathrm{~ms}$ Öffnungszeit und 750 mbar Stagnationsdruck konnte unter Verwendung des idealen Gasgesetzes das am Gratin-Jet benötigte Reservoirvolumen damit auf $150 \mathrm{~L}$ abgeschätzt werden. Mit einem Sicherheitsaufschlag wurde deshalb ein Reservoirvolumen von $200 \mathrm{~L}$ gewählt.

Auch das nötige Puffervolumen lässt sich über die Düsendurchsatzrate bestimmen. Damit die Stoßfronten der Expansion ${ }^{[43]}$ noch außerhalb des Messbereichs der IR-Messung liegen, sollte der lokale Druck auf der Niederdruckseite der Expansion nicht mehr als 2 mbar betragen. Bei einer typischen Düsenöffnungszeit von $150 \mathrm{~ms}$ und dem größten vorgesehenen Stagnationsdruck von 750 mbar beträgt der Druck nach dem Puls bei idealer Verteilung im gesamten Volumen gemäß der Berechnung mit dem Durchsatz aus Gl. 3.2 


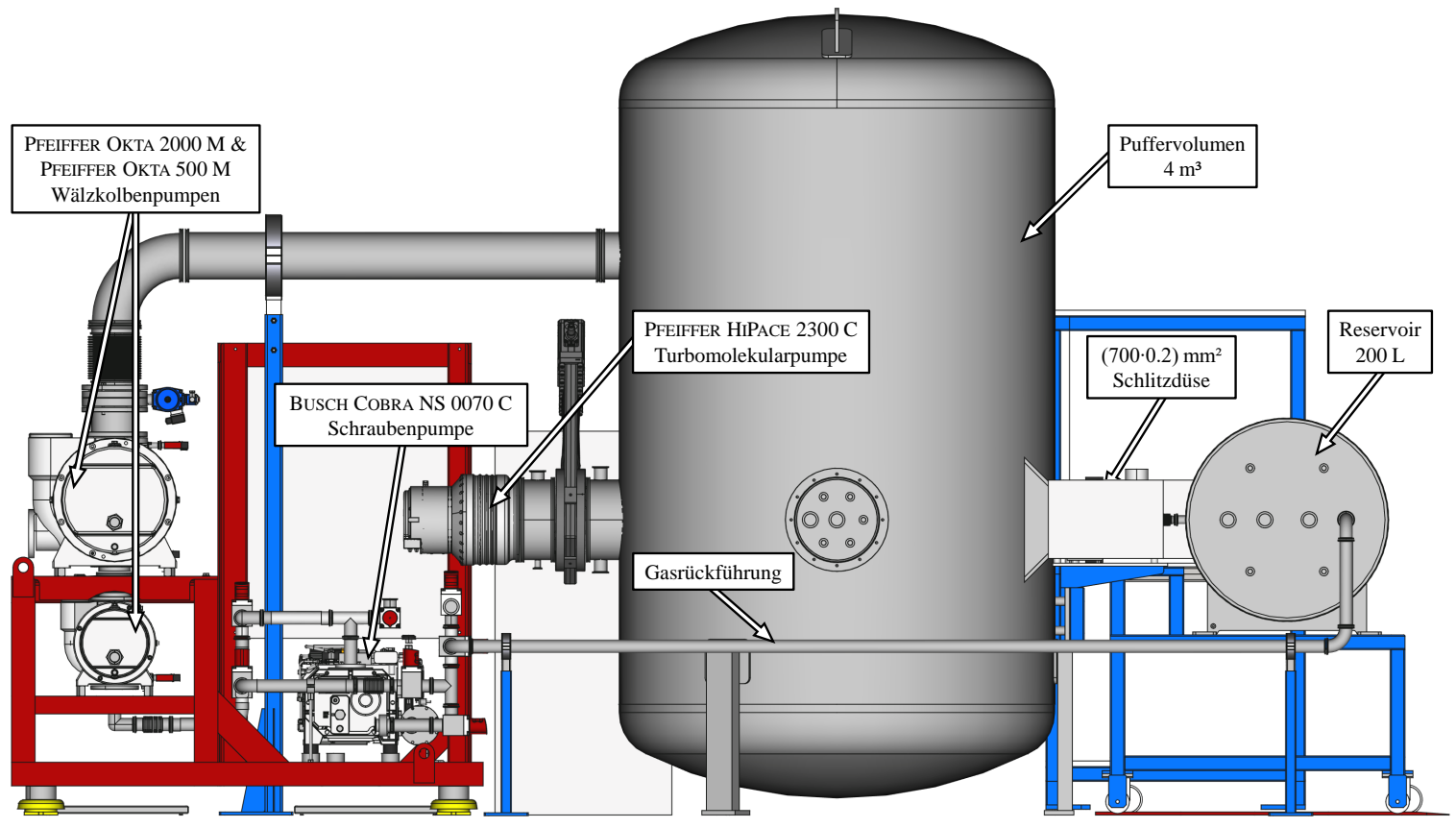

Abbildung 3.2: Seitenansicht des Gratin-Jets mit den für das Gasrecycling wichtigen Komponenten. Nach der gepulsten Expansion vom Reservoir durch die Schlitzdüse ins Puffervolumen wird das Gasgemisch von den bis zu drei Pumpstufen (Pfeiffer Okta 2000 M, Pfeiffer Oктa 500 M, Busch CoBra NS 0070 C) wieder verdichtet und über die Gasrückführung ins Reservoir zurückgeleitet. Bearbeitung von Ref. [247] (lizenziert unter CC BY 4.0).

etwa 2.1 mbar, wenn das Puffervolumen $4 \mathrm{~m}^{3}$ groß ist. Ein solches Volumen wird deshalb als noch ausreichend angesehen, wobei gewisse Einschränkungen für die Pulslänge und den Stagnationsdruck zugunsten der Kompaktheit in Kauf genommen werden.

Mit dem oben bestimmten benötigten Reservoirvolumen von $200 \mathrm{~L}$ kann abgeschätzt werden, dass bei Analytsubstanzkonzentrationen im Bereich von $0.1 \%$, einer Pumpleistung von $2000 \mathrm{~m}^{3} / \mathrm{h}$ und Stagnationsdrücken von 400-750 mbar eine Gesamtleckrate des Vakuumsystems (vakuumführende Komponenten inkl. Pumpen im Betrieb) von 1 . $10^{-3} \mathrm{mbar} \cdot \mathrm{L} \cdot \mathrm{s}^{-1} \mathrm{mehr}$ als $20 \mathrm{~h}$ Messzeit und damit mehr als 2000 Düsenpulse (gleichbedeutend mit mehr als 100 Gasrecycling-Zyklen) ermöglichen sollte, bevor die Konzentration der eindringenden Verunreinigungen die Größenordnung der Analytsubstanzkonzentrationen erreicht. Mit einer Sicherheitsmarge von einer Größenordnung wurde die maximal tolerierbare Gesamtleckrate der Gratin-Jet-Apparatur deshalb auf $1 \cdot 10^{-4} \mathrm{mbar} \cdot \mathrm{L} \cdot \mathrm{s}^{-1}$ festgelegt.

Das komplette Vakuumsystem, bestehend aus Reservoir, Puffervolumen, Gasrückführungsleitung sowie Wälzkolben- und Turbomolekularpumpstand wurde mit dieser spezifizierten Gesamtleckrate von der Firma Pfeiffer Vacuum GмвH angefertigt und geliefert. In dieses System wurde die von der Feinmechanik-Werkstatt der Fakultät für Chemie der Georg-August-Universität Göttingen gefertigte Schlitzdüse eingefügt. Die einzelnen 
Komponenten sind in Abb. 3.2 erkennbar. Im linken Bildbereich ist der mit einem roten Rahmen versehene Wälzkolbenpumpstand sichtbar, der über ein Rohr eines Durchmessers von $160 \mathrm{~mm}$ mit dem Puffervolumen (mittig) und über die Gasrückführung (40 mm Durchmesser) mit dem Reservoir (rechts) verbunden ist. Der Wälzkolbenpumpstand besteht aus drei Pumpstufen. Bei den ersten beiden Pumpstufen kommen magnetgekoppelte Wälzkolbenpumpen (Pfeiffer OKta $2000 \mathrm{M}$ und Pfeiffer OKta $500 \mathrm{M}$ ) zum Einsatz. Für die dritte Pumpstufe wird eine Schraubenpumpe (Busch CoBRa NS 0070 C) verwendet. Alle drei Pumpen kommen ohne Schlepp- oder Sperrgase aus, sodass kein Eintrag solcher Gase in das gepumpte Gasgemisch stattfindet. Die Wälzkolbenpumpen werden luftgekühlt. Die Kühlung der Schraubenpumpe erfolgt über einen externen Kühlwasser- und einen internen Kühlmittelkreislauf (Wasser/Ethylenglykol-Gemisch). In der ersten Pumpstufe wird das Gas aus dem Puffervolumen auf bis zu 10 mbar, in der zweiten Pumpstufe dann weiter auf bis zu 50 mbar verdichtet. In der dritten Pumpstufe wird das Gas abschließend auf bis zu 1000 mbar komprimiert und zum Reservoir zurückgeleitet.

Das Vakuumschema des Wälzkolbenpumpstands ist in Abbildung 3.3 dargestellt. Der Gaseinlass weist einen Durchmesser von $160 \mathrm{~mm}$ auf und kann über ein pneumatisches Schmetterlingsventil (VAT 21044-PE14-0003/0034, PfEIfFER-Modellnr. BVB 160 P) verschlossen werden. Die weiteren Ventile (Eckventile Pfeiffer AVC 040 SX, InlineVentile PfeIffer DVC040 SX) befinden sich in Rohrleitungen mit $40 \mathrm{~mm}$ Durchmesser und sind handbetätigt. Für die Drucküberwachung der einzelnen Pumpstufen stehen drei Druckaufnehmer (Pfeiffer PCR 280) bereit. Diese kombinieren einen kapazitiven Membransensor für eine gasartunabhängige Messung im Druckbereich 10-1000 mbar mit einer gasartabhängigen Pirani-Wärmeleitungsmessung im Druckbereich $5 \cdot 10^{-5}-1$ mbar. Im Übergangsbereich findet ein Mischbetrieb beider Messarten statt. Auslassseitig an der Schraubenpumpe befindet sich ein Rückschlag-Überdruckventil (ÜV, PfEIfFER PK 004499 U), das das Entstehen von unzulässig hohen Drücken im geschlossenen Kreislaufsystem verhindert. Weiterhin stehen an der Schraubenpumpe zwei Spülgaseinlässe, z. B. für Helium und Stickstoff, zur Verfügung.

Die Schraubenpumpe lässt sich durch zwei vor- bzw. nachgelagerte Ventile vom restlichen Pumpstand isolieren und kann durch eine Bypassleitung, die ebenso beidseitig absperrbar ist, umgangen werden. Dies ist nötig, da der Auslassdruck an der letzten Pumpstufe, wie weiter oben bereits beschrieben, dem Stagnationsdruck im Reservoir entspricht. Je nach Forschungsanwendung sind hier verschiedene Drücke nötig. Für die meisten Untersuchungen, in denen auf die Kühlung der Gase durch die Überschallexpansion abgezielt wird, werden Drücke zwischen 400 und 750 mbar angestrebt. Für die Untersuchung wärmerer Expansionen können aber auch geringere Stagnationsdrücke im Bereich $<400$ mbar gewünscht sein. Die Schraubenpumpe erlaubt jedoch nur Auslassdrücke zwischen 300 und 1000 mbar, da andernfalls Betriebsmittel in den Schöpfraum der Pumpe gelangen kann. Bei Verwendung aller drei Pumpstufen können somit nur Stagnationsdrücke im Bereich 300-1000 mbar genutzt werden. Soll bei geringeren Stagnationsdrücken gemessen werden, muss die Schraubenpumpe isoliert und mit dem Bypass umgangen werden, sodass nur die beiden Wälzkolbenpumpen zum Verdichten des Gasgemisches eingesetzt 


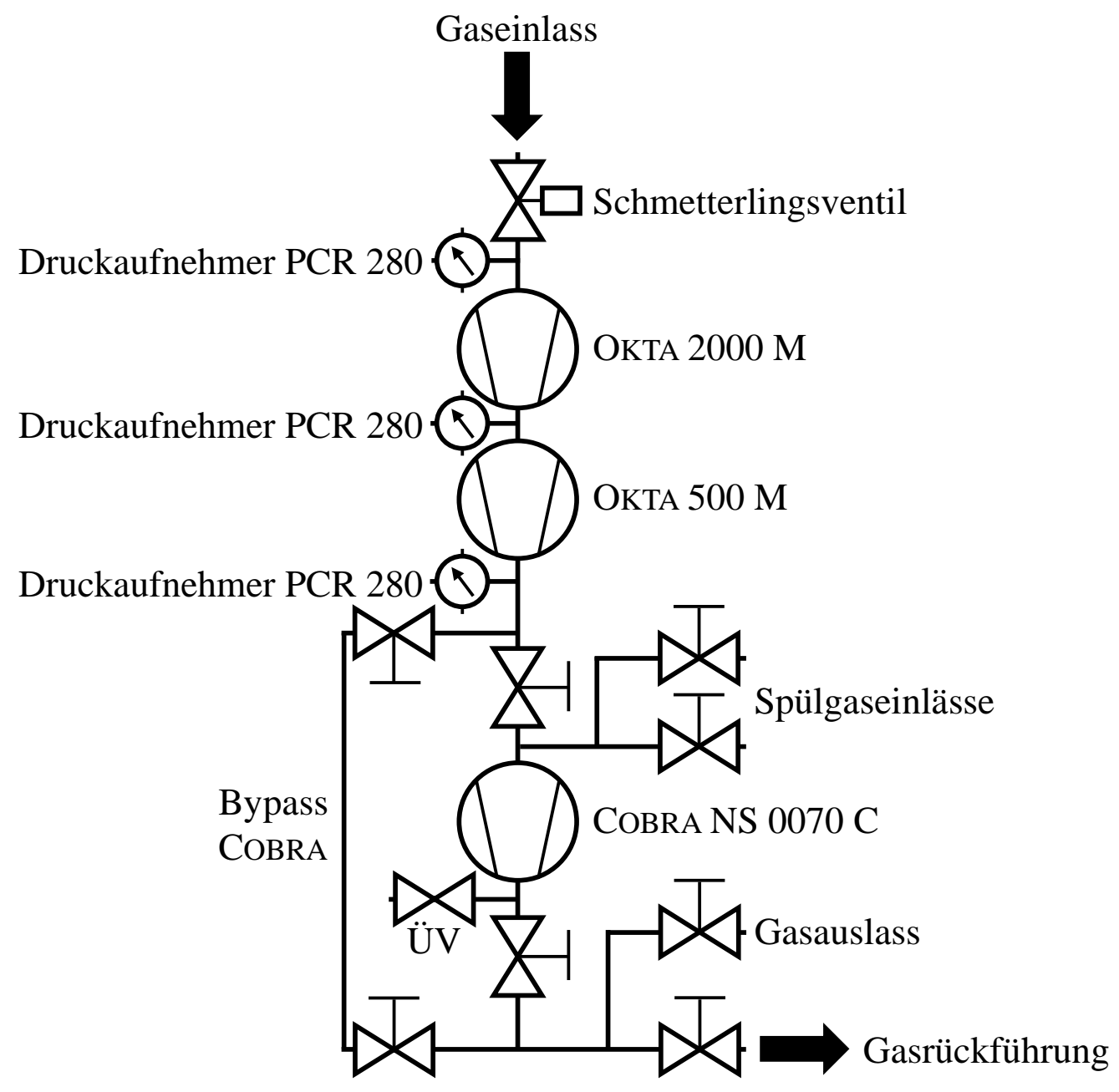

\begin{abstract}
Abbildung 3.3: Vakuumschema des dreistufigen Wälzkolbenpumpstands am Gratin-Jet. Bei den nicht gekennzeichneten Ventilen handelt es sich um handbetätigte Eck- bzw. Inline-Ventile. Die Schraubenpumpe CоBRA NS $0070 \mathrm{C}$ kann mit einem Bypass umgangen werden. Dies ermöglicht einen Kreislaufbetrieb ohne Schraubenpumpe, wenn z. B. Expansionen mit Stagnationsdrücken von $<30$ mbar untersucht werden sollen. Zum Reinigen der nicht evakuierbaren Schraubenpumpe stehen zwei Spülgaseinlässe $\left(\mathrm{He}, \mathrm{N}_{2}\right.$ ) zur Verfügung. Ein Überdruckventil (ÜV) am Auslass der Schraubenpumpe verhindert das Entstehen unzulässiger Überdrücke im geschlossenen Vakuumsystem. Die Drücke zwischen den einzelnen Pumpstufen können mit drei Druckaufnehmern überwacht werden. Die Abbildung wurde auf Basis einer Zeichnung der Firma Pfeiffer VAcuum $\mathrm{GmBH}$ angefertigt.
\end{abstract}


werden. Mit diesen ist im Dauerbetrieb allerdings lediglich eine Verdichtung auf maximal 30 mbar möglich, wodurch Stagnationsdrücke im Bereich 30-300 mbar am Gratin-Jet nur im herkömmlichen „Einweg-“, nicht aber im Kreislaufbetrieb genutzt werden können.

Ein ähnliches Problem besteht beim Evakuieren des Vakuumsystems mit dem Turbomolekularpumpstand (s. Kap. 3.3), bei dem durch Lecks eingedrungene Verunreinigungen oder zuvor gemessene Analyten möglichst restlos aus dem System abgepumpt werden sollen. Während die beiden Wälzkolbenpumpen keine Untergrenze für den minimal zulässigen auslassseitigen Druck aufweisen und somit mit der Turbomolekularpumpe evakuiert werden können, kann die Schraubenpumpe aufgrund des einzuhaltenden Mindestauslassdrucks nicht mit evakuiert werden. Im Turbo-Evakuierbetrieb muss die Schraubenpumpe also vom Vakuumsystem isoliert und getrennt gereinigt werden. Hierzu können die beiden Spülgasanschlüsse genutzt werden. Durch mehrfaches Zuleiten eines Inertgases (hier Helium oder Stickstoff) bei laufender Pumpe werden Verunreinigungen aus der Pumpe gespült.

Aufgrund des vom Hersteller befürchteten Übertritts von Betriebsmittel in den Schöpfraum der Schraubenpumpe bei zu geringen Auslassdrücken wurde überprüft, ob am unteren Ende des noch zulässigen Betriebsbereichs, bei 300-400 mbar Auslassdruck, bereits größere Mengen Öl an der Schraubenpumpe in das Gasgemisch gelangen. Dazu wurde die optische Sensoreinheit eines Rauchmelders in einem Vakuumrohr installiert und direkt am Auslass der Pumpe anstelle des Schalldämpfers angebracht. Im Laufe der ersten Betriebsmonate des Gratin-Jets konnte mit diesem Sensor jedoch kein Ölnebel nachgewiesen werden. Auch sonst ergaben sich keinerlei Hinweise auf Betriebsmitteleintrag an der Schraubenpumpe, sodass die Sensoreinheit wieder entfernt und der Schalldämpfer wieder eingebaut werden konnte.

Wie bereits erwähnt, ist das Reservoir mit einem Volumen von $200 \mathrm{~L}$ so dimensioniert, dass bei den intensivsten vorgesehenen Gaspulsen nicht mehr als 10\% der im Reservoir vorhandenen Gasmenge durch die Schlitzdüse strömen. So wird sichergestellt, dass der Stagnationsdruck über die gesamte Pulsdauer annähernd konstant bleibt, obwohl das Rekomprimieren des Gases durch den Wälzkolbenpumpstand etwa zwei Größenordnungen länger dauert als der Gaspuls durch die Düse.

Für den Pufferbehälter wurde das kleinstmögliche Volumen gewählt, um eine möglichst kleine Innenoberfläche zu erhalten. Von dieser könnten zu einem früheren Zeitpunkt adsorbierte Gase während des Kreislaufbetriebs desorbieren. Geometrisch wäre eine Kugelform angesichts dieser Problematik am günstigsten. Der Durchmesser des Puffervolumens darf jedoch maximal $1.5 \mathrm{~m}$ betragen, damit es noch zum bzw. vom vorgesehenen Aufstellungsort an- und abtransportiert werden kann. Um die mindestens nötigen $4 \mathrm{~m}^{3}$ Volumen (s. oben) trotzdem zu erreichen, wurde eine zylindrische Form gewählt (s. Abb. 3.1 auf S. 34). Die Oberflächen von Reservoir und Puffervolumen wurden glasperlfeingestrahlt, um eine hohe Festigkeit bei geringer Aufrauung zu erreichen. Das Reservoir, das Puffervolumen inkl. der Zuleitung zum Wälzkolbenpumpstand und die Gasrückführungsleitung sind mit Heizmanschetten ummantelt und können geregelt bis $100{ }^{\circ} \mathrm{C}$ geheizt werden (s. Kap. 3.3). 
Zur Umgehung der Düse, beispielsweise zum Abpumpen des Reservoirs, sind Puffervolumen und Reservoir über einen ebenfalls bis zu $100^{\circ} \mathrm{C}$ geregelt heizbaren Bypassschlauch von $40 \mathrm{~mm}$ Durchmesser (Hersteller: Horst GMBH) miteinander verbunden. Dieser kann beidseitig durch handbetätigte Eckventile (PfeIfFEr AVC 040 SX) von Puffervolumen und Reservoir abgetrennt werden.

Die Druckmessung im Vakuumsystem erfolgt über kapazitive Druckaufnehmer, die gasartunabhängig messen. Im Reservoir stehen Druckaufnehmer mit Messbereichen von 0-10 mbar (MKS BARATRON 722B11MGA2FK) sowie von 0-1000 mbar (MKS BARATRON 722B13MGA2FK) zur Verfügung. Im Puffervolumen werden Druckaufnehmer mit Messbereichen von 0-1 mbar (MKS BARATron 722B01MGA2FK) bzw. 0-100 mbar (MKS BARATRON 722B12MGA2FK) eingesetzt. Die Druckaufnehmer sind jeweils über ein Eckventil (Pfeiffer AVC 016 SX) vom Vakuumsystem abtrennbar und können so z. B. für Wartungsarbeiten entfernt werden, ohne größere Volumina belüften zu müssen.

\subsection{Gas- und Substanzbefüllung}

Wie bereits beschrieben, ist durch das Gas-Recycling-Konzept am Gratin-Jet im Messbetrieb kein kontinuierliches Nachfüllen des Reservoirs mit neu produzierter Gasmischung nötig. Deshalb werden für die Befüllung des Reservoirs mit Analytsubstanzen und Trägergasen keine Sättiger, wie sie am Filet-Jet eingesetzt werden (s. Kap. 2.2), benötigt. Für das einmalige Befüllen des Reservoirs zu Beginn einer jeden Messreihe kann auf einen manuellen Mischvorgang zurückgegriffen werden. Dazu stehen am Reservoir und Puffervolumen jeweils zwei Gasanschlüsse und eine Schleuse (s. Abb. 3.4) bereit. Bei den Schleusen handelt es sich um Rohre mit einem Durchmesser von $40 \mathrm{~mm}$, an denen mehrere Anschlüsse für Gaszufuhr, Substanzkolben und eine Vakuumpumpe zur Verfügung stehen. Die Schleusen sind über DN40-ISO-KF-Kleinflansche (Objekt 2 in Abb. 3.4) über Inline-Ventile (Pfeiffer DVC 040 SX) mit den Vakuumbehältern verbunden. Am gegenüberliegenden Ende des Rohrs befindet sich ein weiterer, verschließbarer 40-mm-Kleinflansch (Objekt 5), durch den Feststoffe in einem Schiffchen in die Schleuse eingebracht werden können. Alternativ können feste oder flüssige Stoffe in einem verschließbaren Glaskolben vorgelegt und über einen der fünf DN16-ISO-KF-Flansche (Objekt 7) mit der Schleuse verbunden werden. An beiden Schleusen ist an einen der 16-mm-Kleinflansche eine mehrstufige Wälzkolbenpumpe (Pfeiffer AdiXen ACP 40G) angeschlossen, über die die Schleusen und die damit verbundenen Glaskolben evakuiert werden können. Die Schaltung des Evakuieranschlusses erfolgt über ein handbetätigtes Eckventil (PfEIfFER AVC 016 SX). Über eine zentrale Gasverteilung (s. unten) können die Schleusen über den 6-mm-SwAGELOK-Anschluss (Objekt 4) mit verschiedenen Gasen versorgt werden.

Durch ihre Konstruktion können die Schleusen wie eine Schlenk-Linie verwendet werden. Über die zwischengeschalteten Inline-Ventile sind die Schleusen vom eigentlichen Vakuumsystem des Gratin-Jets abtrennbar und können über die Vakuumpumpe getrennt von diesem evakuiert und über die Gasanschlüsse mit verschiedenen Inertgasen gefüllt 


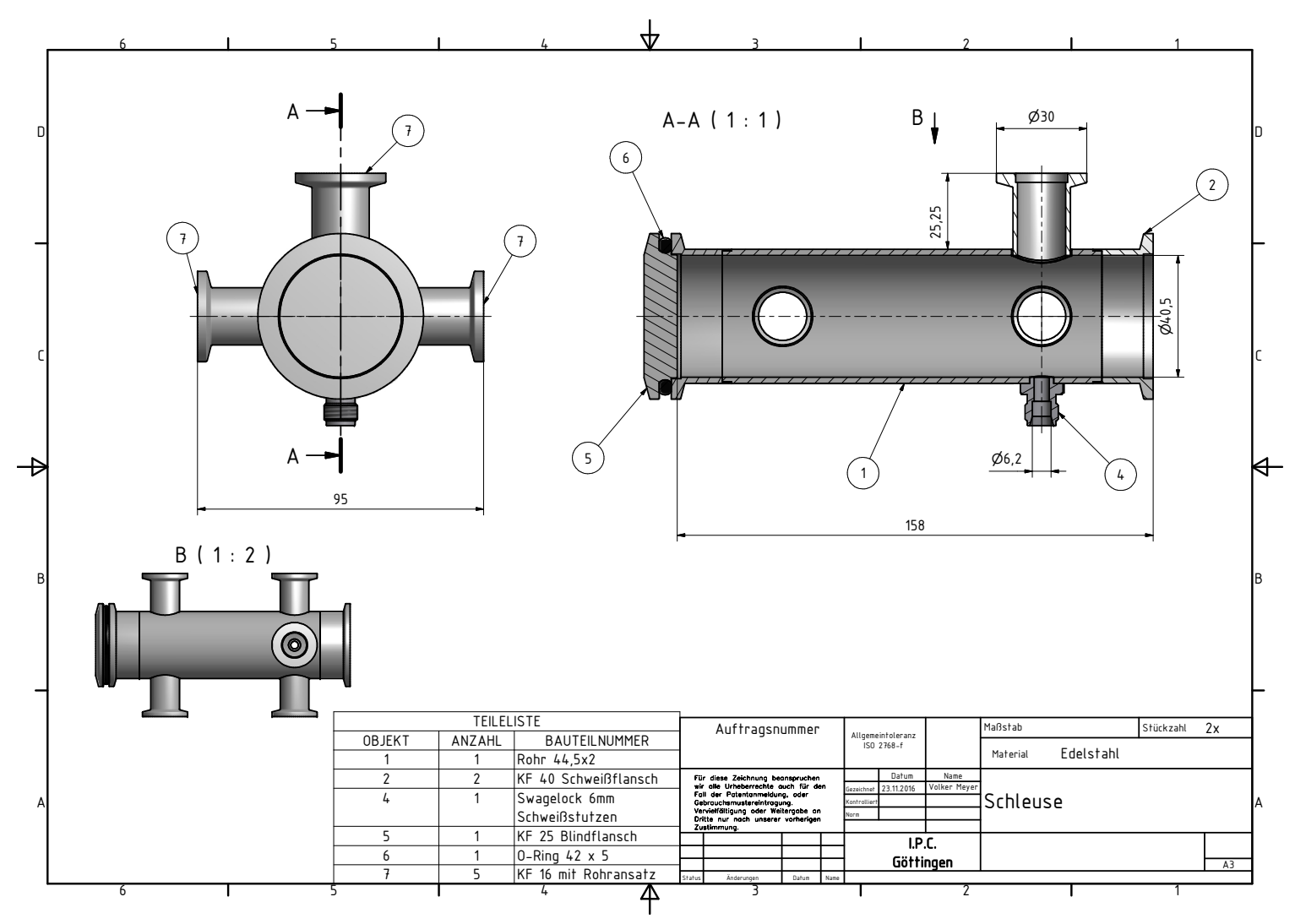

Abbildung 3.4: Technische Zeichnung der am Gratin-Jet eingesetzten Schleusen mit Frontansicht (oben links), Querschnitt (rechts) und Draufsicht (unten links) (@ R. HildEBRandT, Abdruck mit freundlicher Genehmigung).

werden. So können die in den Glaskolben vorgelegten Substanzen über die Schleusen vor Benutzung z.B. durch freeze-pump-thaw-Zyklen entgast und nach Benutzung unter Inertgas im Kolben gelagert werden. Auch ein simples Abdestillieren leichtflüchtigerer Verunreinigungen in den Chemikalien ist über die Schleuse denkbar. Die durch den Kreislaufbetrieb höheren Anforderungen an die Chemikalienreinheit (s. Kap. 3.1) können so zumindest in den meisten Fällen erreicht werden.

Die Gaszufuhr zu den Schleusen und den Anschlüssen direkt an den Vakuumbehältern erfolgt über eine zentrale Gasverteilung, die in Abbildung 3.5 schematisch dargestellt ist. An die Gasverteilung können bis zu drei Gasflaschen angeschlossen werden. Zwei der drei Kanäle sind im Normalzustand mit einer Stickstoff- und einer Helium-Flasche belegt, da diese Gase u. a. für das Spülen der Schraubenpumpe benötigt werden. Am dritten Anschluss können je nach Bedarf verschiedene Gase (Argon, Neon, Mischgasflasche, gasförmige Analytsubstanz, usw.) angeschlossen werden. Die drei Kanäle sind unabhängig voneinander betreibbar. Der Stickstoff-Kanal ist mit der Schraubenpumpe (Schraube) und dem Spektrometer verbunden, der Helium-Kanal mit der Schraubenpumpe sowie dem Re- 


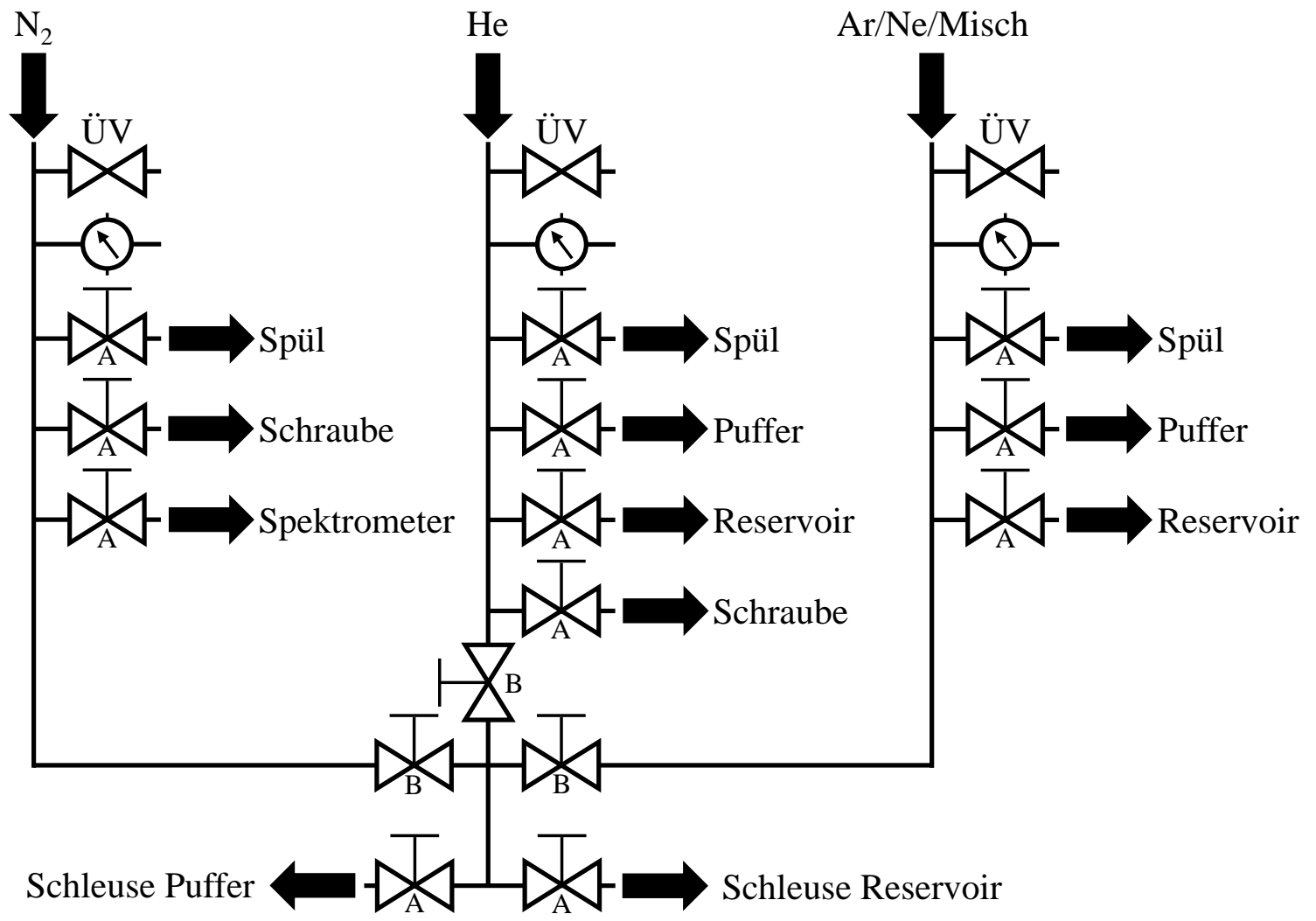

Abbildung 3.5: Schematische Darstellung der Gasverteilung am Gratin-Jet. Die drei Kanäle $\left(\mathrm{N}_{2}\right.$, $\mathrm{He}, \mathrm{Ar} / \mathrm{Ne} / \mathrm{Misch}$ ) können unabhängig voneinander betrieben werden, sind aber allesamt untereinander und mit den beiden Schleusen verbunden. Jeder Kanal ist durch ein Überdruckventil (ÜV, SwAGELOK SS-RL3S6MM), das auf etwa 1.5 bar Überdruck eingestellt ist, gesichert. Die Schaltung der verschiedenen Abgänge und Verbindungen erfolgt über handbetätigte Ventile (A: SwAGELoK SS-6P4T-MM-BK; B: SwAGELOK SS-42GS6MM).

servoir und dem Puffervolumen und der flexible Kanal nur mit dem Reservoir und dem Puffervolumen. Alle drei Kanäle lassen sich über eine gemeinsame Verbindung in die beiden Schleusen einspeisen. Die gemeinsame Verbindung kann auch dazu genutzt werden, die drei Kanäle zusammenzuschalten, sodass z. B. auch Stickstoff direkt in das Reservoir eingeleitet werden kann. Für die stets nur in eine Richtung durchflossenen Ventile (Typ $A$ in Abb. 3.5) werden Kükenhähne (SwAGELOK SS-6P4T-MM-BK) eingesetzt, wohingegen für die in beide Richtungen durchflossenen Ventile (Typ B) Kugelhähne (Swagelok SS-42GS6MM) genutzt werden. Zur Absicherung gegen das Entstehen zu hoher Drücke, z. B. aufgrund des Defekts eines Druckminderers, sind direkt hinter den Druckminderern Überströmventile (ÜV) des Typs Swagelok SS-RL3S6MM verbaut. Die Druckmessung erfolgt mit Rohrfedermanometern des Typs WikA 232.50. In jedem Kanal steht ein unbelegter Ausgang zur Verfügung, der zum Spülen genutzt werden kann (Spül). An den 
jeweils bis zu 10 m langen Leitungen zwischen Gasverteilung und Reservoir bzw. Puffervolumen sind direkt vorm Zugang zum Vakuumsystem 3/2-Wege-Kugelhähne (SwAGELOK SS-43GXS6MM) angebracht, deren unbelegte Ausgänge zum Spülen der Zuleitungen genutzt werden können. Der Zugang zum Vakuumsystem (Puffervolumen bzw. Reservoir) erfolgt dann durch ein handbetätigtes Eckventil (PFEIFfer AVC 016 SX). In den ebenfalls bis zu $10 \mathrm{~m}$ langen Zuleitungen zu den Schleusen sind keine zusätzlichen Spülausgänge nötig, da die Schleusen getrennt vom Vakuumsystem evakuiert werden können (s. oben). Hier erfolgt die Einspeisung aus der Gasverteilung deshalb über Kükenhähne (SwAGELoK SS-6P4T-MM-BK) und ohne zusätzliche 3/2-Wege-Hähne.

\subsection{Evakuieren und Heizen}

Vor Beginn des Messbetriebs mit Gasrecycling muss sichergestellt werden, dass sich möglichst keine unerwünschten Stoffe mehr im Vakuumsystem befinden, da diese andernfalls über die gesamte Messdauer im System verbleiben und damit auch permanent ungewollte Effekte auf das Spektrum ausüben können. In größeren Mengen erwartbare Verunreinigungen sind insbesondere Analytsubstanzen aus vorangegangenen Messungen und eingedrungene Luftbestandteile wie Stickstoff oder Wasser. Da in der Regel wasserstoffbrückengebundene Systeme untersucht werden und deshalb der OH-Streckschwingungsbereich im Spektrum von besonderem Interesse ist, stellt insbesondere das Vorhandensein von Wasser ein Problem für die Jet-FTIR-Spektroskopie dar.

\subsubsection{Feinevakuieren}

Um das Vakuumsystem vor Beginn der Messung möglichst gründlich zu evakuieren, stehen ein Turbomolekularpumpstand und eine Heizanlage zur Verfügung. Der Turbomolekularpumpstand besteht aus einer PfEIfFER HiPACE 2300 C Turbomolekularpumpe (sichtbar auf S. 37 in Abb. 3.2, mittig) und einer mehrstufigen Wälzkolbenpumpe (PfEIffer Adixen ACP 40G) als Vorpumpe. Die Turbomolekularpumpe weist mit einer Flanschgröße von $250 \mathrm{~mm}$ und einer Nenndrehzahl von $525 \mathrm{~Hz}$ ein Saugvermögen von $1900 \mathrm{~L} / \mathrm{s}$ für $\mathrm{N}_{2}$ bzw. $2000 \mathrm{~L} / \mathrm{s}$ für He auf und ist in horizontaler Bauweise am Pufferbehälter angebracht. Über einen Vakuumschieber (VAT SERIES 121) mit einem Öffnungsdurchmesser von $250 \mathrm{~mm}$ kann die Turbomolekularpumpe vom Pufferbehälter abgetrennt werden. Für die Druckmessung stehen zwei Drucksonden Pfeiffer PCR 280 und für den Hochvakuumbereich $\left(2 \cdot 10^{-9}-1 \cdot 10^{-2}\right.$ mbar) zusätzlich ein Kaltkathoden-Druckmesskopf (PFEIfFER IKR 251) zur Verfügung.

Im alltäglichen Messbetrieb erfolgt das Evakuieren des Vakuumsystems in der Regel über Nacht. Dazu wird das Vakuumsystem mit dem Wälzkolbenpumpstand oder der Vorpumpe des Turbomolekularpumpstands auf zunächst $<1 \cdot 10^{-1}$ mbar vorevakuiert, bevor die Turbomolekularpumpe zugeschaltet wird. Anschließend wird für mindestens $12-16 \mathrm{~h}$, bei Substanzwechseln auch mehrere Tage, evakuiert, wobei ein Druck von $<5 \cdot 10^{-7}$ mbar 
erreicht werden kann. Wie bereits erwähnt (Kap. 3.1), kann die Schraubenpumpe nicht mit der Turbomolekularpumpe evakuiert werden und muss stattdessen gespült werden.

\subsection{2 (Aus-)Heizen}

Insbesondere nach einer (Teil-)Belüftung des Vakuumsystems muss - vor allem aufgrund der eingedrungenen Luftfeuchtigkeit - über einen längeren Zeitraum evakuiert werden, bis adsorbierte Substanzen wieder in ausreichendem Maß von den Behälterwänden desorbiert sind. Dieser Prozess kann durch den Einsatz der Heizanlage unterstützt werden. Zusammen mit den Vakuumbehältern und Pumpständen wurde von der Firma Pfeiffer eine Heizanlage der Firma Horst GмвH installiert. Diese besteht aus maßangefertigten Heizmanschetten, die das Reservoir, den Pufferbehälter, die Zuleitung vom Pufferbehälter zum Wälzkolbenpumpstand und die Gasrückführungsleitung umschließen, und einer Steuereinheit, mit der die Manschetten in 8 individuellen Heizkreisen geregelt bis $100^{\circ} \mathrm{C}$ geheizt werden können. Der ebenfalls von der Firma Horsт GмвH angefertigte Bypassschlauch zwischen Reservoir und Puffervolumen kann gleichermaßen geregelt auf bis zu $100^{\circ} \mathrm{C}$ erwärmt werden. Neben der Unterstützung des Evakuiervorgangs durch ein mildes Ausheizen ermöglichen diese Heizungen auch einen Betrieb der Apparatur bei erhöhter Temperatur. So können beispielsweise schwerer flüchtige Substanzen, die bei Raumtemperatur keinen ausreichenden Dampfdruck aufweisen, für den Messbetrieb genutzt werden. Dabei muss jedoch beachtet werden, dass diese an jeder kälteren Oberfläche mit Kontakt zum Gaskreislauf auskondensieren können. Hinsichtlich der geplanten Anwendungen ist das Ziel deshalb, dass die kälteste, mit dem Kreislaufsystem in Kontakt stehende Oberfläche eine Temperatur von mindestens $60^{\circ} \mathrm{C}$ und damit etwa 35-40 K mehr als Raumtemperatur erreicht.

Von den oben genannten Heizmanschetten sind jeweils nur die Behälterwände bedeckt, nicht jedoch die Rohransätze zu den Flanschen bzw. die Flansche selbst. Insbesondere an den größeren Flanschen am Pufferbehälter (Düsenflansch, Turbomolekularpumpenflansch, seitliche 250-mm-Flansche) führt dies zu größeren ungeheizten Flächen. Am Düsenflansch wird dies durch die Heizung der Düse (s. unten) wieder aufgefangen, die restlichen Flansche sind mit Armaflex-Dämmmaterial zumindest isoliert.

Mit einer Wärmebildkamera (FLIR i60) wurden einige innenliegende Oberflächen im belüfteten Zustand untersucht. In Abbildung 3.6(a) ist ein Wärmebild der inneren Oberfläche der Seitenwand des Pufferbehälters gezeigt, das im belüfteten Zustand durch einen der beiden seitlichen DN250-ISO-K-Flansche aufgenommen wurde. Auch wenn die dargestellten absoluten Temperaturwerte nicht kalibriert sind und damit von den Realwerten abweichen können, zeigt sich, dass die relative Abweichung zwischen wärmster und kältester Stelle eine Größenordnung von $10 \mathrm{~K}$ aufweist. Werden die dargestellten Temperaturwerte als näherungsweise zutreffend angesehen, zeigt sich außerdem, dass die Werte allesamt oberhalb der eingestellten Zieltemperatur von $80^{\circ} \mathrm{C}$ liegen. Die Stichprobe zeigt also, dass die Heizung an den aktiv geheizten Stellen die gewünschte Mindesttemperatur von $60^{\circ} \mathrm{C}$ bereits bei einer Regeltemperatur von $80^{\circ} \mathrm{C}$ deutlich überschreitet und dabei eine 


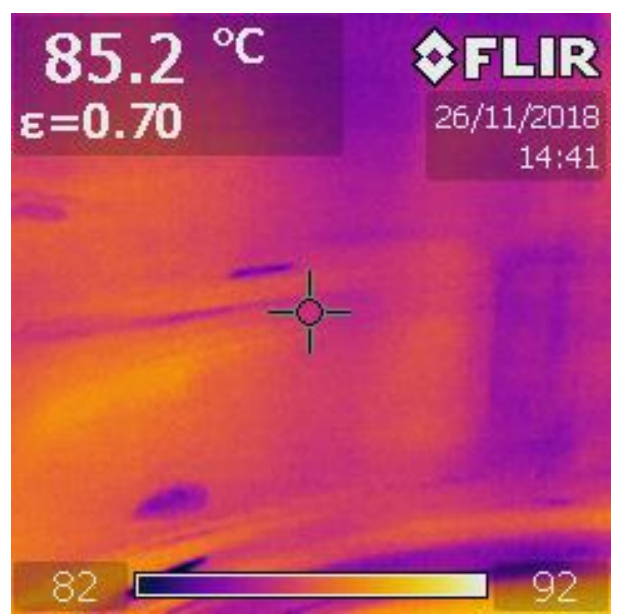

(a)

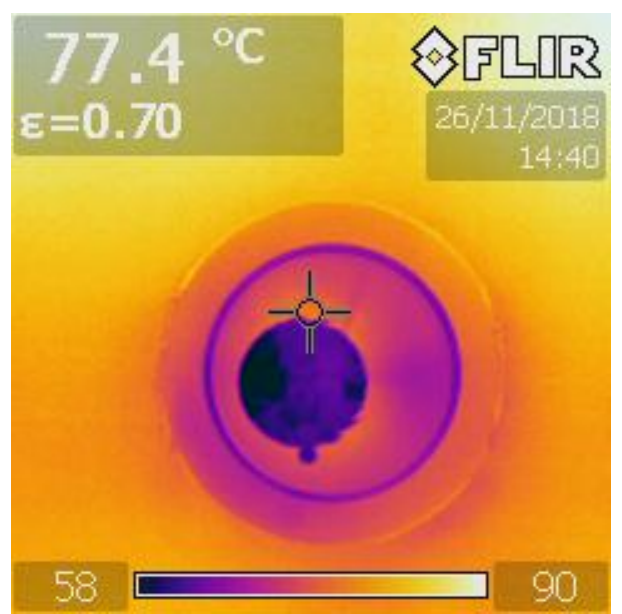

(b)

Abbildung 3.6: Zwei exemplarische Wärmebilder der Innenoberflächen des Pufferbehälters des Gratin-Jets (s. Abb. 3.2 auf S. 37): (a) Seitenwand, vollständig von Heizmanschetten bedeckt; (b) DN250-ISO-K-Flanschdeckel, nur passiv gedämmt. Die runde, kalte Fläche auf dem Bild des Flanschdeckels ist die Reflexion des geöffneten gegenüberliegenden Flansches, durch den das Wärmebild aufgenommen wurde.

recht gleichmäßige Oberflächentemperatur erzeugt.

Auch die für die Wärmebildmessung zugänglichen, mutmaßlich problematischen Stellen im Pufferbehälter wurden mit der Wärmebildkamera untersucht. Als Beispiel ist in Abbildung 3.6(b) einer der beiden seitlichen DN250-ISO-K-Flanschdeckel gezeigt. Dieser ist nur durch eine ARMAFLEX-Dämmschicht isoliert und wird nicht aktiv geheizt. Hier zeigen sich etwas deutlichere Temperaturunterschiede zur aktiv geheizten Behälterwand, wenngleich die im Bild sichtbare, kühle runde Fläche auf dem Flanschdeckel eine Reflexion des geöffneten gegenüberliegenden Flansches darstellt. Der im Wärmebild markierte Punkt auf dem Flanschdeckel weist eine um etwa $13 \mathrm{~K}$ niedrigere Temperatur als die umgebende Behälterwand auf und insbesondere die Randbereiche des Flanschdeckels werden von der Kamera mit noch niedrigeren Temperaturen wiedergegeben. Die Abweichungen zur umgebenden Behälterwand erreichen hier Werte von etwa $20-30 \mathrm{~K}$, sodass die $60^{\circ} \mathrm{C}$ Mindesttemperatur in einigen Bereichen des Flanschdeckels möglicherweise unterschritten werden. Die Regeltemperatur der umgebenden Heizmanschette muss also gegenüber den bei Bildaufnahme eingestellten $80^{\circ} \mathrm{C}$ eventuell etwas erhöht werden, um über Wärmeleitung auch den Flanschdeckel etwas stärker zu erwärmen.

Weitere ungeheizte Stellen finden sich z. B. an den zahlreichen Ventilen, die über die vorhandenen Kleinflansche mit dem Puffervolumen bzw. dem Reservoir verbunden sind. Bis zu ihrer Dichtfläche haben diese Ventile Kontakt mit dem Kreislaufsystem. Die Innentemperatur der Oberflächen kann nicht über die Wärmebildkamera ermittelt werden, da diese Flächen im eingebauten Zustand nicht für eine Messung zugänglich sind. Da 
die Außentemperaturen der Metallteile der Ventile allerdings nur wenige K über der Umgebungstemperatur liegen, ist davon auszugehen, dass nicht alle innenliegenden Oberflächen mit Systemkontakt mindestens $60^{\circ} \mathrm{C}$ beim Heizen von Reservoir bzw. Puffervolumen erreichen. Die Ventile müssten also von außen eventuell isoliert oder sogar aktiv geheizt werden, um „kalte“ Stellen mit Kreislaufsystemkontakt zu vermeiden.

Ein größerer problematischer Bereich befindet sich am Wälzkolbenpumpstand. Hier sind sämtliche Verrohrungen zwischen Gaseinlass und -auslass nicht isoliert oder geheizt. Zwar erreichen die Pumpen im laufenden Betrieb erhöhte Temperaturen (z. B. $>70^{\circ} \mathrm{C}$ außen an Окта $2000 \mathrm{M}$ messbar), die Verrohrungen rund um die Schraubenpumpe werden jedoch an den Außenflächen nicht signifikant wärmer als Raumtemperatur. Hier wäre für einen Betrieb des Gratin-Jets bei erhöhten Temperaturen oder für ein Einbeziehen des Wälzkolbenpumpstands in den Ausheizbetrieb zumindest eine Isolierung, vermutlich aber sogar eine aktive Heizung nötig.

Im Gegensatz zu diesen problematischen Stellen wurden die von den Feinmechanikund Elektronikwerkstätten der Fakultät für Chemie der Georg-August-Universität Göttingen gefertigten Bestandteile der Düseneinheit (Düsenkörper und Düsenkammer) mit einer Heizung versehen. Diese besteht aus in die Edelstahlkörper der Düseneinheit eingelassenen Heizpatronen (HS-Heizelemente Typ 1255), die eine Heizbarkeit auf bis zu $>250{ }^{\circ} \mathrm{C}$ ermöglichen. Mit zwei Eurotherm-Reglern (Eurotherm 2208) kann die Heizung von Düsenkörper und Düsenkammer in zwei getrennten Heizkreisen geregelt werden. Die Heizbarkeit der Düseneinheit verhindert nicht nur, dass anderswo desorbierte Substanzen im Ausheizbetrieb in der Düseneinheit auskondensieren, sondern ermöglicht auch einen Forschungsbetrieb bei erhöhter Düsentemperatur. Hierzu ist insbesondere die getrennte Heizbarkeit von Düsenkammer und -körper von Vorteil, da so selektiv die Düse selbst geheizt werden kann. Bei Durchströmen der geheizten Düse können energetisch höherliegende Zustände untersuchter Analyt-Moleküle thermisch populiert und bei ausreichend hoher Umwandlungsbarriere in der folgenden Expansion unter Depopulierung angeregter Rotationszustände eingefroren werden. Ein entsprechendes Vorgehen unter Verwendung von Jet-RAMAN-Spektroskopie ermöglichte beispielsweise kürzlich die schwingungsspektroskopische Untersuchung des cis-Ameisensäurerotamers in der Gasphase. ${ }^{[34]}$ In der Jet-FTIR-Spektroskopie finden geheizte Düsen ebenso Anwendung ${ }^{[225,229,239]}$ und können z. B. dazu eingesetzt werden, das Konzentrationsverhältnis von Mono-, Di- und Oligomeren in der Expansion zu beeinflussen. ${ }^{[187]}$ Der Gratin-Jet kombiniert nun erstmals Jet-FTIR-Spektroskopie mit einer heizbaren Düse von mehreren hundert Millimetern Länge.

Beim Messbetrieb mit geheizter Düse muss allerdings auf eine möglichst konstante Düsentemperatur geachtet werden. Erste Testaufnahmen von NOTCH-Leerspektren ${ }^{*}$ bei geheiztem Düsenkörper und ebenfalls geheizter Düsenkammer zeigen eine sinusförmige

"NOTCH: vom englischen „Noise Test Challenge“, ein Programm zum Rauschvergleich zwischen verschiedenen Spektren, bei dem standardisiert für je 1 min erst Hintergrund- und anschließend Probespektren aufgenommen werden. ${ }^{[243]}$ 


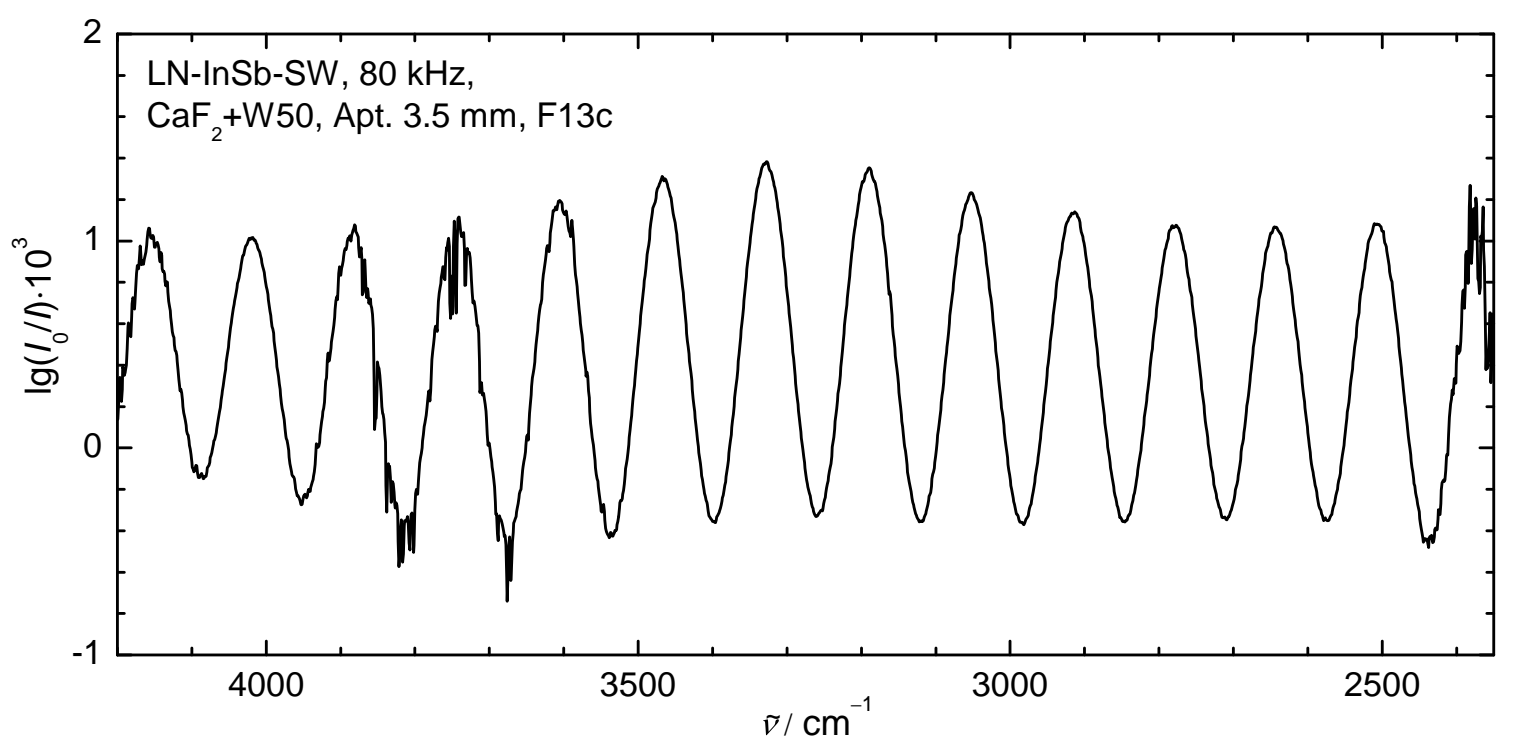

Abbildung 3.7: Ein NOTCH-Spektrum des Gratin-Jets bei ansteigender Düsentemperatur. Die Grundlinie ist von einer sinusförmigen Schwingung überlagert. Die wichtigsten Optikparameter sind in der Reihenfolge Detektor, Spiegelgeschwindigkeit, Strahlteiler/Fenster+Lichtquelle, Apertur, Filter im Spektrum angegeben.

Schwingung auf der Grundlinie (s. Abb. 3.7). Eine solche Schwingung im Absorbanzspektrum weist auf eine scharfe Linie abseits des zentralen Maximums im Interferogramm hin, die sich zwischen Hintergrund- und Probeninterferogramm verschiebt oder in ihrer Intensität verändert. Phase und Periode der Schwingung sind bei mehreren NOTCH-Messungen verschieden. Die Störung wurde bisher ausschließlich in Messungen durch die Düsenkammer bei sich ändernder Düsentemperatur beobachtet, nicht aber bei Messungen mit einem intern im Spektrometer eingesetzten Detektor. Zudem scheint die Amplitude mit steigendem Düsentemperaturgradienten zuzunehmen, sodass die wahrscheinlichste Ursache Reflexionseffekte sind, die eine temperaturabhängige Wegdifferenz verschiedener am Detektor eintreffender Strahlen verursachen. Ändert sich die Wegdifferenz zwischen Hintergrund- und Probescan, tritt im Absorbanzspektrum die beschriebene Schwingung auf. Anhand der beobachteten Schwingungsperiode $\Delta \tilde{v}$ von etwa $120-150 \mathrm{~cm}^{-1}$ lässt sich die Wegdifferenz auf eine Größenordnung von mehreren $10 \mu \mathrm{m}$ abschätzen.

Bei Messungen mit der Düse bei Raumtemperatur tritt die Schwingung nicht auf. Bei erhöhten Temperaturen können anscheinend selbst kleinere Temperaturschwankungen, die durch die Temperaturregelung der Heizung verursacht werden, zu den beobachteten Störungen führen, allerdings mit wesentlich geringerer Intensität als im Aufheizbetrieb. Auch in Jet-Spektren ist die Schwingung deutlich geringer ausgeprägt, da im Jet-Messmodus die Hintergrundspektren jeweils wenige Sekunden vor dem zugehörigen Probespektrum aufgenommen werden. Im Gegensatz dazu werden beim NOTCH-Programm zunächst eine Minute lang Hintergrund- und dann eine weitere Minute lang Probespek- 


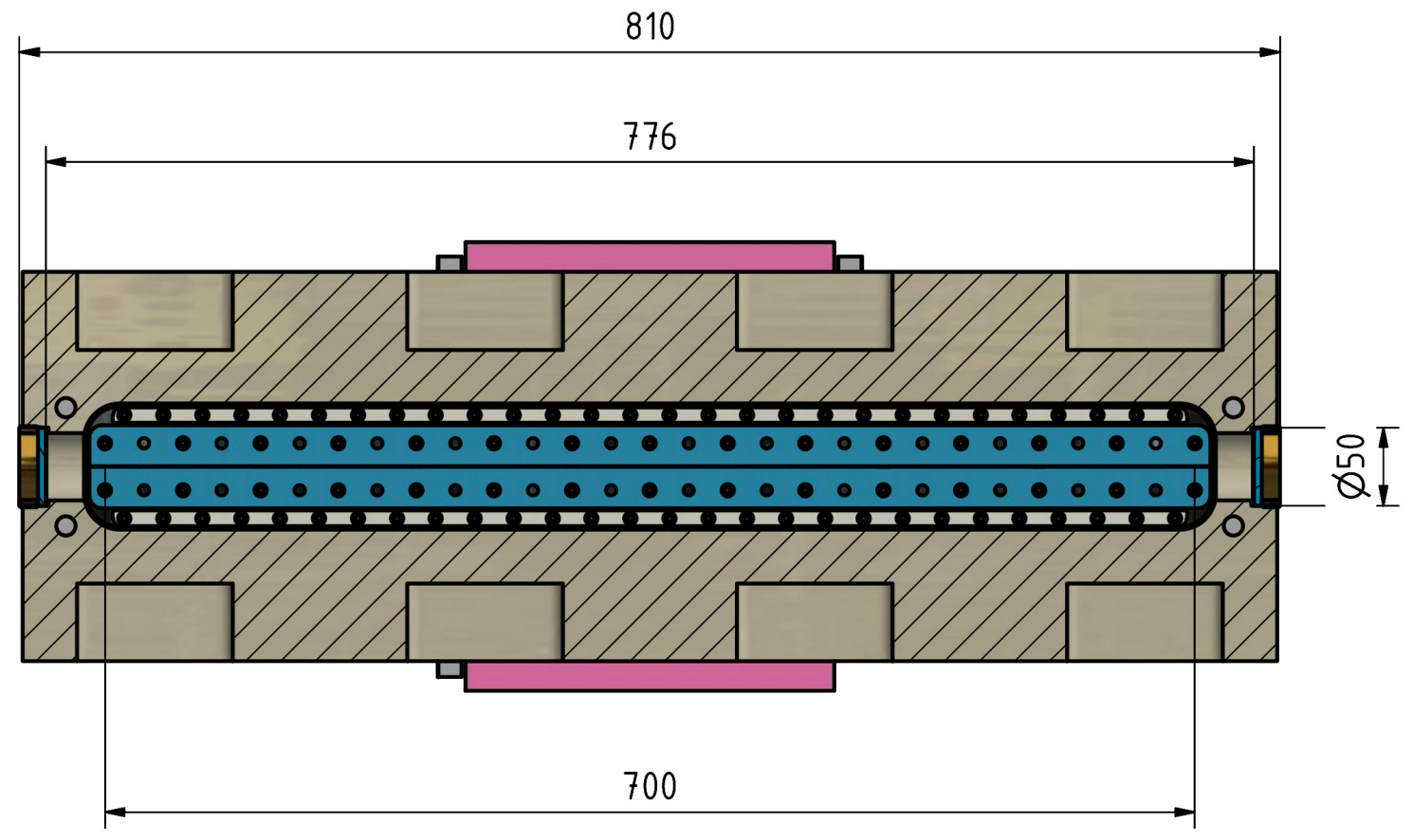

Abbildung 3.8: Technische Zeichnung der Schlitzdüse (blau) mit Düsenkammer (ocker) am Gratin-Jet. Blick von der Niederdruckseite frontal auf die Düse. Mittig ist in blau die $700 \mathrm{~mm}$ lange und $0.2 \mathrm{~mm}$ breite Schlitzdüse dargestellt. Seitlich sind messingfarben die Flansche der beiden $50 \mathrm{~mm}$ weiten Fenster-/Linsenöffnungen sichtbar, die in der grau dargestellten Düsenkammer sitzen. Die Öffnungen haben mit 776 mm den gleichen Abstand wie am Filet-Jet. (๑ R. HildebrandT, Abdruck mit freundlicher Genehmigung.)

tren aufgezeichnet. Da die Störung nur bei Messungen durch die Düsenkammer und nur bei sich ändernder Düsentemperatur auftritt, könnte es sich um einen Reflex an der Düse handeln. Dieser sorgt für einen Gangunterschied zu nicht an der Düse reflektiertem Licht, wobei der Gangunterschied mit der thermischen Ausdehnung oder einer temperaturbedingten Positionsänderung der Düse variiert. Alternativ könnte auch eine Reflexion an den Phasengrenzflächen eines Ölfilms, der sich beim Heizen der Düse auf den kälteren Fenstern niederschlägt und dessen Schichtdicke schwankt, ursächlich sein. Beide Erklärungen wären sowohl mit der beobachteten Temperaturabhängigkeit als auch mit der Größenordnung der Wegdifferenz kompatibel. Ein Düsenreflex könnte durch die Beschichtung der Düse mit einem nicht-reflektierenden Lack unterdrückt werden.

\subsection{Schlitzdüse und Expansionszone}

Die Düse des Gratin-Jets basiert wie viele andere Teile der Gratin-Jet-Apparatur auf dem Konzept der entsprechenden Gegenstücke am Filet-Jet. ${ }^{[55]}$ Allerdings wurde die Schlitz- 


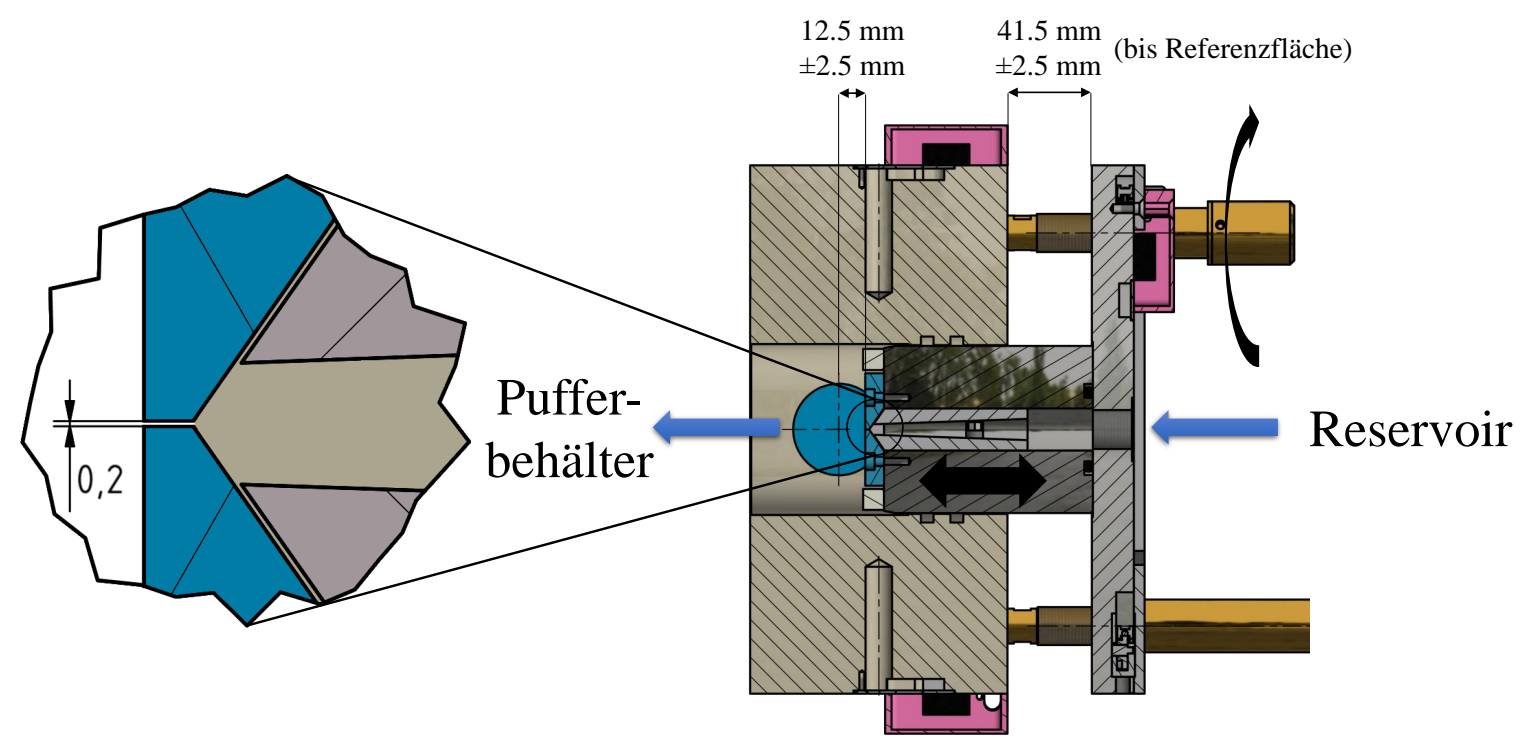

Abbildung 3.9: Seitlicher Querschnitt des Düsenkörpers (grau) und der Düsenkammer (ocker) am Gratin-Jet. Das Gas durchströmt die Einheit von rechts nach links (blaue Pfeile). Der Düsenkörper ist durch Betätigen des Kettenzugmechanismus relativ zur Düsenkammer verschiebbar, sodass der Abstand zwischen Düse und Fenstermitte (blauer Bereich mittig) verändert werden kann (schwarze Pfeile). In der Detailansicht links sind in blau die Blenden erkennbar, die in Flussrichtung $35^{\circ}$ angeschrägt sind. Da der Abstand zwischen Fenster-/Linsenmitte und Düse im Labor nicht exakt bestimmt werden kann, steht eine Referenzfläche zur Verfügung, an der stattdessen der Abstand zwischen Düsenkammer und Düsenkörper gemessen werden kann (gekennzeichnete Abstände). Ein Abstand von $41.5 \mathrm{~mm}$ entspricht dabei einem Düsenabstand von $12.5 \mathrm{~mm}$ und Änderungen erfolgen gleichförmig. (Für die Abbildung verwendete technische Zeichnungen $\odot$ R. HildEBRANDT, V. MEYer und M. ZipPERT, Abdruck mit freundlicher Genehmigung.)

länge von $600 \mathrm{~mm}$ auf $700 \mathrm{~mm}$ erhöht, wodurch sich der für die IR-Spektroskopie nutzbare Absorptionspfad entsprechend verlängert (s. Abb. 3.8). Die Schlitzbreite zwischen den beiden Blenden (blau) kann im Bereich 0.1-0.3 mm variiert werden. Der IR-Strahl wird durch zwei runde Fenster-/Linsenflansche mit einem Durchmesser von jeweils $50 \mathrm{~mm}$ durch die Düsenkammer geleitet. Der Abstand der Fenster-/Linsenflächen beträgt hierbei $776 \mathrm{~mm}$ und ist damit identisch zum Abstand am Filet-Jet, wodurch dieselben Linsen mit Brennweiten von 500 und $250 \mathrm{~mm}$ genutzt werden können. Durch die längere Düse bei gleichbleibendem Fenster-/Linsenabstand konnten der optische Weg durch warmes Hintergrundgas in der Jetkammer im Vergleich zum Filet-Jet verringert und der Weg durch den Expansionsbereich vergrößert werden. Der Anteil des Expansionsbereichs am Gesamtweg zwischen den Öffnungen beträgt mehr als 90\%, verglichen mit ca. $77 \%$ am FiletJet.

Die Konzeption der Düse ist im Querschnitt in Abbildung 3.9 genauer dargestellt. Die gesamte Düseneinheit besteht aus zwei einzelnen Teilen: zum einen dem Düsenkörper 
(rechts, grau), der die Anschlüsse der Zuleitungen, die Präexpansionskammer und die eigentliche Düse, gebildet aus den beiden Schlitzblenden (blau), enthält, und zum anderen der Düsenkammer (mittig, ocker), die den Düsenkörper aufnimmt, über einen Rechteckflansch mit dem Pufferbehälter verbunden ist und die Fenster-/Linsenöffnungen für den IR-Strahl enthält. Beide Teile sind aus massivem Edelstahl gefertigt, da sie eine hohe mechanische Stabilität aufweisen müssen.

In der Abbildung strömt das Gasgemisch bei einem Puls zunächst von rechts über sieben gleichmäßig über die Schlitzlänge verteilte Zuleitungen aus dem Reservoir in die Präexpansionskammer und dann durch die Düse in die Düsenkammer und weiter in den Pufferbehälter (blaue Pfeile). Die Präexpansionskammer mit einem Volumen von etwa 0.6 L sorgt dabei wie beim Filet-Jet (s. Kap. 2.2) für eine Durchmischung des Gases nach Durchqueren der diskreten Zuleitungen, um eine gleichmäßige Expansion über die gesamte Schlitzlänge zu ermöglichen. Die Geometrie der Blenden ist in der Detailansicht links in Abbildung 3.9 erkennbar. Die Blenden sind in Flussrichtung $35^{\circ}$ angeschrägt, wodurch sich die Dicke von $9.0 \mathrm{~mm}$ im Außen- auf $2.0 \mathrm{~mm}$ im Schlitzbereich verringert. Die Form entspricht damit den derzeit am Filet-Jet verbauten Blenden der Form B. ${ }^{[76]}$

Die Düseneinheit ist mit einem Kettenzugmechanismus ausgestattet, der es erlaubt, den Düsenkörper im eingebauten Zustand relativ zur Düsenkammer und damit auch zum IRStrahl um $\pm 2.5 \mathrm{~mm}$ zu verschieben (schwarze Pfeile in Abb. 3.9). Dies erlaubt das Messen der Expansion bei verschiedenen Düsenabständen im Bereich von 10 bis 15 mm (Abstand zwischen Fenster-/Linsenmitte und Düse). Zur Verringerung der auf den Mechanismus wirkenden Kräfte muss die Einheit (und damit auch das gesamte Puffervolumen) für die Abstandsänderung allerdings belüftet werden. Zur Bestimmung des Abstands steht am Düsenkörper eine ausgefräste Referenzfläche an der Ecke der Abdeckplatte zur Verfügung. Hier kann ein Messschieber angesetzt werden, um im Labor den Abstand zwischen Fenster-/Linsenmitte und Düse exakt zu bestimmen. Dieser ist bei eingebauter Düse nicht direkt zugänglich. Ein Abstand von $41.5 \mathrm{~mm}$ zwischen Düsenkammer und Referenzfläche am Düsenkörper entspricht dabei einem Abstand von $12.5 \mathrm{~mm}$ zwischen Fenster-/Linsenmitte und Düse. Für die im Rahmen dieser Arbeit durchgeführten IR-spektroskopischen Messungen wurde der Düsenabstand über diese Referenzfläche auf den am Filet-Jet genutzten Wert von $10 \mathrm{~mm}$ eingestellt. Tests bei weiteren Düsenabständen, z. B. hinsichtlich einer möglichen abstandsabhängigen Signalabschwächung durch eine in den Strahlengang ragende Düse, sind für die Zukunft geplant.

In Abbildung 3.10 ist ein größerer Ausschnitt des Bereichs von Reservoir, Düse und Pufferbehälter dargestellt. Im rechten Bildbereich ist die Zuleitung vom Reservoir zur Düse sichtbar. Neben einem Federungskörper zum Ausgleich der oben beschriebenen variablen Düsenposition beinhaltet jede der sieben Verbindungen ein Magnetventil vom Typ PARKER LUCIFER 221J3301E-299560-483816C2. Die sieben Magnetventile dienen zur Steuerung des Gaspulses und vereinen eine große Nennweite von $8 \mathrm{~mm}$ mit geringen Öffnungs- und Schließzeiten von 10-14 ms bzw. 4-7 ms. ${ }^{[76]}$ Abgesehen von der mit der größeren Düsenlänge hochskalierten Anzahl an Zuleitungen entspricht diese Konstruktion weitgehend derjenigen am Filet-Jet. 


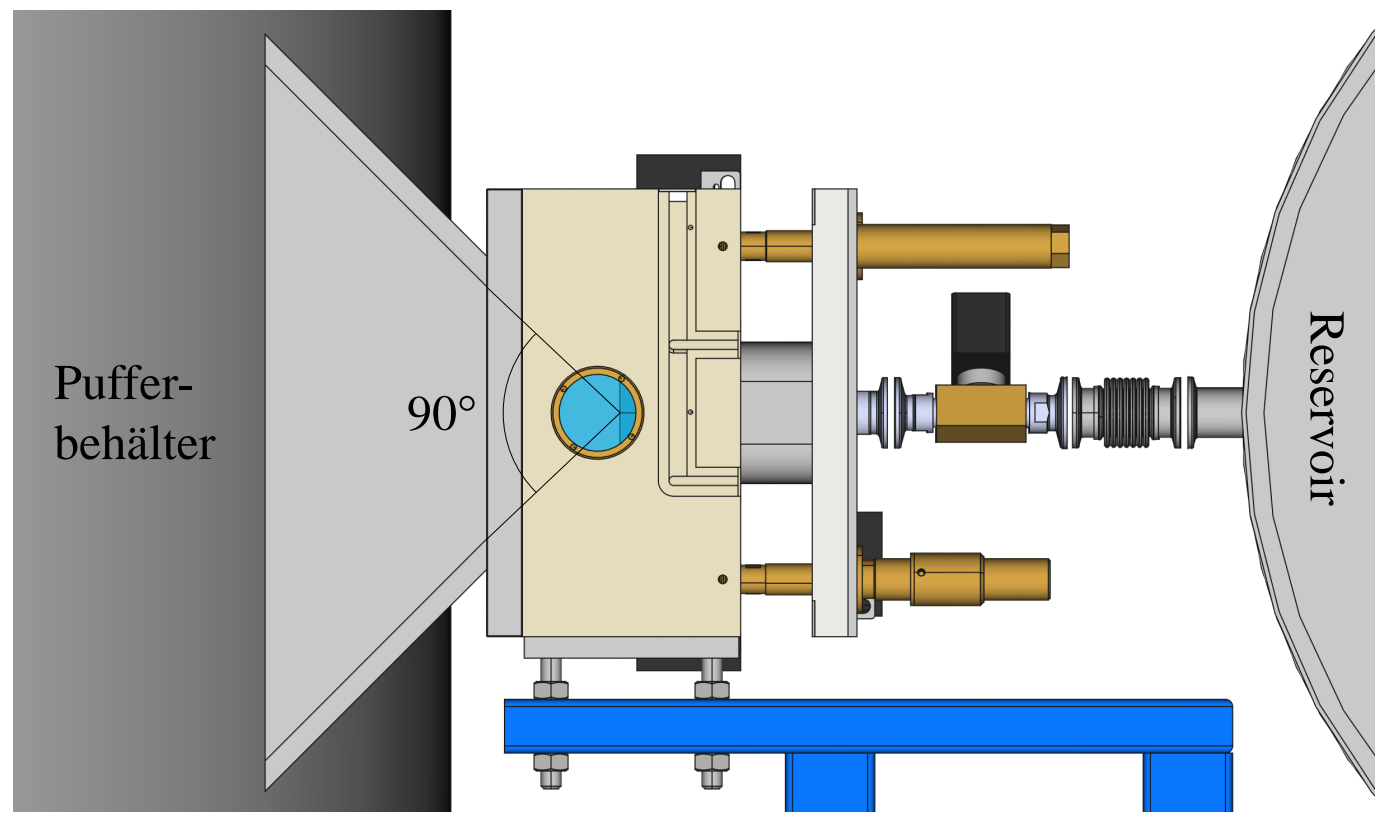

Abbildung 3.10: Seitliche Ansicht der Verbindung von Reservoir, Düse, Düsenkammer und Pufferbehälter (von rechts nach links) am Gratin-Jet. Das Gasgemisch gelangt durch sieben parallele Leitungen mit einem Durchmesser von je $25 \mathrm{~mm}$, in denen jeweils ein Magnetventil (PARKER LucIFER 221J3301E-299560-483816C2) sitzt, aus dem Reservoir in die Präexpansionskammer der Düse. Die Düsenkammer ist über einen trichterförmigen Flanschansatz mit dem Pufferbehälter verbunden, wodurch in vertikaler Richtung ein Öffnungswinkel von $90^{\circ}$ für die Expansion zur Verfügung steht. (Für die Abbildung verwendetes 3D-Modell $\odot$ R. Hildebrandt, V. Meyer und M. Zippert, Abdruck mit freundlicher Genehmigung.)

Im Gegensatz dazu wurde der Aufbau auf der Niederdruckseite der Düse stark verändert und optimiert. Aufgrund des überarbeiteten Verstellmechanismus, der auf einem Kettenzug basiert, konnte die Düse des Gratin-Jets mit horizontaler Strömungsrichtung ausgerichtet werden. Dadurch konnte die Düse direkt seitlich an den Pufferbehälter angebracht werden. Die Verbindung erfolgt über einen eigens konstruierten Rechteckflansch, der mit einem FKM-O-Ring gedichtet ist. Zur Fixierung der Düsenkammer am Pufferbehälterflansch dienen acht Schrauben. Am Filet-Jet ist ein direktes Anbringen der Düse am Puffervolumen aufgrund der vertikalen Bauweise dagegen nicht möglich. Es ist eine Jetkammer nötig, auf die die Düse von oben aufgesetzt ist. Um die Düse in einer für den Laboralltag praktikablen Arbeitshöhe zu halten, ist der Durchmesser der Jetkammer auf $400 \mathrm{~mm}^{[76]}$ beschränkt, sodass die Expansionsgeometrie nicht optimal ist. Das expandierte Gas staut sich an der der Düse gegenüberliegenden Kammerwand, wodurch lokal höhere Drücke als im Puffervolumen entstehen. Zur Abfederung dieses Staudrucks sind sehr große Puffervolumina von mindestens $12 \mathrm{~m}^{3}$ nötig.

Am Gratin-Jet ist durch die seitliche Anbringung der Düse direkt am Puffervolumen 
die gegenüberliegende Behälterwand mehr als $1.5 \mathrm{~m}$ entfernt. Durch einen trichterförmigen Flanschansatz am Pufferbehälter soll der lokale Druck auf der Niederdruckseite der Expansion weiter verringert werden. Wie auf der linken Seite in Abbildung $3.10 \mathrm{zu}$ sehen ist, ermöglicht dieser Aufbau einen freien Expansionswinkel von $90^{\circ}$ in vertikaler Richtung. Aufgrund der Krümmung des Pufferbehälters und der nah an der Düse gelegenen seitlichen Fenster-/Linsenöffnungen ist ein ähnlicher Winkel in horizontaler Richtung nicht möglich. Dennoch wird durch die vorhandene Geometrie das Entstehen eines Staudrucks so weit wie möglich verhindert, sodass der lokale Hintergrunddruck der Expansion näherungsweise dem Druck im Puffervolumen entsprechen sollte. Dadurch ist die Verwendung eines kompakten Puffervolumens von nur ca. $4 \mathrm{~m}^{3}$ (s. Kap. 3.1) möglich, was Vorteile hinsichtlich des Platzbedarfs und des vorgesehenen Gasrecycling-Betriebs mit sich bringt. Durch eine möglichst geringe Behälteroberfläche werden Adsorption und spätere -desorption von Verunreinigungen und Analytsubstanzen und damit das „Substanzgedächtnis“ des Kreislaufsystems so gering wie möglich gehalten.

\subsection{Spektrometer, Optik und Synchronisation}

Einer der zentralen Bestandteile für die Gratin-Jet-Spektroskopie ist die von der Firma BRUKER gelieferte Baugruppe bestehend aus Spektrometer und Detektorkammer. Über eine Transistor-Transistor-Logik-(TTL-)Schnittstelle kann die Datenaufnahme des Spektrometers mit den Gaspulsen durch die Schlitzdüse synchronisiert werden.

\subsubsection{Spektrometer und Detektorkammer}

Das am Gratin-Jet eingesetzte Spektrometer (BRUKER VERTEX 70v) ist mit mehreren Lichtquellen für den Nah- (NIR) und den mittleren Infrarotbereich (MIR), einem Apertur- und einem Filterrad, einem Interferometer zur Modulation des Lichtes, einem Helium-NeonLaser zur Wellenzahlkalibrierung und mehreren Detektoren ausgestattet. Für spektroskopische Messungen können die Detektoren an zwei internen und einem in einer externen Detektorkammer untergebrachten dritten Port betrieben werden. Der Strahlengang vom Spektrometer zur Detektorkammer ist dabei so konzipiert, dass die Düsenkammer des Gratin-Jets (s. Kap. 3.4) zur Untersuchung der Überschallexpansion durchstrahlt werden kann. Die einzelnen Bestandteile der optischen Baugruppe sollen im Folgenden vorgestellt werden.

Die Basis des Spektrometers bildet ein sog. RockSoLID ${ }^{\text {TM }}$-Interferometer. ${ }^{[249]}$ In diesem kommen zwei auf den beiden Armen eines Doppelpendels angebrachte Winkelreflektoren (Cube-Corner-Spiegel) zum Einsatz. Der Gangunterschied zwischen den am Strahlteiler aufgeteilten Strahlengängen kommt durch das Bewegen des Pendels zustande. In dieser Art von Interferometer muss kein Spiegel auf einem Luftkissen bewegt werden, wodurch keine permanente Druckluftzufuhr zum Spektrometer notwendig ist. Darüber hinaus ist das Interferometer unempfindlich gegenüber Dejustierung durch Spiegel- oder Strahl- 
Tabelle 3.1: Zur Verfügung stehende optische Komponenten und Eigenschaften des BRUKER VERTEX 70v Spektrometers.

\begin{tabular}{ll}
\hline Komponente/Parameter & Verfügbare Varianten/Werte \\
\hline Lichtquelle & Globar (intern); Wolframlampen: $50 \mathrm{~W}$ (intern) \& 150 W (extern) \\
Strahlteiler & ${\text { Breitband, } \mathrm{KBr}, \mathrm{CaF}_{2}}$ \\
Fenster/Linsen & $\mathrm{KBr}, \mathrm{CaF}_{2}$ \\
Spiegelgeschw. / kHz & $1.6,2.5,5,7.5,10,20,40,60,80,120,140,160$ \\
Max. Auflösung / cm & \\
Int. Blende / mm & 0.5 \\
Ext. Blende / mm & $0.25,0.5,1,1.5,2,2.5,3,3.5,4,5,6,8$ \\
Detektor & $3,3.5,4,6,8,10,12,14,16,18$ \\
Filterhalterungen & LN-InSb/MCT-SW, RT-DLaTGS (2x), InGaAs \\
\hline
\end{tabular}

teilerverstellung, ${ }^{[246]}$ z. B. durch Temperaturschwankungen. Da der Massenschwerpunkt des Doppelpendels in dessen Drehachse liegt, ist das Interferometer außerdem unempfindlich gegenüber Erschütterungen. Angesichts der räumlichen Nähe des Spektrometers zur Behälterheizung und zum Wälzkolbenpumpstand des Gratin-Jets (s. Abb. 3.1 auf S. 34) sind diese Eigenschaften für einen erfolgreichen Messbetrieb vorteilhaft.

In Tabelle 3.1 sind die verschiedenen zur Verfügung stehenden optischen Komponenten und nutzbaren Eigenschaften des Spektrometers aufgelistet. Als Lichtquellen können ein Globar sowie zwei Wolframlampen ( $50 \mathrm{~W}$ bzw. $150 \mathrm{~W}$ Leistung) genutzt werden. Der Globar und die $50 \mathrm{~W}$ starke Wolframlampe (W50) sind dabei intern verbaut. Die 150-WWolframlampe (W150) sitzt dagegen in einem externen, wassergekühlten Gehäuse. Diese externe Position bringt einige Nachteile mit sich. So können nur mit den internen Lichtquellen das Blenden- und das Filterrad genutzt werden. Das Licht der internen Lichtquellen wird dabei auf die Position des Blendenrads fokussiert, sodass diese als kreisscheibenförmige Lichtquellen von der Größe der eingestellten Apertur genutzt werden können. Bei der externen Lichtquelle findet im Gegensatz dazu keine Fokussierung des Lichtes statt, wodurch kein Fokuspunkt als ideale Blendenposition existiert. Zwar können trotzdem Blenden in den Strahlengang zwischen externer Lichtquelle und Interferometer eingebracht werden, allerdings kann deren Durchmesser nicht direkt mit den Durchmessern der intern genutzten Blenden verglichen werden. Um einen Lichtstrahl vergleichbaren Durchmessers im Interferometer und weiteren Strahlengang zu erzeugen, sind mit der externen Lichtquelle aufgrund des fehlenden Fokuspunkts wesentlich größere Blendendurchmesser nötig. Die Blenden für die Nutzung der externen Lichtquelle wurden von den Chemie-Werkstätten in diversen Größen maßangefertigt und können spektrometerseitig an den Durchlassflansch zwischen Lichtquellengehäuse und Spektrometer angesetzt werden.

Auch das Filterrad, das ebenso wie das Aperturrad zwischen internen Lichtquellen und Interferometer sitzt, kann mit der externen Lichtquelle nicht genutzt werden. Aus diesem 
Grund wurde durch die Chemie-Werkstätten eine zusätzliche Filterhalterung unmittelbar vor der Detektorposition in der Detektorkammer installiert. In diese können optische Filter in der Standardgröße von $25.4 \mathrm{~mm}$ Durchmesser eingesetzt werden. Ein Austausch der Filter zwischen verschiedenen Apparaturen ist damit ohne größeren Aufwand (Ausbau des Filterrads inkl. Belüftung des Spektrometers) möglich.

In der gelieferten Konfiguration können mit dem VERTEX 70v spektrale Bereiche zwischen 130 und $15500 \mathrm{~cm}^{-1}$ abgedeckt werden. Dazu stehen ein Breitband-, ein Kaliumbromid- $(\mathrm{KBr}-)$ und ein Calciumfluorid- $\left(\mathrm{CaF}_{2}-\right)$ Strahlteiler zur Verfügung. Zur Detektion des Lichts können zwei Raumtemperatur-(RT-)DLaTGS-Detektoren (davon einer mit CsIFenster für Messungen im ferninfraroten (FIR) Bereich), ein InGaAs- oder ein InSb/MCTSandwich-Detektor genutzt werden. Letzterer weist im für die geplanten Forschungsanwendungen wichtigen Spektralbereich von $4000-800 \mathrm{~cm}^{-1}$ eine besonders hohe Detektivität auf. Die gesockelten Detektoren können dabei flexibel sowohl an den beiden internen Ports als auch in der (externen) Detektorkammer eingesetzt werden. Das einfallende Licht wird an den internen Positionen durch elliptische Spiegel bzw. in der externen Detektorkammer durch einen Parabolspiegel (Brennweite $f=43 \mathrm{~mm}$ ) auf den Detektor fokussiert.

Der mit flüssigem Stickstoff $\left(\mathrm{N}_{2}(\mathrm{l}), \mathrm{LN}\right)$ gekühlte InSb/MCT-Detektor (INFRARED Associates INC.) bietet einen Messbereich von ca. 10000 bis $600 \mathrm{~cm}^{-1}$ und ist in Sandwich-(SW-)Bauweise ausgeführt. Bei dieser sitzt das runde InSb-Detektorelement (ca. $10000-2000 \mathrm{~cm}^{-1}$ ) von $2 \mathrm{~mm}$ Durchmesser oberhalb des quadratischen, $(2 \cdot 2) \mathrm{mm}^{2}$ großen HgCdTe-(MCT-)Elements (ca. 2000-600 $\mathrm{cm}^{-1}$ ) und dient damit zugleich als optisches Filter für das MCT-Element. Das Spektrometer VerTex 70v erlaubt eine Parallelmessung mit beiden Detektorelementen im Sandwichbetrieb. Trotz der Verwendung eines Detektors gleicher Bauart ist ein solcher Parallelbetrieb aufgrund des älteren Spektrometers am Filet-Jet nicht möglich. Für den Sandwich-Messbetrieb im MIRBereich am Gratin-Jet wurde ein optisches Tiefpassfilter angeschafft. Das gruppenintern als F20 bezeichnete Filter weist im Bereich $4025-1250 \mathrm{~cm}^{-1}$ eine Transmission von $>75 \%$ und im Bereich $4000-1550 \mathrm{~cm}^{-1}$ von $>87 \%$ auf (s. Abb. 3.11). Damit ist das Tiefpassfilter F20 für InSb/MCT-SW-Messungen im Bereich unterhalb von $4000 \mathrm{~cm}^{-1}$ geeignet.

Um den zur Verfügung stehenden Wertebereich des Analog-Digital-Wandlers (engl. analog-digital converter, ADC) optimal auszunutzen, kann die (analoge) Vorverstärkung des InSb-Detektorelements angepasst werden. Die Vorverstärkung besteht aus zwei Verstärkerstufen, die jeweils durch den Austausch eines gesockelten Widerstands (Auslieferungszustand: $10 \mathrm{k} \Omega$ in Stufe am Detektorelement, $51 \mathrm{k} \Omega$ in zweiter Stufe) und eines gesockelten Kondensators (Auslieferungszustand: $390 \mathrm{pF}$ in erster, $82 \mathrm{pF}$ in zweiter Stufe) angepasst werden können.

Spektrometer und Detektorkammer können über eine Scrollpumpe (EDwARDS nXDS 15i) permanent evakuiert werden. Am Spektrometer steht zusätzlich ein Spülgasanschluss mit einem Durchflussregler zur Verfügung, der sowohl zum Betrieb des Spektrometers ohne Vakuum als auch zum Belüften genutzt werden kann. Dieser Anschluss wird am Gratin-Jet über die oben beschriebene Gasverteilung (s. Kap. 3.2) mit Stickstoff versorgt, um beim Belüften oder Spülen ausreichend trockenes, sowie öl- und partikelfreies Gas 


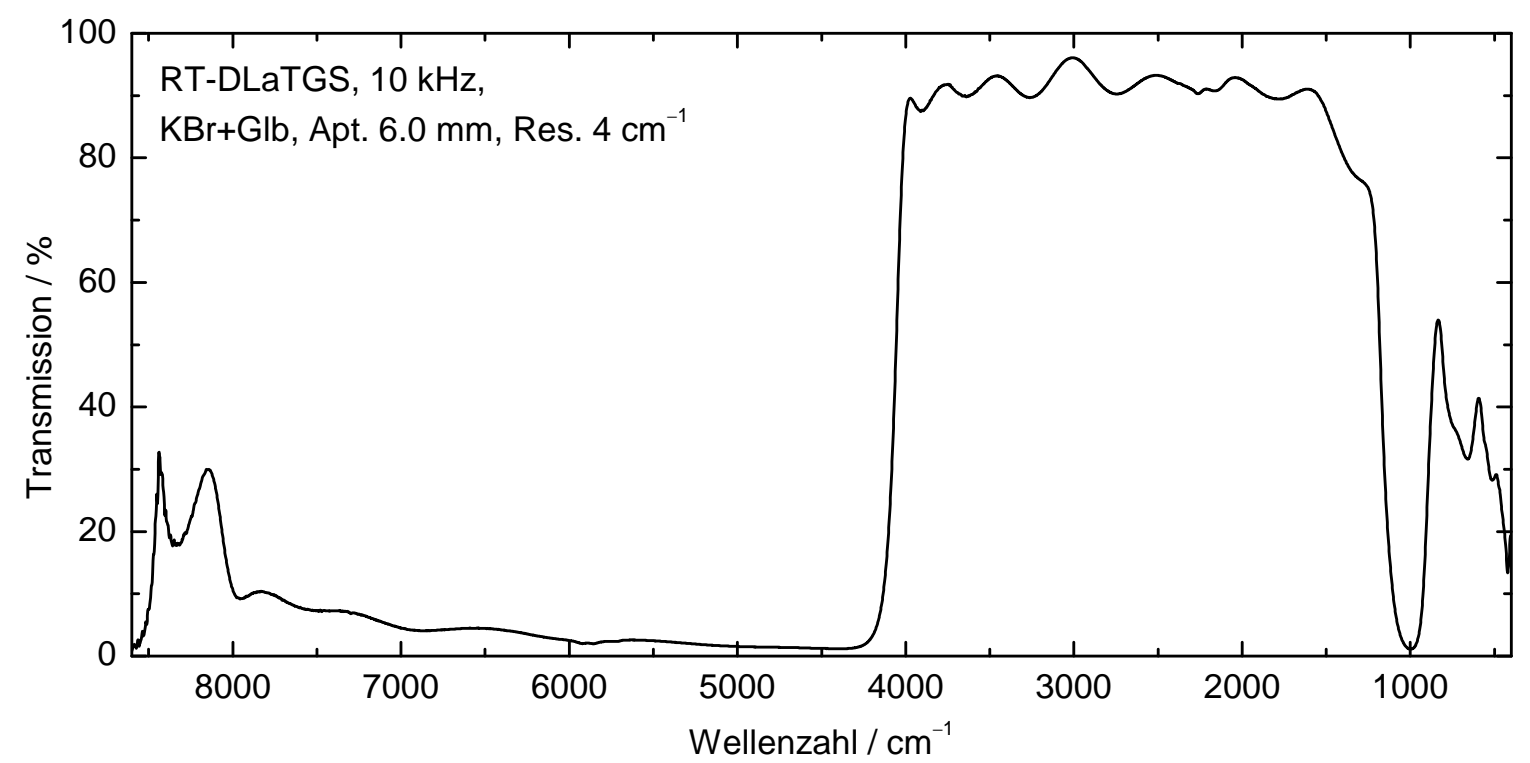

Abbildung 3.11: Die Transmissionskurve vom Tiefpassfilter F20 im Bereich $8600-400 \mathrm{~cm}^{-1}$, gemessen mit dem internen RT-DLaTGS-Detektor, $10 \mathrm{kHz}$ Spiegelgeschwindigkeit, KBr-Strahlteiler, Globar (Glb), $6.0 \mathrm{~mm}$ Blende und einer Auflösung (Res.) von $4 \mathrm{~cm}^{-1}$.

zu verwenden. Für den InSb/MCT-SW-Detektor steht eine Flüssigstickstoffdurchführung bereit, sodass der Detektor im Vakuum betrieben werden kann.

Der im Interferometer modulierte IR-Strahl kann für die Jet-FTIR-Spektroskopie seitlich aus dem Spektrometer ausgekoppelt werden. So durchquert der IR-Strahl den Expansionsbereich der Schlitzdüse, bevor er die Detektorkammer erreicht (s. Abb. 3.1 auf S. 34). Aufgrund des langen optischen Pfades entlang der 700-mm-Schlitzdüse ist eine leichte Fokussierung des Strahls dabei vorteilhaft. Analog zum Filet-Jet kann diese Fokussierung mit zwei Linsen (Brennweiten $f_{1}=250 \mathrm{~mm}$ und $f_{2}=500 \mathrm{~mm}$ ) erfolgen, die in die Fenster-/Linsenflansche der Düsenkammer eingesetzt werden. Da der Flanschabstand, wie in Kapitel 3.4 bereits erwähnt, mit 776 mm exakt dem Abstand am Filet-Jet entspricht, können am Gratin-Jet dieselben Linsen verwendet werden. Alternativ können am Gratin-Jet zwei goldbeschichtete Parabolspiegel (Brennweiten jeweils $f=500 \mathrm{~mm}$ ) zur Strahlfokussierung genutzt werden, die anstelle zweier Planspiegel am Ausgang des Spektrometers und am Eingang der Detektorkammer eingesetzt werden können. An der Düsenkammer können dann Fenster für die Strahldurchführung verwendet werden. Alle Fenster- bzw. Linsenflansche an der Düsenkammer, dem Spektrometer und der Detektorkammer weisen Durchmesser von $50 \mathrm{~mm}$ auf, sodass überall baugleiche Fenster genutzt werden können. Für den MIR- bzw. den NIR-Bereich stehen Fenster und Linsen aus $\mathrm{KBr}$ bzw. $\mathrm{CaF}_{2}$ zur Verfügung.

Auf dem Weg zwischen Spektrometer und Detektorkammer kreuzt der IR-Strahl zwischen Spektrometer und Düsenkammer sowie zwischen Düsenkammer und Detektor- 
Tabelle 3.2: Standardmäßig genutzte Messparameter am Bruker Vertex 70v.

\begin{tabular}{|c|c|}
\hline Parameter & Einstellung \\
\hline Phasenauflösung / $\mathrm{cm}^{-1}$ & 16 \\
\hline Phasen-Korrekturmodus & Mertz \\
\hline Apodisationsfunktion & NORTON-BEER, medium \\
\hline Zerofilling-Faktor & 2 \\
\hline Nichtlinearitätskorrektur des Inter- & \\
\hline ferogramms vor der FT durchführen & ja \\
\hline Hochpassfilter & offen \\
\hline Tiefpassfilter & offen \\
\hline
\end{tabular}

kammer jeweils $0.5-2 \mathrm{~cm}$ breite Abschnitte in der Laborluft. Dies kann zu nicht vollständig kompensierten, störenden Banden bzw. Linien von Kohlenstoffdioxid oder Wasser im Absorbanzspektrum führen, wenn sich die Konzentrationen dieser Stoffe in der Laborluft zwischen Hintergrund- und Probemessung ändern. Insbesondere der oft für die Forschung relevante $\mathrm{OH}$-Streckschwingungsbereich kann hierbei durch schlecht kompensierte Linien atmosphärischen Wassers betroffen sein. Bei Bedarf bestünde die Möglichkeit, eine Trockenluftspülung für die beiden Abschnitte zu installieren, um die Wasserkonzentration zu verringern. Bisher wurden in den Jet-Spektren allerdings noch keine Linien atmosphärischen Wassers in einem störenden Ausmaß beobachtet, sodass eine Trockenluftspülung zur Zeit nicht unbedingt notwendig erscheint.

Die Steuerung des Spektrometers erfolgt über einen Computer mit der Software BRUKER Opus in der Version 7.8. Die im Rahmen dieser Arbeit standardmäßig verwendeten Einstellungen sind in Tabelle 3.2 zusammengefasst. Falls nicht explizit anders erwähnt, wurden die gezeigten Spektren mit diesen Einstellungen aufgenommen. Als Phasenkorrekturmodus wird dabei die Opus-Standardeinstellung MerTz ${ }^{[250]}$ mit einer Auflösung von $16 \mathrm{~cm}^{-1}$ verwendet. Für die Apodisierung wird auf die bewährte ${ }^{[246]}$ NorTON-BEERMedium-Funktion ${ }^{[251]}$ zurückgegriffen, die auch an den anderen Jet-FTIR-Setups in der Arbeitsgruppe Suнm eingesetzt wird. Elektronische Hoch- und Tiefpassfilter werden für die Messungen nicht aktiviert. Dafür wird der auf den Detektor eintreffende Spektralbereich in der Regel durch optische Filter wie das oben erwähnte Filter F20 oder das für InSb-Messungen häufig verwendete Filter F13 $\left(4200-2450 \mathrm{~cm}^{-1}\right)$ begrenzt.

Für die Datenaufnahme können verschiedene Spiegelgeschwindigkeiten zwischen 1.6 und $160 \mathrm{kHz}$ (jeweils bezogen auf die Modulationsfrequenz des HeNe-Lasers) gewählt werden (s. Tab. 3.1). Im Gegensatz zum Spektrometer IFS 66v/S am Filet-Jet wird vom VerTEX 70v aufgrund der hohen Auflösung (24 bit) des ADC bei keiner Spiegelgeschwindigkeit gain ranging (s. Kap. 2.2) verwendet, sodass Geschwindigkeiten von mehr als $80 \mathrm{kHz}$ in dieser Hinsicht nicht gegenüber niedrigeren Geschwindigkeiten benachteiligt sind. Bei Spiegelgeschwindigkeiten von mehr als $80 \mathrm{kHz}$ muss aufgrund der auf $80 \mathrm{kHz}$ begrenzten Samplingrate des ADC lediglich die obere Aufzeichnungsgrenze für Daten auf die 
halbe HeNe-Laserwellenzahl $\left(7899 \mathrm{~cm}^{-1}\right)$ beschränkt werden, damit das NYQUIST-Kriterium $^{[252]}$ erfüllt bleibt. In diesem Fall muss dann entsprechend auch der Spektralbereich des einfallenden Lichts auf $<7899 \mathrm{~cm}^{-1}$ begrenzt werden, um Aliasing-Artefakte durch Faltung zu vermeiden. ${ }^{[246]}$

Analog zum Filet-Jet ${ }^{[55]}$ wird am Gratin-Jet für die Jet-FTIR-Spektroskopie ein schnellscannendes Messverfahren mit mehreren, rasch aufeinander folgenden Scans geringer Auflösung (typischerweise $2 \mathrm{~cm}^{-1}$ ) genutzt. Dazu wird eine sogenannte TRS-Methode verwendet, wobei das Akronym TRS für time-resolved spectroscopy, also zeitaufgelöste Spektroskopie, steht. Die TRS-Methode ist so konfiguriert, dass zunächst 20 Hintergrundspektren aufgenommen werden, die zu einem Leerscan $l$ gemittelt werden. Anschließend folgt die Aufnahme zweier Vorscans $v 1$ und v2 unmittelbar vor Auslösung des Gaspulses, die zur Kontrolle der vor dem Gaspuls herrschenden Bedingungen genutzt werden können. Während des Gaspulses werden ein Probescan $p$ und während des abklingenden Gaspulses zwei Nachscans $n 1$ und $n 2$ angefertigt. Die Nachscans zeigen wärmer werdende Analytgase in der schwindenden Expansion und damit im Übergang zur Gasphase. Die zu den sechs verschiedenen Interferogrammen (l, v1, v2, p, n1, n2) zusammengefügten 25 Scans werden zunächst in einer gemeinsamen IgSm/Multiple-Datei gespeichert. Für die Berechnung der Absorbanzspektren werden die Vor-, Probe- und Nachscans dann jeweils mit dem Hintergrund verrechnet. Durch die Aufnahme neuer Hintergrundspektren unmittelbar vor jedem Gaspuls wird der Einfluss von zeitabhängigen Störungen, wie z. B. Fluktuationen in der Strahlungsintensität der Lichtquelle oder Änderungen der Luftfeuchtigkeit in der vom IR-Strahl durchquerten Laborluft, möglichst gering gehalten. Die hohe Zahl der Hintergrundscans stellt sicher, dass der Einfluss möglicher Störungen während der Hintergrundmessung auf die Resultatspektren möglichst gering ist.

Die Synchronisation zwischen Spektrometerscans und Gaspuls erfolgt über eine TTLSchnittstelle. Die hierbei verwendeten Signale nutzen Gleichspannungen von entweder 0 oder 5 V, um zwei Zustände 0 und 1 darzustellen. Die TTL-Schaltkreise ermöglichen dabei schnelle Spannungswechsel und damit eine hohe zeitliche Auflösung der Zustände. Über die Schnittstelle können vom Spektrometer automatisierte oder manuell konfigurierte Signale z. B. für die Repräsentation von Spiegelbewegung oder Datenaufnahme ausgegeben werden. Ebenso stehen Eingänge für TTL-Signale zur Verfügung, über die dem Spektrometer z. B. das Erreichen eines zuvor definierten Zieldrucks im Puffervolumen signalisiert (zur Zeit noch nicht implementiert) oder Triggersignale eingespeist werden können (am Gratin-Jet nicht vorgesehen).

Die Ansteuerung der Magnetventile der Düse erfolgt über einen Pulsgenerator des Typs Model 505-2C der Firma Berkeley Nucleonics Corp (BNC). Dieser nutzt als Triggersignal ein TTL-Signal des Spektrometers und gibt dann das Steuersignal aus, dessen Verzögerung (Delay) und Länge (Ontime) in Abhängigkeit von der genutzten Spiegelgeschwindigkeit und dem Akquisitionsmodus eingestellt werden. Zur Schaltung der Magnetventile, die eine Gleichspannung von $24 \mathrm{~V}$ bei einer Leistung von $11 \mathrm{~W}$ pro Ventil benötigen, wird das Steuersignal durch ein von den Chemie-Werkstätten gebautes Gerät verstärkt und auf die sieben Ventile verteilt. Zur Ermittlung der benötigten Delay- und Ontime-Einstel- 
lungen können die TTL-Signale des Spektrometers und die am Pulsgenerator erzeugten Signale mit einem Oszilloskop (PICOScOPE 3403D) ausgelesen werden. Dieses ist über USB mit dem Messrechner verbunden.

Experimentell begrenzt die maximal nutzbare Gaspulsdauer die Datenaufnahmezeit für das Probespektrum. Aufgrund des relativ kleinen Puffervolumens von $4 \mathrm{~m}^{3} \mathrm{kann}$ die Düse am oberen Ende des vorgesehenen Stagnationsdruckbereichs ( $>700 \mathrm{mbar}$ ) nicht wesentlich länger als $150 \mathrm{~ms}$ geöffnet bleiben, da andernfalls der Hintergrunddruck im Pufferbehälter zu stark ansteigt (s. Kap. 3.1). Hintergrunddrücke von mehr als etwa 2 mbar verursachen zum einen das Problem, dass die Stoßfronten der Expansion ${ }^{[43]}$ dem IR-Messbereich nahe kommen können, ${ }^{[226]}$ und zum anderen sorgen Druckstöße einer solchen Amplitude für eine hohe mechanische Belastung der für die Rekompression genutzten Pumpen. Durch die somit auf die genannten ca. $150 \mathrm{~ms}$ beschränkte Pulslänge und die daraus resultierende maximale Datenaufnahmezeit von etwa 100-110 ms (s. unten) sind nur bestimmte Kombinationen aus Akquisitionsmodus und Spiegelgeschwindkeit für die Gratin-Jet-FTIR-Spektroskopie nutzbar.

Bezüglich des Akquisitionsmodus, also der Art, wie das Interferogramm aufgezeichnet wird, gibt es zwei sinnvoll in der Jet-FTIR-Spektroskopie nutzbare Varianten: 1. die einseitige (single sided) Variante, in der asymmetrisch um das zentrale Intensitätsmaximum im Interferogramm, den sog. center burst, nur eine Seite des Interferogramms bis zur vollen gewählten Auflösung aufgezeichnet wird; oder 2. die zweiseitige (double sided) Variante, in der symmetrisch beide Seiten des Interferogramms bis zur vollen gewählten Auflösung gemessen werden. Der einseitige Messmodus führt dabei zu kürzeren Aufnahmezeiten. Im Gegensatz dazu werden beim zweiseitigen Messmodus quasi zwei Interferogramme auf einmal erhalten, wodurch pro Gaspuls ein besseres Signal-zu-Rausch-Verhältnis erzielt werden kann. Die weiteren Varianten der Akquisition, die eine Richtungsänderung der Spiegelbewegung während der Datenaufnahme (forward-backward-Akquisitionsmodi) beinhalten, benötigen für die Datenaufnahme zu lange und können damit nicht in Kombination mit einer gepulsten Überschallexpansion genutzt werden.

Die Optimierung der Messparameter für Messungen mit dem InSb-Detektor hinsichtlich optimaler Blende, Spiegelgeschwindigkeit und Akquisitionsmodus, sowie der Synchronisation von Spektrometerscan und Düsenpuls soll im folgenden Abschnitt beschrieben werden.

\subsubsection{Optimierung von Synchronisation und Messparametern}

Da nach der Erstinbetriebnahme des Gratin-Jets zuerst Spektren im OH- und CH-Streckschwingungsbereich aufgenommen werden sollen, erfolgte die Optimierung für Messungen in diesem Spektralbereich. Somit wurden für die Optimierungen der InSb-Detektor und die externe Wolframlampe mit einer Leistung von $150 \mathrm{~W}$ (W150) verwendet. Der Spektralbereich des einfallenden Lichts wurde durch den Einsatz des optischen Filters F13c auf den Bereich $4200-2450 \mathrm{~cm}^{-1}$ begrenzt. Die Vorverstärkung des InSb-Detektors wurde durch Reduzierung des Widerstandes in der ersten Verstärkerstufe von $10 \mathrm{k} \Omega$ auf 
Tabelle 3.3: Parameter und Messdaten für die Optimierung der Apertur der externen Wolframlampe (W150). In allen Fällen wurden der InSb-Detektor, $\mathrm{CaF}_{2}$-Optiken und Filter F13c verwendet und es wurde bei einer Auflösung von $2 \mathrm{~cm}^{-1}$ „single sided“ gemessen. Wegen Übersättigung des Detektors bei Blenden $>10 \mathrm{~mm}$ in Kombination mit $80 \mathrm{kHz}$ Spiegelgeschwindigkeit wurden die Messungen bei größeren Blendenöffnungen mit einer höheren Spiegelgeschwindigkeit bzw. einem geringeren Vorwiderstand durchgeführt und die ADC-Werte entsprechend korrigert (s. Fließtext). In der Tabelle bezeichnet $d$ den Blendendurchmesser, $S$ die Blendenöffnungsfläche, $v$ die Spiegelgeschwindigkeit, $R_{\mathrm{v}}$ den Vorwiderstand, $\mathrm{ADC}_{\text {real }}$ die gemessene Maximalintensität des Interferogramms (in beliebigen Einheiten) und $\mathrm{ADC}_{\mathrm{korr}}$ den entsprechenden korrigierten Wert.

\begin{tabular}{rrrrrr}
\hline $\boldsymbol{d} / \mathbf{~ m m}$ & $\boldsymbol{S} / \mathbf{m m}^{2}$ & $\boldsymbol{v} / \mathbf{k H z}$ & $\boldsymbol{R}_{\mathbf{v}} / \mathbf{k} \boldsymbol{A}$ & $\mathbf{A D C}_{\text {real }}$ & $\mathbf{A D C}_{\mathbf{k o r r}}$ \\
\hline 6 & 28 & 80 & 0.47 & 15185 & 15185 \\
8 & 50 & 80 & 0.47 & 22842 & 22842 \\
10 & 79 & 80 & 0.47 & 30756 & 30756 \\
10 & 79 & 120 & 0.47 & 25989 & 30756 \\
12 & 113 & 120 & 0.47 & 30536 & 36137 \\
21 & 346 & 80 & 0.30 & 26616 & 41698 \\
\hline
\end{tabular}

$0.47 \mathrm{k} \Omega$ verringert, um eine Übersättigung des Detektors in dieser Kombination aus Lichtquelle und Detektor zu vermeiden.

Aufgrund der weiter oben beschriebenen Problematik der fehlenden Fokussierung der externen Lichtquelle musste die Blendenöffnung empirisch optimiert werden. Dazu wurden von den Chemie-Werkstätten runde Blenden verschiedener Durchmesser gefertigt. Anhand von $\mathrm{NOTCH}$-Spektren (s. Kap. 3.3) wurde dann jeweils die Maximalamplitude im Interferogramm für die verschiedenen Blendengrößen bestimmt. Dabei wurde neben dem bereits erwähnten InSb-Detektor, dem Vorwiderstand von $0.47 \mathrm{k} \Omega$ und Filter F13c auf einen $\mathrm{CaF}_{2}$-Strahlteiler und -Fenster, eine Spiegelgeschwindigkeit von $80 \mathrm{kHz}$, den einseitigen (single sided) Akquisitionsmodus und eine Auflösung von $2 \mathrm{~cm}^{-1}$ zurückgegriffen. Es wurden Blenden von 6, 8, 10 und $12 \mathrm{~mm}$, sowie ohne Blende (Öffnungsdurchmesser der Flanschdurchführung zwischen Lichtquellengehäuse und Spektrometer: $21 \mathrm{~mm}$ ) getestet. Für die Aperturen von mehr als $10 \mathrm{~mm}$ Durchmesser ergab sich dabei das Problem, dass in der genannten Konfiguration der Detektor übersättigt war. Deshalb wurde bei einer höheren Spiegelgeschwindigkeit $v=120 \mathrm{kHz}$ (bei Blendendurchmessern $d=10,12 \mathrm{~mm}$ ) bzw. bei einer nochmals geringeren Vorverstärkung (Vorwiderstand $R_{\mathrm{v}}=0.30 \mathrm{k} \Omega$ bei $d=21 \mathrm{~mm}$ ) gemessen, um die Übersättigung des Detektors zu verhindern.

Da die Maximalamplitude im Interferogramm (in der OpUs-Software als $A D C$-Wert bezeichnet) sowohl von der Vorverstärkung als auch von der Spiegelgeschwindigkeit abhängig ist, muss die Änderung dieser Größen für einen einheitlichen Vergleich berücksichtigt werden. Die real gemessenen Werte $\left(\mathrm{ADC}_{\text {real }}\right)$ wurden mit Opus aus den NOTCHSpektren ausgelesen und sind in Tabelle 3.3 dargestellt. Für den Vergleich der ADC-Werte über die Messungen bei verschiedenen Spiegelgeschwindigkeiten und Vorverstärkun- 


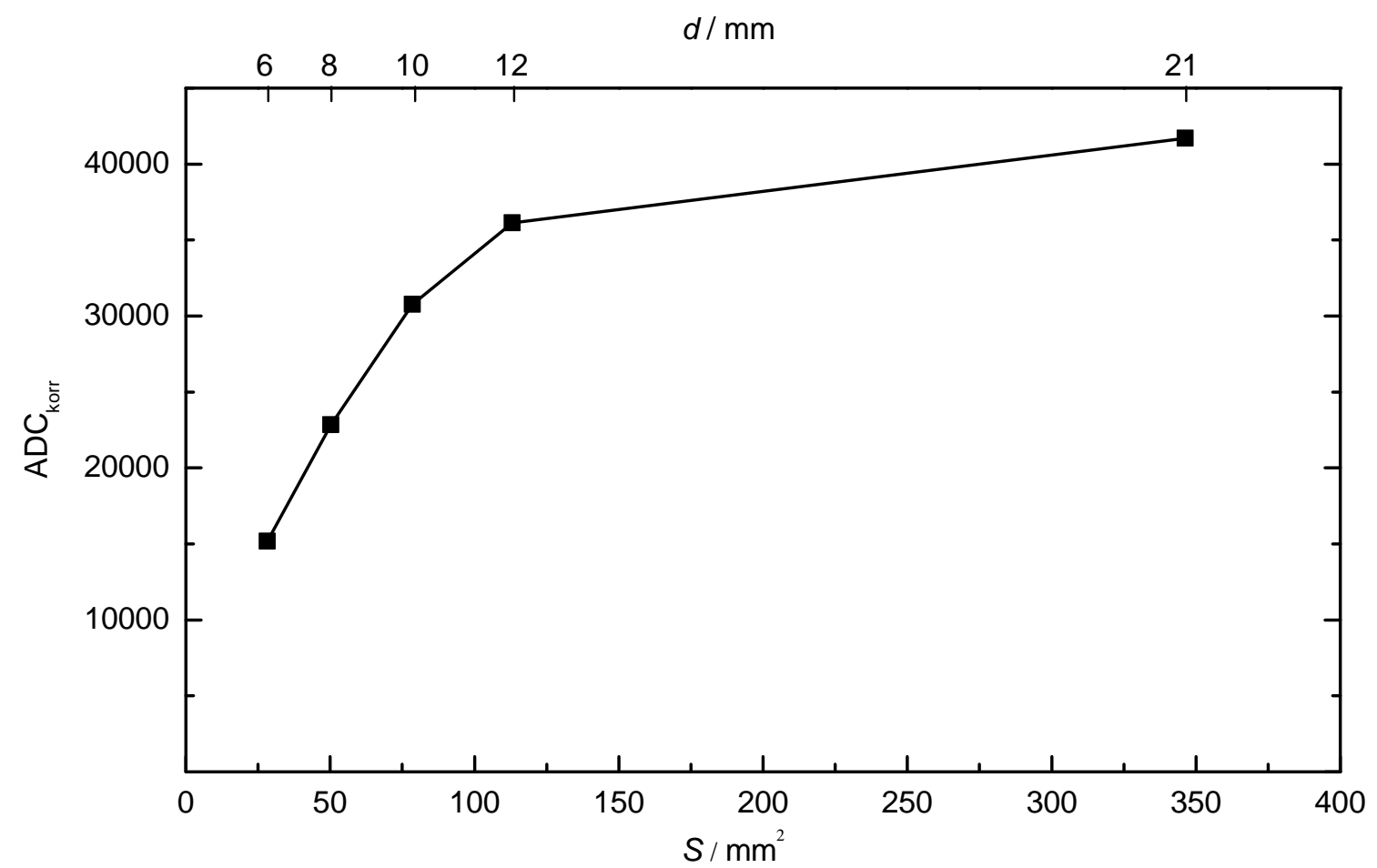

Abbildung 3.12: Graphische Darstellung der Abhängigkeit der korrigierten (s. Fließtext) maximalen Amplitude des Interferogramms $\left(\mathrm{ADC}_{\mathrm{korr}}\right)$ vom Öffnungsquerschnitt der Apertur der W150Lichtquelle.

gen hinweg wurde eine grobe Korrektur vorgenommen. Als Basis dienten dabei $80 \mathrm{kHz}$ Spiegelgeschwindigkeit und der Vorwiderstand von $0.47 \mathrm{k} \Omega$. Für die veränderte Vorverstärkung wurde dann eine lineare Abhängigkeit zwischen Vorwiderstand und ADC-Wert angenommen, sodass der Wert für eine Apertur von $21 \mathrm{~mm}$ (letzte Zeile) um den Faktor $k_{R}=0.47 / 0.30$ korrigiert wurde. Für die Spiegelgeschwindigkeit wurden die Messwerte bei $10 \mathrm{~mm}$ Blende und $80 \mathrm{bzw} .120 \mathrm{kHz}$ Spiegelgeschwindigkeit herangezogen und das Verhältnis der ADC-Werte aus diesen beiden Messungen $\left(k_{v}=30756 / 25989\right)$ wurde als Korrekturfaktor verwendet. Definitionsgemäß wird der Messwert bei $d=10 \mathrm{~mm}$ und $v=120 \mathrm{kHz}$ also auf den entsprechenden Messwert von $80 \mathrm{kHz}$ Spiegelgeschwindigkeit korrigiert. Mit demselben Korrekturfaktor $k_{v}$ kann der ADC-Messwert bei $d=12 \mathrm{~mm}$ und $v=120 \mathrm{kHz}$ dann auf 36137 korrigiert werden, sodass ein vereinheitlichter Satz an ADCWerten für den parameterübergreifenden Vergleich zur Verfügung steht.

In Abbildung 3.12 ist die Abhängigkeit des $\mathrm{ADC}$-Werts $\mathrm{ADC}_{\mathrm{korr}}$ von der Blendenöffnungsfläche $S$ (und damit dem Strahlquerschnitt in der Blendenebene) dargestellt. In der Theorie sollte die Zahl der Photonen und damit auch die Strahlungsintensität linear mit einem steigenden Strahlquerschnitt zunehmen. In den Messungen zeigt sich jedoch eine deutliche Abflachung der Korrelation bei größeren Blendenöffnungen. Dies ist hauptsächlich darauf zurückzuführen, dass der am Detektor eintreffende Strahlquerschnitt bei 
größeren Blenden nicht mehr durch die Blendenöffnung, sondern durch die weiteren im Strahlengang vorhandenen optischen Komponenten wie Strahlteiler und Spiegel begrenzt wird. ${ }^{[26]}$ Größere Blendenöffnungen bieten also kaum noch Intensitätsvorteile, sondern beinhalten vielmehr die Gefahr, dass die erzielbare Auflösung durch die Blende limitiert wird. Eine Untersuchung der Halbwertsbreite mehrerer OH-Streckschwingungslinien des Wassers in bei einer Auflösung von $2 \mathrm{~cm}^{-1}$ gemessenen Jet-FTIR-Spektren ergab bei einer Apertur von $12 \mathrm{~mm}$ jedoch keine Hinweise auf eine Auflösungsbegrenzung durch die Blende. Die Halbwertsbreite lag in allen Fällen im Bereich von $2 \mathrm{~cm}^{-1}$ und wurde somit noch durch die gewählte Auflösung begrenzt.

Die Erkenntnisse hinsichtlich der Nützlichkeit größerer Blendenöffnungen können bei Betrachtung des Rauschniveaus $N_{\mathrm{RMS}}$ nur zum Teil bestätigt werden. Für die Bestimmung des Rauschniveaus in den $\mathrm{NOTCH}$-Absorbanz-Spektren wurde der Bereich 3500-3300 $\mathrm{cm}^{-1}$ gewählt, der zwischen den für die geplanten Forschungsanwendungen relevanten $\mathrm{OH}$ - und $\mathrm{CH}$-Streckschwingungsbereichen liegt, jedoch nicht von möglicherweise schlecht kompensierten Linien atmosphärischen Wassers verfälscht wird. Als Wert des Rauschniveaus wurde die Wurzel der mittleren quadratischen (engl. root-meansquare, RMS) Abweichung gewählt, die für den genannten Bereich jeweils mit dem OpusProgrammpaket bestimmt wurde. Während sich bei einer Vergrößerung der Blende von 6 auf $8 \mathrm{~mm}$ noch eine Verbesserung des beobachteten Rauschniveaus einstellt, verringert es sich für die weitere Blendenvergrößerung auf 10, 12 oder $21 \mathrm{~mm}$ nicht mehr signifikant. Eine Blendenöffnung von $21 \mathrm{~mm}$ scheint also für die beiden analysierten Größen Maximalamplitude und Rauschniveau keine nennenswerten Vorteile gegenüber kleineren Aperturen zu bieten. $12 \mathrm{~mm}$ Blende zeigen hingegen noch eine merkliche Verbesserung der Maximalamplitude gegenüber $10 \mathrm{~mm}$. Da für $12 \mathrm{~mm}$ Blendenöffnung zudem keine Probleme durch Auflösungsbegrenzung festgestellt wurden, scheint die Kombination aus einer Blende von $12 \mathrm{~mm}$ Durchmesser mit dem Vorwiderstand von $0.47 \mathrm{k} \Omega$ das Optimum für Messungen mit der externen Lichtquelle in Verbindung mit dem $\mathrm{CaF}_{2}$-Strahlteiler, dem Filter F13c und dem InSb-Detektor darzustellen.

Für die Ermittlung der optimalen Kombination aus Spiegelgeschwindigkeit und Akquisitionsmodus müssen hingegen Jet-FTIR-Spektren herangezogen werden. Wie in Kapitel 3.5.1 bereits beschrieben, begrenzen die experimentellen Rahmenbedingungen die maximal nutzbare Pulslänge und damit die maximale Datenaufnahmezeit für den Probescan, mit dem die Überschallexpansion untersucht wird. Je größer der Stagnationsdruck, desto geringer ist dabei die maximal anwendbare Pulslänge, da bei höherem Stagnationsdruck mehr Gas pro Zeit durch die Düse strömt. Sollen einheitliche Messbedingungen gefunden werden, muss also vom höchsten für die Nutzung geplanten Stagnationsdruck von 750 mbar ausgegangen werden. Bei diesem ist die verwendbare Pulslänge auf etwa $150 \mathrm{~ms}$ beschränkt. Zunächst sollten deshalb Länge und zeitliche Lage des Gaspulses so optimiert werden, dass ein möglichst großer Anteil dieser $150 \mathrm{~ms}$ zur Datenaufnahme genutzt werden kann.

Für die Einstellung der relativen zeitlichen Lage von Gaspuls und Spektrometer-Probescan werden mehrere Parameter benötigt, die in den Metadaten der aufgezeichneten 


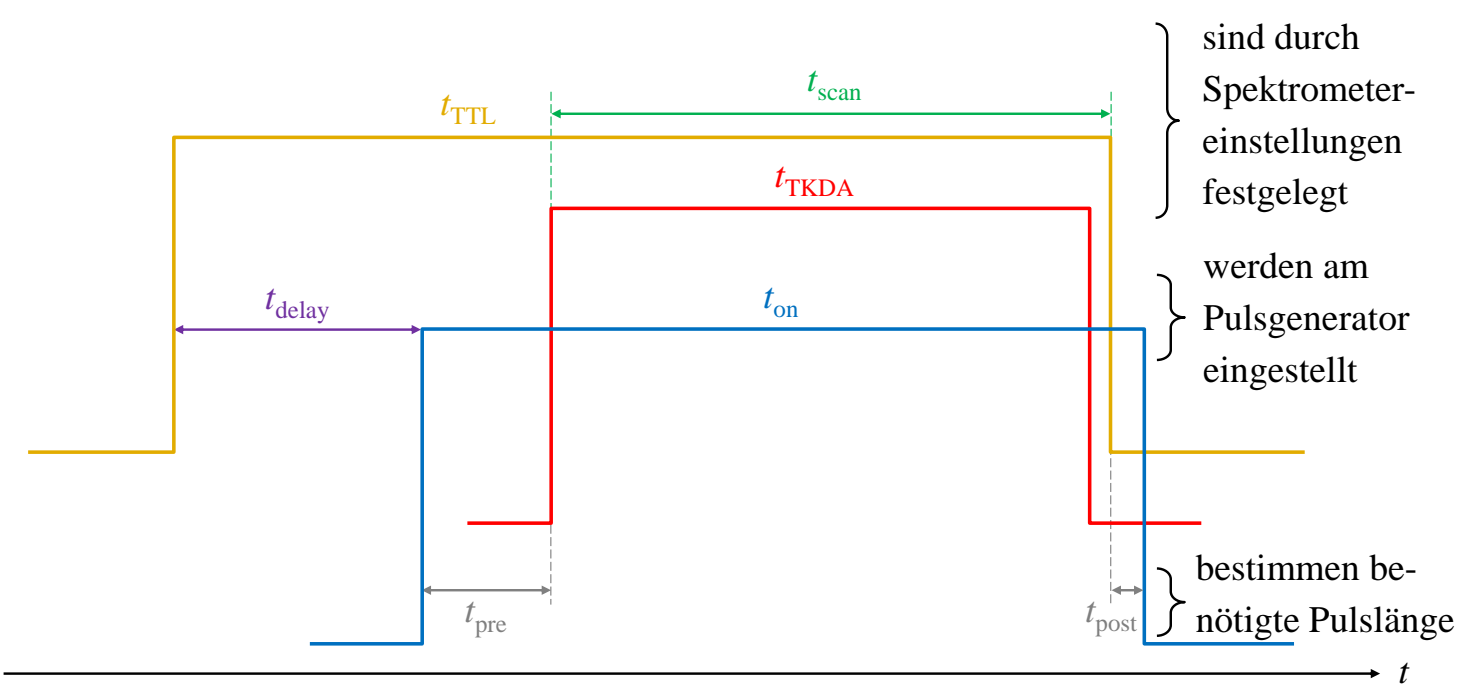

\begin{abstract}
Abbildung 3.13: Graphische Darstellung der für die Synchronisation zwischen Düsenpuls und Spektrometerscan benötigten Signale (Rechteck-Spannungen) und der Zeiten $t_{\mathrm{TTL}}$ (Messvorgangszeit, gelb), $t_{\text {scan }}\left(\right.$ Scanzeit, grün), $t_{\mathrm{TKDA}}$ (Datenaufnahmezeit, rot), $t_{\text {delay }}$ (Verzögerungszeit fürs Öffnen der Magnetventile, violett), $t_{\text {on }}$ (Düsenöffnungszeit, blau), $t_{\text {pre }}$ (Pulsvorlaufzeit, grau) und $t_{\text {post }}$ (Pulsnachlaufzeit, ebenfalls grau). Bearbeitung einer entsprechenden Darstellung in den Betriebsanweisungen des Filet-Jets.
\end{abstract}

Spektren hinterlegt sind oder am Pulsgenerator eingestellt werden. Die wichtigsten Parameter werden in Abbildung 3.13 gezeigt. Die Signale für $t_{\mathrm{TTL}}$ (gelb) und $t_{\mathrm{TKDA}}$ (rot) werden vom Spektrometer ausgegeben und sind über den Zeitraum der gesamten Spiegelbewegung eines Messvorgangs ( $\left.t_{\mathrm{TTL}}\right)$ bzw. während der eigentlichen Datenaufnahme ( $t_{\text {TKDA }}$, TKDA von „take data") aktiv. Das Signal $t_{\text {TTL }}$ beginnt dabei ausreichend rechtzeitig vor der Datenaufnahme, sodass sein Beginn als Triggersignal für den Pulsgenerator genutzt werden kann. Am Pulsgenerator, der die Magnetventile der Schlitzdüse steuert, werden die Verzögerungszeit für das Öffnen der Düsen-Magnetventile nach Beginn des Triggersignals, die Delay-Zeit $t_{\text {delay }}$ (violett), und die Öffnungsdauer der Magnetventile, $t_{\text {on }}$ (blau), eingestellt. Das Signal für die Düsenöffnung kann ebenso wie das Spiegelbewegungs- (gelb) und das Datenaufnahmesignal (rot) am Oszilloskop ausgelesen werden.

Die Synchronisation zwischen Düsenöffnung und Spektrometerscan wird also direkt auf dem Oszilloskop dargestellt. Die Erfahrungen vom Filet-Jet zeigen, dass die Düsenöffnungszeit $t_{\text {on }}$ für eine erfolgreiche Synchronisation mit einer Vorlaufzeit $t_{\text {pre }}$ (grau) von etwa 20-40 ms vor der Datenaufnahme beginnen und mit einer Nachlaufzeit $t_{\text {post }}$ (ebenfalls grau) von wenigen Millisekunden nach Ende der Datenaufnahme enden sollte. Das Signal für die Datenaufnahmezeit, $t_{\mathrm{TKDA}}$, ist am Gratin-Jet interessanterweise stets etwa $5 \mathrm{~ms}$ kürzer als die im Spektrum hinterlegte Scanzeit $t_{\text {scan }}$ (grün), die die tatsächlich verstrichene Zeit für die Datenaufnahme wiedergibt. Da das Signal $t_{\text {TKDA }}$ allerdings auch immer ca. $5 \mathrm{~ms}$ vor dem Spiegelbewegungssignal $t_{\mathrm{TTL}}$ endet, wird davon ausgegangen, 
dass die Datenaufnahme zeitgleich mit $t_{\mathrm{TKDA}}$ beginnt und zeitgleich mit $t_{\mathrm{TTL}}$ endet (vgl. Lage $t_{\text {scan }}$ (grün) in Abb. 3.13).

Für die Optimierung der Synchronisation müssen die Einstellungen am Pulsgenerator $\left(t_{\text {delay }}\right.$ und $\left.t_{\text {on }}\right)$ so gewählt werden, dass die Düse möglichst spät vor Beginn der Datenaufnahme geöffnet und möglichst schnell danach wieder geschlossen wird, ohne dass im Spektrum Störungen auftreten oder Signale an Intensität verlieren. Dazu wurden spektrometerseitig die in der Blendenoptimierung ermittelten Messparameter (LN-InSb-SWDetektor, $\mathrm{CaF}_{2}$-Optiken + W150-Lichtquelle, $120 \mathrm{kHz}$ Spiegelgeschwindigkeit, single sided Akquisitionsmodus, Apertur (Apt.) $12 \mathrm{~mm}$, Auflösung (Res.) $2 \mathrm{~cm}^{-1}$ ) gewählt. Die asymmetrische Aufnahme des Interferogramms hat im Zusammenhang mit der Pulsoptimierung den Vorteil, dass der center burst am Anfang des Gaspulses aufgenommen wird. Niedrig aufgelöste Informationen zu breiten Banden z. B. unspezifischer, größerer Cluster, deren spektrale Intensität besonders stark von der Konzentration des Analyten abhängig ist, werden somit zu Beginn des Gaspulses ermittelt. Beginnt der Gaspuls bezogen auf den Zeitpunkt der Datenaufnahme also zu spät, sollten schnell starke Konzentrationsabnahmen dieser Banden im Spektrum zu beobachten sein.

Die Optimierung wurde mit Ethanol als Analytmolekül durchgeführt. Dazu wurde eine Mischung von $0.17 \%$ Ethanol (SigmA-AldRICH, absolut, $\geq 99.8 \%$ (GC)) in Helium (Linde, 99.996\%) in das Reservoir gefüllt und bei einem Stagnationsdruck von 750 mbar durch die Schlitzdüse expandiert. Das expandierte Gas wurde entsprechend des Kreislaufkonzepts rekomprimiert und ins Reservoir zurückgeleitet. Dieser Kreislaufbetrieb wurde während der Testmessungen für etwa 5 Stunden aufrechterhalten. Sowohl das Jet-Spektrum von reinem Ethanol als auch die entsprechenden Spektren von Ethanol mit verschiedenen Luftbestandteilen, die im Laufe der Messzeit möglicherweise in den Kreislauf eindringen könnten, sind gut erforscht. ${ }^{[189,218]}$ Somit stellt Ethanol eine nützliche Referenz dar. Als Startpunkt der Optimierung wurden dabei die Einstellungen des Filet-Jets übernommen, da die Geometrie zwischen Düsen-Magnetventilen und eigentlicher Düse ebenfalls von diesem übernommen wurde (s. Kap. 3.4). Die Zeit, die das Gas benötigt, bis es von den Magnetventilen durch die Düse in die mit dem IR-Strahl untersuchte Expansionszone geströmt ist, sollte also vergleichbar sein. Dementsprechend wurden $t_{\text {delay }}$ und $t_{\text {on }}$ am Pulsgenerator zunächst so eingestellt, dass die Magnetventile $40 \mathrm{~ms}$ vor Beginn der Datenaufnahme geöffnet und $7 \mathrm{~ms}$ nach Ende der Datenaufnahme geschlossen werden. Bei einer mit Opus ausgelesenen Scanzeit $t_{\text {scan }}$ von $71 \mathrm{~ms}$ und am Oszilloskop abgelesenen Werten von $t_{\mathrm{TTL}}=209 \mathrm{~ms}$ sowie $t_{\mathrm{TKDA}}=66 \mathrm{~ms}$ entspricht dies Einstellungen von $t_{\text {delay }}=98 \mathrm{~ms}$ und $t_{\mathrm{on}}=118 \mathrm{~ms}$. Das daraus resultierende Spektrum ist in Abbildung 3.14a) dargestellt (rot). Die Vorlaufzeit $t_{\text {pre }}$, also die Zeitspanne zwischen Öffnen der Magnetventile und Beginn der Datenaufnahme, ist oberhalb der Grundlinie des Spektrums eingetragen (40 ms). In Teil a) der Abbildung sind die für eine Voroptimierung genutzten Spektren dargestellt. Dabei handelt es sich um die während der Gaspulse gemessenen Probespektren. Für diese wurden jeweils 10 Pulse bei identischen Einstellungen durchgeführt und zu einem Spektrum gemittelt. Die Nachlaufzeit $t_{\text {post }}$, also die Zeit zwischen Ende der Datenaufnahme und Schließen der Magnetventile, beträgt in a) jeweils $7 \mathrm{~ms}$. Die wichtigsten Ethanol- 


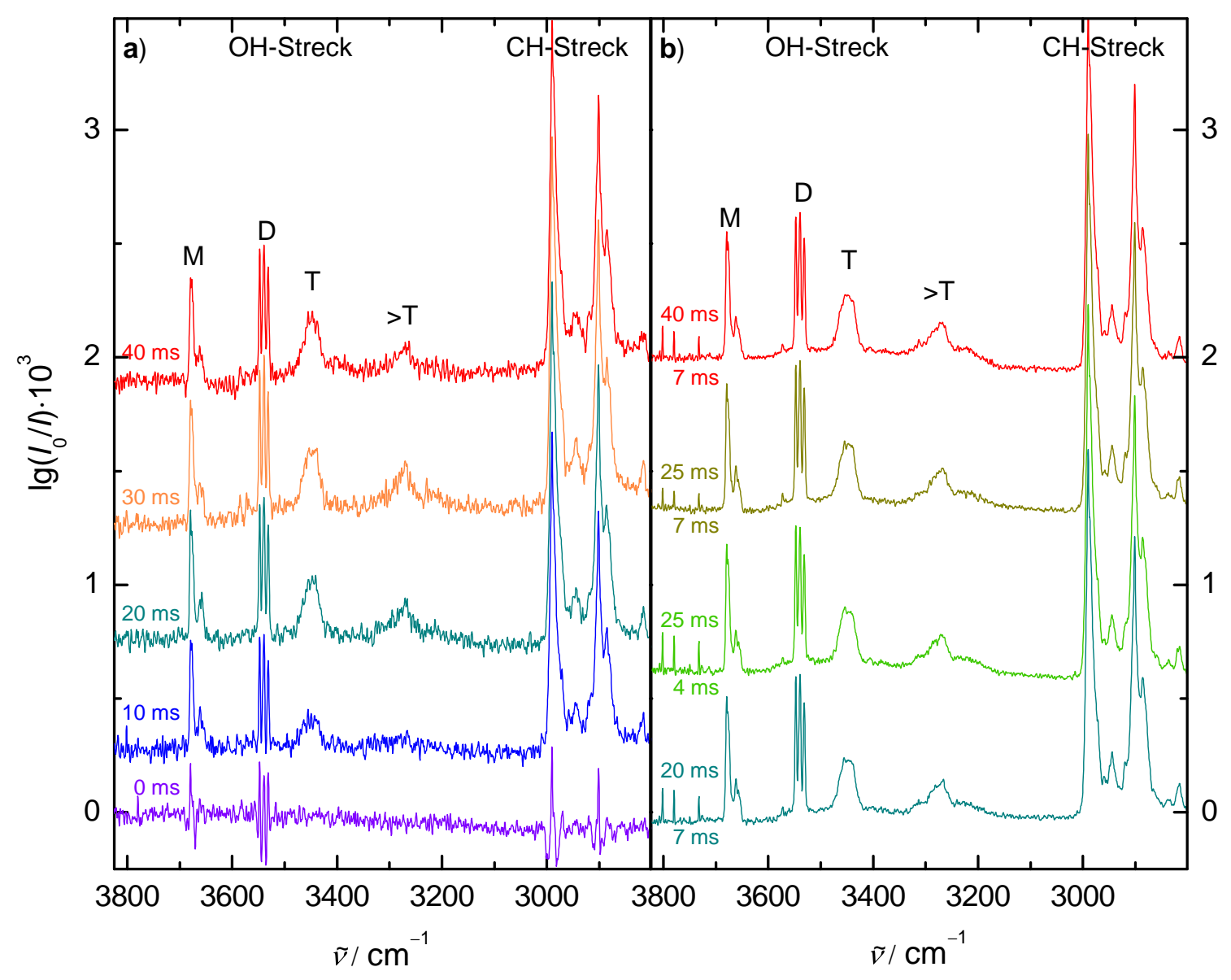

Abbildung 3.14: Optimierung der Synchronisation zwischen Spektrometerscan und Düsenpuls am Gratin-Jet anhand von Ethanol-Jet-Spektren. Es ist jeweils das während des Pulses gemessene Probespektrum dargestellt. a) Grobe Voroptimierung mit aus jeweils 10 Pulsen gemittelten Spektren. b) Feinoptimierung mit Spektren aus jeweils 100 Pulsen. $\mathrm{OH}-$ und $\mathrm{CH}-S t r e c k s c h w i n g u n g s-$ bereich sind jeweils gekennzeichnet, wobei die wichtigsten Banden des OH-Streckschwingungsbereichs (Ethanol-Monomer M, -Dimer D, -Trimer T und größere Cluster $>\mathrm{T}$ ) zugeordnet wurden. Die Vorlaufzeit $t_{\text {pre }}$ wurde jeweils in der Farbe des Spektrums oberhalb der Grundlinie eingefügt. Im rechten Teil der Abbildung (b) wurde zusätzlich die Nachlaufzeit $t_{\text {post }}$ entsprechend unterhalb des Spektrums eingetragen. In a) beträgt $t_{\text {post }}$ jeweils $7 \mathrm{~ms}$. Zur Verbesserung der Übersichtlichkeit wurden die Spektren entlang der Ordinate verschoben. 


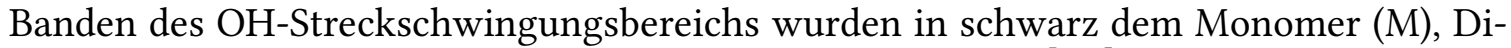
mer (D), Trimer (T) bzw. größeren Clustern $(>\mathrm{T})$ zugeordnet. ${ }^{[189]}$ Die Konzentration der größeren Cluster sollte dabei, wie weiter oben bereits beschrieben, am empfindlichsten auf Änderungen in der Synchronisation reagieren.

In einem ersten Schritt wurde die Vorlaufzeit um $10 \mathrm{~ms}$ auf $30 \mathrm{~ms}$ verkürzt (orange). Da die Nachlaufzeit unverändert beibehalten wurde, wurde dies durch eine Erhöhung des Delays $_{\text {delay }}$ um $10 \mathrm{~ms}$ auf $108 \mathrm{~ms}$ bei einer gleichzeitigen Verkürzung der Düsenöffnungszeit $t_{\text {on }}$ um $10 \mathrm{~ms}$ auf ebenfalls $108 \mathrm{~ms}$ erreicht. Spektral lassen sich keine Intensitätsunterschiede zum Spektrum bei $40 \mathrm{~ms}$ Vorlauf feststellen. Auch bei einer weiteren Verkürzung von $t_{\text {pre }}$ auf $20 \mathrm{~ms}\left(t_{\text {delay }}=118 \mathrm{~ms}, t_{\mathrm{on}}=98 \mathrm{~ms}\right.$, türkis) lassen sich noch keine signifikanten Veränderungen feststellen. Erst bei $10 \mathrm{~ms}$ Vorlauf $\left(t_{\text {delay }}=128 \mathrm{~ms}, t_{\mathrm{on}}=88 \mathrm{~ms}\right.$, blau) sinkt die Konzentration der größeren Cluster deutlich. Bei $0 \mathrm{~ms}$ Vorlauf $\left(t_{\text {delay }}=138 \mathrm{~ms}\right.$, $t_{\mathrm{on}}=78 \mathrm{~ms}$, violett) werden dann Artefakte im Spektrum sichtbar. Hier wird der center burst des Interferogramms vermutlich aufgezeichnet, bevor das Gas der Expansion den IR-Strahl kreuzt, was die Nützlichkeit des einseitigen Akquisitionsmodus für diese Tests verdeutlicht.

In Teil b) der Abbildung 3.14 wurde ausgehend von den Ergebnissen aus Teil a) eine Feinoptimierung vorgenommen. Hierzu wurden für die gezeigten Spektren jeweils 100 Pulse gemittelt, um mögliche statistische Konzentrationsschwankungen zu minimieren. Neben der Vorlauf- wurde hier auch die Nachlaufzeit optimiert und unterhalb des zugehörigen Spektrums aufgetragen. Als Referenz wurde nochmals ein Spektrum bei einer Vorlaufzeit von $40 \mathrm{~ms}$ und einer Nachlaufzeit von $7 \mathrm{~ms}$ (rot) aufgenommen. Nun wurde $t_{\text {pre }}$ jedoch auf $25 \mathrm{~ms}\left(t_{\text {delay }}=113 \mathrm{~ms}, t_{\mathrm{on}}=103 \mathrm{~ms}\right.$, dunkelgelb) bzw. $20 \mathrm{~ms}\left(t_{\text {delay }}=118 \mathrm{~ms}\right.$, $t_{\mathrm{on}}=98 \mathrm{~ms}$, türkis) verringert. In beiden Fällen lassen sich keine signifikanten Änderungen im Vergleich zum roten Referenzspektrum feststellen. 20 ms scheinen als Vorlauf somit ausreichend zu sein. Für den langfristig erfolgreichen Messbetrieb sollte jedoch ein Sicherheitsaufschlag vorgesehen werden, falls es im Laufe der Betriebszeit des GratinJets zu Schwankungen bei der Synchronisation kommen sollte. Mit einem Sicherheitsaufschlag von $5 \mathrm{~ms}$ ergibt sich die optimierte Vorlaufzeit damit zu $t_{\text {pre }}=25 \mathrm{~ms}$.

Mit dieser Vorlaufzeit wurde zusätzlich die Nachlaufzeit vorsichtig optimiert. Hier gilt der Grundsatz, dass das Schließen der Magnetventile nach Ende der Datenaufnahme erfolgen sollte, um mögliche Störsignale im Spektrum, die durch das Schalten der Magnetventile verursacht werden könnten, zu vermeiden. Da sämtliche mit dem Oszilloskop beobachteten Signale in ihrer Länge um bis zu $\pm 1 \mathrm{~ms}$ schwanken, sollte $t_{\text {post }}$ mindestens 2 , besser $4 \mathrm{~ms}$ betragen. Der Nachlauf wurde deshalb auf $4 \mathrm{~ms}\left(t_{\text {delay }}=113 \mathrm{~ms}, t_{\mathrm{on}}=100 \mathrm{~ms}\right.$, grün) verringert. Es wurden keine Veränderungen der Intensitäten und keine Störsignale im Spektrum festgestellt, sodass $4 \mathrm{~ms}$ als Nachlauf ausreichend zu sein scheinen.

Wie weiter oben bereits beschrieben, ist die maximal nutzbare Pulslänge durch die Größe des Puffervolumens und den für die Pumpen zulässigen Eingangsdruck beim maximalen Stagnationsdruck von 750 mbar auf $<150$ ms beschränkt. Wird auch hier eine Sicherheitsmarge berücksichtigt, sollte die Pulslänge für den dauerhaften Messbetrieb bei solchen Stagnationsdrücken möglichst unter 140 ms bleiben. Bei 25 ms Vor- und 4 ms Nach- 
Tabelle 3.4: Datenaufnahmezeiten $t_{\text {scan }}$ verschiedener Kombinationen aus Spiegelgeschwindigkeit $v$ und Akquisitionsmodus. Werte im für den Jet-Messbetrieb optimalen Bereich (100-110 ms) sind fett gedruckt.

\begin{tabular}{rrr}
\hline & \multicolumn{2}{c}{$\boldsymbol{t}_{\text {scan }} / \mathbf{m s}$} \\
\cline { 2 - 3 } $\boldsymbol{v} / \mathbf{k H z}$ & single sided & double sided \\
\hline 80 & $\mathbf{1 0 7}$ & 184 \\
120 & 71 & 123 \\
140 & 60 & $\mathbf{1 0 5}$ \\
160 & 55 & 95 \\
\hline
\end{tabular}

lauf ergibt sich daraus eine maximale Datenaufnahmezeit $t_{\text {scan }}$ von etwa $110 \mathrm{~ms}$ pro Puls. Um das beste Signal-zu-Rauschverhältnis in möglichst kurzer Jet-FTIR-Messzeit zu erreichen, sollte diese maximale Datenaufnahmezeit ausgereizt werden. Kürzere Datenaufnahmezeiten würden zwar kürzere Pulse und damit kürzere Wartezeiten zwischen zwei Pulsen ermöglichen, da weniger Gas aus dem Puffervolumen zurück ins Reservoir gepumpt werden muss. Allerdings nimmt die Wartezeit nicht linear, sondern deutlich langsamer mit sinkender Pulslänge ab, sodass die beste Mess-Effizienz bei möglichst langen Datenaufnahmezeiten pro Puls erreicht wird.

Für einen effizienten Messbetrieb, der bei allen Stagnationsdrücken genutzt werden kann, sollte also eine Kombination aus Spiegelgeschwindigkeit und Akquisitionsmodus gefunden werden, die bei der gewünschten Auflösung von $2 \mathrm{~cm}^{-1}$ eine Datenaufnahmezeit von etwa 100-110 ms aufweist. Die Datenaufnahmezeiten mehrerer solcher Kombinationen sind in Tabelle 3.4 aufgelistet. Spiegelgeschwindigkeiten unterhalb von $80 \mathrm{kHz}$ wurden nicht getestet, da bei dieser Geschwindigkeit bereits im schnelleren, einseitigen Akquisitionsmodus nahezu das obere Limit von 110 ms für die Datenaufnahmezeit erreicht wird. Höhere Spiegelgeschwindigkeiten weisen im einseitigen Modus dann Scanzeiten deutlich unterhalb von $100 \mathrm{~ms}$ auf und führen damit nicht zu einem effizienten JetMessbetrieb. Im doppelseitigen Akquisitionsmodus tritt dagegen das Problem auf, dass für 80 und $120 \mathrm{kHz}$ die Datenaufnahmezeiten zu lang sind. Nur Spiegelgeschwindigkeiten von 140 und $160 \mathrm{kHz}$ sind in diesem Modus bei allen Stagnationsdrücken für die JetSpektroskopie nutzbar. $160 \mathrm{kHz}$ stellen die höchste mit dem VerTEx 70v nutzbare Spiegelgeschwindigkeit dar. Bei Tests mit dieser Spiegelgeschwindigkeit wurden Probleme mit der Grundlinienstabilität beobachtet (s. Abb. 3.15). Wie anhand der dargestellten, nicht verschobenen NOTCH-Testspektren deutlich wird, weicht die Grundlinienabsorbanz bei $160 \mathrm{kHz}$ Spiegelgeschwindigkeit oftmals stark von $0 \mathrm{ab}$ (grün, blau) oder weist einen diagonalen Verlauf entlang des beobachteten Wellenzahlbereichs $\left(4200-2450 \mathrm{~cm}^{-1}\right.$ ) auf (rot, orange). Bei niedrigeren Spiegelgeschwindigkeiten treten diese Probleme nicht in vergleichbarer Stärke auf, wie das abgebildete 140-kHz-Beispielspektrum (schwarz) verdeutlicht. Das Betreiben des Spektrometers an der Grenze der Spezifikation scheint also nicht 


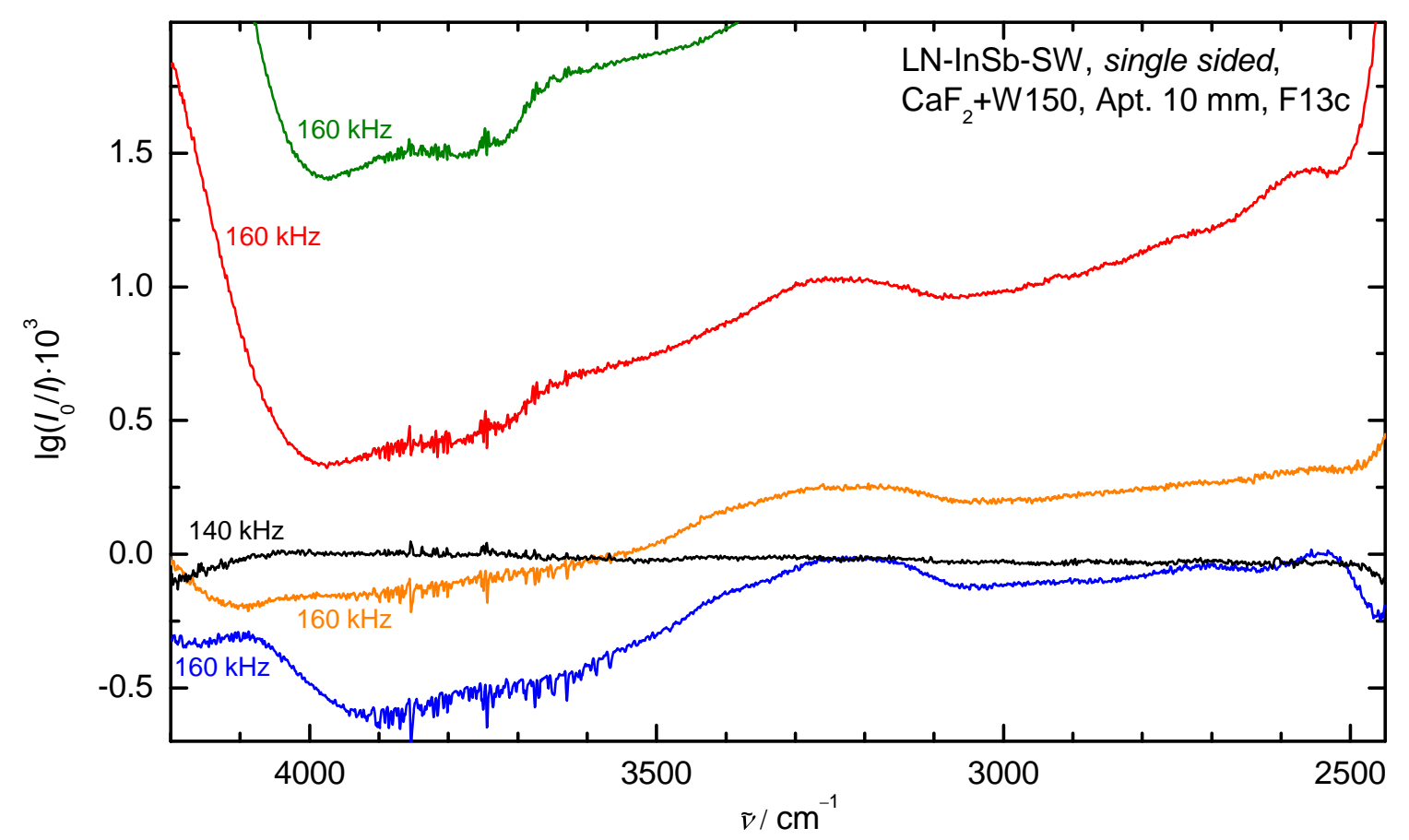

Abbildung 3.15: Veranschaulichung der instabilen Grundlinie im Filterbereich (4200-2450 $\left.\mathrm{cm}^{-1}\right)$ bei $160 \mathrm{kHz}$ Spiegelgeschwindigkeit anhand von NOTCH-Test-Spektren, für die jeweils 1 min lang Hintergrund- und 1 min lang Probescans aufgezeichnet und gemittelt wurden. Im Vergleich zum Referenz-Spektrum bei $140 \mathrm{kHz}$ Spiegelgeschwindigkeit (schwarz) zeigen die an mehreren Messtagen bei jeweils $160 \mathrm{kHz}$ aufgenommenen Spektren (grün, rot, orange, blau) stark von 0 abweichende Grundlinien-Positionen. Die Spiegelgeschwindigkeiten sind an den jeweiligen Spektren und die wichtigsten sonstigen Messparameter oben rechts in der Abbildung eingetragen.

empfehlenswert zu sein.

Als geeignetste Kandidaten für die Jet-FTIR-Spektroskopie wurden damit die Messmodi $80 \mathrm{kHz}$ single sided und $140 \mathrm{kHz}$ double sided identifiziert. In den Spektren mit $80 \mathrm{kHz}$ Spiegelgeschwindigkeit ist jedoch im Bereich $3320-3100 \mathrm{~cm}^{-1}$ ein Störsignal zu beobachten (s. Abb. 3.16). Im gezeigten NOTCH-Spektrum ist das Signal besonders ausgeprägt und zeigt seine extremsten Intensitäten bei etwa 3162 (Maximum) bzw. $3160 \mathrm{~cm}^{-1}$ (Minimum). Die Intensität des Störsignals unterscheidet sich von Spektrum zu Spektrum, das Signal tritt jedoch nicht nur in NOTCH-, sondern auch in Jet-Spektren auf. Durch ein Ändern der Spiegelgeschwindigkeit lässt sich das Signal aus dem hier betrachteten $\mathrm{CH}$ - und $\mathrm{OH}-$ Streckschwingungsbereich in nicht untersuchte Spektralbereiche verschieben. Basierend auf Tests bei ein- bzw. abgeschalteten Pumpen können zwar die Turbomolekular-, Schrauben- und Wälzkolbenpumpen als Quelle ausgeschlossen werden, die Ursache des Störsignals konnte bisher allerdings nicht ermittelt werden.

Bei $140 \mathrm{kHz}$ Spiegelgeschwindigkeit sind hingegen keine Störsignale im betrachteten Spektralbereich erkennbar. Darüber hinaus weist der in Kombination mit dieser Spiegel- 


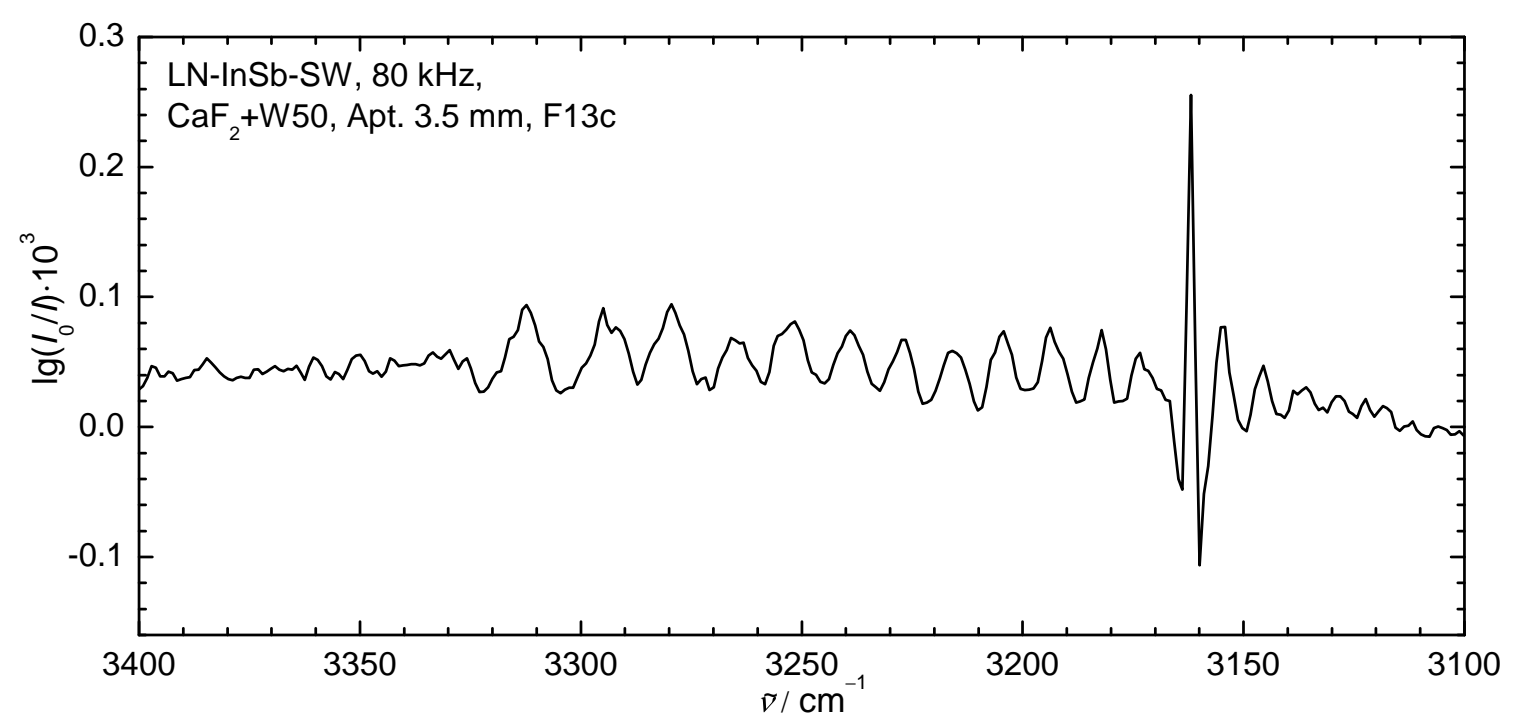

Abbildung 3.16: Das bei $80 \mathrm{kHz}$ Spiegelgeschwindigkeit im Bereich $3320-3100 \mathrm{~cm}^{-1}$ beobachtete Störsignal. Die wichtigsten Messparameter des hier gezeigten NOTCH-Spektrums sind im Spektrum angegeben. Das Störsignal wird auch bei Nutzung der externen Lichtquelle beobachtet und konnte bisher keiner Quelle zugeordnet werden.

geschwindigkeit in der Jet-FTIR-Spektroskopie nutzbare doppelseitige Akquisitionsmodus im Vergleich zum einseitigen Modus eine bessere Pulslage des center bursts auf. Die Aufnahme des zentralen Intensitätsmaximums des Interferogramms erfolgt beim doppelseitigen Messmodus zeitlich in der Mitte des Gaspulses und damit zu einem Zeitpunkt, an dem die Expansion ausreichend Zeit hatte, sich vollständig aufzubauen, aber noch vor Erreichen des maximalen Hintergrunddrucks kurz vor Ende des Pulses. Die Aufnahme von zwei Interferogrammen pro Spektrometerscan wirkt sich zudem positiv auf das pro Puls erreichbare Signal-zu-Rausch-Verhältnis aus. Für den Messmodus $140 \mathrm{kHz}$, double sided wurden die Einstellungen für Delay und Ontime zu $t_{\text {delay }}=146 \mathrm{~ms}$ und $t_{\mathrm{on}}=133 \mathrm{~ms}$ ermittelt, um 25 ms Vor- und 4 ms Nachlauf des Gaspulses rund um die Datenaufnahme zu erreichen.

Zusammenfassend wurden als Optimum für die Jet-FTIR-Spektroskopie des $\mathrm{OH}$ - und $\mathrm{CH}$-Streckschwingungsbereichs bei Stagnationsdrücken von bis zu 750 mbar am GratinJet die in Tabelle 3.5 aufgelisteten Parameter ermittelt. Diese Parameter wurden auch für die Aufzeichnung der in den folgenden Kapiteln gezeigten Gratin-Jet-Spektren verwendet.

Wie in Abbildung 3.14 auf S. 65 bei genauerer Betrachtung bereits sichtbar wird, sind in den am Gratin-Jet aufgezeichneten Jet-Spektren nach einigen Stunden Messzeit Jet-Wasser-Linien zu beobachten. In der rechten Abbildungshälfte (Teil b) sind Spektren dargestellt, die nach mehreren Stunden Kreislaufbetrieb aufgenommen wurden. Die Jet-WasserLinien sind bei 3802, 3780 und $3732 \mathrm{~cm}^{-1}$ zu erkennen und gewinnen mit fortschreitender Messzeit stetig an Intensität. Im Laufe der Zeit sammelt sich im Kreislaufbetrieb also 
Tabelle 3.5: Optimierte Parameter für Messungen im $\mathrm{OH}$ - und $\mathrm{CH}$-Streckschwingungsbereich am Gratin-Jet.

\begin{tabular}{ll}
\hline Komponente/Parameter & Einstellung/Auswahl \\
\hline Lichtquelle & $\mathrm{W} 150$ \\
Optiken & $\mathrm{CaF}_{2}$ \\
Detektor & $\mathrm{LN}-\mathrm{InSb}-\mathrm{SW}$ \\
Apertur & $12 \mathrm{~mm}$ \\
Spiegelgeschwindigkeit & $140 \mathrm{kHz}$ \\
Akquisitionsmodus & double sided \\
Synchronisation & $t_{\text {delay }}=146 \mathrm{~ms}, t_{\text {on }}=133 \mathrm{~ms}$ \\
\hline
\end{tabular}

Wasser in der Apparatur, das dann spektral nachgewiesen werden kann. Die Quelle des Wassers konnte bisher nicht eindeutig identifiziert werden. Über Lecks in das Kreislaufsystem eindringende Luft kann jedoch als Hauptursache ausgeschlossen werden, wie im Folgenden erläutert werden soll.

\subsection{Untersuchung der im Laufe der Messzeit steigenden Wasserkonzentration}

Wird der Gratin-Jet mit allen drei Pumpen des Wälzkolbenpumpstands im Kreislaufbetrieb genutzt, lassen sich nach etwa 30-60 min erste Anzeichen von Wasser in den Jet-FTIR-Spektren feststellen. Die Intensität der beobachteten Wasserlinien steigt dabei mit fortschreitender Zeit gleichmäßig an. In Abbildung 3.17 ist der relevante Spektralbereich eines Spektrums von 1.30 mbar Ethanol in 750 mbar Helium dargestellt. Neben dem Ethanol-Monomer und -Dimer sind die drei bereits erwähnten Linien bei 3802, 3780 und $3732 \mathrm{~cm}^{-1}$ erkennbar, die verschiedenen Rotations-Vibrationsübergängen des Wassers zugeordnet werden können (s. Tab. 3.6). Da diese Linien allesamt nur die Besetzung des jeweils tiefsten erlaubten Rotationszustands von ortho- und para-Wasser involvieren und keine weiteren Wasserlinien signifikanter Intensität beobachtet werden können, handelt es sich hier nicht um warmes, atmosphärisches, sondern um rotationskaltes Wasser in der Überschallexpansion.

Die drei in Abbildung 3.17 dargestellten Spektren wurden entlang der Ordinate so verschoben, dass die Grundlinien im Bereich der Wasserlinien möglichst gut überlappen. So lässt sich der Anstieg der Wasserkonzentration im laufenden Messbetrieb im Vergleich des blauen (gemessen 0:15-1:05 h nach Beginn des Kreislaufbetriebs), grünen (2:35-3:25 h) und roten Spektrums (3:30-4:20 h) deutlich erkennen.

Für die Analyse der beobachteten Stoffmengenzunahme von Wasser sollte zunächst die Leckrate quantifiziert werden. Dazu muss die absolute Wasserkonzentration in einem Spektrum bestimmt und mit der seit Kreislaufbetriebsbeginn verstrichenen Zeit verrech- 


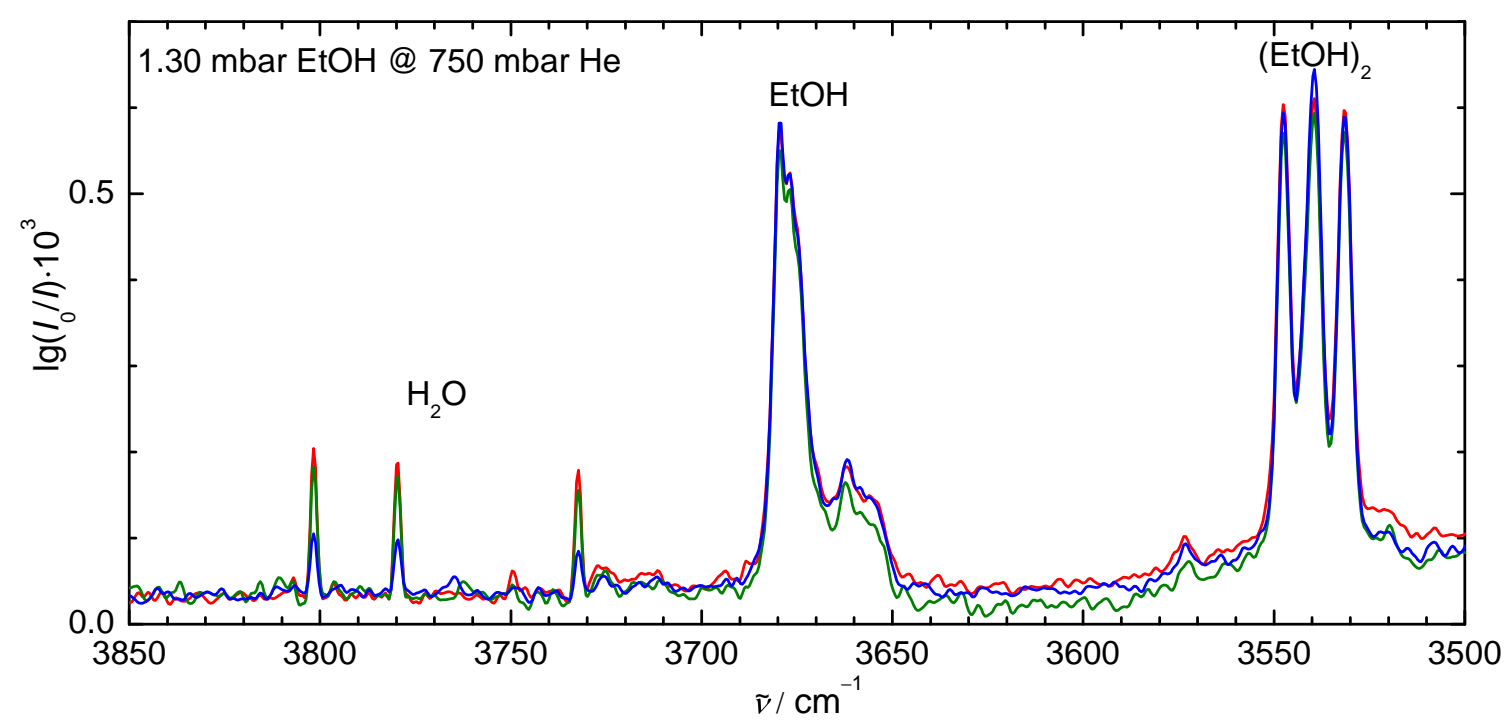

Abbildung 3.17: Teil des OH-Streckschwingungsbereichs eines Ethanol-Spektrums von 1.30 mbar Ethanol in 750 mbar Helium am Gratin-Jet. Neben Ethanol-Monomer- (EtOH) und Ethanol-Dimer-Banden $\left((\mathrm{EtOH})_{2}\right)$ sind bei 3802, 3780 und $3732 \mathrm{~cm}^{-1}$ drei charakteristische Linien zu erkennen, die auf in der Überschallexpansion vorhandenes Wasser zurückgeführt werden können. Die Intensität dieser Linien steigt mit fortschreitender Messzeit (blau über grün zu rot) an. Durch ein Verschieben entlang der Ordinate (rot, grün: +0.09 ; blau: +0.15$)$ wurden die Grundlinien der gezeigten Spektren im Bereich der Wasserlinien möglichst gut zur Überlappung gebracht.

Tabelle 3.6: Zuordnung der beobachteten $\left(\tilde{v}_{\text {exp }}\right)$ Wasserlinien zu den Literaturwerten $\left(\tilde{v}_{\text {Lit }}\right)$ mit zugehörigen Rotationsübergängen nach Ref. [253]. Der beteiligte Schwingungsübergang ist in allen Fällen der Fundamentalübergang der asymmetrischen Streckschwingung. $J^{\prime}, K_{a}^{\prime}$ und $K_{c}^{\prime}$, sowie $J^{\prime \prime}$, $K_{a}^{\prime \prime}$ und $K_{c}^{\prime \prime}$ sind die Rotationsquantenzahlen des angeregten bzw. Vibrationsgrundzustands.

\begin{tabular}{rcrrrrrr}
\hline$\tilde{v}_{\exp } / \mathbf{c m}^{-1}$ & $\tilde{\nu}_{\text {Lit }} / \mathbf{c m}^{-1}$ & $J^{\prime}$ & $K_{\boldsymbol{a}}^{\prime}$ & $\boldsymbol{K}_{\boldsymbol{c}}^{\prime}$ & $J^{\prime \prime}$ & $\boldsymbol{K}_{\boldsymbol{a}}^{\prime \prime}$ & $\boldsymbol{K}_{\boldsymbol{c}}^{\prime \prime}$ \\
\hline 3732.3 & 3732.13539 & 0 & 0 & 0 & 1 & 0 & 1 \\
3779.7 & 3779.49376 & 1 & 0 & 1 & 0 & 0 & 0 \\
3801.7 & 3801.41958 & 2 & 0 & 2 & 1 & 0 & 1 \\
\hline
\end{tabular}


net werden. Zur Bestimmung der Wasserkonzentration wurden zwei verschiedene Ansätze genutzt:

A. Berechnung aus der (bekannten) Konzentration des Ethanols unter Zuhilfenahme der Absorptionsquerschnitte der betrachteten Banden bzw. Linien

B. Vergleich mit einem Referenzspektrum bekannter Wasserkonzentration

Nach dem Lambert-Beer'schen Gesetz (Gl. 3.3) ist die im Spektrum beobachtete Absorbanz bzw. Extinktion $\left(\lg \left(E_{0} / E\right)\right.$, mit $E$ : Strahlungsintensität) einer Bande proportional zur Konzentration $c$ des Stoffes:

$$
\lg \left(\frac{E_{0}}{E}\right)=\epsilon \cdot c \cdot d
$$

Im Folgenden wird für die Auswertung anstelle der Extinktion das dazu proportionale Integral I der betrachteten Bande genutzt. Zur absoluten Bestimmung der Konzentration müssten neben dem gemessenen Bandenintegral auch die Schichtdicke $d$ und der Extinktionskoeffizient $\epsilon$ bekannt sein. Soll hingegen die Konzentration relativ zu einer zweiten Konzentration bestimmt werden, ergibt sich aus Gleichung 3.3:

$$
\frac{c_{1}}{c_{2}}=\frac{I_{1}}{I_{2}} \cdot \frac{\epsilon_{2}}{\epsilon_{1}} \cdot \frac{d_{2}}{d_{1}}
$$

Werden beide Spektren mit demselben Aufbau aufgezeichnet, gilt weiterhin $d_{1}=d_{2}$, sodass sich umgestellt nach $c_{1}$ Gl. 3.5 ergibt:

$$
c_{1}=\frac{I_{1}}{I_{2}} \cdot \frac{\epsilon_{2}}{\epsilon_{1}} \cdot c_{2}
$$

Für die Berechnung von $c_{1}$ ist nun also nicht mehr die Kenntnis der einzelnen Extinktionskoeffizienten erforderlich, sondern lediglich ihres Verhältnisses zueinander. Dieses kann durch die Verwendung berechneter IR-Bandenstärken angenähert werden. Wird für deren Berechnung eine Schwingungsrechnung in doppelt harmonischer Näherung durchgeführt, wie sie für viele quantenchemische Methoden effizient genutzt werden kann, sind die Bandenstärken zwar absolut gesehen stark fehlerbehaftet, das Verhältnis profitiert aber von Fehlerkompensation und kann semi-quantitativ verwendet werden. Dies gilt insbesondere, wenn vergleichbare Schwingungen, z. B. in beiden Fällen eine OH-Streckschwingung, betrachtet werden. Soll also nach Ansatz A die Wasserkonzentration aus der des Ethanols berechnet werden, können die Bandenstärken der jeweiligen $\mathrm{OH}$-Streckschwingungen aus einer quantenmechanischen Rechnung $\left(A_{\mathrm{OH}, x}\right)$ eingesetzt werden. Bei Ethanol liegen allerdings zwei Konformere (gauche $(g)$ und trans $(t)$, bezogen auf den Diederwinkel der OH-Gruppe zur C-C-Bindung des Moleküls) vor, deren $\mathrm{OH}-$ Streckschwingungen unterschiedliche Bandenstärken aufweisen. Als effektive Bandenstärke wird deshalb ein nach der Boltzmann-Verteilung gewichteter Mittelwert dieser beiden Bandenstärken berechnet. Der dafür nötige Energieunterschied 
$\Delta E_{t-g}^{0}=-0.14 \mathrm{~kJ} \cdot \mathrm{mol}^{-1}$ wird ebenso wie die individuellen Bandenstärken von $g$ $(22.4 \mathrm{~km} / \mathrm{mol})$ und $t$-Isomer $(28.7 \mathrm{~km} / \mathrm{mol})$ aus einer DFT-Rechnung (B3LYP-D3(BJ,ABC)/ def2-QZVP, doppelt harmonische Näherung) bezogen. Der Boltzmann-gewichtete Mittelwert der Bandenstärken ergibt sich zu $24.9 \mathrm{~km} / \mathrm{mol}$, wenn für die Berechnung der relativen Häufigkeit von $g$ - und $t$-Isomer der genannte Energieunterschied, die relative Entartung $g_{t} / g_{g}=0.5$ und eine auf $60 \mathrm{~K}$ geschätzte Konformationstemperatur $T_{c}$ verwendet werden (s. Kap. 2.3.2). Der auf demselben Rechenniveau bestimmte Wert für die Bandenstärke der asymmetrischen Streckschwingung von Wasser beläuft sich auf $58.5 \mathrm{~km} / \mathrm{mol}$. Zwar sind die Absolutkonzentrationen aller beteiligten Stoffe direkt in der Überschallexpansion unbekannt, es können jedoch die Konzentrationen im Reservoir genutzt werden. Durch Integration der experimentell beobachteten OH-Streckschwingungsbanden des Wassers und des Ethanol-Monomers (s. Abb. 3.17) im Probespektrum lässt sich damit die Konzentration bzw. proportional dazu der Partialdruck des Wassers im Reservoir aus dem bekannten Partialdruck des Ethanols im Reservoir bestimmen.

Gleichung 3.5 lässt sich noch weiter zu Gleichung 3.6 vereinfachen, wenn nach Ansatz B ein Referenzspektrum derselben Substanz zur Konzentrationsbestimmung genutzt wird, da dann bei Betrachtung gleicher Vibrationsübergänge $\epsilon_{1}=\epsilon_{2}$ gilt:

$$
c_{1}=\frac{I_{1}}{I_{2}} \cdot c_{2}
$$

Hier müssen bei bekannter Konzentration $c_{2}$ lediglich die entsprechenden Banden bzw. Linien in beiden Spektren integriert werden, um die Konzentration $c_{1}$ zu berechnen. Hierzu muss in Ansatz B ein zusätzliches Referenzspektrum, das eine bekannte Wasserkonzentration beinhaltet, aufgezeichnet werden. In diesem Fall wurde dazu ein Spektrum von 1.30 mbar Ethanol und 7.49 mbar Luft in Helium (Stagnationsdruck 750 mbar) aufgenommen. Um den Wasserpartialdruck $p_{\mathrm{H}_{2} \mathrm{O}}$ in dieser Mischung aus der relativen Luftfeuchtigkeit $(22.7 \%)$ zu bestimmen, musste zunächst der Sättigungsdampfdruck des Wassers bei den zum Einfüllzeitpunkt vorherrschenden Bedingungen berechnet werden. Da der Luftdruck zum Einfüllzeitpunkt nicht gemessen wurde, musste der Normluftdruck von 1013.25 mbar $^{[254,255]}$ verwendet werden, um über die MAGNUS-Formel nach SonNTAG ${ }^{\text {[256] }}$ den Sättigungsdampfdruck bei der bestehenden Temperatur $\left(23.8^{\circ} \mathrm{C}\right)$ zu $29.4 \mathrm{mbar}$ zu berechnen. Mithilfe der relativen Luftfeuchte konnte dann der im Reservoir vorliegende Wasserpartialdruck $p_{\mathrm{H}_{2} \mathrm{O}}$ zu 0.05 mbar bestimmt werden.

Für die Abschätzung der Leckrate des Wassers wurde der Wasserpartialdruck in einem Spektrum von 1.30 mbar Ethanol in Helium mit einem Stagnationsdruck von 750 mbar über die beiden vorgestellten Ansätze bestimmt. Da für dieses Spektrum 100 Gaspulse gemittelt wurden, ergibt sich auch für den sich im Laufe der Messung ändernden Wasserpartialdruck eine Mittelung über die gesamte Messdauer. Als der Leckrate $L_{\mathrm{H}_{2} \mathrm{O}}$ zugrundeliegende Zeiten wurden deshalb zwei Grenzwerte verwendet: zum einen die bei Messbeginn verstrichene Zeit seit Aufnahme des Kreislaufbetriebs $\left(\Delta t_{1}\right)$ und zum anderen die entsprechende bei Messende verstrichene Zeit $\left(\Delta t_{2}\right)$. Als Volumen der Gasmischung wird das Volumen des Reservoirs und seiner Zuleitung $\left(V_{R+Z}=205 \mathrm{~L}\right)$ verwendet. Für die 
Tabelle 3.7: Wasserleckrate im Messbetrieb am Gratin-Jet. Die mit „p“indizierten Werte sind Werte aus dem untersuchten Probespektrum für Ansatz A und B, die mit „"“ indizierten Werte sind die entsprechenden Werte des Wassers im Referenzspektrum für Ansatz B.

\begin{tabular}{lrr}
\hline & Ansatz A & Ansatz B \\
\hline$A_{\mathrm{OH}, \mathrm{H}_{2} \mathrm{O}} / \mathrm{km} \cdot \mathrm{mol}^{-1}$ & 58.5 \\
$A_{\mathrm{OH}, \mathrm{EtOH}} / \mathrm{km} \cdot \mathrm{mol}^{-1}$ & 24.9 & \\
$I_{\mathrm{EtOH}, \mathrm{p}} / \mathrm{cm}^{-1}$ & $6.46 \cdot 10^{-3}$ & \\
$I_{\mathrm{H}_{2} \mathrm{O}, \mathrm{r}} / \mathrm{cm}^{-1}$ & \multicolumn{2}{c}{$6.45 \cdot 10^{-4}$} \\
$p_{\mathrm{EtOH}, \mathrm{p}} / \mathrm{mbar}$ & 1.30 & \\
$p_{\mathrm{H}_{2} \mathrm{O}, \mathrm{r}} / \mathrm{mbar}$ & \multicolumn{2}{c}{0.049} \\
$\Delta t_{1} / \mathrm{s}$ & 10200 & \\
$\Delta t_{2} / \mathrm{s}$ & 13020 \\
$I_{\mathrm{H}_{2} \mathrm{O}, \mathrm{p}} / \mathrm{cm}^{-1}$ & $4.50 \cdot 10^{-4}$ \\
\hline$p_{\mathrm{H}_{2} \mathrm{O}, \mathrm{p}} / \mathrm{mbar}$ & 0.039 & 0.034 \\
$L_{\mathrm{H}_{2} \mathrm{O}, \mathrm{min}} / \mathrm{mbar} \cdot \mathrm{L} \cdot \mathrm{s}^{-1}$ & $5.4 \cdot 10^{-4}$ \\
$L_{\mathrm{H}_{2} \mathrm{O}, \mathrm{max}} / \mathrm{mbar} \cdot \mathrm{L} \cdot \mathrm{s}^{-1}$ & $7.8 \cdot 10^{-4}$ & \\
\hline
\end{tabular}

Leckrate $L_{x}$ eines Stoffes $x$ in einen Behälter des Volumens $V$ gilt Gleichung 3.7:

$$
L_{x}=\frac{\Delta p_{x} \cdot V}{\Delta t}
$$

Für die hier durchgeführten Rechnungen wird angenommen, dass zu Beginn des Kreislaufbetriebs kein Wasser im Reservoir vorliegt, sodass für $\Delta p_{\mathrm{H}_{2} \mathrm{O}}$ der jeweilige Wasserpartialdruck zum Zeitpunkt der Messung, $p_{\mathrm{H}_{2} \mathrm{O}, \mathrm{p}}$, eingesetzt werden kann. Mit Gleichung 3.7 und den genannten Daten kann eine Grenzabschätzung der Leckrate erfolgen. Als obere Grenze wird der Quotient aus dem größeren ermittelten Wasserpartialdruck (aus Ansatz A) und der kürzeren Zeitdifferenz $\left(\Delta t_{1}\right)$ verwendet, als untere Grenze entsprechend der Quotient aus dem kleineren Partialdruck (aus Ansatz B) und der längeren Zeitdifferenz $\left(\Delta t_{2}\right)$. Die Ergebnisse sind in Tabelle 3.7 dargestellt. Es zeigt sich, dass über beide Ansätze vergleichbare Werte für den Partialdruck des Wassers im Probespektrum erhalten werden. Das Verhältnis der berechneten Bandenstärken der OH-Streckschwingungen von Wasser und Ethanol, das für Ansatz A benötigt wird, scheint also in der Tat durch Fehlerkompensation trotz Verwendung der doppelt harmonischen Näherung einen realistischen Wert zu liefern. Die über die beiden Ansätze ermittelte Wasserleckrate liegt im Bereich 5.4-7.8 $10^{-4} \mathrm{mbar} \cdot \mathrm{L} \cdot \mathrm{s}^{-1}$. Analoge Auswertungen wurden für weitere Probespektren durchgeführt. Dabei ergaben sich Wasserleckraten im Bereich von etwa $3 \cdot 10^{-4}$ bis $1 \cdot 10^{-3} \mathrm{mbar} \cdot \mathrm{L} \cdot \mathrm{s}^{-1}$.

Die Größenordnung der Wasserleckrate liefert nützliche Hinweise für die Ursachensuche des beobachteten Wasserkonzentrationsanstiegs. So kann zunächst die Masse des 
eindringenden Wassers abgeschätzt werden. Wird als erste Näherung dazu das ideale Gasgesetz verwendet, so sind in den ersten $500 \mathrm{~h}$ Messzeit ca. 0.4-1.3 g Wasser im Kreislaufbetrieb in die untersuchte Gasmischung übergegangen. Neben einem Eindringen von außen ist angesichts dieser Größenordnung auch Desorption von den Behälterwänden und Dichtmaterialien als Quelle denkbar. Die Vakuumbehälter wurden allerdings letztmalig im Dezember 2018 belüftet und wurden zwischen den Messkampagnen nahezu immer mit dem Turbomolekularpumpstand evakuiert und mehrfach bei $80{ }^{\circ} \mathrm{C}$ ausgeheizt. Die Schraubenpumpe, die nicht evakuiert werden kann (s. Kap. 3.1), wird vor und nach jeder Benutzung mit Helium gespült und anschließend befüllt. Wasser wurde vor und nach der einmaligen Messung mit Luftbeimischung für Ansatz B (März 2019) nicht gezielt als Probemolekül in das System eingebracht. Bei Desorption als Ursache wäre aus diesen Gründen mit zunehmendem zeitlichen Abstand zur letzten (Teil-)Belüftung eine Verringerung bzw. ein vollständiges Verschwinden der Wassergasphasenkonzentrationszunahme zu erwarten. Seit der letzten Belüftung wurde zudem mit großen Mengen von Alkoholen (vor allem Ethanol) im Vakuumsystem gearbeitet. Eine längerfristige, langsame Desorption von Alkoholen oder ein Effekt auf die vermeintliche Desorptionsrate von Wasser durch Austausch adsorbierten Wassers durch Alkohol wurde jedoch nicht beobachtet.

Auch eindringende Luft wäre als Verursacher der ansteigenden Wasserkonzentration denkbar. Bei der Aufnahme der Probespektren betrug der Stoffmengenanteil des Wassers in der Luft stets weniger als $1 \%$. Die beobachteten Wasserleckraten müssten also durch Luftleckraten verursacht sein, die zwei Größenordnungen darüber liegen und damit 3 . $10^{-2}$ bis $1 \cdot 10^{-1} \mathrm{mbar} \cdot \mathrm{L} \cdot \mathrm{s}^{-1}$ betragen. Dies liegt deutlich oberhalb der in mehreren Tests bestimmten Gesamtleckrate von weniger als $1 \cdot 10^{-3} \mathrm{mbar} \cdot \mathrm{L} \cdot \mathrm{s}^{-1}$. Weiterhin müsste eine solche Luftleckrate in den Probemessungen zu einem Druckanstieg von mehreren mbar im Reservoir führen. Auch ein solcher Druckanstieg wird nicht beobachtet.

Weitere Hinweise darauf, dass eindringende Luft nicht die Hauptursache der steigenden Wasserkonzentration sein kann, liefert der in Abbildung 3.18 dargestellte Vergleich eines Ethanol-Spektrums nach mehreren Stunden Kreislaufbetrieb (schwarz) mit einem Ethanol-Spektrum, dem Luft beigemischt wurde (grau). Die Spektren weisen einen identischen Ethanol-Partialdruck und vergleichbare Wasserkonzentrationen auf. Im schwarzen Spektrum aus dem Kreislaufbetrieb haben die drei Ethanol-Dimer-Banden bei 3548, 3540 und $3532 \mathrm{~cm}^{-1}$ eine vergleichbare Intensität, während im grauen Spektrum mit Luftbeimischung die Intensität der Dimerbande bei $3532 \mathrm{~cm}^{-1}$ deutlich höher als die der anderen beiden Banden ist. Das Vorhandensein schwerer Moleküle wie Stickstoff verbessert hier die Stoßkühlung im Vergleich zu einer reinen Heliumexpansion, wodurch die metastabilen Dimere ins stabilste Ethanol-Homodimer (gauche ${ }^{+}$-gauche ${ }^{+}$-Konformer) ${ }^{[189,198,218]}$ relaxieren. Darüber hinaus werden im Spektrum mit Luftbeimischung zwei Banden bei 3520 und $3508 \mathrm{~cm}^{-1}$ beobachtet, die Clustern von Ethanol-Dimeren mit Stickstoff zugeordnet werden können. ${ }^{[189]}$ Im schwarzen Spektrum ohne explizite Luftbeimischung sind hingegen keine Banden oder Effekte vorhanden, die auf die Anwesenheit größerer Mengen Stickstoff und damit Luft hindeuten. Ein Luftleck kann damit als Hauptursache für 


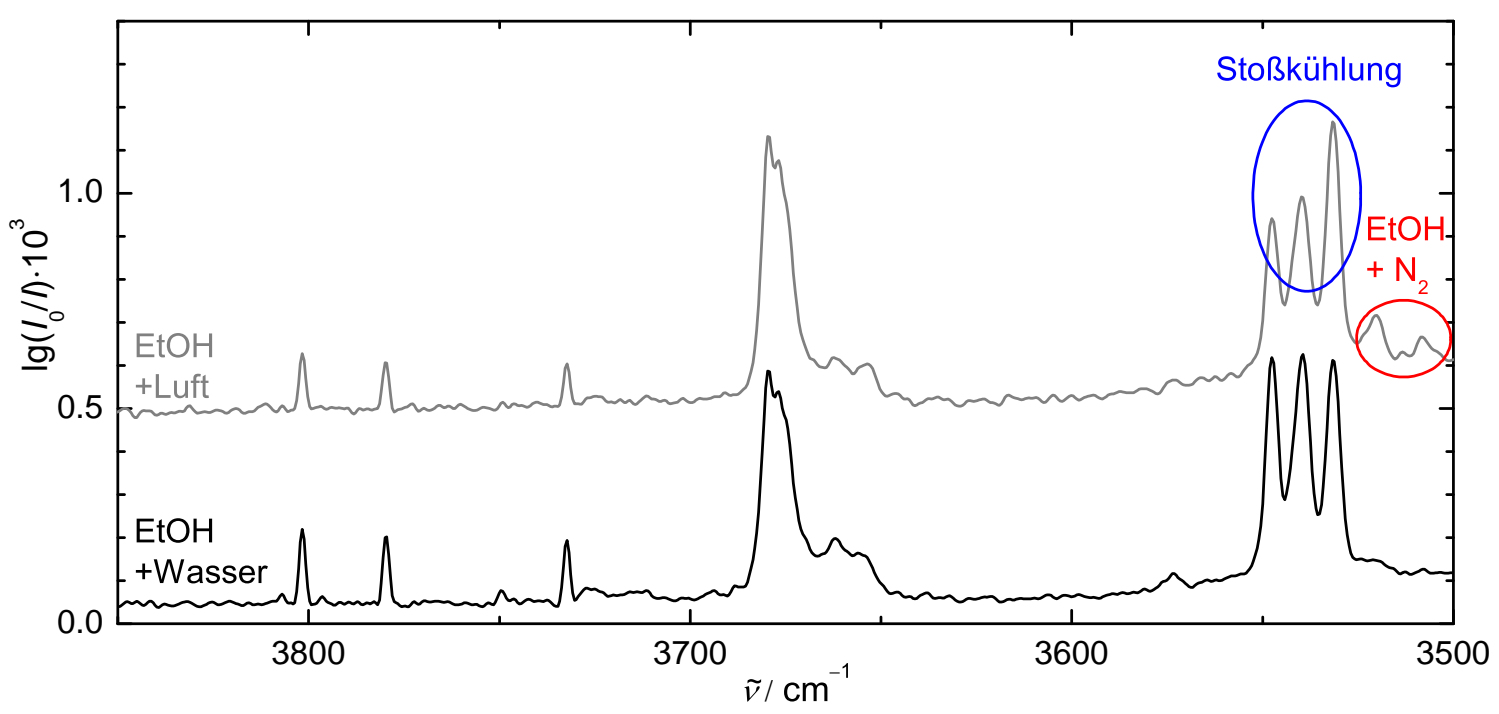

Abbildung 3.18: Vergleich eines nach mehreren Stunden (3:30-4:20 h) Kreislaufbetrieb aufgenommenen Ethanol-Spektrums (schwarz) mit einem Ethanol-Spektrum, dem 7.50 mbar Luft beigemischt wurden (grau). Der Ethanol-Partialdruck beträgt in beiden Fällen 1.30 mbar. Die beigemischte Luftmenge im grauen Spektrum wurde so gewählt, dass eine dem schwarzen Spektrum ähnliche Wasserkonzentration vorliegt. Die bessere Stoßkühlung im Luftspektrum ist in der Intensitätsverteilung des Ethanol-Dimers (3555-3525 $\mathrm{cm}^{-1}$, blau gekennzeichnet) gut erkennbar, ebenso wie die zusätzlichen Banden bei 3520 und $3508 \mathrm{~cm}^{-1}$ (rot gekennzeichnet). Zur Verbesserung der Übersichtlichkeit wurden die Spektren entlang der Ordinate verschoben (schwarz: +0.11 ; grau: $+0.74)$.

das sich im Laufe des Kreislaufbetriebs anreichernde Wasser ausgeschlossen werden.

Wird die Wasserkonzentrationszunahme nicht durch ein Luftleck verursacht, könnte das Wasser an anderer Stelle direkt in das Kreislaufsystem eindringen. Während der Großteil des Vakuumsystems von Luft umgeben ist, gibt es eine Stelle, an der das umgebende Medium zu einem großen Teil Wasser ist: die Schraubenpumpe. Der Schöpfraum dieser Pumpe befindet sich in einem Kühlmittelbad, wobei das Kühlmittel (Busch Zitrec M-25) aus Wasser (60\%) und Ethylenglykol (40\%) besteht. Dieses interne Kühlmittel wird wiederum durch einen externen Wasserkreislauf gekühlt. Bei einem Leck im Bereich des Schöpfraums bestünde also die Möglichkeit, dass an der Schraubenpumpe Wasser in das Vakuumsystem eindringt.

Einen Hinweis darauf, dass die Wasserkonzentration bei Betrieb des Wälzkolbenpumpstands schneller zunimmt als bei abgeschalteten Pumpen, liefert eine Messung, bei der der Kreislaufbetrieb an zwei Messtagen in Folge mit derselben Gasmischung durchgeführt wurde. Dabei wurde das Vakuumsystem zu Beginn des ersten Tages mit einer Gasmischung von $0.1 \%$ Ethanol in Helium befüllt. Anschließend wurden über mehrere Stunden im Kreislaufbetrieb Jet-Spektren aufgezeichnet. Über Nacht wurde das Gasgemisch bei abgeschaltetem Pumpstand in der Apparatur belassen und der Kreislaufbetrieb wurde am 


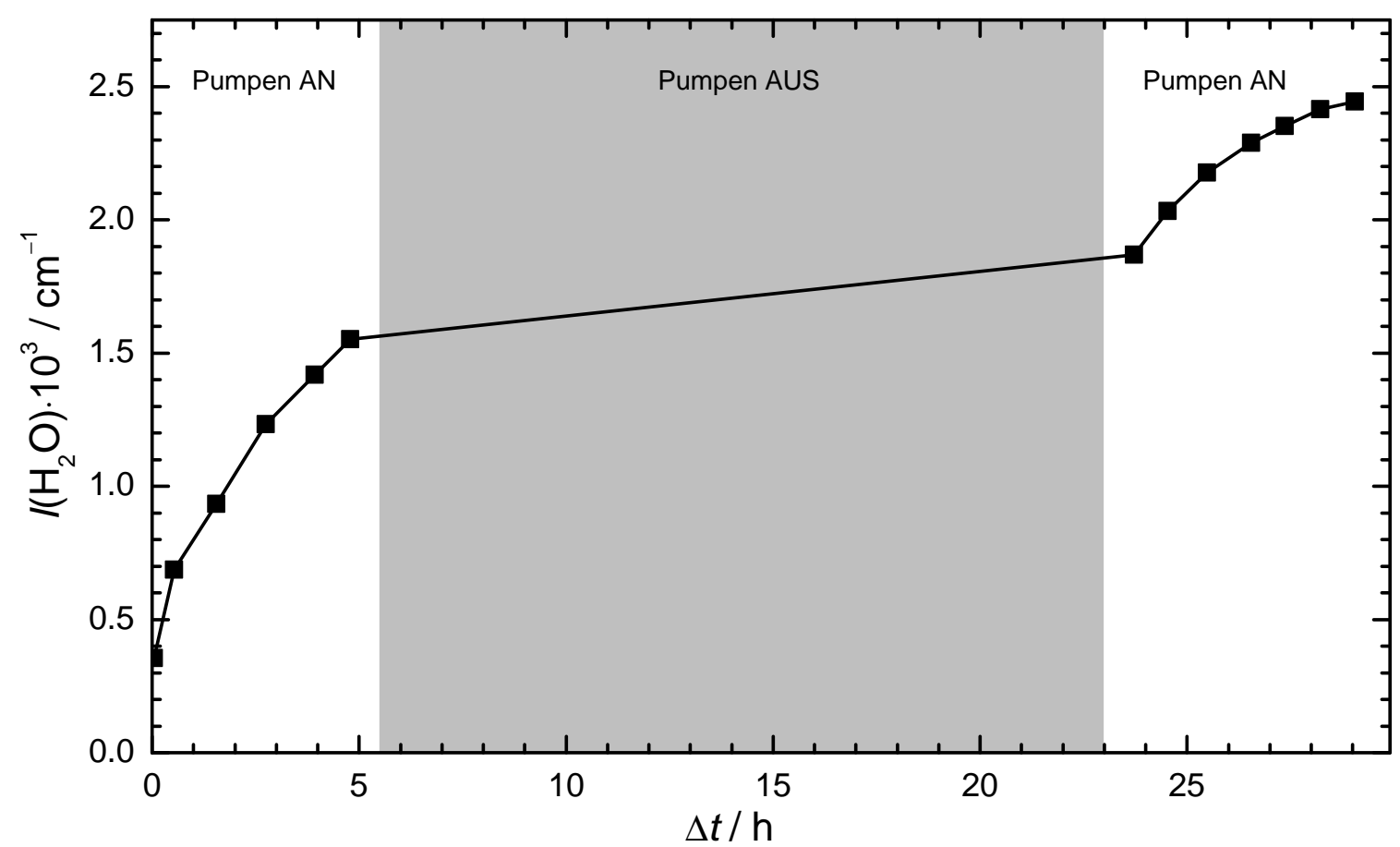

Abbildung 3.19: Verlauf der Intensität $I\left(\mathrm{H}_{2} \mathrm{O}\right)$ der Jet-Wasser-Linien in den Spektren eines Ethanol-Helium-Gemischs in Abhängigkeit von der Zeit $\Delta t$ nach Beginn der Messungen. Das Gemisch wurde bei abgeschaltetem Wälzkolbenpumpstand (grauer Bereich) über Nacht im Reservoir belassen.

nächsten Messtag mit diesem Gasgemisch fortgesetzt. Für die Analyse der Wasserkonzentration wurden die Jet-Wasser-Linien bei 3802, 3780 und $3732 \mathrm{~cm}^{-1}$ mit der Methode B des Opus-Programmpakets in den aufgezeichneten Spektren integriert und die Integrale wurden addiert. Die so erhaltenen Intensitäten $I\left(\mathrm{H}_{2} \mathrm{O}\right)$ sind in Abbildung 3.19 gegen die Zeitdifferenz $\Delta t$ zum Beginn der ersten Messung aufgetragen. Da für jedes Spektrum 10-150 Pulse und damit auch die während der Messung ansteigenden Wasserintensitäten gemittelt wurden, wurden die aus den Spektren ermittelten Intensitäten jeweils dem Zeitpunkt in der Mitte der Messung zugeordnet. Da die ermittelten Werte nur zu qualitativen Analyse dienen sollen, wurde auf eine Fehlerabschätzung verzichtet. Es zeigt sich, dass die Intensität der Jet-Wasser-Linien und damit die Wasserkonzentration während des Betriebs des Wälzkolbenpumpstands signifikant schneller ansteigt als bei Stillstand der Pumpen. Die Wasserleckrate scheint somit maßgeblich durch den Betrieb des Wälzkolbenpumpstands beeinflusst zu werden. Dies könnte allerdings auch auf einen durch die Betriebswärme der laufenden Pumpen ausgelösten Desorptionseffekt zurückzuführen sein, ist also kein Beweis für ein Wasserleck im Bereich der Schraubenpumpe.

Zur genaueren Eingrenzung der Ursache wurden Messungen bei 30 mbar Stagnationsdruck mit reinem Ethanol bzw. einem Gemisch aus 15 mbar Ethanol und 15 mbar Helium im Kreislaufbetrieb ohne Schraubenpumpe durchgeführt. Zwei Spektren des letzteren Ge- 


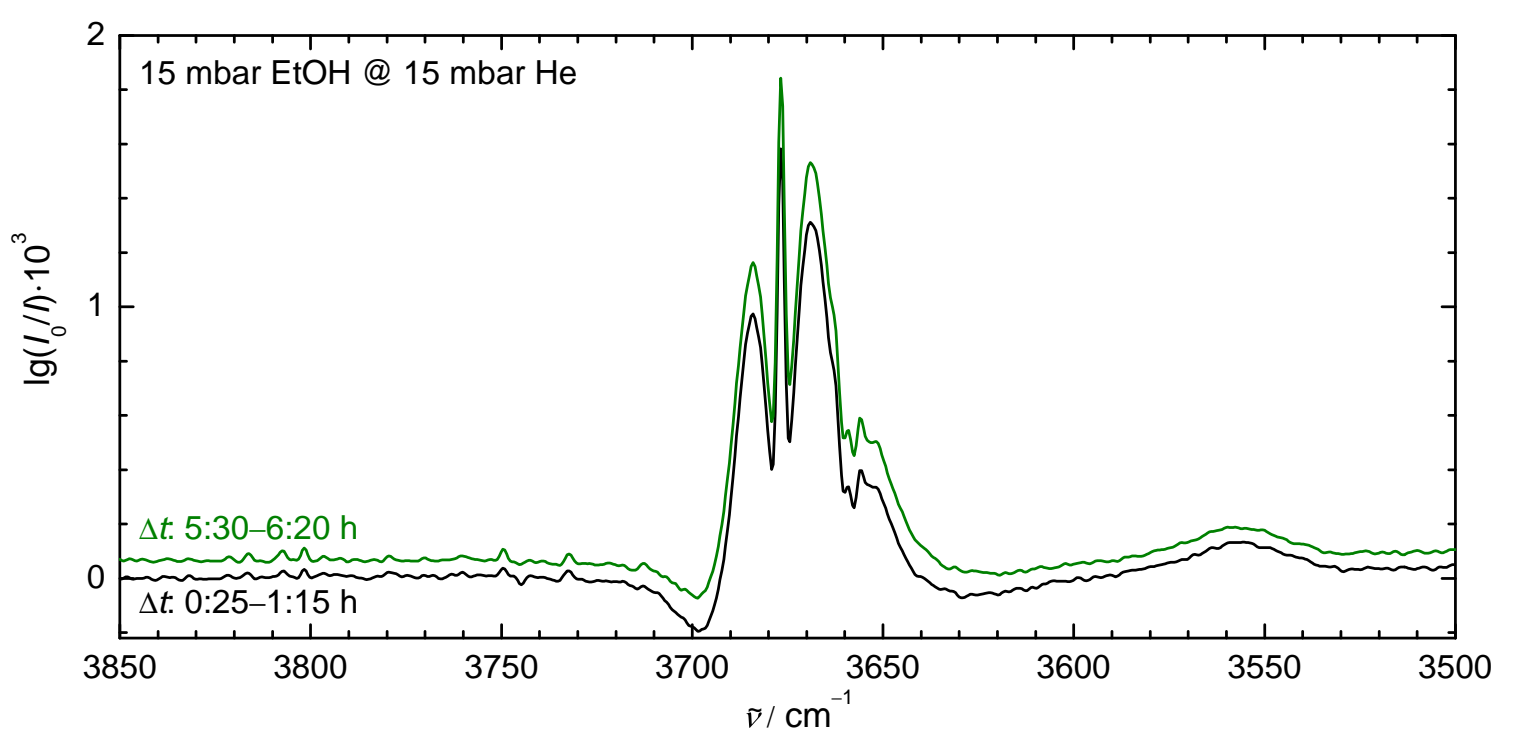

Abbildung 3.20: Ohne Schraubenpumpe gemessene Spektren von $15 \mathrm{mbar}$ Ethanol in $15 \mathrm{mbar}$ Helium, aufgezeichnet im Zeitraum 0:25-1:15 h (schwarz, +0.07 Skaleneinheiten entlang der Ordinate verschoben) bzw. 5:30-6:20 h (grün, +0.21) nach Beginn des Kreislaufbetriebs. Im Rahmen der Schwankungen schlecht kompensierten atmosphärischen Wassers ist kein Anstieg der Wasserkonzentration im Laufe des Kreislaufbetriebs erkennbar.

mischs sind in Abbildung 3.20 dargestellt. Im Vergleich zu den zuvor gezeigten Jet-Spektren, die bei einem Stagnationsdruck von 750 mbar aufgezeichnet wurden, ist die deutlich schwächere Kühlung der Rotationszustände zu erkennen. Die Ethanol-Monomer-Bande (Q-Zweig bei $3677 \mathrm{~cm}^{-1}$ ) weist gut erkennbare P-, Q- und R-Zweige auf. Zwischen den kurz nach Beginn des Kreislaufbetriebs (schwarz) bzw. am Ende des Messtages (grün) aufgezeichneten Spektren lässt sich kein signifikanter Anstieg der Wasserkonzentration erkennen. Durch die schwächere Kühlung bei den vergleichsweise warmen Expansionsbedingungen sind auch beim möglicherweise vorhandenen Wasser zahlreiche verschiedene Rotationszustände populiert, sodass sich Jet-Wasser hier nicht eindeutig von nicht vollständig kompensiertem atmosphärischen Wasser unterscheiden lässt. Darüber hinaus strömt durch die geringe Druckdifferenz zwischen Reservoir und Puffervolumen nur wenig Gas beim Puls durch die Düse, wodurch die zu erwartenden Intensitäten von eventuell vorhandenem Wasser im Jet-Spektrum klein ausfallen. Die in ihrer Intensität schwankenden Linien atmosphärischen Wassers überlagern damit eventuell auftretende Linien von Jet-Wasser. Ein vergleichbares Bild zeigt sich in den Spektren der reinen 30-mbar-Ethanol-Expansionen. Auch hier lassen sich, möglicherweise aufgrund des atmosphärischen Wassers, keine signifikanten Mengen von Jet-Wasser nachweisen. Es ergeben sich somit keine Hinweise auf die Anreicherung von Wasser im Gasgemisch beim Kreislaufbetrieb ohne Schraubenpumpe. Aufgrund der angesprochenen Überlagerung der Jet-Wasser-Linien mit denen des atmosphärischen Wassers lässt sich das Vorhandensein allerdings auch 
nicht ausschließen.

Eine Möglichkeit, die hohe Nachweisschwelle bei geringen Stagnationsdrücken zu umgehen, wäre ein zweistufiger Test: Es wird jeweils über mehrere Stunden gemessen, wobei beim ersten Teil des Test zunächst nur die Wälzkolbenpumpen für den Kreislaufbetrieb bei einem Stagnationsdruck von 30 mbar genutzt werden. Nach einer festgelegten Zeit, z. B. 4 h nach Beginn des Kreislaufbetriebs, wird die Mischung nach Zuschalten der Schraubenpumpe dann mit Helium auf 750 mbar aufgefüllt und es wird ein Testspektrum aufgenommen. Durch den hohen Stagnationsdruck lässt sich vorhandenes Jet-Wasser eindeutig von atmosphärischem Wasser unterscheiden. Für den zweiten Teil des Tests wird die Apparatur zunächst wieder gründlich evakuiert und ausgeheizt. Dann wird direkt mit allen Pumpen bei einem Stagnationsdruck von 750 mbar über denselben Zeitraum von $4 \mathrm{~h}$ gemessen. Anschließend wird erneut ein Testspektrum bei 750 mbar Stagnationsdruck aufgezeichnet, sodass zwei Testspektren beim selben Stagnationsdruck und nach derselben Kreislaufbetriebszeit vorliegen. Die ermittelten Werte für die Wasserkonzentration können anschließend miteinander verglichen werden. Ist die Konzentration nach dem Messbetrieb ohne Schraubenpumpe signifikant kleiner als nach dem Messbetrieb mit Schraubenpumpe, wäre dies ein deutlicher Hinweis darauf, dass das Wasserleck bzw. -reservoir zumindest zum Teil im Bereich der Schraubenpumpe zu vermuten ist.

Parallel zu den IR-spektroskopischen Untersuchungen wurden Versuche unternommen, das in den Jet-FTIR-Spektren sichtbare Wasser auch massenspektrometrisch nachzuweisen. Dazu wurde ein System, bestehend aus einem Massenspektrometer (STANFORD Research Systems RGA 200) und einer Turbomolekular- (Leybold LH Turbovac 50) und Vorpumpeneinheit (vacUUbrand RS-4), über die Schleuse an das Puffervolumen angeschlossen. Es wurden jeweils unmittelbar nach einem Düsenpuls Proben entnommen, um die relative Wasserkonzentration im Laufe eines Messtages im Kreislaufbetrieb zu bestimmen. Durch Lecks direkt am Massenspektrometer eindringende Luft bzw. im Massenspektrometer von den Wänden desorbierendes Wasser verursachten allerdings schwankende Wasserkonzentrationen und schwankende Wasser-zu-Stickstoff- oder auch Wasser-zu-Helium-Verhältnisse in den Massenspektren, wodurch ein Nachweis von Wasser im Gratin-Jet nicht möglich war. Ein Problem war dabei der geringe nutzbare, dynamische Druckbereich des Massenspektrometeraufbaus. Zwischen dem minimal erreichbaren Hintergrunddruck und dem maximal für eine Messung nutzbaren Druck lag weniger als eine Größenordnung. Durch eine Abdichtung möglicher Leckstellen im Bereich des massenspektrometrischen Aufbaus und durch Austausch der Pumpen durch eine leistungsfähigere Pumpeneinheit (PfEIfFER TSH 071E) wurde der dynamische Bereich um mehr als eine Größenordnung erweitert. Ein weiteres Problem stellt die geringe Wasserkonzentration im Kreislaufsystem dar. Der vergleichsweise hohe für den Betrieb der Schraubenpumpe nötige Stagnationsdruck von $>300$ mbar in Verbindung mit dem Reservoirvolumen von $200 \mathrm{~L}$ sorgt für geringe Anteile des eindringenden Wassers an der Gesamtstoffmenge im System, selbst nach längeren Messzeiten. Bei der oben bestimmten Maximal-Wasserleckrate von $1 \cdot 10^{-3} \mathrm{mbar} \cdot \mathrm{L} \cdot \mathrm{s}^{-1}$ beträgt die Stoffmengenkonzentration des Wassers nach 6 h Kreislaufbetrieb z. B. etwa $0.01 \%$. Bei höheren Stagnationsdrücken liegt der Anteil 
dementsprechend noch niedriger. In einem kleineren Volumen lassen sich bei gleicher Leckrate in kürzerer Zeit höhere Wasserkonzentrationen erreichen. Beträgt das Volumen nur $5 \mathrm{~L}$, liegt der Stoffmengenanteil des Wassers nach $6 \mathrm{~h}$ bei ca. $0.4 \%$. Ein Nachweis sollte so also leichter möglich sein.

Da die wahrscheinlichste Leckstelle für den Wassereintrag im Bereich der Schraubenpumpe liegt, wurde im Rahmen der Masterarbeit von TAIJA FISCHER ein verkleinertes Modell des Kreislaufsystems unter Einbeziehung der Schraubenpumpe gebaut. Dazu wurde der Auslass über Rohre mit 16 mm Durchmesser mit dem Einlass der Pumpe verbunden. Ein Drosselventil in der Verrohrung dient dabei als Simulation der Düse und ermöglicht die Regulierung der Druckdifferenz zwischen niedrigerem Druck auf der Einlass- und höherem Druck auf der Auslassseite der Pumpe. Die Befüllung und das Spülen des Systems können über ein Ventil auf der Niederdruckseite erfolgen. Zum Absenken des auslassseitigen Drucks auf den gewünschten simulierten Stagnationsdruck kann eine Vakuumpumpe (Pfeiffer Adixen ACP 40G) angeschlossen werden. Da die Schlitzdüse nicht mehr Teil des Gaskreislaufs ist, kann der Wassernachweis im Modellsystem nur massenspektrometrisch erfolgen. Dazu kann die massenspektrometrische Einheit an ein weiteres Ventil auf der Niederdruckseite angeschlossen werden. Inklusive des Schöpfraums der Schraubenpumpe beträgt das Volumen des Modell-Gaskreislaufs weniger als 5 L. Auch mit dem größeren dynamischen Bereich und dem deutlich verkleinerten Volumen des Gaskreislaufs konnte kein Wasser im Kreislaufsystem nachgewiesen werden. Beispielhaft ist in Abbildung 3.21 das Wasser-zu-Stickstoff-Verhältnis im Verlauf der Messzeit dargestellt. Dringt Wasser in das Vakuumsystem ein, sollte das Verhältnis größer werden, bei Lufteintrag sollte es gleich bleiben. Für die Messung wurde die Pumpe nach anfänglichem Spülen und Befüllung mit Helium bei einem Druck von etwa 650 mbar auf der Hochdruckseite und etwa 20 mbar auf der Niederdruckseite über einen Zeitraum von 50 h betrieben. Dabei wurden während der Stunden 0-6, sowie 25-30 und 47-49 jeweils mehrfach Proben ins Massenspektrometer geleitet und analysiert. In den Zeiträumen zwischen diesen Messungen wurde die gesamte Massenspektrometereinheit inklusive der Pumpen vom Kreislaufmodell getrennt. Es zeigt sich, dass beim ersten täglichen Öffnen der Verbindung zwischen Massenspektrometer und Kreislaufsystem innerhalb von etwa $1 \mathrm{~h}$ das Wasserzu-Stickstoff-Verhältnis größer wird. Im Laufe des Messtags und bei länger geschlossener Verbindung über Nacht sinkt das Verhältnis dann bis zum nächsten Öffnen stetig. Das beim Öffnen mit dem Gasgemisch einströmende Wasser wird zum Teil an den Wänden im massenspektrometrischen Teil des Aufbaus adsorbiert, desorbiert anschließend langsam wieder und wird von der Turbomolekularpumpe abgepumpt, sodass das Wasser-zu-Stickstoff-Verhältnis sinkt. Beim ersten Öffnen der Verbindung am folgenden Messtag dringt dann wieder eine Gasmischung mit einem höheren Wasseranteil ein und der Vorgang wiederholt sich. Mögliche Trends im Wasser-zu-Stickstoff-Verhältnis im Kreislaufsystem werden deshalb durch den Desorptionsvorgang im Massenspektrometer überlagert, sodass auch mit diesem vereinfachten und optimierten Aufbau ein massenspektrometrischer Nachweis der steigenden Wasserkonzentration im Kreislaufsystem nicht möglich war. Eine Referenzmessung einer Probe mit einem bekannten Wasser-zu-Stickstoff- oder 


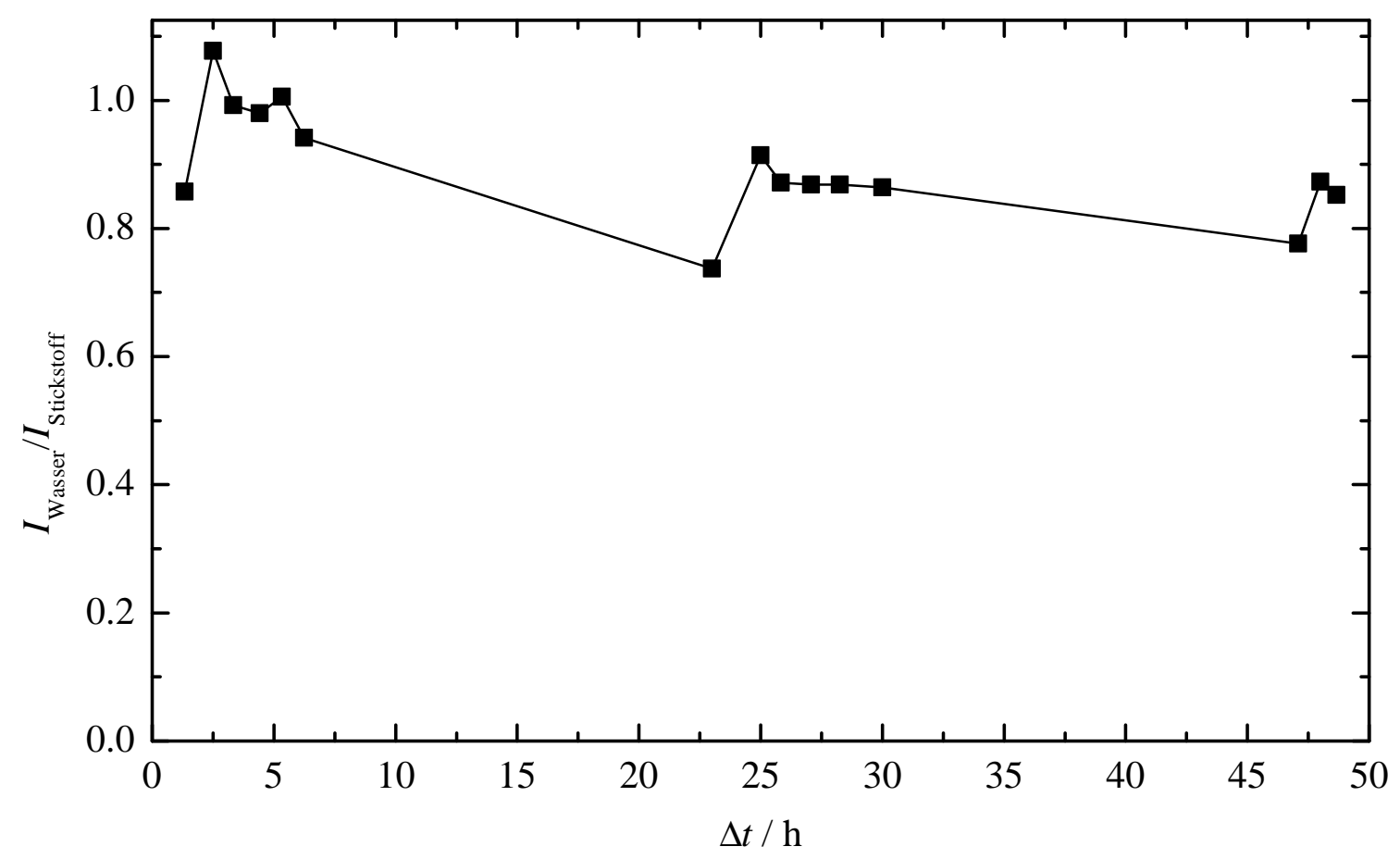

Abbildung 3.21: Mit dem Massenspektrometer ermitteltes Verhältnis der Intensitäten des Wasser-Peaks $(m / z=18)$ zum Stickstoff-Peak $(m / z=28)$ in Abhängigkeit von der Betriebszeit der Schraubenpumpe. Über Nacht wurde die Verbindung zwischen Massenspektrometer und Schraubenpumpe jeweils geschlossen. Bei geschlossener Verbindung lässt sich eine kontinuierliche Verringerung des Verhältnisses feststellen, während das Verhältnis beim darauffolgenden Öffnen der Verbindung wieder ansteigt.

Wasser-zu-Helium-Verhältnis könnte für die Analyse des Problems hilfreich sein und ist für die Zukunft geplant.

Einfacher wäre der Nachweis von Substanzen, die nicht in der Umgebung vorkommen. Sollte das Leck zwischen dem internen Kühlkreislauf der Schraubenpumpe und dem Schöpfraum bestehen, müsste auch der zweite Bestandteil des Kühlmittels, Ethylenglykol, in das Vakuumsystem eindringen. In keinem der untersuchten Massen- oder IR-Spektren wurden jedoch Hinweise auf das Vorhandensein von Ethylenglykol gefunden. Der erwartete Stoffmengenanteil von Ethylenglykol in der Gasphase ist bei einem Kühlmittelleck jedoch auch nochmals geringer als der von Wasser, da Ethylenglykol bei Raumtemperatur $\left(25^{\circ} \mathrm{C}\right)$ mit $0.12 \mathrm{hPa}^{[257]}$ nur etwa $0.4 \%$ des Dampfdrucks von Wasser aufweist. Ein Leck zwischen Schöpfraum und internem Kühlkreislauf in der Schraubenpumpe kann also nicht ausgeschlossen werden. Ein sicherer Nachweis bzw. ein Ausschluss der Schraubenpumpe als Quelle des eindringenden Wassers würde also wohl nur durch den Austausch eines Teils des Wasseranteils der Kühlflüssigkeit durch schweres Wasser $\left(\mathrm{D}_{2} \mathrm{O}\right)$ gelingen. Alkohole wie Isopropanol können aufgrund der zu geringen Wärmekapazität aus technischen Gründen nicht eingesetzt werden. 
Zusammenfassend gestaltet sich der Nachweis von Wasser im Kreislaufsystem sowohl massenspektrometrisch als auch IR-spektroskopisch aufgrund des in der Umgebung überall vorhandenen atmosphärischen Wassers abseits von Jet-FTIR-Spektren bei hohem Stagnationsdruck schwierig. Eine Eingrenzung der Ursache ist mit Ausnahme des Ausschlusses von eindringender Luft als Hauptverursacher damit zum jetzigen Zeitpunkt nicht möglich. Weitere Tests mit einem weiter optimierten massenspektrometrischen Aufbau, der mit einer leistungsfähigeren Turbomolekularpumpe (PfeIffER HiCube Eco 300) einen nochmals größeren dynamischen Bereich bieten sollte, sind für die Zukunft geplant.

\subsection{Leistungsfähigkeit der Gratin- im Vergleich zur Filet-Jet-Spektroskopie}

Nachdem in den vorangegangenen Abschnitten der Gratin-Jet und seine einzelnen Bestandteile detailliert vorgestellt und erläutert wurden, soll abschließend eine Analyse der Leistungsfähigkeit erfolgen. Ziel der Entwicklung, der Anschaffung und der Inbetriebnahme der neuen Gratin-Jet-Apparatur war es, einen in zahlreichen Details verbesserten Nachfolger für den Filet-Jet zu kreieren, der Spektren vergleichbarer Qualität bei deutlich verringertem Substanzverbrauch liefert.

Für den Vergleich der Leistungsfähigkeit der beiden Apparaturen werden Spektren eines Gemisches aus Methanol (MeOH) und 2-Fluoracetophenon (2FAP) im Trägergas Helium, wie es für die intermolekularen Carbonylwaagen in Kapitel 5 verwendet wird, untersucht. Zunächst soll dabei überprüft werden, ob trotz der unterschiedlichen Expansionsgeometrien eine ähnlich gute Kühlung des Konformerengleichgewichts der HeteroDimere erreicht wird (Kap. 3.7.1). Anschließend soll ein Vergleich der Spektrenqualität über eine Rauschanalyse erfolgen (Kap. 3.7.2).

\subsubsection{Vergleich der erreichten Kühlung}

Die sogenannte Konformationstemperatur $T_{\mathrm{c}}$, also die in der Expansion erreichte Temperatur für die Umwandlung zwischen zwei Cluster-Konformeren, stellt eine wichtige Größe für das Benchmarking quantenmechanischer Methoden dar (vgl. Kap. 2.3.2). Neben der Barrierenhöhe und -breite für die Umwandlung der Konformere ineinander ist sie auch von der in der Expansion erreichten Kühlung abhängig. Werden in zwei verschiedenen Expansionen dieselben Aggregate - also z. B. die Hetero-Dimere von MeOH und 2FAP betrachtet, ist die Umwandlungsbarriere identisch. Somit kann $T_{\mathrm{c}}$ als Maß für den Grad der Kühlung des Konformerengleichgewichts in der jeweiligen Expansion herangezogen werden. Bei Untersuchung derselben Konformere können Unterschiede in der Konformationstemperatur durch den identischen Energieunterschied sowie identische Entartungsfaktoren und Infrarotbandenstärken zudem direkt am Intensitätsverhältnis der betrachteten Banden im IR-Spektrum abgelesen werden, ohne dass die Konformationstemperatur explizit berechnet werden muss. 
Tabelle 3.8: Vergleich der ermittelten Intensitätsverhältnisse $I_{\mathrm{Me}} / I_{\mathrm{Ph}}$ der beiden Hetero-Dimere von $\mathrm{MeOH}+2 \mathrm{FAP}$ (s. Kap. 5) im Filet- und Gratin-Jet-Spektrum.

\begin{tabular}{lr}
\hline Jet & $\boldsymbol{I}_{\mathbf{M e}} / \mathbf{I}_{\mathbf{P h}}$ \\
\hline Filet & 2.28 \\
Gratin & 2.27 \\
\hline
\end{tabular}

In Tabelle 3.8 sind die Intensitätsverhältnisse der Hetero-Dimere von $\mathrm{MeOH}+2 \mathrm{FAP}$, die aus bei gleichem Stagnationsdruck und vergleichbarer Konzentration aufgenommenen Spektren ermittelt wurden, dargestellt. Es ergeben sich an beiden Apparaturen nahezu identische Verhältnisse und damit nahezu identische Konformationstemperaturen. Trotz der unterschiedlichen Expansionsgeometrien an Filet- und Gratin-Jet liegen also zumindest bei diesem System keine nennenswerten Unterschiede in der erreichten Kühlung des Konformerengleichgewichts vor.

\subsubsection{Vergleich der Spektrenqualität}

Für die Verwendbarkeit der am Gratin-Jet aufgenommenen Spektren ist neben einer ausreichend guten Kühlung vor allem die Qualität der Spektren an sich entscheidend. Je besser das Signal-zu-Rausch-Verhältnis (S/N), desto genauer lassen sich Größen wie das zuvor bereits erwähnte Intensitätsverhältnis aus den Spektren bestimmen.

Aufgrund ungleicher Strahlquerschnitte durch verschiedene Strahlfokussierungen (s. Kap. 3.5.1) und nicht direkt vergleichbare Blenden im Strahlengang (s. Kap. 3.5.2) werden am Filet- und Gratin-Jet unterschiedliche Bereiche der Überschallexpansion untersucht. Bei gleicher beobachteter Monomerintensität in einem Filet- und einem Gratin-Jet-Spektrum können dadurch beispielsweise abweichende Konzentrationen von Dimeren vorliegen. Ein Vergleich über S/N würde somit durch die verschiedenen Signalintensitäten je nach Clustergröße unterschiedliche Ergebnisse liefern. Die vergleichende Analyse der Spektrenqualität soll deshalb unabhängig von den Signalintensitäten nur über die Höhe des Rauschens in bandenfreien Spektralbereichen erfolgen. Zusätzlich werden die Stabilität der Grundlinie im betrachteten Spektralbereich und das Vorhandensein möglicher Störsignale qualitativ mit in die Beurteilung einbezogen.

Dazu wurden mit dem bereits genannten Gemisch von Methanol und 2-Fluoracetophenon im Trägergas Helium Spektren mit möglichst ähnlicher Konzentration am Filet- und Gratin-Jet aufgezeichnet. Da die Substanzkonzentration am Filet-Jet aufgrund der Verwendung der Sättiger zur Substanzeinbringung nicht exakt bestimmbar ist (s. Kap. 2.2), kann hier nur eine ungefähre Übereinstimmung erreicht werden, was eine Analyse über $\mathrm{S} / \mathrm{N}$ zusätzlich erschweren würde. Für beide Apparaturen wurden dabei die Messparameter gewählt, die sich im Laufe des langjährigen Betriebs (Filet-Jet) bzw. während der Optimierung im Rahmen der vorliegenden Arbeit (Gratin-Jet) für Messungen im $\mathrm{CH}$ - und $\mathrm{OH}$-Streckschwingungsbereich als bestmöglich erwiesen haben. Die Parameter sind in 
Tabelle 3.9: Vergleich der verwendeten Messparameter für die Messung von $\mathrm{MeOH}+2 \mathrm{FAP}$ am Filet- und am Gratin-Jet.

\begin{tabular}{lll}
\hline Parameter & Filet-Jet & Gratin-Jet \\
\hline Lichtquelle & $150-\mathrm{W}$-Wolframlampe & $150-\mathrm{W}$-Wolframlampe \\
Blende & $3.5 \mathrm{~mm}$ & $12 \mathrm{~mm}$ \\
Strahlteiler & $\mathrm{CaF}_{2}$ & $\mathrm{CaF}_{2}$ \\
Optiken & $\mathrm{CaF}_{2}$ & $\mathrm{CaF}_{2}$ \\
Detektor & $2 \mathrm{~mm} \mathrm{InSb}$ & $2 \mathrm{~mm} \mathrm{InSb}$ \\
Filter & $\mathrm{F} 13\left(4200-2450 \mathrm{~cm}^{-1}\right)$ & $\mathrm{F} 13\left(4200-2450 \mathrm{~cm}^{-1}\right)$ \\
Auflösung & $2 \mathrm{~cm}^{-1}$ & $2 \mathrm{~cm}^{-1}$ \\
Spiegelgeschwindigkeit & $80 \mathrm{kHz}$ & $140 \mathrm{kHz}$ \\
Akquisitionsmodus & single sided & double sided \\
Scanzeit pro Puls & $100 \mathrm{~ms}$ & $105 \mathrm{~ms}$ \\
Pulsdauer & $147 \mathrm{~ms}^{3}$ & $133 \mathrm{~ms}$ \\
Pumpleistung & $2500 \mathrm{~m}^{3} / \mathrm{h}$ & $2000 \mathrm{~m}^{3} / \mathrm{h}$ \\
Zyklusdauer & $31 \mathrm{~s}$ & $28 \mathrm{~s}$ \\
\hline
\end{tabular}

Tabelle 3.9 gegenübergestellt.

Das Rauschen der für den Vergleich herangezogenen Spektren wird mit dem NOTCHProgramm ${ }^{[243]}$ analysiert. Dabei werden in einem laufenden $50-\mathrm{cm}^{-1}$-Fenster die Grundlinie des Spektrums quadratisch angefittet und die Wurzel der mittleren quadratischen Abweichung (engl. „root-mean-square error“, RMSE) des Ist-Werts zum Wert aus dem Fit für die jeweilige Wellenzahl bestimmt. Anschließend wird der negative dekadische Logarithmus des RMSE-Werts, - $\lg (R M S E)$, für die jeweilige Wellenzahl berechnet. Im Gegensatz zum normalen Vorgehen bei der Noise Test Challenge werden die RMSE-Werte an dieser Stelle nicht für Leerspektren, sondern für Jet-Spektren der MeOH+2FAP-Mischung bestimmt. Prinzipbedingt können deshalb nur Bereiche mit einem Abstand von mindestens $25 \mathrm{~cm}^{-1}$ zu benachbarten Banden für die Rauschanalyse herangezogen werden.

Wie anhand der sich unterscheidenden Messparameter (Tab. 3.9) deutlich wird, sind die einzelnen Scans am Filet- und Gratin-Jet nicht äquivalent. Für einen sinnvollen Vergleich der Spektrenqualität der beiden Apparaturen sollten also nicht einfach Spektren, die aus gleich vielen gemittelten Einzelscans bestehen, gegenübergestellt werden. Vielmehr sollten die Spektren über die jeweilige Anzahl der gemittelten Pulse so aufeinander skaliert werden, dass bei Gleichstand eines bestimmten Parameters verglichen werden kann. Für den hier durchgeführten Vergleich wurden drei solcher Parameter gewählt: Gleiche Gesamt-Scanzeit, gleiche Realmessdauer und gleicher Substanzverbrauch. Für die Gegenüberstellung wurden Spektren mit 150 Pulsen am Filet- bzw. mit 1200 Pulsen am Gratin-Jet aufgezeichnet, wobei nach Möglichkeit die Zahl der berücksichtigten GratinJet-Pulse für den jeweiligen Vergleich auf die 150 Filet-Jet-Pulse angepasst wurde. Wurde entsprechend nur eine Untermenge der Gesamtmenge der aufgezeichneten Pulse für 
einen Vergleich herangezogen, wurden jeweils die ersten Pulse der Messung verwendet.

\section{Gleiche Gesamt-Scanzeit}

Der Vergleich bei übereinstimmender Gesamt-Scanzeit $\Sigma t_{\text {scan }}$ ist die direkteste Möglichkeit für eine Gegenüberstellung der Leistungsfähigkeit des spektrometrischen Teils der beiden Apparaturen. Unabhängig von Neuerungen oder Optimierungen bei SubstanzRecycling, Pulslänge, Expansionsgeometrie oder Pumptechnik, die vor allem den Substanzverbrauch bzw. die Zeit zwischen zwei Pulsen am Gratin-Jet positiv beeinflussen, kann hier analysiert werden, welcher spektrometrische Aufbau das geringere Rauschen in einer bestimmten Scan- (also Datenaufnahme-) Zeit erzielt. Durch die ausschließliche Betrachtung des Rauschens ohne Berücksichtigung der Signalintensität wird zudem der Vorteil durch die verlängerte Düse und den damit längeren Absorptionspfad am GratinJet ebenfalls nicht berücksichtigt. Der Vergleich bei gleicher Gesamt-Scanzeit ist damit durch die Nichtberücksichtigungen der genannten Optimierungen für den Gratin-Jet am ungünstigsten. Bei einer Scanzeit $t_{\text {scan }}$ von 100 ms pro Puls (s. Tab. 3.9) und 150 gemittelten Pulsen ergibt sich für das betrachtete Filet-Jet-Spektrum eine Gesamt-Scanzeit von 15.0 s. Bei den gewählten Einstellungen beträgt die Scanzeit am Gratin-Jet 105 ms, sodass dort in derselben Gesamt-Scanzeit nur 142 Pulse aufgezeichnet werden können.

Das auf diese Pulszahl reduzierte Gratin-Jet-Spektrum (rot) ist dem Filet-Jet-Spektrum (schwarz) im oberen Teil der Abbildung 3.22 gegenübergestellt. Zur Verbesserung der Übersichtlichkeit wurde das Gratin-Jet-Spektrum entlang der Ordinate nach oben verschoben. Die Zuordnung der Banden zu verschiedenen MeOH-2FAP-Aggregaten ist hier zunächst nicht relevant und erfolgt in Kapitel 5. Anhand der Intensitäten der im gemessenen Spektralbereich sichtbaren Banden zeigt sich jedoch, dass $\mathrm{MeOH}$ und 2FAP wie gewünscht tatsächlich in vergleichbaren Konzentrationen in den an beiden Apparaturen untersuchten Gemischen vorliegen. Beide Spektren weisen abseits der Ränder des Filterbereichs eine ähnliche Grundlinienstabilität auf und es sind keine Störsignale erkennbar.

Das Ergebnis der Rauschanalyse ist im unteren Teil der Abbildung 3.22 dargestellt. Ein höherer Wert für den negativen dekadischen Logarithmus des ermittelten RMSE-Rauschwerts, $-\lg (R M S E)$, bedeutet dabei ein kleineres Rauschniveau. Die für die Analyse nicht nutzbaren, von Banden des Substanzgemischs belegten Bereiche des Spektrums sind in der Abbildung grau hinterlegt. In den weiß hinterlegten Abschnitten zeigt sich, dass vor allem im Bereich zwischen $\mathrm{OH}$ - und $\mathrm{CH}$-Streckschwingungsbanden (ca. 3350-3125 cm ${ }^{-1}$ ) der Filet-Jet das geringere Rauschniveau aufweist. Im Bereich oberhalb von etwa $3800 \mathrm{~cm}^{-1}$ zeigt der Gratin-Jet bereits ohne Berücksichtigung der Optimierungen z. B. bei Pulslänge und Substanzverbrauch allerdings ein vergleichbares bis leicht besseres Rauschniveau. Insgesamt bleiben die Unterschiede gering. Insbesondere im Bereich zwischen 3350 und $3125 \mathrm{~cm}^{-1}$ ist das kleinere Rauschen am Filet-Jet jedoch auch in den Spektren im oberen Teil der Abbildung erkennbar. 


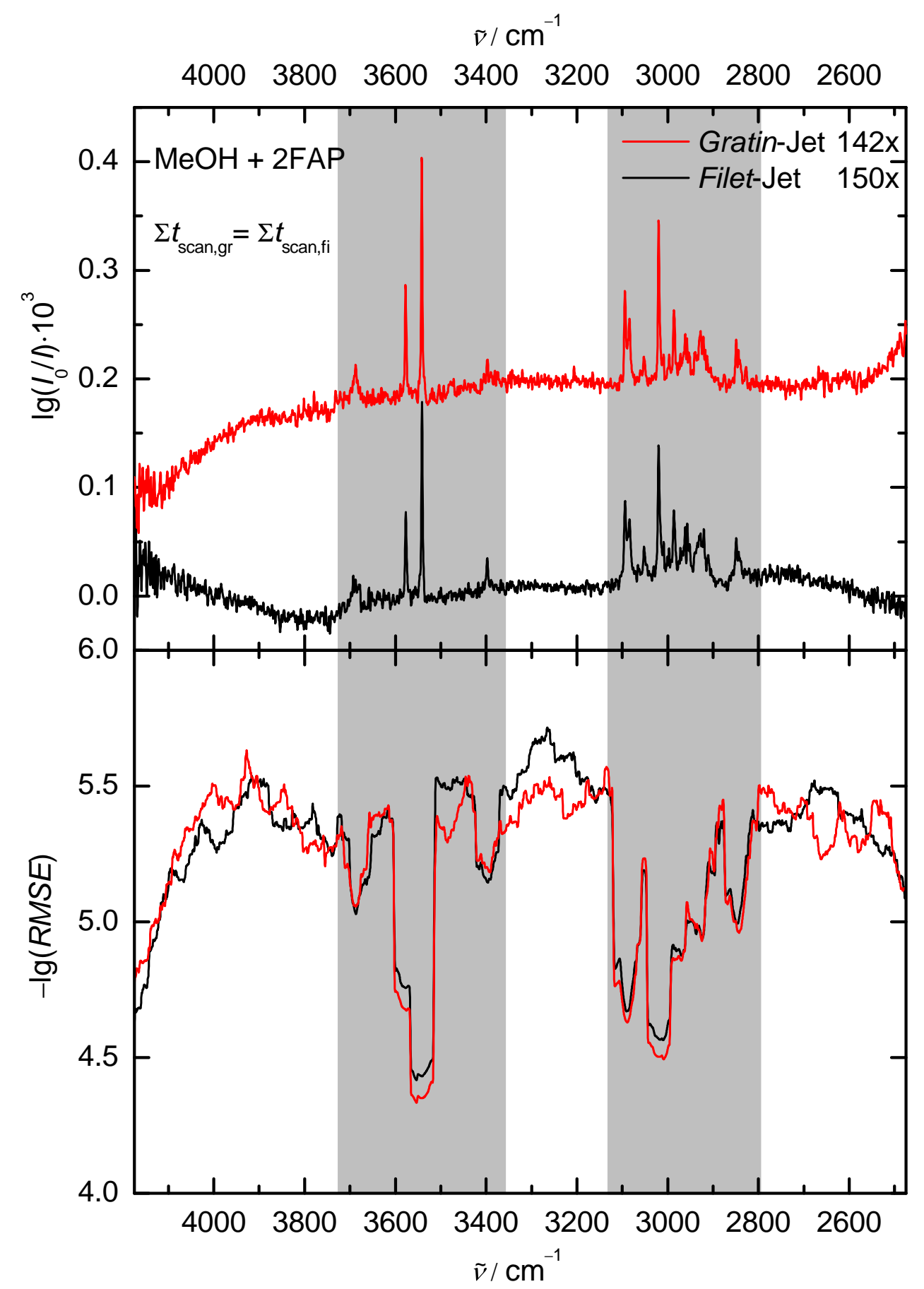

Abbildung 3.22: Vergleich der über die Pulszahl (oben rechts) auf die gleiche Gesamt-Scanzeit $\Sigma t_{\text {scan }}$ reduzierten Spektren (oben) und zugehörigen $-\lg (R M S E)$-Werte (unten) einer Mischung von $\mathrm{MeOH}$ und 2FAP in He am Gratin- (rot) und Filet-Jet (schwarz). Das Gratin-Jet-Spektrum wurde entlang der Ordinate verschoben (+0.26). Die von Absorptionsbanden des Gemischs belegten Spektralbereiche, die nicht zur Rauschanalyse herangezogen werden können, sind grau eingefärbt. 


\section{Gleiche Realmessdauer}

Sollen die Optimierungen hinsichtlich der Messeffizienz, also der nutzbaren Scanzeit pro Realzeit, mit berücksichtigt werden, bietet sich ein Vergleich bei identischer Realmessdauer an. Durch Optimierungen bei Pulsvor- und Pulsnachlauf (s. Kap. 3.5.2) dauert ein Puls am Gratin-Jet trotz der etwas längeren Scanzeit nur 133 ms (s. Tab. 3.9), verglichen mit 147 ms am Filet-Jet. Zusammen mit weiteren Verbesserungen, unter anderem bei der Größe des Puffervolumens (Kap. 3.1), führt dies am Gratin-Jet zu einer Zyklusdauer $t_{\mathrm{zyk}}$ (zeitlicher Abstand zwischen zwei Pulsen) von $28 \mathrm{~s}$, wohingegen beim Filet-Jet $31 \mathrm{~s}$ benötigt werden. Die Messeffizienz $t_{\text {scan }} / t_{\mathrm{zyk}}$ beträgt beim Filet-Jet dadurch $0.32 \%$, beim GratinJet dagegen $0.38 \%$.

Der Vergleich der Spektren und des Rauschwerts ist in Abbildung 3.23 dargestellt. Die Realmesszeiten $\Delta t_{\text {real }}$ wurden dabei aus dem Produkt der Zyklusdauer und der Zahl der für die Spektren gemittelten Pulse ermittelt. Die für Vor- und Nachbereitung der Messungen benötigte Zeit wurde der Einfachheit halber nicht mit berücksichtigt. Die Zahl der in den Vergleich einbezogenen Gratin-Jet-Pulse wurde analog zum vorherigen Vergleich reduziert, im aktuellen Fall aber so, dass dieselbe Realmesszeit wie am Filet-Jet erreicht wird.

Durch die angesprochenen Optimierungsmaßnahmen lassen sich am Gratin-Jet in derselben Zeit mehr Pulse (166) als am Filet-Jet (150) aufzeichnen, sodass im Gegensatz zum Vergleich über die Scandauer nun mehr Pulse des Gratin- als des Filet-Jets berücksichtigt werden. Dennoch zeigt sich qualitativ das gleiche Bild wie zuvor: Vor allem im Bereich zwischen $\mathrm{OH}$ - und $\mathrm{CH}$-Streckschwingungsbanden weist der Filet-Jet das (sichtbar) geringere Rauschniveau auf und erst oberhalb von 3800-3900 $\mathrm{cm}^{-1}$ kann sich der Gratin-Jet leicht vom Filet-Jet absetzen. Signifikante Unterschiede in der Grundlinienstabilität oder Störsignale sind erneut nicht erkennbar.

\section{Gleicher Substanzverbrauch}

Deutliche Unterschiede zwischen beiden Apparaturen lassen sich wie erwartet erkennen, wenn der Hauptvorteil des Gratin- gegenüber dem Filet-Jet berücksichtigt wird: der durch das Gasrecycling deutlich verringerte Substanzverbrauch. Beispielhaft sind in Abbildung 3.24 die Verbräuche des Trägergases Helium, das mehr als $99.9 \%$ des betrachteten Gasgemischs ausmacht und das deshalb stellvertretend für den Gesamt-Substanzverbrauch betrachtet wird, in Abhängigkeit von der Zahl der Pulse am Filet- (schwarz) bzw. Gratin-Jet (rot) dargestellt. Hierbei zeigen sich aufgrund der unterschiedlichen Betriebsarten grundlegend verschiedene Verläufe.

Initial muss bei beiden Apparaturen zunächst einmal das Reservoir mit dem betrachteten Substanzgemisch befüllt werden. Aufgrund des hohen Helium-Anteils kann die dazu benötigte Stoffmenge näherungsweise über das ideale Gasgesetz (Gl. 3.8) berechnet werden:

$$
n=\frac{p_{s} V_{\mathrm{R}}}{R T}
$$




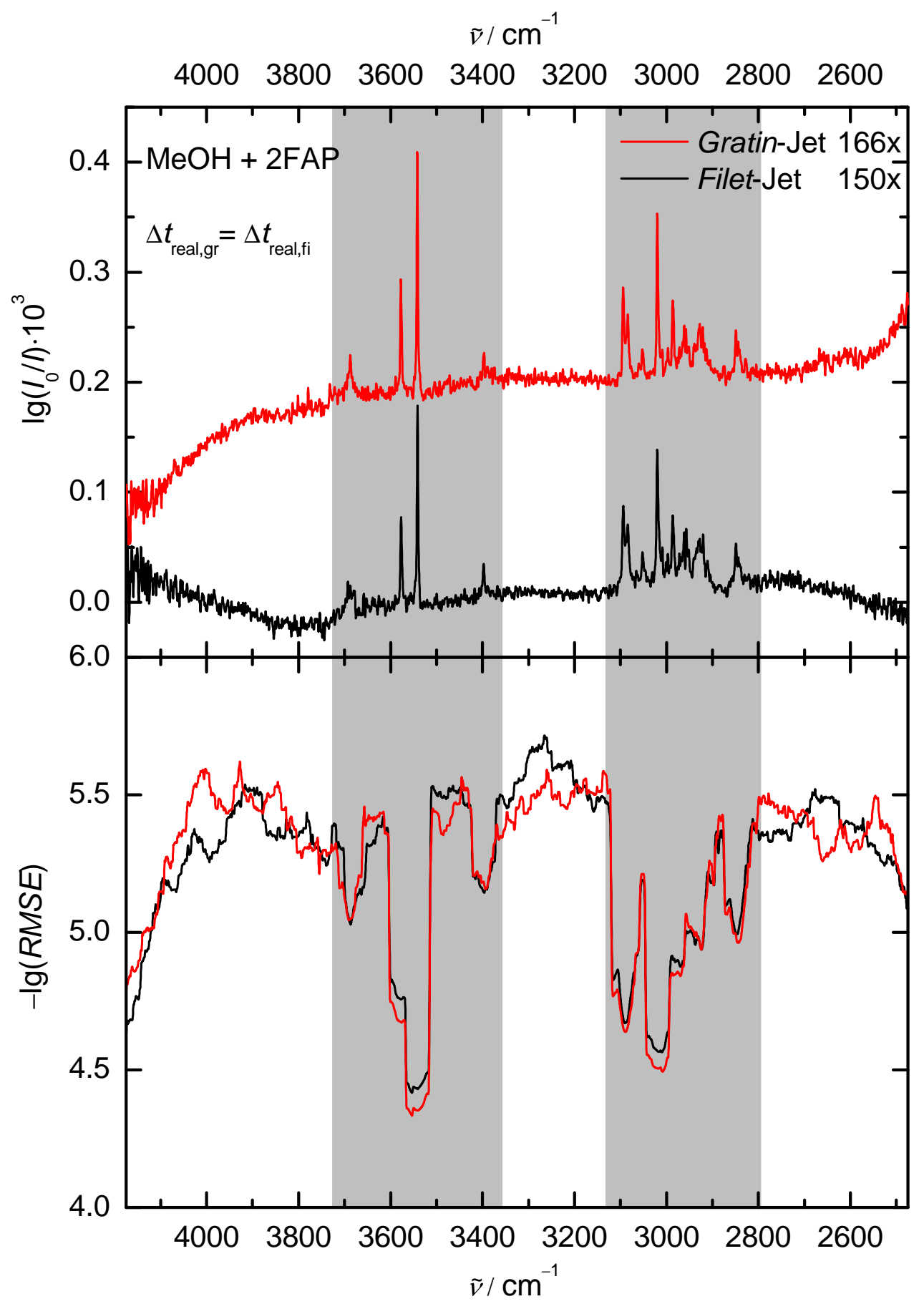

Abbildung 3.23: Vergleich analog zu Abb. 3.22, hier aber reduziert auf die gleiche Realmessdauer $\Delta t_{\text {real }}$. Das Gratin-Jet-Spektrum (rot) wurde auch hier entlang der Ordinate verschoben $(+0.30)$. 


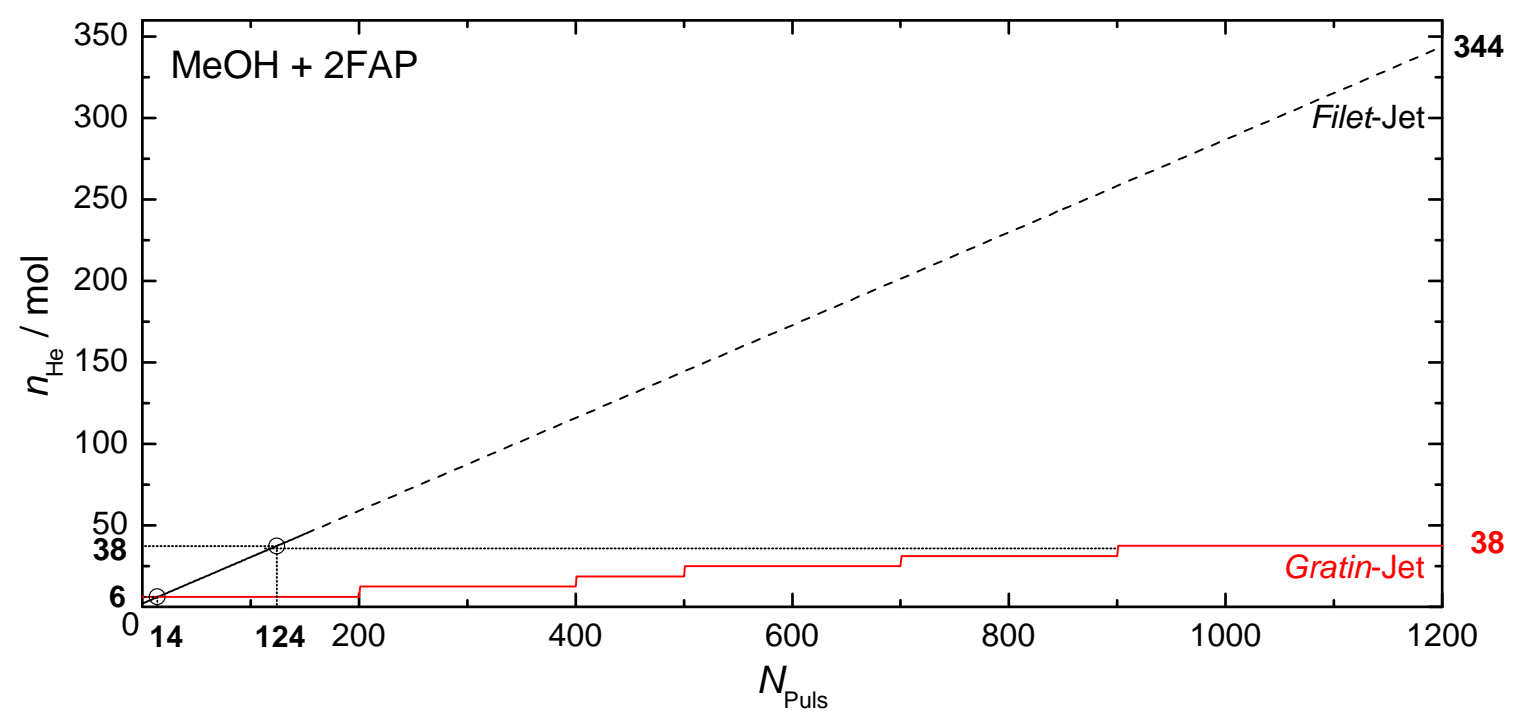

Abbildung 3.24: Vergleich des Trägergas-Substanzverbrauchs in Abhängigkeit von der Zahl der Gaspulse am Filet- (schwarz) und Gratin-Jet (rot) bei Messungen eines Gasgemischs aus $\mathrm{MeOH}$ und 2FAP in Helium. Am Gratin-Jet wurden dabei 1200 Pulse mit mehrfach neu befülltem Reservoir aufgenommen, wohingegen am Filet-Jet nur 150 Pulse durchgeführt wurden. Für die für 200 bzw. 1200 Pulse am Gratin-Jet benötigten Trägergasmengen von 6 bzw. 38 mol (fettgedruckte Zahlen an der Ordinate) wurde die maximal mögliche Zahl von Gaspulsen am Filet-Jet ermittelt (fettgedruckte Zahlen an der Abszisse).

Bei einem Stagnationsdruck $p_{s}$ von jeweils 750 mbar und einer Raumtemperatur $T$ von $298 \mathrm{~K}$ ergeben sich mit der universellen Gaskonstante $R=N_{\mathrm{A}} \cdot k_{\mathrm{B}}=$ $8.31446 \ldots \mathrm{J} \cdot \mathrm{mol}^{-1} \cdot \mathrm{K}^{-1[258-260]}$ und den Reservoirvolumina $V_{\mathrm{R}}$ von $67 \mathrm{~L}$ (Filet) bzw. $205 \mathrm{~L}$ (Reservoir inkl. Zuleitung am Gratin-Jet) Stoffmengen von $2.03 \mathrm{~mol}$ am Filet- bzw. $6.24 \mathrm{~mol}$ am Gratin-Jet.

Am Filet-Jet muss anschließend nach jedem Gaspuls neue Substanz nachgefüllt werden, bis der gewünschte Stagnationsdruck wieder erreicht ist. Der Substanzverbrauch pro Puls ergibt sich also aus der durch die Düse geströmten Gasmenge. Diese kann unter Verwendung von Gleichung 3.1 auf S. 36 beim verwendeten Stagnationsdruck von 750 mbar und der Pulslänge von $147 \mathrm{~ms}$ auf $0.28 \mathrm{~mol}$ bestimmt werden. Der Substanzverbrauch am FiletJet ergibt sich also zu $n_{\mathrm{He}}=\left(2.03+N_{\text {Puls }} \cdot 0.28\right)$ mol und zeigt damit den in Abbildung 3.24 dargestellten linearen Verlauf. Da im betrachteten Fall lediglich 150 Pulse gemessen wurden, ist der hypothetische weitere Verlauf in der Abbildung gestrichelt dargestellt.

Am Gratin-Jet wird dagegen aufgrund des Gasrecyclings nach der initialen Füllung keine zusätzliche Substanz für die einzelnen Pulse benötigt. Durch die im Laufe der Zeit ansteigende Wasserkonzentration (s. Kap. 3.6) muss das Reservoir allerdings etwa alle 90-200 min und damit etwa alle 200-400 Pulse mit „frischem“ Substanzgemisch befüllt werden, wobei jedes Mal erneut 6.24 mol Substanz benötigt werden. Bei der betrachteten Messung von $\mathrm{MeOH}+2 \mathrm{FAP}$ in He wurde das Reservoir nach 200, 400, 500, 700 und 
900 Pulsen neu befüllt, sodass sich der in Abbildung 3.24 rot dargestellte, stufenförmige Vebrauchsverlauf ergibt.

Obwohl am Gratin-Jet 1200 Pulse durchgeführt und damit ebenso viele Scans aufgenommen wurden, reicht der erzielte Substanzverbrauch von ca. $38 \mathrm{~mol}$ nicht aus, um den für 150 Scans am Filet-Jet benötigten Substanzverbrauch von $45 \mathrm{~mol}$ zu erreichen. Ein Vergleich des Rauschniveaus bei 150 Filet-Jet-Pulsen, wie sie für die beiden bisherigen Gegenüberstellungen genutzt wurden, ist also in diesem Fall nicht möglich. Stattdessen werden zwei andere Verbräuche für den Vergleich genutzt (Kreissymbole in Abb. 3.24): der minimal mögliche Substanzverbrauch am Gratin-Jet (eine Füllung, $6.24 \mathrm{~mol}$ ) und der maximale Substanzverbrauch der betrachteten Gratin-Jet-Messung (sechs Füllungen, $37.5 \mathrm{~mol}$ ). Bei diesen Verbräuchen sind 14 Pulse am Filet- und 200 Pulse am Gratin- (minimaler Verbrauch) bzw. 124 Pulse am Filet- und 1200 Pulse am Gratin-Jet (maximaler Verbrauch) möglich. Anhand dieser Zahlen zeigt sich bereits, dass die genaue Höhe der Substanzverbrauchsreduktion durch das Gasrecycling am Gratin-Jet von der Zahl der betrachteten Pulse abhängt. Bei einer Neubefüllung nach (im Durchschnitt) jeweils 200 Pulsen beträgt die Reduktion im Limit einer hohen Pulszahl ca. 89\%, wie die an der rechten OrdinatenSkala eingetragenen Verbräuche zeigen. Sind durch eine langsamere Wasseranreicherung oder bei Betrachtung eines auf Wasser unempfindlicheren Systems am Gratin-Jet mehr Pulse ohne Neubefüllung möglich, werden die Substanzeinsparungen entsprechend größer. Bei einem im Rahmen der Masterarbeit von TAIJA Fischer durchgeführten Spektrenvergleich von Filet- und Gratin-Jet mit einer Mischung von Cyclopentanol $(\mathrm{CyPeOH})$ und Pinakolon (Pin) in He (s. Kap. 3.7.3), bei der an beiden Apparaturen 375 Pulse aufgenommen wurden, wurden alle Pulse am Gratin-Jet ohne Neubefüllung durchgeführt. Hier stehen sich damit ein Verbrauch von $109 \mathrm{~mol}$ am Filet- und $6.24 \mathrm{~mol}$ am Gratin-Jet gegenüber, was einer Verbrauchsreduktion um $94 \%$ entspricht.

Die Spektrenqualität bei gleichem Substanzverbrauch soll jedoch - wie in den vorherigen Vergleichen - anhand des $\mathrm{MeOH}+2 \mathrm{FAP}-S y s t e m s$ analysiert werden. Abbildung 3.25 zeigt zunächst den Vergleich beim kleinstmöglichen Substanzverbrauch. Das aufgrund der geringeren Zahl an Pulsen deutlich größere Rauschen im Filet-Jet-Spektrum ist bereits im oberen Teil der Abbildung deutlich erkennbar. Im Rahmen des Rauschens ist die Methanol-Monomer-Bande bei $3686 \mathrm{~cm}^{-1}$ im Filet-Jet-Spektrum kaum zu erkennen, tritt im Gratin-Jet-Spektrum aber deutlich hervor. Hier lassen sich somit signifikante Unterschiede zwischen beiden Spektren feststellen, die bereits bei der qualitativen Auswertung relevant werden. Aufgrund der deutlich höheren Pulszahl weist das Gratin-Jet-Spektrum auch einen stabileren Verlauf der Grundlinie auf. Der im unteren Teil der Abbildung dargestellte - $\lg (R M S E)$-Wert bestätigt die qualitativen Befunde aus den Spektren noch einmal.

Beim Vergleich mit maximal möglichem Substanzverbrauch (Abb. 3.26) zeigt sich ein ähnliches Bild, wenngleich entsprechend der höheren Zahl an Pulsen auf einem niedrigeren Rauschniveau. In den Spektren selbst sind nun in beiden Fällen die relevanten Banden klar erkennbar. Das Rauschen ist im roten Gratin-Jet-Spektrum jedoch sichtbar kleiner, wie auch durch den Rauschwert im unteren Teil der Abbildung bestätigt wird. Im Be- 


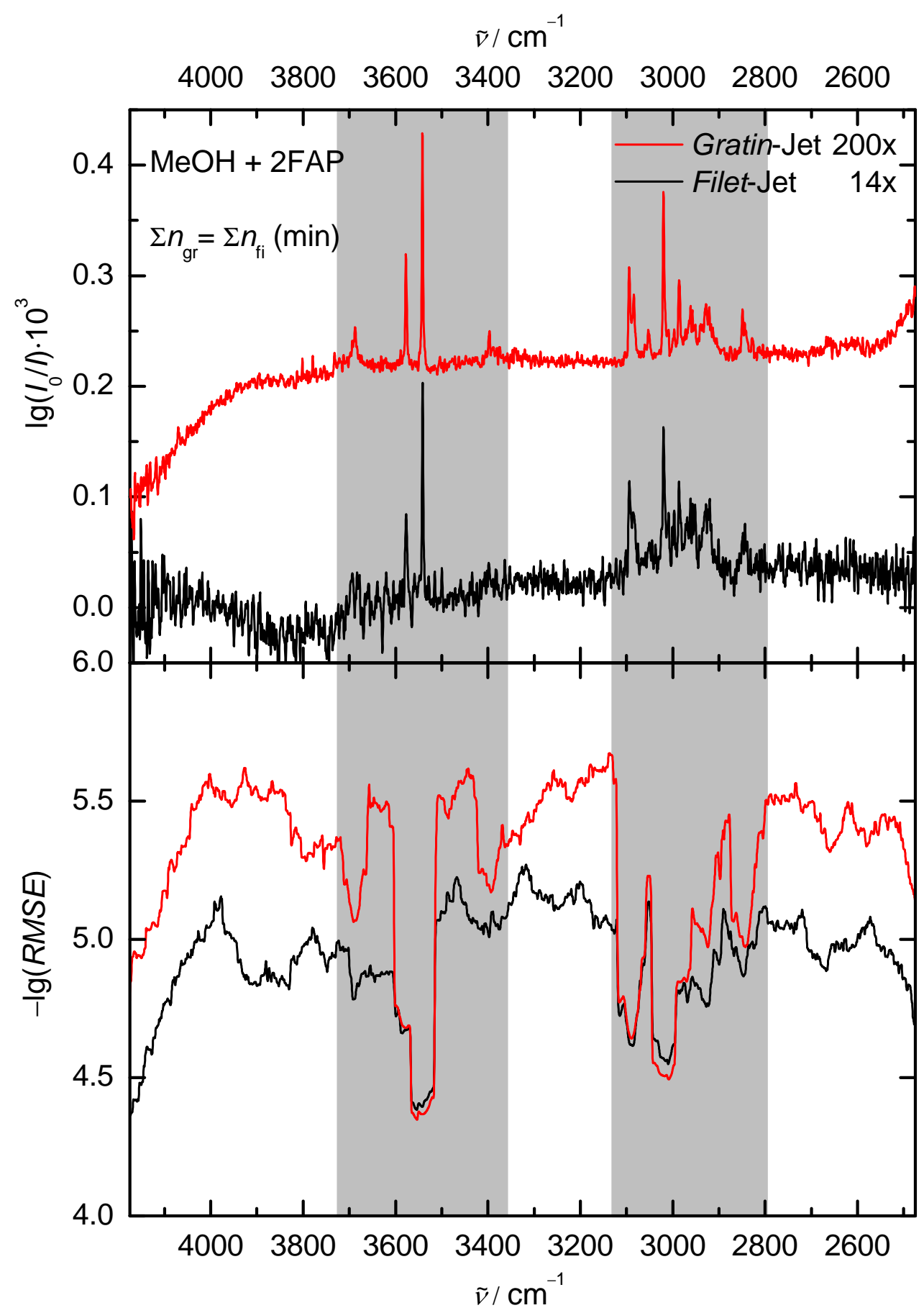

Abbildung 3.25: Vergleich analog zu Abb. 3.22, hier aber reduziert auf den gleichen Substanzverbrauch $\Sigma n$. Als Vergleichspunkt wurde der kleinstmögliche Substanzverbrauch am Gratin-Jet (eine Füllung, ca. 6 mol, s. Abb. 3.24) gewählt. Das Gratin-Jet-Spektrum (rot) wurde erneut entlang der Ordinate verschoben $(+0.28)$. 


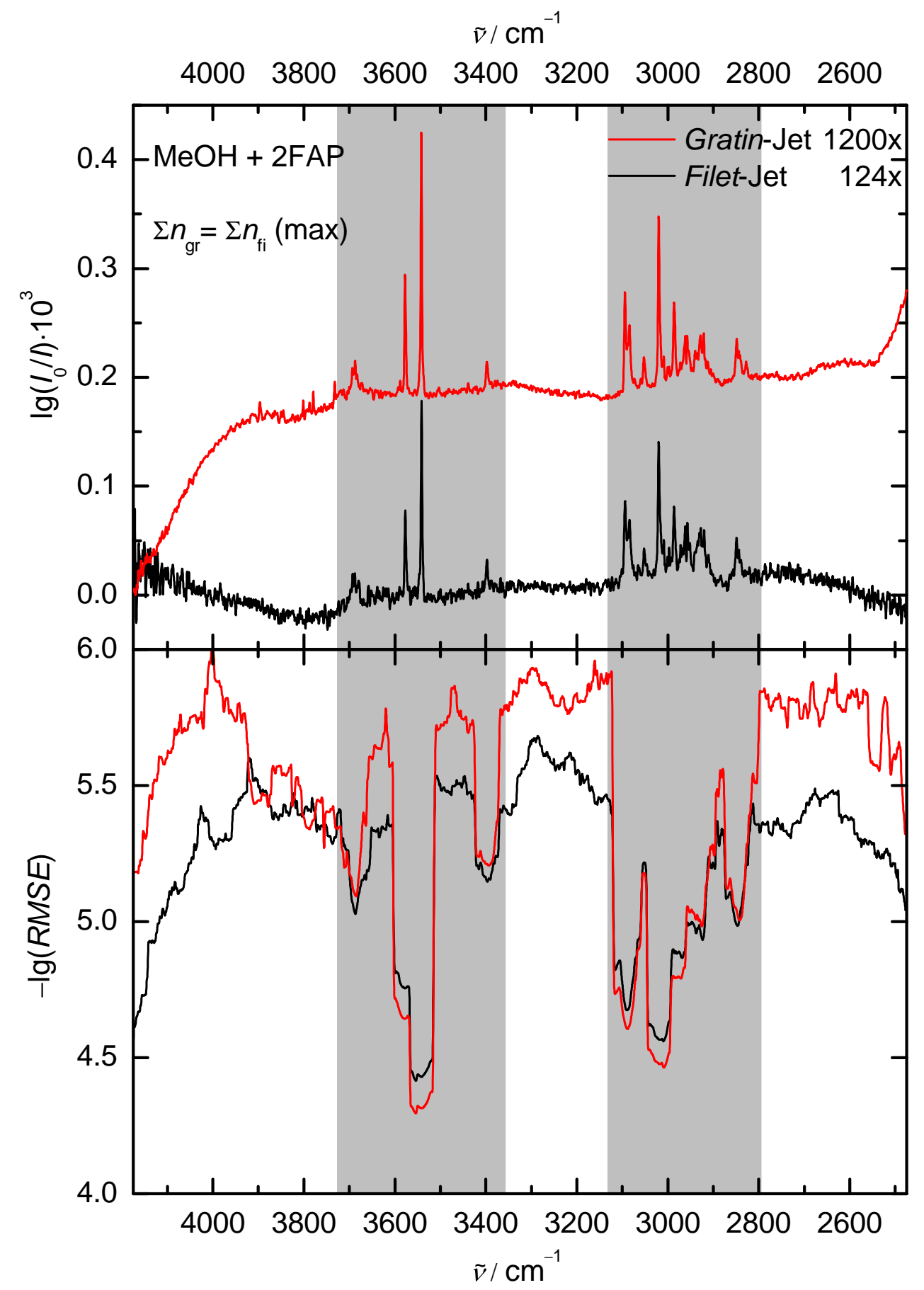

Abbildung 3.26: Weiterer Vergleich bei gleichem Substanzverbrauch $\Sigma n$, analog zu Abb. 3.25. Als Vergleichspunkt wurde hier der für die gesamte Messreihe am Gratin-Jet benötigte Substanzverbrauch (sechs Füllungen, ca. 38 mol, s. Abb. 3.24) gewählt. Das Gratin-Jet-Spektrum (rot) wurde entlang der Ordinate verschoben $(+0.25)$. 
Tabelle 3.10: Vergleich der gemessenen Intensitätsverhältnisse $I_{\mathrm{Me}} / I_{\mathrm{Ph}}$ der Hetero-Dimere von $\mathrm{MeOH}+2 \mathrm{FAP}$ an Gratin- und Filet- Jet (s. Tab. 3.8), erweitert um Informationen zur Zahl der berücksichtigten Scans $\left(N_{\text {scans }}\right)$, dem daraus resultierenden (Helium-)Substanzverbrauch $\left(n_{\mathrm{He}}\right)$, der resultierenden Messzeit $\left(\Delta t_{\text {real }}\right)$ sowie zum Messfehler für das Intensitätsverhältnis.

\begin{tabular}{lrrrr}
\hline Jet & $\boldsymbol{N}_{\text {scans }}$ & $\boldsymbol{n}_{\mathrm{He}} / \mathbf{m o l}$ & $\Delta \boldsymbol{t}_{\text {real }} / \mathbf{h : m m}$ & $\boldsymbol{I}_{\mathbf{M e}} / \boldsymbol{I}_{\mathbf{P h}}$ \\
\hline Filet & 150 & 44.8 & $1: 18$ & $2.28 \pm 0.21$ \\
Gratin & 1200 & 37.5 & $9: 20$ & $2.27 \pm 0.11$ \\
\hline
\end{tabular}

reich zwischen $3900 \mathrm{~cm}^{-1}$ und dem Methanol-Monomer leidet der ermittelte Rauschwert des Gratin-Jet-Spektrums allerdings unter den Jet-Wasser-Linien, die im Spektrum sichtbar werden. Hier zeigt sich, dass zumindest beim momentanen Wassereintrag am GratinJet stets ein Kompromiss zwischen Reduzierung des Substanzverbrauchs (möglichst viele Pulse ohne Neubefüllung) und geringer Jet-Wasser-Konzentration in den Spektren (möglichst häufige Neubefüllung) gefunden werden muss.

Ein handfester Vorteil des deutlich reduzierten Substanzverbrauchs ergibt sich für das Auslesen möglichst akkurater Messwerte aus den Spektren. In Tabelle 3.10 wurde der ursprüngliche Vergleich der gemessenen Intensitätsverhältnisse der gemischten $\mathrm{MeOH}$ 2FAP-Dimere (vgl. Tab. 3.8) um zusätzliche Informationen erweitert. Bei immer noch niedrigerem Substanzverbrauch können am Gratin-Jet wesentlich mehr Gaspulse und damit Spektrometerscans durchgeführt werden. Dies führt zu einem besseren $\mathrm{S} / \mathrm{N}$ und damit zu kleineren Fehlerbalken für verschiedene Messgrößen, wie dem hier dargestellten, für Benchmarks quantenchemischer Methoden wichtigen Intensitätsverhältnis. Da die Zyklusdauer allerdings nicht im selben Maß wie der Substanzverbrauch von Filet- zu GratinJet verringert werden konnte, ist ein entsprechender Zeitaufwand für die Messung nötig.

\subsubsection{Fazit}

Der Vergleich der Spektrenqualität über das Rauschniveau zeigt, dass der Gratin-Jet insgesamt nicht ganz die Leistungsfähigkeit des Filet-Jets erreicht, wenn bei gleicher Scanzeit bzw. bei gleicher Realmesszeit verglichen und der Hauptvorteil - der reduzierte Substanzverbrauch durch das Gasrecycling - damit nicht berücksichtigt wird. Bereits bei diesen Vergleichen zeigen sich jedoch im Spektralbereich oberhalb von ca. 3800-3900 cm ${ }^{-1}$ leichte Vorteile für den Gratin-Jet. Erfolgt die Gegenüberstellung bei gleichem Substanzverbrauch, liegt der Gratin-Jet erwartungsgemäß klar vorn. Hier wird deutlich, dass mit dem Gratin-Jet genauere Ergebnisse mit weniger Chemikalieneinsatz erzielt werden können der um bis zu 95\% verringerte Substanzverbrauch ermöglicht also z. B. die Verwendung teurer Chemikalien, die am Filet-Jet bisher nicht zugänglich waren.

Selbst beim für den Gratin-Jet ungünstigsten Vergleich bei gleicher Gesamt-Scanzeit ist der Vorsprung des Filet-Jets jedoch nicht definitiv. Beim Gratin-Jet wurde in den bishe- 
rigen Messungen eine schnelle Alterung der W150-Lichtquelle festgestellt, die sich in einem Signalverlust von bis zu 30\% innerhalb weniger Wochen nach Einbau äußert. Die hier für den Vergleich herangezogenen Spektren wurden mit einer solchen „gealterten“ Lichtquelle aufgenommen. Zudem scheint auch die Qualität der am Filet-Jet aufgenommenen Spektren von der genauen Konfiguration und einer sorgfältigen Optimierung abhängig zu sein, wie der Vergleich der Spektren eines Gemischs aus Cyclopentanol $(\mathrm{CyPeOH})$ und Pinakolon (Pin) in Helium (Abb. 3.27) zeigt.

Im Rahmen der Masterarbeit von TAIJA Fischer wurde am Gratin-Jet ein Spektrum dieses Gemischs mit frisch eingebauter W150-Lichtquelle und damit maximaler Lichtintensität aufgenommen und mit einem von TAIJA Fischer und CHARLOTTE ZimmermanN am Filet-Jet aufgenommenen Spektrum verglichen. Bei diesem Filet-Jet-Spektrum liegt das Rauschniveau bei gleicher Scanzahl höher als bei dem für die oberen Gegenüberstellungen herangezogenen $\mathrm{MeOH}+2 \mathrm{FAP}-$ Spektrum. Einer der Gründe hierfür könnte möglicherweise in den genutzten Vorwiderständen $(2.0 \mathrm{k} \Omega$ im $\mathrm{CyPeOH}+\mathrm{Pin}-\mathrm{vs} .2 .2 \mathrm{k} \Omega \mathrm{im}$ $\mathrm{MeOH}+2 \mathrm{FAP}-$ Spektrum) liegen, dies wurde bisher allerdings nicht systematisch untersucht. Der Vergleich des so aufgenommenen Filet-CyPeOH+Pin-Spektrums mit dem entsprechenden, auf die gleiche Gesamt-Scanzeit reduzierten Gratin-Jet-Spektrum (Abb. 3.27) zeigt jedoch, dass bereits bei leichten Veränderungen am Zustand der jeweiligen Apparaturen der Gratin-Jet ein geringeres Rauschniveau und damit eine bessere Spektrenqualität als der Filet-Jet erreichen kann.

Zusammenfassend wird pro Scan- bzw. Realmesszeit am neuen Gratin-Jet ein vergleichbares Rauschniveau wie am über die Jahre immer weiter optimierten Filet-Jet erreicht. Darüber hinaus sind am Gratin-Jet abgesehen vom sich anreichernden Jet-Wasser im betrachteten Filterbereich keine Störsignale in den Spektren zu beobachten und auch die Grundlinienstabilität liegt auf dem Niveau des Filet-Jets. Die Spektrenqualität ist somit vergleichbar gut. Bei diesen Vergleichen noch nicht berücksichtigt ist der Einfluss der von Filet- zu Gratin-Jet um ca. 17\% verlängerten Düse, der über eine entsprechende Intensitätserhöhung der spektralen Signale zusätzlich zu einer Verbesserung des S/N führen sollte. Unberücksichtigt bleibt hier zudem das Verbesserungspotential des Gratin-Jets durch Heizung der Düse. Diese könnte bei unwesentlich verschlechterter Expansionskühlung die Bildung großer Aggregate verhindern und bei höherer Moleküldichte verbesserte Monomer- und Dimersignale liefern. Ebenfalls ungetestet bleiben der Einsatz einer Linsenoptik am Gratin-Jet sowie der direkte Leistungsvergleich der beiden eingesetzten Spektrometer. 


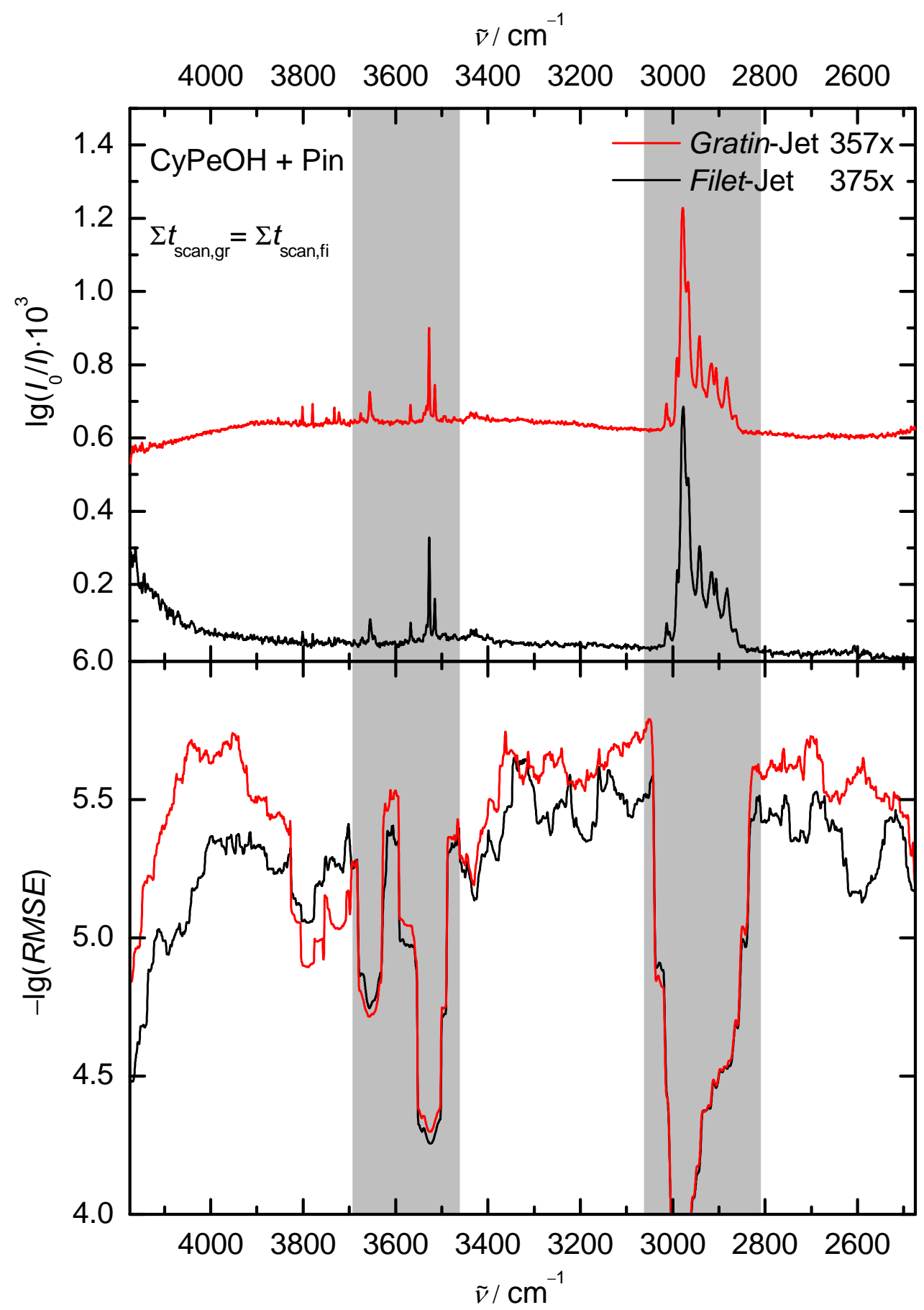

Abbildung 3.27: Ein zweiter Vergleich der Apparaturen bei gleicher Gesamt-Scanzeit $\Sigma t_{\text {scan }}$ (vgl. Abb. 3.22), hier aber anhand von Messungen mit einem Gemisch aus $\mathrm{CyPeOH}$ und Pin in He. Das Gratin-Jet-Spektrum (rot) wurde auch hier entlang der Ordinate verschoben $(+0.60)$. (FiletJet-Spektrum (schwarz) freundlicherweise zur Verfügung gestellt von C. ZimmermanN.) 



\section{Kapitel 4}

\section{O/C-Waagensysteme}

Mikrosolvatation ${ }^{[47,48]}$ bezeichnet das stufenweise Hinzufügen von Lösungsmittelmolekülen zu einem zu lösenden Molekül. Wird dieser Prozess durch eine quantenchemische Methode modelliert, können sich mögliche Fehler in der Beschreibung der individuellen Wechselwirkungen bei Vergrößerung der Solvathülle sowohl addieren als auch wieder auslöschen, sodass eine gute Modellierung der Gesamtsolvatation unter Umständen nur durch Fehlerkompensation zustande kommt. Soll also untersucht werden, ob eine Methode aus den richtigen Gründen eine gute Leistung zeigt, sollte beim ersten Mikrosolvatationsschritt angesetzt werden. ${ }^{[75]}$

Werden für die Beurteilung der Leistungsfähigkeit der betrachteten quantenchemischen Methoden experimentelle Referenzdaten herangezogen, ${ }^{[7]}$ bietet sich hierfür die spektroskopische Untersuchung der Gelöstes-Lösungsmittelcluster in einer Überschallexpansion $^{[261]}$ an (s. Kap. 2). Die quantitativen Bindungsenergien dieser Cluster sind nur in speziellen Fällen ermittelbar, ${ }^{[64,178,262,263]}$ auch wenn es hierzu hybride Ansätze gibt, die experimentelle und theoretische Daten für die Berechnung vereinen. ${ }^{[264,265]}$ Bei Vorhandensein mehrerer metastabiler Konformere kann ein Benchmark quantenchemischer Methoden aber auch auf Grundlage der relativen Energien erfolgen, die in der linearen Jet-FTIR-Spektroskopie etwa über die relativen Häufigkeiten der Konformere zugänglich sind. ${ }^{[31]}$ Je nach relativer Stabilität, Barrierenhöhe für die Umwandlung ineinander und Trägergas können mehrere dieser metastabilen Konformere in der Überschallexpansion koexistieren. ${ }^{[42,44,170]}$

Prinzipiell ist besonders Mikrowellen-(MW-)Spektroskopie gut für die Unterscheidung verschiedener Strukturen geeignet. Unter den kalten Bedingungen der Überschallexpansion kann jedoch auch FTIR-Spektroskopie ${ }^{[55]}$ zu einer solchen Unterscheidung in der Lage sein, insbesondere wenn über Wasserstoffbrücken verbundene Gelöstes-Lösemittelcluster untersucht werden. Die Ausbildung einer Wasserstoffbrückenbindung kann in der FTIRSpektroskopie oft über eine Rotverschiebung - also eine Verschiebung zu niedrigeren Wellenzahlen - der O-H-Streckschwingungsbande des Wasserstoffbrückenbindungsdonors nachgewiesen werden. ${ }^{[59,266]}$ Sind die Wasserstoffbrückenbindungen unterschiedlich stark, zeigen sich verschieden große Rotverschiebungen, was eine Unterscheidung der Cluster auf Basis ihrer Struktur ermöglicht. 
Damit eine Zuordnung der im FTIR-Spektrum beobachteten Banden zu unterschiedlichen Konformeren möglich ist, sollten die betrachteten Konformere ausreichend verschieden stark rotverschobene OH-Streckschwingungsbanden aufweisen. In der vorliegenden Arbeit werden im Sinne der Fokussierung auf den ersten Schritt der Mikrosolvatation (s. oben) dabei ausschließlich Dimere betrachtet. Damit überhaupt mehrere DimerKonformere - für das Benchmarking quantenchemischer Methoden idealerweise zwei (s. Kap. 2.3.2) - in der Expansion vorliegen, sollte die Energiedifferenz weniger als ca. $4 \mathrm{~kJ} \cdot \mathrm{mol}^{-1}$ betragen und die Umwandlungsbarriere zwischen den Konformeren flach und schmal sein. ${ }^{[31,267]}$ Zudem sollten sich Wasserstoffbrückenbindungsdonor und -akzeptor über Substitution systematisch verändern lassen. So können die relativen Häufigkeiten der Dimer-Konformere für eine ganze Reihe ähnlicher Systeme untersucht werden, um eine größere Zahl experimenteller Referenzdatenpunkte für den Vergleich mit den Daten aus der quantenchemischen Rechnung zu erhalten. Ein solche Reihe von Systemen stellt dann eine Art intermolekulare Waage dar, deren Gleichgewichtslage bezüglich der Konformeren-Häufigkeitsverteilung durch die Variation an Donor und Akzeptor beeinflusst werden kann. Je kleiner dabei die Umwandlungsbarriere ist, desto niedriger ist die Temperatur für das Konformerengleichgewicht in der Überschallexpansion und desto empfindlicher reagiert die intermolekulare Waage auf Änderungen im Energieunterschied. Somit können solche Waagen genutzt werden, um quantenchemische Methoden hinsichtlich ihrer Leistungsfähigkeit zu untersuchen (s. Kap. 2.3.2), in günstigen Fällen bis in den sub$\mathrm{kJ} /$ mol-Bereich. ${ }^{[31,37]}$

Eine Möglichkeit, die quantenchemischen Methoden daraufhin zu untersuchen, ob sie ein Gleichgewicht aus verschiedenen Wechselwirkungen adäquat beschreiben können, ist die Wahl eines Systems, das chemisch ungleiche Andockstellen für eine Wasserstoffbrückenbindung aufweist. Furan und seine Derivate wie 2,5-Dimethylfuran sind ein Beispiel für solche Wasserstoffbrückenbindungsakzeptoren und bieten zwei verschiedene Andockstellen: zum einen das delokalisierte, aromatische $\pi$-System des kohlenstoffbasierten Fünfrings und zum anderen die $\pi$ - bzw. $\sigma$-Valenzelektronenpaare des Sauerstoffatoms. ${ }^{[49]}$ Anisol weist vergleichbare Andockstellen auf: das delokalisierte, kohlenstoffbasierte $\pi$-System einerseits und die freien Elektronenpaare des Sauerstoffatoms andererseits. ${ }^{[50]}$ In beiden Fällen konnte bereits die Koexistenz O- (Wasserstoffbrückenbindung zum Sauerstoffatom) und C-gebundener (Wasserstoffbrückenbindung zum kohlenstoffbasierten $\pi$-System) Dimere in Überschallexpansionen und die Beeinflussbarkeit dieses Gleichgewichts durch Variation von Donor und/oder Akzeptor nachgewiesen werden. ${ }^{[31,36,37,49,50,239]}$ Sowohl das Furan-Alkohol- als auch das Anisol-Alkohol-System stellen somit intermolekulare Waagen dar, die gemäß ihrer Wasserstoffbrückenbindungsandockstellen als $\mathrm{O} / \mathrm{C}$-Waagensysteme bezeichnet werden können.

Im vorliegenden Kapitel sollen anhand dieser O/C-Waagensysteme Benchmarks quantenchemischer Methoden durchgeführt werden. In der Furan-Mikrosolvatations-Leistungsüberprüfung (Kap. 4.1) soll zunächst basierend auf dem überschaubaren experimentellen Datensatz der Kombination eines Alkohols (Methanol ${ }^{[268]}$ ) als Wasserstoffbrückenbindungsdonor mit drei Furanderivaten als -akzeptoren eine größere Zahl quantenchemi- 
scher Methoden hinsichtlich ihrer Leistungsfähigkeit überprüft werden. Im Gegensatz dazu soll dann im folgenden Unterkapitel (Kap. 4.2) mit dem Anisol-O/C-Waagensystem ein umfassenderer experimenteller Datensatz genutzt werden, um drei beispielhafte quantenchemische Methoden zu untersuchen.

\subsection{Furan-Mikrosolvatations-Leistungsüberprüfung (Göbench)}

Die Inhalte dieses Kapitels wurden in weiten Teilen bereits veröffentlicht. ${ }^{[75,80]}$ Teile der Abbildungen, Tabellen und des Texts können sich somit mit den Veröffentlichungen decken und Zitate aus den genannten Veröffentlichungen, inklusive Paraphrasierungen und wörtlicher Übersetzungen, werden in diesem Kapitel nicht noch einmal gesondert gekennzeichnet (Hinweis zum Urheberrecht: „Reprinted from f. Chem. Phys. 2018, 148, $014301^{[75]}$ and f. Chem. Phys. 2020, 152, 164303, ${ }^{[80]}$ with the permission of AIP Publishing.").

Basierend auf den Ergebnissen einer früheren Untersuchung ${ }^{[49]}$ wurde für die FuranMikrosolvatations-Leistungsüberprüfung das O/C-Waagensystem aus den Heterodimeren von Methanol mit verschiedenen Furanderivaten ausgewählt. Wie bereits erwähnt, bieten Furane zwei energetisch nah zusammenliegende, aber chemisch unterschiedliche Andockstellen für protische Lösungsmittelmoleküle: das polare Sauerstoffatom $(\mathrm{O})$, dessen Attraktivität durch Delokalisierung seiner Elektronendichte über das aromatische $\pi$-System verringert ist, und das Kohlenstoffgerüst (C), das zusammen mit dem Sauerstoffatom den Fünfring bildet und das durch das delokalisierte $\pi$-System als Andockstelle attraktiver wird. Auf Grundlage der genannten Eigenschaften konnten die Furane in der Vergangenheit bereits als für Benchmarks interessante Systeme identifiziert werden. ${ }^{[36,49,269-271]}$ Furan (Fu), ${ }^{[272,273]}$ 2-Methylfuran $(\mathrm{MFu})^{[274,275]}$ und 2,5-Dimethylfuran $(\mathrm{DMFu})^{[275,276]}$ (s. Abb. 4.1) sollen deshalb in der vorliegenden Untersuchung mit dem Lö-
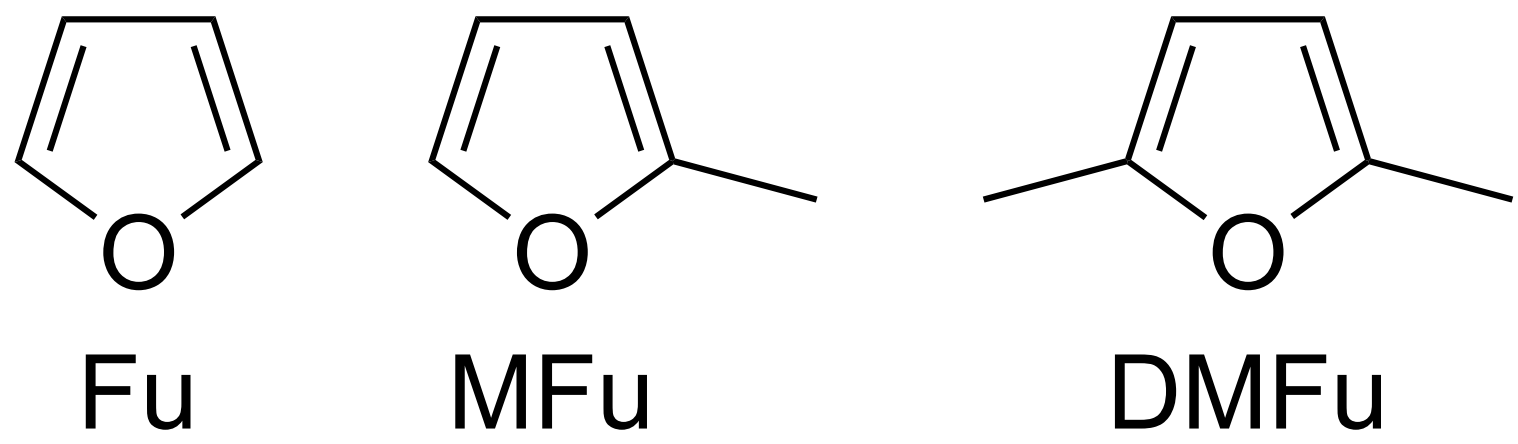

Abbildung 4.1: Lewis-Strukturen der in der Leistungsüberprüfung betrachteten Furane mit ihren im Text verwendeten Kürzeln: Furan (Fu), 2-Methylfuran (MFu) und 2,5-Dimethylfuran (DMFu). Bearbeitung von Fig. 1 in Ref. [75] (lizenziert unter CC BY 4.0). 


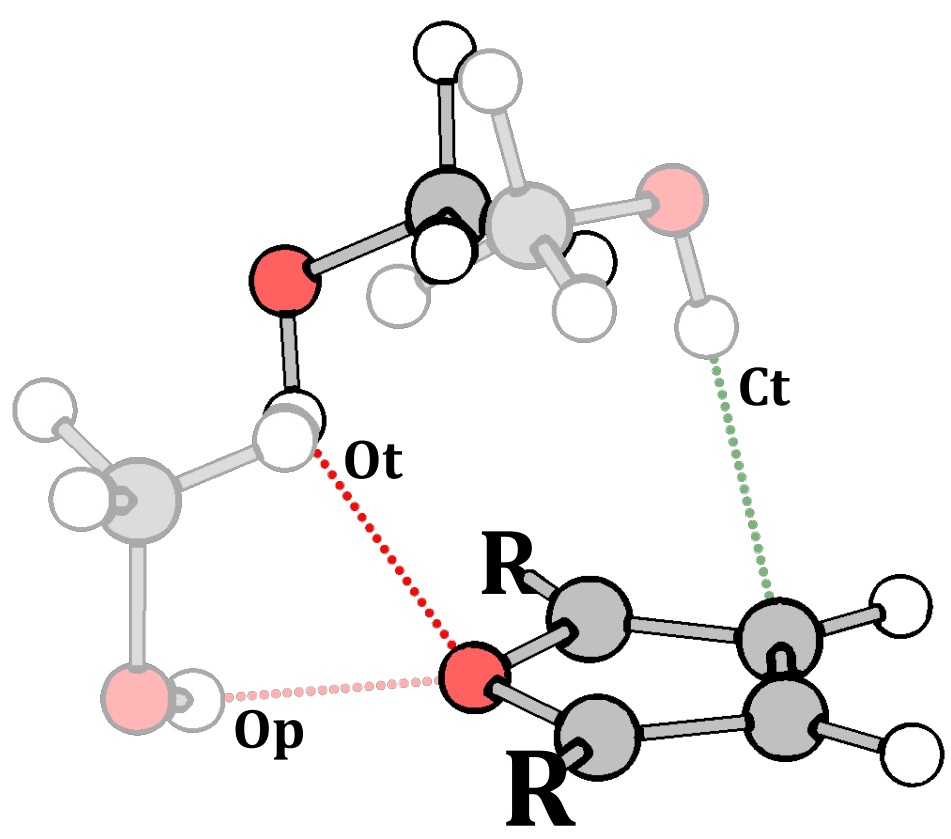

Abbildung 4.2: Schematische Übersicht zur Illustration der wichtigsten Strukturen in der Bindungssituation eines Lösungsmittelmoleküls $(\mathrm{X}-\mathrm{H})$ wie Methanol $(\mathrm{X}=\mathrm{MeO})$ mit Furan-Derivaten. Das Lösungsmittelmolekül kann eine Wasserstoffbrückenbindung zum $\pi$-System oberhalb eines der Kohlenstoffatome (C in der Kennzeichnung) oder zu den $\pi$ - bzw. $\sigma$-Elektronen des Sauerstoffatoms ausbilden $(\mathbf{O})$. Das kleingedruckte $\mathbf{t}$ in der Kennzeichnung steht für eine Wasserstoffbrückenbindung oberhalb der Furan-Ebene (engl. „on top“), also zum $\pi$-System des Furan-Moleküls, wohingegen $\mathbf{p}$ eine Wasserstoffbrückenbindung in der Furan-Ebene bezeichnet (engl. „in plane“), also zu einem $\sigma$-Orbital. Die Positionen, an denen Fu substituiert wird, um MFu oder DMFu zu bilden, sind mit $\mathbf{R}$ gekennzeichnet. Hinweis zum Urheberrecht: „Reprinted from f. Chem. Phys. 2020, 152, 164303, ${ }^{[80]}$ with the permission of AIP Publishing.“.

sungsmittelmolekül Methanol (MeOH) bzw. seinem deuterierten Analogon Methanol-OD (MeOD) kombiniert werden und damit als Modellsystem für die grundlegenden Wechselwirkungen zwischen organischem Solvat und Solvens dienen.

Wie Abbildung 4.2 veranschaulicht, sind für die Furane mehrere Bindungssituationen mit einem protischen Lösungsmittelmolekül $\mathrm{X}-\mathrm{H}$ wie Methanol $(\mathrm{X}=\mathrm{MeO})$ möglich. So kann das Lösungsmittelmolekül, wie bereits beschrieben, entweder eine Wasserstoffbrückenbindung zu einem der Kohlenstoffatome (Kennzeichnung „C“) oder zum Sauerstoffatom (Kennzeichnung „O“) ausbilden. Während die Bindung zu den C-Atomen immer zum delokalisierten $\pi$-System und deshalb von oberhalb der Ringebene (Kennzeichnung „t" für engl. „on top“) erfolgt, bietet das Sauerstoffatom zwei Andockstellen: der sauerstoffzentrierte Teil des $\pi$-Systems, was erneut zu einer Bindung von oberhalb der Ringebene („t") führt oder alternativ das freie $\sigma$-Elektronenpaar, das in der Ringebene liegt (Kennzeichnung „p“ für engl. „in plane“). In allen Fällen kann der Solvens-Rest X sich so anordnen, dass sekundäre Wechselwirkungen mit dem Furan-Ring bzw. den Substitu- 
enten $\mathrm{R}$ maximiert werden. Ist die primäre Bindungspräferenz nur schwach zugunsten der einen oder anderen Stelle ausgeprägt, wie es für die Furane der Fall ist, kann das Gleichgewicht durch die sekundären R $\cdots$ X-Wechselwirkungen entscheidend beeinflusst werden. ${ }^{[31,277]}$

Um eine möglichst große Unabhängigkeit zwischen experimentellen Referenz- und zu untersuchenden berechneten Daten zu gewährleisten, ${ }^{[278]}$ wurde die Furan-Mikrosolvatations-Leistungsüberprüfung - prägnanter auch Göbench („Göttingen Benchmark for Numerical Quantum Chemistry“) genannt - in einem ersten Schritt mit einem Doppelblindcharakter begonnen. Während die experimentellen Daten ohne Kenntnis der Ergebnisse der quantenchemischen Rechnungen in der SuHM-Gruppe aufgenommen und interpretiert wurden, waren Computerchemie-Forschungsgruppen dazu aufgerufen, die Gleichgewichtssituation zwischen C- und O-Heterodimer in den genannten MethanolFuran(derivat)-Kombinationen rechnerisch zu untersuchen und ihre Ergebnisse an den unabhängigen Koordinator (RICARDo MATA) einzureichen. Erst anschließend wurden die Ergebnisse zusammengeführt und gemeinsam ausgewertet. ${ }^{[75]}$

In einer zweiten Runde der Untersuchung wurden einige Daten ergänzt (z. B. zusätzliche Jet-FTIR-Spektren zur Verbesserung des Signal-zu-Rausch-Verhältnisses, detaillierter aufgeschlüsselte Daten aus den quantenchemischen Rechnungen) bzw. erneuert (Errata oder technische Verbesserungen aufseiten der quantenchemischen Rechnungen). Für diese überarbeiteten Methodenteile geht somit zwar der Doppelblindcharakter der ursprünglichen Studie ein Stück weit verloren, allerdings wird durch die zusätzlichen Daten eine wesentlich detailliertere Auswertung ermöglicht. Darüber hinaus wurden für diese zweite Göbench-Runde durch das Hinzufügen mikrowellenspektroskopischer Daten neue Informationen zu den Dimerstrukturen von $\mathrm{MeOH}$ mit Fu und DMFu eingebracht. ${ }^{[80]}$ In der vorliegenden Arbeit sollen diese Ergebnisse jedoch nicht explizit dargestellt und analysiert, wohl aber zur Vorauswahl der für den Vergleich zum Experiment herangezogenen quantenchemischen Methoden (s. unten) genutzt werden.

Wie in Kapitel 2.3 beschrieben, kann das aus Jet-FTIR-Spektren gewonnene relative Intensitätsverhältnis zweier verschiedener Aggregate zueinander über die BolTzMANN-Verteilung mit dem Energieunterschied zwischen den beiden Aggregaten verknüpft werden. Zwar ist letztendlich nur das Verhältnis $\Delta E^{0} / R T_{\mathrm{c}}$ unkompliziert im Experiment zugänglich, aber bereits dies kann hilfreich dazu sein, Grenzen von DFT- oder wellenfunktionsbasierten theoretischen Methoden ${ }^{[18,19,138,279-283]}$ aufzudecken. Mit einer realistischen Abschätzung für die Konformationstemperatur (s. Kap. 2.3) können die Ergebnisse aus der Jet-FTIR-Spektroskopie (Kap. 4.1.1) auch zum Benchmarking berechneter Energieunterschiede (Kap. 4.1.2) verwendet werden. Hierzu sind mehrere Herangehensweisen möglich (s. Kap. 2.3.2), von denen zwei im Folgenden für die Auswertung genutzt werden sollen (Kap. 4.1.3). Dabei kann die Last zu bestimmender Größen entweder mehr auf die Seite des Experiments (Bestimmung rein experimenteller Energiedifferenzen) oder mehr auf die Seite der Theorie (Bestimmung abgeleiteter Konformationstemperaturen unter Einbeziehung quantenchemisch berechneter Daten) gelegt werden. Die Leistungsfähigkeit der theoretischen Methoden hinsichtlich der Beschreibung der Schwingungsnullpunktsener- 
gie (engl. „zero-point vibrational energy“, ZPVE) soll durch die Betrachtung verschiedener Isotopologe (über Deuterierungseffekte und Isotopenaustauschtemperaturen) untersucht werden. Auch schwingungsspektroskopisch direkt observable Größen wie Bandenpositionen und Rotverschiebungen werden für den Benchmark berücksichtigt (Kap. 4.1.4).

Zur Überprüfung der mit linearer Jet-FTIR-Spektroskopie gewonnenen Daten z.B. auf technische Artefakte wäre die Einbindung weiterer spektroskopischer Techniken interessant. Die leistungsfähige UV/IR-Technik wird jedoch durch ultraschnelle Dynamiken in elektronisch angeregten Zuständen des Furans ${ }^{[284]}$ in ihrer Anwendbarkeit eingeschränkt. In warmen IR-spektroskopischen Gasphasenmessungen sind die Rotverschiebungen der $\mathrm{OH}$-Streckschwingungsbanden aufgrund thermischer Anregung der Wasserstoffbrückenbindung zu klein, um Dimer- von Monomerbanden abzugrenzen und die Banden sind zu breit, um unterschiedlich gebundene Isomere zu unterscheiden. Daten aus matrixisolationsspektroskopischen Messungen hingegen sind durch Wechselwirkungen mit den Matrixmolekülen verfälscht. ${ }^{[191]}$ Jet-RAMAN-Spektroskopie könnte eine höhere Empfindlichkeit auf C-gebundene Dimere aufweisen und bietet eine bessere räumliche Auflösung der Konzentrationsentwicklung entlang der Expansion, ${ }^{[188]}$ aber die Berechnung von RAMAN-Streuquerschnitten könnte zu einer geringeren Genauigkeit als für IR-Bandenstärken führen.

Mikrowellenspektroskopie ist als ergänzende experimentelle Technik äußerst attraktiv, da direkt strukturelle Informationen zu den vorliegenden Bindungssituationen erhalten ${ }^{[285]}$ und die Koexistenz von C- und O-gebundenen Dimeren in einer Überschallexpansion direkt nachgewiesen werden können. ${ }^{[187,286-289]}$ Eine quantitative Analyse der relativen Häufigkeit der Dimere ist allerdings anspruchsvoller als in Jet-FTIR-spektroskopischen Messungen. Aus diesem Grund wurden für die genauere Bestimmung der in der initialen Blindstudie ${ }^{[75]}$ der Furan-Mikrosolvatations-Untersuchung ermittelten O/CIntensitätsverhältnisse erneut Jet-FTIR-Spektren angefertigt. Völlig neue Daten zu den Dimerstrukturen konnten für $\mathrm{MeOH}$ mit Fu und DMFu dann durch mikrowellenspektroskopische ${ }^{[290]}$ Messungen gewonnen werden, die in der ScHNELL-Gruppe am Deutschen Elektronen-Synchrotron (DESY) in Hamburg durchgeführt wurden. Diese experimentellen Strukturdaten können ebenfalls zur Überprüfung der in den Theoriegruppen quantenchemisch berechneten Werte herangezogen werden. Das vorliegende Kapitel konzentriert sich, wie bereits erwähnt, jedoch auf die schwingungsspektroskopischen Aspekte des Benchmarks.

Eine kürzlich veröffentlichte $\mathrm{MeOH}-\mathrm{Fu}$-Studie ${ }^{[291]}$ kann kaum Erkenntnisse zum vorliegenden Benchmark beitragen. Die in der Studie verwendete Argon-Matrix beeinflusst die schwach gebundenen $\mathrm{MeOH}-\mathrm{Fu}-K o m p l e x e$, beispielsweise hinsichtlich ihrer relativen Häufigkeit. Zudem wurde der Vergleich von Theorie und Experiment auf Grundlage von nicht-dispersionskorrigierten DFT-Rechnungen durchgeführt, was zu einer systematischen Benachteiligung der stärker von Dispersion profitierenden C-Dimere führt. Die von den Autoren festgestellte Präferenz für das O-gebundene Dimer sollte somit noch einmal kritisch überprüft werden.

Während der aktuelle, systematische Vergleich experimenteller und theoretischer 
Tabelle 4.1: Experimentell vor (oben) und nach (unten) der aktuellen Furan-MikrosolvatationsStudie ${ }^{[49,75]}$ untersuchte XOH-Furan-Dimere. Die O/C-Bindungsstellenpräferenz ändert sich subtil mit dem Lösungsmittelmolekül (C(6) kennzeichnet den Benzolring; Fu, MFu und DMFu s. Fließtext). Tabelle zusammengestellt von A. Ровцотzкі. Hinweis zum Urheberrecht: „Reprinted from f. Chem. Phys. 2020, 152, 164303, ${ }^{[80]}$ with the permission of AIP Publishing...

\begin{tabular}{|c|c|c|c|}
\hline $\mathrm{XOH}$ & Furan & Referenz & Präferenz \\
\hline $\mathrm{HCOOH}$ & $\mathrm{Fu}$ & [292] & $\mathrm{O}^{a}$ \\
\hline $\mathrm{H}_{2} \mathrm{O}$ & 2,3-Benzofuran & [36] & \\
\hline $\mathrm{CH}_{3} \mathrm{OH}$ & 2,3-Benzofuran & {$[36,49]$} & $C(6)$ \\
\hline $\mathrm{CH}_{3} \mathrm{OH}$ & DMFu & {$[49,75]$} & $\mathrm{O}$ \\
\hline $\mathrm{CH}_{3} \mathrm{OD}$ & DMFu & {$[49,75]$} & $\mathrm{O}$ \\
\hline $\mathrm{CH}_{3} \mathrm{OH}$ & 2-t-Butylfuran & [31] & $\mathrm{C}$ \\
\hline $\mathrm{CH}_{3} \mathrm{OH}$ & MFu & [75] & $\mathrm{O}$ \\
\hline $\mathrm{CH}_{3} \mathrm{OD}$ & MFu & [75] & $\mathrm{O}$ \\
\hline $\mathrm{CH}_{3} \mathrm{OH}$ & $\mathrm{Fu}$ & [75] & $\mathrm{O}$ \\
\hline $\mathrm{CH}_{3} \mathrm{OD}$ & $\mathrm{Fu}$ & [75] & $\mathrm{O}$ \\
\hline $\mathrm{H}_{2} \mathrm{O}$ & $\mathrm{Fu}$ & [293] & $\mathrm{O}^{a}$ \\
\hline $\mathrm{D}_{2} \mathrm{O}$ & $\mathrm{Fu}$ & [293] & $\mathrm{O}^{a}$ \\
\hline HOD & $\mathrm{Fu}$ & [293] & $\mathrm{O}^{a}$ \\
\hline $\mathrm{CF}_{3} \mathrm{CH}_{2} \mathrm{OH}$ & $\mathrm{Fu}$ & [294] & $\mathrm{O}^{a}$ \\
\hline 1-Naphthol & $\mathrm{Fu}$ & {$[295,296]$} & - \\
\hline $\mathrm{CF}_{3} \mathrm{CH}_{2} \mathrm{OH}$ & $\mathrm{MFu}$ & [294] & $\mathrm{O}^{a}$ \\
\hline 1-Naphthol & DMFu & {$[295,296]$} & - \\
\hline $\mathrm{H}_{2} \mathrm{O}$ & Dibenzofuran & [239] & $\mathrm{O}$ \\
\hline $\mathrm{CH}_{3} \mathrm{OH}$ & Dibenzofuran & [239] & $C(6)$ \\
\hline$\left(\mathrm{CH}_{3}\right)_{3} \mathrm{OH}$ & Dibenzofuran & [239] & $C(6)$ \\
\hline$\left(\mathrm{CH}_{3}\right)_{3} \mathrm{OH}$ & 2-t-Butylfuran & [296] & $\mathrm{C}$ \\
\hline
\end{tabular}

${ }^{a}$ Daten basieren nicht auf kalten Gasphasenspektren 
Ergebnisse sich auf $\mathrm{MeOH}$ mit Fu, DMFu und mit Einschränkungen (s. Kap. 4.1.1) MFu konzentriert, soll der Benchmark in Zukunft auf weitere Systeme erweitert werden. Initiale Furanderivatstudien ${ }^{[36,49]}$ und die 2018 publizierten, ersten Ergebnisse der Furan-Mikrosolvatations-Untersuchung ${ }^{[75]}$ haben bereits neue Forschungsvorhaben ausgelöst. ${ }^{[239,293,294]}$ Diese und weitere Studien furanbasierter Systeme ${ }^{[269,282,292,295,296]}$ sind in Tab. 4.1 zusammengefasst.

\subsubsection{Experimentelle Ergebnisse}

Die Jet-FTIR-Spektren von Methanol (Sigma-Aldrich, $\geq 99.8 \%$ ) bzw. Methanol-d (euriso-Top, 99\% D, HDO $+\mathrm{D}_{2} \mathrm{O}<0.1 \%$ ) mit Furan (Alfa Aesar, 99\%, 250 ppm BHT), 2-Methylfuran (RоTн, $\geq 99 \%$ ) und 2,5-Dimethylfuran (Acros Organics, 99\%) wurden an der Filet-Jet-Apparatur ${ }^{[55]}$ (s. Kap. 2.2) aufgenommen. Als Trägergas kam Helium (Linde, 99.996\%) zum Einsatz und in einigen Fällen wurde Argon (Linde, 99.999\%) als Relaxationsförderer beigemischt. Für alle Donor-Akzeptor-Gemische wurden Spektren bei verschiedenen Konzentrationen angefertigt, wobei generell im Bereich von $\approx 0.1 \%$ gearbeitet wurde, um die Bildung von Trimeren und größeren Clustern zu vermeiden. Insbesondere Methanol musste in hoher Verdünnung eingesetzt werden, um die bevorzugte Selbstaggregation zu umgehen. Doch auch die Bildung gemischter Trimere mit zwei Furan(derivat)-Molekülen sollte unterbunden werden, da deren Signale mit den 1:1-Dimerbandensignalen aufeinander fallen und so die für die Auswertung wichtigen Intensitäten subtil verfälschen können. Eine zu hohe Verdünnung sorgt allerdings einerseits für schlechte Signal-zu-Rausch-Verhältnisse und führt damit zu größeren Fehlern im bestimmten Intensitätsverhältnis (s. Kap. 2.3.3) und kann andererseits auch zu Änderungen in Aggregations- und Kühlungsmechanismen und damit zu einer Reduktion von Lösungsmittelaustauschprozessen führen. ${ }^{[220,267]}$

Um zunächst einmal die Signale der gesuchten Methanol-Furan(derivat)-Heterodimere von denen höherer Cluster zu unterscheiden, wurden für jedes Furanderivat Konzentrationsreihen aufgenommen. Dabei wurden Donor- und Akzeptorkonzentration variiert und die relative Skalierung verschiedener Signale zueinander wurde beobachtet. Signale, die von Aggregaten mit mehr als einem Molekül des betrachteten Stoffes verursacht werden, sollten mit einer höheren Ordnung mit der Konzentration dieses Stoffes skalieren als 1:1-Dimere. Anhand der Konzentrationsreihen konnten Signale bei 3654 und $3636 \mathrm{~cm}^{-1}$ für $\mathrm{MeOH}+\mathrm{Fu}$, bei 3645, 3623 und (schwächer) bei $3617 \mathrm{~cm}^{-1}$ für $\mathrm{MeOH}+\mathrm{MFu}$, sowie bei 3636 und $3612 \mathrm{~cm}^{-1}$ für $\mathrm{MeOH}+\mathrm{DMFu}$ als 1:1-Dimersignale identifiziert werden, wobei die Signale mit der höchsten Wellenzahl jeweils auch die größte Intensität aufwiesen. Für $\mathrm{MeOH}+\mathrm{DMFu}$ bestätigt dies die Zuordnung der Signale zu 1:1-Heterodimeren in Ref. [49]. Die ähnliche Aufspaltung zwischen den beiden (bzw. im Fall von MFu den beiden intensiveren) Heterodimersignalen von $18(\mathrm{Fu}), 21(\mathrm{MFu})$ und $24 \mathrm{~cm}^{-1}$ (DMFu) und die jeweils größere Intensität des weniger rotverschobenen Signals sprechen für eine einheitliche Zuordnung der Signale über die verschiedenen Furanderivate hinweg.

Prinzipiell könnte es sich dabei für beide Signalgruppen (höhere und niedrigere Wel- 


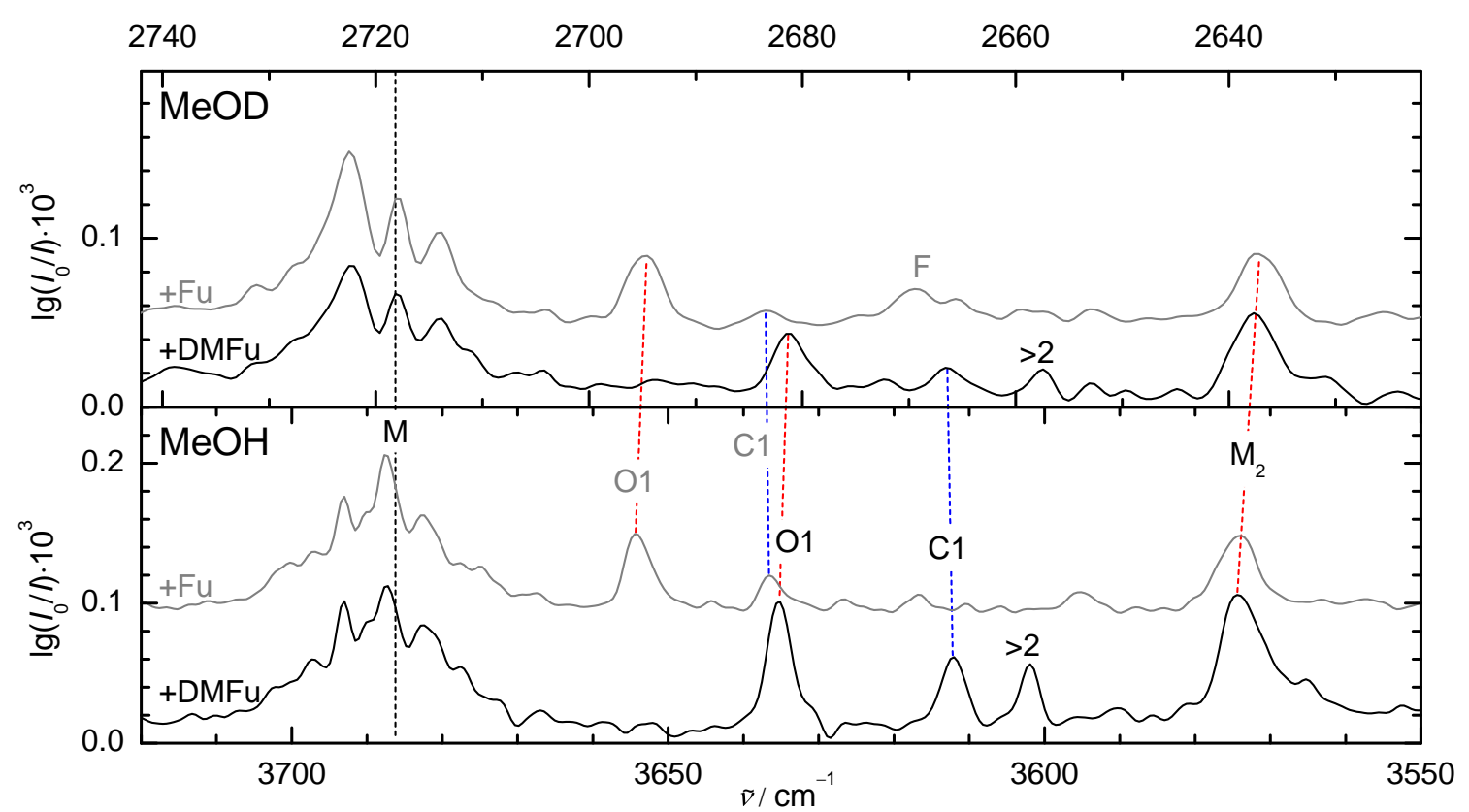

Abbildung 4.3: Spektren von $\mathrm{MeOD}$ (oben) bzw. $\mathrm{MeOH}$ (unten) mit Fu (grau) bzw. DMFu (schwarz).

lenzahlen) jeweils um O- oder C-gebundene Dimere handeln. Zwei C-gebundene Dimere widersprächen jedoch früheren Befunden für ähnliche Komplexe ${ }^{[36,37,49,187]}$ (s. auch Kap. 4.2). Außerdem wäre bei zwei C-Dimeren ein größerer Einfluss der zunehmenden Ringmethylierung von Fu bis DMFu auf das beobachtete Bandenmuster zu erwarten. Gleiches gilt für die für Fu durchgeführte Argon-Beimischung. Mit dieser wäre bei zwei durch eine flache Barriere getrennten C-Dimeren ein starker Relaxationseffekt ins stabilere der beiden Dimere zu erwarten, der jedoch nicht beobachtet wurde. Auch die große Aufspaltung von $18-24 \mathrm{~cm}^{-1}$ zwischen beiden Dimersignalen wäre für zwei C-gebundene Dimere, die an dasselbe $\pi$-System des jeweiligen Furanderivats gebunden sind, sehr ungewöhnlich.

Zwei O-gebundene Dimere sind schwerer auszuschließen, da hier Dimere mit unterschiedlichen $\mathrm{OH}$ ‥O-Wasserstoffbrückenbindungen (Op und Ot, s. Abb. 4.2 auf S. 100) möglich sind, die durchaus die große Aufspaltung zwischen den beobachteten Dimerbanden erklären könnten. Auch der relativ geringe Einfluss der Ringmethylierung auf das vorliegende Bandenmuster würde zu einer Beobachtung zweier O-Dimere passen. Angesichts der viermal so hohen Zahl an C- wie O-Atomen im Furan-Ring, dessen $\pi$-System im ausgedehnten Bereich über den C-Atomen eine attraktive Andockstelle für Wasserstoffbrückenbindungen darstellt, wäre das Fehlen von C-Dimeren allerdings durchaus überraschend. Entscheidende Daten für die Unterscheidung zwischen C- und O-gebundenen Dimeren liefern Spektren mit Methanol-OD (MeOD) als Donor.

In Abbildung 4.3 ist der $\mathrm{OH}$-Streckschwingungsbereich von Beispielspektren von Me- 
thanol (MeOH, unten) mit Furan (Fu, grau) und 2,5-Dimethylfuran (DMFu, schwarz) dargestellt und wird dem entsprechenden OD-Streckschwingungsbereich von Spektren mit MeOD (oben) als Donor gegenübergestellt. Dazu wurden die Bandenpositionen (M) des $\mathrm{MeOH}-$ Monomers bei $3686 \mathrm{~cm}^{-1[154,297]}$ und des MeOD-Monomers bei $2718 \mathrm{~cm}^{-1[154]}$ aufeinandergelegt und die Wellenzahlachse des OD-Bereichs wurde um den Faktor $\sqrt{2}$, dem idealen harmonischen Isotopeneffekt bei unendlicher Sauerstoffmasse und exaktem D/H-Massenverhältnis von 2, gestreckt. Zur Verbesserung der Darstellung wurden Spektren höherer Konzentration ausgewählt, wodurch sowohl das Methanol-Dimer $\left(\mathrm{M}_{2}\right)$ bei 2637 (MeOD) bzw. $3575 \mathrm{~cm}^{-1}(\mathrm{MeOH})$ als auch ein Signal eines größeren Aggregats $(>2)$ im Fall von DMFu (2658 bzw. $3602 \mathrm{~cm}^{-1}$ ) sichtbar werden. Im OD-Streckschwingungsbereich des Fu-Spektrums ist zudem eine mit „F“ beschriftete Bande sichtbar, die einer Kombinationsbande zweier $\mathrm{CH}$-Scherschwingungsmoden des Furans zugeordnet werden kann, ${ }^{[273]}$ jedoch nicht mit den untersuchten 1:1-Dimerbanden überlappt.

Durch die Skalierung der Wellenzahlskala des OD-Bereichs anhand des idealen harmonischen Isotopeneffekts werden anharmonische Einflüsse auf die Rotverschiebung der gemischten Dimerbanden sofort in der Abbildung sichtbar. In einer eindimensionalen Näherung werden Hydrid-Streckschwingungsbanden mit einer größeren Anharmonizität als der des isolierten Methanols in einer solchen Darstellung bei Deuterierung nach rechts verschoben, wie anhand des Methanol-Dimers ${ }^{[181]}$ deutlich wird (positive Steigung der gestrichelten Verbindungslinie zwischen den $\mathrm{M}_{2}$-Signalen). Banden mit einer geringeren Anharmonizität werden dahingegen bei Deuterierung mitunter sogar nach links verschoben, sodass die Verbindungslinie eine negative Steigung aufweist. ${ }^{[154]}$ Dies sollte für CDimere der Fall sein, die eine geringere Zunahme der diagonalen und außer-diagonalen Anharmonizitäten als O-Dimere verursachen, ${ }^{[50,185]}$ wie es für Benzofuran auch beobachtet wurde. ${ }^{[36,49]}$ Eine ergänzende Interpretation dieser Effekte ordnet $\mathrm{OH}$-Gruppen in $\mathrm{OH} \cdots \mathrm{O}$-Bindungen eine größere Librationssteifheit als in $\mathrm{OH} \cdots \mathrm{C}$-Bindungen zu. ${ }^{[50]} \mathrm{Die}$ resultierende Schwingungsnullpunktsenergie schwächt die Wasserstoffbrücke und ist für O-Dimere größer, bei Deuterierung aber um einen geringeren Betrag. Die Rotverschiebung nimmt bei Deuterierung für O-Dimere in Bezug zu C-Dimeren damit zu. In der beschriebenen Darstellungsart sollten O-gebundene Dimere also Verbindungslinien mit positiver Steigung (in Abb. 4.3 rot eingefärbt), C-Dimere dagegen Verbindungslinien mit negativer Steigung (blau eingefärbt) aufweisen. Zwar wären auch für Op- und Ot-Dimere unterschiedliche Anharmonizitäten vorstellbar, aber in diesem Fall sollte die weiter rotverschobene Bande mit der stärkeren, gerichteten Wasserstoffbrückenbindung $(\mathrm{Op})$ auch die größere Anharmonizität aufweisen. ${ }^{[4]}$ Die beobachteten Anharmonizitäten geben damit also subtile Hinweise auf die Zuordnung der beobachteten Heterodimersignale zu Ound C-gebundenen Dimeren. Sowohl für Fu als auch für DMFu sind jeweils ein O- (O1, also stabilstes O-gebundenes Dimer) und ein C-Dimer (C1) in den Spektren erkennbar, wobei die O-gebundenen Dimere jeweils die höhere Wellenzahl aufweisen. Die Zuordnung der Heterodimerbanden von $\mathrm{MeOH}$ und DMFu in Ref. [49] kann somit bestätigt werden.

Auf die gleiche Art und Weise können analog zu Fu und DMFu auch für MFu die bei- 


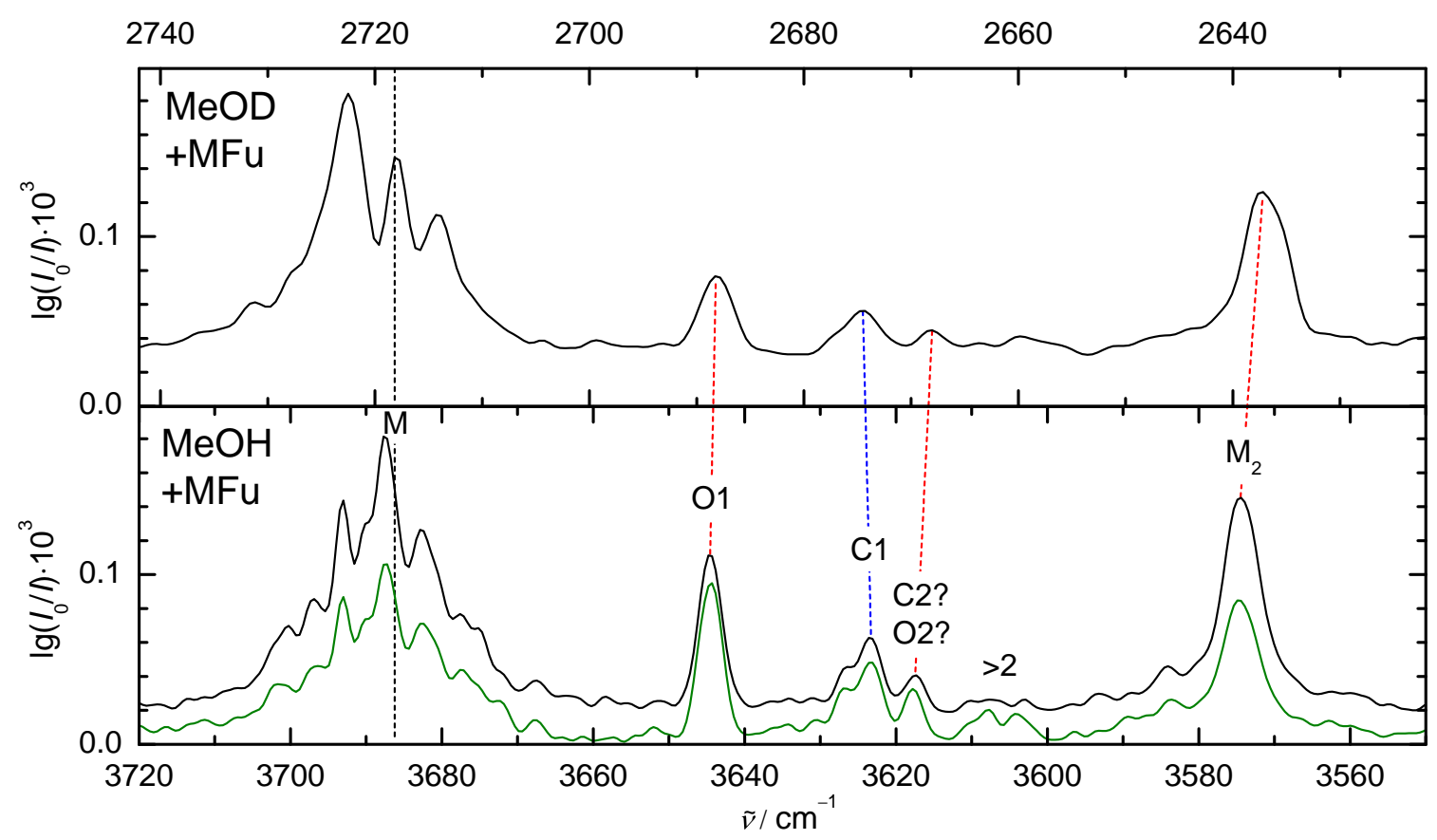

Abbildung 4.4: Spektren von MeOD (oben) bzw. MeOH (unten) mit MFu. Ein höher konzentriertes Spektrum (grün) ermöglicht die Zuordnung der Banden bei 3609 und $3604 \mathrm{~cm}^{-1}$ zu höheren Clustern ( $>2$ ). Bearbeitung von Fig. IR1 in Ref. [80] (lizenziert unter CC BY 4.0).

den intensiveren Heterodimerbanden dem O1- (weniger rotverschoben) und C1-Dimer (stärker rotverschoben) zugeordnet werden (s. Abb. 4.4). Wie die Abbildung verdeutlicht, zeigen sich hier jedoch zusätzliche Banden. Bei höherer Konzentration (grünes Spektrum) sichtbare Banden bei 3608 und $3604 \mathrm{~cm}^{-1}$ können aufgrund ihrer Konzentrationsskalierung zwar größeren Aggregaten zugeordnet werden $(>2)$, die weiter oben bereits erwähnte Bande bei $3617 \mathrm{~cm}^{-1}$ skaliert jedoch zumindest in Teilen wie ein Heterodimer und bleibt auch bei Deuterierung detektierbar. Während die beobachtete Anharmonizität eher auf ein zweites O-Dimer hindeutet (O2), kann aufgrund der beobachteten Rotverschiebung auch ein zweites C-Dimer (C2) nicht ausgeschlossen werden. Eine eindeutige Zuordnung ist somit nicht möglich. Die vor allem im MeOH-Spektrum sichtbare Schulter bei $3626 \mathrm{~cm}^{-1}$ gewinnt bei Argon-Beimischung an Intensität (nicht gezeigt) und kann ebenfalls nicht eindeutig zugeordnet werden.

Um die aufgezeichneten Jet-FTIR-spektroskopischen Daten für das Benchmarking des berechneten Energieunterschieds zwischen dem stabilsten O-Dimer O1 und dem stabilsten C-Dimer C1 zu nutzen, muss das Intensitätsverhältnis der O1- und C1-Dimerbanden zueinander ermittelt werden (s. Kap. 2.3). Wie in Kapitel 2.3.3 beschrieben, werden dazu für jede Donor-Akzeptor-Kombination mehrere Spektren herangezogen, die wiederum jeweils aus 100-1000 Spektrometerscans bestehen. In allen Fällen wurde dabei ein Teil der Spektren nach Kenntnisnahme der Ergebnisse der quantenchemischen Rechnungen 
Tabelle 4.2: Experimentell bestimmte Intensitätsverhältnisse der Komplexe von Methanol mit Fu, MFu und DMFu. Die in Klammern angegebenen Fehlerintervalle wurden mit dem in Kapitel 2.3.3 beschriebenen Verfahren ermittelt. Bearbeiteter Ausschnitt aus Tab. IV in Ref. [80] (lizenziert unter CC BY 4.0).

\begin{tabular}{lllr}
\hline Intensitätsverhältnis & Fu & MFu & DMFu \\
\hline$I_{\mathrm{O}} / I_{\mathrm{C}}(\mathrm{OH})$ & $2.3(3)$ & $>1.5(1)$ & $2.0(1)$ \\
$I_{\mathrm{O}} / I_{\mathrm{C}}(\mathrm{OD})$ & $3.7(8)$ & $>1.4(2)$ & $2.4(2)$ \\
\hline
\end{tabular}

(s. Kap. 4.1.2) aufgenommen, sodass der ursprünglich vorgesehene Doppelblindcharakter strenggenommen reduziert ist. Die zuvor vorgestellte Interpretation der Spektren erfolgte allerdings jeweils ohne Kenntnis der quantenchemischen Daten.

Für jedes Spektrum werden das Intensitätsverhältnis und sein Fehler bestimmt und aus diesen Einzelwerten wird dann ein mit der inversen Varianz gewichteter Mittelwert gebildet, der für die weiteren Benchmarks genutzt werden kann. Aufgrund der zusätzlichen, nicht eindeutig zuordenbaren Banden kann im Fall von MFu keine vollständig quantitative Auswertung erfolgen. Für die Integration der Bandenintensitäten werden sowohl die Schulter bei $3627 \mathrm{~cm}^{-1}$ als auch die Bande bei $3617 \mathrm{~cm}^{-1}$ in das Integral des C1-Peaks einbezogen, sodass sich eine untere Grenze für das O/C-Intensitätsverhältnis ergibt.

Die erhaltenen Ergebnisse für die experimentellen Intensitätsverhältnisse $I_{\mathrm{O}} / I_{\mathrm{C}}$ für Me$\mathrm{OH}$ und MeOD als Donor sind in Tabelle 4.2 zusammengefasst. Da für MFu wie beschrieben nur untere Grenzwerte ermittelt werden können, sind die Werte mit einem „> “ versehen. Wie in den Spektren bereits optisch erkennbar ist, weist die weniger rotverschobene Bande, die dem O-Dimer zugeordnet wurde, die größere Intensität auf. Bei Methylierung des Furan-Rings nimmt die relative Intensität der C-Bande von Fu zu DMFu zu, wobei dieser Trend im Rahmen der ermittelten Fehlerbereiche nur für MeOD als Donor signifikant ist. Beim Vergleich der Donoren zeigt sich, dass für die beiden quantitativ auswertbaren Akzeptoren Fu und DMFu das Intensitätsverhältnis bei Austausch von $\mathrm{MeOH}$ mit $\mathrm{MeOD}$ jeweils signifikant größer wird. Dies bestätigt noch einmal eindeutig die getroffene Bandenzuordnung. Wie weiter oben bereits erläutert, ist die ZPVE für O-Dimere größer als für C-Dimere. Dieser Unterschied ist mit Deuterium in der Wasserstoffbrücke weniger ausgeprägt als mit Protium, sodass die relative Stabilität des O-Dimers und damit auch das O-zu-C-Intensitätsverhältnis bei Deuterierung zunehmen.

Zusätzlich zu den Jet-FTIR-Messungen, aus denen neben den Daten zu den Intensitätsverhältnissen auch die Wellenzahlen und Rotverschiebungen der Dimerbanden zum Benchmarking genutzt werden können, werden auch Mikrowellen-(MW-)spektroskopische Ergebnisse für die Furan-Mikrosolvatations-Untersuchung herangezogen. So können experimentelle Informationen zur Struktur der beobachteten Heterodimere gewonnen werden. Die MW-Spektroskopie wurde von der ScHNELL-Gruppe am DESY in Hamburg für $\mathrm{MeOH}$ mit Fu und DMFu an einem Chirped-Pulse-FourIER-Transform-Mikrowellen- (CP-FTMW-)Aufbau ${ }^{[290,298,299]}$ durchgeführt. 
Für Fu konnten dabei ein dominierendes $\mathrm{O}$ - und ein in geringerer Menge vorkommendes C-Dimer in der Expansion beobachtet werden, sodass der IR-spektroskopische Befund bestätigt wird. Durch Vergleich der spektralen Daten mit den Ergebnissen der für die Göbench-Untersuchung eingereichten quantenchemischen Rechnungen (s. Kap. 4.1.2) können die beobachteten Dimere eindeutig Ot- und Ct-Strukturen zugeordnet werden. Das Vorkommen signifikanter Mengen von Op-Dimer kann dabei ausgeschlossen werden, da es aufgrund einer größeren $A$-Rotationskonstante spektral gut sichtbar sein müsste, jedoch nicht nachgewiesen werden konnte. Die MW-Spektroskopie ermöglicht somit eine eindeutige Identifizierung der experimentell vorliegenden O-Dimerstruktur, sodass hieran eine Selektion der eingereichten Rechendaten erfolgen kann: Rechenmethoden, die für $\mathrm{MeOH}+\mathrm{Fu}$ die Stabilität des Op-Dimers höher einstufen als die des Ot-Dimers, sind nicht mit den experimentellen Befunden kompatibel und werden somit in den späteren quantitativen Untersuchungen (Kap. 4.1.3, 4.1.4) nicht weiter berücksichtigt.

Durch Verwendung von ${ }^{13} \mathrm{C}$-isotopenmarkiertem Furan konnten weiterhin Hinweise auf die Symmetrie der MeOH-Fu-Dimere gewonnen werden. Während das Ct-Dimer $C_{1^{-}}$ Symmetrie aufweist, wurde für das Ot-Dimer im Experiment eine effektive $C_{s}$-Symmetrie festgestellt. Nahezu alle quantenchemischen Rechnungen liefern jedoch mehr oder weniger leicht asymmetrische Ot-Strukturen. Diese Abweichung könnte mehrere Ursachen haben. Quantenchemische Rechnungen liefern Strukturen im elektronischen Potentialminimum. Im vorliegenden Fall könnte z. B. ein Potential mit einer Doppelmulde vorliegen, das zwei enantiomere Minima aufweist, die von einem nur wenig höherliegenden, $C_{s}$-symmetrischen Übergangszustand getrennt werden. Liegt die Umwandlungsbarriere unterhalb der Nullpunktsenergie in diesem Freiheitsgrad, wäre im Experiment die höchste Aufenthaltswahrscheinlichkeit unter Umständen bei der $C_{s}$-symmetrischen Struktur $\mathrm{zu}$ beobachten. Ist die Barriere nur etwas höher als die Schwingungsnullpunktsenergie, könnte sich auf der Zeitskala des Experiments immer noch effektive $C_{s}$-Symmetrie einstellen. Dies gilt insbesondere, da die Übergänge zwischen einer exakt symmetrischen und leicht unsymmetrischen Strukturen fließend und damit nur schwer zu beobachten sind. Effektive experimentelle $C_{s}$-Symmetrie widerspricht also nicht unbedingt quantenchemisch vorhergesagten $C_{1}$-symmetrischen Strukturen, wie die Beispiele vergleichbarer, quasi- $C_{S}$-symmetrischer Moleküle wie 2-Oxazolin ${ }^{[300]}$, Oxetan ${ }^{[301]}$ oder auch des WasserPentamers ${ }^{[302]}$ zeigen. Sogar extrem flexible Moleküle können bei tiefen Temperaturen recht reguläre Spektren aufweisen. ${ }^{[303]}$

Für MeOH+DMFu konnte im Gegensatz zur FTIR- in der MW-Spektroskopie nur das O-gebundene Dimer nachgewiesen werden. Dies bestätigt den FTIR-spektroskopischen Befund, dass das O-Dimer stabiler als das C-Dimer ist. Auch für DMFu konnte die Geometrie des beobachteten Dimers der Ot-Struktur zugeordnet werden, sodass auch hier ein qualitatives Merkmal für den Benchmark gewonnen wurde. Die sich durch die drei Methyl-Rotoren ergebende, reichhaltige Feinstruktur des MW-Spektrums erschwert die Bestimmung der Symmetrie des Komplexes. Auf Grundlage einer Analyse der internen Dynamiken und der Dipolmomentkomponenten ergeben sich jedoch auch hier Hinweise auf eine effektive $C_{s}$-Symmetrie auf der Zeitskala des Experiments. Gut die Hälfte der im 
Benchmark untersuchten quantenchemischen Methoden liefert für das MeOH-DMFu-OtDimer $C_{s}$-symmetrische Strukturen, doch auch hier ist eine vorhergesagte $C_{1}$-Symmetrie aus den oben genannten Gründen durchaus mit den experimentellen Befunden vereinbar.

\subsubsection{Quantenchemische Rechnungen}

Insgesamt wurden von 8 verschiedenen Gruppen Beiträge zur Göbench-Leistungsüberprüfung eingereicht. Da einige Gruppen mehrere Beiträge beigesteuert haben, liegen insgesamt 11 verschiedene Methoden für die Untersuchung vor, die mit den Buchstaben A bis K indiziert wurden (s. „Supplementary Material (TH)“von Ref. [80]). Methode J basiert dabei allerdings auf Methode I und liefert im Wesentlichen nur eine bessere Struktur für $\mathrm{MeOH}+\mathrm{Fu}$ und wird deshalb im Folgenden nicht weiter berücksichtigt. Einige Methoden verwenden anharmonische Korrekturen für die Berechnung der Schwingungsnullpunktsenergie. Diese Methoden werden mit einem „" "gekennzeichnet, wohingegen harmonische ZPVE-Berechnungen nicht gesondert markiert werden. Für alle Methoden werden auch die harmonischen Schwingungsnullpunktsenergien für die Auswertung genutzt, sodass A, C, D, F und K mit je zwei Einträgen im Göbench vertreten sind.

Zusammenfassend werden also folgende Beiträge der jeweils genannten Forschenden für den Vergleich mit den experimentellen Daten herangezogen:

- A, $\mathbf{A}^{*}$, B: Beiträge von M. N. Pereira und L. Baptista.

- C, $\mathbf{C}^{*}$ : Beiträge von D. M. Benoit und I. S. Ulusoy.

- D, D*: Beiträge von R. Dahmani, H. Mouhib, M. Prakash, M. Mogren Al-Mogren und M. Hochlaf.

- E: Beitrag von M. Krasowska, G. Bistoni, A. A. Auer und F. Neese.

- F, F* : Beiträge von F. Bohle, A. Hansen, J. Antony und S. Grimme.

- G, H: Beiträge von G. JANSEN.

- I: Beitrag von M. E. Harding, C. Holzer und W. Klopper.

- K, K*: Beiträge von D. Firaha, W. A. Kopp, L. C. Kröger und K. Leonhard.

Die für die einzelnen Beiträge verwendeten Theoriemethoden sind in Tabelle $4.3 \mathrm{zu}-$ sammengefasst. Nahezu alle Beiträge kombinieren verschiedene Theorieniveaus für die Geometrieoptimierung und Berechnung der ZPVE einerseits und die Berechnung der elektronischen Energie andererseits. Für Ersteres wird überwiegend auf dispersionskorrigierte ${ }^{[83]}$ Hybrid-Dichtefunktionale zurückgegriffen. Der Beitrag $\mathbf{A}\left({ }^{*}\right)$ setzt hingegen auf das B3LYP-Funktional ${ }^{[85,86]}$ ohne Dispersionskorrektur und zwei weitere Beiträge verwenden wellenfunktionsbasierte Methoden (SCS(1.1;0.66)-MP2 ${ }^{[304]}$ in $\mathbf{F}\left(^{*}\right)$; SCS-CC2 ${ }^{[305]}$ in I). Für die Berechnung der elektronischen Energie kommen überwiegend $\operatorname{CCSD}(\mathrm{T})$ - 
basierte Methoden (Beiträge $\mathbf{C}-\mathbf{F}, \mathbf{I}, \mathbf{K})$ zum Einsatz, am häufigsten in Form der lokalen DLPNO-CCSD(T)-Methode ${ }^{[122-125]}$ (Beiträge $\left.\mathbf{C}\left(^{*}\right), \mathbf{E}, \mathbf{K}\left({ }^{*}\right)\right)$ und in den anderen Fällen in verschiedenen explizit korrelierten $\operatorname{CCSD}(\mathrm{T})$-Varianten $\left(\operatorname{CCSD}(\mathrm{T})-\mathrm{F} 12^{[141,306,307]}\right.$ in $\mathbf{D}\left(^{*}\right)$; W2-F12 ${ }^{[308]}$ in $\left.\mathbf{F}^{*}\right)$, CCSD(T) in Kombination mit dem CABS-Ansatz ${ }^{[309,310]}$ in I). Die verbleibenden Beiträge nutzen DFT-basierte Methoden zur Berechnung der elektronischen Energien. In $\mathbf{A}\left(^{*}\right)$ wird dabei die CBS-QB3-Methode ${ }^{[311,312]}$ und in $\mathbf{B}$ das $\omega$ B97xD-Funktional $^{[313]}$ verwendet, wohingegen in $\mathbf{G}$ und $\mathbf{H}$ jeweils SAPT ${ }^{[17-19]}$ eingesetzt wird. Für die Berechnung der ZPVE werden neben der doppelt harmonischen Näherung (harmonischer Oszillator, HO) in einigen Fällen, wie bereits erwähnt, auch anharmonische Korrekturen implementiert. Hierbei wird in den Beiträgen $\mathbf{A}^{*}$ und $\mathbf{D}^{*}$ die VPT2-Methode ${ }^{[314-316]}$ (vibrational second-order perturbation theory, Schwingungs-Störungstheorie zweiter Ordnung) und in Beitrag $C^{*}$ die VCI-Methode ${ }^{[317]}$ (Vibrational-Configuration-Interaction-Methode) verwendet. Letztere wird in Beitrag $\mathbf{C}^{*}$ jedoch nur auf die OH-Streckschwingung angewandt, wohingegen alle weiteren Schwingungen in der harmonischen Näherung berechnet werden. In Beitrag $\mathbf{F}^{*}$ wird ein Skalierungsfaktor über einen auf einem niedrigeren Niveau berechneten anharmonischen VPT2-Modell-Hamilton-Operator bestimmt (anharmonische Skalierung, AS) und in Beitrag $\mathbf{K}^{*}$ kommt das HR-Modell ${ }^{[318]}$ (hindered rotor, gehinderter Rotor) zum Einsatz. Details zu den verwendeten Rechenmethoden sind im „Supplementary Material (TH)“ von Ref. [80] zu finden.

Im Vergleich zur ursprünglichen Doppelblindstudie ${ }^{[75]}$ wurden für die im Folgenden dargestellte Auswertung, wie bereits erwähnt, mehrere der Beiträge überarbeitet oder ergänzt. Für Beitrag A hat sich der Energieunterschied $E^{0}(\mathrm{O})-E^{0}(\mathrm{C})$ für DMFu aufgrund eines Erratums geändert. Während in Beitrag $\mathbf{C}^{*}$ ursprünglich alle Schwingungsmoden anharmonisch korrigiert wurden, kommt nun ein gemischter harmonischer/anharmonischer Ansatz zum Einsatz (s. oben). In Beitrag D werden nun alle Schwingungsmoden zur Berechnung der ZPVE herangezogen, während zuvor nur die intermolekularen Moden berücksichtigt wurden. Beitrag $\mathbf{E}$ verwendet nun einen verbesserten Algorithmus für die Berechnung der störungstheoretischen Triples-Korrektur und einen anderen Basissatz und lokalisiert eine etwas stabilere Ot-Struktur für Fu. Bei den ursprünglich von den Beiträgen $\mathbf{G}$ und $\mathbf{H}$ gemeldeten $C_{s}$-symmetrischen Ot-Strukturen für $\mathrm{MeOH}-\mathrm{Fu}$ handelt es sich um Sattelpunkte, die nun durch etwas stabilere, unsymmetrische Minimumsstrukturen ersetzt wurden. Diese wurden durch Reoptimierungen nach Auslenkung entlang der imaginären Moden der originalen Strukturen erhalten. Streng genommen reduzieren diese Änderungen somit den Doppelblindcharakter der ursprünglichen Studie ${ }^{[75]}$ für die genannten Methoden $\left(\mathbf{C}^{*}, \mathbf{D}, \mathbf{E}, \mathbf{G}\right.$ und $\left.\mathbf{H}\right)$.

Für jeden Beitrag konnten Daten für die bis zu zwei jeweils energetisch tiefstliegenden $\mathrm{C}-(\mathrm{C} 1, \mathrm{C} 2)$ und O-gebundenen $(\mathrm{O} 1, \mathrm{O} 2)$ Methanol-Furan(derivat)-Dimere eingereicht werden (s. „Supplementary Material (TH)“ von Ref. [80]). Weiterhin konnte ein beliebiges weiteres gemischtes Dimer (C3/O3/...) berücksichtigt werden, etwa ein bei Verwendung von MeOD besonders stabiles Dimer. Für jedes der Dimere wurden relative elektronische Energien $\Delta E^{\mathrm{el}}$ und die harmonisch bzw. optional anharmonisch schwingungsnullpunktskorrigierten Energien $\Delta E_{\mathrm{h}}^{0}$ bzw. $\Delta E_{\text {anh }}^{0}$ abgefragt. Für den Vergleich mit MW- 
Tabelle 4.3: Für die zweite Runde der Göbench-Leistungsüberprüfung eingereichte schwingungsnullpunktskorrigierte Energiedifferenzen $E^{0}(\mathrm{O})-E^{0}(\mathrm{C})\left(\mathrm{in} \mathrm{kJ} \cdot \mathrm{mol}^{-1}\right)$ der Komplexe aus Me$\mathrm{OH}$ (obere Tabelle) bzw. MeOD (untere Tabelle) mit Fu und DMFu. Die Differenz wurde jeweils aus den stabilsten O- und C-Strukturen gebildet. Ist eine dieser Strukturen qualitativ nicht mit den experimentellen Befunden vereinbar, ist dies durch „,-“ gekennzeichnet. Die Art der Berechnung der Schwingungsnullpunktsenergie ist über die Kürzel „HO“ (harmonischer Oszillator), „VPT2“ (Schwingungs-Störungstheorie zweiter Ordnung), „VCI“ (Vibrational-configuration-interaction-Methode), „AS“ (anharmonische Skalierung) und „HR“ (gehinderter Rotor) angegeben. Einträge, die dabei anharmonische Korrekturen verwenden, sind mit einem „" " gekennzeichnet. Für jede Donor-Akzeptor-Kombination sind zwei experimentelle Schätzungen für die Energiedifferenz angegeben (s. Kap. 4.1.3), die unterschiedliche Symmetriefälle berücksichtigen. Dabei wird im einen Fall $C_{1}$-Symmetrie für O- und C-Struktur angenommen und im anderen Fall $C_{1}$-Symmetrie für die C- in Verbindung mit $C_{s}$-Symmetrie für die O-Struktur. Um Rundungsfehler zu vermeiden, sind alle Energiewerte unabhängig von ihrer Signifikanz mit zwei Nachkommastellen angegeben. Hinweis zum Urheberrecht: „Reprinted from F. Chem. Phys. 2020, 152, 164303, ${ }^{[80]}$ with the permission of AIP Publishing...

\begin{tabular}{|c|c|c|c|c|c|}
\hline \multirow{2}{*}{$\begin{array}{l}\text { MeOH } \\
\text { Eintrag }\end{array}$} & \multirow[b]{2}{*}{ Optimierung } & \multirow[b]{2}{*}{ ZPVE } & \multirow[b]{2}{*}{ Energie } & $+\mathbf{F u}$ & +DMFu \\
\hline & & & & \multicolumn{2}{|c|}{$E^{0}(\mathrm{O})-E^{0}(\mathrm{C})$} \\
\hline A & B3LYP/CBSB7 & $\mathrm{HO}$ & CBS-QB3 & - & 1.50 \\
\hline$A^{*}$ & B3LYP/CBSB7 & VPT2 & CBS-Q̄B3 & - & 1.23 \\
\hline B & $\omega \mathrm{B} 97 \times \mathrm{x} / 6-311 \mathrm{G}(\mathrm{df}, \mathrm{pd})$ & $\mathrm{HO}$ & $\omega \mathrm{B} 97 \mathrm{xD} / 6-311 \mathrm{G}(\mathrm{df}, \mathrm{pd})$ & 0.77 & -1.32 \\
\hline $\mathbf{C}$ & B3LYP-D3/aug-cc-pVTZ & $\mathrm{HO}$ & DLPNO-CCSD(T)/aug-ano-pV5Z & 0.33 & -1.31 \\
\hline $\mathbf{C}^{*}$ & B3LYP-D3/aug-cc-pVTZ & $\mathrm{HO} / \mathrm{VCI}$ & DLPNO-CCSD(T)/aug-ano-pV5Z & 0.41 & -1.18 \\
\hline D & PBE0-D3/aug-cc-pVTZ & $\mathrm{HO}$ & CCSD(T)-F12/aug-cc-pVTZ & 0.01 & -0.72 \\
\hline $\mathbf{D}^{*}$ & PBE0-D3/aug-cc-pVTZ & VPT2 & CCSD(T)-F12/aug-cc-pVTZ & 1.17 & -0.23 \\
\hline $\mathbf{E}$ & B3LYP-D3/def2-QZVPPD & $\mathrm{HO}$ & DLPNO-CCSD(T)/aug-cc-pwCV5Z & -0.30 & -1.06 \\
\hline $\mathbf{F}$ & SCS(1.1;0.66)-MP2/aug-cc-pVQZ & $\mathrm{HO}$ & W2-F12 & 0.20 & -1.22 \\
\hline $\mathbf{F}^{*}$ & SCS(1.1;0.66)-MP2/aug-cc-pVQZ & AS & W2-F12 & 0.56 & -2.17 \\
\hline G & PBE0-D3/QZVG & $\mathrm{HO}$ & DFT-SAPT(PBE0AC)/CBS[3:4] & 1.15 & 1.05 \\
\hline $\mathbf{H}$ & B3LYP-D3/QZVG & $\mathrm{HO}$ & DFT-SAPT(B3LYPAC)/CBS[3:4] & 0.53 & 0.25 \\
\hline $\mathbf{I}$ & SCS-CC2/def2-TZVP & $\mathrm{HO}$ & $\operatorname{CCSD}(\mathrm{T}) / \mathrm{cc}-\mathrm{pV}[\mathrm{T} / \mathrm{Q}] \mathrm{Z}-\mathrm{F} 12$ & -0.03 & -0.80 \\
\hline $\mathbf{K}$ & B2PLYP-D3/6-311++g(d,p) & $\mathrm{HO}$ & DLPNO-CCSD(T)/CBS[3:4] & - & -0.73 \\
\hline $\mathbf{K}^{*}$ & B2PLYP-D3/6-311++g(d,p) & $\mathrm{HR}$ & DLPNO-CCSD(T)/CBS[3:4] & -0.04 & -0.84 \\
\hline \multicolumn{4}{|c|}{$\begin{array}{l}\text { exp. Abschätzung (C- und O-Strukturen } C_{1} \text {-symmetrisch) } \\
\text { exp. Abschätzung (C-Struktur } C_{1} \text {-, O-Struktur } C_{s} \text {-symmetrisch) }\end{array}$} & $\begin{array}{l}-0.42 \pm 0.58 \\
-0.77 \pm 0.81\end{array}$ & $\begin{array}{l}-0.34 \pm 0.50 \\
-0.69 \pm 0.73\end{array}$ \\
\hline MeOD & & & & $+\mathbf{F u}$ & +DMFu \\
\hline Eintrag & Optimierung & ZPVE & Energie & \multicolumn{2}{|c|}{$E^{0}(\mathrm{O})-\overline{E^{0}(\mathrm{C})}$} \\
\hline $\mathrm{C}$ & B3LYP-D3/aug-cc-pVTZ & $\mathrm{HO}$ & DLPNO-CCSD(T)/aug-ano-pV5Z & 0.13 & \\
\hline $\mathrm{C}^{*}$ & B3LYP-D3/aug-cc-pVTZ & $\mathrm{HO} / \mathrm{VCI}$ & DLPNO-CCSD(T)/aug-ano-pV5Z & 0.11 & \\
\hline $\mathbf{E}$ & B3LYP-D3/def2-QZVPPD & $\mathrm{HO}$ & DLPNO-CCSD(T)/aug-cc-pwCV5Z & -0.51 & -1.32 \\
\hline $\mathbf{F}$ & SCS(1.1;0.66)-MP2/aug-cc-pVQZ & $\mathrm{HO}$ & $\mathrm{W} 2-\mathrm{F} 12$ & -0.05 & -1.45 \\
\hline $\mathbf{F}^{*}$ & SCS(1.1;0.66)-MP2/aug-cc-pVQZ & AS & W2-F12 & 0.27 & -2.43 \\
\hline G & PBE0-D3/QZVG & $\mathrm{HO}$ & DFT-SAPT(PBE0AC)/CBS[3:4] & 0.96 & 0.80 \\
\hline $\mathbf{H}$ & B3LYP-D3/QZVG & $\mathrm{HO}$ & DFT-SAPT(B3LYPAC)/CBS[3:4] & 0.31 & 0.00 \\
\hline I & SCS-CC2/def2-TZVP & $\mathrm{HO}$ & $\mathrm{CCSD}(\mathrm{T}) / \mathrm{cc}-\mathrm{pV}[\mathrm{T} / \mathrm{Q}] \mathrm{Z}-\mathrm{F} 12$ & -0.23 & -0.99 \\
\hline $\mathbf{K}$ & B2PLYP-D3/6-311++g(d,p) & $\mathrm{HO}$ & DLPNO-CCSD(T)/CBS[3:4] & -0.46 & -0.96 \\
\hline $\mathbf{K}^{*}$ & B2PLYP-D3/6-311++g(d,p) & HR & DLPNO-CCSD(T)/CBS[3:4] & -0.27 & -1.06 \\
\hline \multicolumn{4}{|c|}{$\begin{array}{l}\text { exp. Abschätzung (C- und O-Strukturen } C_{1} \text {-symmetrisch) } \\
\text { exp. Abschätzung (C-Struktur } C_{1}-, \text { O-Struktur } C_{S} \text {-symmetrisch) }\end{array}$} & $\begin{array}{l}-0.66 \pm 0.79 \\
-1.01 \pm 1.02\end{array}$ & $\begin{array}{l}-0.44 \pm 0.58 \\
-0.79 \pm 0.81\end{array}$ \\
\hline
\end{tabular}


Spektroskopie waren weiterhin Rotationskonstanten im elektronischen Potentialminimum $\left((A, B, C)_{\mathrm{el}}\right)$ bzw. optional nach anharmonischer Schwingungsnullpunktsmittelung $\left((A, B, C)_{0}\right)$ und ergänzend dazu die Dipolmomentkomponenten $\mu_{(A, B, C), \mathrm{el} / 0}$ anzugeben. Entsprechend wurden für den Vergleich mit den Ergebnissen aus der IR-spektroskopischen Untersuchung die harmonischen $\left(\omega_{\mathrm{OH}}\right)$ bzw. optional anharmonischen $\left(\tilde{v}_{\mathrm{OH}}\right)$ Donor-OH-Streckschwingungswellenzahlen und die zugehörigen IR-Bandenstärken $\left(A_{\mathrm{OH}}^{(\mathrm{anh})}\right)$ des jeweiligen Dimers sowie des Methanol-Monomers abgefragt. Um Hinweise auf die Starrheit bzw. den Übergangszustandscharakter des jeweiligen Dimers zu erhalten, war zusätzlich jeweils die tiefstliegende Schwingungswellenzahl $\left((\omega, \tilde{v})_{\text {low }}\right)$ anzugeben. Alle Größen, die sich bei Isotopenaustausch des Hydroxyl- ${ }^{1} \mathrm{H}-$ Wasserstoffatoms des Methanols mit Deuterium ändern, konnten auch für diesen MeOD-Fall angegeben werden. Die als optionale Angabe ebenfalls abgefragten Umwandlungsbarrieren $E_{B, \text { el }}$ für die Umwandlung zwischen zwei Dimeren wurden in keinem Fall ausgewiesen. Als letzte Größe wurde die relative Entartung zwischen den eingetragenen Dimeren abgefragt. Die hier genannten Werte konnten jedoch mehrfach anhand der ebenfalls jeweils mitgelieferten DimerStrukturen (im $x y z$-Dateiformat) nicht nachvollzogen werden, sodass die relative Entartung anhand der Symmetrie der Strukturen im Rahmen der Auswertung neu bestimmt wurde (s. Tab. 4.4). Dabei wurde in einigen Fällen für die O-gebundenen Strukturen $C_{s}$ Symmetrie erkannt, wohingegen die C-Dimere in allen Fällen $C_{1}$-Symmetrie aufweisen. Somit ergeben sich für mehrere Beiträge in jeweils ein oder zwei Donor-Akzeptor-Kombinationen relative Entartungen $g_{\mathrm{O}} / g_{\mathrm{C}}$ von 0.5 .

Der Vergleich der in den verschiedenen Beiträgen erhaltenen, berechneten Werte mit den experimentellen Ergebnissen kann im Fall von MFu infolge der unklaren IR-spektroskopischen Befunde (s. Kap. 4.1.1) und damit wegen experimenteller Einschränkungen nicht erfolgen. Im Gegensatz dazu können für Fu und DMFu einige theoretische Beiträge aufgrund qualitativer Abweichungen von den experimentellen Ergebnissen vom quantitativen Vergleich ausgeschlossen werden. Die schwingungsnullpunktskorrigierten Energieunterschiede $\Delta E^{0}=E^{0}(\mathrm{O})-E^{0}(\mathrm{C})$ für $\mathrm{MeOH}$ (oberer Teil) bzw. MeOD (unterer Teil) mit Fu und DMFu sind in Tabelle 4.3 dargestellt. Zur Berechnung des Energieunterschieds wurden die jeweils unter Berücksichtigung der ZPVE stabilsten C- und O-gebundenen Dimere verwendet. Liegen für eine Donor-Akzeptor-Kombination keine Daten vor, so ist dies durch ein leeres Feld gekennzeichnet. Ergebnisse, die qualitativ hinsichtlich der bevorzugten Struktur bei Bindung an eine gegebene Atomsorte ( $\mathrm{C}$ oder $\mathrm{O}$ ) nicht mit den experimentellen Ergebnissen vereinbar sind, sind durch einen Strich „-' ersetzt. Solche qualitativ inkompatiblen Ergebnisse liegen vor, wenn durch die Theorie kein C-Dimer $\left(\mathbf{A}, \mathbf{A}^{*}\right)$ oder die Op- anstelle der MW-bestätigten Ot-Struktur als das stabilere O-Dimer für $\mathrm{MeOH}+\mathrm{Fu}\left(\mathbf{A}, \mathbf{A}^{*}, \mathbf{C}^{*}, \mathbf{K}\right)$ vorhergesagt wird. $\mathbf{K}^{*}$ ist hier ein Grenzfall, da die anharmonische Analyse für die in $\mathbf{K}$ stabilere Op-Struktur nicht verfügbar ist. Dennoch wird $\mathbf{K}^{*}$ in der Analyse weiter mit berücksichtigt, da nicht ausgeschlossen werden kann, dass Ot auf dem anharmonischen Theorieniveau die globale Minimumsstruktur darstellt. 
Tabelle 4.4: Vergleich der in den Beiträgen angegebenen (org) und der für die Auswertung verwendeten (eval) relativen Entartung $g_{\mathrm{O}} / g_{\mathrm{C}}$ (stabilste O- und stabilste C-Struktur). Abweichungen sind fett hervorgehoben. Freie Zellen deuten auf fehlende Angaben zur Entartung bzw. in den Beiträgen nicht betrachtete Donor-Akzeptor-Kombinationen hin. Hinweis zum Urheberrecht: „Reprinted from f. Chem. Phys. 2020, 152, 164303, ${ }^{[80]}$ with the permission of AIP Publishing...

\begin{tabular}{|c|c|c|c|c|c|c|c|c|}
\hline \multirow[b]{3}{*}{ Beitrag } & \multicolumn{4}{|c|}{$\mathbf{F u}$} & \multicolumn{4}{|c|}{ DMFu } \\
\hline & \multicolumn{2}{|c|}{$+\mathrm{MeOH}$} & \multicolumn{2}{|c|}{$+\mathrm{MeOD}$} & \multicolumn{2}{|c|}{$+\mathrm{MeOH}$} & \multicolumn{2}{|c|}{$+\mathrm{MeOD}$} \\
\hline & org & eval & org & eval & org & eval & org & eval \\
\hline B & 1 & 1 & & & 1 & 0.5 & & \\
\hline C & 1 & 0.5 & $\mathbf{1}$ & 0.5 & 1 & 0.5 & & \\
\hline$C^{*}$ & 1 & 0.5 & 1 & 0.5 & 1 & 0.5 & & \\
\hline D & 1 & 1 & & & & 0.5 & & \\
\hline$D^{*}$ & 1 & 1 & & & & 0.5 & & \\
\hline $\mathbf{E}$ & 1 & 1 & 1 & 1 & & 0.5 & & 0.5 \\
\hline $\mathbf{F}$ & 1 & 1 & 1 & 1 & 1 & 0.5 & 1 & 0.5 \\
\hline $\mathbf{F}^{*}$ & 1 & 1 & 1 & 1 & 1 & 0.5 & 1 & 0.5 \\
\hline G & 1 & 1 & 1 & 1 & 1 & 1 & 1 & 1 \\
\hline $\mathbf{H}$ & 1 & 1 & 1 & 1 & 1 & 1 & 1 & 1 \\
\hline I & 1 & 1 & 1 & 1 & 1 & 1 & 1 & 1 \\
\hline $\mathbf{K}$ & 1 & 1 & 1 & 1 & 1 & 1 & 1 & 1 \\
\hline $\mathbf{K}^{*}$ & 1 & 1 & 1 & 1 & 1 & 1 & 1 & 1 \\
\hline
\end{tabular}

\subsubsection{Auswertung des O/C-Konformerengleichgewichts}

Für das Benchmarking der theoretischen (Kap. 4.1.2) anhand der experimentellen (Kap. 4.1.1) Daten zum O/C-Konformerengleichgewicht sollen zwei der in Kapitel 2.3.2 vorgestellten Ansätze genutzt werden: Zum einen soll der Vergleich über einen aus den experimentellen Intensitätsverhältnissen bestimmten Energieunterschied erfolgen, zum anderen über die Berechnung einer auf den experimentellen und theoretischen Daten basierenden Konformationstemperatur. Aufgrund des nicht quantitativ bestimmbaren Intensitätsverhältnisses im Fall von MFu wird dieses Furanderivat in dieser Auswertung, wie bereits erwähnt, nicht weiter berücksichtigt.

\section{Experiment kommt Theorie entgegen: Experimenteller Energieunterschied}

Soll die Last auf die Seite des Experiments gelegt werden, kann, wie in Kapitel 2.3.2 beschrieben, rein aus den experimentellen Daten ein Energieunterschied $\Delta E_{\mathrm{O}-\mathrm{C}}^{0}$ gebildet werden, wenn für die experimentell nicht bzw. nicht exakt bestimmbaren Größen Annahmen getroffen werden. Für die Umwandlung der O- und C-gebundenen Dimere ineinander werden relativ kleine Barrieren erwartet, zumal die MW-Ergebnisse zeigen, dass 
Methanol für beide Andockstellen von oberhalb der Ringebene an das jeweilige Furanderivat bindet („on top“-Konformere t). Die Konformationstemperatur $T_{\mathrm{c}}$ wird deshalb auf $60 \pm 40 \mathrm{~K}$ geschätzt. ${ }^{[31]}$ Aufgrund der ähnlichen Konformationen in C- und O-Heterodimer wird auch von vergleichbaren IR-Bandenstärken der OH-Streckschwingungen in Ctund Ot-Dimer ausgegangen, sodass für das Bandenstärkenverhältnis $A_{\mathrm{O}} / A_{\mathrm{C}}$ mit einem konservativen Fehler von $\pm 50 \%$ ein Wert von $1.0 \pm 0.5$ verwendet wird. Während die rotationsspektroskopischen Ergebnisse den Ct-Dimeren $C_{1}$-Symmetrie bescheinigen, ergibt sich für die Ot-Dimere mit $\mathrm{MeOH}$ als Donor, wie weiter oben beschrieben, jeweils effektive $C_{s}$-Symmetrie. In diesem Fall ergäbe sich eine relative Entartung $g_{\mathrm{O}} / g_{\mathrm{C}}$ von 0.5 . Da jedoch auch ein Potential mit zwei äquivalenten $C_{1}$-symmetrischen Ot-Struktur-Minima nicht ausgeschlossen werden kann (s. Kap. 4.1.1) und für MeOD keine experimentellen Daten zur Symmetrie vorliegen, muss der Fall $C_{1}$-symmetrischer Ot-Dimere ebenfalls berücksichtigt werden. In diesem Fall betrüge das Entartungsfaktorenverhältnis $g_{\mathrm{O}} / g_{\mathrm{C}}=1$. Ein solches Verhältnis von 1 erfasst auch den Fall, dass der statistische Nachteil eines $C_{s^{-}}$ symmetrischen Ot-Dimers durch die Relaxation möglicherweise zu Beginn der Expansion vorhandenen Op-Dimers in das stabilere Ot-Dimer zum Teil wieder ausgeglichen werden könnte. Die unterschiedlichen Symmetriefälle (C- und O-Dimer beide $C_{1}$-symmetrisch; CDimer $C_{1}$ - und O-Dimer $C_{s}$-symmetrisch) werden in der Auswertung getrennt betrachtet.

Aus den genannten Schätzungen, den jeweiligen relativen Entartungen und den experimentellen Intensitätsverhältnissen und ihren Fehlern ergeben sich über die Gleichungen 2.13 und 2.14 (s. S. 25) die in Tabelle 4.3 (s. S. 112) aufgeführten experimentellen Energieunterschiede und die zugehörigen Fehler. Negative Werte entsprechen dabei einem stabileren O-, positive einem stabileren C-Dimer. Durch die große Ungewissheit in der abgeschätzten Konformationstemperatur ergeben sich für die Energieunterschiede Fehlerbereiche, die jeweils auch kleine positive Werte umfassen. Sowohl in der Schwingungsals auch in der Rotationsspektroskopie dominiert in allen Fällen jedoch eindeutig das ODimer, das damit die Minimumsstruktur darstellt.

Die Ergebnisse des Vergleichs mit den berechneten, schwingungsnullpunktskorrigierten Energieunterschieden (ebenfalls Tab. 4.3) sind für den Donor $\mathrm{MeOH}$ in Abbildung 4.5 und für MeOD in Abbildung 4.6 graphisch dargestellt. Dabei wurde jeweils der schwingungsnullpunktskorrigierte Energieunterschied für DMFu gegen jenen für Fu aufgetragen. Der untere linke Quadrant, der einer Bevorzugung des O-Dimers für beide Furanderivate entspricht, wurde hellgrau eingefärbt. Die sich für beide Symmetriefälle aufspannenden Rechtecke aus den berechneten experimentellen Energieunterschieden sind in weiß eingezeichnet. Für eine zumindest qualitative Übereinstimmung mit dem Experiment sollte ein Datenpunkt aus einem Paar berechneter Energieunterschiede in den hellgrauen Quadranten fallen, für eine quantitative Übereinstimmung in eins der beiden weiBen Rechtecke.

Qualitativ mit den experimentellen Befunden hinsichtlich der Dimerstrukturen nicht vereinbare Ergebnisse werden, wie bereits erwähnt, in der quantitativen Auswertung nicht berücksichtigt. Da jedoch nur für $\mathrm{MeOH}$ experimentelle Daten vorliegen, kann für MeOD auf diese Weise aktuell keine Methoden-Vorauswahl getroffen werden. Sollte sich 


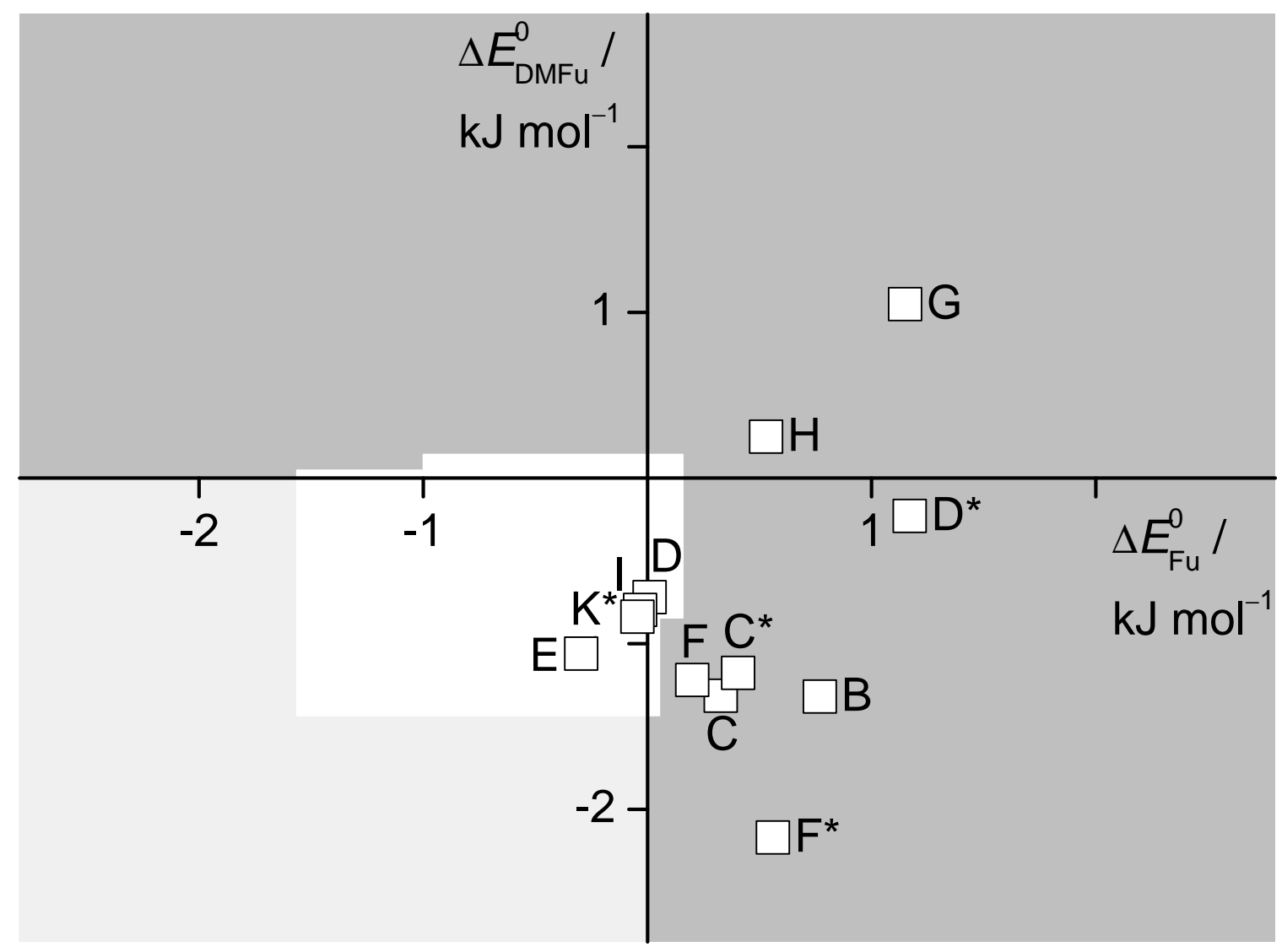

Abbildung 4.5: Korrelation der Energiedifferenzen der Heterodimere von $\mathrm{MeOH}$ mit Fu (Abszisse) bzw. DMFu (Ordinate) für verschiedene harmonische und anharmonische $\left(^{*}\right)$ Theorieniveaus. Es sind nur die Daten dargestellt, deren jeweils niederenergetischste C- und O-Dimerstrukturen mit den experimentellen Befunden vereinbar sind. Der hellgraue Quadrant entspricht qualitativer Übereinstimmung mit der experimentell bestimmten C- bzw. O-Bindungsstellenpräferenz, die überlappenden weißen Rechtecke kennzeichnen die experimentell ermittelten Energieunterschiede für zwei unterschiedliche Symmetrieszenarien (s. Fließtext). Hinweis zum Urheberrecht: „Reprinted from f. Chem. Phys. 2020, 152, 164303, ${ }^{[80]}$ with the permission of AIP Publishing... 


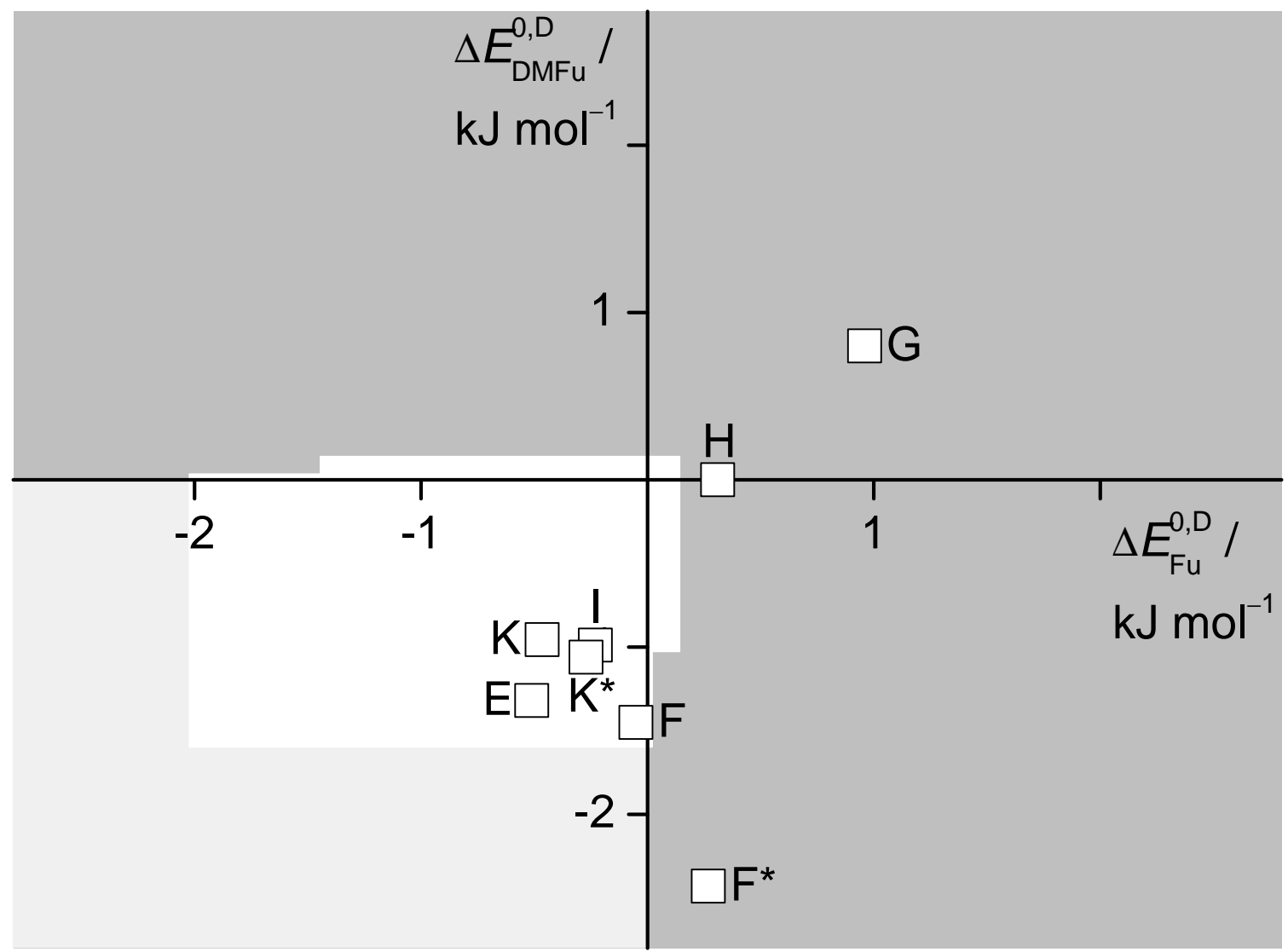

Abbildung 4.6: Darstellung entsprechend Abb. 4.5, aber für MeOD als Donor. Hinweis zum Urheberrecht: „Reprinted from 7. Chem. Phys. 2020, 152, 164303, ${ }^{[80]}$ with the permission of AIP Publishing.".

in späteren mikrowellenspektroskopischen Untersuchungen analog zu $\mathrm{MeOH}$ auch für $\mathrm{MeOD}+\mathrm{Fu}$ das Ot-Dimer als globales Minimum erweisen, müssten die Methoden $\mathbf{C}, \mathbf{F}$ und $\mathbf{K}$ von der Analyse ausgeschlossen werden.

Für die mit den vorliegenden rotationsspektroskopischen Daten qualitativ kompatiblen Methoden zeigt sich, dass nur wenige Ergebnisse in den weißen, quantitativ mit dem Experiment vereinbaren Bereich fallen. Wird keine Symmetrie-Entartung angenommen, liegen für $\mathrm{MeOH}(\mathrm{Abb} .4 .5)$ nur $\mathbf{D}$, $\mathbf{I}$ und $\mathbf{K}^{*}$ in dieser Zone, für MeOD (Abb. 4.6) nur I und K. Unter der Annahme eines $C_{s}$-symmetrischen Ot-Dimers fällt für $\mathrm{MeOH}$ zusätzlich $\mathbf{E}$ in den weißen Bereich, für MeOD kommen $\mathbf{E}, \mathbf{F}$ und $\mathbf{K}^{*}$ dazu. Durchgehend gute Ergebnisse für beide Donoren liefern also nur $\mathbf{E}$ und I, wobei nur I unabhängig von der Symmetrie des Ot-Dimers im weißen Bereich liegt. Die Methode D, die für $\mathrm{MeOH}$ gut abschneidet, hat für MeOD keine Daten. Die für MeOD erfolgreiche Methode K sagt für $\mathrm{MeOH} / \mathrm{D}+\mathrm{Fu}$ jeweils das Ot-Dimer als globales Minimum vorher und ist für $\mathrm{MeOH}$ deshalb vom Vergleich ausgeschlossen (s. oben). Auffällig ist, dass die außerhalb des weißen 
Bereichs liegenden Methoden allesamt die relative Stabilität des C-Dimers beider Donoren mit Fu überschätzen. Für DMFu zeigt sich insgesamt eine bessere Übereinstimmung mit dem experimentellen Energieunterschied, wobei die nicht mit den experimentellen Ergebnissen vereinbaren Methoden hier in beide Richtungen abweichen.

Fast alle für beide Donoren erfolgreichen Methoden nutzen die harmonische Näherung zur Berechnung der ZPVE, nur $\mathbf{K}^{*}$ nutzt eine anharmonische Korrektur. Generell zeigen sich bei Berücksichtigung anharmonischer Korrekturen wenig systematische Effekte. Zum Teil kann dies auf Schwierigkeiten bei der Behandlung der stark gekoppelten und delokalisierten Bewegung großer Amplitude zurückgeführt werden, für die keine Systematik über die verschiedenen Isomere hinweg besteht. So konnte vor über 20 Jahren bereits gezeigt werden, ${ }^{[319]}$ dass VSCF für niederfrequente Moden in neutralen wasserstoffbrückengebundenen Systemen mitunter eine schlechte Leistung aufweist, was kürzlich für das gut untersuchte Ameisensäuredimer bestätigt werden konnte. ${ }^{[320,321]}$ Wird Methode D anharmonisch korrigiert $\left(\mathbf{D}^{*}\right)$, landet der Datenpunkt aufgrund einer Überschätzung der relativen Stabilität des C-Dimers für $\mathrm{MeOH}+\mathrm{Fu}$ so z. B. außerhalb der experimentellen Grenzen, wohingegen mit der anharmonischen Korrektur bei $\mathbf{F}^{*}$ die relative Stabilität des O-Dimers von $\mathrm{MeOH} / \mathrm{D}+\mathrm{DMFu}$ überschätzt wird. Ob die harmonischen Methoden aufgrund von Fehlerkompensation zufällig gut abschneiden oder ob es schlicht an ausgereiften Methoden zur anharmonischen Betrachtung von Systemen dieser Größe fehlt, kann angesichts des kleinen Datensatzes allerdings nicht aufgeklärt werden. Das beobachtete, über Methylierung und Deuterierung hinweg relativ gleichmäßige Bandenmuster deutet allerdings auf die Abwesenheit bedeutender anharmonischer Effekte hin.

Die vorangegangene Analyse legt den Großteil der Last auf die Seite des Experiments, da von den Theoriemethoden nur elektronische Energieunterschiede und eine Abschätzung des Effekts der ZPVE auf die relativen Energien verlangt wird. Noch vorteilhafter für die Theorie wäre es, wenn die Einflüsse der elektronischen Energien und der ZPVE experimentell aufgeschlüsselt werden könnten, was allerdings nicht ohne Weiteres möglich ist. Die experimentellen Daten für MeOD kommen näher ans elektronische Limit als $\mathrm{MeOH}$ und deuten darauf hin, dass das O-Isomer für beide Furanderivate bezogen auf die elektronischen Energien etwa $1 \pm 1 \mathrm{~kJ} \cdot \mathrm{mol}^{-1}$ stabiler als das C-Dimer ist. Dieses recht grobe experimentelle Fenster wird von den Methoden C, D, E, F, H, I und K für beide Furanderivate getroffen, wohingegen A, B und $\mathbf{G}$ in jeweils mindestens einem Fall das CDimer bevorzugen.

\section{Theorie kommt Experiment entgegen: Konformationstemperatur}

Ein strikteres Benchmarking wird möglich, wenn die Last stärker auf die Seite der Theorie verschoben wird. Wie in Kapitel 2.3.2 beschrieben, können aus der Kombination des gemessenen Intensitätsverhältnisses und den Ergebnissen der verschiedenen quantenchemischen Rechnungen theoriebasierte Konformationstemperaturen $T_{\mathrm{c}}$ bestimmt werden. Neben dem schwingungsnullpunktskorrigierten Energieunterschied werden dabei auch das IR-Bandenstärkenverhältnis und - basierend auf den Symmetrien der C- und O-Mi- 
nimumsstrukturen - die relative Entartung der jeweiligen quantenchemischen Rechnung entnommen (verwendete Werte s. Tab. 4.4 auf S. 114). Somit sind hier zum einen keine Schätzung für das Bandenstärkenverhältnis und keine getrennte Betrachtung verschiedener Symmetriefälle mehr nötig, sondern schlechte Werte gehen zulasten der jeweiligen Theoriemethode und führen unter Umständen zu unrealistischen Konformationstemperaturen. Gegebenenfalls könnten sie andere Fehler der theoretischen Methode jedoch auch kompensieren. Zum anderen dient die experimentell am schlechtesten bestimmbare Größe $T_{\mathrm{c}}$ direkt als Zielgröße für das Benchmarking.

Bei der Verwendung der Konformationstemperatur als Benchmarking-Zielwert lässt sich ein zweistufiger Zielbereich definieren. Ein größerer Zielbereich ergibt sich aus den physikalischen Grenzen der Konformationstemperatur, die in jedem Fall zwischen der Rotationstemperatur (hier $\approx 10 \mathrm{~K}$ ) und der Düsentemperatur (hier $300 \mathrm{~K}$ ) liegen muss. Ein engerer Zielbereich resultiert aus der im vorherigen Abschnitt bereits verwendeten Schätzung von 20-100 K für die Barrierensituation im C-O-Konformerengleichgewicht der MeOH-Furan(derivat)-Dimere. Für ein gutes Abschneiden sollte eine Theoriemethode für alle getesteten Donor-Akzeptor-Kombinationen konsistent im weiteren Zielbereich liegen, für ein sehr gutes Abschneiden möglichst durchgehend im engeren Zielbereich. Ähnliche Konformationstemperaturen für $\mathrm{MeOH}$ und MeOD deuten auf eine zutreffende Beschreibung der Schwingungsnullpunktseffekte durch das eingesetzte Modell zur Schwingungsrechnung hin. Sind die Konformationstemperaturen für Fu und DMFu vergleichbar groß, ist dies ein Hinweis auf einheitliche Barrierenhöhen. Gibt es zwischen Fu und DMFu größere Abweichungen in $T_{c}$, ändert sich die Barrierenhöhe entweder durch die Substitution oder die Theorie beschreibt den Effekt der Substitution nicht ausreichend genau.

In Abbildung 4.7 sind die aus der Kombination der gemessenen Intensitätsverhältnisse mit den Daten aus den jeweiligen Rechnungen ermittelten Konformationstemperaturen für $\mathrm{MeOH}$ mit Fu (Abszisse) und DMFu (Ordinate) aufgetragen. Der unphysikalische Bereich $(<10 \mathrm{~K},>300 \mathrm{~K})$ ist dunkelgrau eingefärbt und der erwartete Zielbereich für ein Konformerengleichgewicht mit einer flachen Barriere (20-100 K) ist weiß hervorgehoben. Die Daten sind mit auf dem experimentellen Fehler für das Intensitätsverhältnis basierenden Fehlerbalken (s. Kap. 2.3.2) versehen. Wie schon zuvor werden qualitativ nicht mit den experimentell ermittelten Strukturen vereinbare Ergebnisse auch für die Auswertung über die Konformationstemperatur ignoriert. Im Vergleich zu Abbildung 4.5, die auf denselben Daten für Intensitätsverhältnisse und berechnete schwingungsnullpunktskorrigierte Energieunterschiede basiert, zeigt sich, dass die diskriminierende Leistung des Benchmarks tatsächlich zunimmt, wenn mehr Last auf die Theorie gelegt wird. Während beim Benchmark über den experimentellen Energieunterschied noch die Methoden D, E, I und $\mathbf{K}^{*}$ zumindest für einen der betrachteten Symmetriefälle im Zielbereich lagen, liegt nun mit Methode $\mathbf{E}$ nur noch ein Beitrag überhaupt im physikalischen Bereich. Während Methode $\mathbf{E}$ dabei sogar den engeren Zielbereich trifft, liegen $\mathbf{D}, \mathbf{I}$ und $\mathbf{K}^{*}$ knapp im unphysikalischen Bereich, was vor allem auf Probleme bei der Beschreibung des Fu-Systems zurückzuführen ist. Generell zeigt sich erneut, dass die Daten für Fu häufiger vom 


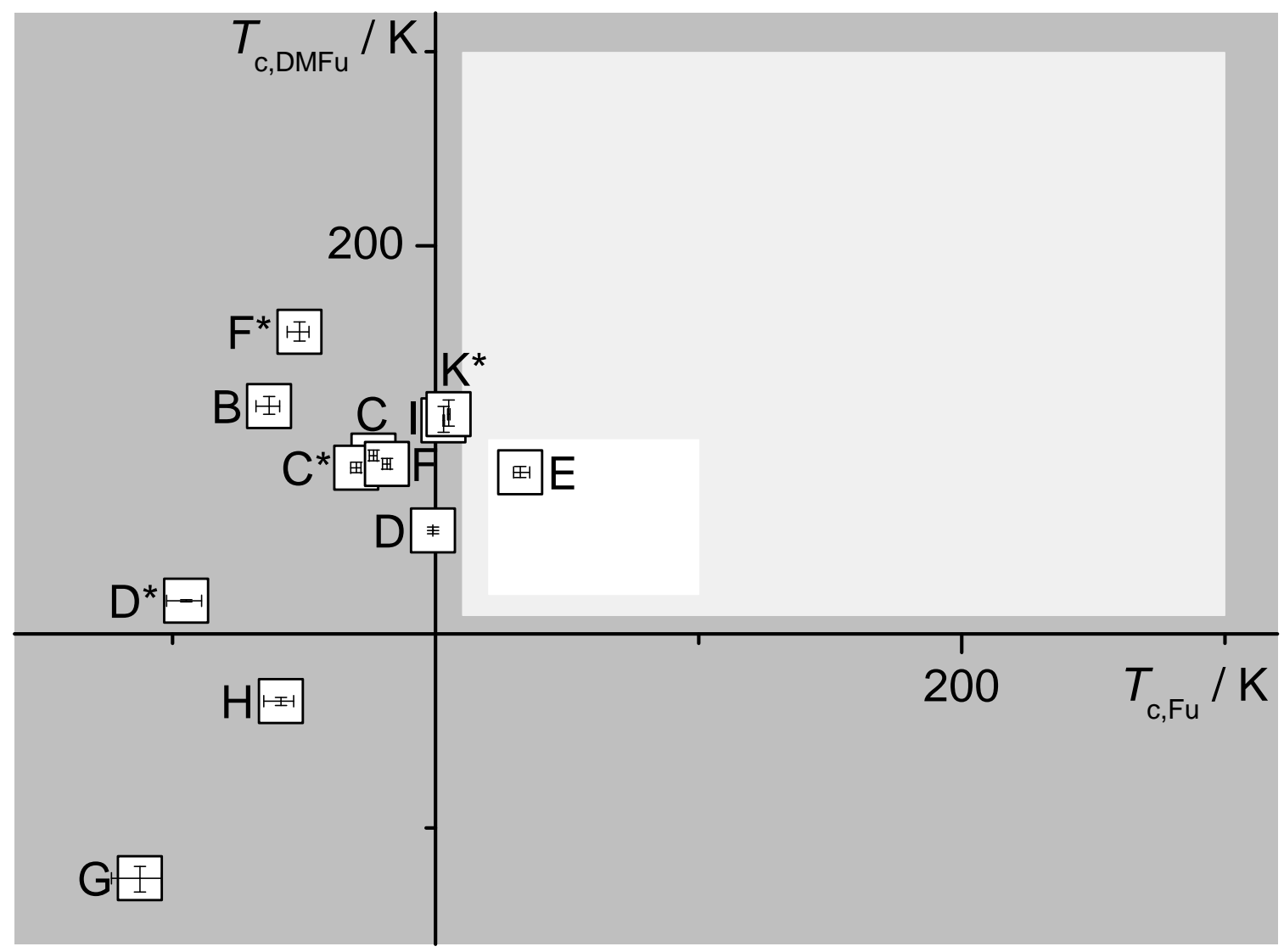

Abbildung 4.7: Korrelation der Konformationstemperaturen für die Aggregation von $\mathrm{MeOH}$ mit $\mathrm{Fu}$ (Abszisse) und DMFu (Ordinate). Die Datenpunkte sind mit auf dem experimentellen Fehler für das Intensitätsverhältnis basierenden Fehlerbalken versehen und es sind nur die mit dem Experiment qualitativ vereinbaren (s. Fließtext) Ergebnisse für harmonische und anharmonische ${ }^{*}$ ) Rechenmethoden abgebildet. Für kleine Umwandlungsbarrieren werden Temperaturen zwischen 20 und $100 \mathrm{~K}$ erwartet (weißer Bereich), Temperaturen außerhalb von 10-300 K sind unphysikalisch (dunkelgrau). Hinweis zum Urheberrecht: „Reprinted from f. Chem. Phys. 2020, 152, 164303, ${ }^{[80]}$ with the permission of AIP Publishing.". 


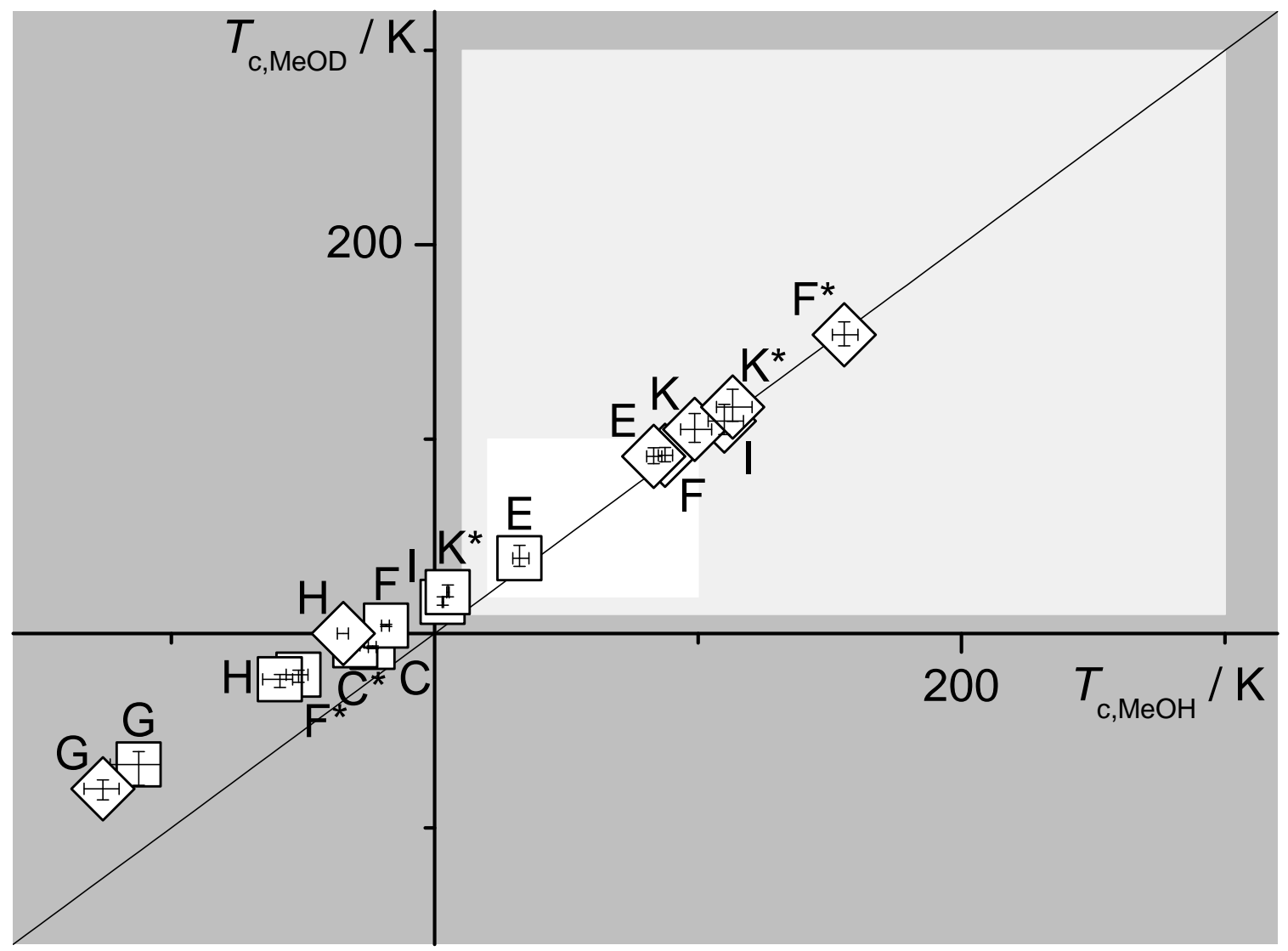

Abbildung 4.8: Korrelation der Konformationstemperaturen für die Aggregation von $\mathrm{MeOH}$ (Abszisse) und MeOD (Ordinate) mit Fu (Quadrate) und DMFu (Rauten). Fehlerbalken der aufgetragenen Werte und Markierung der Zielbereiche analog zu Abbildung 4.7. Hinweis zum Urheberrecht: „Reprinted from f. Chem. Phys. 2020, 152, 164303, ${ }^{[80]}$ with the permission of AIP Publishing...

Zielbereich abweichen als jene für DMFu. In keinem Fall werden dabei Werte oberhalb der Düsentemperatur erreicht. Vielmehr liegen für Fu alle unphysikalischen Ergebnisse bei zu kleinen bzw. negativen Konformationstemperaturen, wohingegen für DMFu nur zwei negative ( $\mathbf{G}$ und $\mathbf{H}$ ), eine noch physikalische, aber zu kleine $\left(\mathbf{D}^{*}\right)$ und mehrere noch physikalische, aber oberhalb des erwarteten Bereichs gelegene $\left(\mathbf{B}, \mathbf{F}^{*}, \mathbf{I}, \mathbf{K}^{*}\right)$ Konformationstemperaturen vorliegen.

Um den Einfluss der Beschreibung der ZPVE auf die Ergebnisse hervorzuheben, können die Konformationstemperaturen für MeOD gegen diejenigen für $\mathrm{MeOH}$ aufgetragen werden (s. Abb. 4.8). Die Werte reichen von $-130 \mathrm{~K}$ bis $+160 \mathrm{~K}$, wobei insbesondere die positiven Werte nah an der Winkelhalbierenden liegen. Dies deutet auf eine akkurate Beschreibung der Schwingungsnullpunktseffekte hin, sodass bedeutende Abweichungen vom Experiment vermutlich durch Schwächen im Bereich der elektronischen Energiedifferenz verursacht werden. Nur E liegt sowohl für Fu (Quadrate) als auch für DMFu 
(Rauten) im optimalen Bereich, wobei auch $\mathbf{I}$ und $\mathbf{K}^{*}$ noch akzeptable Ergebnisse liefern. Weiterhin liegen die DMFu-Datenpunkte für $\mathbf{F}$ im optimalen, für $\mathbf{K}$ im Rahmen der Fehlerbalken ebenfalls im optimalen und für $\mathbf{F}^{*}$ im akzeptablen Bereich, wohingegen die FuDatenpunkte für $\mathbf{F}$ und $\mathbf{F}^{*}$ und alle weiteren Datenpunkte der Methoden $\mathbf{C}, \mathbf{G}$ und $\mathbf{H}$ durch die Überschätzung der Stabilität des C-Dimers für mindestens eins der Donor-Akzeptorpaare in den dunkelgrauen, unphysikalischen Bereich fallen. Auch hier zeigt sich, dass die quantenchemischen Rechnungen insgesamt für DMFu bessere Ergebnisse als für Fu liefern. Anharmonische Fehlerkompensation scheint für DMFu also besser zu funktionieren als für Fu.

Um die Leistungsfähigkeit der theoretischen Methoden bezogen auf die ZPVE möglichst unabhängig von der elektronischen Energiedifferenz zu begutachten, kann die Isotopenaustauschtemperatur $T_{\Delta}$ herangezogen werden, die aus dem Deuterierungseffekt auf den berechneten Energieunterschied einerseits und auf das experimentelle Intensitätsverhältnis andererseits erhalten wird. Da die elektronische Energie im Rahmen der genutzten Näherungen unabhängig von der Isotopenmasse ist, spielt die Differenz der elektronischen Energien für $T_{\Delta}$ keine Rolle und es wird nur untersucht, wie gut der Einfluss der Deuterierung auf die ZPVE durch die Theoriemethoden erfasst wird. Ist $T_{\Delta}$ negativ oder größer als die Düsentemperatur, liegt eine Diskrepanz zwischen ZPVE-Berechnung und experimentellen Befunden vor. In Abbildung 4.9 sind die $\Delta$-Konformationstemperaturen für die Beiträge aufgetragen, die Daten für alle vier Donor-Akzeptorkombinationen aus $\mathrm{MeOH}$ und MeOD mit Fu und DMFu aufweisen. Für Datenpunkte, die in Abbildung 4.8 genau auf der Winkelhalbierenden liegen, entspricht $T_{\Delta}$ dem dort vorliegenden $T_{\mathrm{c}}$-Wert. Andernfalls ergeben sich starke Abweichungen, sodass die experimentellen Intensitätsfehler zu großen Fehlerbalken für $T_{\Delta}$ führen.

Für alle sieben Methoden, die über $T_{\Delta}$ analysiert werden können, ergeben sich Ergebnisse im physikalischen Bereich (hellgrau), wobei alle Fehlerbalken den Erwartungsbereich (weiß) berühren. Die SCS-CC2-basierte Methode I kommt dem weißen Bereich dabei am nächsten, wobei die SCS-MP2-Methode $\mathbf{F}$ und ihre anharmonische Variante $\mathbf{F}^{*}$ die konsistenteren Ergebnisse über beide Furanderivate hinweg aufweisen. Doch auch die DFTbasierten Methoden E, G, $\mathbf{H}$ und $\mathbf{K}^{*}$ sind im Rahmen der großen Fehlerbalken vergleichbar leistungsfähig.

Zusammenfassend können über die Auswertung per Konformationstemperatur I, $\mathbf{K}^{*}$, D und insbesondere $\mathbf{E}$ als vielversprechendste Methoden zur Beschreibung der Strukturen, elektronischen und schwingungsnullpunktskorrigierten Energien, sowie der IRBandenstärken identifiziert werden. Mit $\mathbf{D}, \mathbf{E}$ und $\mathbf{K}^{*}$ nutzen drei dieser Methoden Dichtefunktionale und eine Methode (I) die SCS-CC2-Methodik zur Geometrieoptimierung und Schwingungsrechnung, wohingegen für die Berechnung der elektronischen Energien verschiedene $\operatorname{CCSD}(\mathrm{T})$-Varianten verwendet werden. Mit $\mathbf{K}^{*}$ bedient sich nur eine der erfolgreichen Methoden anharmonischer Korrekturen bei der Berechnung der ZPVE. Inwiefern das gute Abschneiden auf Fehlerkompensation zwischen berechneten Strukturen, elektronischen Energien, ZPVE und Bandenstärken beruht, kann angesichts der geringen Zahl der betrachteten Systeme nicht beurteilt werden. Für eine umfassendere Analyse 


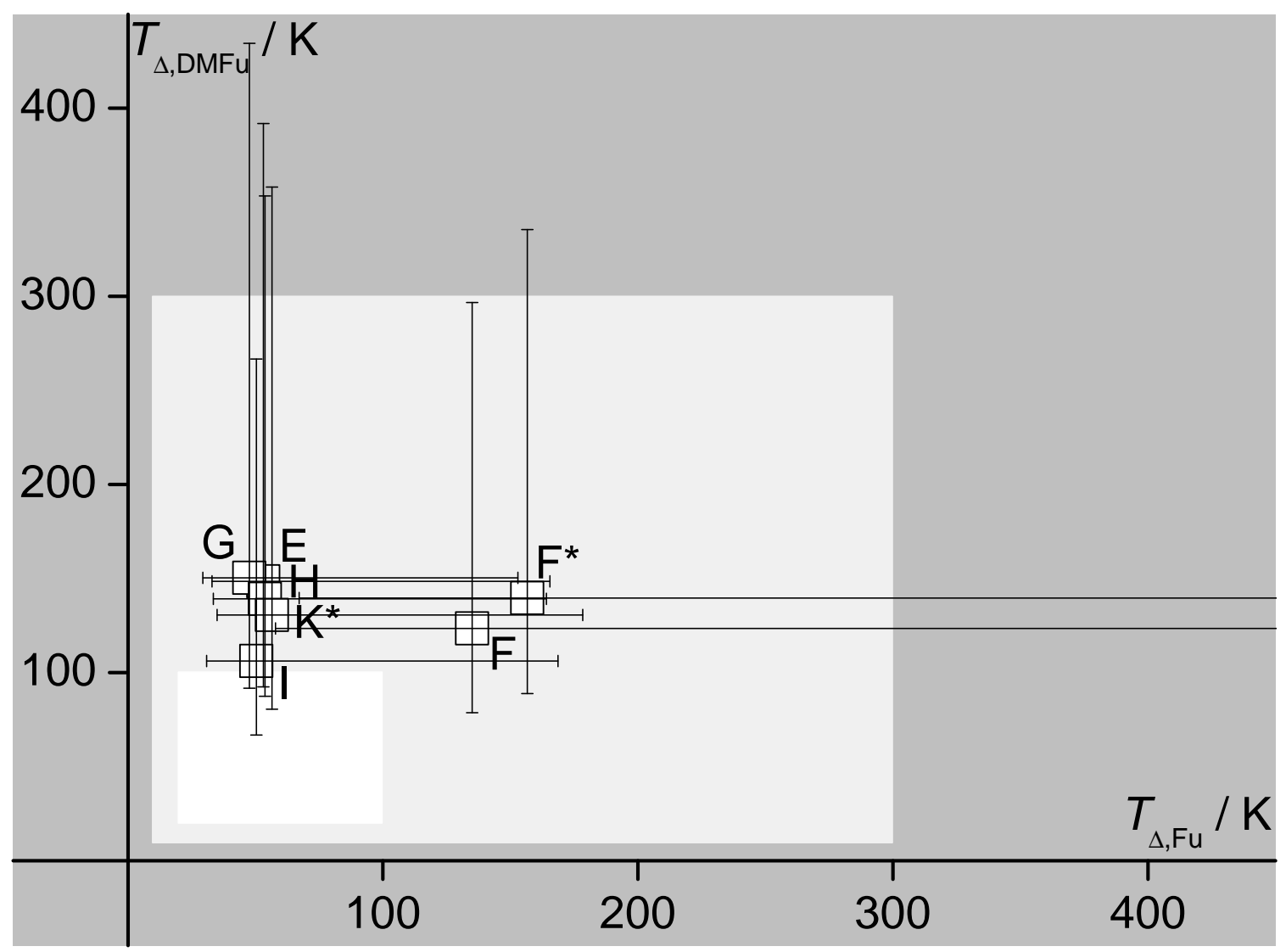

Abbildung 4.9: Korrelation der effektiven Isotopenaustauschtemperatur $T_{\Delta}$ (s. Fließtext) für die Aggregation von $\mathrm{MeOH} / \mathrm{D}$ mit Fu (Abszisse) und DMFu (Ordinate). Die Fehlerbalken ergeben sich aus den experimentellen Fehlern der Intensitätsverhältnisse. Kennzeichnung der Methoden und der Zielbereiche analog zu Abbildung 4.7. Hinweis zum Urheberrecht: „Reprinted from f. Chem. Phys. 2020, 152, 164303, ${ }^{[80]}$ with the permission of AIP Publishing.“.

wäre eine Erweiterung des Benchmarks auf weitere Donor-Akzeptorpaare somit ebenso wünschenswert wie MeOD-Daten für die Methode $\mathbf{D}$.

\subsubsection{Auswertung IR-spektroskopischer Parameter}

Auch wenn der Kern der vorliegenden Untersuchung die Überprüfung der berechneten Energieunterschiede anhand der experimentell ermittelten Intensitätsverhältnisse der jeweils betrachteten Dimer-Gleichgewichte ist, bietet es sich an, auch die in der FTIR-Spektroskopie leicht zugänglichen Bandenpositionen bzw. Rotverschiebungen oder Aufspaltungen mit in den Benchmark einzubeziehen. Aufgrund der nicht zuordenbaren, zusätzlichen Dimerbanden in den MFu-Spektren (s. Kap. 4.1.1) wird dieses Furanderivat auch in der folgenden Auswertung nicht weiter berücksichtigt.

Während es für die Schwingungsrechnungen in der harmonischen Näherung nicht 
sinnvoll ist, die absoluten Werte für die $\mathrm{OH}$-Streckschwingungs-Bandenpositionen $\omega_{\mathrm{OH}}$ oder den Betrag der Rotverschiebung $\Delta \omega_{\mathrm{OH}}$ der $\mathrm{OH}$-Streckschwingungsbanden im Dimer den anharmonischen experimentellen Werten $\tilde{v}_{\mathrm{OH}}$ und $\Delta \tilde{v}_{\mathrm{OH}}$ gegenüberzustellen, können für den Vergleich experimentelle Trends herangezogen werden, bei denen sich anharmonische Effekte zu einem hohen Grad kompensieren. Ein solcher Trend ist die Veränderung der $\mathrm{OH}$-Streckschwingungsrotverschiebung bei zweifacher Methylierung des $\mathrm{Fu}$ ranrings, $\Delta \Delta(\tilde{v}, \omega)_{\mathrm{OH}}(\mathrm{DMFu}-\mathrm{Fu})$. Dieser Wert gibt wieder, wie viel weiter rotverschoben eine DMFu-Bande im Vergleich zur entsprechenden Fu-Bande ist. Da sich für die hier betrachteten Systeme gleichförmige Trends der Rotverschiebungen für C- und O-Dimer bei zunehmender Methylierung des Furanrings von Fu über MFu zu DMFu zeigen (s. oben), kompensieren sich anharmonische Effekte bei Betrachtung des Rotverschiebungsunterschieds $\Delta \Delta \tilde{v}_{\mathrm{OH}}(\mathrm{DMFu}-\mathrm{Fu})$ zwischen DMFu und $\mathrm{Fu}$ anscheinend zu einem großen Teil. Somit sollte bereits ein ausgewogenes harmonisches Modell diesen Rotverschiebungsunterschied reproduzieren können.

Die Rotverschiebungsunterschiede $\Delta \Delta \omega_{\mathrm{OH}, \mathrm{D}}(\mathrm{DMFu}-\mathrm{Fu})$ für harmonische Rechnungen bzw. $\Delta \Delta \tilde{v}_{\mathrm{OH}, \mathrm{D}}(\mathrm{DMFu}-\mathrm{Fu})$ für anharmonische Rechnungen $\left({ }^{*}\right)$ und das Experiment sind in Abbildung 4.10 für $\mathrm{MeOH}$ und in Abbildung 4.11 entsprechend für MeOD für die C(Dreiecke) und O-Dimere (Kreise) gegen die Rotverschiebung $\Delta(\tilde{v}, \omega)_{\mathrm{OH}, \mathrm{D}}(\mathrm{Fu})$ des entsprechenden Dimers für Fu aufgetragen. Die experimentellen Referenzwerte sind dabei jeweils mit schwarz ausgefüllten Symbolen dargestellt, die berechneten Werte mit leeren Symbolen. Die C- und O-Datenpunkte jeder Rechenmethode (gestrichelt) bzw. des Experiments (durchgezogen) sind mit Linien verbunden. Die zugrundeliegenden berechneten und gemessenen Rotverschiebungen $\Delta(\tilde{v}, \omega)_{\mathrm{OH}, \mathrm{D}}$ der C- und O-Dimere von $\mathrm{MeOH}$ und MeOD mit Fu und DMFu sind in Tabelle 4.5 aufgelistet. Wie bereits im vorangegangenen Abschnitt wurden Fälle, in denen die berechnete Struktur qualitativ den mikrowellenspektroskopischen Befunden widerspricht, nicht berücksichtigt. Erneut wurde die Vorauswahl nur für $\mathrm{MeOH}$ als Donor getroffen, da keine experimentellen MeOD-Strukturdaten vorliegen.

Werden zunächst einmal die Ergebnisse für $\mathrm{MeOH}$ als Donor betrachtet (Abb. 4.10), können folgende Schlüsse für die zur Berechnung der Struktur und der Hesse-Matrix genutzten Methoden gezogen werden:

- Wo anharmonische Werte zur Verfügung stehen, zeigen diese eine schlechtere Übereinstimmung mit dem Experiment als die zugehörigen harmonischen Ergebnisse. Der pragmatische Ansatz, als Zuordnungshilfe in der Schwingungsspektroskopie harmonische Rechnungen heranzuziehen, scheint für den vorliegenden Fall also bestätigt zu werden. Anharmonische Beiträge kompensieren sich $^{[65-67]}$ zwischen den eng verwandten Fu- und DMFu-Dimeren zu einem großen Teil.

- Die Methoden B und $\mathbf{D}^{*}$ versagen qualitativ und geben den experimentellen Trend nicht wieder. 


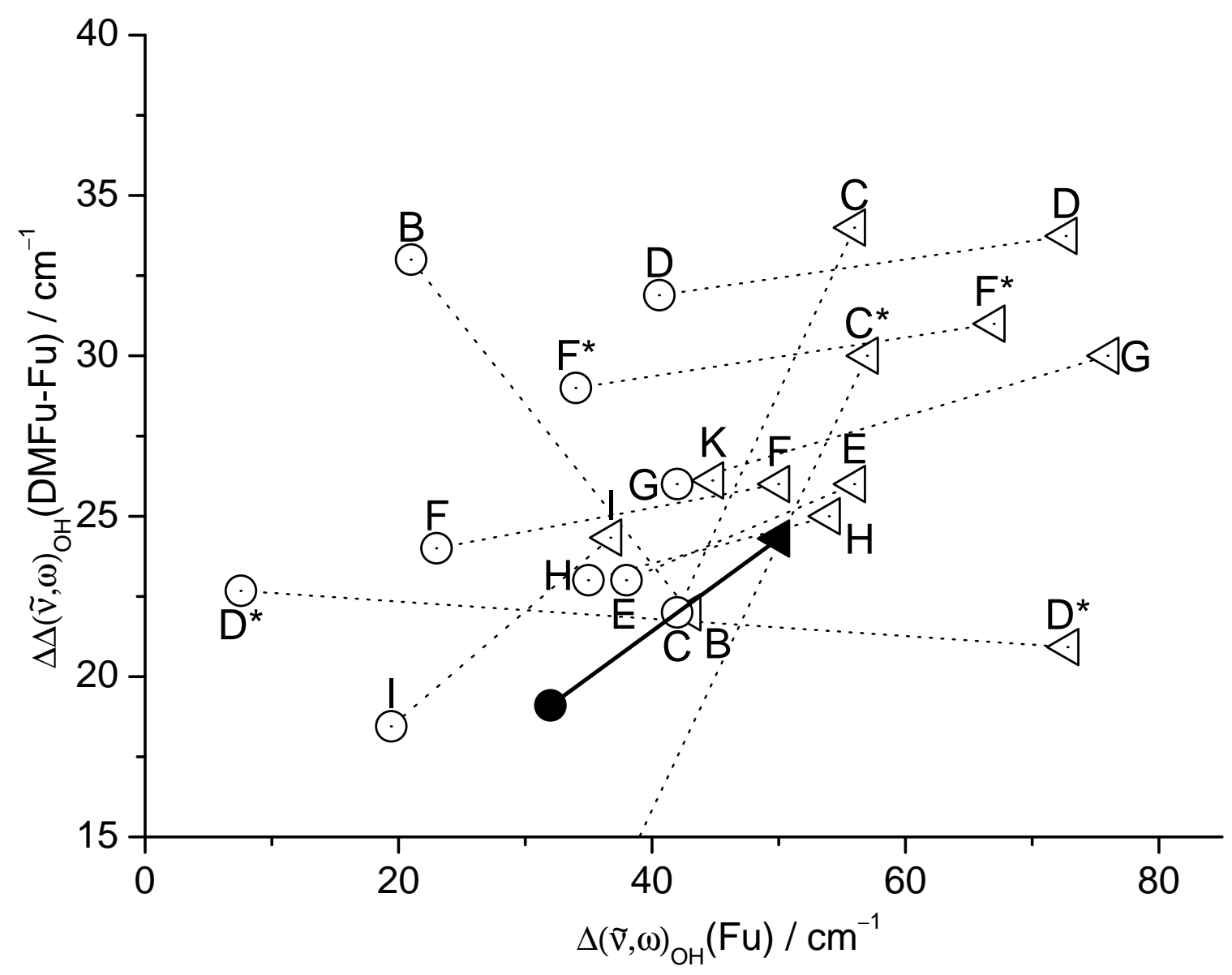

Abbildung 4.10: Korrelation der gemessenen und berechneten OH-Rotverschiebungsdifferenzen

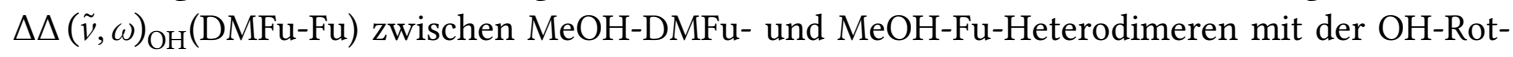
verschiebung $\Delta(\tilde{v}, \omega)_{\mathrm{OH}}(\mathrm{Fu})$ der Fu-Komplexe für C- (Dreiecke) und O-Dimere (Kreise). Die experimentellen Werte sind mit ausgefüllten und die berechneten Werte mit leeren Symbolen dargestellt. C- und O-Datenpunkte jeder Rechenmethode sind mit gestrichelten Linien und die experimentellen Datenpunkte mit einer durchgezogenen Linie verbunden. Hinweis zum Urheberrecht: „Reprinted from F. Chem. Phys. 2020, 152, 164303, ${ }^{[80]}$ with the permission of AIP Publishing.“. 
Tabelle 4.5: Rotverschiebungen $\Delta \omega_{\mathrm{OH}, \mathrm{D}}$ (für harmonische Rechnungen) und $\Delta \tilde{v}_{\mathrm{OH}, \mathrm{D}}$ (für anharmonische Rechnungen und Experiment) der $\mathrm{OH}(\mathrm{D})$-Streckschwingung von Methanol(-OD) im Heterodimer im Vergleich zum freien Methanol in $\mathrm{cm}^{-1}$. Fälle, in denen die berechnete Struktur qualitativ den experimentellen Befunden widerspricht, sind mit ,"- gekennzeichnet. Hinweis zum Urheberrecht: „Reprinted from f. Chem. Phys. 2020, 152, 164303, ${ }^{[80]}$ with the permission of AIP Publishing...

\begin{tabular}{|c|c|c|c|c|c|c|c|c|}
\hline \multirow[b]{3}{*}{ Beitrag } & \multicolumn{4}{|c|}{$\mathrm{MeOH}$} & \multicolumn{4}{|c|}{ MeOD } \\
\hline & \multicolumn{2}{|c|}{$+\mathrm{Fu}$} & \multicolumn{2}{|c|}{$+\mathrm{DMFu}$} & \multicolumn{2}{|c|}{$+\mathbf{F u}$} & \multicolumn{2}{|c|}{$+\mathrm{DMFu}$} \\
\hline & $\mathrm{C}$ & $\mathrm{O}$ & $\mathrm{C}$ & $\mathrm{O}$ & $\mathrm{C}$ & $\mathrm{O}$ & $\mathrm{C}$ & $\mathrm{O}$ \\
\hline A & - & - & 50 & 57 & & & & \\
\hline$A^{*}$ & - & - & 35 & 32 & & & & \\
\hline B & 43 & 21 & 65 & 54 & & & & \\
\hline C & 56 & 42 & 90 & 64 & 41 & 32 & & \\
\hline$C^{*}$ & 57 & 21 & 87 & 21 & 43 & 30 & & \\
\hline D & 73 & 41 & 106 & 72 & & & & \\
\hline $\mathbf{D}^{*}$ & 73 & 8 & 94 & 30 & & & & \\
\hline $\mathbf{E}$ & 56 & 38 & 82 & 61 & 40 & 28 & 60 & 44 \\
\hline $\mathbf{F}$ & 50 & 23 & 76 & 47 & 38 & 24 & 57 & 37 \\
\hline $\mathbf{F}^{*}$ & 67 & 34 & 98 & 63 & 44 & 23 & 65 & 43 \\
\hline G & 76 & 42 & 106 & 68 & 55 & 32 & 77 & 40 \\
\hline $\mathbf{H}$ & 54 & 35 & 79 & 58 & 39 & 26 & 40 & 43 \\
\hline I & 37 & 19 & 61 & 38 & 27 & 15 & 45 & 29 \\
\hline $\mathbf{K}$ & 45 & - & 71 & 51 & 33 & 27 & 51 & 38 \\
\hline $\exp$ & 50 & 32 & 74 & 51 & 33 & 23 & 52 & 37 \\
\hline
\end{tabular}

- B3LYP-D3 (E, H) zeigt für die Strukturoptimierung und harmonische Schwingungsrechnung eine bessere Leistung als PBE0-D3 $(\mathbf{D}, \mathbf{G})$ oder insbesondere $\omega \mathrm{B} 97 \mathrm{xD}$ (B). Besonders gute Ergebnisse liefern die wellenfunktionsbasierten Methoden SCSMP2 (F) bzw. SCS-CC2 (I).

- Methode $\mathbf{C}$ weist eine erstaunlich große Rotverschiebung für das $\mathrm{MeOH}+\mathrm{DMFu}-\mathrm{C}-$ Dimer auf (s. Tab. 4.5), was zu einer im Vergleich zu den anderen B3LYP-D3-Metho$\operatorname{den}(\mathbf{E}, \mathbf{H})$ schwachen Leistung führt. Die anharmonische Variante $\mathbf{C}^{*}$ zeigt dagegen für die O-Dimere von $\mathrm{MeOH}$ eine unerwartet kleine Rotverschiebungsdifferenz von $0 \mathrm{~cm}^{-1}$ (außerhalb des in Abb. 4.10 dargestellten Bereichs).

Für den Donor MeOD (s. Abb. 4.11) liegen nur für eine Teilmenge der Beiträge Ergebnisse vor. Für die vorhandenen Daten ergeben sich jedoch ähnliche Resultate. Erneut zeigen $\mathbf{F}$ und $\mathbf{I}$ die beste Leistung, gefolgt von den DFT-Methoden E, H, G und K. Die 


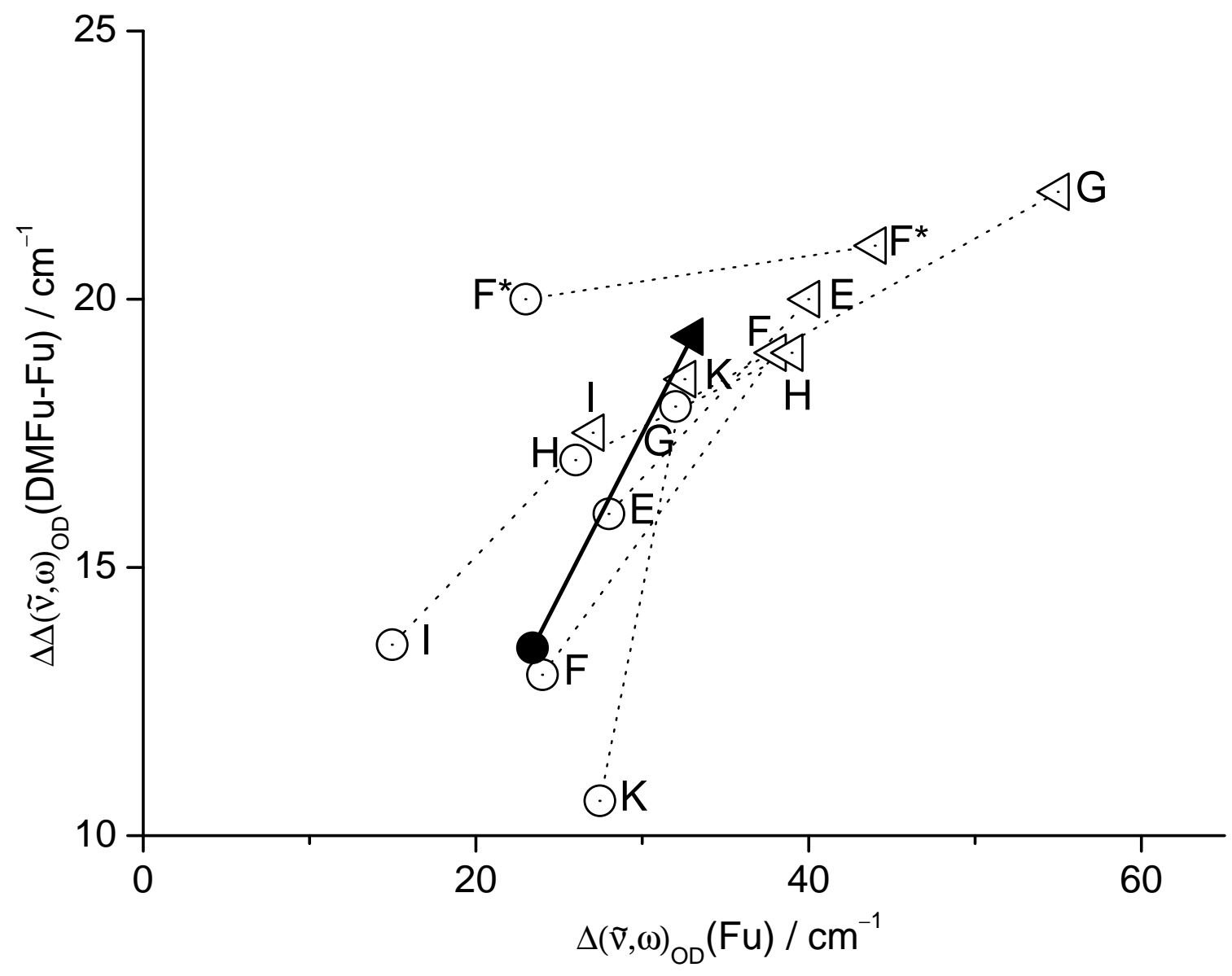

Abbildung 4.11: Korrelation der OD-Rotverschiebungsdifferenzen $\Delta \Delta(\tilde{v}, \omega)_{\mathrm{OD}}(\mathrm{DMFu}-\mathrm{Fu})$ mit der OD-Rotverschiebung $\Delta(\tilde{v}, \omega)_{\mathrm{OD}}(\mathrm{Fu})$ analog zu Abbildung 4.10, aber für MeOD. Hinweis zum Urheberrecht: „Reprinted from F. Chem. Phys. 2020, 152, 164303, ${ }^{[80]}$ with the permission of AIP Publishing...

anharmonisch korrigierte Methode $\mathbf{F}^{*}$ gibt den experimentellen Trend auch für MeOD weniger gut wieder als die zugehörige rein harmonische Methode $\mathbf{F}$. Bezogen auf die hier betrachteten $\mathrm{OH}-$ bzw. OD-Streckschwingungs-Rotverschiebungen und die im vorherigen Abschnitt 4.1.3 untersuchten relativen Energien scheint der Ansatz, Strukturen und Energien mit unterschiedlichen theoretischen Methoden zu beschreiben, für die Methodenkombinationen in $\mathbf{E}$ und $\mathbf{I}$ gut zu funktionieren.

Die anharmonischen Beiträge wie $\mathbf{F}^{*}$ können zusätzlich zu einem Vergleich der absoluten Bandenpositionen mit dem Experiment herangezogen werden. Die verfügbaren, strukturell mit dem Experiment kompatiblen Daten sind in Tabelle 4.6 den experimentellen Werten gegenübergestellt. Durch die Berücksichtigung der diagonalen Anharmonizität des OH-Oszillators ergibt sich zwar eine Verbesserung. Diese könnte jedoch auch über eine simple Skalierung der harmonischen Werte erreicht werden. Abgesehen davon 
Tabelle 4.6: Berechnete absolute Bandenpositionen $\tilde{v}_{\mathrm{OH}, \mathrm{D}}$ der $\mathrm{OH}(\mathrm{D})$-Streckschwingung von Methanol(-OD) im Heterodimer für die anharmonischen Methoden im Vergleich zu den experimentellen Werten (exp), jeweils in $\mathrm{cm}^{-1}$. Erneut sind Fälle, in denen die berechnete Struktur qualitativ den experimentellen Befunden widerspricht, mit ,-, "' gekennzeichnet. Hinweis zum Urheberrecht: „Reprinted from F. Chem. Phys. 2020, 152, 164303, ${ }^{[80]}$ with the permission of AIP Publishing.“.

\begin{tabular}{|c|c|c|c|c|c|c|c|c|}
\hline \multirow[b]{3}{*}{ Beitrag } & \multicolumn{4}{|c|}{$\mathrm{MeOH}$} & \multicolumn{4}{|c|}{ MeOD } \\
\hline & \multicolumn{2}{|c|}{$+\mathrm{Fu}$} & \multicolumn{2}{|c|}{$+\mathrm{DMFu}$} & \multicolumn{2}{|c|}{$+\mathbf{F u}$} & \multicolumn{2}{|c|}{$+\mathrm{DMFu}$} \\
\hline & $\mathrm{C}$ & $\mathrm{O}$ & $\mathrm{C}$ & $\mathrm{O}$ & $\mathrm{C}$ & $\mathrm{O}$ & $\mathrm{C}$ & $\mathrm{O}$ \\
\hline$A^{*}$ & - & - & 3608 & 3611 & & & & \\
\hline $\mathrm{C}^{*}$ & 3631 & 3667 & 3601 & 3667 & 2677 & 2690 & & \\
\hline $\mathrm{D}^{*}$ & 3618 & 3683 & 3597 & 3660 & & & & \\
\hline $\mathbf{F}^{*}$ & 3644 & 3677 & 3613 & 3648 & 2690 & 2711 & 2669 & 2691 \\
\hline $\exp$ & 3636 & 3654 & 3612 & 3635 & 2685 & 2695 & 2666 & 2681 \\
\hline
\end{tabular}

zeigen sich keine systematischen Vorteile über die verschiedenen Methoden und Heterodimere hinweg. Für die O-Dimere liegen die berechneten Werte überwiegend oberhalb der experimentellen Referenz, bei den C-Dimeren gibt es hingegen Abweichungen in beide Richtungen. Methode $\mathbf{A}^{*}$ zeigt in zwei Fällen qualitative strukturelle Abweichungen vom Experiment und lässt aufgrund der wenigen verbleibenden Daten keine systematische Analyse der Bandenpositionen zu. Die Methoden $\mathbf{C}^{*}$ und $\mathbf{D}^{*}$ überschätzen für $\mathrm{MeOH}$ die O-Dimer-Bandenpositionen und unterschätzen diejenigen der C-Dimere, wobei Methode $\mathbf{C}^{*}$ für MeOD+Fu für C- und O-Dimer zu geringe Wellenzahlen liefert. Somit weist einzig Methode $\mathbf{F}^{*}$ eine systematische Abweichung auf. Sämtliche berechneten Wellenzahlen sind für $\mathbf{F}^{*}$ zu groß, wobei die Abweichungen für die O-Dimere jeweils stärker ausgeprägt sind. Insgesamt führt jedoch auch dies zu einer schwächeren Leistung als im zugehörigen unkorrigierten harmonischen Fall (Methode F), wie die vorherige Analyse der Rotverschiebungsunterschiede $\Delta \Delta(\tilde{v}, \omega)_{\mathrm{OH}, \mathrm{D}}(\mathrm{DMFu}-\mathrm{Fu})$ gezeigt hat.

Die Ursachen für die beobachteten Schwächen der anharmonischen Methoden können ohne weitere systematische Untersuchungen nicht sicher bestimmt werden, sind vermutlich aber sowohl auf außerdiagonale Beiträge zur Anharmonizität als auch auf Unzulänglichkeiten der zugrundeliegenden HESSE-Matrizen zurückzuführen.

\subsubsection{Zusammenfassung}

Das Benchmarking - also die Überprüfung der Leistungsfähigkeit - theoretischer Methoden anhand experimenteller Referenzdaten bedarf aufgrund seiner Komplexität großer Sorgfalt, sowohl vonseiten des Experiments als auch vonseiten der Theorie. Zum einen können experimentelle Daten leicht fehlinterpretiert werden, ${ }^{[322]}$ beispielsweise wenn 


Eigenschaft
Energiedifferenz
Konformationstemperatur
Deuterierungseffekt
Isotopenaustauschtemperatur
Wellenzahlkorrelation

Charakter
Energie
Energie
HESSE-Matrix
HESSE-Matrix
HESSE-Matrix

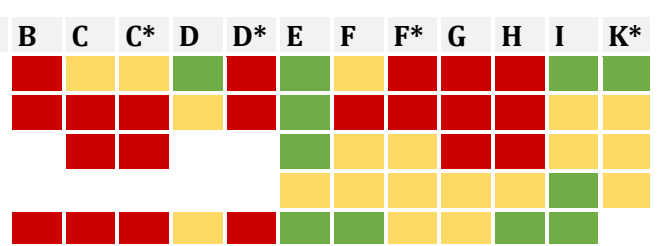

Abbildung 4.12: Bewertung der zusammengesetzten Methoden B-K* (s. Tab. 4.3 für Details), die in den Abbildungen 4.5, 4.6, 4.7, 4.8, 4.9, 4.10 und 4.11 auf der Basis ausgewählter Eigenschaften bezüglich ihrer Energie- und Hesse-Matrix-Leistungsfähigkeit untersucht wurden. Ein gutes Abschneiden ist grün, ein akzeptables Abschneiden gelb und ein schlechtes Abschneiden rot markiert. Keine Farbe bedeutet, dass keine Daten vorliegen oder die vorliegenden Daten qualitativ den experimentellen Befunden widersprechen. Bearbeitung von Fig. 11 in Ref. [80] (lizenziert unter CC BY 4.0).

die vom Forschenden erwarteten Ergebnisse sich scheinbar bestätigen. Zum anderen sind auf theoretischer Seite viele Lagen von Näherungen nötig, insbesondere wenn ein Vergleich zum Experiment erfolgen soll. ${ }^{[7]}$ So kann die ZPVE experimentell nicht umgangen werden, sodass für die Theoriemethoden Schwingungsrechnungen in der harmonischen oder einer anharmonischen Näherung nötig werden. Sollen Fehlinterpretationen aufgrund einer Vorbeeinflussung wechselseitig ausgeschlossen werden, bietet es sich an, Vergleichsstudien als Doppelblindstudien auszulegen. Diese haben für Kristallstrukturen, wo die Primärgrößen Struktur und Energie für nicht-kovalente Wechselwirkungen auch bei tiefen Temperaturen gut zugänglich sind, ${ }^{[323]}$ schon länger Tradition. ${ }^{[30]}$ Überschallexpansionstechniken machen die entsprechenden Größen auch für nicht-periodische, wesentlich kleinere Modellsysteme zugänglich und können deshalb einen wertvollen Beitrag zum Benchmarking theoretischer Methoden liefern, wenn Nicht-Gleichgewichtsaspekte ausreichend kontrolliert werden können. Die Ergebnisse zeigen, dass die Furan-Mikrosolvatation in dieser Hinsicht vielversprechend ist.

Die vorliegende Leistungsüberprüfung wurde in einem ersten Schritt als Doppelblindstudie durchgeführt, um unbeeinflusste experimentelle und rechnerische Ergebnisse zu erhalten. ${ }^{[75]}$ Erst im zweiten Schritt wurden alle Daten zusammengeführt, teilweise ergänzt und gemeinsam ausgewertet. Für den Vergleich der experimentellen und theoretischen Ergebnisse wurden dabei verschiedene Verfahren genutzt, die die Last der Bestimmung diverser Größen wie der IR-Bandenstärken oder der relativen Entartung zum Teil auf die Seite des Experiments („Experiment kommt Theorie entgegen“), zum Teil auf die Seite der Theorie („Theorie kommt Experiment entgegen“) legen. Ein direkterer Vergleich ist für die spektroskopischen Observablen wie die Rotverschiebungsdifferenz $\Delta \Delta(\tilde{v}, \omega)_{\mathrm{OH}, \mathrm{D}}(\mathrm{DMFu}-\mathrm{Fu})$ möglich.

Die Ergebnisse für die verschiedenen untersuchten Methoden sind in der farbmarkierten Abbildung 4.12 zusammengefasst. Nur zwei Methoden (E und I) zeigen eine gute (grün) bzw. akzeptable (gelb) Leistung für alle untersuchten Eigenschaften. Die weniger ressourcenintensive Methode $\mathbf{D}$ schneidet ebenfalls gut ab, hat aber keine Daten für Me- 
OD und konnte deshalb nicht in allen Analysen berücksichtigt werden. Ähnliches gilt für $\mathbf{K}^{*}$, das nur hinsichtlich der Energien und Deuterierungseffekte untersucht werden konnte. Methode $\mathbf{F}$ weist für die Konformationstemperatur eine unzureichende (rot) Leistung auf und alle weiteren Methoden versagen für mindestens zwei der getesteten Eigenschaften. Wenig überraschend wird eine striktere Abgrenzung der Leistungsfähigkeit der Methoden möglich, wenn die Last auf die Seite der Theorie gelegt wird, wie der Vergleich der Benchmarks der relativen Energien über Energiedifferenz und Konformationstemperatur zeigt. Insgesamt ergibt sich für die Energie-Benchmarks eine stärkere Diskrimination stärkerer von schwächeren Methoden als für die Benchmarks der Hesse-Matrix. Wo ein Vergleich möglich ist, schneiden anharmonische Methoden $\left({ }^{*}\right)$ konsistent schlechter ab als ihre harmonischen Pendants.

Eine längerfristige Herausforderung scheint somit die anharmonische Modellierung der Lösungsmittel-Bindungsstellenpräferenz in den Furanderivaten zu sein, dessen verschiedene Andockstellen für O-Wasserstoffbrücken in der Molekülebene (Op), bzw. O$(\mathrm{Ot})$ oder C-Wasserstoffbrücken $(\mathrm{Ct})$ von oberhalb der Ebene durch eher flache Barrieren getrennt sind. Auch wenn die aktuellen Befunde darauf hindeuten, dass die Energiedifferenzen sich bereits in der harmonischen Näherung gut beschreiben lassen, kann ohne eine Erweiterung der betrachteten Systeme nicht ausgeschlossen werden, dass Anharmonizität einen quantitativen oder sogar qualitativen Einfluss auf die Bindungsstellenpräferenz haben kann. Die Problematik der möglicherweise nur effektiven Symmetrie einiger experimentell ermittelter Strukturen deutet auf nicht-triviale Effekte großer Amplituden hin. Darüber hinaus kann ein erfolgreiches Abschneiden für zwei sehr ähnliche Furan(derivat)-Methanol-Komplexe und ihrer Isotopologe durchaus auf zufälliger Fehlerkompensation beruhen.

Soll diesem Umstand durch die Erweiterung der Furan-Mikrosolvatations-Leistungsüberprüfung Rechnung getragen werden, könnten einige der in Tabelle 4.1 (s. S. 103) aufgeführten weiteren Systeme herangezogen werden. Hierbei wäre vor allem das Abschneiden der bisher vielversprechendsten Methoden interessant. Einschränkungen ergeben sich vor allem durch die Molekülgröße einiger Systeme und durch den Umstand, dass in vielen Fällen die Bindungspräferenzen im Experiment nur qualitativ geordnet, nicht aber quantitativ erfasst werden können. Doch selbst auf Grundlage solcher qualitativen Befunde kann noch eine Diskrimination der Leistungsfähigkeit verschiedener Methoden möglich sein, wie die zahlreichen negativen Konformationstemperaturen im vorliegenden Benchmark zeigen. Das Endziel der Leistungsüberprüfung ist es, ein physikalisch plausibles Rechenprotokoll zu finden, das im Vergleich zum Experiment eine systematisch gute Leistungsfähigkeit aufweist.

\subsection{Alkohol-Anisol}

Wie zu Beginn des Kapitels 4 bereits erwähnt, bietet auch Anisol ${ }^{[324]}$ mit den freien Elektronenpaaren am Sauerstoffatom und dem kohlenstoffbasierten $\pi$-System seines Benzol- 
rings $\mathrm{O}$ - und C-zentrierte Andockstellen für Wasserstoffbrückenbindungsdonoren. Durch den mesomeren Effekt sind diese Andockstellen vergleichbar attraktiv. ${ }^{[36]}$ Anisol, seine Derivate und seine Komplexe gehören zu den bestuntersuchten aromatischen Gasphasen-Systemen. ${ }^{[4,262,264,322,325-333]}$ Für das Anthracen-Analogon von Anisol, 9-Methoxyanthracen, konnten schon 1992 mittels spektralen Lochbrennens O- und C-Dimere mit Methanol in einer Überschallexpansion nachgewiesen werden und die Untersuchung der Methanol-Anisol-Komplexe wurde dort bereits von den Autoren vorgeschlagen. ${ }^{\text {[34] }}$ Tatsächlich wurde das Methanol-Anisol-Waagensystem jedoch erst 2015 Jet-FTIR-spektroskopisch und theoretisch betrachtet. ${ }^{[50]}$ Dort zeigte sich eine Bevorzugung des ODimers gegenüber dem C-Dimer um etwa $1 \mathrm{~kJ} \cdot \mathrm{mol}^{-1}$. Analog zum Methanol-FuranWaagensystem ergaben sich aufgrund der unterschiedlichen Direktionalität der O- und C-Wasserstoffbrückenbindungen dabei für O- und C-Dimer systematisch unterschiedlich hohe Schwingungsnullpunktsenergien, sodass diese für die Berechnung des Energieunterschieds berücksichtigt werden mussten. Anhand von VPT2-Modellrechnungen auf B3LYP-D3(BJ)-Niveau wurden anharmonische Beiträge zu den betrachteten Energiedifferenzen von den Autoren jedoch auf weniger als $0.1 \mathrm{~kJ} \cdot \mathrm{mol}^{-1}$ abgeschätzt, sodass eine Berechnung der Schwingungsnullpunktsenergien in der harmonischen Näherung ausreichend zu sein scheint. ${ }^{[50]}$ Basierend auf den in dieser Studie ermittelten Minimumsstrukturen für O- und C-Dimer ist aufgrund der räumlichen Nähe zwischen den Bindungsstellen zudem eine kleine Umwandlungsbarriere und damit eine effektive Kühlung des Konformerengleichgewichts in der Überschallexpansion zu erwarten.

Die gute Eignung des Methanol-Anisol-Systems als intermolekulares Waagensystem (s. oben) konnte durch eine weitergehende, systematische Studie bestätigt werden. ${ }^{\text {[37] }}$ Durch Alkylierung des Anisolrings ließ sich das O/C-Gleichgewicht in Richtung des Cgebundenen Dimers verschieben. Auf Basis der so gewonnenen experimentellen Daten zur relativen Häufigkeit von O- und C-Konformeren konnte anschließend eine Bewertung der Leistung dreier beispielhafter quantenchemischer Methoden bezogen auf dieses Gleichgewicht angefertigt werden. Hierbei konnte gezeigt werden, dass basierend auf den experimentellen Daten zu den verschiedenen Methanol-Anisol(derivat)-Kombinationen eine Unterscheidung der Leistungsfähigkeit dieser Methoden möglich ist. Der Datensatz wurde später erweitert. ${ }^{\text {[31] }}$

Nachdem im vorangegangenen Kapitel 4.1 anhand eines relativ kleinen experimentellen Datensatzes eine größere Zahl quantenchemischer Methoden detailliert hinsichtlich ihrer Leistungsfähigkeit untersucht wurde, soll der Fokus im vorliegenden Kapitel auf der Verwendung des umfangreicheren, in den Referenzen [37] und [31] vorgestellten experimentellen Referenzdatensatzes liegen. Einen Überblick über die betrachteten Anisolderivate, sortiert nach Substitutionsgrad, gibt Abbildung 4.13. Methanol $(\mathrm{MeOH})$ wurde mit Anisol (An), 2-Methylanisol (2MAn), 3-Methylanisol (3MAn), 4-Methylanisol (4MAn), 2-Chloranisol (2ClAn), 4-Fluoranisol (4FAn), 4-Chloranisol (4ClAn), 4Bromanisol (4BrAn), 2,3-Dimethylanisol (23DMAn), 2,6-Dimethylanisol (26DMAn), 3,5Dimethylanisol (35DMAn), 2,3,5-Trimethylanisol (235TMAn) und 4-tert-Butylanisol (4tBuAn) kombiniert. Zusätzlich wurde Methanol-OD (MeOD) mit 35DMAn untersucht. 


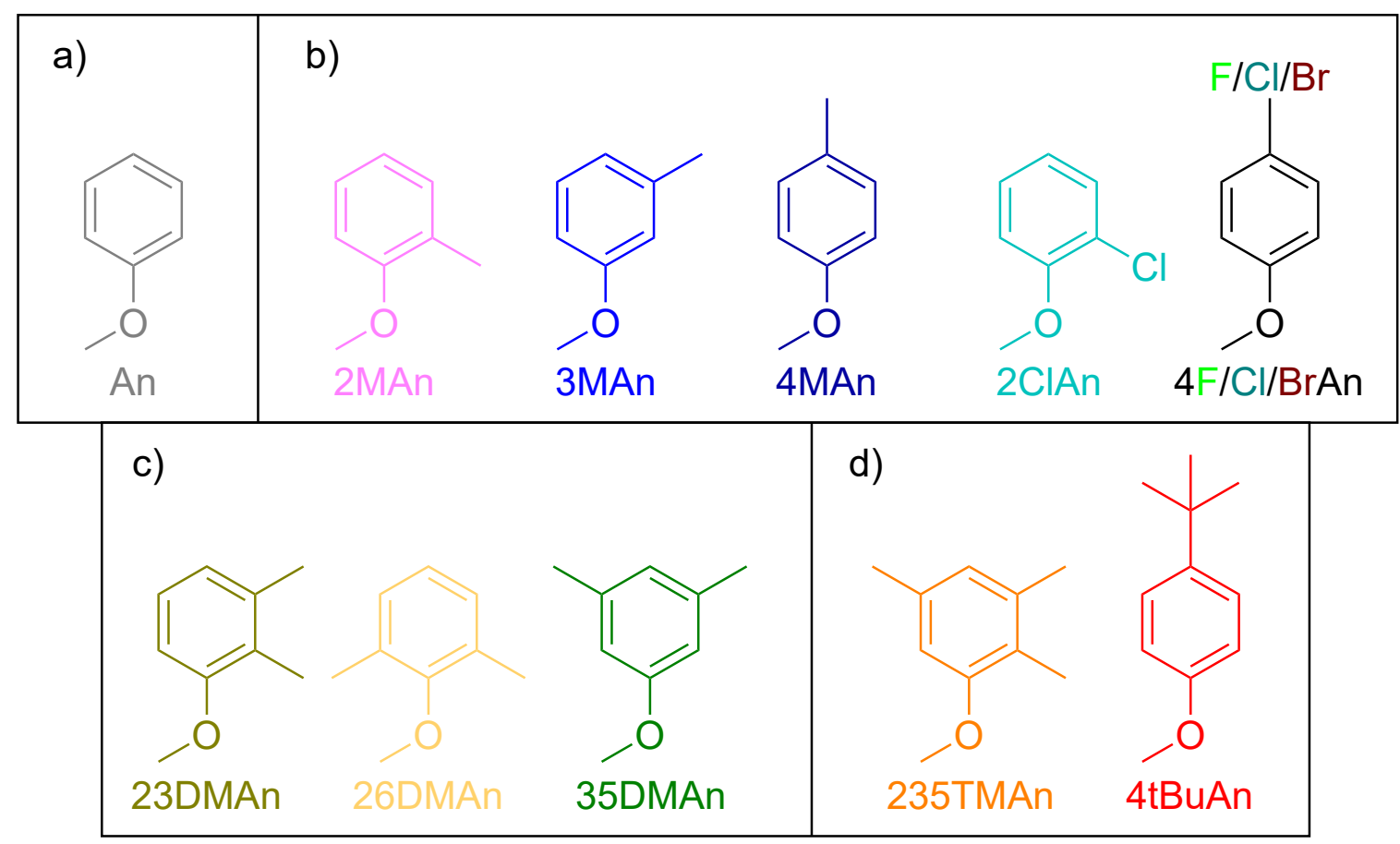

Abbildung 4.13: Lewis-Formeln der untersuchten Anisolderivate. a) unsubstituiertes Anisol (An, grau); b) einfach alkylierte (2-Methylanisol (2MAn, magenta), 3-Methylanisol (3MAn, hellblau), 4Methylanisol (4MAn, dunkelblau)) und einfach halogenierte Anisolderivate (2-Chloranisol (2ClAn, türkis), 4-Fluoranisol (hellgrün), 4-Chloranisol (4ClAn, blau-grün), 4-Bromanisol (4BrAn, braun)); c) zweifach alkylierte Anisolderivate (2,3-Dimethylanisol (23DMAn, dunkelgelb), 2,6-Dimethylanisol (26DMAn, hellgelb), 3,5-Dimethylanisol (35DMAn, dunkelgrün)); d) höher alkylierte Anisolderivate (2,3,5-Trimethylanisol (235TMAn, orange), 4-tert-Butylanisol (4tBuAn, rot)). Die hier verwendeten Farben werden im gesamten Kapitel 4.2 für die Zuordnung der Daten zum jeweiligen Anisolderivat genutzt.

Diese bereits vorhandenen experimentellen Daten werden im Rahmen der vorliegenden Arbeit vollständig neu ausgewertet (Kap. 4.2.1). Zudem wurden neue Messungen von tert-Butanol ${ }^{*}$ als Donormolekül mit An, 4MAn, 4FAn, 23DMAn, 26DMAn, 35DMAn und 235TMAn sowie von tert-Butanol-OD mit An, 4MAn, 4FAn, 35DMAn und 235TMAn angefertigt (ebenfalls Kap. 4.2.1). Die in Referenz [37] demonstrierte diskriminatorische Leistung des Anisol-Datensatzes soll anhand beispielhafter Benchmarks neuer quantenchemischer Rechnungen (Kap. 4.2.2) noch einmal überprüft werden (Kap. 4.2.3). Mit den experimentellen und berechneten $\mathrm{OH}$-Streckschwingungs-Rotverschiebungen soll eine weitere Größe ebenfalls erneut für den Benchmark genutzt werden (Kap. 4.2.4). Dazu werden mit B3LYP-D3 und M06-2X zwei populäre DFT-Funktionale nochmals in abgewandelter Form (u.a. mit anderen Basissätzen und Integrationsgittern) und mit DLPNO-

"Besser eigentlich „tert-Butylalkohol“, da kein tert-Butan existiert. Im Sinne der Vereinfachung wird im Folgenden dennoch die kompaktere Bezeichnung „tert-Butanol“ verwendet. 
$\operatorname{CCSD}(\mathrm{T})$ zusätzlich eine neue, wellenfunktionsbasierte lokale Korrelationsmethode herangezogen. Anhand der für letztere Methode verfügbaren local energy decomposition (LED) soll abschließend auch der Einfluss sekundärer Dispersionswechselwirkungen auf das O/C-Gleichgewicht analysiert werden (Kap. 4.2.5).

\subsubsection{Experimentelle Ergebnisse}

In den folgenden Abschnitten sollen die Ergebnisse der Jet-FTIR-spektroskopischen Untersuchungen der verschiedenen Alkohol-Anisol(derivat)-Kombinationen dargestellt werden. Alle Spektren wurden an der Filet-Jet-Apparatur (s. Kap. 2.2) aufgezeichnet. Für die Spektren der einzelnen Donor-Akzeptor-Kombinationen wurden dabei jeweils 100-700 Einzelscans gemittelt. Methanol (Roth, >99.9\%; SigmA-AldRich, $\geq 99.8 \%$ ), Methanol-OD (EURISO-TOP, $\left(\mathrm{HDO}+\mathrm{D}_{2} \mathrm{O}\right)<0.1 \%, 99.00 \% \mathrm{D}$ ), tert-Butanol (RoTH, $\geq 99 \%$ ), tert-Butanol-OD (CAmbridge Isotope Laboratories, OD 99\%), Anisol (Fluka, >99\%), 2-Methylanisol (Alfa Aesar, 99\%), 3-Methylanisol (Alfa Aesar, 99\%), 4-Methylanisol (Alfa Aesar, 99\%), 2-Chloranisol (Acros Organics, 98\%), 4-Fluoranisol (Acros OrgaNiCs, 99\%), 4-Chloranisol (Alfa AesAr, 99\%), 4-Bromanisol (Sigma-Aldrich, $\geq 99.0 \%$ ), 2,3-Dimethylanisol (Sigma-Aldrich, 97\%), 2,6-Dimethylanisol (Sigma-AldRICH, 98\%), 3,5-Dimethylanisol (Alfa Aesar, 99\%; Acros Organics, 99\%), 2,3,5-Trimethylanisol (TCI, >97.0\%) und 4-tert-Butylanisol (TCI, >98.0\%) wurden ohne weitere Aufbereitung über temperierte Sättiger in das Trägergas Helium (LINDE, 99.996\%) eingebracht. Die Konzentration der Donor- und Akzeptorstoffe konnte über die Temperatur der Sättiger und über zeitlich gesteuerte Magnetventile in den Zuleitungen zum Reservoir variiert werden. Die Überschallexpansion erfolgte dann durch die $(600 \cdot 0.2) \mathrm{mm}^{2}$ große Schlitzdüse bei einem Stagnationsdruck von 0.75 bar. Für die Aufnahme der Spektren im Bereich 4200-2450 $\mathrm{cm}^{-1}$ (Auflösung: $2 \mathrm{~cm}^{-1}$ ) wurde das Spektrometer BRUKer IFS $66 \mathrm{v} / \mathrm{S}$ mit einer $150 \mathrm{~W}$-Wolframlampe, $\mathrm{CaF}_{2}$-Optiken und dem InSb-Element eines InSb/MCTSandwich-Detektors bei einer Spiegelgeschwindigkeit von $80 \mathrm{kHz}$ betrieben.

\section{Methanol als Donor}

Für den einfachsten alkoholischen Wasserstoffbrückenbindungsdonor, Methanol (Me$\mathrm{OH})$, liegt der umfassendere experimentelle Anisol-Datensatz vor. Die hier besprochenen Jet-FTIR-Spektren wurden bereits in den Referenzen [37] (MeOH mit An, 2MAn, 3MAn, 4MAn, 23DMAn, 26DMAn, 35DMAn, 235TMAn, 4tBuAn) und [31] (zusätzlich $\mathrm{MeOH}$ mit 2ClAn, 4FAn, 4ClAn, 4BrAn sowie MeOD mit 35DMAn) verwendet. In Referenz [31] wurde dabei lediglich eine Beispielabbildung gezeigt, die diese experimentellen Daten beinhaltet. Es erfolgte jedoch keine Diskussion der Ergebnisse. Im Vergleich zu den genannten Referenzen wurde für die vorliegende Arbeit eine vollständige Neuauswertung der Spektren vorgenommen, deren Ergebnisse im Folgenden deswegen kurz dargestellt werden sollen.

Wie weiter oben bereits beschrieben, bietet Anisol zwei verschiedene Andockstellen 
(freie Elektronenpaare am O-Atom: O-Dimer; $\pi$-System des kohlenstoffbasierten Benzolrings: C-Dimer) für Wasserstoffbrückenbindungsdonoren wie Methanol, die sich experimentell aufgrund der unterschiedlichen Rotverschiebungen der Donor-OH-Streckschwingungsbande unterscheiden lassen. Während das C-Dimer dabei eine kleinere Rotverschiebung aufweist, die für $\mathrm{MeOH}+\mathrm{An}$ mit $57 \mathrm{~cm}^{-1}$ beispielsweise vergleichbar groß ist wie für das chemisch ähnliche Methanol-Toluol-Dimer, ${ }^{[50]}$ ist die Rotverschiebung für die O-gebundenen Dimere aufgrund der stärker lokalisierten Wasserstoffbrückenbindung größer. So beträgt sie für das O-Dimer von $\mathrm{MeOH}+\mathrm{An}$ beispielsweise $89 \mathrm{~cm}^{-1}$.

In Abbildung 4.14 sind die OH-Streckschwingungsbereiche zwischen Methanol-Monomer $\left(M, 3686 \mathrm{~cm}^{-1[154,297]}\right)$ und -Dimer $\left(\mathrm{MM}, 3575 \mathrm{~cm}^{-1}\right)$ der Spektren von Methanol mit den genannten Anisolderivaten nach steigendem Substitutionsgrad sortiert dargestellt. So sind in Abschnitt a) das unsubstituierte Anisol (grau) abgebildet, in Abschnitt b) die einfach substituierten Alkylanisole 2MAn (magenta), 3MAn (hellblau) und 4MAn (dunkelblau) und Halogenanisole (eingeschobener Ausschnitt) 2ClAn (türkis), 4FAn (hellgrün), 4ClAn (blau-grün) und 4BrAn (braun), in Abschnitt c) die zweifach alkylierten Anisolderivate 23DMAn (dunkelgelb), 26DMAn (hellgelb) und 35DMAn (dunkelgrün), sowie in Abschnitt d) die höher alkylierten Derivate 235TMAn (orange) und 4tBuAn (rot). Auf Basis der bereits vorliegenden Zuordnung der Methanol-Anisol(derivat)-Dimerbanden der dargestellten Alkylanisole zum C- $\left(\mathrm{MA}_{\mathrm{C}}\right)$ und O-Dimer $\left(\mathrm{MA}_{\mathrm{O}}\right)^{[37,50]}$ kann für die Halogenanisole eine analoge Zuordnung zu $\mathrm{C}$ - und $\mathrm{O}-$ Dimer erfolgen. Während die $\mathrm{MA}_{\mathrm{O}^{-}}$ Signale dabei eindeutig zu erkennen sind (Signale im Bereich $3615-3600 \mathrm{~cm}^{-1} \mathrm{im}$ grau unterlegten Kasten in Teil b) von Abb. 4.14), sind die $\mathrm{MA}_{C}$-Banden kaum signifikant intensiver als das umgebende Rauschen, insbesondere im Fall von 4FAn. Die in Tabelle 4.7 angegebenen Wellenzahlen stellen im Fall der Halogenanisole also eher den wahrscheinlichsten Fall einer möglichen $\mathrm{MA}_{\mathrm{C}}$-Bandenposition als einen sicheren experimentellen Wert dar. Entsprechend sind die für die Halogenanisole ermittelten Werte für die Bandenintensität $I_{C}$ als obere und daraus folgend die Verhältnisse $I_{\mathrm{O}} / I_{\mathrm{C}}$ als untere Grenze für den experimentellen Wert zu sehen.

Analog zum Vorgehen in Referenz [50] für Anisol bzw. in Kapitel 4.1.1 für die Furanderivate soll die Bandenzuordnung stichprobenartig noch einmal anhand eines Deuterierungsexperiments überprüft werden. Als Anisolderivat wird für die Stichprobe 35DMAn verwendet, da für dieses im Spektrum mit $\mathrm{MeOH}$ zwei Dimerbanden signifikanter Intensität bei 3619 und $3592 \mathrm{~cm}^{-1}$ erkennbar sind. Diese wurden auf Basis der Anisoldaten $^{[50]}$ dem C- $\left(3619 \mathrm{~cm}^{-1}\right)$ bzw. O-Dimer $\left(3592 \mathrm{~cm}^{-1}\right)$ zugeordnet. ${ }^{[37]}$ In Abbildung 4.15 ist der $\mathrm{OH}$-Streckschwingungsbereich für $\mathrm{MeOH}$ als Donor (unten) dem entsprechenden Spektralbereich für MeOD als Donormolekül gegenübergestellt. Analog zu Abbildung 4.3 (s. S. 105) wurden für den Vergleich die Bandenpositionen des Methanol-Monomers (M; $\mathrm{OH}: 3686 \mathrm{~cm}^{-1}$, OD: $2718 \mathrm{~cm}^{-1[154]}$ ) aufeinandergelegt. Zudem wurde die Wellenzahlachse des OD-Spektrums um den Faktor $\sqrt{2}$ gestreckt, um dem idealen, harmonischen Isotopeneffekt bei unendlicher Sauerstoffmasse und exaktem D/H-Massenverhältnis von 2 Rechnung zu tragen. Wie in Kapitel 4.1.1 erläutert, werden durch diese Skalierung anharmonische Einflüsse auf die Hydrid-Streckschwingungsbandenpositionen sofort sicht- 


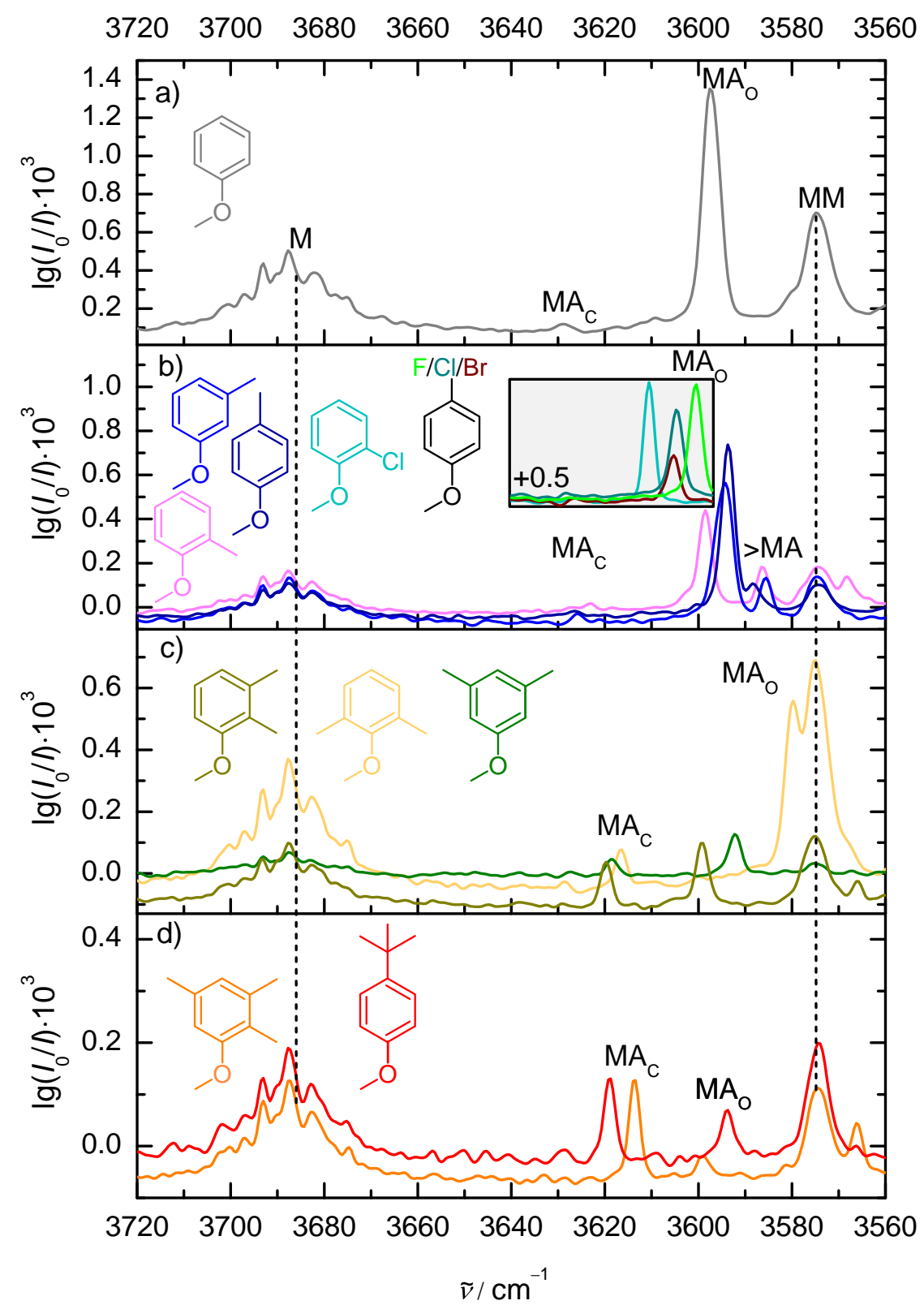

Abbildung 4.14: Übersicht der OH-Streckschwingungsbereiche der für die Auswertung genutzten Spektren von Methanol mit dem jeweils in der Spektrenfarbe abgebildeten Anisolderivat. Der Substitutionsgrad des Anisols nimmt von oben nach unten zu: a) unsubstituiert (An), b) einfach alkyliert (2MAn, 3MAn, 4MAn) bzw. halogeniert (2ClAn, 4FAn, 4ClAn, 4BrAn; grau hinterlegter Einschub), c) zweifach alkyliert (23DMAn, 26DMAn, 35DMAn), d) höher alkyliert (235TMAn, $4 \mathrm{tBuAn}$ ). Alle Spektren sind unskaliert dargestellt. Die Spektren der halogenierten Anisolderivate wurden entlang der Ordinate um 0.5 Einheiten nach oben verschoben. Die Banden des Metha-

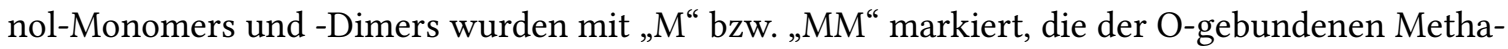
nol-Anisol(derivat)-Dimere mit ",MA $\mathrm{O}_{\mathrm{O}}$, die der entsprechenden C-gebundenen Dimere mit "MA ${ }_{C}$ “ und die höherer Aggregate mit „>MA“. Eine ähnliche Gegenüberstellung (ohne die Spektren von 2ClAn, 4FAn, 4ClAn und 4BrAn, sowie mit anderen Spektren für 35DMAn und 4tBuAn) findet sich in Fig. S6 in Ref. [37]. 


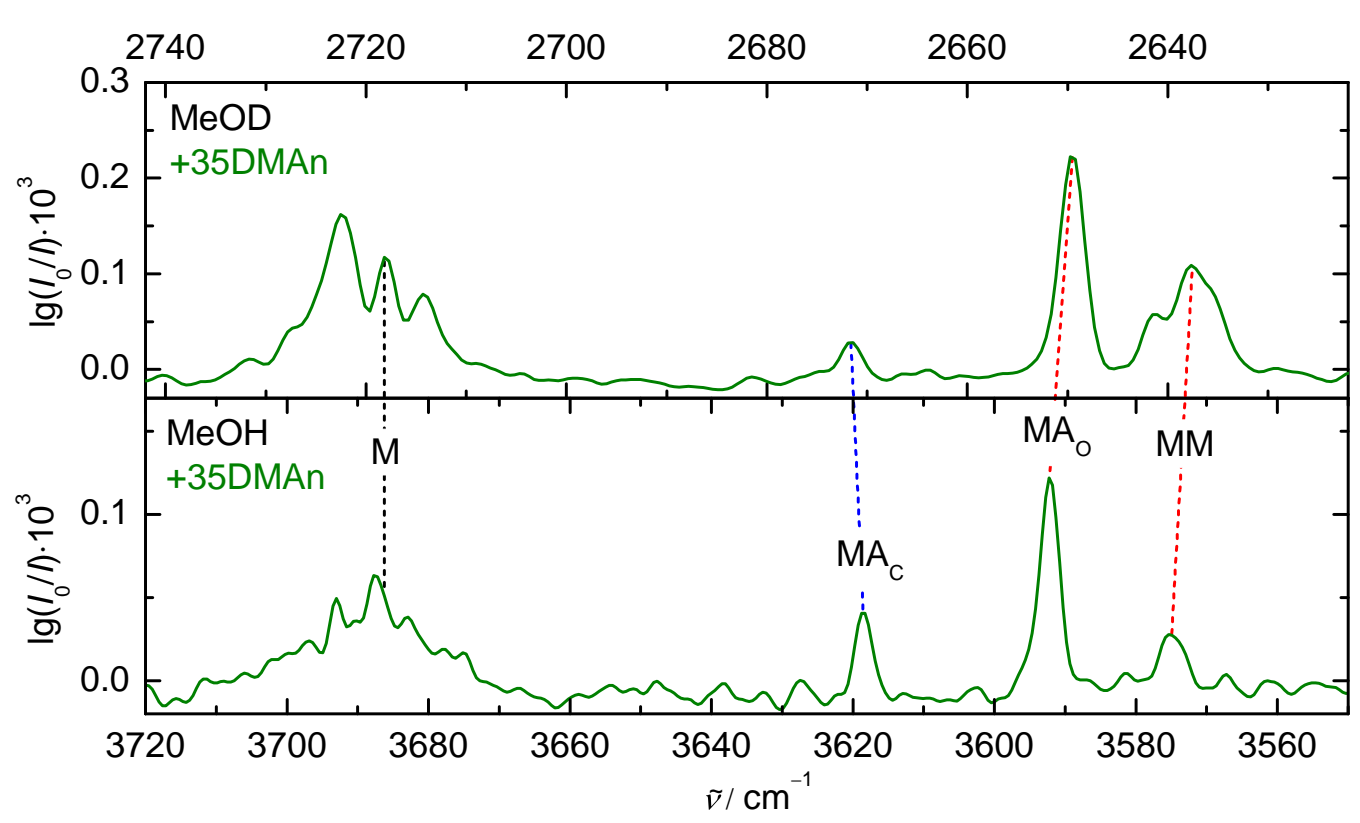

Abbildung 4.15: Gegenüberstellung der $\mathrm{OH}(\mathrm{D})$-Streckschwingungsbereiche der Spektren von $\mathrm{MeOD}$ (oben) und $\mathrm{MeOH}$ (unten) mit 35DMAn. Die Skala des OD-Spektrums wurde im Vergleich zum OH-Spektrum um den Faktor $\sqrt{2}$ gestreckt und beide Skalen wurden auf das Methanol-Monomer-Signal ausgerichtet. Die relevanten in den Spektren sichtbaren Banden wurden analog zu Abbildung 4.14 gekennzeichnet.

bar und erlauben eine Unterscheidung von O- und C-Dimeren. Dimere mit OH...O-Wasserstoffbrücke weisen dabei eine positive Steigung (rot markiert) auf, wie am Methanol-

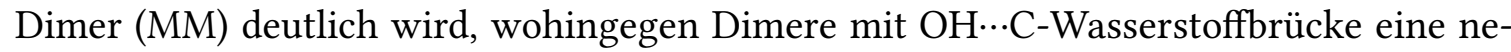
gative Steigung (blau markiert) besitzen. Die Betrachtung der Anharmonizität bestätigt somit die Bandenzuordnung zu $\mathrm{MA}_{\mathrm{C}^{-}}$und $\mathrm{MA}_{\mathrm{O}^{-}}$Dimer. Diese wird auch noch einmal durch die bei Deuterierung abnehmende Intensität der weniger rotverschobenen relativ zur stärker rotverschobenen Dimerbande bestätigt, da sich die Stabilität des C-Dimers im Vergleich zum O-gebundenen Dimer bei Deuterierung verringert (s. Kap. 4.1.1).

Für die weitere Auswertung und den Vergleich mit den Ergebnissen quantenchemischer Rechnungen (Kap. 4.2.3, 4.2.4) wurden die Positionen $\tilde{v}_{\mathrm{OH} / \mathrm{D}, \mathrm{C}}$ der OH-Streckschwingungsbanden des $\mathrm{C}$ - und $\tilde{v}_{\mathrm{OH} / \mathrm{D}, \mathrm{O}}$ des $\mathrm{O}$-gebundenen Dimers sowie das Intensitätsverhältnis $I_{\mathrm{O}} / I_{\mathrm{C}}$ dieser beiden Banden aus den in den Abbildungen 4.14 und 4.15 gezeigten Spektren von $\mathrm{MeOH}$ bzw. MeOD mit den verschiedenen Anisolderivaten bestimmt. Die Ermittlung der Bandenpositionen, Bandenintegrale und Peakhöhen erfolgte mit dem OpusProgrammpaket in der Version 7.8, wobei für die Berechnung der Intensitätsverhältnisse und ihrer Fehler das in Kapitel 2.3.3 beschriebene Verfahren genutzt wurde. Pro DonorAkzeptor-Kombination wurde jeweils nur ein aus 100-300 Einzelscans bestehendes Spektrum zur Auswertung herangezogen. Die Ergebnisse sind in Tabelle 4.7 dargestellt.

Werden die Bandenpositionen der mit $\mathrm{MeOH}$ als Donor gebildeten gemischten Dimere 
Tabelle 4.7: Aus den in den Abbildungen 4.14 und 4.15 dargestellten Spektren bestimmte Positionen der $\mathrm{OH}$-Streckschwingungsbanden des C- $\left(\tilde{v}_{\mathrm{OH} / \mathrm{D}, \mathrm{C}}\right)$ und O-gebundenen $\left(\tilde{v}_{\mathrm{OH} / \mathrm{D}, \mathrm{O}}\right)$ Dimers in $\mathrm{cm}^{-1}$ sowie Intensitätsverhältnisse $I_{\mathrm{O}} / I_{\mathrm{C}}$ dieser Dimerbanden von Methanol(-OD) mit den verschiedenen Anisolderivaten. Die in Klammern angegebenen Fehlerintervalle der Intensitätsverhältnisse wurden mit dem in Kapitel 2.3.3 beschriebenen Verfahren ermittelt.

\begin{tabular}{lrrr}
\hline Stoffgemisch & $\tilde{\boldsymbol{v}}_{\mathrm{OH} / \mathrm{D}, \mathrm{C}}$ & $\tilde{\boldsymbol{v}}_{\mathrm{OH} / \mathrm{D}, \mathrm{O}}$ & \multicolumn{1}{c}{$\boldsymbol{I}_{\mathrm{O}} / \boldsymbol{I}_{\mathrm{C}}$} \\
\hline $\mathrm{MeOH}+\mathrm{An}$ & 3629 & 3597 & $35(9)$ \\
$\mathrm{MeOH}+2 \mathrm{MAn}$ & 3623 & 3599 & $2(2) \cdot 10^{1}$ \\
$\mathrm{MeOH}+3 \mathrm{MAn}$ & 3626 & 3594 & $2(1) \cdot 10^{1}$ \\
$\mathrm{MeOH}+4 \mathrm{MAn}$ & 3626 & 3594 & $5(4) \cdot 10^{1}$ \\
$\mathrm{MeOH}+2 \mathrm{ClAn}$ & 3627 & 3611 & $23(6)$ \\
$\mathrm{MeOH}+4 \mathrm{FAn}$ & 3628 & 3601 & $2(2) \cdot 10^{2}$ \\
$\mathrm{MeOH}+4 \mathrm{ClAn}$ & 3628 & 3605 & $3(2) \cdot 10^{1}$ \\
$\mathrm{MeOH}+4 \mathrm{BrAn}$ & 3626 & 3605 & $1(2) \cdot 10^{1}$ \\
$\mathrm{MeOH}+23 \mathrm{DMAn}$ & 3620 & 3599 & $1.5(3)$ \\
$\mathrm{MeOH}+26 \mathrm{DMAn}$ & 3617 & 3580 & $5(8)$ \\
$\mathrm{MeOH}+35 \mathrm{DMAn}$ & 3619 & 3592 & $2.8(5)$ \\
$\mathrm{MeOH}+235 \mathrm{TMAn}$ & 3614 & 3599 & $0.20(3)$ \\
$\mathrm{MeOH}+4 \mathrm{tBuAn}$ & 3619 & 3594 & $0.6(2)$ \\
\hline $\mathrm{MeOD}+35 \mathrm{DMAn}$ & 2672 & 2650 & $7(2)$ \\
\hline
\end{tabular}

analysiert, so zeigt sich für die C-Dimerbande ausgehend von $3629 \mathrm{~cm}^{-1}$ für das unsubstituierte An mit steigender Alkylierung insgesamt ein Trend zu niedrigeren Wellenzahlen bis hin zu $3614 \mathrm{~cm}^{-1}$ für das dreifach alkylierte 235TMAn. In einem einfachen Modell ließe sich dies durch die aufgrund des +I-Effekts der Alkylsubstituenten steigende Elektronendichte im $\pi$-System des Benzolrings und die damit zunehmende Stärke der Wasserstoffbrückenbindung begründen. Die für die halogenierten Anisolderivate ermittelten Wellenzahlen $\tilde{v}_{\mathrm{OH}, \mathrm{C}}$ der C-Dimerbande liegen mit 3628-3626 cm $\mathrm{cm}^{-1}$ im Bereich der einfach alkylierten Anisole. Wie weiter oben bereits beschrieben, handelt es sich bei diesen Wellenzahlen aufgrund der äußerst schwachen, teilweise kaum gegenüber dem Rauschen signifikanten $\mathrm{MA}_{\mathrm{C}}$-Banden aber eher um beste Schätzungen für die mögliche Bandenposition als um definitive Werte.

Für die für alle Anisolderivate eindeutig vom Rauschen unterscheidbaren $\mathrm{MA}_{\mathrm{O}}$-Banden lässt sich hingegen keine Abhängigkeit der Bandenposition $\tilde{v}_{\mathrm{OH}, \mathrm{O}}$ vom Substitutionsgrad feststellen. Innerhalb eines Substitutionsgrades (vgl. Abschnitte a)-d) in Abb. 4.14) weisen einfach ortho- (also in 2-Position) substituierte Anisolderivate aber stets eine höhere ODimerbandenwellenzahl als nicht-ortho-substituierte Anisolderivate auf. Dies lässt sich durch den sterischen Anspruch der ortho-Substituenten und die dadurch gestörte Wasserstoffbrückenbindungsgeometrie erklären. Insgesamt liegen die $\mathrm{MA}_{\mathrm{O}}$-Banden im $\mathrm{Be}-$ 
reich 3605-3592 $\mathrm{cm}^{-1}$, mit einem Ausreißer zu höheren $\left(3611 \mathrm{~cm}^{-1}, 2 \mathrm{ClAn}\right)$ und einem zu niedrigeren Wellenzahlen $\left(3580 \mathrm{~cm}^{-1}, 26 \mathrm{DMAn}\right)$. Mithilfe der Ergebnisse der quantenchemischen Rechnungen können beide Fälle auf Besonderheiten in den jeweiligen $\mathrm{MA}_{\mathrm{O}^{-}}$ Dimerstrukturen zurückgeführt werden (s. Kap. 4.2.2).

Die ermittelten Intensitätsverhältnisse $I_{\mathrm{O}} / I_{\mathrm{C}}$ bestätigen den bereits in Abbildung 4.14 sichtbaren Trend der relativen Intensitätszunahme der $\mathrm{MA}_{\mathrm{C}}$-Dimerbande mit steigendem Substitutionsgrad des Anisols. Während die $\mathrm{MA}_{\mathrm{O}}$-Bande für un- oder einfach substituiertes Anisol eine um etwa ein bis zwei Größenordnungen höhere Intensität als die $\mathrm{MA}_{\mathrm{C}}$-Bande aufweist, beträgt der Intensitätsvorteil des $\mathrm{MA}_{\mathrm{O}}$-Dimers bei zweifacher Substitution weniger als eine Größenordnung. Für nochmals erhöhte Substitutionsgrade (235TMAn, 4tBuAn) zeigt dann die $\mathrm{MA}_{\mathrm{C}}$-Bande die höhere Intensität im Spektrum. Die relative Stabilität des C-gebundenen Dimers scheint mit steigendem Substitutionsgrad also tendenziell zuzunehmen, wobei für eine quantitative Analyse auch die individuellen Bandenstärken der OH-Streckschwingungen in den Dimeren berücksichtigt werden müssen (s. Kap. 2.3.2). Dies wird für den Vergleich mit den quantenchemischen Daten auch getan (Kap. 4.2.3).

Besonders für die Fälle mit schwacher $\mathrm{MA}_{C}$-Dimerbande ergeben sich aufgrund des schlechten Signal-zu-Rauschverhältnisses dieser Bande große Unsicherheiten in $I_{\mathrm{O}} / I_{\mathrm{C}}$. Dies gilt insbesondere auch für die Halogenanisole 4FAn, 4ClAn und 4BrAn. Für Me$\mathrm{OH}+26 \mathrm{DMAn}$ ergibt sich aufgrund erschwerter Bestimmung der $\mathrm{MA}_{\mathrm{O}}$-Bandenintensität durch partielle Überlappung der $\mathrm{MA}_{\mathrm{O}^{-}}$mit der Methanol-Homodimerbande $\mathrm{MM}$ (s. Abb. 4.14c), hellgelb) ebenfalls ein besonders großer Fehler im Intensitätsverhältnis, der ebenso wie der Fehler für $\mathrm{MeOH}+4 \mathrm{BrAn}$ größer als der eigentliche Wert ist. Die Fehlerbereiche erlauben also negative (und damit unphysikalische) Intensitätsverhältnisse, was verdeutlicht, dass die Bestimmung symmetrischer Fehlerbereiche für die genannten zwei Methanol-Anisol(derivat)-Kombinationen an ihre Grenzen stößt. Im Sinne einer einheitlichen Fehlerbetrachtung über die verschiedenen Systeme hinweg (s. auch Kap. 4.1 und 5) soll das Verfahren trotzdem auch für die beiden problematischen Fälle beibehalten werden.

Neben diesen in der Fehleranalyse erfassten statistischen Fehlern könnten die Intensitätsverhältnisse in einzelnen Fällen auch durch weitere, systematische Fehler verfälscht werden. Im Vergleich zu den Methanol-Furan(derivat)-Spektren (s. Kap. 4.1) mussten einige der Methanol-Anisol(derivat)-Spektren bei deutlich höheren Substanzkonzentrationen aufgezeichnet werden, um das C-gebundene Dimer überhaupt noch nachweisen zu können. In einigen Fällen (insbesondere $\mathrm{MeOH}+\mathrm{An}, \mathrm{MeOH}+2 \mathrm{MAn}, \mathrm{MeOH}+3 \mathrm{MAn}, \mathrm{Me}-$ $\mathrm{OH}+4 \mathrm{MAn}, \mathrm{MeOH}+26 \mathrm{DMAn}$ ) zeigen sich deshalb in den Spektren Hinweise auf größere Methanol-Anisol(derivat)-Aggregate (s. bspw. mit „>MA“ gekennzeichnete Banden in Abb. 4.14b)). Auch wenn es keine direkten Hinweise darauf gibt, kann hier nicht völlig ausgeschlossen werden, dass weitere Signale höherer Aggregate mit den Dimerbanden überlappen und so deren Intensität unbemerkt beeinflussen. Einen solchen ÜberlagerungsEinfluss auf die Bandenintensität zeigt das Beispiel der Überlappung der $\mathrm{MA}_{\mathrm{O}^{-}}$mit der MM-Bande im Fall von 26DMAn. Während die Überlappung der beiden Banden hier offen- 
sichtlich ist und deshalb zumindest zum Teil in der Auswertung der Bandenintensität erfasst werden kann, können weniger offensichtliche Bandenüberlagerungen die Intensität subtiler und unbemerkt verändern. Im gut erkennbaren Beispiel würde eine Messung bei verringerter Methanol-Konzentration und entsprechend weniger intensiver bis vollständig verschwundener $\mathrm{MM}$-Bande einen weniger verfälschten Blick auf die $\mathrm{MA}_{\mathrm{O}}$-Bandenintensität erlauben. Für eine akkurate Bestimmung der $\mathrm{MA}_{\mathrm{C}}$-Bandenintensität wäre dann im Gegenzug aber eine deutliche Reduzierung des Rauschniveaus durch eine Erhöhung der Zahl der gemittelten Scans nötig. Vergleichbares gilt für die anderen hier diskutierten Spektren, in denen Signale höherer Aggregate durch geringere Substanzkonzentrationen ebenfalls reduziert werden könnten, allerdings ebenso zulasten der $\mathrm{MA}_{\mathrm{C}}$-Intensität. Eine Verminderung des Rauschniveaus durch eine höhere Scanzahl ist wiederum jedoch nur zu einem gewissen Grad praktikabel, da der Zahl der pro Spektrum messbaren Scans durch den dafür nötigen Substanz- und Zeitaufwand Grenzen gesetzt sind. Es muss also ein Kompromiss zwischen der Zahl der betrachteten Systeme und dem Messaufwand pro System - und damit der erzielbaren Genauigkeit - gefunden werden, wobei eine höhere Zahl betrachteter Systeme potentiell mehr Vorteile für den Vergleich mit quantenchemischen Methoden mit sich bringt als eine weitere Verbesserung des Signal-zu-RauschVerhältnisses. ${ }^{[196]}$ Aus diesem Grund liegt der Fokus in der vorliegenden Studie auf der Untersuchung einer möglichst großen Zahl relevanter Methanol-Anisol(derivat)-Kombinationen und die teils recht großen Fehlerbereiche für die Intensitätsverhältnisse werden in Kauf genommen.

\section{tert-Butanol als Donor}

Als weiterer Wasserstoffbrückenbindungsdonor wurde tert-Butanol (tBuOH) in Verbindung mit mehreren Anisolderivaten verschiedener Substitutionsgrade untersucht. Auch hier sind $\mathrm{C}$ - und $\mathrm{O}$-gebundene gemischte $\mathrm{tBuOH}$-Anisol-Dimere zu erwarten, die bei ausreichend kleinem Energieunterschied simultan in der Überschallexpansion vorliegen sollten. In Abbildung 4.16 ist jeweils ein Beispielspektrum für jede tert-Butanol-Anisol(derivat)-Kombination dargestellt. Analog zur entsprechenden Methanol-Abbildung 4.14 sind die Spektren von oben beginnend nach steigendem Anisol-Substitutionsgrad sortiert (Abschnitte a)-d)) und die Spektren sind für die Zuordnung zum jeweiligen Anisolderivat farblich gekennzeichnet. Dargestellt ist jeweils der OH-Streckschwingungsbereich der Spektren zwischen dem $\mathrm{tBuOH}$-Monomer (T) und -Dimer (TT). Alle Spektren sind unskaliert abgebildet, einige Spektren sind jedoch zur Verbesserung der Übersichtlichkeit der Abbildung entlang der Ordinate verschoben (4FAn +0.25, 23DMAn +0.10, $26 \mathrm{DMAn}+0.05$ ). Für jede tert-Butanol-Anisol(derivat)-Kombination wurden Spektren bei verschiedenen Alkohol- und Anisolkonzentrationen aufgezeichnet, um die beobachteten gemischten Aggregat-Banden anhand ihrer Konzentrationsskalierung gemischten Dimeren (TA) oder höheren Aggregaten ( $>\mathrm{TA}$ ) zuzuordnen. In allen Fällen werden gemischte Dimerbanden sichtbar. Allerdings treten für jedes der untersuchten Anisolderivate Banden von tert-Butanol-Anisol(derivat)-Aggregaten auf, bei denen anhand ihrer Konzentra- 


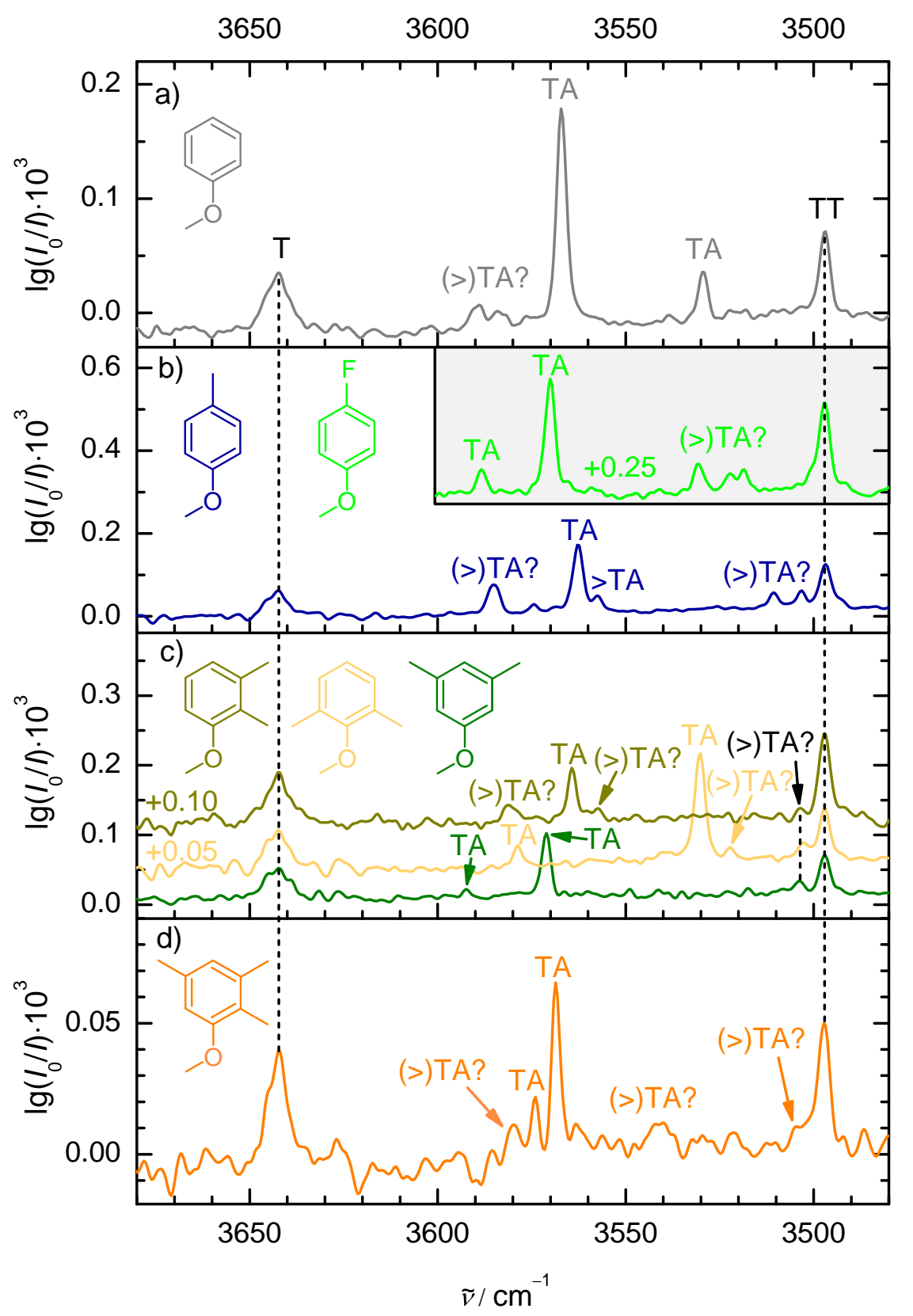

Abbildung 4.16: Abbildung analog zu Abbildung 4.14, aber für tert-Butanol (tBuOH) als Donor, mit dem nicht alle Anisolderivate untersucht wurden. Die unskalierten Spektren wurden teilweise zur Verbesserung der Übersichtlichkeit um die jeweils am Spektrum angegebene Zahl der Skaleneinheiten entlang der Ordinate verschoben. Die Banden des tBuOH-Monomers und -Dimers wurden mit „T“ bzw. „TT“ markiert. Banden, die tBuOH-Anisol-Dimeren zuzuordnen sind, wurden mit „TA“ gekennzeichnet und Banden, die zu höheren Aggregaten gehören, mit „>TA“. Kann keine eindeutige Zuordnung getroffen werden, sind die Banden mit „(>)TA?" beschriftet. Schwarze Beschriftungen beziehen sich auf mehrere Spektren, farbige Beschriftungen hingegen nur auf das Spektrum derselben Farbe. 
tionsskalierung im Rahmen der erzielten Signal-zu-Rausch-Verhältnisse keine eindeutige Unterscheidung von Dimeren und höheren Clustern möglich ist. Zur Verdeutlichung dieses Umstands sind die entsprechenden Banden mit „(>)TA?“ beschriftet. Somit könnten in allen Fällen möglicherweise mehr als zwei gemischte Dimerbanden vorliegen. Während eine der einem gemischten Dimer zugeordneten Banden dabei jeweils im Bereich 3580-3560 $\mathrm{cm}^{-1}$ liegt, weisen die weiteren Dimerbanden bzw. Banden, bei denen ein Dimeranteil nicht ausgeschlossen werden kann, je nach Anisolderivat höhere und/oder niedrigere Wellenzahlen auf. Aufgrund der fehlenden Systematik in den Bandenpositionen und der Vielzahl der jeweils für gemischte Dimere in Frage kommenden Banden ist eine Zuordnung zu C- und O-gebundenem Dimeren auf Grundlage der vorliegenden Daten nicht eindeutig möglich. Auch Messungen mit an der Hydroxylgruppe deuteriertem tert-Butanol (tBuOD) für An, 4MAn, 4FAn, 35DMAn und 235TMAn können nicht entscheidend zur Bandenzuordnung beitragen. Da sich die Spektren ohne eine solche Zuordnung nicht zur weiteren Analyse von O/C-Waagensystemen eignen, werden sie für den folgenden Vergleich mit den quantenmechanischen Rechnungen nicht herangezogen. Das Benchmarking der quantenchemischen Methoden wird somit ausschließlich auf Basis der Methanol-Daten durchgeführt.

Methoden, die sich in den MeOH-Benchmarks bewähren, könnten im Gegenzug als Zuordnungshilfe für die $\mathrm{tBuOH}-S p e k t r e n$ dienen. Vor allem für die Spektren mit einer dominanten Dimerbande (insbesondere $\mathrm{tBuOH}+\mathrm{An}, \mathrm{tBuOH}+35 \mathrm{DMAn}$ ) könnte ein Vergleich mit den berechneten Rotverschiebungen der auf dem Rechenniveau stabilsten Dimerstrukturen nützliche Hinweise liefern. Eine Voraussetzung dafür ist jedoch, dass die verwendete Rechenmethode das $\mathrm{OH}$-Streckschwingungsspektrum und das $\mathrm{O} / \mathrm{C}$-Konformerengleichgewicht für die tert-Butanol-Anisol(derivat)-Dimere treffend beschreiben kann.

\subsubsection{Quantenchemische Rechnungen}

Für die quantenchemische Betrachtung des Methanol-Anisol-O/C-Waagensystems wurden, wie bereits erwähnt, drei beispielhafte Methoden ausgewählt. Analog zur originalen Studie ${ }^{[37]}$ werden dabei die Dichtefunktionale B3LYP ${ }^{[85,86]}$ und M06-2X ${ }^{[82]}$ eingesetzt, wobei das B3LYP-Funktional erneut mit der D3-Dispersionskorrektur ${ }^{[83]}$ mit BECKE-JOHNSON-Damping (BJ) ${ }^{[90-93]}$ kombiniert wird. In der vorliegenden Arbeit wird jedoch zusätzlich der Dreikörperterm $(\mathrm{ABC})^{[83]}$ der Dispersionskorrektur berücksichtigt. Beide Funktionale werden nun mit dem def2-TZVP-Basissatz ${ }^{[103]}$ kombiniert, während zuvor die aug-cc-pVTZ-Basis verwendet wurde. Zudem kommen mit ORCA ${ }^{[107-109]}$ in der Version 4.0.1 und Gaussian16 in der Revision A.03 ${ }^{[111]}$ neue Programme bzw. aktualisierte Programmversionen zum Einsatz und für beide Funktionale werden nun feinere Integrationsgitter genutzt. Weitere Details zu den verwendeten Rechenmethoden finden sich in Kap. 2.1.1. Die Ergebnisse der B3LYP-D3(BJ,ABC)/def2-TZVP-Rechnungen wurden bereits für Fig. 4 in Referenz [31] genutzt.

Mit der DLPNO-CCSD(T)-Methode ${ }^{[122-125]}$ wird darüber hinaus eine wellenfunktionsbasierte Elektronenkorrelationsmethode eingesetzt, die in der Göbench-Leistungsüber- 


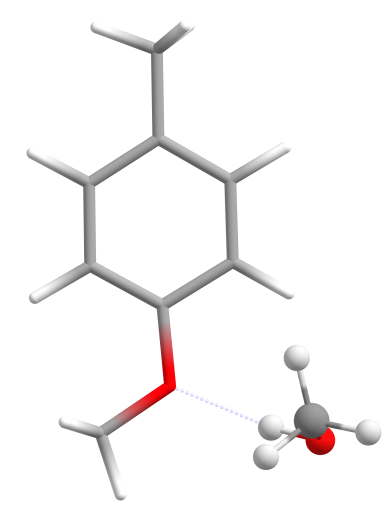

$\mathrm{O}-\mathrm{a}$

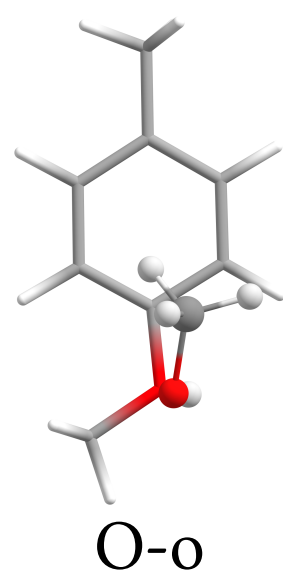

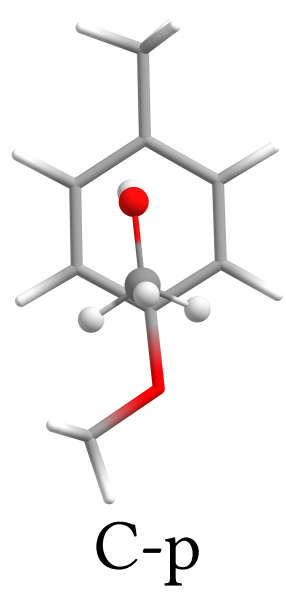

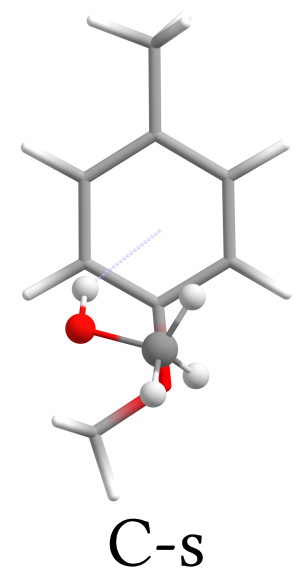

Abbildung 4.17: Die wichtigsten Dimerstrukturtypen der Methanol-Anisol(derivat)-Dimere am Beispiel von $\mathrm{MeOH}+4 \mathrm{MAn}$. Zur Verbesserung der Übersichtlichkeit ist 4MAn in Stäbchenform dargestellt. Die Beschriftung „O “ kennzeichnet eine Wasserstoffbrückenbindung zum Sauerstoffatom, „C“ eine solche zum kohlenstoffzentrierten $\pi$-System des Anisols. Die O-Dimere lassen sich in Strukturen mit Wasserstoffbrücke von außen in der Anisolebene (O-a) bzw. von oberhalb der Anisolebene (O-o) unterteilen. Die C-Dimere werden nach Ausrichtung der C-O-Bindung im Me$\mathrm{OH}$ klassifiziert. In den wichtigsten C-Dimerstrukturen ist diese entweder antiparallel zur $\mathrm{C}_{\text {aryl }}-\mathrm{O}$ Bindung im Anisol ausgerichtet (C-p), oder liegt senkrecht zur Anisol- $\mathrm{C}_{\text {aryl }}-\mathrm{O}$-Bindung, wobei sich das $\mathrm{MeOH}-\mathrm{O}-$ Atom auf der Ringseite der Anisol-Methoxy-Methylgruppe befindet (C-s). Die abgebildeten Strukturen wurden auf dem B3LYP-D3(BJ,ABC)/def2-TZVP-(B3-)Niveau optimiert.

prüfung besonders gut abschneiden konnte (s. Kap. 4.1). Die Betrachtung des Methanol-Anisol-Waagensystems auf konsistentem $\operatorname{CCSD}(\mathrm{T})$-Niveau wurde in der originalen Studie ${ }^{[37]}$ bereits vorgeschlagen und kann hier nun zumindest im Rahmen einer lokalen Näherung erfolgen. Für das Anisol-Waagensystem wird die DLPNO-CCSD(T)-Methode mit dem aug-cc-pVQZ-Basissatz ${ }^{[105,142,143]}$ (kurz aVQZ) verwendet. Die elektronische Energie $E^{\mathrm{el}}$ wird dabei über DLPNO-CCSD(T)-Einzelpunktrechnungen auf den mit der B3LYP-D3(BJ,ABC)/def2-TZVP-Methode optimierten Strukturen ermittelt. Für die Berechnung der schwingungsnullpunktskorrigierten Energie $E^{0}$ wird diese elektronische Energie dann mit der ZPVE aus der B3LYP-D3(BJ,ABC)/def2-TZVP-Rechnung kombiniert. Für die DLPNO-CCSD(T)-Rechnungen wird auf die TightPNO-Einstellung zurückgegriffen. Auf Grundlage der DLPNO-CCSD(T)-Rechnungen kann mit der LED-Methode ${ }^{[20]}$ anschließend eine Analyse der verschiedenen Beiträge zur Bindungsenergie im MethanolAnisol(derivat)-Dimer erfolgen. Weitere Details zur DLPNO-CCSD(T)- und LED-Methode und zu den dafür verwendeten Einstellungen sind in Kapitel 2.1.2 dargelegt.

In den quantenchemischen Rechnungen werden die Dimere der auch experimentell betrachteten Methanol-Anisol(derivat)-Kombinationen untersucht. Für 3MAn ergibt sich dabei eine Besonderheit, da dieses Anisolderivat als einziges der betrachteten Anisole zwei relevante Isomere aufweist. Diese unterscheiden sich in der Stellung der Methoxygruppe relativ zur 3-Methylgruppe. Beträgt der Diederwinkel $d\left(\mathrm{C}_{\text {methoxy }}, \mathrm{O}, \mathrm{C}_{\text {aryl, }, 1}, \mathrm{C}_{\text {aryl,2 }}\right)$ 
$180^{\circ}$, befinden sich die Methoxy- und die 3-Methylgruppe also auf entgegengesetzten „Molekülseiten“, wird dies im Folgenden als E-Isomer bezeichnet. Beträgt der zuvor genannte Diederwinkel hingegen $0^{\circ}$, befinden sich Methoxy- und 3-Methylgruppe also zusammen auf einer „Molekülseite“, resultiert das $Z$-Isomer. Im Rahmen der quantenchemischen Untersuchung des Methanol-Anisol-Waagensystems werden diese beiden Isomere als getrennte Spezies betrachtet.

Für die Optimierung der Methanol-Anisol(derivat)-Dimerstrukturen auf dem B3LYPD3(BJ,ABC)/def2-TZVP- (B3) oder M06-2X/def2-TZVP-Niveau (M6), zeigen sich für die unterschiedlichen Anisolderivate wiederkehrende Strukturmotive, die sich nach ihrer Konformation in verschiedene Dimerstrukturtypen einteilen lassen. Die wichtigsten dieser Strukturtypen sind am Beispiel der auf dem B3-Niveau optimierten Dimere von $\mathrm{MeOH}$ mit 4MAn in Abbildung 4.17 dargestellt. Analog zum Methanol-Furan-System (s. Kap. 4.1) erfolgt die Einteilung dabei zunächst nach der Wasserstoffbrückenbindungsstelle (O oder C). Für die O-Dimere lassen sich dann zwei Strukturmotive unterscheiden. Entweder erfolgt die Bindung von der Außenseite des Anisol-Moleküls, wobei die Wasserstoffbrücke in etwa in der Ringebene liegt (O-a), oder die Bindung erfolgt von oberhalb der Anisolebene (O-o). Wie dargestellt steht die $\mathrm{C}-\mathrm{O}-\mathrm{Bindung}$ im $\mathrm{MeOH}$ im $\mathrm{O}-\mathrm{a}-\mathrm{Dimer}$ etwa senkrecht zur Anisol-Ringebene. Im O-o-Dimer liegt diese Bindung im Gegensatz dazu in etwa parallel zur $\mathrm{C}_{\text {aryl }}-\mathrm{O}$-Bindung im Anisol, sodass die MeOH-Methylgruppe sekundäre Wechselwirkungen mit dem Anisol- $\pi$-System eingehen kann. Für die C-Dimere erfolgt die Unterscheidung nach Ausrichtung der $\mathrm{MeOH}-\mathrm{C}-\mathrm{O}-\mathrm{Bindung}$. So liegt diese im C-p-Dimer antiparallel zur $\mathrm{C}_{\text {aryl }}-\mathrm{O}$-Bindung des Anisols, wohingegen sie im C-s-Dimer senkrecht zur $\mathrm{C}_{\text {aryl }}-\mathrm{O}$-Bindung ausgerichtet ist. Im C-s-Dimer befindet sich das Sauerstoffatom des Methanol-Moleküls dabei auf der Anisol-Ringseite der Methoxy-Methylgruppe. Auf dem B3-Niveau lassen sich die jeweils stabilsten O- bzw. C-Dimerstrukturen für alle Anisolderivate einer dieser Strukturtypen zuteilen, auf dem M6-Niveau für fast alle Anisolderivate (s. unten).

Für einige Anisolderivate treten bei der Geometrieoptimierung weitere wiederkehrende Strukturmotive für das C-Dimer auf, die in Abbildung 4.18 erneut am Beispiel der auf dem B3-Niveau optimierten MeOH-4MAn-Dimere dargestellt sind. Im C-p'-Dimer liegt die Methanol-C-O-Bindung parallel zur Anisol- $\mathrm{C}_{\text {aryl }}-\mathrm{O}-\mathrm{Bindung}$ (vgl. antiparallel im C-p-Dimer), im C-s'-Dimer senkrecht dazu. Im Gegensatz zum C-s-Dimer befindet sich das $\mathrm{MeOH}-\mathrm{O}-$ Atom im C-s'-Dimer auf der der Methoxy-Methylgruppe entgegengesetzten Anisol-Ringseite. Außerdem liegt das Methanol-Molekül im C-s'-Dimer mittiger über dem Anisolring. Weitere C-Dimerstrukturen, bei denen sich andere Winkel zwischen der $\mathrm{MeOH}-\mathrm{C}-\mathrm{O}$ - und der Anisol- $\mathrm{C}_{\text {aryl }}-\mathrm{O}$-Bindung ergeben, werden für die folgende Auswertung als „C-r“-Dimere zusammengefasst.

Für die Halogenanisole ergibt sich am elektronegativen Halogenatom eine neue, zusätzliche Andockstelle für die Wasserstoffbrückenbindung. So gebundene Dimere werden mit „X“ gekennzeichnet. Prinzipiell sind hier analog zu den O-Dimeren zwei Strukturtypen „a" und „० (s. oben) denkbar, doch für die in 4-Position substituierten Halogenanisole (4FAn, 4ClAn, 4BrAn) wurde in den Optimierungen lediglich das in Abbildung $4.18 \mathrm{am}$ 

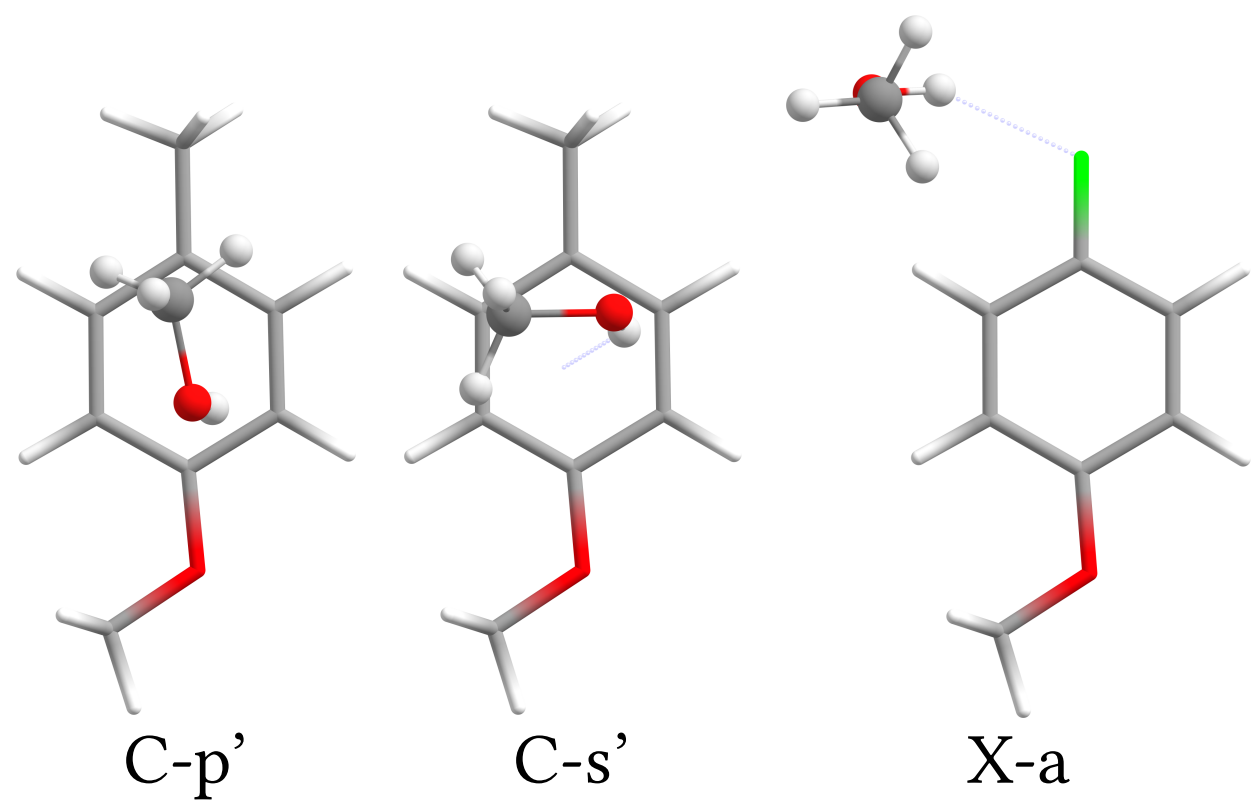

\begin{abstract}
Abbildung 4.18: Weitere Dimerstrukturtypen für die gemischten Methanol-Anisol(derivat)-Dimere. Darstellung analog zu Abbildung 4.17. Die Beschriftung „C" kennzeichnet erneut eine Wasserstoffbrücke zum kohlenstoffbasierten Anisol- $\pi$-System, „X“ eine solche zum Halogenatom in den halogenierten Anisolderivaten (hier 4FAn). Die C-gebundenen Dimerstrukturtypen werden erneut nach der Lage der C-O-Bindung im Methanol klassifiziert: „p'“: parallel zur Anisol- $\mathrm{C}_{\text {aryl }}-\mathrm{O}$ Bindung; ,"s": senkrecht zur Anisol- $\mathrm{C}_{\text {aryl }}-\mathrm{O}$-Bindung, mit dem Methanol-O-Atom auf der der Anisol-Methoxy-Methylgruppe abgewandten Ringseite. Die abgebildeten Strukturen wurden auch hier auf dem B3LYP-D3(BJ,ABC)/def2-TZVP-(B3-)Niveau optimiert.
\end{abstract}

Beispiel des auf dem B3-Niveau optimierten $\mathrm{MeOH}-4 \mathrm{FAn}$-Dimers dargestellte X-a-Dimer gefunden.

Neben den gezeigten Strukturtypen, die für mehrere bzw. alle Anisolderivate vorliegen, ergeben sich für zwei Anisolderivate spezifisch abweichende Strukturen. Diese sind in Abbildung 4.19 dargestellt. 2ClAn ist das einzige in 2-Position substituierte Anisolderivat, für das auf dem B3-Niveau ein O-a-Dimer gefunden wurde. Dieses hat sowohl auf B3- als auch auf M6-Niveau eine Struktur, die neben einer Wasserstoffbrückenbindung zum O- auch eine weitere, stark verzerrte Wasserstoffbrücke zum Cl-Atom aufzuweisen scheint. Der $\mathrm{OH} \cdots \mathrm{O}-$ Winkel in der Wasserstoffbrückenbindung zum O-Atom weicht dafür mit $140^{\circ}$ (B3) bzw. $131^{\circ}$ (M6) stärker von $180^{\circ}$ ab als für die O-a-Dimere der anderen Ani-

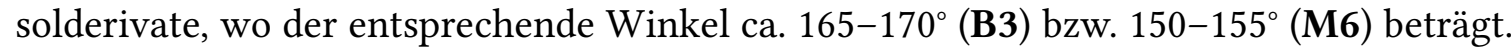
Mit beiden Dichtefunktionalen wird für $\mathrm{MeOH}+2 \mathrm{ClAn}$ weiterhin eine X-o-Dimerstruktur gefunden, bei der die Wasserstoffbrückenbindung zum Chloratom von oberhalb der Anisol-Ringebene erfolgt.

Weitere Besonderheiten zeigen sich für die Dimere mit 26DMAn. Aufgrund der beiden Methylgruppen in ortho- (also 2- und 6-) Position ist die Methoxygruppe in 26DMAn 


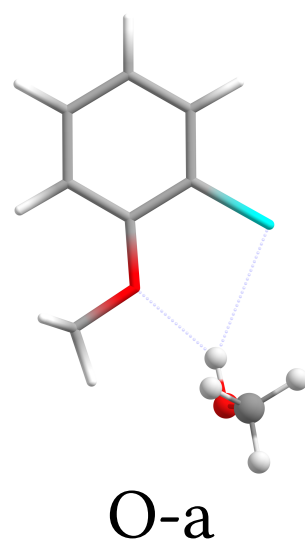

$\mathrm{MeOH}+2 \mathrm{ClAn}$

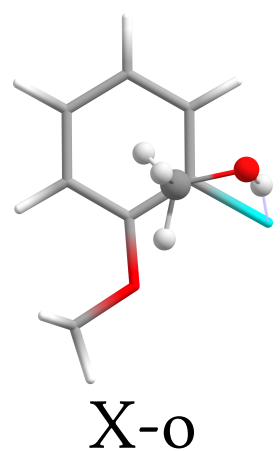

$\mathrm{MeOH}+2 \mathrm{ClAn}$

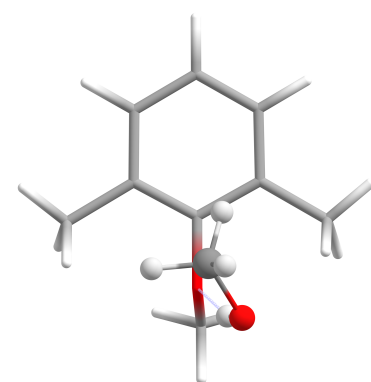

$\mathrm{O}-\mathrm{O}$

$\mathrm{MeOH}+26 \mathrm{DMAn}$

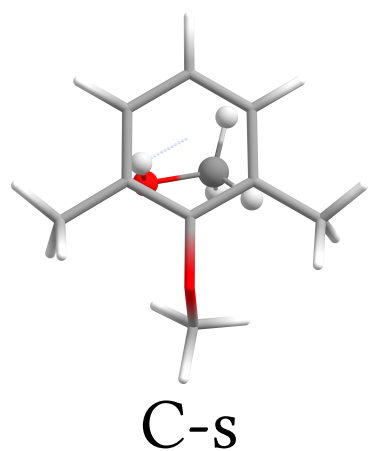

$\mathrm{MeOH}+26 \mathrm{DMAn}$

Abbildung 4.19: Relevante besondere Methanol-Anisolderivat-Dimerstrukturen. Von links nach rechts: Als einziges in 2-Position substituiertes Anisolderivat weist 2ClAn auf dem B3-Niveau ein O-a-Dimer auf, das eine Wasserstoffbrückenbindung zu O- und Cl-Atom besitzt. Als einziges Halogenanisol wurde für 2ClAn ein X-Dimer mit einer Wasserstoffbrückenbindung von oberhalb der Anisolebene gefunden. Im Fall von 26DMAn ist die Methoxygruppe aus der Ringebene gedreht, sodass eine besonders stabile Wasserstoffbrückenbindung zum Methoxy-O-Atom von oberhalb der Ringebene möglich wird. Im stabilsten $\mathrm{C}$-Dimer ist $\mathrm{MeOH}$ dann von der anderen Ringseite an 26DMAn gebunden. Erneut wurden die abgebildeten Strukturen auf dem B3LYP-D3(BJ,ABC)/ def2-TZVP-(B3-)Niveau optimiert.

aus der Ringebene gedreht. Die daraus resultierende O-o-Dimerstruktur ist am Beispiel der auf B3-Niveau optimierten Struktur ebenfalls in Abbildung 4.19 dargestellt. Das Methanol-Molekül ist darin von oberhalb der Anisol-Ringebene an das 26DMAn-Molekül gebunden, während die Methoxy-Methylgruppe unterhalb der Ringebene liegt. Im Gegensatz dazu liegt das $\mathrm{MeOH}-M o l e k u ̈ l$ im ebenfalls abgebildeten $\mathrm{C}$-s-Dimer, das für alle betrachteten Rechenniveaus die stabilste C-Dimerstruktur darstellt (s. unten), auf derselben Ringseite wie die Methoxy-Methylgruppe. In der dargestellten Ansicht entspricht dies einer Positionierung unterhalb der Ringebene. Für eine Umwandlung vom O-o- ins C-sDimer müsste das Methanol-Molekül somit die Ringseite wechseln, was eine im Vergleich zu den anderen Anisolderivaten deutlich erhöhte Umwandlungsbarriere erwarten lässt.

Um die Bedeutung der verschiedenen Dimerstrukturen für den Vergleich mit den experimentellen Daten besser beurteilen zu können, sollen zunächst die elektronischen Energien der optimierten Strukturen betrachtet werden. Die relativen elektronischen Energien $E^{\text {el }}$ sind für die verwendeten Methoden B3LYP-D3(BJ,ABC)/def2-TZVP (B3), DLPNOCCSD(T)/aVQZ//B3LYP-D3(BJ,ABC)/def2-TZVP (B3+) und M06-2X/def2-TZVP (M6) in den Tabellen $4.8\left(E^{\mathrm{el}, \mathrm{B} 3}\right), 4.9\left(E^{\mathrm{el}, \mathrm{B} 3+}\right)$ und $4.10\left(E^{\mathrm{el}, \mathrm{M} 6}\right)$ zusammengefasst. Die Energien sind jeweils relativ zum stabilsten $C$-Dimer angegeben. Die relative Energie des stabilsten O-Dimers entspricht damit direkt der Energiedifferenz $\Delta E_{\mathrm{O}-\mathrm{C}}^{\mathrm{el}}=E_{\mathrm{O}}^{\mathrm{el}}-E_{\mathrm{C}}^{\mathrm{el}}$ zwischen stabilstem O- und stabilstem C-Dimer. 
Tabelle 4.8: Elektronische Energien $E^{\text {el,B3 }}$ relativ zum stabilsten C-Dimer für das B3LYP-D3(BJ, ABC)/def2-TZVP-Theorieniveau (B3). Die Bezeichnungen der Dimerstrukturen folgen der in den Abbildungen 4.17 und 4.18 eingeführten Nomenklatur. C-Dimere, die durch die Klassifizierung in C-p, C-p', C-s und C-s' nicht erfasst werden, sind unter „C-r“ zusammengefasst. Die jeweils stabilste Struktur für jede Wasserstoffbrückenbindungsandockstelle ist fett hervorgehoben. Wurde eine Dimerstruktur für eine $\mathrm{MeOH}$-Anisol-Kombination nicht gefunden, bleibt das Feld leer.

\begin{tabular}{|c|c|c|c|c|c|c|c|c|c|}
\hline$E^{\mathrm{el}, \mathrm{B} 3} / \mathrm{kJ} \cdot \mathbf{m o l}^{-1}$ & O-a & O-o & C-p & C-p' & C-s & C-s' & C-r & $\mathrm{X}-\mathbf{o}$ & $\mathrm{X}-\mathbf{a}$ \\
\hline $\mathrm{MeOH}+\mathrm{An}$ & -1.58 & 0.04 & 2.44 & & 0.00 & 3.71 & & & \\
\hline $\mathrm{MeOH}+2 \mathrm{MAn}$ & & -1.65 & 1.98 & & 0.00 & & & & \\
\hline $\mathrm{MeOH}+3 \mathrm{MAn}(E)$ & -1.77 & & 0.91 & & 0.00 & 2.37 & & & \\
\hline $\mathrm{MeOH}+3 \mathrm{MAn}(Z)$ & -0.98 & 0.35 & 1.16 & & 0.00 & 2.52 & & & \\
\hline $\mathrm{MeOH}+4 \mathrm{MAn}$ & -1.74 & -0.07 & 1.57 & 2.75 & 0.00 & 1.57 & & & \\
\hline $\mathrm{MeOH}+2 \mathrm{ClAn}$ & -2.89 & -1.82 & 3.52 & & 0.00 & & 2.84 & 5.48 & 5.00 \\
\hline $\mathrm{MeOH}+4 \mathrm{FAn}$ & -2.68 & -0.70 & 4.37 & & 0.00 & & 4.38 & & 3.29 \\
\hline $\mathrm{MeOH}+4 \mathrm{ClAn}$ & -2.07 & -0.27 & 4.20 & 2.60 & 0.00 & & & & 2.48 \\
\hline $\mathrm{MeOH}+4 \mathrm{BrAn}$ & -1.95 & -0.20 & 4.19 & 2.34 & 0.00 & & 2.33 & & 1.71 \\
\hline $\mathrm{MeOH}+23 \mathrm{DMAn}$ & & -1.67 & 0.52 & 2.29 & 0.00 & & & & \\
\hline $\mathrm{MeOH}+26 \mathrm{DMAn}$ & & -4.23 & 4.15 & 5.86 & 0.00 & & 2.80 & & \\
\hline $\mathrm{MeOH}+35 \mathrm{DMAn}$ & -1.11 & 0.39 & 0.19 & & 0.00 & 1.44 & 0.42 & & \\
\hline $\mathrm{MeOH}+235 \mathrm{TMAn}$ & & -1.01 & 0.31 & & 0.00 & 1.64 & & & \\
\hline $\mathrm{MeOH}+4 \mathrm{tBuAn}$ & 0.44 & 1.87 & 1.86 & & 0.00 & 2.02 & 1.82 & & \\
\hline
\end{tabular}

Für die B3-Methode (s. Tab. 4.8) zeigt sich dabei, dass das C-s-Dimer in allen Fällen die stabilste C-Dimerstruktur darstellt. Für alle Anisolderivate wurden jeweils ein bis drei weitere C-Dimerstrukturen gefunden, die jedoch ca. 0.2 (C-p von $\mathrm{MeOH}+35 \mathrm{DMAn})$ bis $5.9 \mathrm{~kJ} \cdot \mathrm{mol}^{-1}$ (C-p' von $\left.\mathrm{MeOH}+26 \mathrm{DMAn}\right)$ weniger stabil sind. Bei den O-Dimeren zeigt sich ein vergleichbar systematisches Bild. Existiert eine O-a-Dimerstruktur, stellt diese das O-Minimum dar. O-a-Dimerstrukturen wurden für alle Anisolderivate gefunden, die nicht in 2- (also ortho-) Position substituiert sind. Eine Ausnahme bildet hier das 2ClAn, für das trotz der Halogenierung in 2-Position eine O-a-Dimerstruktur (s. Abb. 4.19) entdeckt wurde. O-o-Dimerstrukturen wurden für alle Anisole bis auf das E-Konformer von $3 \mathrm{MAn}$ gefunden. Außer für $4 \mathrm{tBuAn}$ ist mindestens eine der O-Dimerstrukturen stabiler als die stabilste C-Dimerstruktur. Für die halogenierten Anisolderivate sind die X-Dimere in keinem Fall stabiler als das jeweils stabilste O- und C-Dimer. Im Fall von 2ClAn, wo zwei verschiedene X-Dimerstrukturen gefunden wurden, stellt X-a die elektronisch stabilere Struktur dar.

Leichte Änderungen ergeben sich, wenn die elektronischen Energien der mit der B3Methode optimierten Strukturen auf dem deutlich höheren DLPNO-CCSD(T)-Niveau berechnet werden (s. Tab. 4.9). So löst auf dem B3+-Niveau in drei Fällen C-p C-s als stabilste Dimerstruktur ab, wenn auch nur um 0.02 (23DMAn), 0.39 (35DMAn) bzw. $0.52 \mathrm{~kJ} \cdot \mathrm{mol}^{-1}$ (235TMAn). Aufseiten der O-Dimere ergeben sich zwar keine qualitativen Änderungen, 
Tabelle 4.9: Analog zu Tabelle 4.8, aber für elektronische Energien $E^{\text {el,B3+ }}$ (Theorieniveau: DLPNO-CCSD(T)/aVQZ//B3LYP-D3(BJ,ABC)/def2-TZVP (B3+)).

\begin{tabular}{|c|c|c|c|c|c|c|c|c|c|}
\hline$E^{\mathrm{el}, \mathrm{B} 3+} / \mathrm{kJ} \cdot \mathrm{mol}^{-1}$ & O-a & O-o & C-p & $C-p^{\prime}$ & C-s & C-s' & C-r & $\mathbf{X}-\mathbf{o}$ & $\mathbf{X}-\mathbf{a}$ \\
\hline $\mathrm{MeOH}+\mathrm{An}$ & -2.42 & -1.43 & 1.27 & & 0.00 & 3.08 & & & \\
\hline $\mathrm{MeOH}+2 \mathrm{MAn}$ & & -2.29 & 0.97 & & 0.00 & & & & \\
\hline $\mathrm{MeOH}+3 \mathrm{MAn}(E)$ & -2.48 & & 0.25 & & 0.00 & 2.25 & & & \\
\hline $\mathrm{MeOH}+3 \mathrm{MAn}(Z)$ & -1.71 & -1.25 & 0.50 & & 0.00 & 1.58 & & & \\
\hline $\mathrm{MeOH}+4 \mathrm{MAn}$ & -2.27 & -1.60 & 0.30 & 1.93 & 0.00 & 1.95 & & & \\
\hline $\mathrm{MeOH}+2 \mathrm{ClAn}$ & -3.51 & -2.61 & 2.21 & & 0.00 & & 1.57 & 3.82 & 4.59 \\
\hline $\mathrm{MeOH}+4 \mathrm{FAn}$ & -3.14 & -2.08 & 2.96 & & 0.00 & & 2.85 & & 2.79 \\
\hline $\mathrm{MeOH}+4 \mathrm{ClAn}$ & -2.54 & -1.53 & 3.03 & 1.90 & 0.00 & & & & 2.46 \\
\hline $\mathrm{MeOH}+4 \mathrm{BrAn}$ & -2.24 & -1.54 & 2.43 & 0.53 & 0.00 & & 0.66 & & 0.16 \\
\hline $\mathrm{MeOH}+23 \mathrm{DMAn}$ & & -2.24 & 0.00 & 1.59 & 0.02 & & & & \\
\hline $\mathrm{MeOH}+26 \mathrm{DMAn}$ & & -4.42 & 3.98 & 5.39 & 0.00 & & 1.84 & & \\
\hline $\mathrm{MeOH}+35 \mathrm{DMAn}$ & -1.35 & -0.82 & 0.00 & & 0.39 & 1.20 & 0.82 & & \\
\hline $\mathrm{MeOH}+235 \mathrm{TMAn}$ & & -1.14 & 0.00 & & 0.52 & 1.43 & & & \\
\hline $\mathrm{MeOH}+4 \mathrm{tBuAn}$ & -0.16 & 0.44 & 0.94 & & 0.00 & 1.70 & 0.80 & & \\
\hline
\end{tabular}

insgesamt werden die O-Dimere durch die Berücksichtigung der elektronischen DLPNO$\operatorname{CCSD}(\mathrm{T})$-Energie im Vergleich zur reinen B3-Methode gegenüber dem jeweils stabilsten C-Dimer jedoch um ca. $0.2-1.6 \mathrm{~kJ} \cdot \mathrm{mol}^{-1}$ stabilisiert. Insbesondere die O-o-Dimere profitieren dabei auf dem B3+-Niveau. Auch die X-Dimere zeigen eine Stabilisierung gegenüber der C-Minimumsstruktur. Während die Änderungen für 2ClAn, 4FAn und 4ClAn dabei entweder klein sind oder bei relativen Energien oberhalb von $2 \mathrm{~kJ} \cdot \mathrm{mol}^{-1}$ erfolgen und damit für das experimentelle Konformerengleichgewicht vermutlich nicht von Bedeutung sind, ergibt sich für das X-a-Dimer von 4BrAn eine Stabilisierung um knapp $1.6 \mathrm{~kJ} \cdot \mathrm{mol}^{-1}$ von $1.71 \mathrm{~kJ} \cdot \mathrm{mol}^{-1}$ auf dem B3- zu $0.16 \mathrm{~kJ} \cdot \mathrm{mol}^{-1}$ auf dem B3+-Niveau. Zwar ist das X-aDimer damit weiterhin weniger stabil als beide O- und die stabilste C-Dimerstruktur von $\mathrm{MeOH}+4 \mathrm{BrAn}$, aber relativ zum $\mathrm{C}$-Dimer nur um einen vergleichsweise geringen Betrag.

Ein deutlich anderes Bild zeigt sich bei Betrachtung der auf dem M6-Niveau berechneten elektronischen Energien, die in Tabelle 4.10 zusammengefasst sind. Bei der Geometrieoptimierung mit der M6-Methode wurden für alle Anisolderivate sowohl O-a- als auch O-o-Dimerstrukturen gefunden. Analog zur B3-Methode liegt die stabilere O-Dimerstruktur dabei zwar erneut für alle Anisolderivate bis auf 4tBuAn bei einer niedrigeren elektronischen Energie als die stabilste C-Dimerstruktur, allerdings entspricht diese stabilere O-Dimerstruktur auf dem M6-Theorieniveau in allen Fällen dem O-o-Strukturtyp. Das O-a-Dimer wird dagegen bei deutlich höheren Energien vorhergesagt und liegt in den meisten Fällen sogar oberhalb der jeweils stabilsten C-Dimerstruktur. Letztere entspricht auf dem M6-Niveau nur in 7 Fällen der C-s-, in 5 Fällen dagegen der C-p- und in 2 Fällen einer C-r-Struktur (235TMAn und 4tBuAn). Die beiden relevanten C-r-Strukturen sind 
Tabelle 4.10: Analog zu Tabelle 4.8, aber für elektronische Energien $E^{\mathrm{el}, \mathrm{M} 6}$ (Theorieniveau: M06-2X/def2-TZVP (M6)).

\begin{tabular}{|c|c|c|c|c|c|c|c|c|c|}
\hline$E^{\mathrm{el}, \mathrm{M} 6} / \mathbf{k J} \cdot \mathbf{m o l}^{-1}$ & O-a & O-o & C-p & C-p' & C-s & C-s' & C-r & X-o & $\mathbf{X}-\mathbf{a}$ \\
\hline $\mathrm{MeOH}+\mathrm{An}$ & 0.33 & -1.39 & 0.64 & & 0.00 & 3.36 & & & \\
\hline $\mathrm{MeOH}+2 \mathrm{MAn}$ & 0.13 & -1.43 & 0.00 & & 0.04 & & 1.63 & & \\
\hline $\mathrm{MeOH}+3 \mathrm{MAn}(E)$ & 0.68 & -0.75 & 0.00 & 3.15 & 0.63 & 3.13 & & & \\
\hline $\mathrm{MeOH}+3 \mathrm{MAn}(Z)$ & 1.07 & -1.00 & 0.00 & 1.96 & 0.45 & 1.10 & & & \\
\hline $\mathrm{MeOH}+4 \mathrm{MAn}$ & 0.03 & -1.40 & 0.00 & 1.15 & 0.00 & 1.73 & & & \\
\hline $\mathrm{MeOH}+2 \mathrm{ClAn}$ & -2.82 & -3.00 & 2.06 & & 0.00 & 7.25 & & 5.43 & 6.45 \\
\hline $\mathrm{MeOH}+4 \mathrm{FAn}$ & -0.84 & -1.90 & 2.71 & 3.18 & 0.00 & & & & 4.04 \\
\hline $\mathrm{MeOH}+4 \mathrm{ClAn}$ & -0.22 & -1.71 & 2.93 & 1.93 & 0.00 & & & & 3.64 \\
\hline $\mathrm{MeOH}+4 \mathrm{BrAn}$ & -0.10 & -1.69 & 3.04 & 1.84 & 0.00 & & & & 3.00 \\
\hline $\mathrm{MeOH}+23 \mathrm{DMAn}$ & 1.91 & -0.76 & 0.00 & 3.31 & 0.59 & & & & \\
\hline $\mathrm{MeOH}+26 \mathrm{DMAn}$ & 2.43 & -0.28 & 6.41 & & 0.00 & & 3.20 & & \\
\hline $\mathrm{MeOH+35DMAn}$ & 1.68 & -0.11 & 0.00 & 2.29 & 1.31 & 1.13 & & & \\
\hline $\mathrm{MeOH}+235 \mathrm{TMAn}$ & 2.60 & -0.25 & 0.08 & 3.03 & & 2.12 & 0.00 & & \\
\hline $\mathrm{MeOH}+4 \mathrm{tBuAn}$ & 2.80 & 0.68 & 0.56 & & 2.58 & 0.88 & 0.00 & & \\
\hline
\end{tabular}

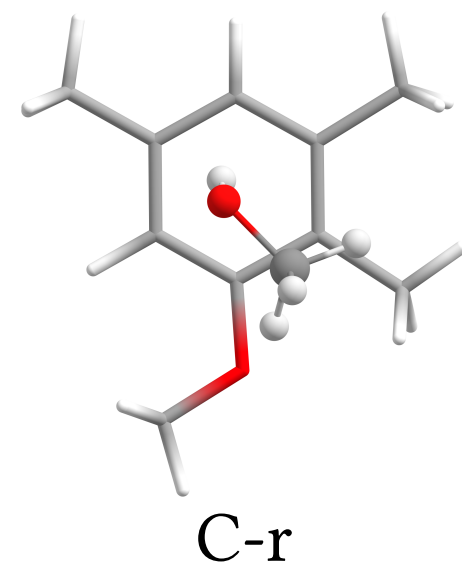

$\mathrm{MeOH}+235 \mathrm{TMAn}$

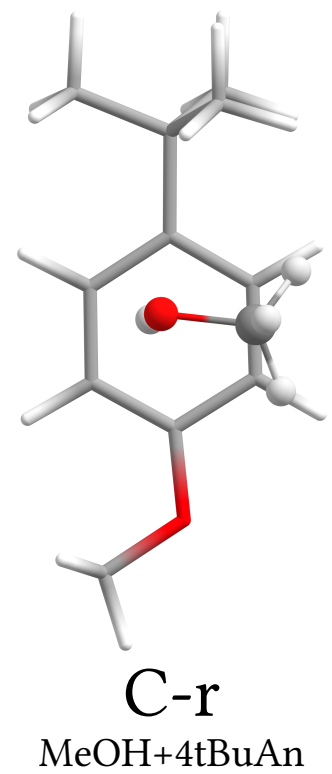

$\mathrm{MeOH}+4 \mathrm{tBuAn}$

Abbildung 4.20: Ausgewählte, auf dem M06-2X/def2-TZVP-Niveau optimierte C-r-Dimerstrukturen. Für 235TMAn und 4tBuAn stellen die gezeigten Strukturen auf diesem Rechenniveau jeweils das (elektronische) Minimum für C-Dimere dar (vgl. Tab. 4.10). 
Tabelle 4.11: Analog zu Tabelle 4.8, aber für schwingungsnullpunktskorrigierte Energien $E^{0, B 3}$ (Theorieniveau: B3LYP-D3(BJ,ABC)/def2-TZVP (B3)).

\begin{tabular}{|c|c|c|c|c|c|c|c|c|c|}
\hline$E^{0, \mathrm{~B} 3} / \mathrm{kJ} \cdot \mathrm{mol}^{-1}$ & $\mathbf{O}-\mathbf{a}$ & O-o & C-p & C-p' & C-s & C-s' & $\mathrm{C}-\mathbf{r}$ & X-o & $\mathrm{X}-\mathbf{a}$ \\
\hline $\mathrm{MeOH}+\mathrm{An}$ & -0.93 & 0.38 & 1.28 & & 0.00 & 2.41 & & & \\
\hline $\mathrm{MeOH}+2 \mathrm{MAn}$ & & -0.82 & 0.87 & & 0.00 & & & & \\
\hline $\mathrm{MeOH}+3 \mathrm{MAn}(E)$ & -1.12 & & 0.29 & & 0.00 & 1.52 & & & \\
\hline $\mathrm{MeOH}+3 \mathrm{MAn}(Z)$ & -0.40 & 0.66 & 0.37 & & 0.00 & 1.63 & & & \\
\hline $\mathrm{MeOH}+4 \mathrm{MAn}$ & -1.06 & 0.25 & 0.25 & 1.42 & 0.00 & 0.25 & & & \\
\hline $\mathrm{MeOH}+2 \mathrm{ClAn}$ & -2.96 & -1.88 & 2.34 & & 0.00 & & 1.98 & 4.57 & 4.71 \\
\hline $\mathrm{MeOH}+4 \mathrm{FAn}$ & -1.78 & -0.29 & 3.17 & & 0.00 & & 2.79 & & 3.38 \\
\hline $\mathrm{MeOH}+4 \mathrm{ClAn}$ & -1.20 & 0.07 & 2.38 & 1.20 & 0.00 & & & & 2.35 \\
\hline $\mathrm{MeOH}+4 \mathrm{BrAn}$ & -1.06 & 0.13 & 2.41 & 0.92 & 0.00 & & 1.06 & & 1.56 \\
\hline $\mathrm{MeOH}+23 \mathrm{DMAn}$ & & -0.38 & 0.00 & 1.53 & 0.32 & & & & \\
\hline $\mathrm{MeOH}+26 \mathrm{DMAn}$ & & -2.99 & 3.34 & 4.49 & 0.00 & & 2.03 & & \\
\hline $\mathrm{MeOH}+35 \mathrm{DMAn}$ & 0.12 & 1.29 & 0.00 & & 0.55 & 1.19 & 0.60 & & \\
\hline $\mathrm{MeOH}+235 \mathrm{TMAn}$ & & 0.60 & 0.00 & & 0.59 & 1.19 & & & \\
\hline $\mathrm{MeOH}+4 \mathrm{tBuAn}$ & 1.44 & 2.58 & 1.32 & & 0.00 & 1.44 & 1.05 & & \\
\hline MeOD+35DMAn & -0.33 & 1.02 & 0.00 & & 0.45 & 1.21 & 0.57 & & \\
\hline
\end{tabular}

in Abbildung 4.20 dargestellt. Für $\mathrm{MeOH}+235 \mathrm{TMAn}$ zeigt sich dabei eine diagonale Ausrichtung der $\mathrm{MeOH}-\mathrm{C}-\mathrm{O}-$ relativ zur Anisol- $\mathrm{C}_{\text {aryl }}-\mathrm{O}$-Bindung, wobei der Methylrest des Methanols in Richtung des Sauerstoffatoms und der 2-Methylgruppe des Anisols zeigt. Im Fall von $\mathrm{MeOH}+4 \mathrm{tBuAn}$ ist das $\mathrm{MeOH}-$ Molekül relativ zur Anisol- $\mathrm{C}_{\text {aryl }}-\mathrm{O}$-Bindung zwar wie im C-s-Dimer ausgerichtet, liegt aber deutlich mittiger über dem Anisolring (vgl. C-s in Abb. 4.17). Bei den X-Dimeren zeigen sich im Gegensatz zu O- und C-Dimeren keine qualitativen Unterschiede zu B3 und B3+, wobei die relativen Energien für die M6Methode in allen Fällen $\geq 3 \mathrm{~kJ} \cdot \mathrm{mol}^{-1}$ sind.

Für den im Kapitel 4.2.3 folgenden Vergleich mit den weiter oben vorgestellten experimentellen Daten (s. Kap. 4.2.1) auf Basis der BoltzmanN-Verteilung (s. Kap. 2.3.2) werden die schwingungsnullpunktskorrigierten Energieunterschiede zwischen dem (ebenfalls schwingungsnullpunktskorrigiert) stabilsten O- und C-Dimer einer jeden MethanolAnisol(derivat)-Kombination benötigt. Darum sollen im Folgenden zunächst die schwingungsnullpunktskorrigierten, relativen Energien $E^{0}$ für die verschiedenen Dimerstrukturen auf dem B3- ( $E^{0, \mathrm{~B} 3}$, Tab. 4.11), B3+- $\left(E^{0, \mathrm{~B} 3+}\right.$, Tab. 4.12) und M6-Niveau ( $E^{0, \mathrm{M} 6}$, Tab. 4.13) analysiert werden. Als Bezugspunkt dient dabei erneut das jeweils stabilste C-Dimer.

In Tabelle 4.11 sind die relativen Energien $E^{0, \mathrm{~B} 3}$ für die B3-Methode zusammengefasst. Während sich für die elektronischen Energien im Rahmen der BORN-OpPENHEIMER-Näherung ${ }^{[335]}$ keine Unterschiede für verschiedene Isotopenmassen ergeben, sind die Schwingungsnullpunktsenergien massenabhängig. Somit müssen für die Energien $E^{0} \mathrm{MeOH}$ und $\mathrm{MeOD}$ als getrennte Donoren betrachtet werden. Da für MeOD nur mit 35DMAn expe- 
rimentelle Daten vorliegen (s. Kap. 4.2.1), wird auch nur dieser Fall einbezogen. Im Vergleich zu den elektronischen Energien $E^{\mathrm{el}, \mathrm{B} 3}$ (s. Tab. 4.8) zeigen sich bei Berücksichtigung der ZPVE auf dem B3-Niveau einige Unterschiede. Aufseiten der O-Dimere stellt zwar immer noch in allen Fällen, in denen eine solche Struktur gefunden wurde, O-a das energetische Minimum dar, aufseiten der C-Dimere ist nun jedoch in 4 Fällen (Me$\mathrm{OH}+23 \mathrm{DMAn}, \mathrm{MeOH}+35 \mathrm{DMAn}, \mathrm{MeOH}+235 \mathrm{TMAn}, \mathrm{MeOD}+35 \mathrm{DMAn}$ ) C-p anstelle von C-s die C-Minimumsstruktur. Der wichtigste Unterschied ergibt sich jedoch beim Vergleich der O- mit den C-Dimeren. Durch die Berücksichtigung der ZPVE nimmt die relative Stabilität der O- gegenüber den C-Dimeren systematisch ab. Die ZPVE ist also wie im Fall des MeOH-Furan-Waagensystems (s. Kap. 4.1) für die O-Dimere systematisch größer als für die C-Dimere. Die Unterschiede zum jeweils stabilsten C-Dimer betragen hier ca. $0.3 \mathrm{~kJ} \cdot \mathrm{mol}^{-1}$ für das O-o-Dimer von $\mathrm{MeOH}+3 \mathrm{MAn}(Z)$ bis $1.9 \mathrm{~kJ} \cdot \mathrm{mol}^{-1}$ für das O-oDimer von $\mathrm{MeOH}+235 \mathrm{TMAn}$. Diese systematische Begünstigung der C- gegenüber der O-Andockstelle bewirkt, dass schwingungsnullpunktskorrigiert nun für 3 verschiedene zwei- oder mehrfach substituierte Anisolderivate mit $\mathrm{MeOH}$ als Donor (MeOH+35DMAn, $\mathrm{MeOH}+235 \mathrm{TMAn}, \mathrm{MeOH}+4 \mathrm{tBuAn})$ ein C-gebundenes Dimer die stabilste Struktur ${ }^{\dagger}$ dar- $^{-}$ stellt, während dies für die elektronischen Energien nur für ein Anisolderivat der Fall war. Beim Vergleich von $\mathrm{MeOH}+35 \mathrm{DMAn}$ mit MeOD+35DMAn zeigt sich, dass der Unterschied in der ZPVE für MeOD um etwa $0.6 \mathrm{~kJ} \cdot \mathrm{mol}^{-1}$ kleiner ist als für $\mathrm{MeOH}$, was für einen Wechsel der Bindungsstellenpräferenz zwischen $\mathrm{MeOH}$ und $\mathrm{MeOD}$ sorgt. Einen Ausreißer stellt 2ClAn dar. Hier ergeben sich nur kleine Unterschiede in der ZPVE zwischen dem stabilsten C- und den beiden O-gebundenen Dimeren, die jeweils weniger als $0.1 \mathrm{~kJ} \cdot \mathrm{mol}^{-1}$ betragen und im Gegensatz zu den anderen Anisolderivaten zugunsten des O-gebundenen Dimers ausfallen. Für die X-Dimerstrukturen zeigen sich bei Berücksichtigung der ZPVE größtenteils leichte Stabilisierungen gegenüber dem stabilsten C-gebundenen Dimer. Die schwingungnullpunktskorrigierten Energieunterschiede zu diesen C-Dimeren betragen aber jeweils mehr als $1.5 \mathrm{~kJ} \cdot \mathrm{mol}^{-1}$, wobei die O-Minimumsstrukturen noch einmal um ca. $1-3 \mathrm{~kJ} \cdot \mathrm{mol}^{-1}$ stabiler sind. Somit ergeben sich keine Hinweise darauf, dass eins der im Experiment beobachteten Signale (s. Kap. 4.2.1) einem X- anstelle eines O- oder C-Dimers zuzuordnen wäre.

Da die B3+-Methode ebenfalls auf den mit der B3-Methode optimierten Dimerstrukturen basiert und somit auch die auf B3-Niveau berechneten Schwingungsnullpunktsenergien verwendet, ergeben sich hier für den Vergleich der schwingungsnullpunktskorrigierten Energien $E^{0, \mathrm{~B} 3+}$ (s. Tab. 4.12) mit den elektronischen Energien $E^{\text {el,B3+ }}$ (s. Tab. 4.9) dieselben Unterschiede wie für B3. Im Fall der B3+-Rechnungen führt dies dazu, dass für die Mehrheit der betrachteten $\mathrm{MeOH} / \mathrm{D}$-Anisol-Kombinationen (8) C-p das C-Minimum darstellt, während dieses für 6 Kombinationen einer C-s- und für eine Kombination $(\mathrm{MeOH}+4 \mathrm{BrAn})$ einer C-p'-Struktur entspricht. Erneut ist das O-a-Dimer, wenn es gefunden wurde, immer stabiler als das O-o-Dimer und erneut sorgt der Unterschied in der

$\dagger$ Obwohl hier Nullpunktsauslenkungen vom elektronischen Minimum vorliegen, wird im Folgenden der Einfachheit halber auch bei Berücksichtigung der ZPVE der Begriff „Minimumsstruktur“ verwendet. 
Tabelle 4.12: Analog zu Tabelle 4.8, aber für schwingungsnullpunktskorrigierte Energien $E^{0, B 3+}$ (Theorieniveau: DLPNO-CCSD(T)/aVQZ//B3LYP-D3(BJ,ABC)/def2-TZVP mit ZPVE aus B3LYPD3(BJ,ABC)/def2-TZVP (B3+)).

\begin{tabular}{|c|c|c|c|c|c|c|c|c|c|}
\hline$E^{0, \mathrm{~B} 3+} / \mathrm{kJ} \cdot \mathrm{mol}^{-1}$ & $\mathbf{O}-\mathbf{a}$ & O-o & C-p & C-p' & C-s & C-s' & C-r & $\mathbf{X}-\mathbf{o}$ & $\mathbf{X}-\mathbf{a}$ \\
\hline $\mathrm{MeOH}+\mathrm{An}$ & $-\mathbf{1 . 7 7}$ & -1.09 & 0.11 & & 0.00 & 1.78 & & & \\
\hline $\mathrm{MeOH}+2 \mathrm{MAn}$ & & -1.33 & 0.00 & & 0.13 & & & & \\
\hline $\mathrm{MeOH}+3 \mathrm{MAn}(E)$ & -1.46 & & 0.00 & & 0.37 & 1.77 & & & \\
\hline $\mathrm{MeOH}+3 \mathrm{MAn}(Z)$ & -0.83 & -0.65 & 0.00 & & 0.29 & 0.98 & & & \\
\hline $\mathrm{MeOH}+4 \mathrm{MAn}$ & -0.57 & -0.27 & 0.00 & 1.61 & 1.02 & 1.65 & & & \\
\hline $\mathrm{MeOH}+2 \mathrm{ClAn}$ & -3.58 & -2.68 & 1.03 & & 0.00 & & 0.71 & 2.91 & 4.30 \\
\hline $\mathrm{MeOH}+4 \mathrm{FAn}$ & -2.24 & -1.67 & 1.76 & & 0.00 & & 1.26 & & 2.89 \\
\hline $\mathrm{MeOH}+4 \mathrm{ClAn}$ & -1.67 & -1.20 & 1.20 & 0.50 & 0.00 & & & & 2.33 \\
\hline $\mathrm{MeOH}+4 \mathrm{BrAn}$ & -0.46 & -0.32 & 1.54 & 0.00 & 0.89 & & 0.28 & & 0.89 \\
\hline $\mathrm{MeOH}+23 \mathrm{DMAn}$ & & -0.43 & 0.00 & 1.35 & 0.86 & & & & \\
\hline $\mathrm{MeOH}+26 \mathrm{DMAn}$ & & -3.18 & 3.18 & 4.02 & 0.00 & & 1.06 & & \\
\hline $\mathrm{MeOH}+35 \mathrm{DMAn}$ & 0.06 & 0.27 & 0.00 & & 1.13 & 1.13 & 1.18 & & \\
\hline $\mathrm{MeOH}+235 \mathrm{TMAn}$ & & 0.78 & 0.00 & & 1.42 & 1.30 & & & \\
\hline $\mathrm{MeOH}+4 \mathrm{tBuAn}$ & 0.84 & 1.15 & 0.40 & & 0.00 & 1.12 & 0.03 & & \\
\hline MeOD+35DMAn & -0.39 & 0.01 & 0.00 & & 1.03 & 1.15 & 1.15 & & \\
\hline
\end{tabular}

ZPVE für einen Wechsel der Bindungsstellenpräferenz bei Isotopenaustausch zwischen $\mathrm{MeOH}+35 \mathrm{DMAn}$ und MeOD+35DMAn. Auch für das B3+-Rechenniveau ergeben sich keine Hinweise darauf, dass die Zuordnung der beobachteten Banden zu C- und O-Dimer für die Halogenanisole (s. Kap 4.2.1) unter Einbeziehung der X-Dimere überarbeitet werden müsste.

Vollständig unabhängige Ergebnisse resultieren für die M6-Methode, da hier alle Bestandteile der Rechnung (Geometrieoptimierung, elektronische Energie, ZPVE) auf dem M6-Niveau erfolgen. Bei Berücksichtigung der ZPVE, also Betrachtung der relativen Energien $E^{0, \mathrm{M} 6}$, ergeben sich auch hier systematische Unterschiede im Vergleich zu den elektronischen Energien $E^{\mathrm{el}, \mathrm{M} 6}$ (s. Tab. 4.10). Wie in Tabelle 4.13 ersichtlich wird, stellt der O-o-Dimertyp für die schwingungsnullpunktskorrigierten Energien zwar weiterhin für fast alle Fälle die O-Minimumsstruktur dar, es gibt mit 2ClAn jedoch eine Ausnahme. Die besondere Stabilität dieser O-a-Struktur ist eventuell auf die spezifische Geometrie (s. oben) zurückzuführen. Bei den C-Dimeren stellt C-p für 7 Anisolderivate (in 8 Kombinationen, wenn MeOD+35DMAn mit berücksichtigt wird) die C-Minimumsstruktur dar, während dies für die elektronischen Energien nur für 5 Anisolderivate der Fall war. Die X-Dimere sind allesamt mehr als $3 \mathrm{~kJ} \cdot \mathrm{mol}^{-1}$ weniger stabil als die für das $\mathrm{O} / \mathrm{C}$ Gleichgewicht bedeutsamen O- und C-Dimere und sind somit nicht weiter relevant. Der interessanteste Unterschied zwischen $E^{\mathrm{el}, \mathrm{M} 6}$ und $E^{0, \mathrm{M} 6}$ ist allerdings die analog zur B3-Methode auftretende systematisch höhere ZPVE für die O-Dimerstrukturen. Die 
Tabelle 4.13: Analog zu Tabelle 4.8, aber für schwingungsnullpunktskorrigierte Energien $E^{0, M 6}$ (Theorieniveau: M06-2X/def2-TZVP (M6)).

\begin{tabular}{lrrrrrrrrr}
\hline $\boldsymbol{E}^{\mathbf{0 , \mathbf { M }}} / \mathbf{~ k J} \cdot \mathbf{m o l}^{-\mathbf{1}}$ & $\mathbf{O}-\mathbf{a}$ & $\mathbf{O}-\mathbf{o}$ & $\mathbf{C}-\mathbf{p}$ & $\mathbf{C}-\mathbf{p}^{\prime}$ & $\mathbf{C}-\mathbf{s}$ & $\mathbf{C}-\mathbf{s}^{\prime}$ & $\mathbf{C}-\mathbf{r}$ & $\mathbf{X}-\mathbf{o}$ & $\mathbf{X}-\mathbf{a}$ \\
\hline MeOH+An & 1.16 & $-\mathbf{0 . 4 1}$ & 0.33 & & $\mathbf{0 . 0 0}$ & 2.31 & & & \\
MeOH+2MAn & 1.35 & $-\mathbf{0 . 1 9}$ & $\mathbf{0 . 0 0}$ & & 0.35 & & 1.37 & & \\
MeOH+3MAn $(E)$ & 1.71 & $\mathbf{0 . 3 2}$ & $\mathbf{0 . 0 0}$ & 2.55 & 1.03 & 2.41 & & & \\
MeOH+3MAn $(Z)$ & 2.26 & $\mathbf{0 . 3 0}$ & $\mathbf{0 . 0 0}$ & 2.28 & 0.83 & 1.40 & & & \\
MeOH+4MAn & 1.76 & $\mathbf{0 . 2 2}$ & $\mathbf{0 . 0 0}$ & 1.50 & 0.89 & 1.97 & & & \\
MeOH+2ClAn & $-\mathbf{2 . 6 7}$ & -2.50 & 1.74 & & $\mathbf{0 . 0 0}$ & 5.62 & & $\mathbf{4 . 9 4}$ & 6.40 \\
MeOH+4FAn & 0.19 & $-\mathbf{0 . 9 1}$ & 2.07 & 2.31 & $\mathbf{0 . 0 0}$ & & & & $\mathbf{4 . 4 6}$ \\
MeOH+4ClAn & 0.92 & $-\mathbf{0 . 8 1}$ & 2.05 & 1.13 & $\mathbf{0 . 0 0}$ & & & & $\mathbf{3 . 9 3}$ \\
MeOH+4BrAn & 0.85 & $-\mathbf{1 . 0 1}$ & 1.89 & 1.02 & $\mathbf{0 . 0 0}$ & & & & $\mathbf{3 . 0 5}$ \\
MeOH+23DMAn & 3.94 & $\mathbf{0 . 9 2}$ & $\mathbf{0 . 0 0}$ & 3.03 & 1.15 & & & & \\
MeOH+26DMAn & 3.67 & $\mathbf{0 . 9 4}$ & 5.95 & & $\mathbf{0 . 0 0}$ & & 3.11 & & \\
MeOH+35DMAn & 2.90 & $\mathbf{1 . 0 4}$ & $\mathbf{0 . 0 0}$ & 2.39 & 1.69 & 1.28 & & & \\
MeOH+235TMAn & 4.22 & $\mathbf{0 . 9 4}$ & $\mathbf{0 . 0 0}$ & 2.07 & & 2.08 & 0.67 & & \\
MeOH+4tBuAn & 3.94 & $\mathbf{1 . 9 8}$ & 0.05 & & 2.89 & 0.61 & $\mathbf{0 . 0 0}$ & & \\
\hline MeOD+35DMAn & 2.43 & $\mathbf{0 . 8 2}$ & $\mathbf{0 . 0 0}$ & 2.31 & 1.55 & 1.30 & & & \\
\hline
\end{tabular}

Unterschiede betragen für M6 ca. $0.7 \mathrm{~kJ} \cdot \mathrm{mol}^{-1}$ für das O-o-Dimer von $\mathrm{MeOH}+4 \mathrm{BrAn}$ bis $2.0 \mathrm{~kJ} \cdot \mathrm{mol}^{-1}$ für das $\mathrm{O}$-a-Dimer von $\mathrm{MeOH}+23 \mathrm{DMAn}$ und sind damit tendenziell etwas größer als für B3. Beim „Ausreißer“ $\mathrm{MeOH}+2 \mathrm{ClAn}$ ist die ZPVE auf dem M6- im Gegensatz zum B3-Niveau für die O-Dimere analog zu den anderen Anisolderivaten größer, allerdings nur um ca. $0.2-0.5 \mathrm{~kJ} \cdot \mathrm{mol}^{-1}$. Bei Deuterierung der Wasserstoffbrücke wird der ZPVE-Unterschied zwischen O- und C-Dimer auch auf dem M6-Niveau kleiner, wie der Vergleich von $\mathrm{MeOH}+35 \mathrm{DMAn}$ und MeOD+35DMAn zeigt.

Wie weiter oben bereits erwähnt, werden die beiden Isomere des 3MAn ( $E$ und $Z$ ) im Rahmen der quantenchemischen Betrachtung wie getrennte Spezies behandelt. Im Experiment konnte jedoch keine Unterscheidung der beiden Isomere erfolgen, sodass die Einzelwerte für die beiden Isomere für die folgenden Benchmarks (Kap. 4.2.3) zunächst gemittelt werden müssen. Hierfür sollen BoltzMANN-gewichtete Mittelwerte herangezogen werden, d. h. die Gewichtungsfaktoren sollen dem Anteil des jeweiligen Konformers in der experimentell untersuchten Überschallexpansion entsprechen. Dabei wird davon ausgegangen, dass in der Überschallexpansion aufgrund der zu erwartenden Barrierenhöhe keine Isomerenumwandlung erfolgt. Näherungsweise sollten $E$ - und $Z$-Isomer in der Expansion also in dem Verhältnis vorliegen, das sich zuvor im Reservoir eingestellt hat. Als Temperatur für die Gleichgewichtseinstellung wird somit die Raumtemperatur von etwa $298 \mathrm{~K}$ angenommen. Die Energieunterschiede zwischen den beiden Isomeren werden mit den quantenchemischen Methoden B3, B3+ und M6 berechnet. Über die BoltzManNVerteilung lassen sich dann die Anteile $x_{E}$ und $x_{Z}$ von $E$ - bzw. $Z$-Isomer (und damit die 
Tabelle 4.14: Über eine Boltzmann-Verteilung errechnete Anteile $x$ der 3MAn-Isomere $E$ und $Z$ bei Raumtemperatur. Zugrundeliegende Energiedifferenzen $\Delta E_{E-Z}^{0}: \mathbf{B} 3:-0.44 \mathrm{~kJ} \cdot \mathrm{mol}^{-1}$, B3+: $-0.43 \mathrm{~kJ} \cdot \mathrm{mol}^{-1}$, M6: $-0.47 \mathrm{~kJ} \cdot \mathrm{mol}^{-1}$. Als Raumtemperatur wurden $298 \mathrm{~K}$ angenommen. Für die Naturkonstanten wurde auf die Werte aus der aktuellen CODATA-Empfehlung ${ }^{[258-260]}$ zurückgegriffen. Verwendete Rechenniveaus: B3LYP-D3(BJ,ABC)/def2-TZVP (B3), DLPNO-CCSD(T)/aVQZ//B3LYP-D3(BJ,ABC)/def2-TZVP mit ZPVE aus B3LYP-D3(BJ,ABC)/def2TZVP (B3+), M06-2X/def2-TZVP (M6).

\begin{tabular}{lrrr}
\hline $\boldsymbol{x}$ & B3 & B3+ & M6 \\
\hline $\mathrm{MeOH}+3 \mathrm{MAn}(E)$ & 0.54 & 0.54 & 0.55 \\
$\mathrm{MeOH}+3 \mathrm{MAn}(Z)$ & 0.46 & 0.46 & 0.45 \\
\hline
\end{tabular}

zu verwendenden Gewichtungsfaktoren) gemäß Gleichung 2.11 (s. S. 24) bestimmen. Basierend auf den berechneten Energieunterschieden $\Delta E_{E-Z}^{0}=E_{E}^{0}-E_{Z}^{0}$ von $-0.44 \mathrm{~kJ} \cdot \mathrm{mol}^{-1}$ (B3), $-0.43 \mathrm{~kJ} \cdot \mathrm{mol}^{-1}(\mathbf{B} 3+)$ und $-0.47 \mathrm{~kJ} \cdot \mathrm{mol}^{-1}$ (M6) ergeben sich die in Tabelle $4.14 \mathrm{zu}-$ sammengefassten Isomerenanteile, die für die Mittelwertbildung der Daten der jeweiligen Methode herangezogen werden sollen.

Die für den Vergleich mit dem Experiment nötigen schwingungsnullpunktskorrigierten Energiedifferenzen zwischen dem jeweils stabilsten $\mathrm{O}$ - und $\mathrm{C}$-gebundenen Dimer, $\Delta E_{\mathrm{O}-\mathrm{C}}^{0}=E^{0}(\mathrm{O})-E^{0}(\mathrm{C})$, sind in Tabelle 4.15 zusammengefasst und noch einmal den entsprechenden elektronischen Energiedifferenzen $\Delta E_{\mathrm{O}-\mathrm{C}}^{\mathrm{el}}$ gegenübergestellt. Negative Werte entsprechen hierbei einer Präferenz für die O-Bindungsstelle, positive einer solchen für die C-Bindungsstelle. Für 3MAn sind neben den grau eingefärbten Einzelwerten für die beiden Isomere auch die BoltzMAnN-gewichteten Mittelwerte (MW), die für die Benchmarks genutzt werden, in Tabelle 4.15 aufgelistet.

Im Gesamtvergleich der elektronischen mit den schwingungsnullpunktskorrigierten Energien der jeweiligen Methode zeigt sich noch einmal der Einfluss der systematisch höheren ZPVE der O-Dimere. Bei Betrachtung der elektronischen Energien lässt sich erkennen, dass die B3+-Methode durchgehend niedrigere Werte für die Energiedifferenz $\Delta E_{\mathrm{O}-\mathrm{C}}^{\mathrm{el}}$ vorhersagt als die B3-Methode. Dies entspricht einer stärker ausgeprägten O-Bindungsstellenpräferenz. Werden die Schwingungsnullpunktsenergien berücksichtigt, wird der Trend von B3 zu B3+ weniger systematisch, obwohl die ZPVE in beiden Fällen auf dem B3-Niveau berechnet wird. Dies liegt darin begründet, dass in B3 und B3+ teilweise unterschiedliche C-Dimerstrukturen das C-Bindungsstellenminimum darstellen. Die M6Methode sagt im Vergleich zu den beiden anderen Methoden tendenziell eher höhere Werte für die O/C-Energiedifferenzen voraus, insbesondere nach Berücksichtigung der ZPVE. Während B3 und B3+ schwingungsnullpunktskorrigiert für 11 Methanol-Anisol(derivat)Kombinationen eine Präferenz für die O- und für 3 Kombinationen eine Präferenz für die C-Bindungsstelle vorhersagen, stellt $\mathrm{C}$ auf dem M6-Niveau mit 8 Kombinationen deutlich häufiger die präferierte Bindungsstelle dar. Hier zeigen sich somit deutliche Unterschiede in den Methoden. Nur ein Vergleich mit den experimentellen Daten, wie er in den folgen- 
Tabelle 4.15: Elektronische $\left(\Delta E^{\mathrm{el}}\right)$ und schwingungsnullpunktskorrigierte $\left(\Delta E^{0}\right)$ Energiedifferenzen $\Delta E_{\mathrm{O}-\mathrm{C}}=E(\mathrm{O})-E(\mathrm{C})$ zwischen stabilstem O- und stabilstem C-Dimer aus Methanol(-OD) mit den verschiedenen Anisolderivaten. Aus den grau eingefärbten Einzelwerten für die 3MAnIsomere $E$ und $Z$ werden Boltzmann-gewichtete Mittelwerte (MW) gebildet. Verwendete Rechenniveaus: B3LYP-D3(BJ,ABC)/def2-TZVP (B3), DLPNO-CCSD(T)/aVQZ//B3LYP-D3(BJ,ABC)/def2TZVP mit ZPVE aus B3LYP-D3(BJ,ABC)/def2-TZVP (B3+), M06-2X/def2-TZVP (M6).

\begin{tabular}{|c|c|c|c|c|c|c|}
\hline \multirow[b]{2}{*}{$\Delta E_{\mathrm{O}-\mathrm{C}} / \mathrm{kJ} \cdot \mathrm{mol}^{-1}$} & \multicolumn{3}{|c|}{$\Delta E^{\mathrm{el}}$} & \multicolumn{3}{|c|}{$\Delta E^{0}$} \\
\hline & B3 & B3+ & M6 & B3 & B3+ & M6 \\
\hline $\mathrm{MeOH}+\mathrm{An}$ & -1.58 & -2.42 & -1.39 & -0.93 & -1.77 & -0.41 \\
\hline $\mathrm{MeOH}+2 \mathrm{MAn}$ & -1.65 & -2.29 & -1.43 & -0.82 & -1.33 & -0.19 \\
\hline $\mathrm{MeOH}+3 \mathrm{MAn}(E)$ & -1.77 & -2.48 & -0.75 & -1.12 & -1.46 & 0.32 \\
\hline $\mathrm{MeOH}+3 \mathrm{MAn}(Z)$ & -0.98 & -1.71 & -1.00 & -0.40 & -0.83 & 0.30 \\
\hline $\mathrm{MeOH}+3 \mathrm{MAn}(\mathrm{MW})$ & -1.41 & -2.13 & -0.86 & -0.79 & -1.17 & 0.31 \\
\hline $\mathrm{MeOH}+4 \mathrm{MAn}$ & -1.74 & -2.27 & -1.40 & -1.06 & -0.57 & 0.22 \\
\hline $\mathrm{MeOH}+2 \mathrm{ClAn}$ & -2.89 & -3.51 & -3.00 & -2.96 & -3.58 & -2.67 \\
\hline $\mathrm{MeOH}+4 \mathrm{FAn}$ & -2.68 & -3.14 & -1.90 & -1.78 & -2.24 & -0.91 \\
\hline $\mathrm{MeOH}+4 \mathrm{ClAn}$ & -2.07 & -2.54 & -1.71 & -1.20 & -1.67 & -0.81 \\
\hline $\mathrm{MeOH}+4 \mathrm{BrAn}$ & -1.95 & -2.24 & -1.69 & -1.06 & -0.46 & -1.01 \\
\hline $\mathrm{MeOH}+23 \mathrm{DMAn}$ & -1.67 & -2.24 & -0.76 & -0.38 & -0.43 & 0.92 \\
\hline $\mathrm{MeOH}+26 \mathrm{DMAn}$ & -4.23 & -4.42 & -0.28 & -2.99 & -3.18 & 0.94 \\
\hline $\mathrm{MeOH}+35 \mathrm{DMAn}$ & -1.11 & -1.35 & -0.11 & 0.12 & 0.06 & 1.04 \\
\hline $\mathrm{MeOH}+235 \mathrm{TMAn}$ & -1.01 & -1.14 & -0.25 & 0.60 & 0.78 & 0.94 \\
\hline $\mathrm{MeOH}+4 \mathrm{tBuAn}$ & 0.44 & -0.16 & 0.68 & 1.44 & 0.84 & 1.98 \\
\hline MeOD+35DMAn & -1.11 & -1.35 & -0.11 & -0.33 & -0.39 & 0.82 \\
\hline
\end{tabular}

den Abschnitten (Kap. 4.2.3 und 4.2.4) erfolgen soll, kann jedoch quantitativ aufklären, welche dieser Vorhersagen dabei am besten zutrifft.

\subsubsection{Auswertung des $\mathrm{O} / \mathrm{C}$-Konformerengleichgewichts}

Zunächst sollen die im vorherigen Abschnitt vorgestellten, quantenchemisch berechneten Energiedifferenzen anhand der experimentell bestimmten $\mathrm{O} / \mathrm{C}$-Intensitätsverhältnisse einem Benchmark unterzogen werden. Wie in Kapitel 2.3.2 erläutert, werden für diesen Vergleich nicht nur die schwingungsnullpunktskorrigierten Energiedifferenzen, sondern auch das Verhältnis der berechneten $\mathrm{OH}$-Streckschwingungsbandenstärken der betrachteten Dimere benötigt. Die doppelt-harmonisch genäherten Bandenstärken $A_{\mathrm{OH}}$ der $\mathrm{OH}$ Streckschwingungen des jeweils stabilsten O- und C-Dimers sowie deren Verhältnisse $A_{\mathrm{OH}}(\mathrm{O}) / A_{\mathrm{OH}}(\mathrm{C})$ sind in Tabelle 4.16 zusammengefasst. Da für die B3- und die B3+-Methode die B3-Schwingungsrechnungen herangezogen werden, ergeben sich hier prinzipi- 


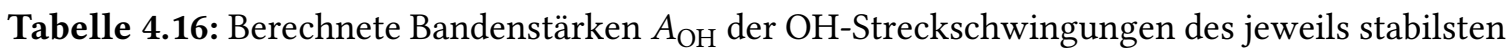
O- $\left(A_{\mathrm{OH}}(\mathrm{O})\right)$ bzw. C-Dimers $\left(A_{\mathrm{OH}}(\mathrm{C})\right)$ der verschiedenen Methanol-Anisol(derivat)-Kombinationen. Da die Daten für den Vergleich zum Experiment herangezogen werden sollen, werden hier die relativen Stabilitäten auf Grundlage der schwingungsnullpunktskorrigierten Energien verwendet (s. Tabs. 4.11, 4.12, 4.13). Aus den grau eingefärbten Einzelwerten für die 3MAn-Isomere $E$ und $Z$ werden erneut Boltzmann-gewichtete Mittelwerte (MW) gebildet. Verwendete Rechenniveaus: B3LYP-D3(BJ,ABC)/def2-TZVP (B3), DLPNO-CCSD(T)/aVQZ//B3LYP-D3(BJ,ABC)/def2TZVP mit ZPVE aus B3LYP-D3(BJ,ABC)/def2-TZVP (B3+), M06-2X/def2-TZVP (M6).

\begin{tabular}{|c|c|c|c|c|c|c|c|c|c|}
\hline \multirow[b]{2}{*}{$A / \mathrm{km} \cdot \mathrm{mol}^{-1}$} & \multicolumn{3}{|c|}{$A_{\mathrm{OH}}(\mathrm{O})$} & \multicolumn{3}{|c|}{$A_{\mathrm{OH}}(\mathrm{C})$} & \multicolumn{3}{|c|}{$A_{\mathrm{OH}}(\mathrm{O}) / A_{\mathrm{OH}}(\mathrm{C})$} \\
\hline & B3 & B3+ & M6 & B3 & B3+ & M6 & B3 & B3+ & M6 \\
\hline $\mathrm{MeOH}+\mathrm{An}$ & 361 & s. B3 & 81 & 130 & s. B3 & 105 & 2.77 & 2.77 & 0.77 \\
\hline $\mathrm{MeOH}+2 \mathrm{MAn}$ & 271 & s. B3 & 146 & 136 & 133 & 139 & 1.99 & 2.04 & 1.04 \\
\hline $\mathrm{MeOH}+3 \mathrm{MAn}(E)$ & 350 & s. B3 & 89 & 149 & 164 & 151 & 2.35 & 2.13 & 0.59 \\
\hline $\mathrm{MeOH}+3 \mathrm{MAn}(Z)$ & 383 & s. B3 & 81 & 180 & 169 & 153 & 2.13 & 2.27 & 0.53 \\
\hline $\mathrm{MeOH}+3 \mathrm{MAn}(\mathrm{MW})$ & & & & & & & 2.25 & 2.20 & 0.56 \\
\hline $\mathrm{MeOH}+4 \mathrm{MAn}$ & 377 & s. B3 & 90 & 146 & 165 & 154 & 2.59 & 2.28 & 0.58 \\
\hline $\mathrm{MeOH}+2 \mathrm{ClAn}$ & 245 & s. B3 & 202 & 92 & s. B3 & 76 & 2.66 & 2.66 & 2.66 \\
\hline $\mathrm{MeOH}+4 \mathrm{FAn}$ & 342 & s. B3 & 81 & 104 & s. B3 & 90 & 3.28 & 3.28 & 0.90 \\
\hline $\mathrm{MeOH}+4 \mathrm{ClAn}$ & 337 & s. B3 & 66 & 103 & s. B3 & 86 & 3.27 & 3.27 & 0.77 \\
\hline $\mathrm{MeOH}+4 \mathrm{BrAn}$ & 335 & s. B3 & 62 & 102 & 122 & 85 & 3.27 & 2.76 & 0.73 \\
\hline $\mathrm{MeOH}+23 \mathrm{DMAn}$ & 253 & s. B3 & 141 & 149 & s. B3 & 139 & 1.70 & 1.70 & 1.01 \\
\hline $\mathrm{MeOH}+26 \mathrm{DMAn}$ & 358 & s. B3 & 242 & 160 & s. B3 & 153 & 2.23 & 2.23 & 1.58 \\
\hline $\mathrm{MeOH}+35 \mathrm{DMAn}$ & 372 & s. B3 & 88 & 196 & s. B3 & 164 & 1.90 & 1.90 & 0.54 \\
\hline $\mathrm{MeOH}+235 \mathrm{TMAn}$ & 258 & s. B3 & 140 & 167 & s. B3 & 154 & 1.54 & 1.54 & 0.91 \\
\hline $\mathrm{MeOH}+4 \mathrm{tBuAn}$ & 362 & s. B3 & 81 & 179 & s. B3 & 163 & 2.02 & 2.02 & 0.49 \\
\hline MeOD+35DMAn & 210 & s. B3 & 56 & 115 & s. B3 & 98 & 1.83 & 1.83 & 0.57 \\
\hline
\end{tabular}

ell die gleichen Werte. Bei Berücksichtigung der elektronischen Energie aus der DLPNO$\operatorname{CCSD}(\mathrm{T})$-Rechnung weisen jedoch in einigen Fällen andere C-Dimerstrukturen eine höhere Stabilität auf (vgl. Tab. 4.11 und Tab. 4.12), sodass teilweise unterschiedliche Bandenstärken in das Verhältnis eingehen. In diesen Fällen zeigen sich im resultierenden Bandenstärkenverhältnis allerdings nur geringe Abweichungen von weniger als 20\%. Generell weisen die O-Dimere auf dem B3- bzw. B3+-Niveau höhere Bandenstärken als die C-Dimere auf, wobei die Bandenstärken der O-o- etwas kleiner als die der O-a-Dimere sind. Eine Ausnahme stellt das O-o-Dimer von $\mathrm{MeOH}+26 \mathrm{DMAn}$ dar, was durch die besondere Struktur dieses Dimers (s. Abb. 4.19 auf S. 145) erklärt werden kann. Im Ergebnis weisen die einfach in 2-Position alkylierten Anisolderivate, für die O-o-Dimere das O-Minimum darstellen, im Dimer mit MeOH damit kleinere Bandenstärkenverhältnisse (1.54-2.04) als die übrigen Anisolderivate (1.90-3.28), für die das O-a-Dimer stabiler ist, auf. 
Auf dem M6-Niveau ergibt sich ein deutlich anderes Bild. Abhängig von der Bewertung der relativen Stabilität der Dimerstrukturtypen ergeben sich für die O-Dimere auf M6- etwa 20\% (für alle Methoden O-a O-Minimum) bzw. 30-50\% (für alle Methoden O-o O-Minimum) bis etwa 75\% (O-a für B3 und B3+, O-o für M6 O-Minimum) kleinere Bandenstärken $A_{\mathrm{OH}}(\mathrm{O})$ als auf $\mathbf{B 3}$ bzw. B3+-Niveau. Für die C-Dimere zeigen sich deutlich geringere Abweichungen zwischen M6 und den anderen Methoden. Hier sind die M6Bandenstärken maximal um $20 \%$ kleiner, größtenteils vergleichbar groß und vereinzelt sogar um bis zu 5\% größer. Im Ergebnis resultieren aufgrund der deutlich kleineren Ound vergleichbar großen C-Bandenstärken für die M6-Methode deutlich kleinere Bandenstärkenverhältnisse als für B3 oder B3+. Auch hier kann erst durch den Vergleich mit den experimentellen Daten beurteilt werden, welche der berechneten Verhältnisse dabei den realen Werten näher kommen. Fehler in der Bandenstärke und in der Energievorhersage können sich hierbei jedoch kompensieren.

Für den Vergleich der berechneten mit den experimentellen Daten werden die in Kapitel 2.3.2 beschriebenen Boltzmann-Plots genutzt. In diesen wird der Anteil des Cgebundenen Dimers, $x_{C}$, in den das experimentelle Intensitätsverhältnis und das quantenchemisch berechnete Bandenstärkenverhältnis zwischen O- und C-Dimer eingehen (s. Gl. 2.11 auf S. 24), gegen den berechneten Energieunterschied $\Delta E_{\mathrm{O}-\mathrm{C}}^{0}$ aufgetragen. Anhand realistischer Abschätzungen für die Konformationstemperatur kann dann auf Basis der Boltzmann-Verteilung ein Bereich definiert werden, in den die Datenpunkte $\left(\Delta E_{\mathrm{O}-\mathrm{C}}^{0}, x_{\mathrm{C}}\right)$ fallen müssen, um mit den experimentellen Befunden kompatibel zu sein. Für das Methanol-Anisol-O/C-Waagensystem wird analog zum ähnlichen Methanol-Furan-O/C-Waagensystem (s. Kap. 4.1) eine Konformationstemperatur von (60 \pm 40$) \mathrm{K}$ abgeschätzt. ${ }^{[31]}$ Zudem wird für besondere Fälle mit höherer O/C-Umwandlungsbarriere wie sie z. B. anhand der stabilsten O- und C-Dimerstrukturen für $\mathrm{MeOH}+26 \mathrm{DMAn}$ erwartet werden kann - bis zu $20 \%$ kinetisches Trapping erlaubt. ${ }^{[37]}$ In diesen Fällen kann die Umwandlungsbarriere in der Überschallexpansion möglicherweise nicht überwunden werden, sodass die Relaxation ins stabilere Dimer unabhängig vom Energieunterschied zwischen den Konformeren kinetisch gehemmt ist. ${ }^{[31]}$ Im Gegensatz zum MeOH-FuranSystem sind die in den Geometrieoptimierungen erhaltenen Methanol-Anisol(derivat)Dimere allesamt unsymmetrisch, sodass keinerlei unterschiedliche Entartungsfaktoren berücksichtigt werden müssen.

In Abbildung 4.21 ist der Boltzmann-Plot für das B3-Rechenniveau dargestellt. Die einzelnen Datenpunkte sind dabei in den in Abbildung 4.13 eingeführten Farben für die einzelnen Anisolderivate eingezeichnet. Über die Füllfarbe können die Donormoleküle $\mathrm{MeOH}$ (weiß) und MeOD (grün) für den Akzeptor 35DMAn unterschieden werden. Die Fehlerbalken beruhen ausschließlich auf den in Tabelle 4.7 aufgelisteten Fehlern der experimentellen Intensitätsverhältnisse (s. Kap. 2.3.2).

Für das B3-Rechenniveau liegen alle 14 Datenpunkte im Rahmen ihrer experimentellen Fehlerbalken im weißen, also mit den experimentellen Daten vereinbaren, Bereich. Die Datenpunkte selbst liegen in 12 von 14 Fällen im Zielbereich. Die beiden Ausnahmen sind hier $\mathrm{MeOH}+26 \mathrm{DMAn}$ (hellgelb), bei dem die Bestimmung des experimentellen In- 


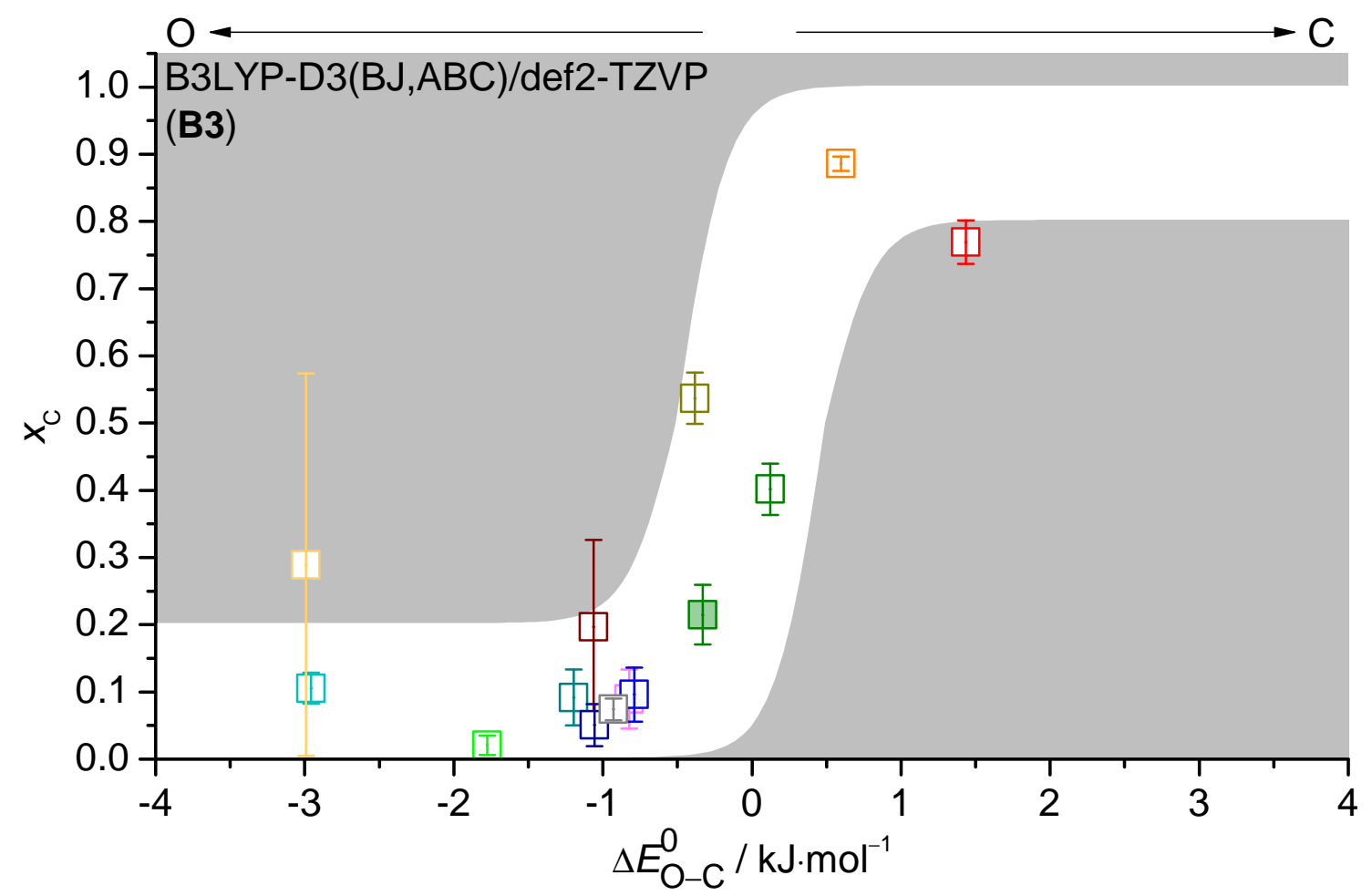

Abbildung 4.21: Boltzmann-Plot des C-Dimeranteils in der Überschallexpansion, $x_{\mathrm{C}}$, gegen den quantenchemisch berechneten Energieunterschied $\Delta E_{\mathrm{O}-\mathrm{C}}^{0}=E^{0}(\mathrm{O})-E^{0}(\mathrm{C})$, hier für das B3LYP-D3(BJ,ABC)/def2-TZVP-Rechenniveau (B3). Die eingezeichneten Fehlerbalken basieren ausschließlich auf dem Fehler der experimentellen Intensitätsverhältnisse. Die Datenpunkte sind in der in Abb. 4.13 eingeführten Farbkennzeichnung dargestellt und lassen sich so den verschiedenen Anisolderivaten zuordnen. Weiß gefüllte Symbole gehören zu MeOH als Donor, das farbig gefüllte Symbol zu MeOD. Für $\Delta E_{\mathrm{O}-\mathrm{C}}^{0}$ s. Tab. 4.11. $x_{\mathrm{C}}$ berechnet nach Gl. 2.11 (S. 24) aus den experimentellen Intensitätsverhältnissen in Tab. 4.7 und den berechneten Bandenstärkenverhältnissen in Tab. 4.16. Eine ähnliche Gegenüberstellung, die teilweise auf anderen experimentellen Daten beruht und weitere Akzeptormoleküle beinhaltet, findet sich in Fig. 4 in Ref. [31]. 
tensitätverhältnisses aufgrund der partiellen Überlappung der $\mathrm{MA}_{\mathrm{O}^{-}}$mit der MethanolHomodimer-Bande deutlich erschwert ist (s. Kap. 4.2.1), und $\mathrm{MeOH}+4 \mathrm{tBuAn}$ (rot). Der experimentell beobachtete generelle Trend der Zunahme der relativen C-DimerbandenIntensität bei steigendem Anisol-Substitutionsgrad bleibt bei Berücksichtigung der auf B3-Niveau berechneten Bandenstärkenverhältnisse auch im C-Dimeranteil $x_{C}$ ersichtlich, insbesondere für 23DMAn (dunkelgelb), 26DMAn (hellgelb), 35DMAn (dunkelgrün), 235TMAn (orange) und 4tBuAn (rot). Mit Ausnahme von 26DMAn wird dieser Trend durch die berechneten Energieunterschiede treffend erfasst, auch wenn sich der durch die B3-Methode vorhergesagte Wechsel der Bindungsstellenpräferenz bei O-Deuterierung zwischen $\mathrm{MeOH}+35 \mathrm{DMAn}$ (dunkelgrün, weiße Füllung) und MeOD+35DMAn (grüne Füllung) (s. Kap. 4.2.2) im Experiment nicht zeigt. Dennoch liegen beide Datenpunkte im Rahmen des für die Energiedifferenz erlaubten Fehlers von $\pm 0.5 \mathrm{~kJ} \cdot \mathrm{mol}^{-1}$ (s. Kap. 2.3.2) im weißen Zielbereich. Für den „Ausreißer“ 26DMAn sind zwei Erklärungen möglich. Zum einen liegt der Punkt im Rahmen seines großen experimentellen Fehlers im Zielbereich. Zum anderen ist auf Basis der quantenchemisch ermittelten Dimerstrukturen im Fall von 26DMAn für die O/C-Umwandlung eine deutlich größere Barriere zu erwarten (s. Kap. 4.2.2), sodass hier ein Fall von kinetischem Trapping, also einem unabhängig vom Energieunterschied kinetisch bestimmten Dimerverhältnis in der Expansion, vorliegen könnte. Im Rahmen der im Experiment erzielbaren thermodynamischen Kontrolle des Gleichgewichts und der Genauigkeit der ermittelten Intensitätsverhältnisse kann die B3Methode das O/C-Gleichgewicht hinsichtlich der berechneten Energieunterschiede und Bandenstärkenverhältnisse also treffend beschreiben.

Werden die elektronischen Energien aus der B3-Rechnung durch auf dem DLPNO$\mathrm{CCSD}(\mathrm{T}) / \mathrm{aVQZ}-\mathrm{Niveau}$ bestimmte elektronische Energien ersetzt, ergibt sich die B3+Methode (s. oben). Der Boltzmann-Plot für dieses Theorieniveau ist in Abbildung 4.22 dargestellt. Erneut liegen alle Datenpunkte im Rahmen ihrer Fehlerbalken im weißen Zielbereich, wobei nun 13 der 14 Punkte direkt in diesen Bereich fallen. Der einzige außerhalb liegende Punkt ist $\mathrm{MeOH}+26 \mathrm{DMAn}$ (hellgelb), was auf die bereits beschriebenen Gründe zurückgeführt werden kann. Alle weiteren Datenpunkte fallen auf dem B3+-Niveau inklusive ihrer Fehlerbalken vollständig in den Zielbereich, während auf dem B3-Niveau $\mathrm{MeOH}+4 \mathrm{tBuAn}$ (rot) und ein Teil des Fehlerbereichs für $\mathrm{MeOH}+4 \mathrm{BrAn}$ (braun) außerhalb des Zielbereichs liegen. Bei Berechnung der elektronischen Energie auf dem höheren Theorieniveau ergeben sich mit der B3+- gegenüber der B3-Methode somit leichte Verbesserungen hinsichtlich der Beschreibung des O/C-Waagensystems.

Ein deutlich anderes Bild zeigt sich bei Betrachtung des M6-Niveaus, dessen BoltzMANN-Plot in Abbildung 4.23 wiedergegeben ist. Nur die Hälfte der 14 Datenpunkte liegt direkt bzw. im Rahmen der Fehlerbalken im Zielbereich $(\mathrm{MeOH}+\mathrm{An}, \mathrm{MeOH}+2 \mathrm{MAn}, \mathrm{Me}-$ $\mathrm{OH}+2 \mathrm{ClAn}, \mathrm{MeOH}+4 \mathrm{FAn}, \mathrm{MeOH}+4 \mathrm{ClAn}, \mathrm{MeOH}+4 \mathrm{BrAn}, \mathrm{MeOH}+235 \mathrm{TMAn}$ ), wohingegen die andere Hälfte vollständig außerhalb liegt $(\mathrm{MeOH}+3 \mathrm{MAn}, \mathrm{MeOH}+4 \mathrm{MAn}, \mathrm{MeOH}+$ 23DMAn, $\mathrm{MeOH}+26 \mathrm{DMAn}, \mathrm{MeOH}+35 \mathrm{DMAn}, \mathrm{MeOH}+4 \mathrm{tBuAn}, \mathrm{MeOD}+35 \mathrm{DMAn})$. Während sich die halogenierten Anisolderivate allesamt innerhalb des Zielbereichs befinden, zeigt sich für die in den grauen Bereich fallenden Datenpunkte alkylierter Anisole eine 


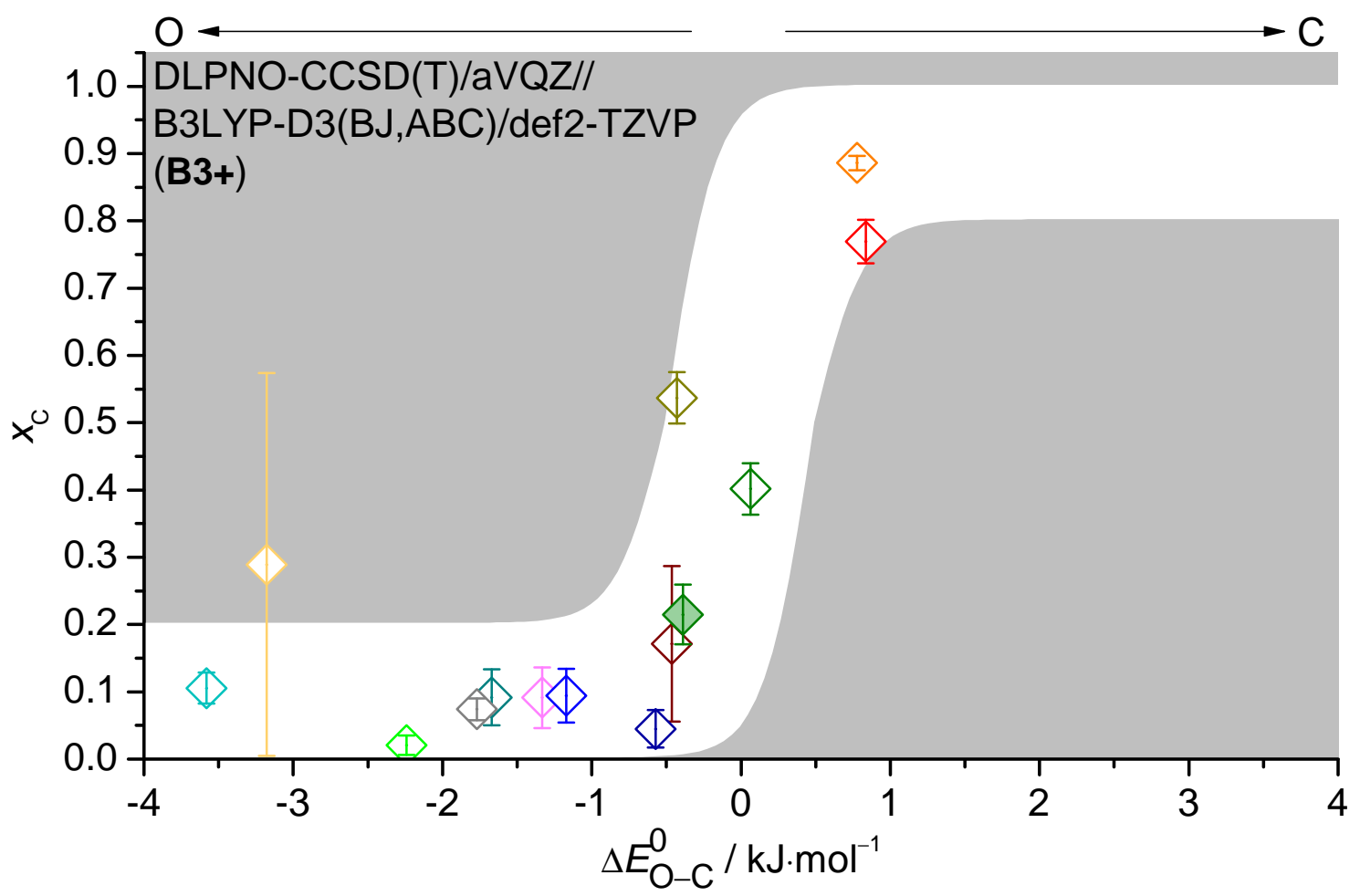

Abbildung 4.22: Analog zu Abbildung 4.21, aber für das DLPNO-CCSD(T)/aVQZ//B3LYP-D3(BJ, $\mathrm{ABC}$ )/def2-TZVP-Rechenniveau (B3+). Für $\Delta E_{\mathrm{O}-\mathrm{C}}^{0}$ s. Tab. 4.12, Quellen der restlichen Werte wie bei Abb. 4.21.

systematische Überschätzung der relativen Stabilität des C-gebundenen Dimers, wie sie für das M06-2X/aVTZ-Niveau bereits in der originalen Studie beobachtet werden konnte. ${ }^{[37]}$ Analog zu Referenz [37] lassen sich auch hier leichte Unterschiede im Abschneiden des M06-2X-Funktionals zwischen in 2- (also ortho-)Position substituierten und nicht-substituierten Anisolderivaten erkennen, wobei die nicht-ortho-substituierten Anisolderivate tendenziell eine stärkere Überschätzung der relativen C-Stabilität aufweisen. Im Vergleich zum B3- bzw. B3+-Niveau fallen zudem die kleineren $x_{c}$-Werte auf, die durch die auf dem M6-Niveau kleineren Bandenstärkenverhältnisse bedingt sind (s. oben). Beides lässt sich auf eine gemeinsame Ursache zurückführen: der Unterschätzung der relativen Stabilität der O-a-gebundenen Dimere mit der M6-Methode. Während dieser Strukturtyp für B3 und B3+ für alle nicht-ortho-substituierten Anisolderivate das O-Minimum darstellt, ist auf dem M6-Niveau in diesen Fällen stets O-o die stabilere Dimerstruktur (s. Kap. 4.2.2). Dies führt zu den systematisch kleineren Bandenstärkenverhältnissen und begünstigt die Verzerrung hin zu positiven Energieunterschieden, also zur C-Seite der intermolekularen Waage, die für diese nicht-ortho-substituierten Anisolderivate besonders ausgeprägt ist. Die für die ortho-substituierten Anisolderivate in vielen Fällen ebenfalls vorhandene Verschiebung in Richtung C-Dimer zeigt aber, dass die Unterschätzung der relativen O-a- 


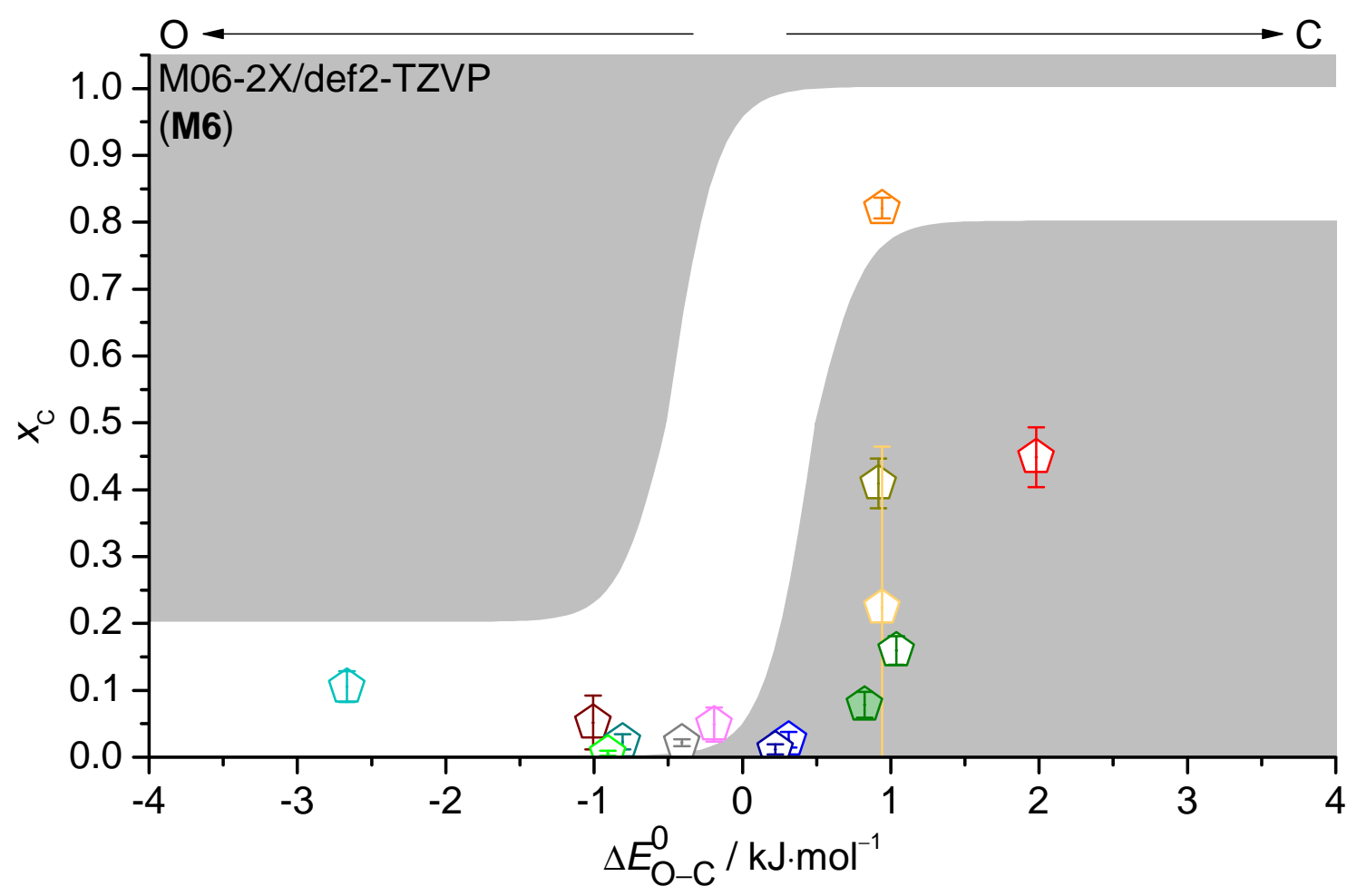

Abbildung 4.23: Analog zu Abbildung 4.21, aber für das M06-2X/def2-TZVP-Rechenniveau (M6). Für $\Delta E_{\mathrm{O}-\mathrm{C}}^{0}$ s. Tab. 4.13, Quellen der restlichen Werte wie bei Abb. 4.21.

Stabilität nicht die einzige Ursache für das schlechte Abschneiden der M6-Methode ist. Hinsichtlich der im Experiment tatsächlich vorliegenden Dimerstrukturen, vor allem in den O-gebundenen Dimeren, könnte Mikrowellenspektroskopie wie schon für das Methanol-Furan-System (s. Kap. 4.1) auch hier klärend wirken.

Insgesamt verdeutlicht die Auswertung des O/C-Konformerengleichgewichts, dass mit dem Methanol-Anisol-Waagensystem ein experimenteller Datensatz zur Verfügung steht, anhand dessen eine Überprüfung der Leistungsfähigkeit quantenchemischer Methoden möglich ist. Über die verschiedenen Anisolderivate zeigen sich sowohl eindeutige Ooder C-Präferenzen als auch einige, auf Änderungen im Energieunterschied besonders empfindliche, ausgewogene O/C-Situationen. Der Datensatz ermöglicht damit einen Vergleich zu quantenchemischen Methoden über verschiedene Bindungssituationen hinweg. Bei einem durchweg guten Abschneiden einer untersuchten quantenchemischen Methode für alle untersuchten Methanol-Anisol(derivat)-Kombinationen kann ein Basieren des Erfolges auf Fehlerkompensation deshalb mit sehr viel höherer Wahrscheinlichkeit ausgeschlossen werden als für einzelne Referenzdatenpunkte. ${ }^{[31]}$ Die Ergebnisse für die B3 und B3+-Methoden einerseits und für die M6-Methode andererseits zeigen, dass auf Basis dieses Systems zudem mit hoher Empfindlichkeit zwischen zum Experiment passenden und nicht-passenden berechneten Energieunterschieden - in günstigen Fällen bis in den sub- 
$\mathrm{kJ} /$ mol-Bereich - und Bandenstärkenverhältnissen differenziert werden kann. Die Befunde der originalen Studie ${ }^{[37]}$ hinsichtlich des Abschneidens der B3LYP-D3- und M06-2XDichtefunktionale können somit auch für den um halogenierte Anisole erweiterten Datensatz ${ }^{[31]}$ und für die überarbeiteten Rechenmethoden (u. a. andere Basissätze, s. oben) bestätigt werden.

\subsubsection{Auswertung IR-spektroskopischer Parameter}

Im Gegensatz zum Benchmarking des Energieunterschieds, für das neben den experimentellen Intensitätsverhältnissen auch die berechneten Bandenstärken und fundierte Schätzungen für die Konformationstemperatur benötigt werden, können für das Benchmarking IR-spektroskopischer Parameter direkt Observablen aus dem Experiment verwendet werden. ${ }^{[33]}$ Aufgrund der sich nach Bindungsstelle $(\mathrm{O} / \mathrm{C})$ unterscheidenden Werte sind die OH-Streckschwingungsrotverschiebungen der gemischten Dimere bzw. die Aufspaltung zwischen den entsprechenden Dimerbanden hierzu am besten geeignet und sollen im Folgenden für den Vergleich mit den quantenchemischen Daten herangezogen werden.

Wie in Kapitel 2.3.1 erläutert, können die experimentellen anharmonischen Rotverschiebungen auf die harmonischen Anteile rückkorrigiert werden, ${ }^{[33]}$ da sich diagonale und außerdiagonale Beiträge zur Anharmonizität bis zu einem gewissen Grad kompensieren. ${ }^{[67]}$ Das für die Methanol-Anisol(derivat)-Dimere beobachtete, über die verschiedenen Anisolderivate hinweg relativ regelmäßige Bandenmuster (weniger stark rotverschobene $\mathrm{MA}_{\mathrm{C}^{-}}$und stärker rotverschobene $\mathrm{MA}_{\mathrm{O}^{-}}$-Bande, s. Abb. 4.14) lässt in der Tat darauf schließen, dass die verbleibenden anharmonischen Beiträge zu den Rotverschiebungen analog zum MeOH-Furan-Waagensystem (s. Kap. 4.1) relativ konstant sind. Dies ermöglicht einen sinnvollen Vergleich mit den harmonisch berechneten Werten. Während für die absolute Größe dieser Verschiebungen keine direkte Übereinstimmung zwischen (anharmonischem) Experiment und (harmonischer) Theorie zu erwarten ist, sollte eine „gute" Rechenmethode die experimentellen Trends bei Anisol-Substitution in einem solchen Fall relativ gleichförmiger Anharmonizität systematisch wiedergeben können. Unter Berücksichtigung der in der Rückkorrektur vorgesehenen Sicherheitsmarge von $\pm 50 \%$ kann zudem ein direkter Vergleich mit den berechneten Werten erfolgen. ${ }^{[33]}$ Da für den Donor MeOD lediglich ein Anisolderivat untersucht wurde und somit keine Trends analysiert werden können, werden nur die $\mathrm{MeOH}-D a t e n$ für die Auswertung berücksichtigt.

Die für den Vergleich herangezogenen harmonischen berechneten $\left(\Delta \omega_{\mathrm{OH}}\right.$,theor $)$ bzw. anharmonischen experimentellen $\left(\Delta \tilde{v}_{\mathrm{OH}, \exp }\right)$ Rotverschiebungen $\Delta(\tilde{v}, \omega)_{\mathrm{OH}}=$ $(\tilde{v}, \omega)_{\mathrm{OH}}(\mathrm{M})-(\tilde{v}, \omega)_{\mathrm{OH}}\left(\mathrm{MA}_{\mathrm{O}, \mathrm{C}}\right) \quad\left(\right.$ mit $(\tilde{v}, \omega)_{\mathrm{OH}}(\mathrm{M})$ : Methanol-Monomer-OH-Streckschwingungswellenzahl; $\quad(\tilde{v}, \omega)_{\mathrm{OH}}\left(\mathrm{MA}_{\mathrm{O}, \mathrm{C}}\right): \quad \mathrm{O} / \mathrm{C}$-Dimer-OH-Streckschwingungswellenzahl) aus dem Experiment (Exp) und den Schwingungsrechnungen auf B3LYPD3(BJ,ABC)/def2-TZVP- (B3) bzw. M06-2X/def2-TZVP-Niveau (M6) sind in Tabelle 4.17 aufgelistet. Aufseiten der quantenchemischen Methoden wurden dazu die OH-Streckschwingungswellenzahlen des jeweils schwingungsnullpunktskorrigiert stabilsten $\mathrm{O}$ und C-Dimers herangezogen. Da auf dem DLPNO-CCSD(T)/aVQZ-Rechenniveau ledig- 
Tabelle 4.17: Experimentell bestimmte anharmonische $\left(\Delta \tilde{v}_{\mathrm{OH}, \exp }\right)$ und berechnete harmonische $\left(\Delta \omega_{\mathrm{OH}, \text { theor }}\right)$ Rotverschiebungen der OH-Streckschwingungsbanden relativ zum Methanol-Monomer (Exp: $3686 \mathrm{~cm}^{-1}$, B3: $3810 \mathrm{~cm}^{-1}$, M6: $3893 \mathrm{~cm}^{-1}$ ) für das jeweils beobachtete bzw. schwingungsnullpunktskorrigiert stabilste O- und C-Methanol-Anisol(derivat)-Dimer. Verwendete Rechenniveaus: B3LYP-D3(BJ,ABC)/def2-TZVP (B3), M06-2X/def2-TZVP (M6).

\begin{tabular}{|c|c|c|c|c|c|c|}
\hline \multirow[b]{2}{*}{$\Delta(\tilde{v}, \omega)_{\mathrm{OH}} / \mathrm{cm}^{-1}$} & \multicolumn{3}{|c|}{$\Delta(\tilde{v}, \omega)_{\mathrm{OH}}(\mathrm{O})$} & \multicolumn{3}{|c|}{$\Delta(\tilde{v}, \omega)_{\mathrm{OH}}(\mathrm{C})$} \\
\hline & $\operatorname{Exp}$ & B3 & M6 & $\operatorname{Exp}$ & B3 & M6 \\
\hline $\mathrm{MeOH}+\mathrm{An}$ & 89 & 102 & 36 & 57 & 50 & 42 \\
\hline $\mathrm{MeOH}+2 \mathrm{MAn}$ & 87 & 97 & 48 & 63 & 56 & 43 \\
\hline $\mathrm{MeOH}+3 \mathrm{MAn}(E)$ & 92 & 105 & 37 & 60 & 59 & 41 \\
\hline $\mathrm{MeOH}+3 \mathrm{MAn}(Z)$ & 92 & 104 & 37 & 60 & 70 & 42 \\
\hline $\mathrm{MeOH}+4 \mathrm{MAn}$ & 92 & 106 & 39 & 60 & 56 & 42 \\
\hline $\mathrm{MeOH}+2 \mathrm{ClAn}$ & 75 & 57 & 55 & 59 & 37 & 36 \\
\hline $\mathrm{MeOH}+4 \mathrm{FAn}$ & 85 & 98 & 37 & 58 & 41 & 37 \\
\hline $\mathrm{MeOH}+4 \mathrm{ClAn}$ & 81 & 93 & 34 & 58 & 40 & 36 \\
\hline $\mathrm{MeOH}+4 \mathrm{BrAn}$ & 81 & 91 & 33 & 60 & 39 & 36 \\
\hline $\mathrm{MeOH}+23 \mathrm{DMAn}$ & 87 & 96 & 47 & 66 & 47 & 47 \\
\hline $\mathrm{MeOH}+26 \mathrm{DMAn}$ & 106 & 127 & 80 & 69 & 56 & 51 \\
\hline $\mathrm{MeOH}+35 \mathrm{DMAn}$ & 94 & 107 & 38 & 67 & 61 & 43 \\
\hline $\mathrm{MeOH}+235 \mathrm{TMAn}$ & 87 & 98 & 48 & 72 & 52 & 47 \\
\hline $\mathrm{MeOH}+4 \mathrm{tBuAn}$ & 92 & 105 & 36 & 67 & 53 & 44 \\
\hline
\end{tabular}

lich Einzelpunktrechnungen und keine eigenen Schwingungsrechnungen durchgeführt wurden, werden für diesen Vergleich nur die B3- und M6-Methode berücksichtigt.

Auf Basis dieser Daten sind die berechneten Rotverschiebungen $\Delta \omega_{\mathrm{OH}}$,theor der B3(Quadrate) und M6-Methode (Fünfecke) in Abbildung 4.24 gegen die experimentellen Rotverschiebungen $\Delta \tilde{v}_{\mathrm{OH} \text {,exp }}$ aufgetragen. Die Rotverschiebungen der O-gebundenen Dimere sind darin mit horizontal getrennten farbigen/weißen Füllungen dargestellt, die C-gebundenen Dimere mit vertikal getrennten Füllungen. Der sich aus der Summe der Rückkorrektur $^{[33]}$ der experimentellen anharmonischen auf harmonische Werte $\left(\Delta \tilde{v}_{\mathrm{OH}, \exp } \pm 50 \%\right)$ und dem experimentellen Fehler von $2 \mathrm{~cm}^{-1}$ (s. Kap. 2.3.1) ergebende Bereich ist weiß hervorgehoben. Beim Vergleich der berechneten Daten untereinander zeigt sich zunächst, dass die auf dem M6-Niveau berechneten Rotverschiebungen durchgängig kleiner sind als ihre B3-Gegenstücke. Während die Unterschiede für die C-gebundenen Dimere eher klein sind, ergeben sich für die O-gebundenen Dimere deutlich größere Abweichungen. So sind die mit der B3-Methode berechneten Rotverschiebungen der O-gebundenen Dimere mit Ausnahme des O-Dimers von $\mathrm{MeOH}+2 \mathrm{ClAn}$ (türkis, durch Überlappung mit M6-Datenpunkt teils verdeckt) allesamt größer $\left(91-127 \mathrm{~cm}^{-1}\right)$ als die Rotverschiebungen der C-Dimere (37-70 $\mathrm{cm}^{-1}$ ). Für die M6-Methode weisen die O- und C-Rotverschiebun- 


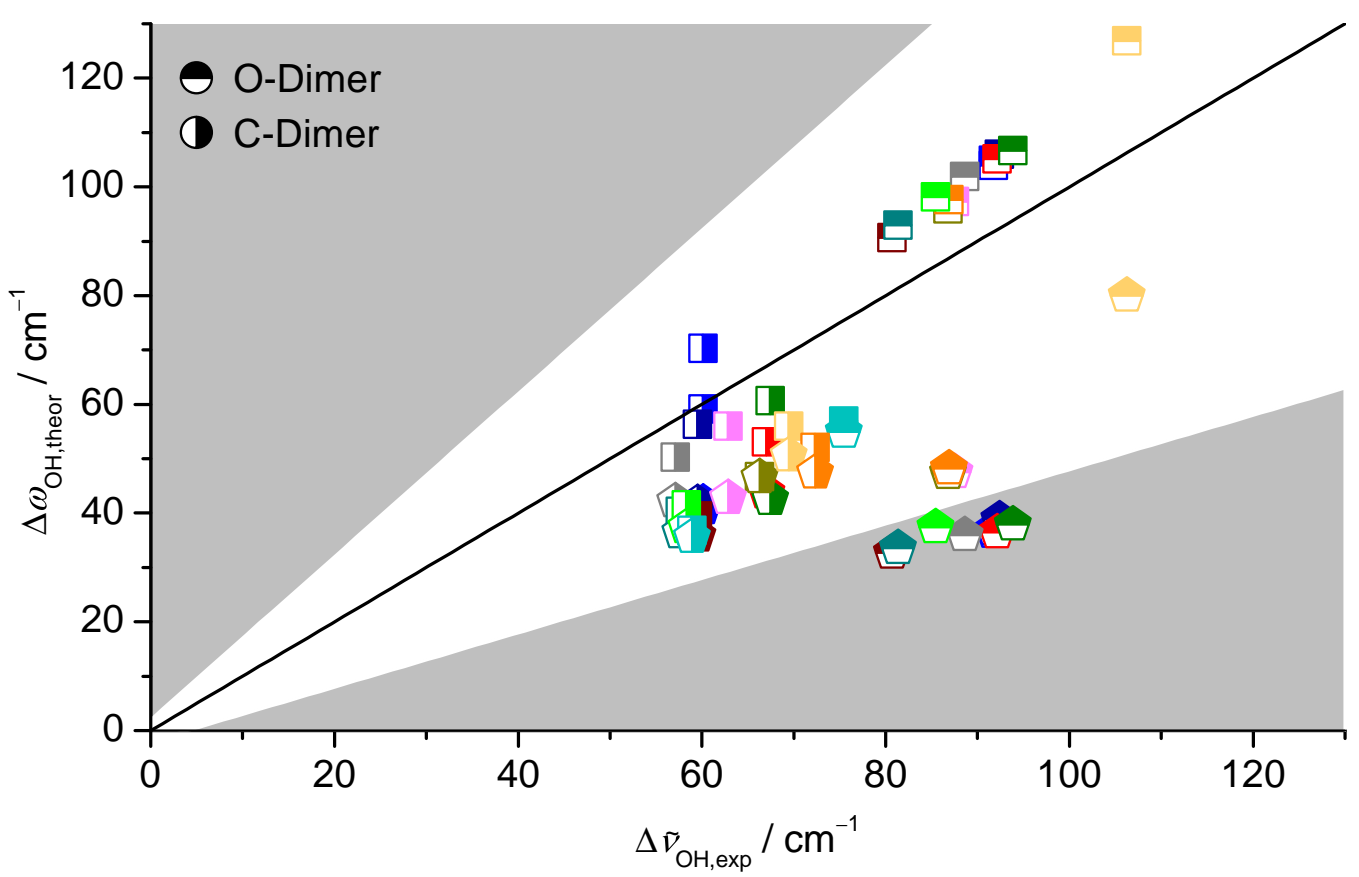

Abbildung 4.24: Auftragung der auf dem B3- (Quadrate) bzw. M6-Niveau (Fünfecke) berechneten Rotverschiebungen $\Delta \omega_{\mathrm{OH}}$,theor der $\mathrm{MeOH}$-Anisol-Dimere gegen die experimentellen Rotverschiebungen $\Delta \tilde{v}_{\mathrm{OH} \text {,exp }}$ der O- (oben/unten farbig/weiß) und C-gebundenen (links/rechts weiß/farbig) Dimere (s. Tab. 4.17). Zur Zuordnung zu den Anisolderivaten wird erneut die in Abbildung 4.13 eingeführte Farbkennzeichnung verwendet. Als Referenz ist die Winkelhalbierende $\Delta \omega_{\mathrm{OH} \text {,theor }}=\Delta \tilde{v}_{\mathrm{OH}, \exp }$ eingezeichnet (schwarz). Der aus der von Oswald und SuHM vorgeschlagenen Rückkorrektur ${ }^{[33]}$ und der Messungenauigkeit resultierende Bereich (s. Kap. 2.3.1) ist weiß hervorgehoben.

gen mit Ausnahme des O-Dimers von $\mathrm{MeOH}+26 \mathrm{DMAn}$ (hellgelb) hingegen vergleichbare Werte auf (O: $33-55 \mathrm{~cm}^{-1}$; C: $\left.36-51 \mathrm{~cm}^{-1}\right)$.

Im Experiment zeigen sich für alle O-Dimere größere Rotverschiebungen $\left(75-106 \mathrm{~cm}^{-1}\right)$ als für die C-Dimere $\left(57-72 \mathrm{~cm}^{-1}\right)$. Die B3-Methode kann den experimentellen Trend also deutlich besser reproduzieren als die M6-Methode, wie die graphische Darstellung auch verdeutlicht. Für die O-Dimere der nicht-ortho-substituierten Anisolderivate lassen sich die geringen Rotverschiebungen auf dem M6-Niveau erneut auf die Unterschätzung der relativen Stabilität der O-a-Dimere zurückführen, da die somit stabiler eingeschätzten $\mathrm{O}-\mathrm{o}$-Dimere durchgehend geringere Rotverschiebungen aufweisen. Interessanterweise zeigen diese Dimere (O-Dimere der nicht-ortho-substituierten Anisolderivate) eine auffallend starke Korrelation zwischen berechneten und experimentellen Rotverschiebungen (Bestimmtheitsmaß $R^{2}=0.9991$ ), unterschätzen die experimentellen Rotverschiebungen dabei jedoch um knapp 60\% und liegen damit außerhalb des mit dem Experiment vereinbaren Zielbereichs (weiß).

Für die B3-Methode liegt, wie bereits erwähnt, insgesamt eine deutlich bessere Re- 
produktion der experimentellen Trends vor. Zwar zeigen die berechneten Rotverschiebungen der C-gebundenen Dimere auch auf dem B3-Niveau keine eindeutige Korrelation mit dem Experiment und weisen größtenteils eine Unter-, teilweise jedoch auch eine Überschätzung der Rotverschiebung auf, es liegen aber alle Datenpunkte innerhalb des weißen Zielbereichs. Für die O-Dimere ergibt sich zudem eine äußerst systematische $\left(R^{2}=0.9996\right)$ Überschätzung der Rotverschiebung um etwa $14 \%$, wenn das O-Dimer von $\mathrm{MeOH}+2 \mathrm{ClAn}$ (türkis) nicht berücksichtigt wird. Möglicherweise kann dieser Ausreißer durch die besondere Struktur dieses Dimers (s. Abb. 4.19 auf S. 145) erklärt werden, die zu anderen anharmonischen Effekten führen könnte. Die ansonsten systematische Überschätzung der $\mathrm{OH} \cdots \mathrm{O}$-Rotverschiebungen durch das B3LYP-Funktional bestätigt frühere Befunde. ${ }^{[33,49,181,336]}$ Zusammen mit den passend zum Experiment für O- im Vergleich zu C-Dimeren (nahezu) konsistent größeren Rotverschiebungen reproduziert die B3-Methode die experimentellen Trends somit gut und liegt zudem hinsichtlich der absoluten Größen der berechneten harmonischen Rotverschiebungen durchgehend im Zielbereich. Insbesondere aufgrund des systematischen Verhaltens für O-gebundene Dimere sollte sich die B3-Methode als zuverlässige Zuordnungshilfe für die Dimerbanden bisher unerforschter Methanol-Anisolderivat-Systeme eignen. Entsprechende Ergebnisse für das B3LYP-Funktional mit dem aVTZ-Basissatz ${ }^{[37]}$ können also bestätigt werden. Die M6-Methode lässt dagegen insgesamt keine eindeutige Korrelation zu den experimentellen Rotverschiebungen erkennen und sagt im Widerspruch zum Experiment zudem vergleichbar große Rotverschiebungen für O- und C-gebundene Dimere vorher. Entsprechend liegt ein Teil der berechneten O-Rotverschiebungen (nicht-ortho-substituierte Anisolderivate) unterhalb des experimentellen Zielbereichs, sodass sich die Methode auf Basis dieser Daten nicht als Hilfe für die Bandenzuordnung zukünftig untersuchter Systeme empfiehlt.

Ein strikteres Benchmarking wird möglich, wenn nicht die Rotverschiebung $\Delta(\tilde{v}, \omega)_{\mathrm{OH}}$, sondern die Bandenaufspaltung $\Delta \Delta(\tilde{v}, \omega)_{\mathrm{OH}}(\mathrm{O}-\mathrm{C})$ zwischen $\mathrm{O}$ - und C-gebundenem Dimer herangezogen wird (s. Kap. 2.3.1). In Abbildung 4.25 ist die Bandenaufspaltung $\Delta \Delta(\tilde{v}, \omega)_{\mathrm{OH}}(\mathrm{O}-\mathrm{C})$ gegen die Rotverschiebung $\Delta(\tilde{v}, \omega)_{\mathrm{OH}}(\mathrm{O})$ des O-gebundenen Dimers aufgetragen. Die experimentellen Werte (farbig gefüllte Kreise) sind jeweils mit ihrem aus Rückkorrektur und Messungenauigkeit erhaltenen Fehlerintervall (entsprechend eingefärbte und hellgrau hervorgehobene Rechtecke) versehen. Fallen die harmonischen berechneten Werte (B3: weiß gefüllte Quadrate; M6: weiß gefüllte Fünfecke) in die rechteckigen Fehlerbereiche des entsprechenden experimentellen Datenpunkts, sind die Ergebnisse mit dem Experiment vereinbar.

Dies ist für die $\mathrm{OH} \cdots \mathrm{O}$-Rotverschiebung (Abszisse), wie weiter oben bereits beschrieben, auf dem B3-Niveau für alle Methanol-Anisol(derivat)-Kombinationen der Fall, auf dem M6-Niveau hingegen nur für die ortho-substituierten Anisolderivate. Aufgrund des kleineren Fehlerintervalls für die Rückkorrektur ergibt sich für die Bandenaufspaltung (Ordinate) eine deutlich stärkere Diskrimination durch das Experiment. Auf dem B3(2ClAn, 3MAn $(Z)$ ) und M6-Niveau (2ClAn, 26DMAn) liegen nun jeweils nur noch zwei Datenpunkte im Zielbereich. Die beste Reproduktion der experimentell beobachteten Aufspaltung gelingt beiden betrachteten Rechenmethoden interessanterweise mit nahezu 


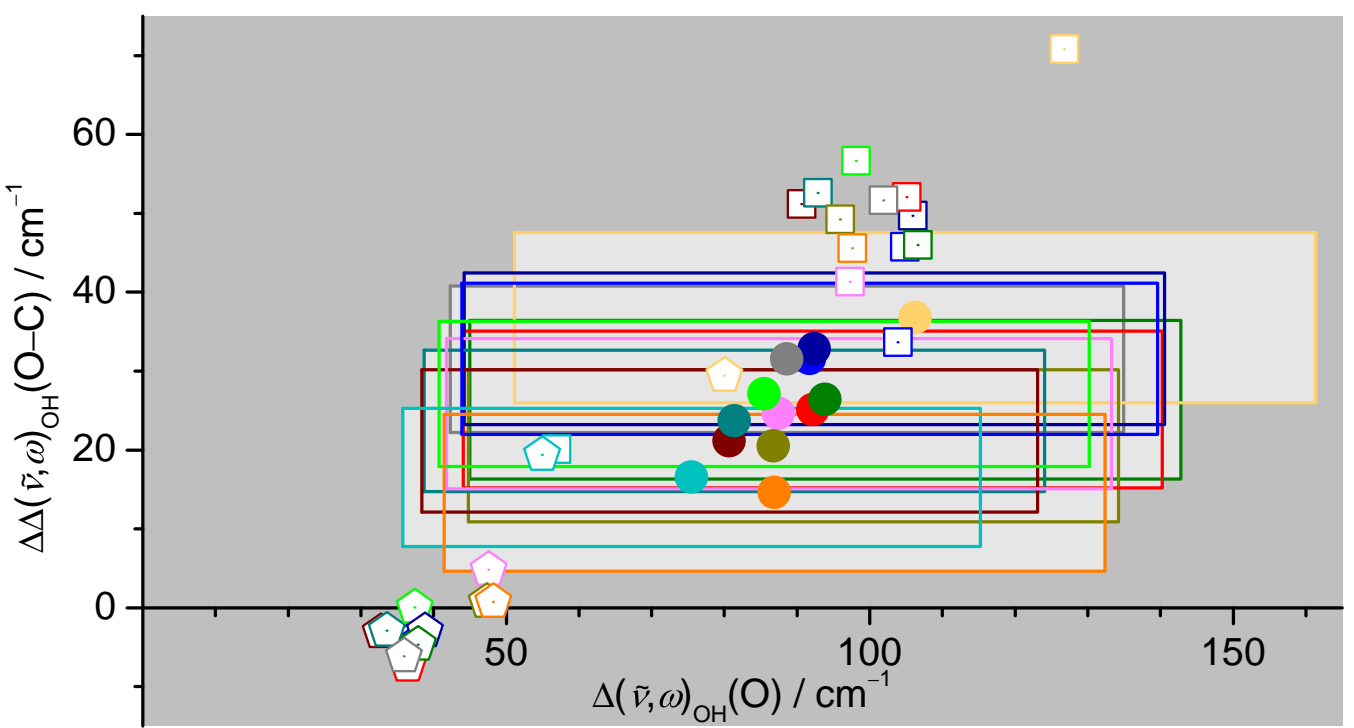

Abbildung 4.25: Auftragung der Bandenaufspaltung $\Delta \Delta(\tilde{v}, \omega)_{\mathrm{OH}}(\mathrm{O}-\mathrm{C})$ zwischen den $\mathrm{OH}$ Streckschwingungsbanden des $\mathrm{O}$ - und $\mathrm{C}$-gebundenen Dimers gegen die Rotverschiebung $\Delta(\tilde{v}, \omega)_{\mathrm{OH}}(\mathrm{O})$ der $\mathrm{OH}$-Streckschwingungsbande des $\mathrm{O}$-gebundenen Dimers für $\mathrm{MeOH}$ mit den verschiedenen Anisolderivaten. Die experimentellen Daten sind als farbig ausgefüllte Kreise dargestellt und die von den jeweiligen experimentellen Fehlern und der Rückkorrektur ${ }^{[33]}$ aufgespannten Bereiche (s. Kap. 2.3.1) sind in der entsprechenden Farbe umrandet und hellgrau eingefärbt. Die berechneten Daten sind als weiß gefüllte Quadrate (B3) bzw. Fünfecke (M6) abgebildet. Die Farbkennzeichnung ermöglicht eine Zuordnung zu den Anisolderivaten gemäß Abb. 4.13.

übereinstimmenden Werten für $\mathrm{MeOH}+2 \mathrm{ClAn}$ (türkis). In den weiteren Fällen wird die Aufspaltung durch die B3-Methode durchgehend über- und durch die M6-Methode durchgehend unterschätzt. Die auf M6-Niveau berechneten Aufspaltungen liegen dabei größtenteils im Bereich um $0 \mathrm{~cm}^{-1}$, wodurch eine Zuordnung der im Spektrum beobachteten Banden zu O- und C-gebundenem Dimer auf Basis dieser Rechendaten nicht möglich wäre. Die systematische Überschätzung der Aufspaltung durch die B3Methode ermöglicht hingegen eine Zuordnung, auch wenn die absoluten Werte in den meisten Fällen ebenso außerhalb des experimentellen Zielbereichs liegen.

Soll die Leistungsfähigkeit der quantenchemischen Methoden hinsichtlich der Berechnung der genannten schwingungsspektroskopischen Größen in Bezug zum Experiment quantifiziert werden, können die ebenfalls in Ref. [33] vorgestellten $\overline{\delta_{\mathrm{Xn}}^{2}}(\mathrm{Q})$-Werte herangezogen werden (s. Kap. 2.3.1). In diesen sind die mit dem inversen experimentellen Fehler gewichteten quadratischen Abweichungen zwischen berechnetem und rückkorrigiertem experimentellen Wert für eine bestimmte Observablenklasse gemittelt.

Diese Mittelwerte sind für die hier betrachteten Observablenklassen O- $\left(\mathrm{S} 1_{\mathrm{O}}\right), \mathrm{C}-\mathrm{Di}$ mer-Rotverschiebung $\left(\mathrm{S}_{\mathrm{C}}\right)$ und Dimerbandenaufspaltung (S2) in Abbildung 4.26 zusammengefasst. Durch die wesentlich kleineren experimentellen Fehler ergeben sich für die 


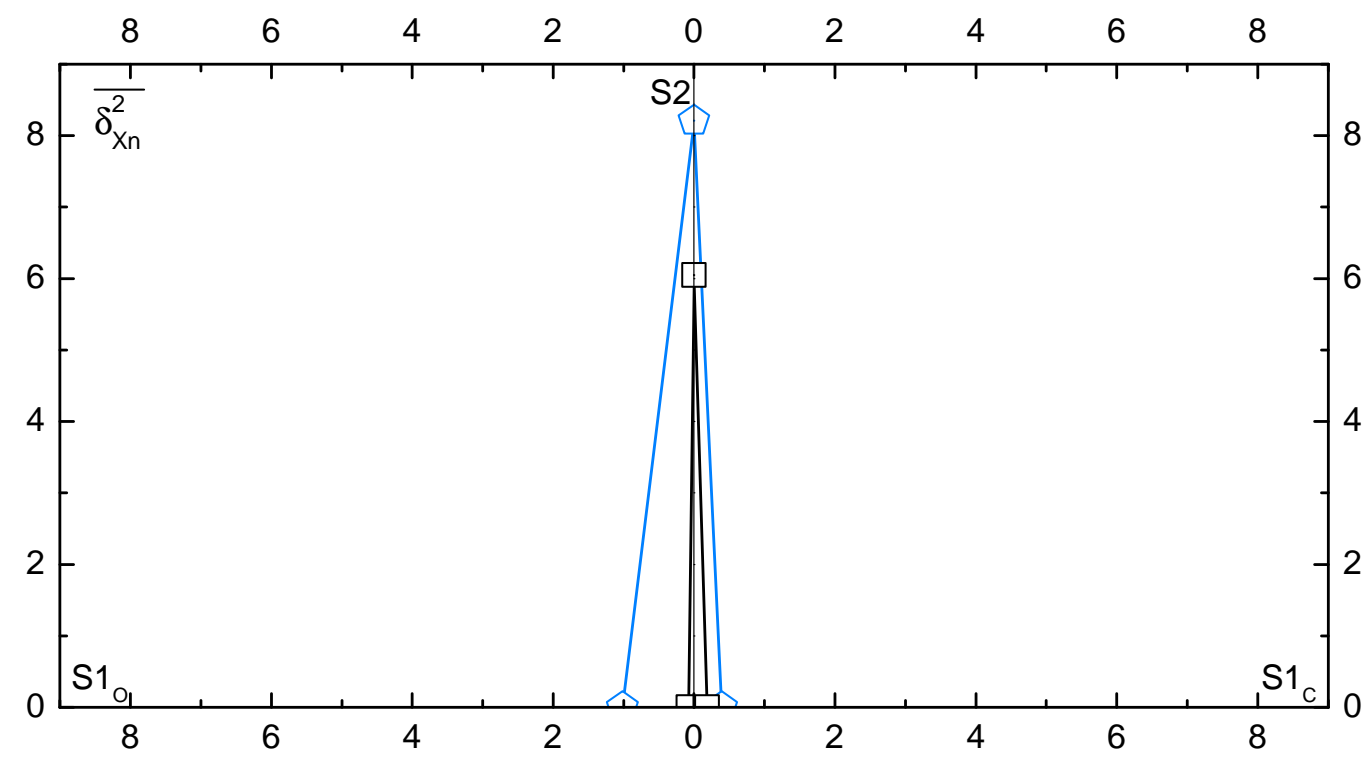

Abbildung 4.26: Über die unterschiedlichen Methanol-Anisol(derivat)-Kombinationen gemittelte Abweichungswerte $\overline{\delta_{\mathrm{Xn}}^{2}}(\mathrm{Q})^{[33]}$ der Ergebnisse der quantenchemischen Methoden B3 (schwarze Quadrate) und M6 (blaue Fünfecke) für die Observablen $\mathrm{OH}-$ Streckschwingungsrotverschiebung im O- $\left(\mathrm{S} 1_{\mathrm{O}}\right)$ bzw. C-gebundenen $\left(\mathrm{S}_{\mathrm{C}}\right)$ Dimer (Abszisse) bzw. O/C-Dimer-OH-Streckschwingungsbandenaufspaltung (S2, Ordinate).

Bandenaufspaltung deutlich größere $\delta^{2}$-Werte als für die nur ungenau aus dem Experiment auf harmonische Werte rückkorrigierbaren Rotverschiebungen. Der Vergleich der $\overline{\delta_{\mathrm{Xn}}^{2}}$-Werte für B3 (schwarze Quadrate) und M6 (blaue Fünfecke) zeigt, dass die B3-Methode nicht nur qualitativ (s. oben) sondern auch quantitativ durchgehend deutlich besser zur experimentellen Referenz passende Ergebnisse liefert, insbesondere für die Rotverschiebungen der O-Dimere. Somit ergibt sich für das Methanol-Anisol-Waagensystem für die untersuchten schwingungsspektroskopischen Größen ein anderes Resultat als für die entsprechenden Observablenklassen im ENCH-51-Referenzdatensatz. Für diesen kann das M06-2X- insgesamt bessere Ergebnisse als das B3LYP-D3-Dichtefunktional (ohne Dreikörperterm in der Dispersionskorrektur) erzielen, wobei die Funktionale in der Studie mit größeren Basissätzen von Quadruple- $\zeta$-Qualität kombiniert wurden. ${ }^{\text {[3] }}$

Zusammenfassend zeigt sich somit auch hinsichtlich der Untersuchung der IR-spektroskopischen Parameter, dass das Methanol-Anisol-Waagensystem für das Benchmarking quantenchemischer Methoden geeignet ist. Das im Experiment beobachtete gleichförmige Bandenmuster lässt auf die Abwesenheit größerer Unterschiede in den Anharmonizitäten zwischen den verschiedenen Dimeren schließen, sodass besonders für die Bandenaufspaltung ein Vergleich zu den berechneten harmonischen Werten erfolgen kann. Analog zum Energie-Benchmarking (s. Kap. 4.2.3) erlaubt das Waagensystem eine empfindliche Differenzierung zwischen der Leistungsfähigkeit verschiedener quantenchemischer Me- 
thoden. Für die beiden beispielhaft untersuchten Methoden konnten so mehr (M6) oder weniger (B3) stark ausgeprägte Schwächen in der Beschreibung der schwingungsspektroskopischen Größen aufgedeckt werden.

\subsubsection{Analyse des Einflusses von Dispersionswechselwirkungen auf das $\mathrm{O} / \mathrm{C}$-Konformerengleichgewicht}

In Kap. 4.2.3 konnte gezeigt werden, dass die B3+-Methode sich gut zur Beschreibung des O/C-Gleichgewichts im Methanol-Anisol-Waagensystem eignet. Das in der B3+-Methode verwendete DLPNO-CCSD(T)-Verfahren bietet mit dem LED-Konzept (s. Kap. 2.1.2) eine Option, mit der unter anderem die Dispersionsbeiträge zur Wechselwirkung zwischen zwei Fragmenten - wie beispielsweise Donor und Akzeptor in einem wasserstoffbrückengebundenen Dimer - bestimmt werden können. Anders als die Ergebnisse der Berechnung der elektronischen Energie auf dem DLPNO-CCSD(T)-Niveau selbst können die Ergebnisse der LED-Analyse nicht anhand experimenteller Daten überprüft werden, sodass ihre Aussagekraft unklar bleibt. Dennoch soll auf Basis der LED-Daten eine grobe Analyse des Einflusses der Dispersion auf das O/C-Gleichgewicht erfolgen.

In Tabelle 4.18 sind die mit dem LED-Verfahren auf dem DLPNO-CCSD(T)/aVQZ-Niveau ermittelten Dispersionsbeiträge $E^{\text {disp }}$ zur Donor-Akzeptor-Wechselwirkung in den verschiedenen auf B3LYP-D3(BJ,ABC)/def2-TZVP-Niveau optimierten Methanol-Anisol(derivat)-Dimerstrukturen und die Differenz zwischen den Beiträgen des jeweils stabilsten O- und C-Dimers, $\Delta E_{\mathrm{O}-\mathrm{C}}^{\text {disp }}$, aufgelistet. Der Beitrag der für eine Bindungsstelle jeweils stabilsten Struktur ist fett hervorgehoben. Für die O-Dimere zeigt sich, dass die O-o-Strukturen generell stärkere Dispersionswechselwirkungen $\left(-16.4 \mathrm{bis}-18.8 \mathrm{~kJ} \cdot \mathrm{mol}^{-1}\right)$ als die Oa-Dimere $\left(-13.8 \mathrm{bis}-14.6 \mathrm{~kJ} \cdot \mathrm{mol}^{-1}\right)$ aufweisen. Dies lässt sich möglicherweise durch den geringeren Abstand zwischen Methanol-Methylgruppe und Anisolmolekül in den O-o-Dimeren erklären. Die O-o-Dimere der ortho-methylierten Anisolderivate weisen besonders hohe Dispersionsbeiträge von $-17.5 \mathrm{bis}-18.8 \mathrm{~kJ} \cdot \mathrm{mol}^{-1}$ auf, während die entsprechenden Werte für die nicht-ortho-methylierten Dimere bei $-16.4 \mathrm{bis}-17.2 \mathrm{~kJ} \cdot \mathrm{mol}^{-1}$ liegen. Dies gilt insbesondere für die nur einfach in ortho-Position methylierten Anisolderivate 2MAn, 23DMAn und 235TMAn $\left(<-18 \mathrm{~kJ} \cdot \mathrm{mol}^{-1}\right)$. Eine Methylgruppe in 2-Position scheint also Dispersionswechselwirkungen im O-o-Dimer zu begünstigen, wohingegen ein Cl-Atom in 2-Position (2ClAn) mit $-17.0 \mathrm{~kJ} \cdot \mathrm{mol}^{-1}$ nicht zu vergleichbar hohen Dispersionsbeiträgen führt. Generell scheint Dispersion für die O-Strukturselektivität jedoch keine entscheidende Rolle einzunehmen. So stellt der O-a-Strukturtyp in allen Fällen, in denen er gefunden wurde, das Minimum dar, obwohl er stets die geringere Dispersionsstabilisierung aufweist. Hinsichtlich des Substitutionsgrades zeigt sich eine schwache Korrelation zur Größe des Dispersionsbeitrags. Zwar liegen die Dispersionsbeiträge für die nicht und einfach substituierten Anisolderivate auf der einen und die höher substituierten Anisolderivate auf der anderen Seite mit $-13.8 \mathrm{bis}-18.4 \mathrm{~kJ} \cdot \mathrm{mol}^{-1}$ (Substitutionsgrad $\leq 1$ ) bzw. $-14.2 \mathrm{bis}-18.8 \mathrm{~kJ} \cdot \mathrm{mol}^{-1}$ (Substitutionsgrad $\geq 2$ ) in vergleichbaren Bereichen, die Me- 
Tabelle 4.18: Über die LED-Analyse bestimmte Dispersionsbeiträge $E^{\text {disp }}$ zur Bindungsenergie der MeOH-Anisol-Dimere, bestimmt auf Basis der DLPNO-CCSD(T)/aVQZ//B3LYP-D3(BJ,ABC)/ def2-TZVP-(B3+-)Rechnungen. Die schwingungsnullpunktskorrigiert auf diesem Rechenniveau jeweils stabilste Struktur einer Wasserstoffbrückenbindungsandockstelle (O bzw. C) ist fett hervorgehoben und die Energiedifferenz $\Delta E_{\mathrm{O}-\mathrm{C}}^{\mathrm{disp}}$ in $\mathrm{kJ} \cdot \mathrm{mol}^{-1}$ zwischen diesen Beiträgen ist in der rechten Spalte abgedruckt (Abweichungen sind auf Rundungsfehler zurückzuführen).

\begin{tabular}{|c|c|c|c|c|c|c|c|c|}
\hline 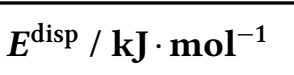 & $\mathbf{O}-\mathbf{a}$ & O-o & C-p & C-p' & C-s & C-s' & C-r & $\Delta E_{\mathrm{O}-\mathrm{C}}^{\text {disp }}$ \\
\hline $\mathrm{MeOH}+\mathrm{An}$ & -14.2 & -16.4 & -17.2 & & -16.9 & -14.6 & & 2.7 \\
\hline $\mathrm{MeOH}+2 \mathrm{MAn}$ & & -18.4 & -18.3 & & -18.3 & & & -0.0 \\
\hline $\mathrm{MeOH}+3 \mathrm{MAn}(E)$ & -14.6 & & -17.2 & & -17.4 & -15.6 & & 2.6 \\
\hline $\mathrm{MeOH}+3 \mathrm{MAn}(Z)$ & -13.9 & -16.8 & -17.6 & & -17.2 & -17.9 & & 3.7 \\
\hline $\mathrm{MeOH}+4 \mathrm{MAn}$ & -13.8 & -16.7 & -17.4 & -16.8 & -17.4 & -16.3 & & 3.6 \\
\hline $\mathrm{MeOH}+2 \mathrm{ClAn}$ & -14.3 & -17.0 & -18.8 & & -18.3 & & -18.1 & 4.0 \\
\hline $\mathrm{MeOH}+4 \mathrm{FAn}$ & -14.1 & -16.5 & -16.9 & & -16.5 & & -16.8 & 2.4 \\
\hline $\mathrm{MeOH}+4 \mathrm{ClAn}$ & -14.0 & -16.4 & -17.3 & -16.9 & -16.6 & & & 2.6 \\
\hline $\mathrm{MeOH}+4 \mathrm{BrAn}$ & -14.0 & -16.6 & -17.4 & -17.3 & -16.6 & & -17.2 & 3.3 \\
\hline $\mathrm{MeOH}+23 \mathrm{DMAn}$ & & -18.8 & -18.2 & -16.7 & -19.1 & & & -0.5 \\
\hline $\mathrm{MeOH}+26 \mathrm{DMAn}$ & & -17.5 & -18.2 & -15.6 & -20.1 & & -18.0 & 2.6 \\
\hline $\mathrm{MeOH+35DMAn}$ & -14.4 & -17.2 & -18.2 & & -17.7 & -18.8 & -17.5 & 3.8 \\
\hline $\mathrm{MeOH}+235 \mathrm{TMAn}$ & & -18.7 & -19.7 & & -19.4 & -18.7 & & 0.9 \\
\hline $\mathrm{MeOH}+4 \mathrm{tBuAn}$ & -14.2 & -17.0 & -19.2 & & -18.5 & -19.4 & -19.1 & 4.4 \\
\hline
\end{tabular}

dianwerte offenbaren jedoch eine Steigerung von $-15.5 \mathrm{~kJ} \cdot \mathrm{mol}^{-1}$ für die niedriger zu $-17.2 \mathrm{~kJ} \cdot \mathrm{mol}^{-1}$ für die höher substituierten Anisolderivate.

Aufseiten der C-Dimere sind keine vergleichbaren systematischen Unterschiede zwischen den verschiedenen Strukturtypen erkennbar. Die Größe der Dispersionsbeiträge liegt hier zwischen -14.6 und $-20.1 \mathrm{~kJ} \cdot \mathrm{mol}^{-1}$ und erneut ist die Dispersion nicht der entscheidende Faktor für die Strukturselektivität. Nur in 3 der 14 untersuchten MethanolAnisol(derivat)-Kombinationen stellt die Struktur mit dem höchsten Dispersionsbeitrag auch das C-Minimum dar. Auch für die C-Dimere zeigt sich insgesamt ein schwacher Trend zu stärkeren Dispersionsbeiträgen für die höher substituierten Anisolderivate. Liegen die Dispersionsbeiträge für die nicht- oder einfach substituierten Anisolderivate zwischen -14.6 und $-18.8 \mathrm{~kJ} \cdot \mathrm{mol}^{-1}\left(\right.$ Median $\left.-17.2 \mathrm{~kJ} \cdot \mathrm{mol}^{-1}\right)$, so nehmen diese für die zweifach oder höher substituierten Anisolderivate Werte zwischen -15.6 und $-20.1 \mathrm{~kJ} \cdot \mathrm{mol}^{-1}$ (Median $-18.6 \mathrm{~kJ} \cdot \mathrm{mol}^{-1}$ ) an.

Für die Analyse der Dispersionswechselwirkungen in Bezug auf das Waagensystem ist der Einfluss von Dispersion auf die O/C-Regioselektivität besonders interessant. Hierzu kann die Dispersions-Differenz $\Delta E_{\mathrm{O}-\mathrm{C}}^{\text {disp }}$ herangezogen werden. Diese ist so definiert, dass das Vorzeichen der bisherigen Konvention folgt - ein negatives Vorzeichen bedeutet also stärkere Dispersionswechselwirkungen im O-, ein positives Vorzeichen stärkere Disper- 
sionswechselwirkungen im C-Dimer.

Bei Betrachtung der Werte für $\Delta E_{\mathrm{O}-\mathrm{C}}^{\text {disp }}$ (s. Tab. 4.18) zeigt sich, dass in 12 der 14 betrachteten Methanol-Anisol(derivat)-Kombinationen das C-Dimer stärker durch Dispersion stabilisiert wird als das O-Dimer. Lediglich für die jeweils einfach in ortho-Position methylierten Anisolderivate 2MAn $\left(-0.04 \mathrm{~kJ} \cdot \mathrm{mol}^{-1}\right)$ und 23DMAn $\left(-0.5 \mathrm{~kJ} \cdot \mathrm{mol}^{-1}\right)$ zeigt sich eine leichte Dispersions-Bevorzugung des O-Dimers. In beiden Fällen stellen besonders dispersionsstabilisierte O-o-Dimere das O-Minimum dar (s. oben). Auch im dritten betrachteten einfach-ortho-methylierten Anisolderivat (235TMAn) ist die Dispersionsbeitragsdifferenz zwischen O- und C-Dimer mit $+0.9 \mathrm{~kJ} \cdot \mathrm{mol}^{-1}$ deutlich kleiner als für die verbleibenden Anisolderivate, für die diese Differenz zwischen +2.4 (4FAn) und $+4.4 \mathrm{~kJ} \cdot \mathrm{mol}^{-1}(4 \mathrm{tBuAn})$ liegt. Insbesondere für diese restlichen Anisolderivate zeigt sich also ein deutlicher Einfluss von Dispersion auf die Regioselektivität, durch den der Nachteil der C-gegenüber der O-Bindungsstelle durch die schwächere Wasserstoffbrückenbindung (vgl. kleinere Rotverschiebung der C-Dimere, Tab. 4.7) zumindest teilweise wieder ausgeglichen werden kann. Selbst für den kleinsten organischen Alkohol MeOH, der mit seinem Methylrest nur wenig Raum für Dispersionswechselwirkungen lässt, ergibt sich somit schon ein signifikanter Einfluss von Dispersion auf das O/C-Gleichgewicht. Eine C-Stabilisierung durch Dispersion konnte in einer vergleichbaren Analyse auch für das $\mathrm{ROH}-$ Dibenzofuran-O/C-Waagensystem (mit $\mathrm{R}=\mathrm{H}, \mathrm{Me}$, $\mathrm{tBu}$ ) ermittelt werden. ${ }^{\text {[239] }}$

Die gegenüber der O-Bindungsstelle steigende C-Attraktivität bei zunehmendem Substitutionsgrad ist nicht hauptsächlich durch Dispersionswechselwirkungen bedingt. Liegt der Median der O-C-Dispersionsbeitragsdifferenz $\Delta E_{\mathrm{O}-\mathrm{C}}^{\text {disp }}$ für das nicht und die einfach substituierten Anisolderivate bei $2.7 \mathrm{~kJ} \cdot \mathrm{mol}^{-1}$, so beträgt der Wert für die zweifach oder höher substituierten Anisolderivate $2.6 \mathrm{~kJ} \cdot \mathrm{mol}^{-1}$. Für höhere Substitutionsgrade ergibt sich somit keine stärkere C-Bevorzugung in den Dispersionsbeiträgen, sodass die Ursache hierfür anderer, z. B. elektrostatischer Natur ${ }^{[337]}$ sein muss.

Eine stärkere Dispersionskontrolle der O/C-Regioselektivität wäre für größere Alkohole wie tert-Butanol (tBuOH) zu erwarten, da hier aufgrund des größeren Alkylrests insgesamt stärkere Dispersionswechselwirkungen im Dimer mit den Anisolen vorliegen sollten. Auch wenn zur Zeit noch keine gesicherten experimentellen Daten zur O/C-Präferenz in den tert-Butanol-Anisol(derivat)-Dimeren als Referenz für die theoretische Untersuchung vorliegen, wäre eine rechnerische Analyse der Dispersionkontrolle im Vergleich zu MeOH interessant. Bereits für diesen kompakteren Donor konnte aufgezeigt werden, dass sekundäre Dispersionswechselwirkungen einen Einfluss auf die Regioselektivität bei der Ausbildung der Wasserstoffbrückenbindung im Methanol-Anisol(derivat)-Dimer nehmen können.

\subsubsection{Zusammenfassung}

Der organische Akzeptor Anisol bietet einem alkoholischen Wasserstoffbrückenbindungsdonor wie $\mathrm{MeOH}$ sowohl $\mathrm{O}$ - als auch C-zentrierte Andockstellen, wobei das in 
einer Überschallexpansion entstehende Gleichgewicht zwischen O- und C-gebundenen Heterodimeren als intermolekulares Waagensystem fungieren kann. Ein solches O/CWaagensystem wurde bereits im vorangegangenen Kapitel 4.1 am Beispiel der MethanolFuran(derivat)-Dimere untersucht. Während der Fokus dort auf dem Benchmarking einer Vielzahl quantenchemischer Methoden anhand eines kleinen experimentellen Datensatzes lag, war die Zielsetzung für das Anisol-Waagensystem, einen möglichst umfassenden Satz von experimentellen Referenzdaten für das Benchmarking heranzuziehen. Dieser sollte sowohl Systeme mit eindeutiger O- als auch eindeutiger C-Bevorzugung, vor allem aber auch Systeme mit einer ausgewogenen O/C-Situation umfassen. Zwar konnten für $\mathrm{tBuOH}$ als Wasserstoffbrückenbindungsdonor keine neuen verwendbaren experimentellen Referenzdaten erhalten werden, nach der Neuauswertung der experimentellen Daten von $\mathrm{MeOH}$ als Donor und diversen Anisolderivaten als Akzeptor ${ }^{[31,37]}$ konnte aber dennoch ein umfassender Datensatz einer fein austarierbaren intermolekularen O/C-Waage für den Benchmark genutzt werden. Das O/C-Bindungsstellengleichgewicht konnte dabei über Alkyl- und Halogen-Substitution am Anisolring kleinschrittig beeinflusst werden. Eine Überprüfung der hier gewonnenen relativen Bindungsenergien mithilfe absoluter, experimenteller Werte, ${ }^{[263]}$ wie sie bereits für das Anisol-Dimer ermittelt werden konnten, ${ }^{[322]}$ wäre allerdings wünschenswert. ${ }^{[37]}$ Eine experimentelle Aufklärung der Dimerstrukturen mittels Mikrowellenspektroskopie könnte zudem analog zum Methanol-Furan-Waagensystem (s. Kap. 4.1) zusätzliche, wichtige Kriterien - z. B. die Zuordnung der O-Dimere zum O-a- oder O-o-Strukturtyp - zum Benchmark beisteuern.

Anhand einer kleinen Auswahl verschiedener populärer und leicht anwendbarer Rechenmethoden konnte gezeigt werden, dass der Datensatz bestehend aus relativen Energien und IR-spektroskopischen Parametern des Methanol-Anisol-Waagensystems die Unterscheidung der Leistungsfähigkeit dieser Rechenmethoden, und somit einen Benchmark derselben, ermöglicht. Während das um die D3-Dispersionskorrektur (inkl. BECKEJoHnson-Damping und Dreikörperterm) ergänzte B3LYP-Funktional hierbei sowohl für die relativen Energien als auch für die untersuchten IR-spektroskopischen Parameter insgesamt gut abschnitt, zeigte das M06-2X-Funktional eine deutlich schwächere Leistung. Dies bestätigt die früheren Ergebnisse ${ }^{[37]}$ und die Befunde weiterer experimenteller ${ }^{[34,89,191,196,197]}$ und theoretischer ${ }^{[338]}$ Untersuchungen. Für den ENCH-51-Datensatz schnitt das M06-2X- hingegen besser als das B3LYP-D3-Funktional ab. ${ }^{\text {[3] }}$

Die schwache Leistung des M06-2X-Funktionals für das Methanol-Anisol-Waagensystem ist trotz der bekannten Basissatzkonvergenzprobleme der MinnesotaFunktionale ${ }^{[339]}$ vermutlich nicht hauptsächlich auf den vergleichsweise kleinen hier verwendeten Basissatz (def2-TZVP) zurückzuführen, da auch mit der größeren aVTZBasis keine besseren Ergebnisse erzielt wurden. ${ }^{[37]}$ Für die B3LYP-D3-Methode spricht das erfolgreiche Abschneiden vor allem im Energie-Benchmark über alle betrachteten Systeme hinweg auf den ersten Blick dagegen, dass die gute Leistung auf Fehlerkompensation beruht. Auch wenn die Ergebnisse der B3LYP-Rechnungen mit auf einem deutlich höheren Theorieniveau (DLPNO-CCSD(T)) berechneten elektronischen Energien kombiniert werden, ergeben sich immer noch durchgehend mit dem Experiment 
vereinbare O-C-Energiedifferenzen. Dieser Befund scheint zunächst ebenfalls gegen eine Fehlerkompensation, z.B. zwischen elektronischen und schwingungsnullpunktskorrigierten Energien, in der B3LYP-Methode zu sprechen. Allerdings sollte bedacht werden, dass in der aktuellen Untersuchung nur ein intermolekulares System betrachtet wurde, das stets aus dem gleichen Donor $(\mathrm{MeOH})$ und mehreren, auf dem gleichen Grundgerüst basierenden Akzeptoren (verschiedene Anisolderivate) besteht. Zudem wurde dabei lediglich das Gleichgewicht zwischen den immer gleichen Bindungsstellen (freie Elektronenpaare des Sauerstoffatoms und kohlenstoffbasiertes $\pi$-System des Anisols) begutachtet. Das dispersionskorrigierte B3LYP-Funktional könnte hierbei also so gleichförmig von Fehlerkompensation profitieren, dass diese durch die leichte Variation des Systems (Substitution am Anisol) nicht aufgedeckt wird. Erst die Erweiterung der Benchmarks auf möglichst viele weitere, vom Methanol-Anisol-Waagensystem möglichst unabhängige Systeme kann somit aufdecken, ob die B3LYP-D3-Methode die richtigen Ergebnisse auch aus den richtigen Gründen ${ }^{[3]}$ liefert. Bereits einzelne Systeme, die eine schwache Leistung seitens der B3LYP-D3-Methode aufdecken, ${ }^{[33,192]}$ lassen daran jedoch Zweifel aufkommen. Schwächen zeigt die Methode auch im hier durchgeführten IR-spektroskopischen Benchmark, in dem sich zwar eine äußerst systematische Überschätzung der $\mathrm{OH} \cdots \mathrm{O}$-Rotverschiebungen durch das dispersionskorrigierte B3LYPFunktional ergibt, der aber auch deutliche Abweichungen der berechneten Werte vom rückkorrigierten, experimentellen Zielbereich für die Bandenaufspaltung zwischen Ound C-gebundenem Dimer offenbart. Andererseits kann auch nicht ausgeschlossen werden, dass die von OswALD und SuHm vorgeschlagene, pauschale Rückkorrektur für Bandenaufspaltungen für den vorliegenden Fall (Aufspaltung zwischen Banden deutlich unterschiedlicher Andockstellen) zu klein gewählt ist. Eventuell sind hier in Zukunft deutlich differenziertere Rückkorrekturen nötig.

Anhand einer Analyse der Dispersionsbeiträge zur Wechselwirkungsenergie in den Methanol-Anisol(derivat)-Dimeren konnten Hinweise auf die Rolle der Dispersion für die O/C-Regioselektivität gewonnen werden. Insgesamt zeigte sich dabei eine Dispersionsstabilisierung der C-gegenüber den O-gebundenen Dimeren, die mit Differenzen von bis $\mathrm{zu} 4.4 \mathrm{~kJ} \cdot \mathrm{mol}^{-1}$ entscheidend zur Attraktivität der C-Bindungsstelle beiträgt. Da keine experimentelle Referenzierung der von der LED-Analyse vorhergesagten Dispersionsbeiträge möglich ist, bleibt die Aussagekraft dieser Ergebnisse unklar. Die zugrundeliegende DLPNO-CCSD(T)-Methode liefert zwar grundsätzlich mit dem Experiment kompatible Ergebnisse für die elektronischen Energien, wenn diese für B3LYP-D3-Geometrien berechnet und mit B3LYP-D3-Schwingungsnullpunktsenergien kombiniert werden, eine Verifizierung der LED-Ergebnisse anhand unabhängiger theoretischer Methoden zur Dekomposition der Bindungsenergie in ihre Bestandteile ${ }^{[16-19,21]}$ wäre aber wünschenswert. Sollten die Ergebnisse zumindest hinsichtlich der relativen Größe der Dispersionsbeiträge zutreffend sein, offenbaren sie jedoch einen möglichen Weg, wie über die gezielte Einbringung dispersionsaffiner Reste am Alkohol-Donor oder Anisol-Akzeptor die Attraktivität der C-Bindungsstelle weiter erhöht werden könnte.

Eine experimentelle Untersuchung verschiedener Anisolderivate mit dem in dieser 
Hinsicht vielversprechenden Donor $\mathrm{tBuOH}$ brachte bisher allerdings keine für das Benchmarking nutzbaren Ergebnisse. Einen Ansatzpunkt für die Spektrenaufklärung könnte eine systematische Betrachtung der tert-Butanol-Anisol(derivat)-Dimere mit der für Methanol als Donor erfolgreichen B3LYP-D3-Methode darstellen. Berechnete Daten zu Rotverschiebungen und relativen Stabilitäten der unterschiedlichen Dimerstrukturen könnten entscheidende Hinweise liefern, falls die Leistungsfähigkeit der DFT-Methode für den größeren Donor vergleichbar gut wie für $\mathrm{MeOH}$ ist.

Eingehendere Untersuchungen mit tert-Butanol oder weiteren dispersionsaffinen Donoren wie iso-Propanol oder Phenol wären hinsichtlich der Analyse des Dispersionseinflusses auf das Bindungsstellengleichgewicht äußerst interessant. Die Einbringung zusätzlicher oder größerer Substituenten auf Akzeptorseite ist für Messungen am bisher eingesetzten Filet-Jet jedoch nicht möglich. Analyten müssen dort einen ausreichend hohen Dampfdruck bei Raumtemperatur aufweisen, um in ausreichender Konzentration im untersuchten Gasgemisch vorzuliegen. Abhilfe könnte hier der neue, auf bis zu $100{ }^{\circ} \mathrm{C}$ heizbare Gratin-Jet (s. Kap. 3) schaffen. Dieser ermöglicht die Messung etwas schwererflüchtiger Substanzen als der Filet-Jet, ohne auf der anderen Seite Nachteile im Signal-zuRausch-Verhältnis durch einen kürzeren Absorptionspfad wie am deutlich höher heizbaren Popcorn-Jet ${ }^{[222]}$ mitzubringen.

Der Vergleich der quantenchemisch berechneten elektronischen und schwingungsnullpunktskorrigierten Energien zeigt, dass die Dimere mit $\mathrm{OH} \cdots \mathrm{O}$ - eine systematisch um bis zu $2 \mathrm{~kJ} \cdot \mathrm{mol}^{-1}$ höhere ZPVE als die Dimere mit OH..C-Wasserstoffbrückenbindungen aufweisen. Für einen Vergleich berechneter Energieunterschiede mit den experimentellen Daten ist also stets eine Berechnung der ZPVE nötig, was das Benchmarking von Methoden, die aufgrund ihrer Rechenanforderungen zurzeit nur im Rahmen von Einzelpunktrechnungen zugänglich sind, erschwert. Für eine direktere Überprüfung elektronischer Energien sollte somit ein System ausgewählt werden, bei dem die ZPVE in den relativen Energien möglichst vollständig kompensiert wird. Eine Möglichkeit hierzu könnte ein Waagensystem, das auf dem Gleichgewicht zwischen zwei gleichartigen Wasserstoffbrückenbindungen beruht, darstellen. Ein solches Waagensystem aus zwei verschiedenen, aber jeweils OH...O-gebundenen, Dimeren soll mit den im folgenden Kapitel 5 betrachteten Carbonylwaagen untersucht werden. Ein Nebeneffekt gleichartiger und ähnlich stabiler primärer Wechselwirkungen (hier der Wasserstoffbrückenbindung) könnte zudem ein größerer Einfluss sekundärer Wechselwirkungen wie der Dispersion sein, was im folgenden Kapitel ebenfalls begutachtet werden soll. 


\section{Kapitel 5}

\section{Carbonylwaagensysteme}

Carbonylverbindungen bieten mit den beiden freien Elektronenpaaren am CarbonylSauerstoffatom zwei attraktive Andockstellen für Wasserstoffbrückenbindungen. Solche $\mathrm{H} \cdots \mathrm{O}=\mathrm{C}-$ Wasserstoffbrückenbindungen ${ }^{[340]}$ sind ein wichtiges Strukturmotiv in Biomolekülen ${ }^{[188,341,342]}$ wie Proteinen und sind entsprechend seit Jahrzehnten Gegenstand von Forschungsbemühungen. ${ }^{[266,343-348]}$ Ist die Carbonyl-Verbindung asymmetrisch mit zwei unterschiedlichen Resten $\mathrm{R}^{1}$ und $\mathrm{R}^{2}$ substituiert, sind auch die beiden freien Elektronenpaare prinzipiell chemisch verschieden (s. Abb. 5.1). Für einen Donor X-H (grau) stehen somit zwei Möglichkeiten zur Ausbildung einer Wasserstoffbrückenbindung zur Verfügung (dunkel- und hellrot). Je nach sterischem Anspruch der Reste $\mathrm{R}^{1}$, $\mathrm{R}^{2}$ und $\mathrm{X}$ resultieren unterschiedliche $\mathrm{C}=\mathrm{O} \cdots \mathrm{H}-$ Winkel $\left(\alpha_{1,2}\right)$ bzw. Diederwinkel zur Carbonyl-Ebene $\left(\tau_{1,2}\right)$. Zusammen mit den sekundären Wechselwirkungen zwischen $\mathrm{R}^{1}$ bzw. $\mathrm{R}^{2}$ und $\mathrm{X}$ können sich für die beiden Bindungsseiten (dunkel- und hellrot) somit verschieden stabile Aggregate ergeben. Wenn diese durch eine ausreichend flache und schmale Barriere getrennt sind, einen relativ geringen Energieunterschied aufweisen und sich spektroskopisch unterscheiden lassen, eignen sich solche wasserstoffbrückengebundenen Aggregate mit Carbonylverbindungen somit analog $\mathrm{zu}$ den im vorherigen Kapitel betrachteten Dimeren als intermolekulares Waagensystem (s. Kap. 4) für das Benchmarking quantenchemischer Methoden. Im Vergleich zu den in Kapitel 4 untersuchten O/C-Waagensystemen basieren die Carbonylwaagen jedoch auf gleichartigen Wasserstoffbrückenbindungen und sollten demnach deutlich geringere systematische Unterschiede in der Schwingungsnullpunktsenergie (ZPVE) aufweisen, sodass ein direkterer Benchmark elektronischer Energien ermöglicht wird.

Aufgrund der besonders ausgeprägten Separation zwischen den freien Elektronenpaaren des Sauerstoffatoms ${ }^{[346]}$ eignen sich Carbonylverbindungen besser als beispielsweise Ether für die Ausbildung eines solchen Waagensystems, da die $\mathrm{H} \cdots \mathrm{O}=\mathrm{C}$-Wasserstoffbrückenbindungen jeweils eindeutig an einem der freien Elektronenpaare und damit auf einer der beiden Carbonylseiten $\mathrm{R}^{1}$ bzw. $\mathrm{R}^{2}$ (s. Abb. 5.1) entstehen. ${ }^{[52]}$ Das Waagensystem lässt sich somit dem Wippentyp (engl. „seesaw balance“) ${ }^{[349-351]}$ zuordnen. ${ }^{[31]}$ Für die experimentelle Unterscheidung der Aggregate kann sich Jet-FTIR-Spektroskopie eignen, wenn die Donor-X-H-Streckschwingungsbanden der in der Überschallexpansion ausgebildeten 

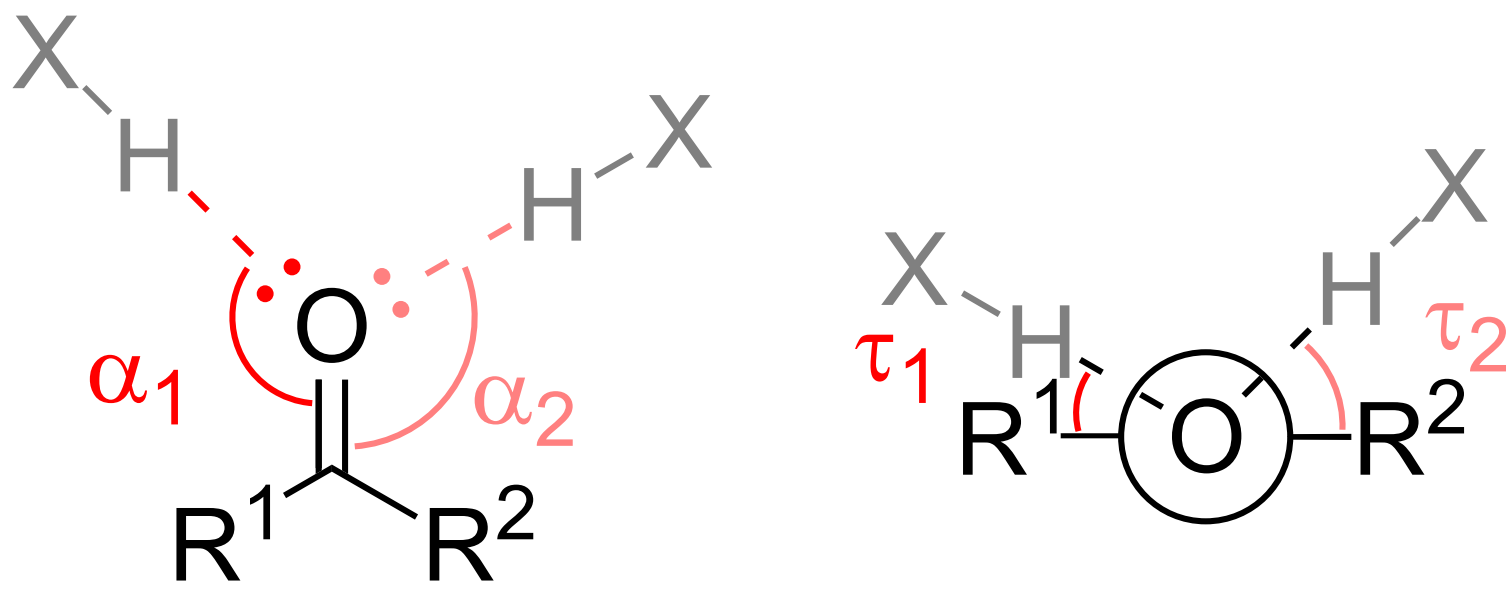

Abbildung 5.1: Grundlegendes Schema der Carbonylwaagen. Bei asymmetrischer Substitution mit unterschiedlichen Resten $\mathrm{R}^{1}$ und $\mathrm{R}^{2}$ sind die beiden freien Elektronenpaare am Carbonyl-Sauerstoffatom chemisch leicht verschieden (dunkelrot und hellrot). Für einen Wasserstoffbrückenbindungsdonor $\mathrm{X}-\mathrm{H}$ (grau) ergeben sich somit zwei Andockstellen für $\mathrm{X}-\mathrm{H} \cdots \mathrm{O}=\mathrm{C}$-Wasserstoffbrückenbindungen (gestrichelte Linien), wobei die Wasserstoffbrückenbindungen je nach sterischem Anspruch der Reste $\mathrm{R}^{1}, \mathrm{R}^{1}$ und $\mathrm{X}$ unterschiedliche $\mathrm{H} \cdots \mathrm{O}=\mathrm{C}$-Bindungswinkel $\alpha_{1,2}$ und Diederwinkel $\tau_{1,2}\left(\mathrm{R}^{1,2}-\mathrm{C}=\mathrm{O} \cdots \mathrm{H}\right)$ zur Carbonyl-Ebene aufweisen können. In der Projektion im rechten Teil der Abbildung liegt das Carbonyl-Sauerstoffatom hinter dem Carbonyl-Kohlenstoffatom.

Aggregate ausreichend unterschiedliche (etwa $>10 \mathrm{~cm}^{-1}$ ) Rotverschiebungen aufweisen. Dies ist der Fall, wenn die Wasserstoffbrückenbindungen unterschiedlich stark sind, z. B. aufgrund verschiedener Bindungswinkel $\alpha_{1,2}$ und $\tau_{1,2}$ (s. Abb. 5.1). ${ }^{[52]}$ Für N-Methylacetamid, einem einfachen Modellsystem für ein Peptid, konnten beispielsweise zwei nahezu isoenergetische, aber auf unterschiedlichen Carbonylseiten $\mathrm{N}-\mathrm{H} \cdots \mathrm{O}=\mathrm{C}$-verbrückte Dimere mittels Jet-FTIR-Spektroskopie nachgewiesen werden. ${ }^{[188]}$ Mitunter kann eine Unterscheidung unterschiedlich gebundener Dimere auch über die Betrachtung der Rotverschiebungen der $\mathrm{C}=\mathrm{O}$-Streckschwingungsbanden möglich sein, wie eine kombinierte Matrix-FTIR- und DFT-Studie zeigt. ${ }^{[352]}$ Jet-Mikrowellen-(MW-)Spektroskopie eignet sich ebenfalls zur Unterscheidung solcher auf verschiedenen Carbonylseiten wasserstoffbrückengebundenen Dimere. ${ }^{[89,290]}$ Die quantitative Analyse der relativen Häufigkeit, wie sie für das Benchmarking berechneter Energieunterschiede benötigt wird (s. Kap. 2.3.2), ist anhand Jet-FTIR-spektroskopischer Daten jedoch leichter möglich. ${ }^{\text {[75] }}$

In der vorliegenden Arbeit soll ein solches Carbonylwaagensystem am Beispiel des einfachsten Alkohols, Methanol $(\mathrm{MeOH})$, als Donor und des einfachsten asymmetrischen aromatischen Ketons, Acetophenon (AP), als Akzeptor untersucht werden. Dabei stehen durch die sich unterscheidenden Substituenten $\mathrm{Ph}$ und Me an der Carbonylgruppe, wie weiter oben bereits beschrieben, unterschiedliche Bindungsseiten für die Mikrosolvatation durch $\mathrm{MeOH}$ zur Verfügung. ${ }^{[31]}$ Analog zu den zuvor betrachteten O/C-Waagensystemen (s. Kap. 4) soll auch hier wieder der Akzeptor systematisch variiert wer- 


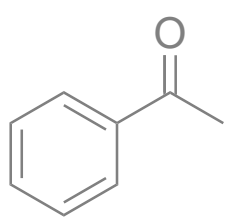<smiles>CC(=O)c1ccccc1F</smiles>

AP<smiles>CC(=O)c1ccc(F)cc1</smiles>

4FAP<smiles>[2H]c1c([2H])c([2H])c(C(C)=O)c([2H])c1[2H]</smiles>

APd5<smiles>[2H]C([2H])(O)C(=O)c1ccccc1</smiles>

APd3

Abbildung 5.2: Lewis-Formeln der untersuchten Acetophenonderivate: Acetophenon (AP, grau), 2-Fluoracetophenon (2FAP, grün), 4-Fluoracetophenon (4FAP, blau), Acetophenon-2',3',4',5',6'- $\mathrm{d}_{5}$ (APd5, rot), Acetophenon-2,2,2- $\mathrm{d}_{3}$ (APd3, orange). Die hier verwendeten Farben werden im gesamten Kapitel 5 für die Zuordnung der Daten zum jeweiligen Acetophenonderivat genutzt.

den, um den Einfluss dieser Substitution auf die Bindungsseitenpräferenz zu untersuchen und quantenchemische Rechnungen auf Basis dieser Daten zu benchmarken. Dazu werden verschiedene Gemische von MeOH bzw. MeOD mit dem unsubstituierten $\mathrm{AP}^{[353-362]}$ (s. Abb. 5.2) sowie den Derivaten 2-Fluoracetophenon (2FAP) ${ }^{[353]}$ und 4-Fluoracetophenon $(4 \mathrm{FAP})^{[353,360]}$ bzw. den teildeuterierten Isotopologen Acetophenon-2',3',4',5',6'- $\mathrm{d}_{5}$ $(A P d 5)^{[354]}$ und Acetophenon-2,2,2- $\mathrm{d}_{3}(\mathrm{APd} 3)^{[354,361]}$ mittels Jet-FTIR-Spektroskopie untersucht.

$\mathrm{AP}^{[363]}$ und auch seine Derivate $2 \mathrm{FAP}^{[364,365]}$ und $4 \mathrm{FAP}^{[366]}$ dienen als prochirale Ausgangsstoffe für zahlreiche Synthesen wie asymmetrische Hydrierungen ${ }^{[367-369]}$ oder Hydrosilylierungen ${ }^{[370]}$ und sind deshalb von großer Bedeutung für diverse Industriezweige. ${ }^{[371]}$ Da diese Synthesen in der Regel in Lösung stattfinden, ist eine Untersuchung der zugrundeliegenden Wechselwirkungen zwischen Lösungsmittel und AP-Solvat ${ }^{[372-375]}$ von Relevanz. So können diese Wechselwirkungen für Ausbeute und Selektivität von entscheidender Bedeutung sein. ${ }^{[371]}$ Entsprechend wurde der Einfluss von $\mathrm{H} \cdots \mathrm{O}=\mathrm{C}-$ Wasserstoffbrückenbindungen auf diverse Molekülmerkmale von AP und seinen Derivaten, wie $\mathrm{C}=\mathrm{O}$-Streckschwingungswellenzahlen ${ }^{[376]}$ oder dielektrische Eigenschaften, ${ }^{[359]}$ bereits vielfältig untersucht. Gemäß des Anwendungsbezugs wurden diese Untersuchungen überwiegend in Lösung oder in flüssiger Phase vorgenommen. ${ }^{[358,377,378]}$

Im Rahmen der vorliegenden Arbeit soll hingegen analog zum vorangegangenen Kapitel 4 nur der erste Schritt der Acetophenon-Mikrosolvatation, also die Bildung der 1:1-Methanol-Acetophenon(derivat)-Heterodimere, betrachtet werden. Dies gestattet eine möglichst isolierte Analyse der grundlegenden Solvens-Solvat-Wechselwirkungen. Erneut werden dabei Überschallexpansionen genutzt, um Spektren der kalten Gasphase $\mathrm{zu}$ erhalten. Eine solche Jet-Spektroskopie-Methode konnte kürzlich erfolgreich dazu eingesetzt werden, die Rotationsspektren der ebenfalls wasserstoffbrückengebundenen Dimere von Acetophenon mit Wasser aufzuzeichnen. ${ }^{[51]}$ Mit Helium als Trägergas konnte dabei für $\mathrm{H}_{2} \mathrm{O}+\mathrm{AP}$ nur das am freien Elektronenpaar auf der Methyl-Seite der Carbonylgruppe gebundene Dimer nachgewiesen werden. Begleitende Rechnungen 
(MP2/6-311++G(2df,2pd)-Niveau) weisen jedoch auf ein weiteres, nur $1.3 \mathrm{~kJ} \cdot \mathrm{mol}^{-1}$ weniger stabiles auf der Phenyl-Seite gebundenes Dimer hin. ${ }^{[51]}$ Für die Dimere von AP mit Methanol konnten mit Jet-FTIR-Spektroskopie bereits auf beiden Seiten gebundene Dimere im Experiment beobachtet werden. ${ }^{[31,52]}$ Dabei konnte gezeigt werden, dass sich bei Alkyl-Substitution am Donor sowie auf der Alkylseite des AP-Akzeptors im Überschallstrahl unterschiedliche Konzentrationsverhältnisse zwischen den Bindungsseiten einstellen und dass anhand dieser Daten und weiterer schwingungsspektroskopischer Observablen eine Beurteilung der Leistungsfähigkeit verschiedener quantenchemischer Methoden hinsichtlich der Beschreibung dieser Systeme erfolgen kann. ${ }^{[52]}$ Wie erwartet zeigen sich dabei nur geringe Unterschiede in den Schwingungsnullpunktsenergien auf Alkyl- und Phenyl-Seite gebundener Dimere, sodass ein recht direkter Benchmark der berechneten relativen elektronischen Energien möglich wird. Zudem zeigt das System trotz der jeweils gleichartigen Wasserstoffbrückenbindungen auf beiden betrachteten Dimerseiten noch ein ausreichend hohes Diskriminationsvermögen, kann also Rechenergebnisse guter und schlechterer Qualität unterscheiden. Die vorliegende Arbeit ergänzt diese Studie $^{[52]}$ um die Betrachtung der systematischen Variation auf der Phenyl-Seite des AP-Akzeptors durch die Fluorierung in ortho- (2FAP) und para-Position (4FAP).

Die prinzipiellen Strukturtypen der Heterodimere von AP (und den Isotopologen APd3 und APd5) bzw. 2FAP und 4FAP mit MeOH(D) sind in Abbildung 5.3 dargestellt. Die wichtigste Unterscheidung betrifft zunächst die Andockstelle der Wasserstoffbrückenbindung. Liegt diese am freien Carbonyl-Sauerstoff-Elektronenpaar auf der Seite der Methylgruppe, wird das Dimer als Me-Dimer bezeichnet. Liegt diese am entsprechenden Elektronenpaar auf der Seite der (Fluor-)Phenylgruppe, ergibt sich ein Ph-Dimer. Diese beiden Dimertypen bilden die weiter oben beschriebene Carbonylwaage. Innerhalb der Dimertypen lassen sich je nach Ausrichtung des Methylrests des Methanolmoleküls zwei generelle Sub-Klassifizierungen vornehmen. Liegt dieser Methylrest in der AP-Molekülebene (engl. „in plane“), wird dies mit „ip“ gekennzeichnet, bei einer Lage außerhalb der AP-Ebene (engl. „out of plane“) mit „op“. Prinzipiell sind zudem OH $\cdots \pi$-gebundene Dimere denkbar, bei denen Wasserstoffbrückenbindungen zum $\pi$-System der Carbonylgruppe oder des Phenylrings ausgebildet werden. ${ }^{[379]}$ Wurden solche Dimere in den quantenchemischen Rechnungen gefunden, lagen diese jedoch stets mehr als $5 \mathrm{~kJ} \cdot \mathrm{mol}^{-1}$ oberhalb des weniger stabilen $\mathrm{OH} \cdots \mathrm{O}$-Dimers. Ähnliches gilt für Dimere mit $\mathrm{OH} \cdots \mathrm{F}-$ Wasserstoffbrückenbindungen.

Für die Umwandlung der auf derselben Seite (Me oder $\mathrm{Ph}$ ) gebundenen ip- und opDimere ineinander werden nur äußerst kleine Barrieren erwartet, da sich das MethanolMolekül für diese Umwandlung nur wenig bewegen muss (s. Abb. 5.3). Somit sollte in der Überschallexpansion jeweils eine Relaxation in das stabilste Dimer einer Seite erfolgen. Die beiden Bindungsseiten wiederum sind von einer größeren Barriere getrennt, wodurch dieser Relaxationsfreiheitsgrad in der Expansion weniger stark gekühlt wird. ${ }^{[52]}$

Für die Untersuchung des Carbonylwaagensystems wurden einerseits Jet-FTIR-Spektren der verschiedenen Methanol-Acetophenon(derivat)-Kombinationen aufgenommen (Kap. 5.1.1) und andererseits 5 Dichtefunktionaltheorie-(DFT-)Methoden genutzt, um re- 


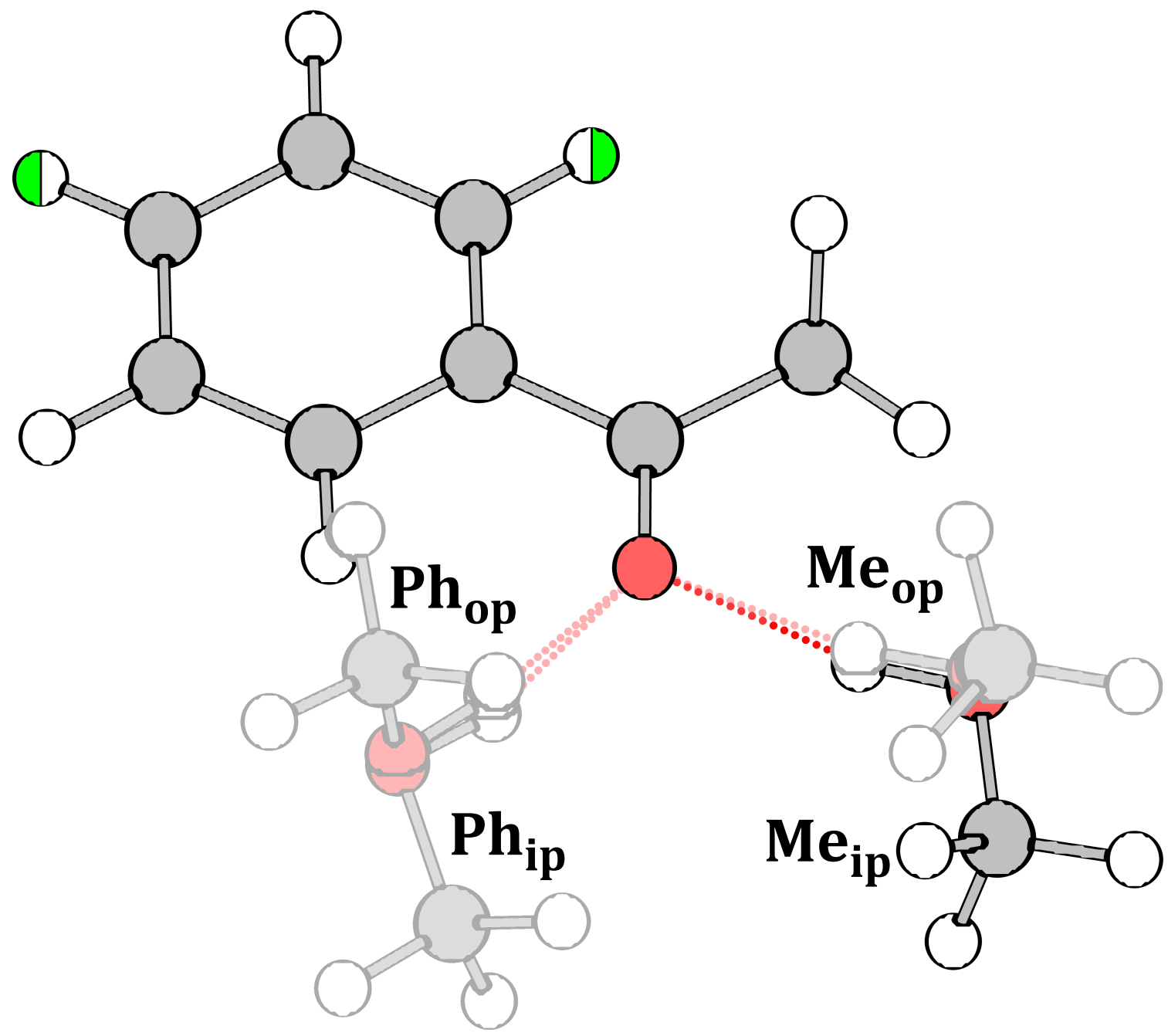

Abbildung 5.3: Bei der Dimerbildung aus Methanol (MeOH) bzw. Methanol-OD (MeOD) und Acetophenon (AP) bzw. seinen Isotopologen Acetophenon-2,2,2- $\mathrm{d}_{3}$ (APd3), Acetophenon$2^{\prime}, 3^{\prime}, 4^{\prime}, 5^{\prime}, 6^{\prime}-\mathrm{d}_{5}$ (APd5) und Derivaten 2-Fluoracetophenon (2FAP, Fluoratom (grün) in ortho-Position am Benzolring) und 4-Fluoracetophenon (4FAP, Fluoratom in para-Position) sind mehrere Strukturtypen unterscheidbar. Die für das hier betrachtete Waagensystem wichtigste Klassifizierung betrifft die Ausbildung der Wasserstoffbrückenbindung von $\mathrm{MeOH}$ entweder zum freien Carbonyl-Sauerstoff-Elektronenpaar auf der Seite des Phenylrests (Kennzeichnung „Ph“) oder alternativ zu jenem auf der Seite des Methylrests („Me“). Innerhalb dieser Strukturtypen kann nach Ausrichtung des Methylrests im $\mathrm{MeOH}$ unterschieden werden. Dieser kann entweder innerhalb der AP-Molekülebene (engl. „in plane“, „ip“) oder außerhalb dieser Ebene (engl. „out of plane“, „op“) liegen. 
lative Energien und die (harmonischen) Schwingungsmoden der MeOH-AP-Dimere zu berechnen (Kap. 5.1.2). Im Hinblick auf den angestrebten Benchmark elektronischer Energien wurden für alle betrachteten DFT-Methoden für die jeweils stabilsten Dimerstrukturen zusätzlich elektronische Energien mit Einzelpunktrechnungen auf Coupled-ClusterNiveau bestimmt. Teile der genannten Messungen und Rechnungen erfolgten dabei im Rahmen der Bachelorarbeit von Julia Stachowiak. Die Ergebnisse der Rechnungen sollen anhand der experimentellen Referenzdaten (Kap. 5.2) anschließend einem Benchmark unterzogen werden, wobei sowohl das $\mathrm{Me} / \mathrm{Ph}$-Konformerengleichgewicht (Kap. 5.3) als auch die Rotverschiebungen und Aufspaltungen der OH-Streckschwingungsbanden der gemischten Dimere (Kap. 5.4) herangezogen werden. Abschließend soll, wie bereits für das Methanol-Anisol-Waagensystem (s. Kap. 4.2.5), auf Grundlage des LED-Konzepts eine Analyse des Einflusses von Dispersionswechselwirkungen auf die Regioselektivität der Wasserstoffbrückenbindungsstelle im Dimer erfolgen (Kap. 5.5). Aufgrund der gleichartigen Wasserstoffbrückenbindungen in Me- und $\mathrm{Ph}$-Dimer ist für die Carbonyl- im Vergleich zu den $\mathrm{O} / \mathrm{C}$-Waagen dabei ein größerer Einfluss sekundärer Wechselwirkungen zu erwarten, weshalb die Analyse des Dispersions-Anteils hier besonders interessant ist.

\subsection{Methoden}

\subsubsection{Experimentelles}

Die Gemische von Methanol (SIgmA-Aldrich, $\geq 99.8 \%$; ABCR, 99\%) mit Acetophenon (TCI, $>98.5 \%$ ), 2-Fluoracetophenon (SigmA-Aldrich, 97\%), 4-Fluoracetophenon (Acros OrgANICs, 99\%), Acetophenon-2,2,2- $\mathrm{d}_{3}$ (ABCR, 98\% D) und Acetophenon-2',3',4',5',6'- $\mathrm{d}_{5}$ (ABCR, $98 \% \mathrm{D})$ sowie von Methanol-OD (EURISO-TOP, $\left.\left(\mathrm{HDO}+\mathrm{D}_{2} \mathrm{O}\right)<0.1 \%, 99.00 \% \mathrm{D}\right)$ mit Acetophenon wurden in Überschallexpansionen mit Helium (LINDE, 99.996\%) als Trägergas an der Filet-Jet-Apparatur (s. Kap. 2.2) FTIR-spektroskopisch untersucht. Die nicht weiter aufbereiteten Chemikalien wurden über temperierte Sättiger in das Trägergas eingebracht, wobei die Analytkonzentrationen über die Sättigertemperaturen und die Schaltzeiten der Magnetventile in den Reservoirzuleitungen variiert werden konnten. Die Expansionen durch die $(600 \cdot 0.2) \mathrm{mm}^{2}$ große Schlitzdüse erfolgten bei einem Stagnationsdruck von 0.75 bar und für die einzelnen Spektren wurden jeweils 150-800 Einzelscans gemittelt. Für die Aufzeichnung des O-H-, C-H- und O-D-Streckschwingungsbereichs (4200-2450 $\mathrm{cm}^{-1}$ ) wurde das Spektrometer (BRUKER IFS $66 \mathrm{v} / \mathrm{S}$ ) mit einer Wolframlampe $(150 \mathrm{~W}), \mathrm{CaF}_{2}$-Optiken und dem InSb-Detektorelement eines InSb/MCT-Sandwich-Detektors betrieben. Die dabei genutzte Spiegelgeschwindigkeit betrug $80 \mathrm{kHz}$ (einseitiger Messmodus) und als Auflösung wurden $2 \mathrm{~cm}^{-1}$ gewählt.

Zusätzlich wurden Spektren des Gemischs von Methanol (ABCR, 99\%) mit 2-Fluoracetophenon (SigmA-AldRICH, 97\%) an der neuen Gratin-Jet-Apparatur (s. Kap. 3) aufgezeichnet. Die Chemikalien wurden vor Einbringen in den Gaskreislauf der Apparatur jeweils durch dreifache freeze-pump-thaw-Zyklen (schnelles Einfrieren durch Eintauchen 
des Kolbens in flüssigen Stickstoff, gefolgt von Evakuieren des Kolbens bei ansteigender Temperatur im Wasserbad, Einleiten von Inertgas (He) als „Schleppgas“ und Wiederholung des Vorgangs) entgast. Anschließend wurde das Reservoir mit den gewünschten Mengen Methanol (ca. 0.01\%) und 2FAP (ca. 0.04-0.07\%) befüllt und mit dem Trägergas Helium (Linde, 99.996\%) auf den Stagnationsdruck von 0.75 bar aufgefüllt. Das Gasgemisch wurde für die Aufnahme der Jet-FTIR-Spektren dann durch die $(700 \cdot 0.2) \mathrm{mm}^{2}$ große Schlitzdüse ins Puffervolumen expandiert, vom Wälzkolbenpumpstand rekomprimiert und ins Reservoir zurückgeleitet. Für das für die Auswertung verwendete Spektrum wurden 1200 Einzelscans gemittelt, wobei das Gasgemisch aufgrund der im Laufe des Kreislaufbetriebs ansteigenden Wasserkonzentration (s. Kap. 3.6) mehrfach abgepumpt und erneuert wurde (für Details s. Abb. 3.24 auf S. 89). Auch am Gratin-Jet wurden die Spektren im Bereich 4200-2450 $\mathrm{cm}^{-1}$ aufgezeichnet, wobei das Spektrometer (BRUKER VerTEX $70 \mathrm{v}$ ) auch hier mit einer $150 \mathrm{~W}$-Wolframlampe, $\mathrm{CaF}_{2}$-Optiken und dem InSb-Detektorelement eines InSb/MCT-Sandwich-Detektors betrieben wurde. Die Spektren wurden bei einer Auflösung von erneut $2 \mathrm{~cm}^{-1}$ bei $140 \mathrm{kHz}$ Spiegelgeschwindigkeit im doppelseitigen Akquisitionsmodus gemessen.

Wie ein Vergleich der Filet- und Gratin-Jet-Spektren der Mischung von $\mathrm{MeOH}$ und 2FAP zeigt (s. Kap. 3.7), ergeben sich in den Überschallexpansionen nahezu identische Bedingungen, sodass bei der Auswertung keine Differenzierung nach verwendeter Apparatur erfolgen muss.

Für jede der untersuchten Methanol(-OD)-Acetophenon(derivat)-Kombinationen werden aus dem Spektrum mit den jeweils besten Analytkonzentrationen (möglichst hohes Signal-zu-Rausch-Verhältnis für die gemischten Dimerbanden ohne größere Mengen von Trimeren) Bandenpositionen und Intensitätverhältnisse der Methanol-Acetophenon(derivat)-Dimer-OH(D)-Streckschwingungsbanden ermittelt. Für die Bestimmung des Intensitätsverhältnisses wird erneut das in Kap. 2.3.3 beschriebene Verfahren verwendet.

\subsubsection{Quantenchemische Rechnungen}

Für den in den folgenden Abschnitten 5.3 und 5.4 vorgestellten Benchmark quantenchemischer Methoden anhand des kompakten experimentellen CarbonylwaagenDatensatzes (ein Donor mit drei nur durch Substitution variierten Akzeptoren sowie drei weitere Isotopolog-Kombinationen) sollen fünf beispielhafte, DFT-basierte Methoden herangezogen werden. Analog zum Methanol-Anisol-Benchmark werden dabei erneut die beliebten Hybrid-Dichtefunktionale $\mathrm{B} 3 \mathrm{LYP}^{[85,86]}$ und $\mathrm{M} 06-2 \mathrm{X}^{[82]}$ berücksichtigt, wobei das B3LYP-Funktional wieder mit der D3-Dispersionskorrektur inklusive Dreikörperterm ${ }^{[83]}(\mathrm{ABC})$ und BECKE-Johnson-Damping ${ }^{[90-93]}$ (BJ) kombiniert wird. Sowohl das B3LYP-D3(BJ,ABC)- als auch das M06-2X-Funktional werden erneut mit dem def2TZVP-Basissatz ${ }^{[103]}$ eingesetzt. Um die Basissatzkonvergenz beispielhaft zu überprüfen, wird die B3LYP-D3(BJ,ABC)-Methode zusätzlich mit dem Quadruple- $\zeta-B a s i s s a t z ~ d e f 2-$ QZVP $^{[103]}$ kombiniert. Die B3LYP-D3(BJ,ABC)/def2- $n$ ZVP-Rechnungen (mit $n=\mathrm{T}, \mathrm{Q}$ ) werden mit dem ORCA-Programmpaket ${ }^{[107-109]}$ in der Version 4.0.1, die M06-2X/def2- 
TZVP-Rechnungen mit GAUSSIAN16 in der Revision A.03 ${ }^{[111]}$ durchgeführt.

Als kostengünstige Alternativen, mit denen auch größere Systeme betrachtet werden können, werden die Kompositmethoden B97-3c ${ }^{[98]}$ und PBEh-3c ${ }^{[97]}$ in den Benchmark integriert, um ihre Leistungsfähigkeit hinsichtlich der Beschreibung wasserstoffbrückengebundener Systeme am Beispiel der Carbonylwaagen zu überprüfen. Im Gegenzug wird auch ein höheres Theorieniveau, das Doppel-Hybrid-Funktional B2PLYP ${ }^{[102]}$ mit D3(BJ,ABC)-Dispersionkorrektur, für den Benchmark herangezogen. In Kombination mit dem Basissatz a'VTZ (cc-pVTZ ${ }^{[104]}$ und Hilfsbasissatz cc-pVTZ/C ${ }^{[106]}$ an H, aug-cc$\mathrm{pVTZ}^{[105]}$ und aug-cc-pVTZ/C ${ }^{[106]}$ an den anderen Atomen) stellt diese Methode trotz Verwendung der "resolution of the identity“-(RI-)Näherung ${ }^{[68]}$ das Maximum dessen dar, was mit den zur Verfügung stehenden Rechenressourcen auf Systeme dieser Größe routinemäßig anwendbar ist (Laufzeit von Geometrieoptimierung und Frequenzrechnung $>100$ h auf 23 (= $N_{\text {atome }}$ ) AMD Opteron 6378 CPU-Kernen). Die B97-3c-, PBEh3c- und B2PLYP-D3(BJ,ABC)/a'VTZ-Rechnungen werden mit ORCA in der Version 4.1.2 durchgeführt. Die Rechnungen der 3c-Kompositmethoden sind dabei potentiell von einem Programmfehler betroffen, der sporadisch zu falschen, z. B. großen imaginären, Frequenzen führt. ${ }^{[380]}$ Der Programmfehler kann bei analytischen Frequenzrechnungen auftreten, bei denen die RI-Näherung zum Einsatz kommt. Im Rahmen dieser Arbeit ist dies nur für die 3c-Methoden der Fall. Die RI-Näherung wird ansonsten nur für die B2PLYP-D3(BJ,ABC)/a'VTZ-Rechnungen verwendet, für die mit OrCA jedoch keine analytischen Frequenzrechnungen möglich sind. Somit wird in diesem Fall auf nicht vom Programmfehler betroffene numerische Frequenzrechnungen zurückgegriffen (s. Kap. 2.1.1). In den hier durchgeführten B97-3c- und PBEh-3c-Rechnungen wurden keine Hinweise auf das Auftreten des Programmfehlers gefunden, sodass die Ergebnisse trotzdem für den Benchmark verwendet werden sollen.

Für alle genannten Methoden werden zusätzlich die elektronischen Energien mit dem DLPNO-CCSD(T)-Verfahren ${ }^{[122-125]}$ mit TightPNO-Vorgabe, das sowohl in der Furan-Mikrosolvatations-Leistungsüberprüfungtersuchung (s. Kap. 4.1) als auch für die MethanolAnisol-Waagensyteme (s. Kap. 4.2) gute Ergebnisse erzielen konnte, berechnet. Dabei wird auf den aug-cc-pVQZ-Basissatz ${ }^{[105]}$ (mit Hilfsbasis aug-cc-pVQZ/C ${ }^{[106]}$ ) zurückgegriffen. Auf Grundlage dieser Rechnungen wird der Einfluss von Dispersionswechselwirkungen auf das Carbonylwaagen-Gleichgewicht mit dem Konzept der „local energy decomposition " (LED), ${ }^{[20]}$ das ebenfalls bereits für die Methanol-Anisol-Waagensysteme eingesetzt wurde (s. Kap. 4.2.5), analysiert.

Die Berechnung der elektronischen Energien der B3LYP-D3(BJ,ABC)/def2- nZVPGeometrien (mit $n=\mathrm{T}$, Q) wird zusätzlich testweise unter Berücksichtigung der Kernelektronenkorrelation durchgeführt. Dazu werden für die Atomsorte $\mathrm{H}$ erneut der aVQZBasissatz und für die Atomsorten C, O und F der aug-cc-pwCVQZ-Basissatz ${ }^{[105,140]}$ (mit Hilfsbasis aug-cc-pwCVQZ/C ${ }^{[106,141]}$ ) verwendet. Auf dem DLPNO-CCSD(T)/ awCVQZ//B3LYP-D3(BJ,ABC)/def2-TZVP-Niveau wird zudem das alternative PIPEKMEzeY-Lokalisierungsverfahren ${ }^{[133]}$ (DLPNO-CCSD(T)-PM) eingesetzt. Die DLPNO$\operatorname{CCSD}(\mathrm{T}) / \mathrm{aVQZ}$ - (inkl. LED) und die DLPNO-CCSD(T)/awCVQZ//B3LYP-D3(BJ,ABC)/ 
Tabelle 5.1: Übersicht der verwendeten quantenchemischen Methoden für die Berechnung der Geometrie und Hesse-Matrix sowie der elektronischen Energie und die für die Methodenkombinationen verwendeten Kürzel. Eine Beschreibung der Detaileinstellungen der durchgeführten Rechnungen befindet sich in Kapitel 2.1.

\begin{tabular}{lll}
\hline Kürzel & Geometrie und Hesse-Matrix & Elektronische Energie \\
\hline B3 & B3LYP-D3(BJ,ABC)/def2-TZVP & B3LYP-D3(BJ,ABC)/def2-TZVP \\
B3+ & B3LYP-D3(BJ,ABC)/def2-TZVP & DLPNO-CCSD(T)/aVQZ \\
B3+c & B3LYP-D3(BJ,ABC)/def2-TZVP & DLPNO-CCSD(T)/awCVQZ \\
B3+cP & B3LYP-D3(BJ,ABC)/def2-TZVP & DLPNO-CCSD(T)-PM/awCVQZ \\
\hline B3Q & B3LYP-D3(BJ,ABC)/def2-QZVP & B3LYP-D3(BJ,ABC)/def2-QZVP \\
B3Q+ & B3LYP-D3(BJ,ABC)/def2-QZVP & DLPNO-CCSD(T)/aVQZ \\
B3Q+c & B3LYP-D3(BJ,ABC)/def2-QZVP & DLPNO-CCSD(T)/awCVQZ \\
\hline M6 & M06-2X/def2-TZVP & M06-2X/def2-TZVP \\
M6+ & M06-2X/def2-TZVP & DLPNO-CCSD(T)/aVQZ \\
\hline B9 & B97-3c & B97-3c \\
B9+ & B97-3c & DLPNO-CCSD(T)/aVQZ \\
\hline PB & PBEh-3c & PBEh-3c \\
PB+ & PBEh-3c & DLPNO-CCSD(T)/aVQZ \\
\hline B2 & B2PLYP-D3(BJ,ABC)/a'VTZ & B2PLYP-D3(BJ,ABC)/a'VTZ \\
B2+ & B2PLYP-D3(BJ,ABC)/a'VTZ & DLPNO-CCSD(T)/aVQZ \\
\hline
\end{tabular}

def2-QZVP-Rechnungen werden unter ORCA in der Version 4.1.2, die DLPNO-CCSD(T)/ awCVQZ//B3LYP-D3(BJ,ABC)/def2-TZVP-Rechnungen unter ORCA in der Version 4.0.1 durchgeführt.

Einen Überblick über die hier verwendeten Rechenmethoden und die im Folgenden verwendeten Methodenkürzel gibt Tabelle 5.1. Die Detaileinstellungen der durchgeführten Rechnungen sind in Kapitel 2.1 dokumentiert.

\subsection{Experimentelle Ergebnisse}

Im Jet-FTIR-Spektrum von $\mathrm{MeOH}$ mit dem unsubstituierten AP (Abb. 5.4 unten) zeigen sich zwei gegenüber dem Methanol-Monomer (M) stark rotverschobene OH-Streckschwingungsbanden bei 3565 und $3528 \mathrm{~cm}^{-1}$, die über ihre Skalierung bei Variation der $\mathrm{MeOH}$ - und AP-Konzentration den Donor-OH-Streckschwingungen in gemischten $\mathrm{MeOH}$-AP-Dimeren (MA) zugeordnet werden können. Mit 121 bzw. $158 \mathrm{~cm}^{-1}$ weisen die beiden gemischten Dimerbanden größere Rotverschiebungen gegenüber der $\mathrm{OH}$ Streckschwingungsbande des Methanol-Monomers $\left(3686 \mathrm{~cm}^{-1[154,297]}\right)$ als das Methanol- 


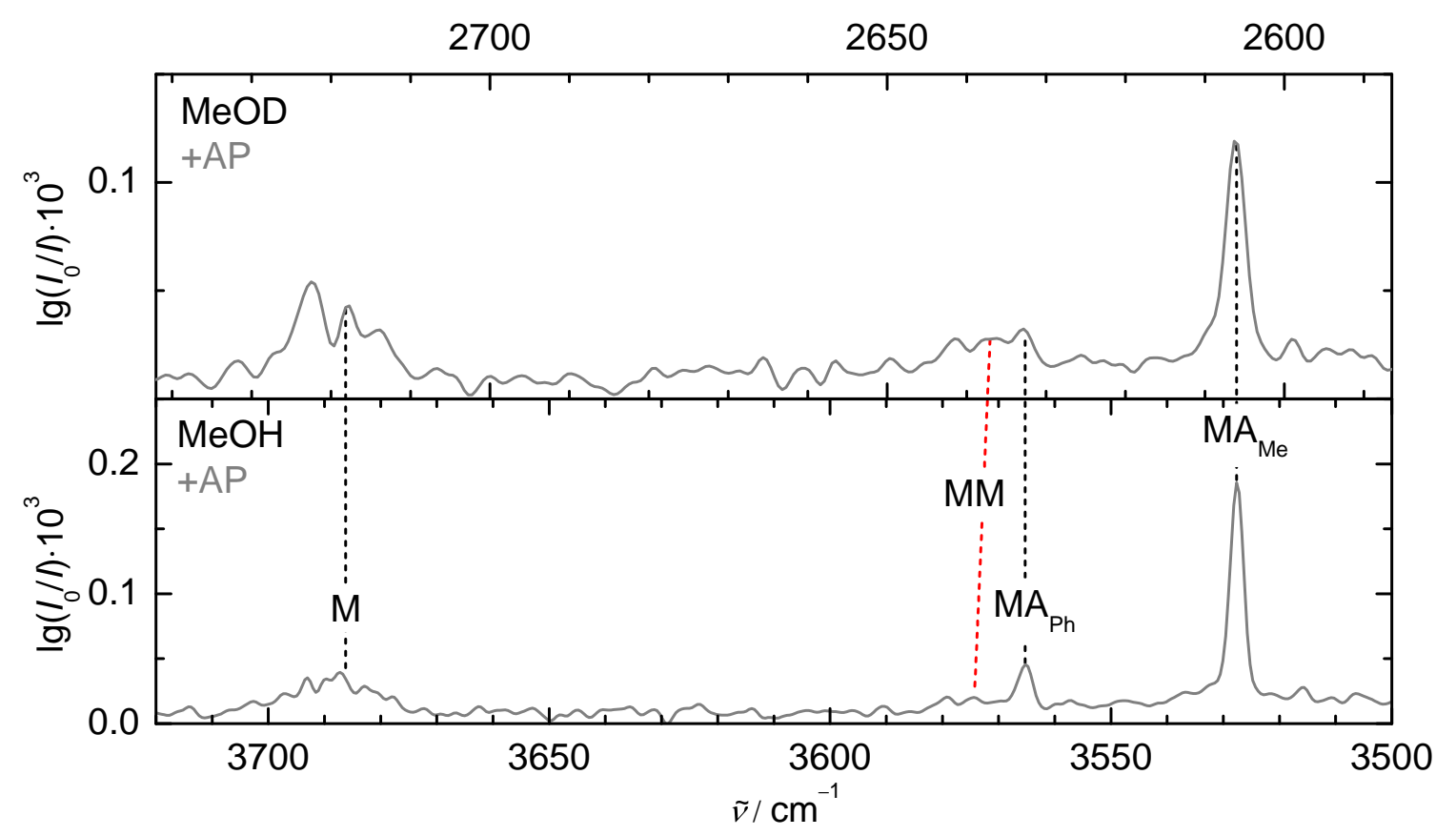

Abbildung 5.4: Vergleich der OH(D)-Streckschwingungsbereiche der Spektren von MeOD (oben) bzw. MeOH (unten) mit AP. Die Wellenzahlachse des OD-Spektrums wurde relativ zum OH-Spektrum um den Faktor $\sqrt{2}$ gestreckt und die Spektren wurden am Methanol-Monomer-Signal ausgerichtet. Die relevanten Banden (Methanol-Monomer (M), Methanol-Homodimer (MM), MethanolAP-Me-Dimer $\left(\mathrm{MA}_{\mathrm{Me}}\right)$ und -Ph-Dimer $\left(\mathrm{MA}_{\mathrm{Ph}}\right)$ wurden im Spektrum gekennzeichnet. Gleichartige Banden wurden mit gestrichelten Linien verbunden, die gemäß ihrer Steigung eingefärbt wurden (rot: positive Steigung; schwarz: keine erkennbare Richtung).

Homodimer (MM, $111 \mathrm{~cm}^{-1}$ ) auf. Die stärker rotverschobene Heterodimerbande besitzt dabei etwa die fünffache Intensität der weniger rotverschobenen Bande. Die Größe der Rotverschiebungen lässt vermuten, dass die Wasserstoffbrückenbindungen der beiden beobachteten gemischten Dimere vom $\mathrm{OH} \cdots \mathrm{O}$-Typ sind, da $\mathrm{OH} \cdots \pi$-Wasserstoffbrückenbindungen analog zu den Methanol-Furan(derivat)- bzw. Methanol-Anisol(derivat)Dimeren zu kleineren Rotverschiebungen führen sollten. In der Tat scheinen sich hier also zwei Carbonyl-gebundene Dimere auszubilden. Die großen Rotverschiebungen der Dimerbanden lassen zudem auf stärkere lokale Wasserstoffbrückenbindungen als für die Methanol-Furan- bzw. Methanol-Anisol-Waagensysteme schließen, bei denen die entsprechenden Dimerbanden stets bei geringeren Rotverschiebungen als das MethanolDimer liegen.

Um die Zuordnung der Banden zu Carbonyl-gebundenen Dimeren weiter abzusichern, kann ein Vergleich mit dem entsprechenden Spektralbereich für MeOD als Donor herangezogen werden. In der oberen Hälfte von Abbildung 5.4 ist der OD-Streckschwingungsbereich des Jet-Spektrums von MeOD mit AP dargestellt. Die Wellenzahlachse wurde da- 
bei gegenüber dem im unteren Teil abgebildeten OH-Spektrum um den Faktor $\sqrt{2}$, dem idealen, harmonischen Isotopeneffekt für H/D-Austausch bei unendlicher Sauerstoffmasse und exaktem D/H-Massenverhältnis von 2, gestreckt. So werden anharmonische Einflüsse auf die Rotverschiebungen sichtbar (s. Kap. 4.1.1), wie die positive Steigung (rot) der Verbindungslinie für das Methanol-Dimer (MM) verdeutlicht. Die beiden Methanol-Acetophenon-Heterodimerbanden (MA) zeigen hingegen keine signifikanten Unterschiede zwischen OD- und OH-Spektrum. Das einheitliche Verhalten der beiden Banden spricht für Wasserstoffbrückenbindungen zu gleichartigen Andockstellen. Weitere Hinweise auf die Bandenzuordnung können prinzipiell aus dem Intensitätsverhältnis beider Banden gewonnen werden. $\mathrm{OH} \cdots \mathrm{O}$-gebundene Dimere weisen eine systematisch größere ZPVE als $\mathrm{OH} \cdots \pi$-Dimere auf, wobei der Unterschied bei deuterierter Wasserstoffbrückenbindung geringer ist (s. Kap. 4.1.1 \& 4.2.2). Die relative Intensität von $\mathrm{OH} \cdots \mathrm{O}-\mathrm{im}$ Vergleich zu $\mathrm{OH} \cdots \pi$-Banden sollte somit bei Deuterierung zunehmen. Aufgrund einer partiellen Überlappung der weniger rotverschobenen Bande mit der Methanol-Dimerbande im OD-Spektrum kann im vorliegenden Fall allerdings nicht beurteilt werden, ob sich das Intensitätsverhältnis bei Deuterierung ändert.

Zusammenfassend ergibt die Analyse des Spektrums, dass zwei Banden gemischter Methanol-Acetophenon-Dimere zu beobachten sind, die beide dem gleichen Wasserstoffbrückenbindungstyp zuzuordnen sind. Aufgrund der großen Rotverschiebungen handelt es sich hierbei um $\mathrm{OH} \cdots \mathrm{O}$-Wasserstoffbrückenbindungen zweier Carbonyl-verbrückter Dimere, auch wenn dies auf Basis der im Deuterierungsexperiment beobachteten Anharmonizitäten rein experimentell nicht mit abschließender Sicherheit bestätigt werden kann.

Offen bleibt zudem die Zuordnung der beiden Dimerbanden zu Me- und Ph-Dimer (s. Abb. 5.3). Erste Hinweise auf eine Zuordnung ergeben sich aus der Teildeuterierung des APs auf Methyl- (APd3) bzw. Phenyl-Seite (APd5). Im eingeschobenen Kasten in Abbildung 5.5 ist der Bereich der beiden Heterodimerbanden für die Spektren von $\mathrm{MeOH}$ mit AP (grau), APd3 (orange) und APd5 (rot) im Detail dargestellt. Es zeigt sich, dass die Heterodimerbanden bei Teildeuterierung des APs insgesamt etwas zu niedrigeren Wellenzahlen verschoben werden. Für APd3 ergibt sich dabei für die intensivere Heterodimerbande eine etwas stärkere Verschiebung $\left(0.6 \mathrm{~cm}^{-1}\right)$ als für APd5 $\left(0.4 \mathrm{~cm}^{-1}\right)$. Die weniger intensive Bande wird hingegen bei Teildeuterierung auf Phenylseite (APd5) mit $0.9 \mathrm{~cm}^{-1}$ stärker verschoben als bei Teildeuterierung auf Methylseite (APd3, $\left.0.4 \mathrm{~cm}^{-1}\right)$. Da bei Bindung auf der jeweils deuterierten Seite (Me-Seite bei APd3, Ph-Seite bei APd5) aufgrund von sekundären Wechselwirkungen, die eins der Deuterium-Atome involvieren, ein stärkerer Effekt auf die Bandenposition zu erwarten ist als bei Bindung auf der anderen Seite, kann auf Basis dieser Daten eine vorsichtige experimentelle Bandenzuordnung vorgenommen werden. Die weniger intensive Bande bei höheren Wellenzahlen wäre demnach dem Ph-gebundenen Dimer $\left(\mathrm{MA}_{\mathrm{Ph}}\right)$ zuzuordnen, die intensivere Bande bei niedrigeren Wellenzahlen dem Me-gebundenen Dimer $\left(\mathrm{MA}_{\mathrm{Me}}\right)$. Da die für diese Zuordnung herangezogenen Verschiebungseffekte bei Teildeuterierung des APs jedoch allesamt weniger als $1 \mathrm{~cm}^{-1}$ betragen und damit unterhalb der Spektrenauflösung von $2 \mathrm{~cm}^{-1}$ liegen, sollte diese Zuordnung zusätzlich abgesichert werden. 


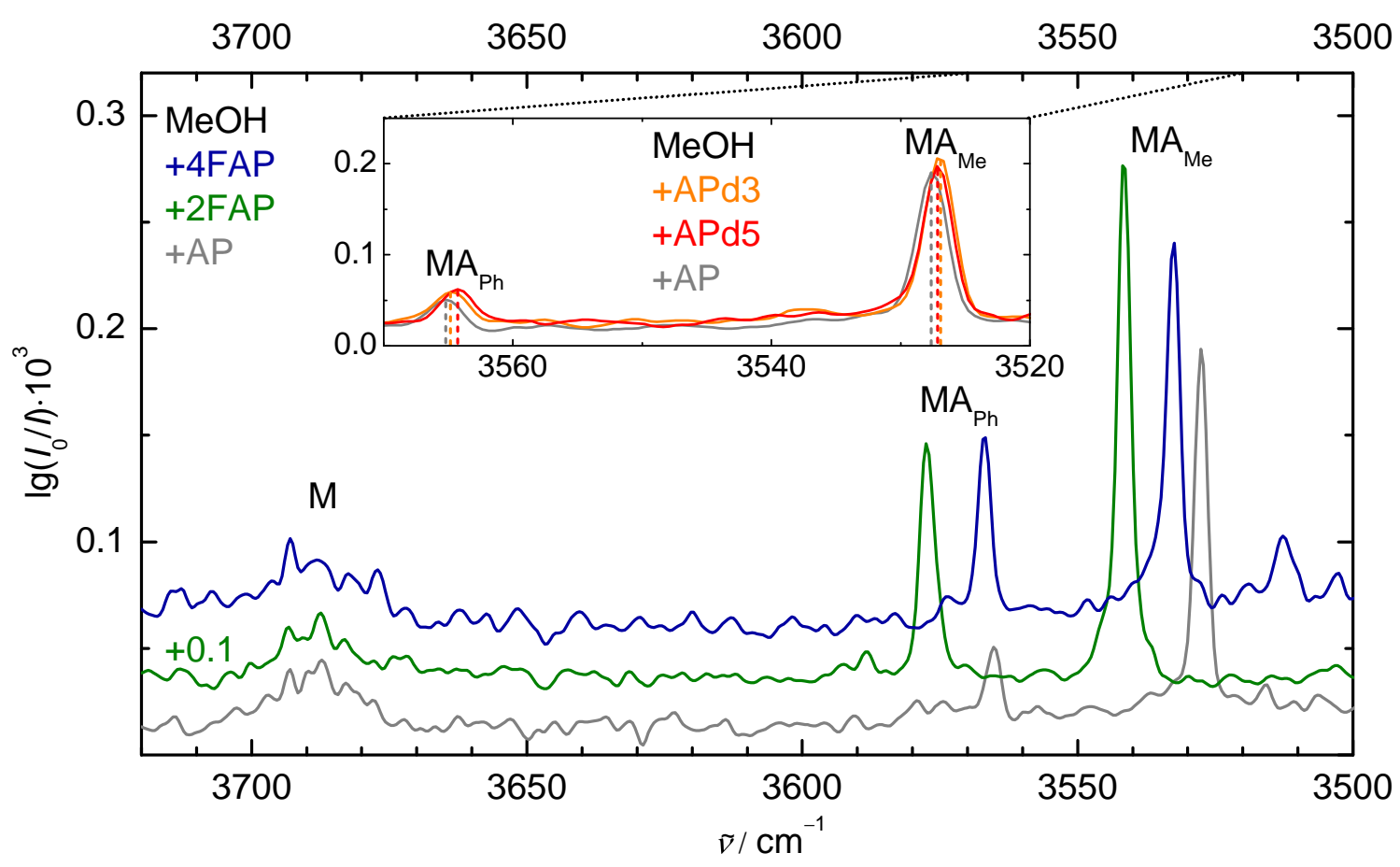

Abbildung 5.5: Übersicht der OH-Streckschwingungsbereiche der Spektren von $\mathrm{MeOH}$ mit AP (grau), 2FAP (grün) und 4FAP (blau). Im eingeschobenen Kasten ist zudem der Spektralbereich der Heterodimerbanden für MeOH mit AP, APd3 (orange) und APd5 (rot) vergrößert dargestellt. Die Methanol-Monomer-OH-Streckschwingungsbande ist mit „M“ gekennzeichnet, die Bande $\mathrm{Ph}$ Heterodimers mit „MA $\mathrm{MA}_{\mathrm{Ph}}$ " und die des Me-Dimers mit „MA $\mathrm{Me}$ “. Zur Verbesserung der Übersichtlichkeit wurde das $\mathrm{MeOH}+2 \mathrm{FAP}$-Spektrum um 0.1 Skaleneinheiten nach oben verschoben.

Dazu können die Daten aus quantenchemischen Rechnungen herangezogen werden. $\mathrm{Da}$ in beiden Fällen vermutlich $\mathrm{OH} \cdots \mathrm{O}$-gebundene Dimere vorliegen, bietet das dispersionskorrigierte B3LYP-Funktional sich als zuverlässige Zuordnungshilfe an. ${ }^{[33,49,181,336]}$ Die B3LYP-D3(BJ,ABC)/def2-TZVP-Methode (B3) zeigt beispielsweise für die MethanolAnisol(derivat)-Dimere eine sehr systematische, leichte Überschätzung der OH $\cdots$ O-Rotverschiebungen (s. Kap. 4.2.4) und soll deshalb hier ebenfalls genutzt werden. Bei Geometrieoptimierung und Frequenzrechnung auf B3-Niveau ergibt sich für die $\mathrm{MeOH}-\mathrm{AP}-$ Heterodimere eine Aufspaltung von $46 \mathrm{~cm}^{-1}$ zwischen stabilstem Me- und Ph-Dimer, wobei das Me-Dimer die größere Rotverschiebung aufweist (für Details s. Folgekapitel 5.3 und 5.4). Bei einer experimentellen Aufspaltung von $38 \mathrm{~cm}^{-1}$ bestätigt dies die zuvor getroffene vorsichtige Bandenzuordnung. Auch der berechnete Energieunterschied von ca. $0.6 \mathrm{~kJ} \cdot \mathrm{mol}^{-1}$ zugunsten des Me-gebundenen Dimers passt zum beobachteten Intensitätsverhältnis, das auf eine Bevorzugung des Me-Dimers hindeutet. Alle weiteren hier genutzten quantenchemischen Methoden liefern darüber hinaus ebenso Aufspaltungen im Bereich 37-57 $\mathrm{cm}^{-1}$, wobei die Me-Dimerbande jeweils stärker rotverschoben ist. Mögli- 
Tabelle 5.2: $\mathrm{OH}(\mathrm{D})$-Streckschwingungsbandenpositionen $\quad\left(\tilde{v}_{\mathrm{OH}(\mathrm{D})}\right)$ und -rotverschiebungen $\left(\Delta \tilde{v}_{\mathrm{OH}(\mathrm{D})}\right)$ der Ph- und Me-Dimere der verschiedenen Methanol-Acetophenon(derivat)-Kombinationen, sowie die Aufspaltung $\left(\Delta \Delta \tilde{v}_{\mathrm{OH}(\mathrm{D})}\right)$ und das mit dem in Kap. 2.3.3 beschriebenen Verfahren bestimmte Intensitätsverhältnis $\left(I_{\mathrm{Me}} / I_{\mathrm{Ph}}\right)$ dieser Banden.

\begin{tabular}{|c|c|c|c|c|c|c|}
\hline & \multicolumn{2}{|c|}{ Ph-Dimer } & \multicolumn{2}{|c|}{ Me-Dimer } & \multicolumn{2}{|c|}{$\mathrm{Me} / \mathrm{Ph}$} \\
\hline & $\begin{array}{l}\tilde{v}_{\mathrm{OH}(\mathrm{D})} \\
/ \mathrm{cm}^{-1}\end{array}$ & $\begin{array}{c}\Delta \tilde{v}_{\mathrm{OH}(\mathrm{D})} \\
/ \mathrm{cm}^{-1}\end{array}$ & $\begin{array}{l}\tilde{v}_{\mathrm{OH}(\mathrm{D})} \\
/ \mathrm{cm}^{-1}\end{array}$ & $\begin{array}{c}\Delta \tilde{v}_{\mathrm{OH}(\mathrm{D})} \\
\quad / \mathrm{cm}^{-1}\end{array}$ & $\begin{array}{r}\Delta \Delta \tilde{v}_{\mathrm{OH}(\mathrm{D})} \\
\quad / \mathrm{cm}^{-1}\end{array}$ & $I_{\mathrm{Me}} / I_{\mathrm{Ph}}$ \\
\hline $\mathrm{MeOH}+\mathrm{AP}$ & 3565 & 121 & 3528 & 158 & 38 & $5.2(4)$ \\
\hline $\mathrm{MeOH}+2 \mathrm{FAP}$ & 3577 & 109 & 3542 & 144 & 36 & $2.3(2)$ \\
\hline $\mathrm{MeOH+4FAP}$ & 3567 & 119 & 3533 & 153 & 34 & $2.3(5)$ \\
\hline $\mathrm{MeOH}+\mathrm{APd} 3$ & 3565 & 121 & 3527 & 159 & 38 & $5.1(9)$ \\
\hline $\mathrm{MeOH}+\mathrm{APd} 5$ & 3564 & 122 & 3527 & 159 & 37 & $4.7(5)$ \\
\hline $\mathrm{MeOD}+\mathrm{AP}$ & 2633 & 85 & 2606 & 112 & 27 & $9(7)$ \\
\hline
\end{tabular}

che $\mathrm{OH} \cdots \pi$-gebundene Dimere weisen zudem in allen Rechnungen mehr als $5 \mathrm{~kJ} \cdot \mathrm{mol}^{-1}$ höhere Energien als das weniger stabile $\mathrm{OH} \cdots \mathrm{O}$-Dimer auf. In der Kombination dieser quantenchemischen mit den experimentellen Daten ist somit eine eindeutige Bandenzuordnung zu den Carbonyl-gebundenen Dimeren auf $\mathrm{Ph}$ - (Bande bei $3565 \mathrm{~cm}^{-1}$ ) und MeSeite $\left(3528 \mathrm{~cm}^{-1}\right)$ möglich. ${ }^{[31,52]}$

Bei Fluorierung des APs in ortho- (2FAP, grün) bzw. para-Position (4FAP, blau) an der Phenylgruppe ergeben sich in der Kombination mit $\mathrm{MeOH}$ sehr ähnliche Heterodimerbandenmuster aus einer weniger intensiven Dimerbande bei höheren und einer intensiveren Dimerbande bei niedrigeren Wellenzahlen (s. Abb. 5.5), die aufgrund der Analogie zum $\mathrm{MeOH}+\mathrm{AP}-$ Spektrum und über den Vergleich mit B3-Rechnungen (berechnete Aufspaltung $46 \mathrm{~cm}^{-1}$ für 2FAP bzw. $41 \mathrm{~cm}^{-1}$ für 4FAP, jeweils mit stärker rotverschobenem Me-Dimer) dem Ph- bzw. Me-Dimer zugeordnet werden können. Die experimentellen Positionen $\left(\tilde{v}_{\mathrm{OH}(\mathrm{D})}(\mathrm{Me}, \mathrm{Ph})\right)$ dieser $\mathrm{OH}-\mathrm{Streckschwingungsbanden} \mathrm{der} \mathrm{Ph-} \mathrm{und} \mathrm{Me-}$ Heterodimerere sind gemeinsam mit den resultierenden Rotverschiebungen $\left(\Delta \tilde{v}_{\mathrm{OH}(\mathrm{D})}=\right.$ $\left.\tilde{v}_{\mathrm{OH}(\mathrm{D})}(\mathrm{M})-\tilde{v}_{\mathrm{OH}(\mathrm{D})}(\mathrm{Me}, \mathrm{Ph})\right)$ gegenüber der entsprechenden Bande des Methanol-Monomers $\left(\tilde{v}_{\mathrm{OH}(\mathrm{D})}(\mathrm{M})\right)$, den Bandenaufspaltungen $\left(\Delta \Delta \tilde{v}_{\mathrm{OH}(\mathrm{D})}(\mathrm{Me}-\mathrm{Ph})=\Delta \tilde{v}_{\mathrm{OH}(\mathrm{D})}(\mathrm{Me})-\right.$ $\left.\Delta \tilde{v}_{\mathrm{OH}(\mathrm{D})}(\mathrm{Ph})\right)$ und den Intensitätsverhältnissen $\left(I_{\mathrm{Me}} / I_{\mathrm{Ph}}\right)$ in Tabelle 5.2 zusammengefasst.

Für die teildeuterierten AP-Isotopologe ergeben sich abgesehen von den geringen Verschiebungen der Bandenpositionen keine signifikanten Unterschiede gegenüber dem nicht-deuterierten AP. Bei Deuterierung des Methanols (MeOD) nimmt das gemessene Intensitätsverhältnis $I_{\mathrm{Me}} / I_{\mathrm{Ph}}$ für $\mathrm{MeOD}+\mathrm{AP}$ zwar gegenüber $\mathrm{MeOH}+\mathrm{AP}$ zu, aufgrund der partiellen Überlappung der $\mathrm{MA}_{\mathrm{Ph}^{-}}$mit der $\mathrm{MM-Bande}$ (s. Abb. 5.4 oben) kann das Verhältnis für MeOD+AP jedoch nur sehr ungenau bestimmt werden, sodass die Zunahme statistisch nicht signifikant ist.

Für $\mathrm{MeOH}$ als Donor werden die Heterodimerbanden bei Fluorierung im Vergleich zum 
unsubstituierten AP zu höheren Wellenzahlen verschoben. Für die $\mathrm{MA}_{\mathrm{Ph}}$-Bande von $\mathrm{Me}$ $\mathrm{OH}+2 \mathrm{FAP}$ bei $3577 \mathrm{~cm}^{-1}$ ergibt sich dabei eine potentielle Überlappung mit der MethanolHomodimerbande bei $3575 \mathrm{~cm}^{-1}$. Hierdurch könnte die Intensität der $\mathrm{MA}_{\mathrm{Ph}}$-Bande subtil verfälscht werden, wobei die Spektren keine Hinweise auf signifikante Mengen von Methanol-Dimer liefern. Die Verschiebungen zwischen den Heterodimerbanden der fluorierten Derivate und des reinen AP reichen von $2 \mathrm{~cm}^{-1}\left(\mathrm{MA}_{\mathrm{Ph}}\right.$ von $\left.4 \mathrm{FAP}\right)$ bis $14 \mathrm{~cm}^{-1}$ $\left(\mathrm{MA}_{\mathrm{Me}}\right.$ von 2FAP) und sind generell für 2- größer als für 4-Substitution sowie für die Methyl- größer als für die Phenylseite. Die Aufspaltung zwischen den Banden verringert sich daher bei Fluorierung nur leicht von $38 \mathrm{~cm}^{-1}$ für $\mathrm{MeOH}+\mathrm{AP}$ auf $36 \mathrm{~cm}^{-1}$ für Me$\mathrm{OH}+2 \mathrm{FAP}$ bzw. $34 \mathrm{~cm}^{-1}$ für $\mathrm{MeOH}+4 \mathrm{FAP}$. Deutlichere Unterschiede zeigen sich hingegen im Intensitätsverhältnis der Heterodimerbanden, das signifikant von $5.2 \pm 0.4(\mathrm{MeOH}+\mathrm{AP})$ auf $2.3 \pm 0.2$ (2FAP) bzw. $2.3 \pm 0.5$ (4FAP) sinkt. Die angegebenen statistischen Fehler erfassen mögliche systematische Abweichungen, z. B. durch potentielle Bandenüberlappung wie im Fall von $\mathrm{MeOH}+2 \mathrm{FAP}$, nicht und stellen somit die untere Grenze der tatsächlichen Messunsicherheit dar. Werden die Bandenstärkenverhältnisse als näherungsweise konstant angenommen, was auf Basis des geringen Einflusses der Fluor-Substitution auf die beobachteten Rotverschiebungen gerechtfertigt erscheint, und wird von gleichbleibender relativer Entartung ausgegangen, ergibt sich bei Fluorierung aus den experimentellen Intensitätsverhältnissen eine relative Stabilisierung des Ph-Dimers gegenüber dem Me-Dimer. Dies passt auch qualitativ zur Verkleinerung der spektralen Aufspaltung. Im folgenden Benchmark der berechneten Energien (Kap. 5.3) soll unter Einbeziehung der ebenfalls berechneten Bandenstärkenverhältnisse und der sich aus den erhaltenen Dimerstrukturen ergebenden relativen Entartung quantitativ überprüft werden, ob die untersuchten Rechenmethoden diesen experimentellen Trend treffend beschreiben können. Die Leistungsfähigkeit der untersuchten quantenchemischen Methoden in Bezug auf die IR-spektroskopischen Parameter Rotverschiebung und Bandenaufspaltung soll anschließend ebenfalls beurteilt werden (Kap. 5.4).

Für das in Abbildung 5.5 gezeigte Spektrum von MeOH+2FAP (grün), das am GratinJet gemessen wurde, können im Gegensatz zu den weiteren, am Filet-Jet aufgezeichneten Spektren genaue Analytkonzentrationen angegeben werden. Bei Partialdrücken von 0.08 mbar für MeOH, 0.30 mbar für 2FAP und 750 mbar für He ergeben sich Konzentrationen von ca. $0.01 \%$ bzw. $0.04 \%$. Da die am Filet-Jet aufgenommenen Spektren ähnliche Intensitäten für die Methanol- und Acetophenon(derivat)-Banden aufweisen, scheinen hier (unter Berücksichtigung der in allen genutzten Rechenmethoden über die AP-Derivate hinweg relativ konstanten IR-Bandenstärken) vergleichbare Analytkonzentrationen vorzuliegen, auch wenn diese aufgrund des Herstellungsverfahrens für das Gasgemisch (s. Kap. 2.2) nicht exakt bestimmt werden können. Die trotz dieser niedrigen Analytkonzentrationen erzielten guten Signal-zu-Rauschverhältnisse der Heterodimerbanden (mit Ausnahme der $\mathrm{MA}_{\mathrm{Ph}}$-Bande von $\mathrm{MeOD}+\mathrm{AP}$ stets $>10$ ) erleichtern im Vergleich zu den Methanol-Furan- bzw. Methanol-Anisol-Waagensystemen die Bestimmung genauer, für die folgenden Benchmarks (Kap. 5.3) wichtiger Intensitätsverhältnisse. Höhere Aggregate, deren Banden unbemerkt mit den Heterodimerbanden überlagern könnten, sollten bei 
den genutzten niedrigen Konzentrationen nur in äußerst geringen Mengen vorliegen.

\subsection{Auswertung des $\mathrm{Me} / \mathrm{Ph}$ - Konformerengleichgewichts}

Zunächst sollen die Ergebnisse für die verschiedenen quantenchemischen Rechenmethoden (s. Tab. 5.1 auf S. 181) hinsichtlich der relativen elektronischen und schwingungsnullpunktskorrigierten Energien detailliert vorgestellt werden (Kap. 5.3.1), bevor im Anschluss nach einer Diskussion möglicher Betrachtungsvarianten für die Ermittlung der Dimer-Symmetrie (Kap. 5.3.2) der Benchmark der quantenchemischen Resultate anhand der experimentellen Referenzdaten erfolgt (Kap. 5.3.3).

\subsubsection{Ergebnisse der quantenchemischen Rechnungen}

Da für jede Bindungsseite im Methanol-Acetophenon(derivat)-Dimer zwei unterschiedliche Strukturtypen (ip und op, s. Abb. 5.3 auf S. 177) möglich sind, sollen für jede DonorAkzeptor-Kombination zunächst die auf den verschiedenen Theorieniveaus berechneten relativen Energien dieser Strukturen betrachtet werden. Die Ergebnisse für die relativen elektronischen Energien $\left(E_{\text {rel }}^{\mathrm{el}}\right)$ für $\mathrm{MeOH}+\mathrm{AP}, \mathrm{MeOH}+2 \mathrm{FAP}$ und $\mathrm{MeOH}+4 \mathrm{FAP}$ sind in Tabelle 5.3 dargestellt, die entsprechenden Ergebnisse für die schwingungsnullpunktskorrigierten Energien $\left(E_{\text {rel }}^{0}\right)$ in Tabelle 5.4. Die Energien sind nach Methode und AP-Derivat getrennt jeweils relativ zum stabilsten Ph-Dimer angegeben. Der fettgedruckte Energiewert für die niederenergetischste Me-Dimerstruktur entspricht damit direkt der Energiedifferenz $\Delta E=E(\mathrm{Me})-E(\mathrm{Ph})$, die für die weitere Auswertung benötigt wird.

Werden zunächst einmal die mit den verschiedenen DFT-Methoden in den Geometrieoptimierungen erhaltenen Strukturtypen der Methanol-Acetophenon(derivat)-Dimere betrachtet, so fällt für die ip-Strukturen $\mathrm{Me}_{\mathrm{ip}}$ und $\mathrm{Ph}_{\mathrm{ip}}$ eine interessante Korrelation auf. Diese stellen nur für die Methoden B3Q und B2, also die Methoden mit den größten verwendeten Basissätzen (def2-QZVP bzw. a'VTZ), lokale Minima dar. Für die weiteren Methoden wurden aus den Geometrieoptimierungen lediglich op-Strukturen erhalten. Auf dem B2-Niveau wurden hingegen für Ph jeweils beide Strukturtypen, für Me jedoch ausschließlich ip-Dimere gefunden. Besonders interessant ist der Vergleich des dispersionskorrigierten B3LYP-Funktionals mit def2-TZVP-(TZ-) und def2-QZVP-(QZ-)Basis. So ist das $\mathrm{Me}_{\mathrm{ip}}$-Dimer, das für $\mathbf{B} 3$ kein lokales Minimum darstellt, mit dem größeren Basissatz sogar der stabilere Me-Strukturtyp (für MeOH+4FAP jedoch erst bei Berechnung der elektronischen Energie mit dem DLPNO-CCSD(T)-Verfahren). Diese von der Basissatzgröße abhängigen Unterschiede verdeutlichen, dass zumindest für das B3LYP-Funktional mit dem TZ-Basissatz noch keine Basissatzkonvergenz erreicht zu sein scheint. Inwiefern dies auch für das M06-2X-Funktional oder die in den Kompositmethoden B97-3c und PBEh-3c verwendeten Basissätze gilt, kann ohne zusätzliche Untersuchungen nicht beurteilt werden. 
Tabelle 5.3: Elektronische Energien $\left(E_{\mathrm{rel}}^{\mathrm{el}}\right)$ der unterschiedlich gebundenen Dimere (s. Abb. 5.3) von Methanol mit AP, 2FAP und 4FAP für die verschiedenen quantenchemischen Methoden (s. Tab. 5.1), jeweils relativ zum stabilsten Ph-Dimer. Die jeweils stabilste Struktur für eine Bindungsseite $(\mathrm{Me}$ oder $\mathrm{Ph}$ ) ist fett hervorgehoben. Existieren für einen der jeweils vier Strukturtypen auf einem quantenchemischen Rechenniveau mehrere lokale Minima, wurde nur das energetisch tiefstliegende Minimum berücksichtigt. Wurde ein Strukturtyp mit einer Rechenmethode nicht als Minimum gefunden, bleibt das Feld leer.

\begin{tabular}{|c|c|c|c|c|c|c|c|c|c|c|c|c|}
\hline \multirow{2}{*}{$\begin{array}{l}E_{\mathrm{rel}}^{\mathrm{el}} \\
/ \mathrm{kJ} \cdot \mathrm{mol}^{-1}\end{array}$} & \multicolumn{4}{|c|}{$\mathrm{MeOH}+\mathrm{AP}$} & \multicolumn{4}{|c|}{$\mathrm{MeOH}+2 \mathrm{FAP}$} & \multicolumn{4}{|c|}{$\mathrm{MeOH}+4 \mathrm{FAP}$} \\
\hline & $\mathrm{Me}_{\mathrm{ip}}$ & $\mathrm{Me}_{\mathrm{op}}$ & $\mathrm{Ph}_{\text {ip }}$ & $\mathrm{Ph}_{\mathrm{op}}$ & $\mathrm{Me}_{\mathrm{ip}}$ & $\mathrm{Me}_{\mathrm{op}}$ & $\mathrm{Ph}_{\mathrm{ip}}$ & $\mathrm{Ph}_{\mathrm{op}}$ & $\mathrm{Me}_{\mathrm{ip}}$ & $\mathrm{Me}_{\mathrm{op}}$ & $\mathrm{Ph}_{\text {ip }}$ & $\mathrm{Ph}_{\mathrm{op}}$ \\
\hline B3 & & -0.48 & & 0.00 & & 0.13 & & 0.00 & & 0.14 & & 0.00 \\
\hline B3+ & & -0.08 & & 0.00 & & 0.63 & & 0.00 & & 0.28 & & 0.00 \\
\hline B3+c & & -0.19 & & 0.00 & & 0.55 & & 0.00 & & 0.20 & & 0.00 \\
\hline $\mathrm{B} 3+\mathrm{cP}$ & & -0.45 & & 0.00 & & 0.19 & & 0.00 & & -0.06 & & 0.00 \\
\hline B3Q & -0.51 & -0.45 & 0.43 & 0.00 & 0.15 & 0.17 & 0.40 & 0.00 & 0.04 & -0.02 & 0.22 & 0.00 \\
\hline B3Q+ & -0.74 & -0.02 & 0.38 & 0.00 & 0.19 & 0.63 & 0.33 & 0.00 & -0.37 & -0.18 & 0.00 & 0.15 \\
\hline B3Q+c & -0.94 & -0.19 & 0.34 & 0.00 & 0.07 & 0.52 & 0.28 & 0.00 & -0.45 & -0.26 & 0.00 & 0.16 \\
\hline M6 & & -0.48 & & 0.00 & & 0.22 & & 0.00 & & 0.01 & & 0.00 \\
\hline M6+ & & 0.17 & & 0.00 & & 0.73 & & 0.00 & & 0.62 & & 0.00 \\
\hline B9 & & -1.02 & & 0.00 & & -0.40 & & 0.00 & & -0.67 & & 0.00 \\
\hline B9+ & & -0.08 & & 0.00 & & 0.50 & & 0.00 & & 0.12 & & 0.00 \\
\hline PB & & -0.57 & & 0.00 & & -0.39 & & 0.00 & & -0.01 & & 0.00 \\
\hline PB+ & & -0.01 & & 0.00 & & 0.45 & & 0.00 & & 0.25 & & 0.00 \\
\hline B2 & -0.88 & & 0.00 & 0.07 & -0.09 & & 0.00 & 0.10 & -0.13 & & 0.00 & 0.18 \\
\hline B2+ & -0.68 & & 0.39 & 0.00 & 0.05 & & 0.30 & 0.00 & -0.42 & & 0.00 & 0.15 \\
\hline
\end{tabular}

Bei Betrachtung der elektronischen Energien (Tab. 5.3) zeigt sich für $\mathrm{MeOH}+\mathrm{AP}$ für nahezu alle Methoden eine Bevorzugung des stabilsten Me-Dimers gegenüber dem jeweils stabilsten Ph-Dimer. Lediglich für das M6+-Niveau liegt das Me- bei höheren Energien als das $\mathrm{Ph}$-Minimum. Existiert ein $\mathrm{Me}_{\mathrm{ip}}$-Dimer, weist dies die niedrigere Energie der $\mathrm{Me}$ Strukturtypen auf, wohingegen auf $\mathrm{Ph}$-Seite $\mathrm{Ph}_{\text {ip }}$ nur für B2 stabiler als $\mathrm{Ph}_{\mathrm{op}}$ ist.

Die gleichen Befunde zeigen sich bei Fluorierung in ortho-Position ( $\mathrm{MeOH}+2 \mathrm{FAP})$, allerdings bei anderen relativen Stabilitäten zwischen den Bindungsseiten. Für die meisten Methoden wird nun die Ph-Seite bevorzugt. Lediglich für die Methoden B9, PB und B2 ergibt sich eine Me-Struktur, die energetisch tiefer als die stabilste $\mathrm{Ph}$-Struktur liegt. Bei para-Fluorierung des AP $(\mathrm{MeOH}+4 \mathrm{FAP})$ zeigt sich in dieser Hinsicht eine ausgewogene Situation, wobei acht Methoden eine Me- und sieben Methoden eine Ph-Bevorzugung vorhersagen. Die ip-Strukturen stellen für $\mathrm{MeOH}+4 \mathrm{FAP}$ sowohl für die $\mathrm{Me}$ - als auch für die Ph-Dimere generell die stabilere Strukturklasse dar, wenn sie in den Geometrieoptimierungen erhalten wurden. Lediglich auf B3Q-Niveau liegen die ip-Dimere energetisch jeweils etwas höher als ihre op-Gegenstücke.

Wird die elektronische Energie auf dem DLPNO-CCSD(T)/aVQZ-Niveau („+“) berechnet, ergibt sich für B3, M6, B9 und PB eine relative Stabilisierung der Ph- gegenüber der Me-Seite. Ein gegenläufiger Trend zeigt sich auf B2-Niveau lediglich für die $\mathrm{Me}_{\mathrm{ip}}{ }^{-}$ Struktur von $\mathrm{MeOH}+4 \mathrm{FAP}$ und auf B3Q-Niveau für die $\mathrm{Me}_{\mathrm{ip}}$-Strukturen von $\mathrm{MeOH}+\mathrm{AP}$ und $\mathrm{MeOH}+4 \mathrm{FAP}$ sowie die $\mathrm{Me}_{\mathrm{op}}$-Struktur von $\mathrm{MeOH}+4 \mathrm{FAP}$. Werden die Kernelektronen 
Tabelle 5.4: Analog zu Tabelle 5.3, aber für schwingungsnullpunktskorrigierte Energien $E_{\text {rel }}^{0}$. Ändert sich die stabilste Struktur innerhalb eines Strukturtyps zwischen elektronischer und schwingungsnullpunktskorrigierter Energie, ist dies durch kursiv gedruckte Werte gekennzeichnet. Dies ist für das $\mathrm{Ph}_{\mathrm{op}}$-Dimer von $\mathrm{MeOH}+4 \mathrm{FAP}$ mit der B9+-Methode der Fall.

\begin{tabular}{|c|c|c|c|c|c|c|c|c|c|c|c|c|}
\hline \multirow{2}{*}{$\begin{array}{l}E_{\mathrm{rel}}^{0} \\
/ \mathrm{kJ} \cdot \mathrm{mol}^{-1}\end{array}$} & \multicolumn{4}{|c|}{$\mathrm{MeOH}+\mathrm{AP}$} & \multicolumn{4}{|c|}{$\mathrm{MeOH}+2 \mathrm{FAP}$} & \multicolumn{4}{|c|}{$\mathrm{MeOH}+4 \mathrm{FAP}$} \\
\hline & $\mathrm{Me}_{\mathrm{ip}}$ & $\mathrm{Me}_{\mathrm{op}}$ & $\mathrm{Ph}_{\mathrm{ip}}$ & $\mathrm{Ph}_{\mathrm{op}}$ & $\mathrm{Me}_{\mathrm{ip}}$ & $\mathrm{Me}_{\mathrm{op}}$ & $\mathrm{Ph}_{\mathrm{ip}}$ & $\mathrm{Ph}_{\mathrm{op}}$ & $\mathrm{Me}_{\mathrm{ip}}$ & $\mathrm{Me}_{\mathrm{op}}$ & $\mathrm{Ph}_{\text {ip }}$ & $\mathrm{Ph}_{\mathrm{op}}$ \\
\hline B3 & & -0.61 & & 0.00 & & -0.02 & & 0.00 & & -0.02 & & 0.00 \\
\hline B3+ & & -0.20 & & 0.00 & & 0.48 & & 0.00 & & 0.13 & & 0.00 \\
\hline $\mathrm{B} 3+\mathrm{c}$ & & -0.31 & & 0.00 & & 0.39 & & 0.00 & & 0.04 & & 0.00 \\
\hline $\mathrm{B} 3+\mathrm{cP}$ & & -0.57 & & 0.00 & & 0.04 & & 0.00 & & -0.22 & & 0.00 \\
\hline B3Q & -0.90 & -0.61 & 0.28 & 0.00 & -0.31 & -0.12 & 0.06 & 0.00 & -0.33 & -0.24 & 0.00 & 0.04 \\
\hline B3 $\tilde{Q}_{+}$ & -1.13 & -0.17 & 0.23 & 0.00 & -0.27 & 0.34 & 0.00 & 0.01 & -0.53 & -0.17 & 0.00 & 0.40 \\
\hline B3 $\widetilde{Q}+c$ & -1.32 & -0.35 & 0.19 & 0.00 & -0.33 & 0.29 & 0.00 & 0.06 & -0.60 & -0.25 & 0.00 & 0.42 \\
\hline M6 & & -0.45 & & 0.00 & & 0.32 & & 0.00 & & -0.10 & & 0.00 \\
\hline M6+ & & 0.20 & & 0.00 & & 0.83 & & 0.00 & & 0.51 & & 0.00 \\
\hline B9 & & -1.30 & & 0.00 & & -0.62 & & 0.00 & & -0.56 & & 0.00 \\
\hline B9+ & & -0.36 & & 0.00 & & 0.28 & & 0.00 & & 0.28 & & 0.00 \\
\hline PB & & -0.85 & & 0.00 & & -0.12 & & 0.00 & & -0.22 & & 0.00 \\
\hline PB+ & & -0.29 & & 0.00 & & 0.72 & & 0.00 & & -0.05 & & 0.00 \\
\hline B2 & -0.78 & & 0.00 & 0.60 & -0.40 & & 0.00 & 0.28 & -0.15 & & 0.00 & 0.89 \\
\hline B2+ & -0.97 & & 0.00 & 0.46 & -0.55 & & 0.00 & 0.16 & -0.44 & & 0.00 & 0.85 \\
\hline
\end{tabular}

in der DLPNO-CCSD(T)-Rechnung mitkorreliert $\left(,+\mathbf{c}^{*}\right)$, tritt für beide betrachteten DFTMethoden (B3 und B3Q) gegenüber der DLPNO-CCSD(T)-Rechnung bei eingefrorenen Kernelektronen („+") eine relative Stabilisierung der Me-Bindungsseite auf. Bei Ersetzen des Foster-Boys- durch das Pipek-Mezey-Lokalisierungsverfahren $(\mathbf{B 3}+\mathbf{c P})$ wird die MeSeite weiter gegenüber der Ph-Seite stabilisiert.

Bei Berücksichtigung der auf dem jeweiligen DFT-Niveau berechneten Schwingungsnullpunktsenergien (s. Tab. 5.4) ergeben sich hinsichtlich der relativen Energien der verschiedenen Strukturtypen einige Unterschiede. So weisen die ip- gegenüber den op-Strukturen derselben Bindungsseite sowohl auf B3Q- als auch auf B2-Niveau in allen Fällen eine niedrigere ZPVE auf. Die Unterschiede bewegen sich dabei zwischen 0.05 (Ph-Seite von $\mathrm{MeOH}+2 \mathrm{FAP}$ auf B2-Niveau) und $0.71 \mathrm{~kJ} \cdot \mathrm{mol}^{-1}$ (Ph-Seite von $\mathrm{MeOH}+4 \mathrm{FAP}$ auf demselben Niveau) zugunsten der ip-Struktur. Dies führt dazu, dass sich der Typ der stabileren Struktur einer Bindungsseite gegenüber der Betrachtung der elektronischen Energien in einigen Fällen von op zu ip ändert. Auch für das Ph-Dimer von $\mathrm{MeOH}+4 \mathrm{FAP}$ auf B9+Niveau ergibt sich bei Berücksichtigung der ZPVE eine Änderung der energetisch günstigsten Struktur gegenüber der Betrachtung der elektronischen Energien, hier aber innerhalb des op-Strukturtyps (in Tab. 5.4 durch Kursivsatz gekennzeichnet). Die elektronisch und schwingungsnullpunktskorrigiert jeweils stabilsten $\mathrm{MeOH}+4 \mathrm{FAP}-\mathrm{Ph}$-Strukturen unterscheiden sich auf diesem Rechenniveau in der Anordnung des $\mathrm{MeOH}$-Moleküls relativ zum 4FAP-Molekül. In allen weiteren Fällen ergeben sich keine Wechsel der jeweils stabilsten Struktur einer Bindungsseite durch Berücksichtigung der ZPVE.

Ein Ziel bei der Betrachtung der Carbonylwaagen als System für das Benchmarking quantenchemischer Methoden ist, den Einfluss der ZPVE auf das Gleichgewicht zwischen 
Tabelle 5.5: Zusammenfassung der wichtigsten, mit den quantenchemischen Methoden berech-

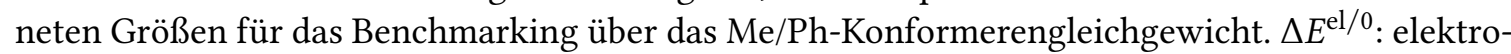
nische (el) bzw. schwingungsnullpunktskorrigierte (0) Energiedifferenz zwischen stabilstem Meund $\mathrm{Ph}$-gebundenem Dimer in $\mathrm{kJ} \cdot \mathrm{mol}^{-1}$. Negative Werte entsprechen einer Me-, positive einer $\mathrm{Ph}$ Bevorzugung. $\triangle Z$ : der sich durch die Berücksichtigung der ZPVE ergebende Unterschied zwischen $\Delta E^{\mathrm{el}}$ und $\Delta E^{0}$ in $\mathrm{kJ} \cdot \mathrm{mol}^{-1}$, wobei hier auch Wechsel der jeweils berücksichtigten Dimerstruktur zwischen $\Delta E^{\mathrm{el}}$ und $\Delta E^{0}$ (s. Tab. 5.3 und 5.4) mit einbezogen werden (kursiv hervorgehoben). $R_{A}=A_{\mathrm{Me}} / A_{\mathrm{Ph}}$ : Verhältnis der berechneten Bandenstärken der OH-Streckschwingungen der jeweils (schwingungsnullpunktskorrigiert) stabilsten $\mathrm{Me}$ - und $\mathrm{Ph}$-Dimere.

\begin{tabular}{|c|c|c|c|c|c|c|c|c|c|c|c|c|}
\hline \multirow[b]{2}{*}{ Methode } & \multicolumn{4}{|c|}{$\mathrm{MeOH}+\mathrm{AP}$} & \multicolumn{4}{|c|}{$\mathrm{MeOH}+2 \mathrm{FAP}$} & \multicolumn{4}{|c|}{$\mathrm{MeOH}+4 \mathrm{FAP}$} \\
\hline & $\Delta E^{\mathrm{el}}$ & $\Delta E^{0}$ & $\Delta Z$ & $R_{A}$ & $\Delta E^{\mathrm{el}}$ & $\Delta E^{0}$ & $\Delta Z$ & $R_{A}$ & $\Delta E^{\mathrm{el}}$ & $\Delta E^{0}$ & $\Delta Z$ & $R_{A}$ \\
\hline B3 & -0.48 & -0.61 & -0.12 & 1.66 & 0.13 & -0.02 & -0.15 & 1.78 & 0.14 & -0.02 & -0.16 & 1.67 \\
\hline B3+ & -0.08 & -0.20 & -0.12 & 1.66 & 0.63 & 0.48 & -0.15 & 1.78 & 0.28 & 0.13 & -0.16 & 1.67 \\
\hline $\mathrm{B} 3+\mathrm{c}$ & -0.19 & -0.31 & -0.12 & 1.66 & 0.55 & 0.39 & -0.15 & 1.78 & 0.20 & 0.04 & -0.16 & 1.67 \\
\hline $\mathrm{B} 3+\mathrm{cP}$ & -0.45 & -0.57 & -0.12 & 1.66 & 0.19 & 0.04 & -0.15 & 1.78 & -0.06 & -0.22 & -0.16 & 1.67 \\
\hline B3Q & -0.51 & -0.90 & -0.39 & 1.92 & 0.15 & -0.31 & -0.46 & 2.00 & -0.02 & -0.33 & -0.31 & 1.41 \\
\hline B3Q+ & -0.74 & -1.13 & -0.39 & 1.92 & 0.19 & -0.27 & -0.45 & 1.43 & -0.37 & -0.53 & -0.16 & 1.41 \\
\hline B3Q+c & -0.94 & -1.32 & -0.39 & 1.92 & 0.07 & -0.33 & -0.40 & 1.43 & -0.45 & -0.60 & -0.16 & 1.41 \\
\hline M6 & -0.48 & -0.45 & 0.03 & 1.68 & 0.22 & 0.32 & 0.10 & 1.72 & 0.01 & -0.10 & -0.11 & 1.70 \\
\hline M6+ & 0.17 & 0.20 & 0.03 & 1.68 & 0.73 & 0.83 & 0.10 & 1.72 & 0.62 & 0.51 & -0.11 & 1.70 \\
\hline B9 & -1.02 & -1.30 & -0.28 & 1.88 & -0.40 & -0.62 & -0.22 & 1.89 & -0.67 & -0.56 & 0.11 & 1.73 \\
\hline B9+ & -0.08 & -0.36 & -0.28 & 1.88 & 0.50 & 0.28 & -0.22 & 1.89 & 0.12 & 0.28 & 0.17 & 1.42 \\
\hline PB & -0.57 & -0.85 & -0.28 & 1.66 & -0.39 & -0.12 & 0.27 & 1.92 & -0.01 & -0.22 & -0.20 & 1.61 \\
\hline PB+ & -0.01 & -0.29 & -0.28 & 1.66 & 0.45 & 0.72 & 0.27 & 1.92 & 0.25 & 0.05 & -0.20 & 1.61 \\
\hline B2 & -0.88 & -0.78 & 0.10 & 1.39 & -0.09 & -0.40 & -0.30 & 1.45 & -0.13 & -0.15 & -0.02 & 1.41 \\
\hline B2+ & -0.68 & -0.97 & -0.29 & 1.39 & 0.05 & -0.55 & -0.60 & 1.45 & -0.42 & -0.44 & -0.02 & 1.41 \\
\hline
\end{tabular}

den verschiedenen Bindungsstellen des Waagensystems zu minimieren. In diesem Sinne sollte die Berücksichtigung der ZPVE möglichst geringe Auswirkungen auf den Energieunterschied zwischen $\mathrm{Me}$ - und $\mathrm{Ph}$-Seite haben, es sollten sich also möglichst gleiche Energiedifferenzen $\Delta E^{\mathrm{el}}$ und $\Delta E^{0}$ zwischen den jeweils stabilsten Strukturen der beiden Bindungsseiten ergeben. Unterschiede in diesen beiden Differenzen können sowohl direkt durch verschieden große Schwingungsnullpunktsenergien der eingehenden Dimerstrukturen als auch durch Wechsel der jeweils stabilsten Struktur einer Bindungsseite bei Berücksichtigung der ZPVE (s. oben) verursacht werden. Die sich aus diesen Effekten insgesamt ergebende Differenz $\Delta Z=\Delta E^{0}-\Delta E^{\mathrm{el}}$ ist gemeinsam mit den elektronischen und schwingungsnullpunktskorrigierten Energieunterschieden und den für die weitere Auswertung benötigten Verhältnissen der berechneten Bandenstärken $A$ der schwingungsnullpunktskorrigiert jeweils stabilsten Me- und Ph-Dimere $\left(R_{A}=A_{\mathrm{Me}} / A_{\mathrm{Ph}}\right)$ in Tabelle 5.5 zusammengefasst. Ein negatives Vorzeichen in $\Delta Z$ kennzeichnet dabei energetische Vorteile für die Me-Seite durch die gesammelten ZPVE-Einflüsse, ein positives Vorzeichen entsprechende Vorteile für die Ph-Seite. Gehen dieselben Strukturen in die Berechnung von $\Delta E^{\mathrm{el}}$ und $\Delta E^{0}$ ein, entspricht $\Delta Z$ der Differenz in den Schwingungsnullpunktsenergien und ist somit für alle auf derselben Schwingungsrechnung basierenden Rechenmethoden (z.B. B3[+,+c,+cP]) gleich. Müssen zusätzlich Wechsel der eingehenden Dimerstrukturen 
Tabelle 5.6: Auf den verschiedenen DFT-Niveaus berechnete Bandenstärken $A_{\mathrm{OH}}$ der OH-Streckschwingungen der jeweils schwingungsnullpunktskorrigiert stabilsten Me- bzw. Ph-Dimere für $\mathrm{MeOH}+\mathrm{AP}, \mathrm{MeOH}+2 \mathrm{FAP}$ und $\mathrm{MeOH}+4 \mathrm{FAP}$. Für das $\mathrm{Ph}-\mathrm{Dimer}$ von $\mathrm{MeOH}+2 \mathrm{FAP}$ auf B3Q+- und B3Q+c-Niveau sowie das Ph-Dimer von $\mathrm{MeOH}+4 \mathrm{FAP}$ auf B9+-Niveau ergeben sich mit 597 bzw. $493 \mathrm{~km} \cdot \mathrm{mol}^{-1}$ von den angegebenen Werten abweichende Bandenstärken, da hier andere Strukturen schwingungsnullpunktskorrigiert die stabilsten $\mathrm{Ph}$-Dimere darstellen als für die gelistete B3Q- bzw. B9-Methode (s. Tab. 5.4).

\begin{tabular}{|c|c|c|c|c|c|c|}
\hline \multirow{2}{*}{$\begin{array}{l}A_{\mathrm{Me}, \mathrm{Ph}} \\
/ \mathrm{km} \cdot \mathrm{mol}^{-1}\end{array}$} & \multicolumn{2}{|c|}{$\mathrm{MeOH}+\mathrm{AP}$} & \multicolumn{2}{|c|}{$\mathrm{MeOH}+2 \mathrm{FAP}$} & \multicolumn{2}{|c|}{$\mathrm{MeOH}+4 \mathrm{FAP}$} \\
\hline & $\mathrm{Me}$ & $\mathrm{Ph}$ & $\mathrm{Me}$ & $\mathrm{Ph}$ & $\mathrm{Me}$ & $\mathrm{Ph}$ \\
\hline B3 & 751 & 452 & 715 & 402 & 741 & 443 \\
\hline B3Q & 899 & 469 & 852 & 427 & 887 & 630 \\
\hline M6 & 471 & 281 & 451 & 262 & 461 & 272 \\
\hline B9 & 703 & 374 & 660 & 349 & 701 & 406 \\
\hline PB & 449 & 271 & 403 & 210 & 434 & 269 \\
\hline B2 & 867 & 622 & 831 & 574 & 848 & 602 \\
\hline
\end{tabular}

in $\Delta Z$ berücksichtigt werden (durch Kursivsatz des $\Delta Z$-Werts in Tab. 5.5 gekennzeichnet), ergeben sich jedoch auch für Methoden mit denselben Schwingungsrechnungen Unterschiede, z. B. für $\mathrm{MeOH}+4 \mathrm{FAP}$ zwischen $\mathbf{B} 3 \mathbf{Q}$ und B3Q+. Insgesamt zeigen sich für die meisten Methoden in den gesammelten ZPVE-Einflüssen $\triangle Z$ leichte energetische Vorteile der $\mathrm{Me}$ - gegenüber der $\mathrm{Ph}$-Seite. In einigen Fällen liegt jedoch auch ein entgegengesetzter Trend vor. Die Unterschiede zwischen $\Delta E^{\mathrm{el}}$ und $\Delta E^{0}$ sind bis auf eine Ausnahme von $-0.60 \mathrm{~kJ} \cdot \mathrm{mol}^{-1}(\mathrm{MeOH}+2 \mathrm{FAP}$ für $\mathbf{B} 2+)$ mit $\Delta Z$-Werten von $-0.46 \mathrm{bis}+0.27 \mathrm{~kJ} \cdot \mathrm{mol}^{-1}$ deutlich kleiner und weniger systematisch als für die $\mathrm{O} / \mathrm{C}$-Waagensysteme, für die die ODimere in allen Fällen eine höhere ZPVE (Unterschiede bis zu $2 \mathrm{~kJ} \cdot \mathrm{mol}^{-1}$ ) als die C-Dimere aufweisen (s. Kap. 4). Diese nahezu vollständige Kompensation harmonischer Schwingungsnullpunktseffekte zwischen Me- und Ph-Dimer (etwa 99.9\% der Schwingungsnullpunktsenergien von je nach Dimer und Methode ca. 470 bis $520 \mathrm{~kJ} \cdot \mathrm{mol}^{-1}$ ) lässt darauf schließen, dass die wesentlich kleineren anharmonischen Beiträge zur ZPVE keinen signifikanten Einfluss auf die Energiedifferenz zwischen Me- und Ph-Seite nehmen sollten. ${ }^{[52]}$ Trotz der hohen Kompensationsrate sorgt die Berücksichtigung der (harmonischen) ZPVE-Beiträge in einigen Fällen für einen Wechsel der Bindungsstellenpräferenz von der Ph- zur Me-Seite (für MeOH+2FAP auf B3-, B3Q-, B3Q+-, B3Q+c- und B2+-Niveau; für MeOH+4FAP auf B3- und M6-Niveau). Wechsel von Me- zu Ph-Präferenz treten hingegen nicht auf.

Die Bandenstärkenverhältnisse $\left(R_{\mathrm{A}}=A_{\mathrm{Me}} / A_{\mathrm{Ph}}\right)$ sind zwischen den verschiedenen, für die doppelt-harmonischen Schwingungsrechnungen eingesetzten Methoden trotz signifikanter Unterschiede in den absoluten Größen der Bandenstärken $A_{\mathrm{Me}, \mathrm{Ph}}$ (s. Tab. 5.6) relativ ähnlich und hängen vor allem von den für die Verhältnisbildung berücksichtigten 
Tabelle 5.7: Energieunterschiede $\Delta E^{0}$ (in $\mathrm{kJ} \cdot \mathrm{mol}^{-1}$ ) und Bandenstärkenverhältnisse $R_{A}$ der deuterierten Isotopologe $\mathrm{MeOD}+\mathrm{AP}, \mathrm{MeOH}+\mathrm{APd} 3$ und $\mathrm{MeOH}+\mathrm{APd}$. Die elektronischen Energiedifferenzen $\Delta E^{\mathrm{el}}$ entsprechen denen für $\mathrm{MeOH}+\mathrm{AP}$ in Tab. 5.5 und sind deshalb hier nicht erneut aufgeführt.

\begin{tabular}{|c|c|c|c|c|c|c|}
\hline \multirow[b]{2}{*}{ Methode } & \multicolumn{2}{|c|}{$\mathrm{MeOD}+\mathrm{AP}$} & \multicolumn{2}{|c|}{$\mathrm{MeOH}+\mathrm{APd} 3$} & \multicolumn{2}{|c|}{$\mathrm{MeOH}+\mathrm{APd} 5$} \\
\hline & $\Delta E^{0}$ & $R_{A}$ & $\Delta E^{0}$ & $R_{A}$ & $\Delta E^{0}$ & $R_{A}$ \\
\hline B3 & -0.64 & 1.65 & -0.71 & 1.66 & -0.46 & 1.67 \\
\hline B3+ & -0.23 & 1.65 & -0.30 & 1.66 & -0.05 & 1.67 \\
\hline B3+c & -0.34 & 1.65 & -0.42 & 1.66 & -0.16 & 1.67 \\
\hline $\mathrm{B} 3+\mathrm{cP}$ & -0.60 & 1.65 & -0.67 & 1.66 & -0.42 & 1.67 \\
\hline B3Q & -0.86 & 1.92 & -0.97 & 1.92 & -0.77 & 1.93 \\
\hline B3Q+ & -1.09 & 1.92 & -1.20 & 1.92 & -1.00 & 1.93 \\
\hline$B 3 Q+c$ & -1.29 & 1.92 & -1.40 & 1.92 & -1.20 & 1.93 \\
\hline M6 & -0.52 & 1.64 & -0.57 & 1.68 & -0.31 & 1.68 \\
\hline M6+ & 0.13 & 1.64 & 0.08 & 1.68 & 0.34 & 1.68 \\
\hline B9 & -1.32 & 1.86 & -1.39 & 1.88 & -1.17 & 1.89 \\
\hline B9+ & -0.37 & 1.86 & -0.45 & 1.88 & -0.22 & 1.89 \\
\hline PB & -0.92 & 1.63 & -0.97 & 1.66 & -0.66 & 1.67 \\
\hline PB+ & -0.36 & 1.63 & -0.41 & 1.66 & -0.10 & 1.67 \\
\hline B2 & -0.78 & 1.37 & -0.84 & 1.39 & -0.63 & 1.40 \\
\hline B2+ & -0.97 & 1.37 & -1.03 & 1.39 & -0.82 & 1.40 \\
\hline
\end{tabular}

Strukturtypen ab. So ergeben sich für die Bandenstärkenverhältnisse (s. Tab. 5.5), bei denen die Me- und Ph-Strukturen vom ip-Typ sind, Werte zwischen 1.39 und 1.45. Ist die Ph-Struktur vom op-Typ, betragen die Bandenstärkenverhältnisse 1.61-1.92 (Me-Struktur op) bzw. 1.92-2.00 (Me-Struktur ip), mit Ausnahme des Wertes für das MeOH+4FAPDimer auf B9+-Niveau. Für dieses ergibt sich ein Bandenstärkenverhältnis von 1.42, obwohl Me- und Ph-Struktur dem op-Typ zugeordnet wurden. Die schwingungsnullpunktskorrigiert stabilste Ph-Struktur stellt jedoch einen Grenzfall zwischen ip- und op-Klasse dar und weist mit $493 \mathrm{~km} \cdot \mathrm{mol}^{-1}$ eine deutlich größere Bandenstärke als beispielsweise die elektronisch stabilere $\mathrm{Ph}_{\mathrm{op}}$-Struktur $\left(406 \mathrm{~km} \cdot \mathrm{mol}^{-1}\right)$ auf, was zum beobachteten kleinen Bandenstärkenverhältnis führt. Größere Unterschiede in den Bandenstärkenverhältnissen zwischen den verschiedenen quantenchemischen Methoden sind also im Wesentlichen auf die unterschiedliche Beurteilung der relativen Stabilität der verschiedenen Dimer-Strukturtypen zurückzuführen. Auf Basis dieser Robustheit der Bandenstärkenverhältnisse über die verschiedenen betrachteten Theorieniveaus hinweg erscheint es somit gerechtfertigt, diese zu nutzen, um die berechneten Energieunterschiede anhand der experimentellen Intensitätsverhältnisse (s. Tab. 5.2 auf S. 185) zu benchmarken.

Für diese Benchmarks sollen auch die bereits im Experiment betrachteten (s. Kap. 5.2) deuterierten Isotopologe der MeOH-AP-Dimere berücksichtigt werden. Da im Rahmen 
der BoRn-Oppenheimer-Näherung ${ }^{[335]}$ die elektronischen Energien von den Atommassen unabhängig sind, ergeben sich für die Isotopologe lediglich in den Schwingungsrechnungen Unterschiede zu MeOH+AP, z. B. in den Schwingungsnullpunktsenergien und Bandenstärken. Die resultierenden schwingungsnullpunktskorrigierten Energieunterschiede $\Delta E^{0}$ und Bandenstärkenverhältnisse $R_{A}$ für $\mathrm{MeOD}+\mathrm{AP}, \mathrm{MeOH}+\mathrm{APd} 3$ und Me$\mathrm{OH}+\mathrm{APd} 5$ sind in Tabelle 5.7 zusammengefasst. Während sich für die Bandenstärkenverhältnisse in allen Fällen mit maximal ca. $2 \%$ und für die Energiedifferenzen $\Delta E^{0}$ bei Deuterierung an der Methanol-OH-Gruppe mit weniger als $0.07 \mathrm{~kJ} \cdot \mathrm{mol}^{-1}$ nur geringe Unterschiede zum nicht-deuterierten Isotopolog ergeben, zeigen sich bei Deuterierung am Acetophenon systematische Abweichungen in $\Delta E^{0}$. Bei Deuterierung der Methylgruppe ergibt sich für alle betrachteten Methoden eine relative Stabilisierung des Me-Dimers $\left(0.06-0.12 \mathrm{~kJ} \cdot \mathrm{mol}^{-1}\right)$, bei Deuterierung der Phenyl-Gruppe eine entsprechende Stabilisierung des Ph-Dimers $\left(0.13-0.20 \mathrm{~kJ} \cdot \mathrm{mol}^{-1}\right)$.

\subsubsection{Symmetrie der Dimerstrukturen}

Für die Benchmarks auf Basis des Me/Ph-Konformerengleichgewichts werden neben den in den Tabellen 5.5 und 5.7 aufgelisteten berechneten schwingungsnullpunktskorrigierten Energieunterschieden $\left(\Delta E^{0}\right)$ und Verhältnissen der berechneten Bandenstärken $\left(R_{\mathrm{A}}\right)$ gemäß Gleichung 2.9 (s. S. 21) auch die Verhältnisse der Entartungsfaktoren ( $\left.g_{\mathrm{Me}} / g_{\mathrm{Ph}}\right) \mathrm{der}$ beiden betrachteten Dimere benötigt. Diese basieren auf der Symmetrie der beteiligten Dimere. Eine Möglichkeit zur Bestimmung der Entartungsfaktorenverhältnisse ist somit, streng die formalen Punktgruppen der in den Geometrieoptimierungen erhaltenen Minimumsstrukturen zu nutzen (Symmetriebetrachtungsvariante „Sym. A“). Dies entspricht dem Vorgehen in der Furan-Mikrosolvatations-Leistungsüberprüfung (s. Kap. 4.1). Für die hier untersuchten Dimere kommen dabei die Punktgruppen $C_{1}$ und $C_{s}$ in Betracht. Während die op-Dimere aufgrund der aus der Acetophenon-Ebene gedrehten MethanolMethylgruppe stets $C_{1}$-Symmetrie aufweisen, ist für die (ausschließlich auf B3Q- und B2Niveau erhaltenen) ip-Dimere eine genauere Analyse nötig. Liegt die Methanol-Methylgruppe exakt in der Acetophenon-Ebene, ist $C_{s}$-Symmetrie möglich. Um zu überprüfen, ob ein lokales, $C_{s}$-symmetrisches Minimum auf der Potentialhyperfläche vorliegt, werden die ip-Strukturen zunächst mit dem CHEMCRAFT-Programmpaket (Version 1.8, Build $562 \mathrm{~b})^{[381]} C_{s}$-symmetrisiert. Dabei werden alle Atome - bis auf die vier Methyl-H-Atome, für die dies nicht möglich ist - in die $x y$-Ebene gebracht. Anschließend wird eine erneute Geometrieoptimierung mit nachfolgender Frequenzrechnung mit ORCA durchgeführt. Da in Geometrieoptimierungen in den hier verwendeten ORCA-Versionen 4.0.1 und 4.1.2 keine bestimmte Symmetrie erzwungen werden kann, muss im Anschluss überprüft werden, ob die $C_{s}$-Symmetrie durch die Optimierung gebrochen wurde. Folgende Kriterien müssen nach der Optimierung erfüllt sein, damit eine Struktur als $C_{s}$-symmetrisch angenommen wird:

- Die $z$-Koordinaten der zuvor in die $x y$-Ebene gelegten Atome sind kleiner als $10^{-4} \AA$. 
Tabelle 5.8: Wellenzahlen $\omega$ der jeweils drei niederfrequentesten Moden der auf B3Q- und B2Niveau erhaltenen $\mathrm{Me}_{\mathrm{ip}}$ - und $\mathrm{Ph}_{\mathrm{ip}}$-Strukturen, getrennt nach $C_{1}$ - und $C_{s}$-Symmetrie.

\begin{tabular}{|c|c|c|c|c|c|c|c|c|c|c|c|c|}
\hline \multirow{3}{*}{$\begin{array}{l}\omega \\
/ \mathrm{cm}^{-1}\end{array}$} & \multicolumn{4}{|c|}{$\mathrm{MeOH}+\mathrm{AP}$} & \multicolumn{4}{|c|}{$\mathrm{MeOH}+2 \mathrm{FAP}$} & \multicolumn{4}{|c|}{$\mathrm{MeOH+4FAP}$} \\
\hline & \multicolumn{2}{|c|}{$\mathrm{Me}_{\mathrm{ip}}$} & \multicolumn{2}{|c|}{$\mathrm{Ph}_{\text {ip }}$} & \multicolumn{2}{|c|}{$\mathrm{Me}_{\mathrm{ip}}$} & \multicolumn{2}{|c|}{$\mathrm{Ph}_{\mathrm{ip}}$} & \multicolumn{2}{|c|}{$\mathrm{Me}_{\mathrm{ip}}$} & \multicolumn{2}{|c|}{$\mathrm{Ph}_{\mathrm{ip}}$} \\
\hline & $C_{1}$ & $C_{s}$ & $C_{1}$ & $C_{s}$ & $C_{1}$ & $C_{s}$ & $C_{1}$ & $C_{s}$ & $C_{1}$ & $C_{s}$ & $C_{1}$ & $C_{s}$ \\
\hline \multirow{3}{*}{ B3Q } & 5.2 & $15.9 \mathrm{i}$ & 15.3 & $32.7 \mathrm{i}$ & 3.9 & $22.8 \mathrm{i}$ & 7.2 & $33.2 \mathrm{i}$ & 2.2 & $18.3 \mathrm{i}$ & 14.1 & $34.4 \mathrm{i}$ \\
\hline & 24.0 & 21.8 & 34.7 & 19.0 & 20.9 & 18.1 & 24.9 & 12.8 & 22.4 & 21.3 & 28.5 & 19.8 \\
\hline & 44.2 & 43.1 & 50.0 & 47.0 & 27.6 & 20.4 & 34.9 & 34.5 & 41.9 & 40.5 & 47.6 & 46.9 \\
\hline \multirow{3}{*}{ B2 } & 17.2 & & 11.9 & $75.1 \mathrm{i}$ & 15.5 & & 18.2 & $21.3 \mathrm{i}$ & 14.5 & 5.8 & 3.7 & $33.2 \mathrm{i}$ \\
\hline & 33.7 & & 27.7 & $28.3 \mathrm{i}$ & 24.3 & & 27.3 & 16.5 & 20.8 & 23.6 & 11.6 & $15.7 \mathrm{i}$ \\
\hline & 36.5 & & 42.0 & 10.1 & 32.2 & & 43.7 & 38.4 & 32.6 & 35.0 & 49.7 & 44.8 \\
\hline
\end{tabular}

- Der Diederwinkel $\tau$ sowie der Diederwinkel zwischen Carbonyl- und Phenylgruppe $\left(\mathrm{O}=\mathrm{C} \ldots \mathrm{C}^{6^{\prime}} \mathrm{H}^{6^{\prime}}\right)$ betragen $0.000^{\circ}$.

- Der Diederwinkel C $=\mathrm{O} \ldots \mathrm{O}_{\mathrm{MeOH}}-\mathrm{C}_{\mathrm{MeOH}}$ beträgt $180.000^{\circ}$.

Weist die Struktur zudem keine imaginäre Schwingungsmode auf, wird sie in der Symmetriebetrachtungsvariante Sym. A als $C_{s}$-symmetrische Minimumsstruktur in die folgende Auswertung einbezogen. Für den Benchmark auf Basis des Me/Ph-Konformerengleichgewichts ist die Struktur dabei jedoch nur von Relevanz, wenn sie das schwingungsnullpunktskorrigiert stabilste Dimer einer Bindungsseite darstellt.

Wie Tabelle 5.8 verdeutlicht, wurden nach diesen Kriterien auf B3Q- für alle und auf B2-Niveau für die meisten betrachteten ip-Dimere $C_{s}$-symmetrische Strukturen erhalten. Allerdings ergibt sich lediglich für das auf dem B2-Niveau optimierte $\mathrm{Me}_{\text {ip }}$-Dimer von $\mathrm{MeOH}+4 \mathrm{FAP}$ eine Struktur ohne imaginäre Schwingungsmoden, sodass nur diese im Rahmen der Betrachtung Sym. A als $C_{s}$-symmetrische Minimumsstruktur angesehen wird. Als schwingungsnullpunktskorrigiert stabilstes Me-Dimer für MeOH+4FAP auf B2- und B2+Niveau wird diese Struktur dabei für die Auswertung des Me/Ph-Konformerengleichgewichts herangezogen. Für die beiden genannten Methoden ergibt sich für $\mathrm{MeOH}+4 \mathrm{FAP}$ somit ein Entartungsfaktorenverhältnis von 0.5. Für alle weiteren Dimere und alle weiteren Rechenmethoden betragen die Entartungsfaktorenverhältnisse für Sym. A 1.

Eine solche strenge, rein auf den berechneten elektronischen Minimumsstrukturen beruhende Symmetrie-Analyse kann jedoch zu kurz gegriffen sein. Im Rahmen der für die Betrachtung des Konformerengleichgewichtes getroffenen Näherungen (s. Kap. 2.3.2), z. B. der Vernachlässigung der Rotations- und Vibrations-Zustandssummen, sind für die Entartungsfaktorenverhältnisse nur diskrete Werte (hier 0.5 oder 1) möglich, wohingegen die Übergänge zwischen verschiedenen Verhältnissen in der Realität fließend sind. Zudem 
Tabelle 5.9: Inertialdefekte $\Delta=I_{C}-I_{A}-I_{B}$ der auf B3Q- bzw. B2-Niveau erhaltenen Dimer-Minimumsstrukturen. Als Referenz (Ref.) für die bei $C_{s}$-Symmetrie erreichbaren Inertialdefekte sind die Werte der auf B3Q-Niveau ermittelten $C_{s}$-symmetrischen Dimer-(Übergangzustands-)strukturen angegeben.

\begin{tabular}{|c|c|c|c|c|c|c|c|c|c|c|c|c|}
\hline \multirow{2}{*}{$\begin{array}{l}\Delta \\
/\left(10^{-46} \mathrm{~kg} \cdot \mathrm{m}^{2}\right)\end{array}$} & \multicolumn{4}{|c|}{$\mathrm{MeOH}+\mathrm{AP}$} & \multicolumn{4}{|c|}{$\mathrm{MeOH}+2 \mathrm{FAP}$} & \multicolumn{4}{|c|}{$\mathrm{MeOH}+4 \mathrm{FAP}$} \\
\hline & $\mathrm{Me}_{\mathrm{ip}}$ & $\mathrm{Me}_{\mathrm{op}}$ & $\mathrm{Ph}_{\text {ip }}$ & $\mathrm{Ph}_{\mathrm{op}}$ & $\mathrm{Me}_{\mathrm{ip}}$ & $\mathrm{Me}_{\mathrm{op}}$ & $\mathrm{Ph}_{\mathrm{ip}}$ & $\mathrm{Ph}_{\mathrm{op}}$ & $\mathrm{Me}_{\mathrm{ip}}$ & $\mathrm{Me}_{\mathrm{op}}$ & $\mathrm{Ph}_{\mathrm{ip}}$ & $\mathrm{Ph}_{\mathrm{op}}$ \\
\hline $\begin{array}{l}\text { B3Q } \\
\text { B2 }\end{array}$ & $\begin{array}{l}-1.25 \\
-1.08\end{array}$ & -5.90 & $\begin{array}{l}-1.88 \\
-1.34\end{array}$ & $\begin{array}{l}-8.36 \\
-9.36\end{array}$ & $\begin{array}{l}-1.90 \\
-1.07\end{array}$ & -5.93 & $\begin{array}{l}-2.14 \\
-2.07\end{array}$ & $\begin{array}{l}-12.48 \\
-12.27\end{array}$ & $\begin{array}{l}-1.26 \\
-1.04\end{array}$ & -2.48 & $\begin{array}{l}-1.56 \\
-1.08\end{array}$ & $\begin{array}{l}-8.02 \\
-3.67\end{array}$ \\
\hline Ref.: $\mathbf{B} 3 \mathbf{Q} C_{s}$ & -1.04 & & -1.05 & & -1.04 & & -1.04 & & -1.05 & & -1.05 & \\
\hline
\end{tabular}

könnte im Experiment Quasi-Planarität ${ }^{*}$ bestehen (vgl. Diskussion der (effektiven) Symmetrie in Kap. 4.1.1). Sind zwei leicht asymmetrische enantiomere Minimumsstrukturen über einen energetisch nur wenig höher liegenden $C_{s}$-symmetrischen Übergangszustand voneinander getrennt, kann eine auf der Zeitskala des Experiments effektiv planare und damit $C_{s}$-symmetrische Struktur vorliegen. Hieraus ergeben sich weitere mögliche Symmetriebetrachtungsvarianten, wie im Folgenden erläutert werden soll.

Als Maß für die Abweichung der erhaltenen Minimumsstrukturen (keine imaginären Moden) von perfekter Planarität (unter Berücksichtigung der Methyl-H-Atome) kann der Inertialdefekt $\Delta$ herangezogen werden, der sich aus den Hauptträgheitsmomenten $I_{A, B, C}$ berechnet:

$$
\Delta=I_{C}-I_{A}-I_{B}
$$

Bei vollständig planaren Strukturen beträgt der Inertialdefekt 0. Da die hier betrachteten Dimere aufgrund der Methyl-H-Atome jedoch nicht vollständig planar sind, ergeben sich auch für die $C_{s}$-symmetrischen Strukturen Inertialdefekte ungleich 0. Die Inertialdefekte der für die Symmetrie-Betrachtung relevanten, mit den quantenchemischen Rechenmethoden B3Q und B2 erhaltenen Minimumsstrukturen sind in Tabelle 5.9 zusammengefasst. Als Referenz für die kleinsten erreichbaren Inertialdefektbeträge sind in der untersten Tabellenzeile die $\Delta$-Werte für die auf Basis der Atomkoordinaten und diverser Diederwinkel (s. oben) als $C_{s}$-symmetrisch eingestuften, mit der B3Q-Methode ermittelten Übergangszustände (je 1 imaginäre Schwingungsmode, s. Tab. 5.8) aufgelistet. Diese ergeben sich $\mathrm{zu}-1.04 \cdot 10^{-46} \mathrm{~kg} \cdot \mathrm{m}^{2}$ bis $-1.05 \cdot 10^{-46} \mathrm{~kg} \cdot \mathrm{m}^{2}$. Einen vergleichbaren Wert weist das mit der B2-Methode optimierte $\mathrm{Me}_{\mathrm{ip}}$-Dimer von $\mathrm{MeOH}+4 \mathrm{FAP}$ auf. Die weiter oben bereits getroffene Zuordnung zur $C_{s}$-Punktgruppe wird durch den Inertialdefekt somit bestätigt. Doch auch die weiteren ip-Dimere besitzen mit $-1.07 \cdot 10^{-46} \mathrm{~kg} \cdot \mathrm{m}^{2} \mathrm{bis}-2.14 \cdot 10^{-46} \mathrm{~kg} \cdot \mathrm{m}^{2}$ betragsmäßig vergleichsweise kleine Inertialdefekte. Dies deutet auf nahezu $C_{s}$-symmetrische Strukturen hin. Die zu Vergleichszwecken ebenfalls abgedruckten, klar $C_{1}$-symmetrischen op-Dimere weisen hingegen deutlich stärker von 0 abweichende Werte auf.

*Im Sinne einer vereinfachten Formulierung werden hier und im Folgenden die vier stets außerhalb der Molekülebene gelegenen Methyl-H-Atome für die Verwendung der Begriffe „planar“ und „Planarität“ ignoriert, sofern nicht anders gekennzeichnet. 


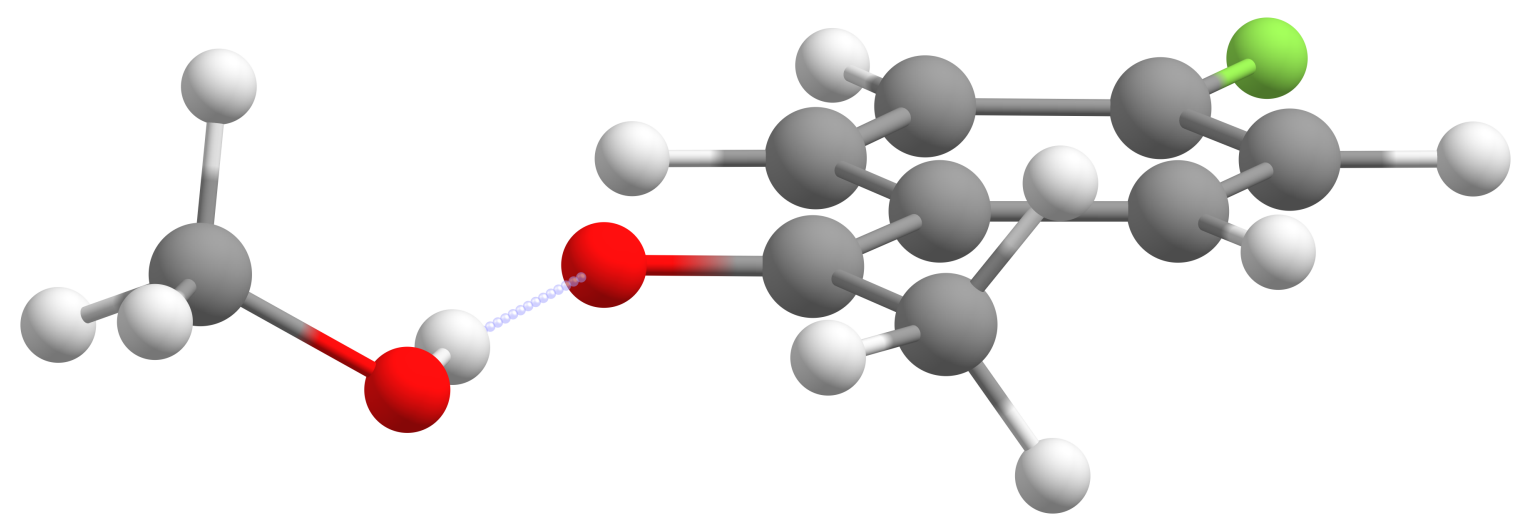

Abbildung 5.6: Die auf B3Q-Niveau optimierte $\mathrm{Me}_{\mathrm{op}}$-Dimerstruktur für $\mathrm{MeOH}+4 \mathrm{FAP}$. Die Struktur stellt einen Grenzfall zwischen $\mathrm{Me}_{\mathrm{ip}}$ - und $\mathrm{Me}_{\mathrm{op}}$-Dimertyp dar.

Eine Ausnahme bildet das auf B3Q-Niveau erhaltene $\mathrm{Me}_{\mathrm{op}}$-Dimer von $\mathrm{MeOH}+4 \mathrm{FAP}$ (s. Abbildung 5.6). Über den Inertialdefekt von $-2.48 \cdot 10^{-46} \mathrm{~kg} \cdot \mathrm{m}^{2} \mathrm{kann}$ dies als Grenzfall zwischen ip- und op-Dimertyp identifiziert werden.

Die kleinen Inertialdefektbeträge der ip-Dimere verdeutlichen die nur leichte Asymmetrie der erhaltenen Minimumsstrukturen und lassen die Vermutung zu, dass für alle ip-Dimere experimentell Planarität oder Quasi-Planarität vorliegen könnte. Ersteres wäre dann der Fall, wenn das „reale“ Minimum bei der $C_{s}$-symmetrischen Struktur liegt (wie für das $\mathrm{Me}_{\mathrm{ip}}$-Dimer von $\mathrm{MeOH}+4 \mathrm{FAP}$ von der B2-Methode auch vorhergesagt). Zweiteres ist möglich, wenn der Energieunterschied zwischen unplanaren Minima und planarem Sattelpunkt so gering ist, dass er z. B. durch die Nullpunktsenergie in der Umwandlungskoordinate überwunden werden kann (s. Kap. 4.1.1). Da bisher keine experimentellen (mikrowellenspektroskopischen) Daten zur Struktur und Symmetrie der hier betrachteten Dimere verfügbar sind, muss die Beurteilung hinsichtlich des möglichen Vorliegens $C_{s}$-symmetrischer Strukturen auf Basis der quantenchemischen Rechendaten erfolgen. Als strenges Kriterium für Quasi-Planarität kann hierbei der Vergleich des Energieunterschieds zwischen der $C_{s}$-symmetrischen Struktur und dem $C_{1}$-symmetrischen Minimum $\left(E_{\mathrm{r}}=E_{\mathrm{el}}\left(C_{s}\right)-E_{\mathrm{el}}\left(C_{1}\right)\right)$ mit dem Betrag $E_{\mathrm{Z}}$ der ZPVE der niederfrequentesten Planarisierungsmode des $C_{1}$-Minimums dienen. Ist $E_{\mathrm{r}} \leq E_{Z}$, kann Quasi-Planarität angenommen werden. In diesem Fall reicht die ZPVE $E_{\mathrm{Z}}$ aus, um den Energieunterschied $E_{\mathrm{r}}$ zu überwinden. Das Aufenthaltswahrscheinlichkeits-Maximum fällt damit (näherungsweise) auf die planare, $C_{s}$-symmetrische Struktur. Für zahlreiche der hier betrachteten Dimerstrukturen wurden mehrere Planarisierungsmoden in den quantenchemischen Rechnungen erhalten, sodass zumeist sogar mehr ZPVE zur Planarisierung zur Verfügung stehen sollte. Reicht bereits die ZPVE der niederfrequentesten Mode aus, um den Energieunterschied zur $C_{s^{-}}$ symmetrischen Struktur auszugleichen, spricht dies also deutlich für Quasi-Planarität.

In Tabelle 5.10 sind die relativen elektronischen Energien $E_{\mathrm{r}}$ den Schwingungsnullpunktsenergien $E_{Z}$ gegenübergestellt. Die berücksichtigten Symmetrisierungsmoden ent- 
Tabelle 5.10: Vergleich der elektronischen Energien $E_{\mathrm{r}}=E_{\mathrm{el}}\left(C_{s}\right)-E_{\mathrm{el}}\left(C_{1}\right)$ der $C_{s}$ - relativ zu den $C_{1}$-symmetrischen Strukturen mit den Schwingungsnullpunktsenergien $E_{\mathrm{Z}}$ der jeweils niederfrequentesten Symmetrisierungsmode $\operatorname{der} C_{1}$-symmetrischen Strukturen.

\begin{tabular}{|c|c|c|c|c|c|c|c|c|c|c|c|c|}
\hline \multirow{3}{*}{$\begin{array}{l}E \\
/ \mathrm{kJ} \cdot \mathrm{mol}^{-1}\end{array}$} & \multicolumn{4}{|c|}{$\mathrm{MeOH}+\mathrm{AP}$} & \multicolumn{4}{|c|}{$\mathrm{MeOH+2FAP}$} & \multicolumn{4}{|c|}{$\mathrm{MeOH}+4 \mathrm{FAP}$} \\
\hline & \multicolumn{2}{|c|}{$\mathrm{Me}_{\mathrm{ip}}$} & \multicolumn{2}{|c|}{$\mathrm{Ph}_{\text {ip }}$} & \multicolumn{2}{|c|}{$\mathrm{Me}_{\mathrm{ip}}$} & \multicolumn{2}{|c|}{$\mathrm{Ph}_{\text {ip }}$} & \multicolumn{2}{|c|}{$\mathrm{Me}_{\mathrm{ip}}$} & \multicolumn{2}{|c|}{$\mathrm{Ph}_{\text {ip }}$} \\
\hline & $E_{\mathrm{r}}$ & $E_{\mathrm{Z}}$ & $E_{\mathrm{r}}$ & $E_{\mathrm{Z}}$ & $E_{\mathrm{r}}$ & $E_{\mathrm{Z}}$ & $E_{\mathrm{r}}$ & $E_{\mathrm{Z}}$ & $E_{\mathrm{r}}$ & $E_{\mathrm{Z}}$ & $E_{\mathrm{r}}$ & $E_{\mathrm{Z}}$ \\
\hline B3Q & 0.02 & 0.03 & 0.22 & 0.09 & 0.10 & 0.02 & 0.36 & 0.04 & 0.01 & 0.01 & 0.19 & 0.08 \\
\hline B3Q+ & -0.06 & 0.03 & -0.08 & 0.09 & -0.14 & 0.02 & 0.04 & 0.04 & -0.08 & 0.01 & -0.04 & 0.08 \\
\hline B3Q+c & -0.04 & 0.03 & -0.15 & 0.09 & -0.15 & 0.02 & 0.03 & 0.04 & -0.07 & 0.01 & -0.01 & 0.08 \\
\hline B2 & & & 0.01 & 0.07 & & & 0.10 & 0.11 & -0.02 & 0.09 & 0.00 & 0.02 \\
\hline B2+ & & & -0.05 & 0.07 & & & 0.03 & 0.11 & -0.01 & 0.09 & -0.01 & 0.02 \\
\hline
\end{tabular}

sprechen dabei jeweils den niederfrequentesten in Tabelle 5.8 aufgeführten Moden der $C_{1}$-symmetrischen Dimere. Werden zunächst die in Tabelle 5.10 aufgelisteten Energien für die mit der B3Q-Methode optimierten Strukturen betrachtet, zeigt sich, dass auf dem reinen DFT-Niveau lediglich in zwei Fällen $\left(\mathrm{Me}_{\text {ip }}\right.$-Dimer von AP und $\left.4 \mathrm{FAP}\right)$ das strenge Kriterium für Quasi-Planarität $\left(E_{\mathrm{r}} \leq E_{\mathrm{Z}}\right)$ erfüllt ist. Ein deutlich anderes Bild ergibt sich jedoch, wenn die elektronischen Energien auf DLPNO-CCSD(T)-Niveau berechnet werden $(\mathbf{B} 3 \mathbf{Q}+, \mathbf{B} 3 \mathbf{Q}+\mathbf{c})$. Hierdurch werden die $C_{s}$-gegenüber den $C_{1}$-symmetrischen Strukturen so stark stabilisiert, dass erstere bis auf eine Ausnahme $\left(\mathrm{Ph}_{\mathrm{ip}}\right.$-Dimer von $\left.2 \mathrm{FAP}\right)$ sogar niedrigere Energien als letztere aufweisen (negative Vorzeichen in $E_{\mathrm{r}}$ ). Dies deutet darauf hin, dass es sich bei den leicht asymmetrischen ip-Minima um ein Artefakt der B3Q-Methode handelt und dass höhere Theorieniveaus hier zu $C_{s}$-symmetrischen Minima führen würden. Für die Ausnahme $\left(\mathrm{Ph}_{\mathrm{ip}}\right.$-Dimer von $\left.\mathrm{MeOH}+2 \mathrm{FAP}\right)$ ist die relative Energie $\operatorname{der} C_{s^{-}}$ symmetrischen Struktur auf B3Q+- und B3Q+c-Niveau zwar positiv, aber dennoch nicht größer als die ZPVE der Symmetrisierungsmode, sodass in allen Fällen die Bedingung für effektive $C_{s}$-Symmetrie erfüllt ist.

Diese Ergebnisse werden durch die B2-Methode im Wesentlichen bestätigt. Zwar konnten hier mit dem OrcA-Programmpaket für die $\mathrm{Me}_{\mathrm{ip}}$-Dimere von $\mathrm{MeOH}+\mathrm{AP}$ und $\mathrm{MeOH}+2 \mathrm{FAP}$ bisher keine $C_{s}$-symmetrischen Dimerstrukturen in den Geometrieoptimierungen erhalten werden, in den anderen Fällen ist jedoch bereits für die reine DFTMethode $E_{\mathrm{r}}$ kleiner als $E_{Z}$. Bei Berechnung der elektronischen Energie mit dem DLPNO$\operatorname{CCSD}(\mathrm{T})$-Verfahren $(\mathbf{B} 2+)$ werden die $C_{s}$-symmetrischen Strukturen nur noch wenig (max. $0.07 \mathrm{~kJ} \cdot \mathrm{mol}^{-1}$ ) gegenüber den $C_{1}$-Minima stabilisiert bzw. in einem Fall sogar leicht destabilisiert, was auf eine im Vergleich zur B3Q- geringere Verzerrung der B2Methode zu asymmetrischen Doppelminima hindeutet. Auch für B2 und B2+ ist im Ergebnis in allen Fällen die Bedingung für Quasi-Planarität erfüllt.

Angesichts dieser Daten scheint effektive $C_{S}$-Symmetrie für die ip-Dimere im Experiment somit ebenso möglich zu sein wie die streng formale Symmetrie auf Basis der in den Optimierungen erhaltenen Minimumsstrukturen. Eine Beurteilung der tatsächlich vor- 
Tabelle 5.11: Aus den beiden Symmetriebetrachtungsvarianten A (Sym. A) und B (Sym. B) für die verschiedenen Theorieniveaus resultierende Entartungsfaktorenverhältnisse $g_{\mathrm{Me}} / g_{\mathrm{Ph}}$ zwischen dem schwingungsnullpunktskorrigiert jeweils stabilsten Me- und Ph-Dimer. Unterschiede zwischen den beiden Symmetrie-Varianten sind fett hervorgehoben.

\begin{tabular}{|c|c|c|c|c|c|c|}
\hline \multirow[b]{2}{*}{$g_{\mathrm{Me}} / g_{\mathrm{Ph}}$} & \multicolumn{2}{|c|}{$\mathrm{MeOH}+\mathrm{AP}$} & \multicolumn{2}{|c|}{$\mathrm{MeOH}+2 \mathrm{FAP}$} & \multicolumn{2}{|c|}{$\mathrm{MeOH}+4 \mathrm{FAP}$} \\
\hline & Sym. A & Sym. B & Sym. A & Sym. B & Sym. A & Sym. B \\
\hline B3 & 1 & 1 & 1 & 1 & 1 & 1 \\
\hline B3+ & 1 & 1 & 1 & 1 & 1 & 1 \\
\hline $\mathrm{B} 3+\mathrm{c}$ & 1 & 1 & 1 & 1 & 1 & 1 \\
\hline $\mathrm{B} 3+\mathrm{cP}$ & 1 & 1 & 1 & 1 & 1 & 1 \\
\hline B3Q & 1 & 0.5 & 1 & 0.5 & 1 & 1 \\
\hline B3Q+ & 1 & 0.5 & 1 & 1 & 1 & 1 \\
\hline B3Q+c & 1 & 0.5 & 1 & 1 & 1 & 1 \\
\hline M6 & 1 & 1 & 1 & 1 & 1 & 1 \\
\hline M6+ & 1 & 1 & 1 & 1 & 1 & 1 \\
\hline B9 & 1 & 1 & 1 & 1 & 1 & 1 \\
\hline B9+ & 1 & 1 & 1 & 1 & 1 & 1 \\
\hline PB & 1 & 1 & 1 & 1 & 1 & 1 \\
\hline PB+ & 1 & 1 & 1 & 1 & 1 & 1 \\
\hline B2 & 1 & 1 & 1 & 1 & 0.5 & $\mathbf{1}$ \\
\hline B2+ & 1 & 1 & 1 & 1 & 0.5 & $\mathbf{1}$ \\
\hline
\end{tabular}

liegenden Symmetrie kann jedoch ohne zusätzliche, mikrowellenspektroskopische Daten nicht erfolgen, sodass für die folgende Auswertung zwei Symmetriebetrachtungsvarianten berücksichtigt werden sollen. In beiden Fällen werden die relevanten Daten (elektronische Energie, ZPVE, IR-Bandenstärken) der in den DFT-Rechnungen erhaltenen Minimumsstrukturen verwendet. In der weiter oben bereits vorgestellten Variante A („Sym. $\mathrm{A}^{\prime \prime}$ ) erfolgt die Bestimmung der Entartungsfaktorenverhältnisse $g_{\mathrm{Me}} / g_{\mathrm{Ph}}$ dabei nach formaler Symmetrie der Minimumsstrukturen, wobei die Punktgruppen dieser Strukturen auf Basis relevanter Winkel und Atomkoordinaten rigoros voneinander abgegrenzt werden. Variante B („Sym. B“) berücksichtigt die mögliche effektive $C_{s}$-Symmetrie der ipStrukturen auf der Zeitskala des Experiments und weist deshalb alle ip-Strukturen $\operatorname{der} C_{s}-$ Punktgruppe zu. Zwar ist durchaus auch denkbar, dass nur einige der ip-Dimere effektiv $C_{s}$-symmetrisch sind, im Rahmen dieser Arbeit können aber nicht alle möglichen sich daraus ergebenden Kombinationen berücksichtigt werden. Die beiden Varianten Sym. A und Sym. B sollten deshalb als (diskrete) Grenzfälle der im Experiment erwartbaren (kontinuierlich variablen) Entartungsverhältnisse angesehen werden.

Wie bereits erwähnt, werden für die Auswertung des Konformerengleichgewichts nur die jeweils schwingungsnullpunktskorrigiert stabilsten Dimerstrukturen einer Bindungs- 
seite (und damit auch deren Entartungsfaktorenverhältnisse) berücksichtigt. Für die beiden hier betrachteten Varianten Sym. A und Sym. B ergeben sich dabei die in Tabelle 5.11 aufgelisteten Verhältnisse $g_{\mathrm{Me}} / g_{\mathrm{Ph}}$. Da die op-Dimere in jedem Fall $C_{1}$-symmetrisch sein müssen, liegen nur dann Änderungen (fett gedruckt) vor, wenn ip-Dimerstrukturen in die Verhältnisse eingehen (für schwingungsnullpunktskorrigierte relative Energien s. Tab. 5.4), diese nach Sym. A und Sym. B unterschiedliche Punktgruppen aufweisen und sich die Unterschiede im Entartungsfaktorenverhältnis nicht wieder herauskürzen. Für diese Fälle ergeben sich in der folgenden Auswertung unterschiedliche Ergebnisse für die Varianten Sym. A und Sym. B.

\subsubsection{Benchmarks auf Basis des Me/Ph- Konformerengleichgewichts}

Das für die Benchmarks nötige Vorgehen wird in Kapitel 2.3.2 erläutert. Für die Wahl einer geeigneten Auswertemethode muss einerseits berücksichtigt werden, dass unterschiedliche Entartungsfaktorenverhältnisse von 0.5 und 1 eingehen, was beim Benchmarking über Boltzmann-Plots eine getrennte Betrachtung nötig machen würde. Die berechneten Bandenstärkenverhältnisse können aufgrund ihrer Robustheit andererseits aber für den Benchmark herangezogen werden. Der Vergleich mit den experimentellen Referenzdaten soll deshalb über die Bestimmung einer Konformationstemperatur $T_{\mathrm{c}, \mathrm{QM}}$, in die die berechneten Energieunterschiede, Bandenstärken- und Entartungsverhältnisse und die experimentell bestimmten Intensitätsverhältnisse zwischen $\mathrm{Me}$ - und $\mathrm{Ph}$-Dimer eingehen (s. Gl. 2.15 auf S. 26), erfolgen. Die erhaltenen Werte können dann mit den im Experiment vorliegenden Konformationstemperaturen $T_{\mathrm{c}, \exp }$ verglichen werden. Diese sind nicht exakt bekannt, müssen in jedem Fall aber zwischen der Düsentemperatur (hier Raumtemperatur, also ca. $300 \mathrm{~K}$ ) und der Rotationstemperatur in der Schlitzdüsen-Überschallexpansion (minimal ca. $10 \mathrm{~K}$ ) liegen. Der (engere) Erwartungsbereich für die Konformationstemperatur der Umwandlung zwischen Me- und Ph-Dimer in der Heliumexpansion hängt von der Barrierensituation entlang der Umwandlungskoordinate ab. In Rechnungen wurde diese Barriere für das $\mathrm{H}_{2} \mathrm{O}$-AP-Dimer zu $5.5 \mathrm{~kJ} \cdot \mathrm{mol}^{-1}$ (Umwandlung $\mathrm{Ph}$ - zu Me-Dimer, Theorieniveau B3LYP/6-31+G(d,p) $)^{[51]}$ und für das $\mathrm{MeOH}-\mathrm{AP}-D i m e r ~ \mathrm{zu} 5.8 \mathrm{~kJ} \cdot \mathrm{mol}^{-1}(\mathrm{er}-$ neut $\mathrm{Ph}$ zu Me, Theorieniveau: B3LYP-D3(BJ,ABC)/def2-TZVP) bestimmt. ${ }^{[52]}$ Ausgehend von diesen Werten sind hier etwas höhere Temperaturen als für die $\mathrm{O} / \mathrm{C}$-Waagensysteme zu erwarten, sodass der Erwartungsbereich auf $90 \pm 60 \mathrm{~K}$ abgeschätzt wird. ${ }^{[31,37,75,80]}$

Für ein gutes Abschneiden im Benchmark sollten die für eine quantenchemische Methode erhaltenen Werte $T_{\mathrm{c}, \mathrm{QM}}$ für die betrachteten Methanol-Acetophenon(derivat)-Kombinationen im physikalischen Bereich (10-300 K), für ein sehr gutes Abschneiden konsistent im Erwartungsbereich (30-150 K) für $T_{\mathrm{c}}$ liegen. Ergeben sich unphysikalische höhere Temperaturen $T_{\mathrm{c}, \mathrm{QM}}$, ist der berechnete Energieunterschied zu groß. Wird durch die Theorie eine falsche Bindungsstellenpräferenz vorhergesagt, resultieren ebenso unphysikalische negative Temperaturen. Eine detaillierte Beschreibung des Benchmarkings über 
die Bestimmung einer Konformationstemperatur findet sich in Kapitel 2.3.2.

In Abbildung 5.7 sind die Konformationstemperaturen für das $\mathrm{Me} / \mathrm{Ph}$-Gleichgewicht von $\mathrm{MeOD}+\mathrm{AP}$ gegen die entsprechenden Temperaturen für $\mathrm{MeOH}+\mathrm{AP}$ aufgetragen. In Teil (a) sind die Daten dabei inklusive der vollständigen auf dem experimentellen Fehler für das Intensitätsverhältnis beruhenden Fehlerbalken dargestellt. Der Erwartungsbereich für die Konformationstemperatur ist dabei weiß, der physikalische Bereich hellgrau eingefärbt. Aufgrund des nur ungenau bestimmbaren Intensitätsverhältnisses (s. Abb. 5.4) sind die (äußerst asymmetrischen, s. Kap. 2.3.2) Fehlerbalken für MeOD+AP sehr groß, während für $\mathrm{MeOH}+\mathrm{AP}$ deutlich kleinere Wertebereiche für die Konformationstemperaturen erhalten werden. Im Abbildungsteil (b) ist der Bereich, in dem sich die Datenpunkte befinden, im Detail gezeigt. Bei einer akkuraten Beschreibung der Schwingungsnullpunktseffekte durch eine Methode sollten sich für $\mathrm{MeOH}$ und MeOD dieselben Konformationstemperaturen ergeben, sodass die Datenpunkte auf der Winkelhalbierenden (schwarze Linie) liegen. Aufgrund der großen experimentellen Unbestimmtheit für MeOD+AP kann jedoch nicht beurteilt werden, welche Methoden in dieser Hinsicht erfolgreich sind und welche nicht. Im Rahmen der großen Fehlerbalken liegen für MeOD+AP zudem nahezu alle Datenpunkte im weißen Erwartungsbereich. Lediglich die M6+-Methode sagt eine nicht zum Experiment passende $\mathrm{Ph}$-Bindungsstellenpräferenz vorher, sodass eine unphysikalische negative Konformationstemperatur resultiert. Hier wird deutlich, dass große experimentelle Unsicherheiten ein empfindliches Benchmarking verhindern.

Für $\mathrm{MeOH}+\mathrm{AP}$ zeigt sich aufgrund der deutlich kleineren Fehlerbalken eine etwas bessere Diskrimination zwischen den Ergebnissen der verschiedenen Rechenmethoden. Während erneut nur der M6+-Datenpunkt im dunkelgrauen unphysikalischen Bereich liegt, fallen für $\mathrm{MeOH}+\mathrm{AP}$ zwei weitere Datenpunkte nicht mehr in den weißen Erwartungsbereich (B3+, B3Q+c für Sym. A). Die Symmetriebetrachtungsvariante Sym. B führt für B3Q+c zu einer Verbesserung in der Beschreibung des Konformerengleichgewichts, sodass die Konformationstemperatur im weißen Zielbereich liegt. Für B3Q und B3Q+ fallen die Datenpunkte unabhängig von der Symmetriebetrachtungsvariante in den Erwartungsbereich. In diesem liegen im Gegensatz zu M6+ auch die Konformationstemperaturen für das unkorrigierte M6-Niveau, sowohl für $\mathrm{MeOH}$ als auch für MeOD. Die Berechnung der elektronischen Energie auf dem höheren DLPNO-CCSD(T)/aVQZNiveau führt im Fall des M06-2X-Funktionals also zu einer schlechteren Beschreibung der Waagensituation. Dies deutet darauf hin, dass das gute Abschneiden des „reinen“ Funktionals zumindest teilweise auf Fehlerkompensation beruht. Ähnliches, wenn auch weniger stark ausgeprägt, gilt für die B3- und PB-Methode, deren MeOH-Ergebnisse mit den elektronischen DLPNO-CCSD(T)-Energien tendenziell weiter zum Rand des Erwartungsbereich bzw. aus diesem heraus rücken.

Für die beiden am AP teildeuterierten Isotopologe von $\mathrm{MeOH}+\mathrm{AP}(\mathrm{MeOH}+\mathrm{APd} 3$ und $\mathrm{MeOH}+\mathrm{APd} 5$ ) ergeben sich entsprechend ähnliche Ergebnisse (s. Abb. 5.8). Für beide APIsotopologe weist erneut nur M6+ aufgrund der falsch vorhergesagten Ph-Bindungsstellenpräferenz negative Konformationstemperaturen auf. Eine positive, aber dennoch unphysikalisch niedrige Konformationstemperatur von weniger als $10 \mathrm{~K}$ ergibt sich für den 


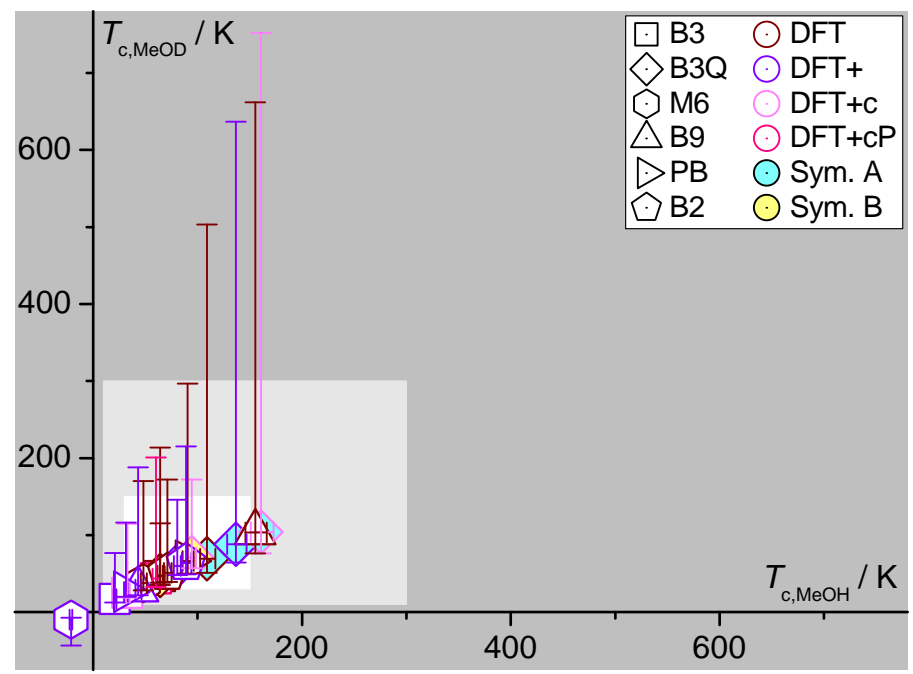

(a)

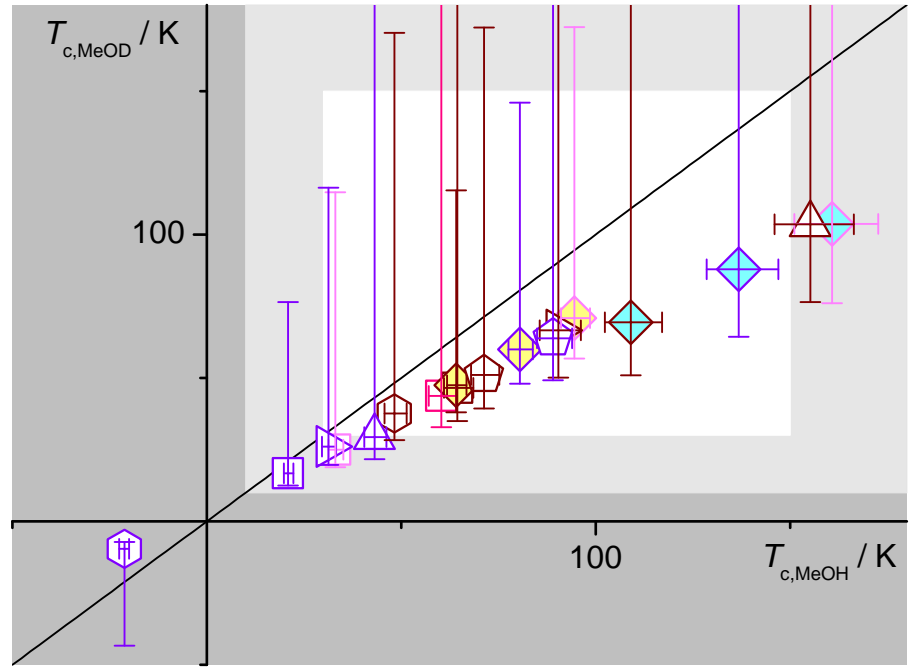

(b)

Abbildung 5.7: Korrelation der Konformationstemperaturen für die Aggregation von $\mathrm{MeOH}$ (Abszisse) und MeOD (Ordinate) mit AP. Die Fehlerbalken basieren auf dem experimentellen Fehler für das Intensitätsverhältnis. Für die Konformerenumwandlung werden Temperaturen zwischen 30 und $150 \mathrm{~K}$ erwartet (weißer Bereich). Temperaturen zwischen 10 und $300 \mathrm{~K}$ sind physikalisch (hellgrau) und unterhalb von $10 \mathrm{~K}$ bzw. oberhalb von $300 \mathrm{~K}$ unphysikalisch (dunkelgrau). Über die Symbolformen können die für die Geometrieoptimierung und Berechnung der ZPVE herangezogenen DFT-Methoden unterschieden werden, über die Symbolfarben die für die Berechnung der elektronischen Energien verwendeten Theorieniveaus und über die Symbolfüllungen die Symmetriebetrachtungsvarianten (weiße Füllungen: keine Unterschiede zwischen Sym. A und Sym. B). Als Referenz ist die Winkelhalbierende $T_{\mathrm{c}, \mathrm{MeOD}}=T_{\mathrm{c}, \mathrm{MeOH}}$ (schwarz) eingezeichnet. (a) Gesamtübersicht, (b) Detailansicht des mit Datenpunkten belegten Bereichs. 


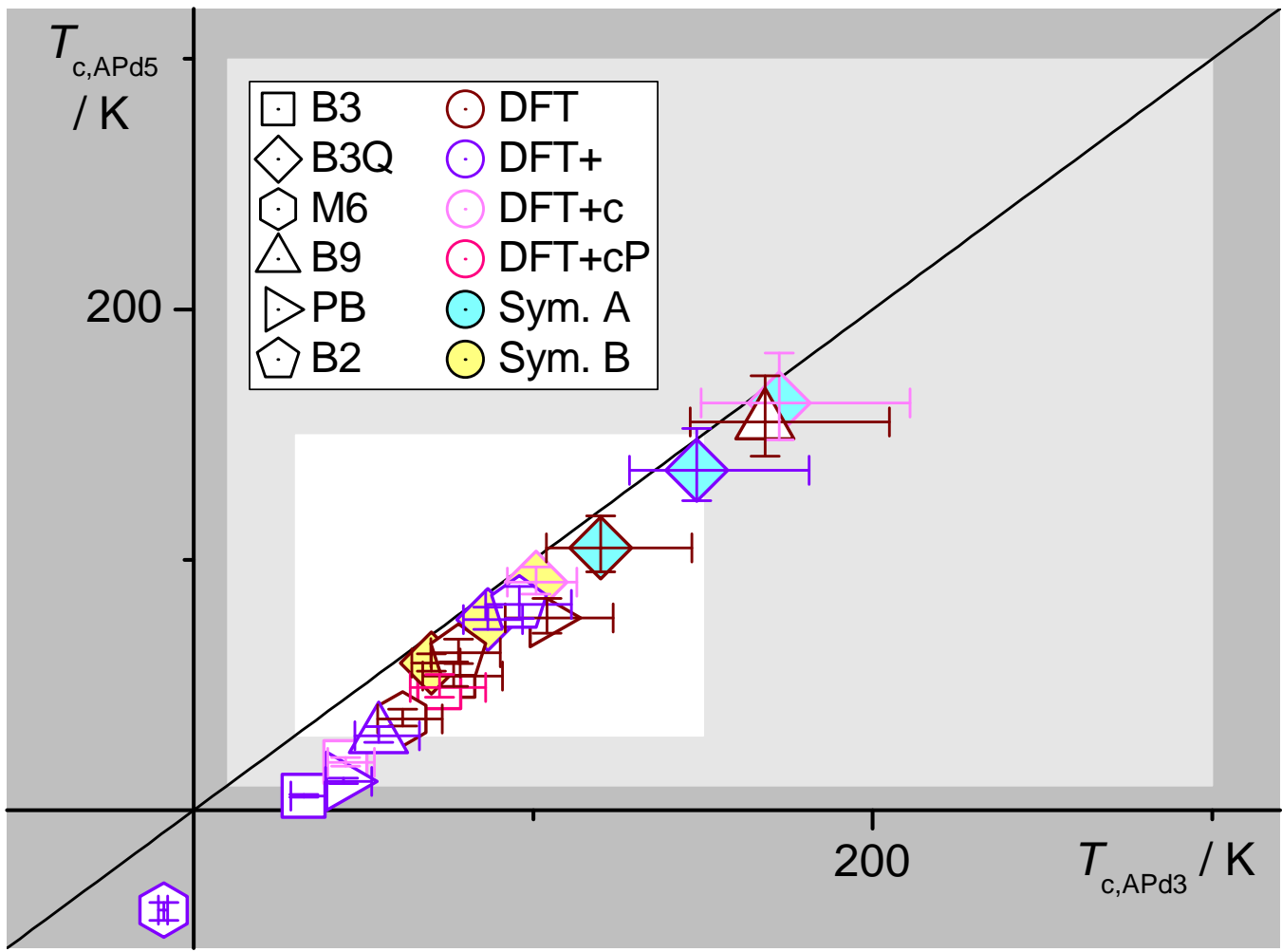

Abbildung 5.8: Korrelation der Konformationstemperaturen analog zu Abb. 5.7, hier aber für die Aggregation von $\mathrm{MeOH}$ mit APd3 (Abszisse) und APd5 (Ordinate).

$\mathrm{MeOH}+\mathrm{APd} 5$-Wert der B3+-Methode, wohingegen die zugehörige $\mathrm{MeOH}+\mathrm{APd} 3-$ Konformationstemperatur im Erwartungsbereich liegt. Die $\mathrm{MeOH}+\mathrm{APd} 5-W e r t e$ von $\mathbf{B} 3+\mathbf{c}$ und PB+ liegen unterhalb des Erwartungsbereichs, dabei aber im physikalischen Bereich. Alle weiteren $T_{\mathrm{c}}$-Werte der betrachteten Methoden fallen im Rahmen der experimentellen Fehlerbalken für beide Isotopologe in den weißen Erwartungsbereich. Die Datenpunkte von B3Q+ und B3Q+c befinden sich für die Symmetriebetrachtungsvariante Sym. A jedoch am oberen Rand bzw. nur noch im Rahmen der Fehlerbalken überhaupt im Erwartungsbereich. Mit Sym. B werden dagegen Konformationstemperaturen mittig im weißen Zielbereich erhalten. Die ermittelten Konformationstemperaturen sind für alle Methoden für $\mathrm{MeOH}+\mathrm{APd} 5$ kleiner und für $\mathrm{MeOH}+\mathrm{APd} 3$ größer als für das undeuterierte Isotopolog $(\mathrm{MeOH}+\mathrm{AP})$, für B3Q+c jedoch nur für Sym. B. Im Rahmen der Fehlerbalken sind die Unterschiede zudem nicht überall statistisch signifikant. Diese systematischen Abweichungen bedeuten (zumindest in den Fällen signifikanter Unterschiede), dass der Effekt der Deuterierung auf das $\mathrm{Me} / \mathrm{Ph}$-Konformerengleichgewicht durch die verwendeten $\mathrm{Me}-$ thoden im Rahmen der ZPVE-Berechnungen nicht treffend wiedergegeben wird. Ob dies auf Schwächen in allen verwendeten Methoden hindeutet oder schlicht der Vernachlässigung anharmonischer Effekte durch die Schwingungsrechnungen in doppelt-harmonischer Näherung geschuldet ist, müsste in weiteren Analysen, z. B. unter Zuhilfenahme 


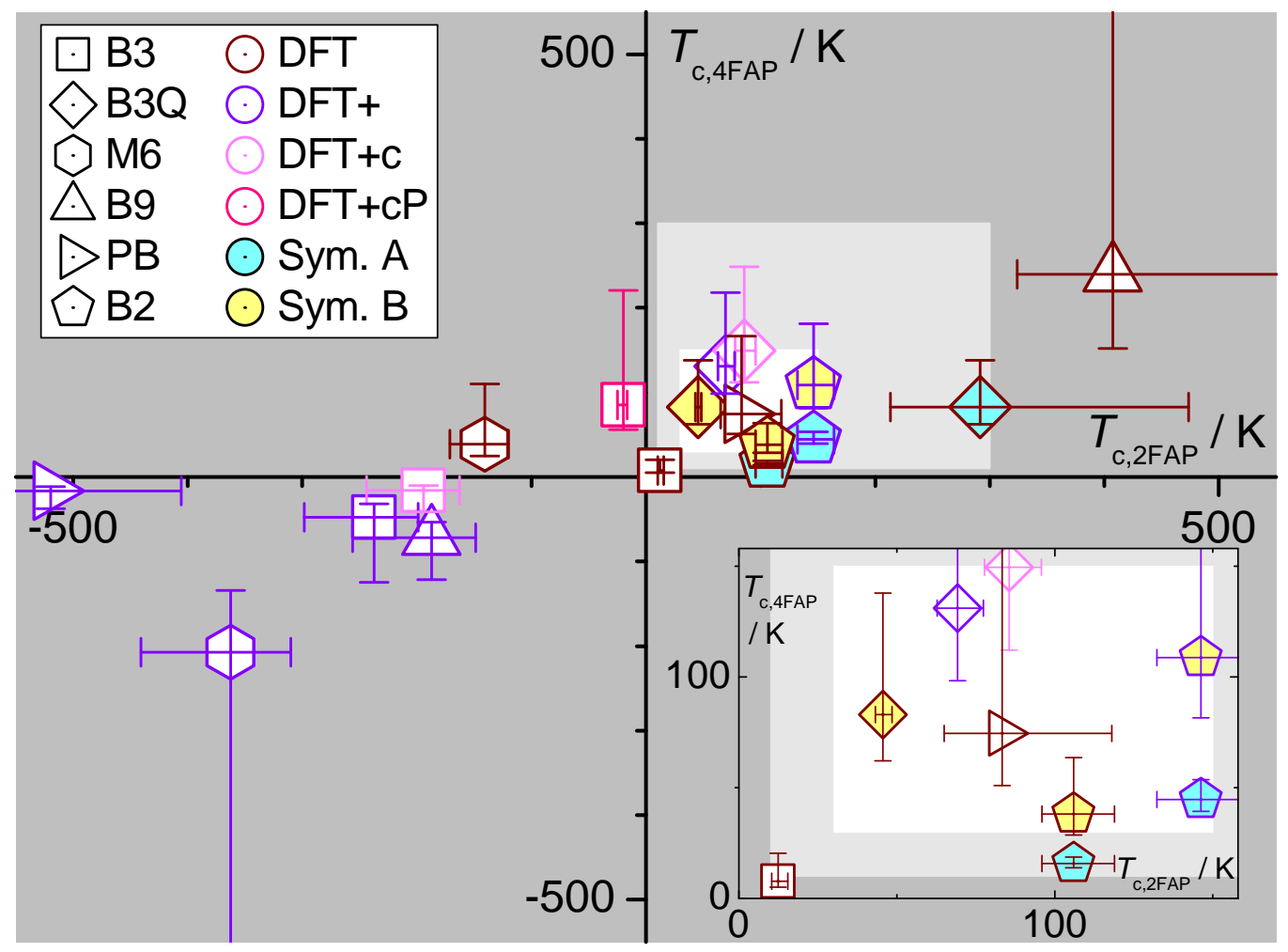

Abbildung 5.9: Korrelation der Konformationstemperaturen analog zu Abb. 5.7, hier aber für die Aggregation von $\mathrm{MeOH}$ mit 2FAP (Abszisse) und 4FAP (Ordinate). Im unteren rechten Quadranten der Abbildung ist der Bereich zwischen 0 und $160 \mathrm{~K}$ noch einmal im Detail dargestellt.

anharmonischer Schwingungsrechnungen, untersucht werden. Eine Unterschätzung des experimentellen Integrationsfehlers ist dagegen unwahrscheinlich, da sich die Integralverhältnisse (vgl. Abb. 5.5, Tab. 5.2) nicht signifikant unterscheiden.

Eine deutlich stärkere Streuung der erhaltenen Konformationstemperaturen ergibt sich für die Betrachtung der fluorierten Acetophenonderivate (s. Abb. 5.9). Nur vier (B3Q+, B3Q+c, PB, B2+) der insgesamt 15 quantenchemischen Methoden liegen unabhängig von den Symmetrie-Varianten und zwei weitere ausschließlich für Sym. B (B3Q, B2) für beide Derivate im weißen Erwartungsbereich. Eine weitere Methode (B3) und die Sym.-AVarianten von B3Q und B2 liefern Konformationstemperaturen im hellgrauen physikalischen Bereich. Vier der sieben genannten Methoden basieren auf dem dispersionskorrigierten B3LYP-Funktional. Für alle weiteren Methoden ist mindestens eine der erhaltenen Konformationstemperaturen unphysikalisch groß (B9) oder negativ (restliche Methoden). Auffällig sind auch die großen Unterschiede in den Konformationstemperaturen, die sich insbesondere für B9, PB, B3 und M6 ergeben, wenn die elektronische Energie mit der DLPNO-CCSD(T)-Methode berechnet wird („+"). So verschiebt sich der Datenpunkt von B9 von unphysikalischen Temperaturen oberhalb der Düsentemperatur zu ebenso unphy- 
sikalisch negativen Temperaturen (B9+). Auch zwischen B3 und B3+ bzw. M6 und M6+ ergibt sich ein Vorzeichenwechsel in $T_{c}$, im Fall von $\mathbf{M 6}[+]$ aber nur für MeOH+4FAP. Für PB+ wird für MeOH+2FAP eine besonders stark negative Konformationstemperatur erhalten, wohingegen PB Konformationstemperaturen im Erwartungsbereich liefert. Das schlechtere Abschneiden bei Berechnung der elektronischen Energie auf einem höheren Theorieniveau verdeutlicht, wie stark die PB-Methode von Fehlerkompensation profitiert. Ein vergleichbares Verhalten ist für diese Methode auch für $\mathrm{MeOH}+\mathrm{AP}$ und seine Isotopologe zu beobachten, wenn auch weniger stark ausgeprägt.

Wird die Kernelektronenkorrelation für die Berechnung der elektronischen Energie auf dem DLPNO-CCSD(T)-Niveau berücksichtigt („+c“), führt dies im Vergleich zur Berechnung bei eingefrorenen Kernelektronen („+") für die betrachteten Konformerengleichgewichte nicht systematisch zu realistischeren Konformationstemperaturen. Der deutlich erhöhte Rechenaufwand scheint also zumindest für die hier untersuchten Systeme nicht lohnenswert zu sein. Die Verwendung der Pipek-Mezey- anstelle der Foster-Boys-Lokalisierungsmethode in den DLPNO-CCSD(T)-Einzelpunktrechnungen auf den B3-Strukturen resultiert hingegen in einer tendenziellen Verbesserung der erhaltenen Konformationstemperaturen, wobei sich für $\mathrm{MeOH}+2 \mathrm{FAP}$ dennoch eine negative und damit unphysikalische Konformationstemperatur ergibt.

Für das B3LYP-D3-Funktional können zudem die unterschiedlich großen Basissätze TZ und QZ verglichen werden. Tendenziell ergeben sich mit dem größeren Basissatz dabei bessere Ergebnisse $(\mathbf{B} 3 \mathbf{Q}[+,+\mathbf{c}])$, insbesondere für Sym. B und bei Berechnung der elektronischen Energien auf DLPNO-CCSD(T)-Niveau. Besonders deutlich wird dies für Me$\mathrm{OH}+2 \mathrm{FAP}$, für das alle B3-Varianten Temperaturen außerhalb des Erwartungs- (B3) bzw. des physikalischen Bereichs $(\mathbf{B} 3+, \mathbf{B} 3+\mathbf{c}, \mathbf{B} 3+\mathbf{c P})$ liefern, B3Q+, B3Q+c und B3Q (letzteres nur für Sym. B) jedoch im Erwartungsbereich liegen.

Dies kann zumindest zum Teil auf die unterschiedlichen, in den Geometrieoptimierungen erhaltenen Strukturen zurückgeführt werden. Da für alle DFT-Methoden, mit denen Strukturoptimierungen durchgeführt wurden, elektronische Energien auf dem DLPNOCCSD(T)/aVQZ-Niveau („+") berechnet wurden, kann die Stabilität der erhaltenen Strukturen auf einer gemeinsamen Basis miteinander verglichen werden. Auch wenn dies nicht zwingend die „realen“ Stabilitäten der ermittelten Strukturen widerspiegelt, sollte die Analyse anhand der wellenfunktionsbasierten Korrelationsmethode zumindest eine grobe Beurteilung ermöglichen.

Die auf dem DLPNO-CCSD(T)/aVQZ-Niveau ermittelten elektronischen Energien für die $\mathrm{Me}$ - und $\mathrm{Ph}$-Dimere der verschiedenen Methanol-Acetophenon(derivat)-Kombinationen sind in Abbildung 5.10 relativ zur entsprechenden Energie der jeweils stabilsten Struktur einer $\mathrm{MeOH}$-Acetophenon(derivat)-Kombination dargestellt. Mit B2 findet das höchste für die Optimierungen verwendete Theorieniveau auch in allen Fällen die elektronisch stabilsten Strukturen, gefolgt von B3Q, dessen Strukturen bei etwa 0.2-0.3 kJ $\cdot \mathrm{mol}^{-1}$ höheren Energien liegen. Mit dem kleineren TZ-Basissatz (B3) werden mit dem B3LYP-D3-Funktional ähnlich stabile Strukturen wie mit dem größeren QZBasissatz (B3Q) erhalten, wenn für beide Basissatzgrößen op die niederenergetischste 


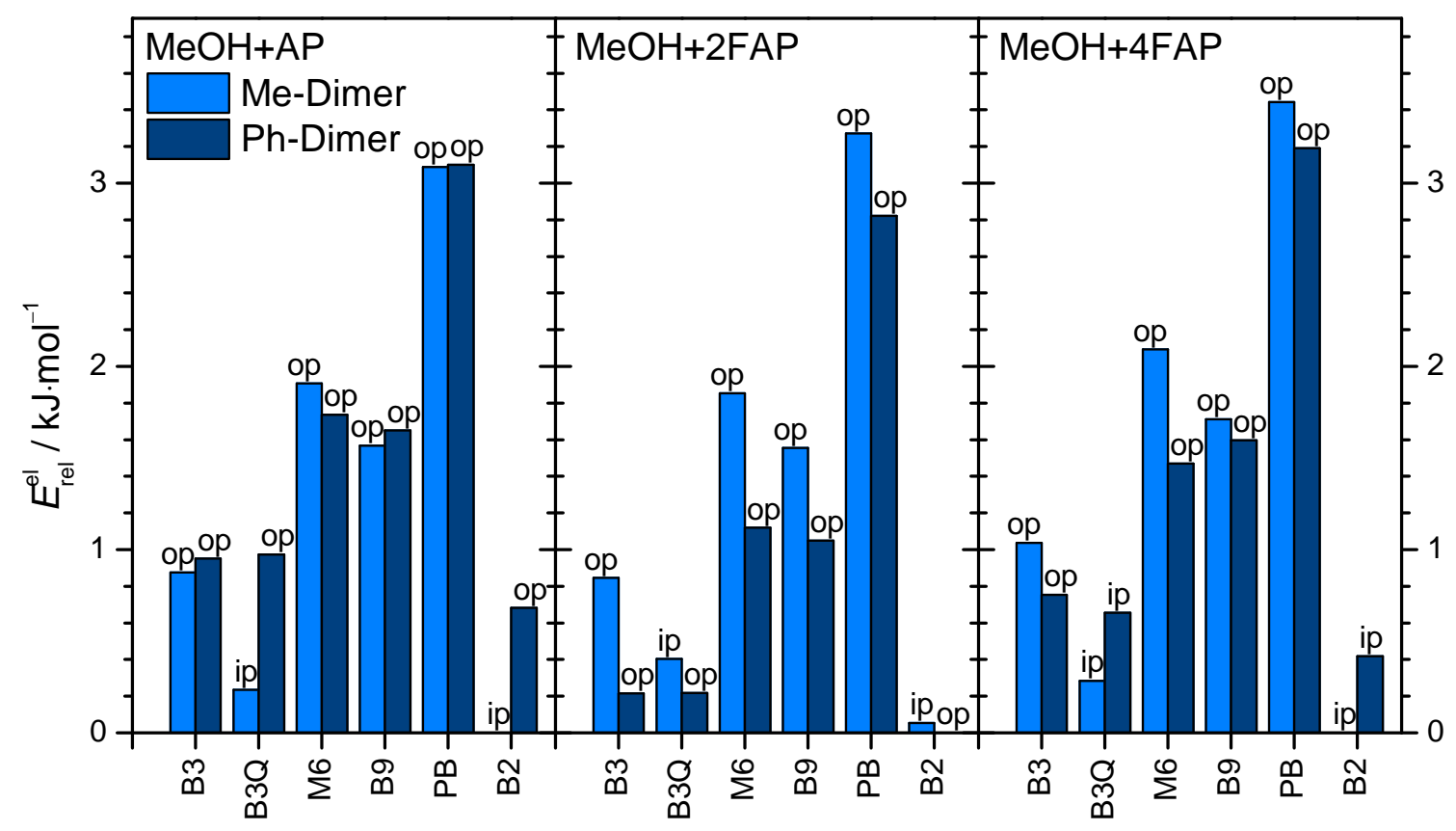

Abbildung 5.10: Auf dem DLPNO-CCSD(T)/aVQZ-Niveau berechnete elektronische Energien der mit den entlang der Abszisse angegebenen Methoden optimierten Strukturen, jeweils relativ zur niederenergetischsten Struktur der Methanol-Acetophenon(derivat)-Kombination. Für den Vergleich werden nur die stabilsten Me- (hellblau) bzw. Ph-Minimumsstrukturen (dunkelblau) jeder Methode berücksichtigt, deren Klassen (ip oder op) angegeben sind.

Strukturklasse einer Bindungsseite darstellt. Ist mit dem QZ-Basissatz ein ip-Dimer das Minimum, ergeben sich insbesondere für die Me-Dimere deutlich größere Unterschiede von bis zu $0.8 \mathrm{~kJ} \cdot \mathrm{mol}^{-1}(\mathrm{MeOH}+4 \mathrm{FAP})$, da ip-Strukturen für den kleineren TZ-Basissatz keine lokalen Minima darzustellen scheinen (s. Kap. 5.3.1). Auch für die weiteren Methoden (M6, B9, PB), die kleine Basissätze von maximal Triple- $\zeta$-Qualität verwenden, ergeben sich, wie bereits erwähnt, ausschließlich op-Strukturen. Die mit M6 und B9 erhaltenen Strukturen weisen dabei vergleichbare relative Energien auf, die etwa $1.0-1.2 \mathrm{~kJ} \cdot \mathrm{mol}^{-1}$ (Ph-Dimere) bzw. 1.5-2.1 $\mathrm{kJ} \cdot \mathrm{mol}^{-1}$ (Me-Dimere) höher als die der entsprechenden B2-Strukturen sind. Die B9- ermittelt somit in weniger als 1/10 der Rechenzeit vergleichbar gute Strukturen wie die M6-Methode. Die energetisch höchstliegenden Minimumsstrukturen werden mit der PB-Methode erhalten. Diese sind auf dem DLPNO-CCSD(T)/aVQZ-Niveau etwa 2.4-2.8 $\mathrm{kJ} \cdot \mathrm{mol}^{-1}$ (Ph-Dimere) bzw. $3.1-3.4 \mathrm{~kJ} \cdot \mathrm{mol}^{-1}$ (Me-Dimere) weniger stabil als die B2-Strukturen.

Zusammenfassend liefern nur drei (B3Q+, PB, B2+) der insgesamt 15 betrachteten Methoden Konformationstemperaturen, die sich für alle untersuchten Derivate und Isotopologe des Methanol-Acetophenon-Gleichgewichts für beide Symmetrie-BetrachtungsVarianten im Erwartungsbereich zwischen 30 und $150 \mathrm{~K}$ befinden. B3Q, B3Q+c und B2 liegen nur für Sym. B stets im Erwartungsbereich, wohingegen mit Sym. A jeweils für 
mindestens eine der Methanol-Acetophenon(derivat)-Kombinationen lediglich der physikalische Bereich erreicht wird. B3 weist ebenfalls durchgängig physikalische Konformationstemperaturen auf, die teilweise jedoch außerhalb des Erwartungsbereichs liegen. Für alle weiteren Methoden treten für mindestens eins der betrachteten $\mathrm{Me} / \mathrm{Ph}$-Konformerengleichgewichte unphysikalische Konformationstemperaturen auf. Insbesondere die fluorierten Acetophenonderivate ermöglichen eine Unterscheidung erfolgreicher von weniger erfolgreichen Methoden. Das unerwartet gute Abschneiden der günstigen PB-Methode kann auf Fehlerkompensation zurückgeführt werden. Durch die hohen relativen Energien der erhaltenen PB-Minimumsstrukturen auf DLPNO-CCSD(T)-Niveau (s. Abb. 5.10) konnten Schwächen in der Geometrieoptimierung aufgedeckt werden. Die deutlichen Änderungen in den Energiedifferenzen $\Delta E^{\mathrm{el}}$ bei Berechnung der elektronischen Energien auf dem Coupled-Cluster-Niveau (vgl. PB mit PB+ in Tab. 5.5) geben zudem Hinweise auf weitere Schwächen in der Beschreibung der elektronischen Energie. Diese Fehler (und mögliche weitere Fehler in der doppelt-harmonischen Berechnung der ZPVE) gleichen sich für die „reine“ PB-Methode für die hier betrachteten $\mathrm{Me} / \mathrm{Ph}$-Konformerengleichgewichte anscheinend hervorragend aus.

Ergeben sich für die Symmetriebetrachtungsvarianten Sym. A und Sym. B unterschiedliche Entartungsfaktorenverhältnisse und damit auch unterschiedliche Konformationstemperaturen, werden mit Sym. B für alle betroffenen Methoden in allen Fällen gleich gute oder bessere Ergebnisse als mit Sym. A erhalten. Dies kann als Indiz dafür angesehen werden, dass, wie in Sym. B angenommen, in der Tat für alle ip-Dimere tatsächliche oder effektive $C_{s}$-Symmetrie vorliegt. Klare experimentelle Belege für diese These fehlen jedoch, könnten aber z. B. über Jet-mikrowellenspektroskopische Untersuchungen ${ }^{[51,290]}$ gewonnen werden.

Das Ziel, ein Waagensystem zu finden, das einen möglichst direkten Benchmark elektronischer Energien ermöglicht, wurde nur zum Teil erreicht. Durch die gleichartigen $\mathrm{OH}$... O-Wasserstoffbrückenbindungen zu beiden Bindungsseiten wird zwar einerseits im Gegensatz zu den O/C-Waagen keine Bindungsstelle systematisch in der ZPVE bevorzugt, wie die in den Rechnungen erhaltenen $\Delta Z$-Werte verdeutlichen. Zudem sind die $\Delta Z$-Korrekturen mit maximal $0.6 \mathrm{~kJ} \cdot \mathrm{mol}^{-1}$ deutlich kleiner als für die $\mathrm{O} / \mathrm{C}-W a a g e n$. Andererseits ergeben sich in einigen Fällen dennoch Wechsel der Bindungsstellenpräferenz durch die Berücksichtigung der ZPVE, sodass diese entscheidenden Einfluss auf das Abschneiden der betroffenen Methoden im Benchmark nimmt.

Insgesamt lassen sich die B2- und B3Q-Methoden als klare Gewinner des Relativenergie-Benchmarks bezeichnen, da diese Methoden sowohl mit als auch ohne DLPNO$\operatorname{CCSD}(\mathrm{T})$-Korrektur zumindest bei einer Quasiplanaritätsbetrachtung des ip-Schweratomgerüsts durchgängig im Erwartungsbereich für alle untersuchten Spezies liegen und auch die tiefsten absoluten DLPNO-CCSD(T)-Energien liefern. Sie unterscheiden sich allerdings in der energetischen Robustheit ihrer ip/op-Strukturvorhersagen, in denen B2 nach Nullpunktskorrektur systematisch ip-Strukturen bevorzugt, während B3Q dies auf der Phenylseite nur in einem Fall tut. Solche energetisch subtilen, aber strukturell signifikanten Unterschiede kann neben der (ausstehenden) Mikrowellenspektroskopie 
Tabelle 5.12: Auf den verschiedenen DFT-Niveaus berechnete Rotverschiebungen $\Delta \omega_{\mathrm{OH}}$ der Methanol-Acetophenon(derivat)-Dimere für die unterschiedlichen Strukturtypen. Die schwingungsnullpunktskorrigiert jeweils stabilste Struktur einer Bindungsseite (s. Tab. 5.4) ist fett hervorgehoben. Wurde ein Strukturtyp mit einer Rechenmethode nicht als Minimum gefunden, bleibt das Feld leer.

\begin{tabular}{|c|c|c|c|c|c|c|c|c|c|c|c|c|}
\hline \multirow{2}{*}{$\begin{array}{l}\Delta \omega_{\mathrm{OH}} \\
/ \mathrm{cm}^{-1}\end{array}$} & \multicolumn{4}{|c|}{ MeOH+AP } & \multicolumn{4}{|c|}{$\mathrm{MeOH}+2 \mathrm{AP}$} & \multicolumn{4}{|c|}{$\mathrm{MeOH}+4 \mathrm{AP}$} \\
\hline & $\mathrm{Me}_{\mathrm{ip}}$ & $\mathrm{Me}_{\mathrm{op}}$ & $\mathrm{Ph}_{\mathrm{ip}}$ & $\mathrm{Ph}_{\mathrm{op}}$ & $\mathrm{Me}_{\mathrm{ip}}$ & $\mathrm{Me}_{\mathrm{op}}$ & $\mathrm{Ph}_{\mathrm{ip}}$ & $\mathrm{Ph}_{\mathrm{op}}$ & $\mathrm{Me}_{\mathrm{ip}}$ & $\mathrm{Me}_{\mathrm{op}}$ & $\mathrm{Ph}_{\mathrm{ip}}$ & $\mathrm{Ph}_{\mathrm{op}}$ \\
\hline B3 & & 191 & & 145 & & 176 & & 130 & & 186 & & 145 \\
\hline B3Q & 208 & 200 & 171 & 153 & 192 & 186 & 153 & 142 & 203 & 202 & 168 & 153 \\
\hline M6 & & 133 & & 93 & & 122 & & 84 & & 129 & & 91 \\
\hline B9 & & 201 & & 144 & & 183 & & 129 & & 198 & & 146 \\
\hline PB & & 113 & & 76 & & 99 & & 60 & & 109 & & 75 \\
\hline B2 & 205 & & 163 & 159 & 189 & & 149 & 143 & 199 & & 160 & 158 \\
\hline
\end{tabular}

gegebenenfalls auch die IR-Trendanalyse beleuchten, die im folgenden Unterkapitel behandelt wird.

\subsection{Auswertung IR-spektroskopischer Parameter}

Die in den Geometrieoptimierungen auf den verschiedenen quantenchemischen Theorieniveaus erhaltenen Dimer-Strukturen weisen verschiedene $\mathrm{C}=\mathrm{O} \cdots \mathrm{H}$-Bindungswinkel $\alpha$ und $\mathrm{C}-\mathrm{C}=\mathrm{O} \cdots \mathrm{H}$-Diederwinkel $\tau$ (s. Abb. 5.1) auf. In der bestehenden Studie der Dimere der auf Alkylseite substituierten Acetophenonderivate mit verschiedenen Alkoholen ergab sich dabei eine ausgeprägte Abhängigkeit der berechneten $\mathrm{OH}$-StreckschwingungsRotverschiebungen $\Delta \omega_{\mathrm{OH}}$, theor von diesen beiden Winkeln, insbesondere für die voluminöseren Donoren und Akzeptoren. ${ }^{[52]}$ Im Folgenden soll untersucht werden, ob auch für die kompakteren hier betrachteten Methanol-Acetophenon(derivat)-Dimere eine solche Korrelation besteht.

Dazu sind in Tabelle 5.12 zunächst die berechneten Rotverschiebungen $\Delta \omega_{\mathrm{OH}}$ für die verschiedenen DFT-Niveaus, auf denen Schwingungsrechnungen durchgeführt wurden, getrennt nach den Strukturtypen $\mathrm{Me}_{\mathrm{ip}}, \mathrm{Me}_{\mathrm{op}}, \mathrm{Ph}_{\mathrm{ip}}$ und $\mathrm{Ph}_{\mathrm{op}}$ dargestellt. Wie bereits beschrieben, sind die Rotverschiebungen für die Me-Seite dabei konsistent größer als für die $\mathrm{Ph}$-Seite. Existieren für eine Bindungsseite eines Methanol-Acetophenon(derivat)-Dimers sowohl ip- als auch op-Minima, weisen die ip-Strukturen stets die größeren Rotverschiebungen und somit die stärkeren Wasserstoffbrückenbindungen auf. Die B3Q-Methode stuft in zwei Fällen (Ph-Dimere von $\mathrm{MeOH}+\mathrm{AP}$ und $\mathrm{MeOH}+2 \mathrm{FAP}$ ) dennoch den op-Typen als schwingungsnullpunktskorrigiert stabilere Dimerstruktur (fettgedruckte Werte) ein. Für die folgende Auswertung werden, soweit nicht anders angegeben, jeweils nur die Werte für die stabilsten Strukturen einer Bindungsseite herangezogen.

In Abbildung 5.11 sind die berechneten Rotverschiebungen dieser Strukturen gegen die Winkel $\alpha$ (Teil (a)) bzw. $\tau$ (Teil (b)) aufgetragen. Da sich für die an der AP-Molekülebene gespiegelten Enantiomere $\operatorname{der} C_{1}$-symmetrischen Strukturen unterschiedliche Vorzeichen 


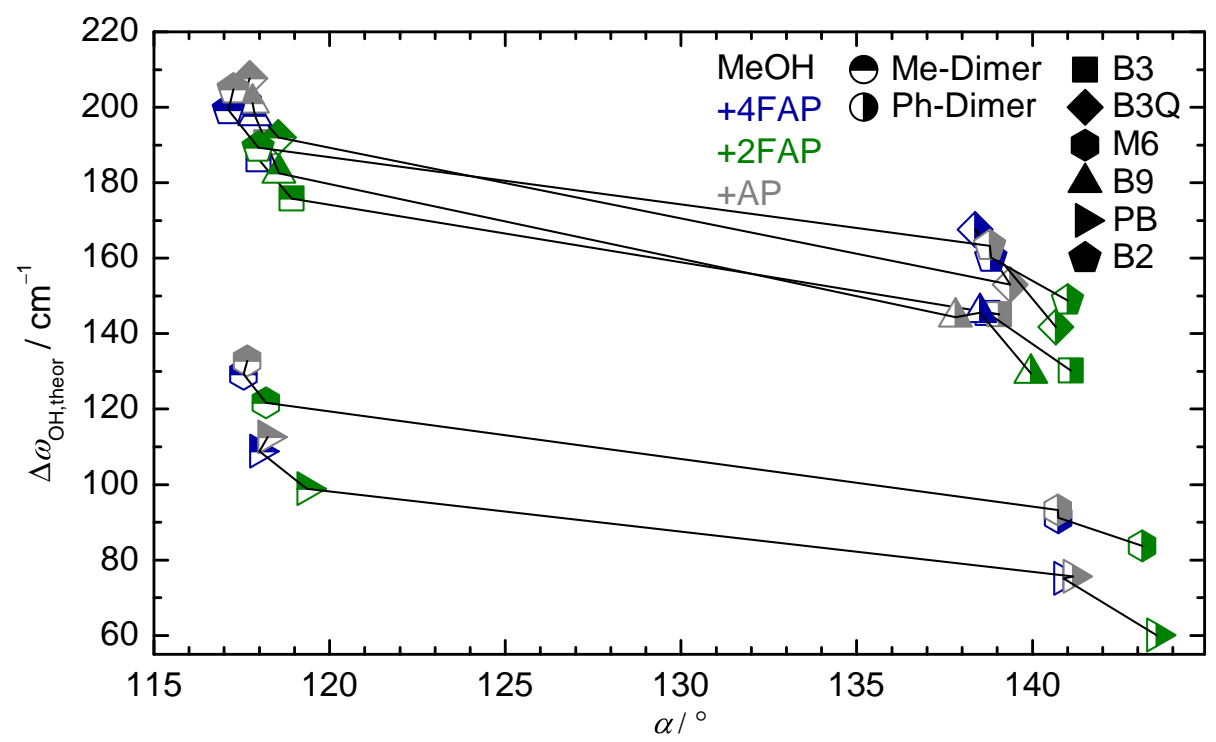

(a)

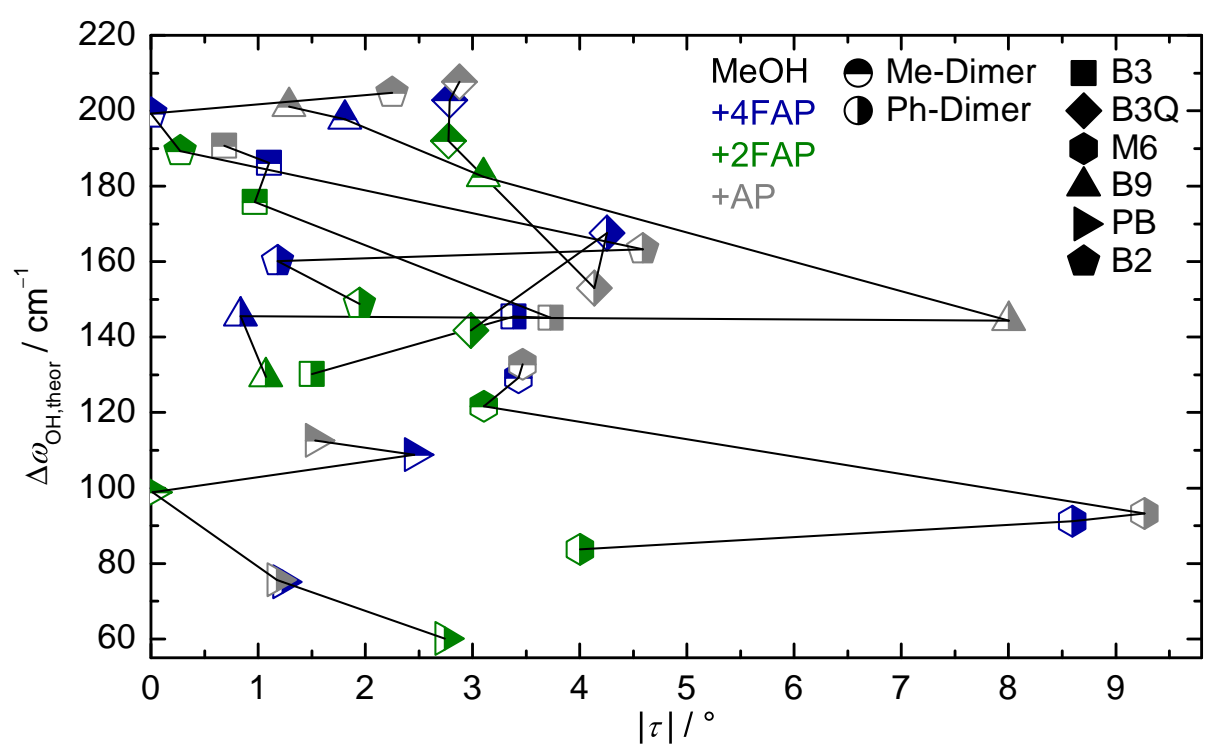

(b)

Abbildung 5.11: Korrelation zwischen den auf den verschiedenen Theorieniveaus berechneten Rotverschiebungen $\Delta \omega_{\mathrm{OH} \text {,theor }}$ und dem Bindungswinkel (s. Abb. 5.1) $\alpha$ (a) bzw. dem Betrag des Winkels $\tau$ (b) der in den Optimierungen ermittelten Strukturen. Die auf demselben Rechenniveau (Quadrate: B3; Rauten: B3Q; Sechsecke: M6; aufrechte Dreiecke: B9; seitliche Dreiecke: PB; Fünfecke: B2) erhaltenen Daten für die verschiedenen Dimere (blau: $\mathrm{MeOH}+4 \mathrm{FAP}$; grün: $\mathrm{MeOH}+2 \mathrm{FAP}$; grau: $\mathrm{MeOH}+\mathrm{AP}$; oben/unten farbig/weiß: Me-Dimer; links/rechts weiß/farbig: Ph-Dimer) sind durch Linien sortiert nach der Größe der experimentellen Rotverschiebungen miteinander verbunden. 
Tabelle 5.13: Experimentelle $(\tilde{v})$ und berechnete $(\omega)$ Unterschiede $\Delta \Delta(\tilde{v}, \omega)_{\mathrm{OH}}(\mathrm{APd} n-\mathrm{AP})$ in den Rotverschiebungen der Dimerbanden $\mathrm{MA}_{\mathrm{Me}}$ und $\mathrm{MA}_{\mathrm{Ph}}$ zwischen den teildeuterierten Isotopologen $\mathrm{MeOH}+\mathrm{APd} 3$ bzw. $\mathrm{MeOH}+\mathrm{APd} 5$ und dem undeuterierten $\mathrm{MeOH}+\mathrm{AP}$.

\begin{tabular}{lrrrrr}
\hline$\Delta \Delta(\tilde{v}, \omega)_{\mathrm{OH}}(\mathrm{APd} n-\mathrm{AP})$ & \multicolumn{2}{c}{$\mathbf{M A}_{\mathbf{M e}}$} & & \multicolumn{2}{c}{$\mathbf{M A}_{\mathbf{P h}}$} \\
\cline { 2 - 3 }$/ \mathrm{cm}^{-1}$ & $\mathrm{APd} 3$ & $\mathrm{APd} 5$ & & $\mathrm{APd} 3$ & $\mathrm{APd} 5$ \\
\hline B3 & 0.02 & 0.00 & & 0.01 & 0.07 \\
B3Q & 0.02 & 0.00 & & 0.00 & 0.05 \\
M6 & 0.02 & 0.00 & & 0.00 & 0.03 \\
B9 & 0.02 & 0.01 & & 0.01 & 0.06 \\
PB & 0.02 & 0.00 & & 0.00 & 0.04 \\
B2 & 0.02 & 0.00 & & 0.01 & 0.06 \\
\hline Exp & 0.6 & 0.4 & 0.4 & 0.9 \\
\hline
\end{tabular}

für den Diederwinkel $\tau$ ergeben, wird für die Untersuchung der Korrelation der Betrag dieses Winkels, $|\tau|$, verwendet. Dieser entspricht der Auslenkung der Wasserstoffbrückenbindung aus der Carbonylebene.

Für den $\mathrm{C}=\mathrm{O} \cdots \mathrm{H}$-Bindungswinkel $\alpha$ zeigt sich eine eindeutige Abhängigkeit von der Bindungsseite. Während $\alpha$ für die Me-Dimere Werte zwischen $117^{\circ}$ und $120^{\circ}$ annimmt, liegt der Winkel für die $\mathrm{Ph}$-Dimere zwischen $137^{\circ}$ und $144^{\circ}$. Der für die Ph-Dimere größere Bindungswinkel ist vermutlich auf den sterischen Anspruch der Ph-Gruppe zurückzuführen. ${ }^{[52]}$ Bei konsistent größeren berechneten Rotverschiebungen für die Me- im Vergleich zu den Ph-Dimeren ergibt sich zwischen den Bindungsseiten eine ausgeprägte Korrelation mit dem Winkel $\alpha$. Auch innerhalb der Bindungsseiten zeigt sich eine solche Korrelation, da die Dimere von $\mathrm{MeOH}$ mit 2FAP (grün) die kleinsten Rotverschiebungen bei den jeweils größten Bindungswinkeln $\alpha$ aufweisen. Zwischen $\mathrm{MeOH}+4 \mathrm{FAP}$ (blau) und $\mathrm{MeOH}+\mathrm{AP}$ (grau) spielen dann anscheinend aber elektronische Effekte für die Größe der Rotverschiebung eine wichtigere Rolle als die relativ geringen Unterschiede in den Bindungswinkeln.

Wie Teil (b) von Abbildung 5.11 verdeutlicht, ergibt sich für den Winkel der Wasserstoffbrückenbindung zur Carbonylebene, $|\tau|$, keine systematische Korrelation mit der Rotverschiebung. Für die verschiedenen Methoden zeigen sich sehr unterschiedliche Trends, wobei in keinem Fall über die Bindungsseiten und Acetophenonderivate hinweg eine eindeutige Korrelation vorliegt. Dies deckt sich mit den Befunden aus Ref. [52], wonach dieser Diederwinkel erst für ausladendere Substituenten an Donor und Akzeptor einen klaren Einfluss auf die Rotverschiebung nimmt.

Im Folgenden sollen direkt aus dem Experiment erhaltene IR-spektroskopische Parameter zum Benchmarking genutzt werden. Auf Basis der subtilen Auswirkungen auf die Größe der Rotverschiebungen der Dimer-OH-Streckschwingungsbanden bei Teildeuterierung des AP (s. Kap. 5.2) kann zunächst ein qualitativer Benchmark erfolgen. In Ta- 
belle 5.13 sind die berechneten Unterschiede in den Rotverschiebungen der $\mathrm{MA}_{\mathrm{Me}}$ - und $\mathrm{MA}_{\mathrm{Ph}}$-Dimerbanden den experimentellen Werten gegenübergestellt. Der experimentelle Trend, dass bei Teildeuterierung die Bande des jeweils auf der deuterierten Seite gebundenen Dimers $\left(\mathrm{MA}_{\mathrm{Me}}\right.$ für $\mathrm{APd} 3, \mathrm{MA}_{\mathrm{Ph}}$ für $\mathrm{APd}$ ) $)$ eine stärkere Erhöhung der Rotverschiebung als das andere Dimer erfährt, kann von allen Methoden qualitativ reproduziert werden. Die berechneten Änderungen sind jedoch mit $0.00-0.07 \mathrm{~cm}^{-1}$ nochmals deutlich kleiner als die experimentellen Werte von $0.4-0.9 \mathrm{~cm}^{-1}$. Für die auf der undeuterierten Molekülseite gebundenen Dimere ergeben sich in den DFT-Rechnungen zum Teil zudem gar keine Änderungen der Rotverschiebung.

Die Rotverschiebungen der OH-Streckschwingungsbanden in den $\mathrm{Me}$ - und $\mathrm{Ph}-\mathrm{Dime}-$ ren (S1) bieten sich, wie bereits in Kapitel 4 beschrieben, ebenso wie die spektralen Aufspaltungen zwischen diesen Banden (S2) auch für ein quantitatives Benchmarking an. ${ }^{[3]}$ Da sämtliche Rechnungen in der doppelt-harmonischen Näherung durchgeführt wurden, müssen die anharmonischen experimentellen Werte zunächst auf ihre harmonischen Anteile rückkorrigiert werden.

Dazu wird wieder das von Oswald und SUHM vorgeschlagene Verfahren ${ }^{[33]}$ genutzt, das in Kapitel 2.3.1 detailliert erläutert wird. Für Rotverschiebungen $\Delta(\tilde{v}, \omega)_{\mathrm{OH}}$, also die Wellenzahldifferenz zwischen der $\mathrm{OH}$-Streckschwingungsbandenposition des freien OH-Donors, $(\tilde{v}, \omega)_{\mathrm{OH}}(\mathrm{M})$, und der Position der entsprechenden Bande im Komplex, $(\tilde{v}, \omega)_{\mathrm{OH}}(\mathrm{Me}, \mathrm{Ph})$, wird aufgrund der zu erwartenden Teilkompensation anharmonischer Beiträge zwischen den beteiligten Banden als beste Schätzung für den harmonischen experimentellen Wert $\Delta \omega_{\mathrm{OH} \text {,exp }}$ der anharmonische Messwert $\Delta \tilde{v}_{\mathrm{OH}, \exp }$ verwendet und mit einer Sicherheitsmarge von $\pm 50 \%$ versehen. Für die Aufspaltung $\Delta \Delta(\tilde{v}, \omega)_{\mathrm{OH}}(\mathrm{Me}-\mathrm{Ph})$ zwischen den Dimer-OH-Streckschwingungsbanden wird ebenfalls der anharmonische Messwert als beste Schätzung für den harmonischen Wert herangezogen. Da für die Aufspaltung zwischen den Bandenpositionen unterschiedlicher Konformationen des gleichen Dimers eine nochmals stärkere Kompensation anharmonischer Beiträge als für die Rotverschiebungen zu erwarten ist, schlagen Oswald und SuHM eine kleinere Sicherheitsmarge von $\pm 10 \%$ des Mittelwerts der Rotverschiebungen der beteiligten Banden vor. Für die Bestimmung des experimentellen Gesamtfehlers werden die aus der Rückkorrektur erhaltenen Fehlerbereiche nach dem Prinzip des Größtfehlers mit der experimentellen Messunsicherheit kombiniert. Diese ergibt sich wiederum als Größtfehler aus der Unbestimmtheit der beitragenden individuellen Bandenpositionen (hier je $1 \mathrm{~cm}^{-1}$, Hälfte der spektralen Auflösung).

In Abbildung 5.12 sind die auf den verschiedenen Theorieniveaus berechneten Rotverschiebungen $\Delta \omega_{\mathrm{OH}}$ gegen die experimentellen Rotverschiebungen $\Delta \tilde{v}_{\mathrm{OH}}$ aufgetragen. Der sich aus Rückkorrektur und Messungenauigkeit ergebende Zielbereich ist weiß hervorgehoben und die Winkelhalbierende $\Delta \omega_{\mathrm{OH}}=\Delta \tilde{v}_{\mathrm{OH}}$ ist schwarz eingezeichnet. Für alle Methoden fallen sämtliche Datenpunkte für die Me- und Ph-Dimere aller Methanol-Acetophenon(derivat)-Kombinationen in den weißen Bereich, wobei die Datenpunkte von B3, M6 und B9 am nächsten an der Winkelhalbierenden liegen. Während die auf B3-, B3Q-, B9- und B2-Niveau berechneten Rotverschiebungen dabei jeweils größer als die (anhar- 


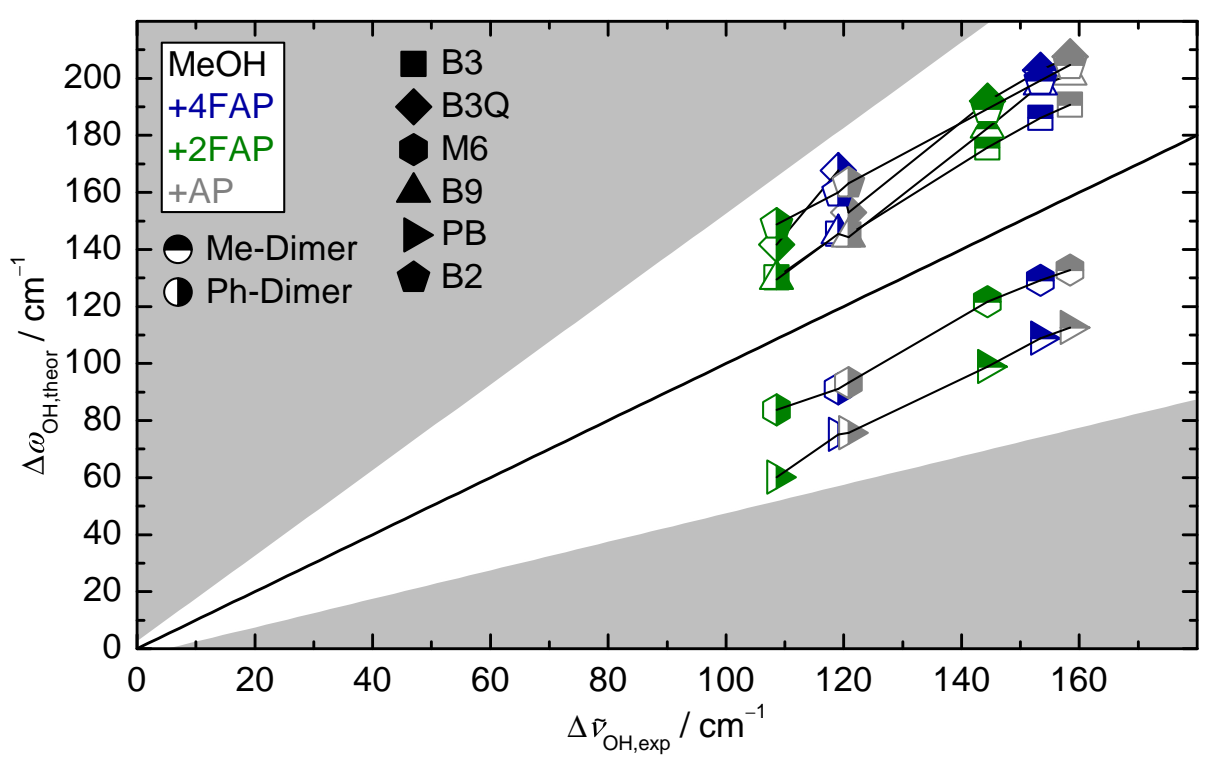

(a)

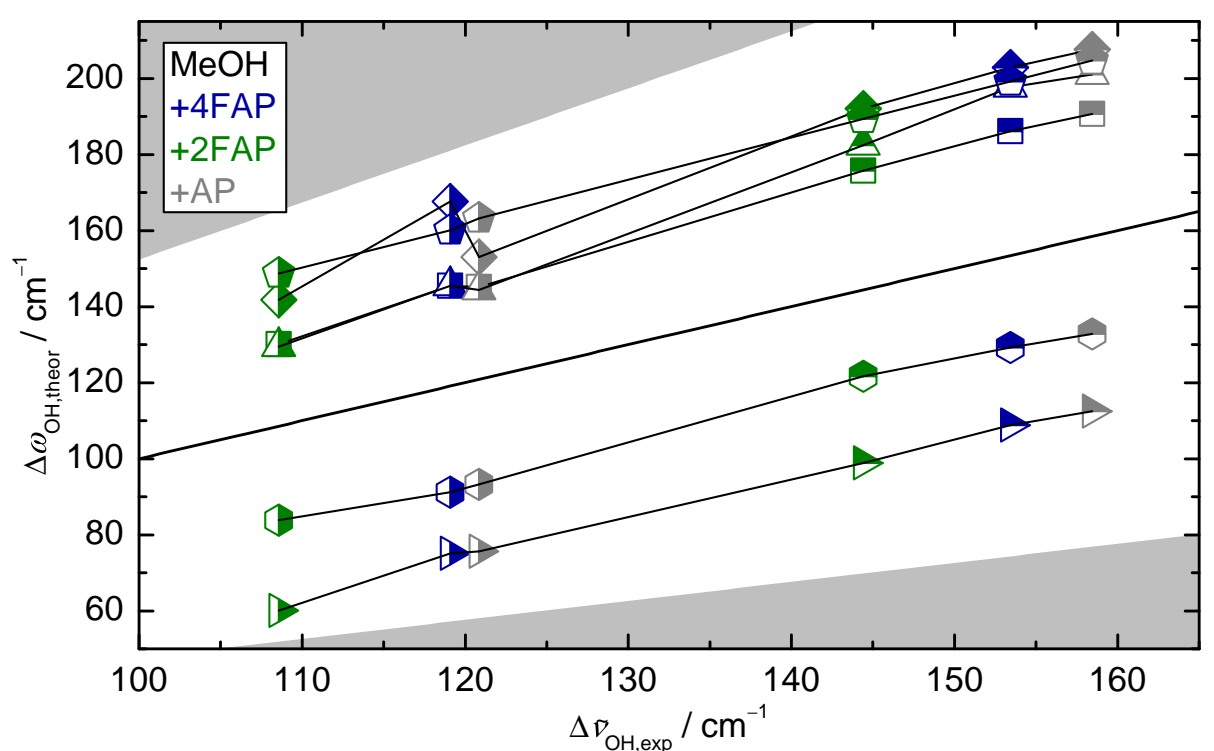

(b)

Abbildung 5.12: Auftragung der auf den verschiedenen Theorieniveaus berechneten Rotverschiebungen $\Delta \omega_{\mathrm{OH}}$,theor gegen die entsprechenden experimentellen Werte $\Delta \tilde{v}_{\mathrm{OH}}$,exp . (a) Gesamtübersicht, (b) Detailansicht des mit Daten belegten Bereichs. Die Symbolfarben, -formen und -füllungen folgen der in Abb. 5.11 vorgestellten Codierung und die Datenpunkte derselben Rechenmethode sind erneut sortiert nach der Größe der experimentellen Rotverschiebungen über schwarze Linien verbunden. Als Referenz ist zudem die Winkelhalbierende $\Delta \omega_{\mathrm{OH} \text {,theor }}=\Delta \tilde{v}_{\mathrm{OH} \text {,exp }}$ eingezeichnet. Der aus der Rückkorrektur und dem Messfehler erhaltene Bereich für den harmonischen Beitrag zur experimentellen Rotverschiebung ${ }^{[33]}$ ist weiß hervorgehoben. 
Tabelle 5.14: Ergebnisse der linearen Regressionen der Korrelation zwischen den auf den verschiedenen Theorieniveaus berechneten und den experimentellen Rotverschiebungen. Für jedes Theorieniveau wurden drei Regressionen mit den in der ersten Spalte angegebenen Geradengleichungen durchgeführt. Darin bezeichnet $m$ die Steigung und $b$ den $y$-Achsenabschnitt (in $\mathrm{cm}^{-1}$ ). $R^{2}$ ist das Bestimmtheitsmaß der jeweiligen Regression.

\begin{tabular}{llrrrrrr}
\hline Regression & Größe & B3 & B3Q & M6 & B9 & PB & B2 \\
\hline$y=m x+b$ & $m$ & $1.22(3)$ & $1.3(2)$ & $1.04(5)$ & $1.49(5)$ & $1.03(4)$ & $1.12(1)$ \\
& $b$ & $-1(4)$ & $0(2) \cdot 10^{1}$ & $-32(6)$ & $-33(7)$ & $-49(5)$ & $27(2)$ \\
& $R^{2}$ & 0.99789 & 0.95827 & 0.99328 & 0.99631 & 0.99531 & 0.99971 \\
\hline$y=m x$ & $m$ & $1.210(4)$ & $1.32(2)$ & $0.81(2)$ & $1.25(2)$ & $0.67(3)$ & $1.32(2)$ \\
& $R^{2}$ & 0.99996 & 0.99918 & 0.99821 & 0.99917 & 0.99408 & 0.99956 \\
\hline$y=x+b$ & $b$ & $28(2)$ & $43(4)$ & $-25.5(9)$ & $33(5)$ & $-45.6(7)$ & $44(2)$ \\
& $R^{2}$ & 0.96571 & 0.90774 & 0.99144 & 0.88888 & 0.99463 & 0.98759 \\
\hline
\end{tabular}

monischen) experimentellen Messwerte sind, liefern die M6- und die PB-Methoden kleinere Werte. Drei der sechs untersuchten Methoden (M6, PB, B2) reproduzieren den experimentellen Trend steigender Rotverschiebungen von $\mathrm{MeOH}+2 \mathrm{FAP}$ über $\mathrm{MeOH}+4 \mathrm{FAP}$ zu $\mathrm{MeOH}+\mathrm{AP}$ für Me- und Ph-Dimere, wohingegen sich auf dem B3-, B9- und B3Q-Niveau für das Ph-Dimer von $\mathrm{MeOH}+4 \mathrm{FAP}$ jeweils eine größere Rotverschiebung als für das PhDimer von $\mathrm{MeOH}+\mathrm{AP}$ ergibt. Für B3Q kann dies auf die Bewertung der relativen Stabilitäten von $\mathrm{Ph}_{\mathrm{ip}}$ - und $\mathrm{Ph}_{\mathrm{op}}$-Dimer für $\mathrm{MeOH}+4 \mathrm{FAP}$ zurückgeführt werden, da für dieses $\mathrm{AP}-$ Derivat $\mathrm{Ph}_{\mathrm{ip}}$ und für die anderen Derivate $\mathrm{Ph}_{\mathrm{op}}$ schwingungsnullpunktskorrigiert das stabilste Ph-Dimer darstellt (s. Tab. 5.4 auf S. 189). Wird für MeOH+4FAP stattdessen ebenfalls die Rotverschiebung des $\mathrm{Ph}_{\mathrm{op}}$-Dimers herangezogen, ergibt sich mit $153 \mathrm{~cm}^{-1}$ eine Rotverschiebung, die in etwa gleich groß wie die des $\mathrm{Ph}$-Dimers von $\mathrm{MeOH}+\mathrm{AP}$ ist. Alle weiteren betrachteten Methoden weisen keine Wechsel des schwingungsnullpunktskorrigiert stabilsten Strukturtyps zwischen den AP-Derivaten auf (überall ip für B2, überall op für B3, M6, B9 und PB).

Soll die Korrelation zwischen berechneten (harmonischen) und gemessenen (anharmonischen) Rotverschiebungen quantifiziert werden, können lineare Regressionen durchgeführt werden. Für maximale Flexibilität können dabei für Steigung $m$ und $y$-Achsenabschnitt $b$ beliebige Werte erlaubt und eine gleichzeitige Anpassung durchgeführt werden. Alternativ können vereinfachte Geradengleichungen mit je nur einem Parameter $(y=m x$ bzw. $y=x+b$ ) für die Regression genutzt werden. Durch das Bestimmtheitsmaß $R^{2}$ kann dabei die Anpassungsgüte der jeweiligen Regression erfasst werden. Je näher der erzielte $R^{2}$-Wert an 1 ist, desto stärker korrelieren die berechneten Werte im jeweiligen Regressionsmodell mit den experimentellen Daten, d. h. desto besser eignet sich eine Methode zur Vorhersage der experimentellen Rotverschiebungen.

Die Ergebnisse dieser linearen Anpassungen sind in Tabelle 5.14 zusammengefasst. In- 
teressanterweise weisen für die gleichzeitige Regression von $m$ und $b(y=m x+b)$ auch die Methoden, deren berechnete Rotverschiebungen kleiner als die experimentellen Werte sind (M6, PB), Steigungen $>1$ auf, auch wenn diese mit 1.04(5) bzw. 1.03(4) innerhalb ihrer Fehlerintervalle auch Werte $<1$ zulassen. Die Unterschätzung der experimentellen Rotverschiebungen wird durch negative $y$-Achsenabschnitte ausgeglichen. Entsprechend der Steigungen nahe an 1 weisen diese beiden Methoden für die Regression über den $y$ Achsenabschnitt $(y=x+b)$ auch die beste Korrelation mit den experimentellen Werten auf $\left(R^{2}\right.$-Werte von 0.991 bzw. 0.995). Generell werden für diesen Regressionsansatz die niedrigsten Bestimmtheitsmaße erzielt, wobei insbesondere B9 $\left(R^{2}=0.88888\right)$ und B3Q $\left(R^{2}=0.90774\right)$ schlecht abschneiden. Für das B3LYP-D3-Funktional ergeben sich mit der kleineren TZ-Basis über die drei verwendeten Regressionsansätze hinweg bessere Ergebnisse als mit dem QZ-Basissatz. M6 und PB weisen in allen Regressionsansätzen hohe Bestimmtheitsmaße von $>0.99$ auf, während B3 für die Regression über die Steigung $(y=m x)$ und B2 für die „volle“ Regression $(y=m x+b)$ das beste Ergebnis erzielen. Insgesamt zeigen aber alle Methoden ausgeprägte Korrelationen mit den experimentellen Daten und eignen sich somit als Hilfe für die Zuordnung der experimentellen Dimerbanden zu den jeweiligen Bindungsseiten. Die einheitliche Art von Wasserstoffbrückenbindungen $(\mathrm{OH} \cdots \mathrm{O})$ in den betrachteten gemischten Methanol-Acetophenon(derivat)-Dimeren scheint also eine systematische Beschreibung der Rotverschiebungen durch die quantenchemischen Rechenmethoden zu erleichtern, wie das gute Abschneiden selbst der günstigen B9- und PB-Methoden verdeutlicht. Für die O/C-Waagensysteme mit ihrem Gleichgewicht aus $\mathrm{OH} \cdots \mathrm{O}$ - und $\mathrm{OH} \cdots \mathrm{C}$-Wasserstoffbrückenbindungen (s. Kap. 4.1.4 und 4.2.4) wurden in dieser Hinsicht zum Teil deutlich schlechtere Ergebnisse erzielt.

Eine etwas stärkere Separation der Ergebnisse zeigt sich für die Me-Ph-Bandenaufspaltung $\Delta \Delta(\tilde{v}, \omega)_{\mathrm{OH}}(\mathrm{Me}-\mathrm{Ph})$, die in Abbildung 5.13 gegen die Rotverschiebung $\Delta(\tilde{v}, \omega)_{\mathrm{OH}}(\mathrm{Ph})$ aufgetragen ist. B2, M6 und insbesondere PB zeigen gute Übereinstimmungen mit den experimentellen Ergebnissen für die Bandenaufspaltung und auch die Werte für B3 befinden sich für die drei Datenpunkte des kleinen CarbonylwaagenDatensatzes in den individuellen Fehlerbereichen, wenn auch bei leicht überschätzten Aufspaltungen. Die B9-Methode liefert für alle drei Acetophenonderivate zu große Bandenaufspaltungen, sodass sich die Datenpunkte oberhalb der Zielbereiche befinden. Für B3Q liegt der $\mathrm{MeOH}+2 \mathrm{FAP}-D a t e n p u n k t$ (grün) zwar innerhalb des Zielbereichs, ist aber am oberen Rand lokalisiert und der $\mathrm{MeOH}+\mathrm{AP}-\mathrm{Datenpunkt} \mathrm{(grau)} \mathrm{liegt} \mathrm{leicht} \mathrm{ober-}$ halb des experimentellen Fehlerbereichs für die Bandenaufspaltung. Für $\mathrm{MeOH}+4 \mathrm{FAP}$ (blau) reproduziert die B3Q-Methode die Bandenaufspaltung hingegen deutlich besser, sodass ein kleinerer Wert resultiert. Das Muster der experimentellen Datenpunkte, das einem liegenden „L“ ähnelt, wird im Ergebnis durch die B3Q-Methode besonders schlecht wiedergegeben. Dies ist, wie weiter oben bereits beschrieben, vermutlich auf den Wechsel der von der B3Q-Methode vorhergesagten ip/op-Präferenz der Ph-Dimere bei AP-Substitution zurückzuführen (ip für $\mathrm{MeOH}+4 \mathrm{FAP}$ bevorzugt, op für $\mathrm{MeOH}+\mathrm{AP}$ und $\mathrm{MeOH}+2 \mathrm{FAP}$, s. Tab. 5.4). Wird für $\mathrm{MeOH}+4 \mathrm{FAP}$ ebenfalls die Rotverschiebung des nur $0.04 \mathrm{~kJ} \cdot \mathrm{mol}^{-1}$ weniger stabilen $\mathrm{Ph}_{\mathrm{op}}$ - anstelle des $\mathrm{Ph}_{\mathrm{ip}}$-Dimers herangezogen (blaue 


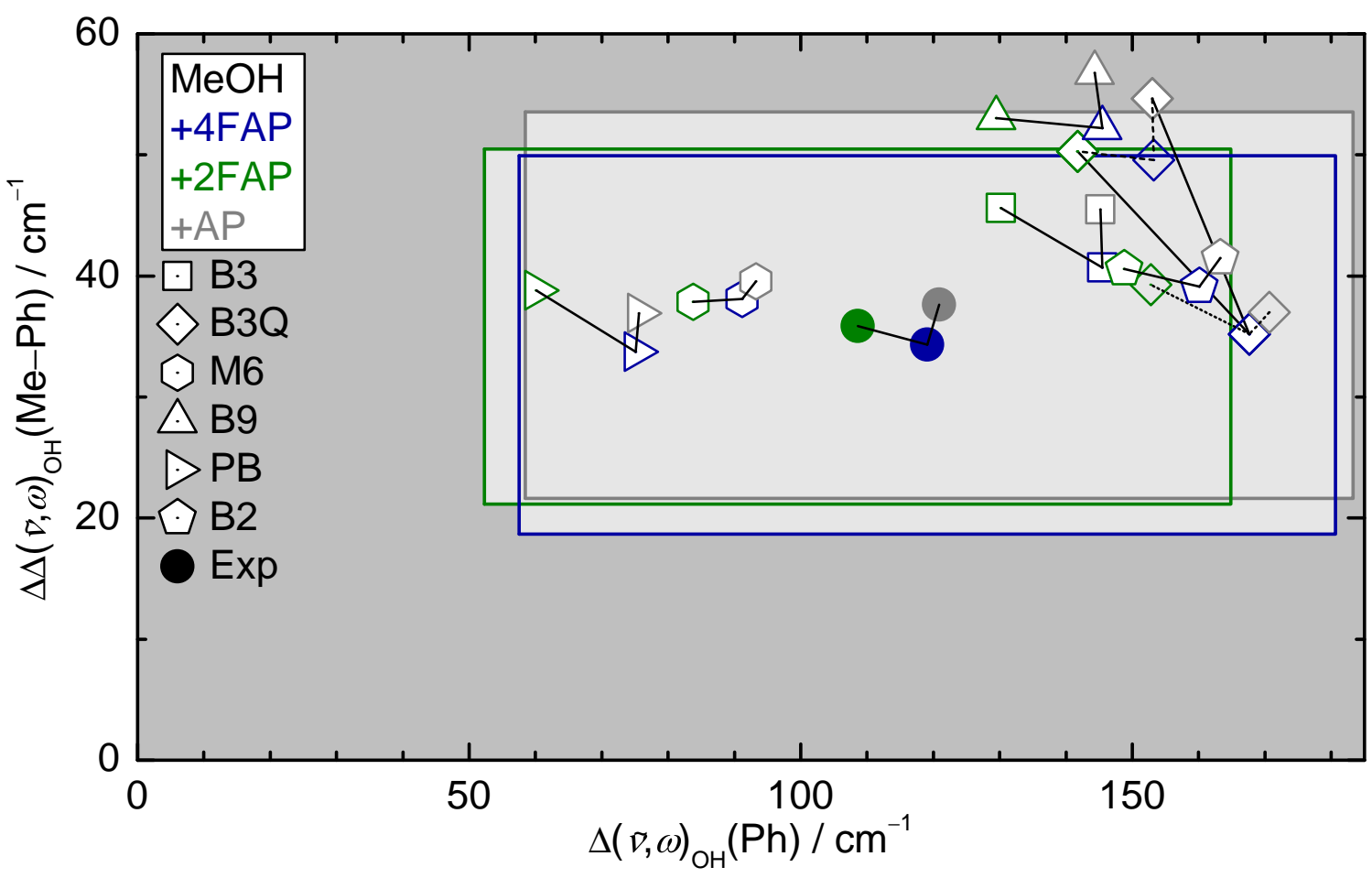

Abbildung 5.13: Auftragung der Aufspaltung der OH-Streckschwingungsbanden der Meund Ph-gebundenen Dimere, $\Delta \Delta(\tilde{v}, \omega)_{\mathrm{OH}}(\mathrm{Me}-\mathrm{Ph})$ (Ordinate), von $\mathrm{MeOH}$ mit 4FAP (blau), 2FAP (grün) und AP (grau) gegen die Rotverschiebungen der jeweiligen Ph-Dimerbanden, $\Delta(\tilde{v}, \omega)_{\mathrm{OH}}(\mathrm{Ph})$ (Abszisse), für die verschiedenen quantenchemischen Methoden (weiß gefüllte Symbole) und das Experiment (farbig gefüllte Kreise). Die Symbolfarben und -formen entsprechen der in Abb. 5.11 vorgestellten Codierung und die Datenpunkte derselben Methode sind auch hier sortiert nach der Größe der experimentellen Rotverschiebungen über schwarze Linien verbunden. Die experimentellen Datenpunkte sind mit den individuellen aus der Rückkorrektur auf die harmonischen Anteile ${ }^{[33]}$ und der Messungenauigkeit erhaltenen Fehlerbereichen (farbig umrandete, hellgrau eingefärbe Rechtecke) versehen. Für die B3Q-Methode (Rauten) sind zusätzliche Datenpunkte (keine Füllung) für weniger stabile Dimerstrukturen (s. Fließtext) eingezeichnet. 
Raute ohne Füllung, gestrichelte Verbindungslinie), ergibt sich eine deutlich bessere Reproduktion des experimentellen Musters. Allerdings liegt der Datenpunkt für Me$\mathrm{OH}+4 \mathrm{FAP}$ dann ebenfalls am oberen Rand des Fehlerbereichs für die Bandenaufspaltung. Eine vergleichbar gute Reproduktion des experimentellen Musters bei gleichzeitig besser zum Experiment passenden Bandenaufspaltungen wird erhalten, wenn stattdessen einheitlich die Rotverschiebungen der $\mathrm{Ph}_{\mathrm{ip}}$-Dimere verwendet werden (grüne und graue Rauten ohne Füllung, gepunktete Verbindungslinie). Diese sind auf dem B3Q-Niveau für $\mathrm{MeOH}+\mathrm{AP}$ und $\mathrm{MeOH}+2 \mathrm{FAP}$ schwingungsnullpunktskorrigiert $0.28 \mathrm{bzw} .0 .06 \mathrm{~kJ} \cdot \mathrm{mol}^{-1}$ weniger stabil als die $\mathrm{Ph}_{\mathrm{op}}$-Strukturen (s. Tab. 5.4). Die deutlich bessere Übereinstimmung mit dem Experiment hinsichtlich der untersuchten IR-spektroskopischen Parameter lässt jedoch vermuten, dass die B3Q-Methode hier die relative Stabilität kompakterer räumlicherer Strukturen (op-Dimere) überbewertet und dass tatsächlich für alle untersuchten Acetophenonderivate die gestreckteren planareren ip-Strukturen vorliegen. Für das B3LYP-D3-Funktional wurde ein solches Verhalten für Carbonylwaagensysteme bereits beobachtet. ${ }^{[52]}$ Weitere Indizien für diese These ergeben sich aus der relativen Stabilisierung der ip- gegenüber den op-Dimeren bei Berechnung der elektronischen Energien auf DLPNO-CCSD(T)-Niveau (B3Q+, B3Q+c) und dem Umstand, dass von der B2- bzw. B2+-Methode eine durchgehende ip-Präferenz vorhergesagt wird. Endgültige Aufklärung der tatsächlich im Experiment vorliegenden ip/op-Präferenz können jedoch nur mikrowellenspektroskopische Untersuchungen leisten.

Insgesamt ermöglichen die kleineren Fehlerbereiche für die Me-Ph-Bandenaufspaltung eine bessere Unterscheidung der Leistungsfähigkeit der betrachteten quantenchemischen Methoden als für die Rotverschiebungen. Im Vergleich zum Methanol-Anisol-Waagensystem, bei dem keine der beiden betrachteten Methoden Ergebnisse liefern konnte, die durchgängig im Zielbereich liegen (s. Kap. 4.2.4, Abb. 4.25), weist das hier untersuchte Carbonyl-Waagensystem aber eine deutlich geringere diskriminatorische Leistung auf.

Dies spiegelt sich auch bei Betrachtung der $\overline{\delta_{\mathrm{Xn}}^{2}}(\mathrm{Q})$-Werte für die Me- und Ph-Rotverschiebungen $\left(\mathrm{S} 1_{\mathrm{Me}}\right.$ und $\left.\mathrm{S} 1_{\mathrm{Ph}}\right)$ und die Me-Ph-Bandenaufspaltung (S2) wider. Der ebenfalls von Oswald und SuHM vorgeschlagene $\delta^{2}$-Wert ${ }^{[33]}$ stellt ein mit dem inversen experimentellen Fehler gewichtetes Maß für die Abweichung zwischen berechnetem und gemessenem Wert dar (s. Kap. 2.3.1). Werden die individuellen $\delta^{2}$-Werte für eine bestimmte quantenchemische Methode Q über eine Observablenklasse Xn (hier S1, S2) gemittelt, ergeben sich die $\overline{\delta_{\mathrm{Xn}}^{2}}(\mathrm{Q})$-Mittelwerte, die in Abbildung 5.14 für den Carbonylwaagen-Datensatz dargestellt sind. Im Vergleich zum Methanol-Anisol-Waagensystem (s. Kap. 4.2.4, Abb. 4.26) werden dabei für das Methanol-Acetophenon-Waagensystem deutlich kleinere $\overline{\delta_{\mathrm{Xn}}^{2}}$-Werte für die Bandenaufspaltung S2 erhalten (max. 1.4 für das Methanol-Acetophenon-, max. 8.2 für das Methanol-Anisol-Waagensystem).

Die $\overline{\delta_{\mathrm{Xn}}^{2}}$-Werte fassen insgesamt nochmal die bereits genannten Ergebnisse der quantenchemischen Methoden in Bezug auf die Rotverschiebungen und Bandenaufspaltungen zusammen und quantifizieren diese. Während für die Rotverschiebungen (S1) alle betrachteten Methoden gute Ergebnisse erzielen, wobei M6, B3 und B9 hier besonders gut ab- 


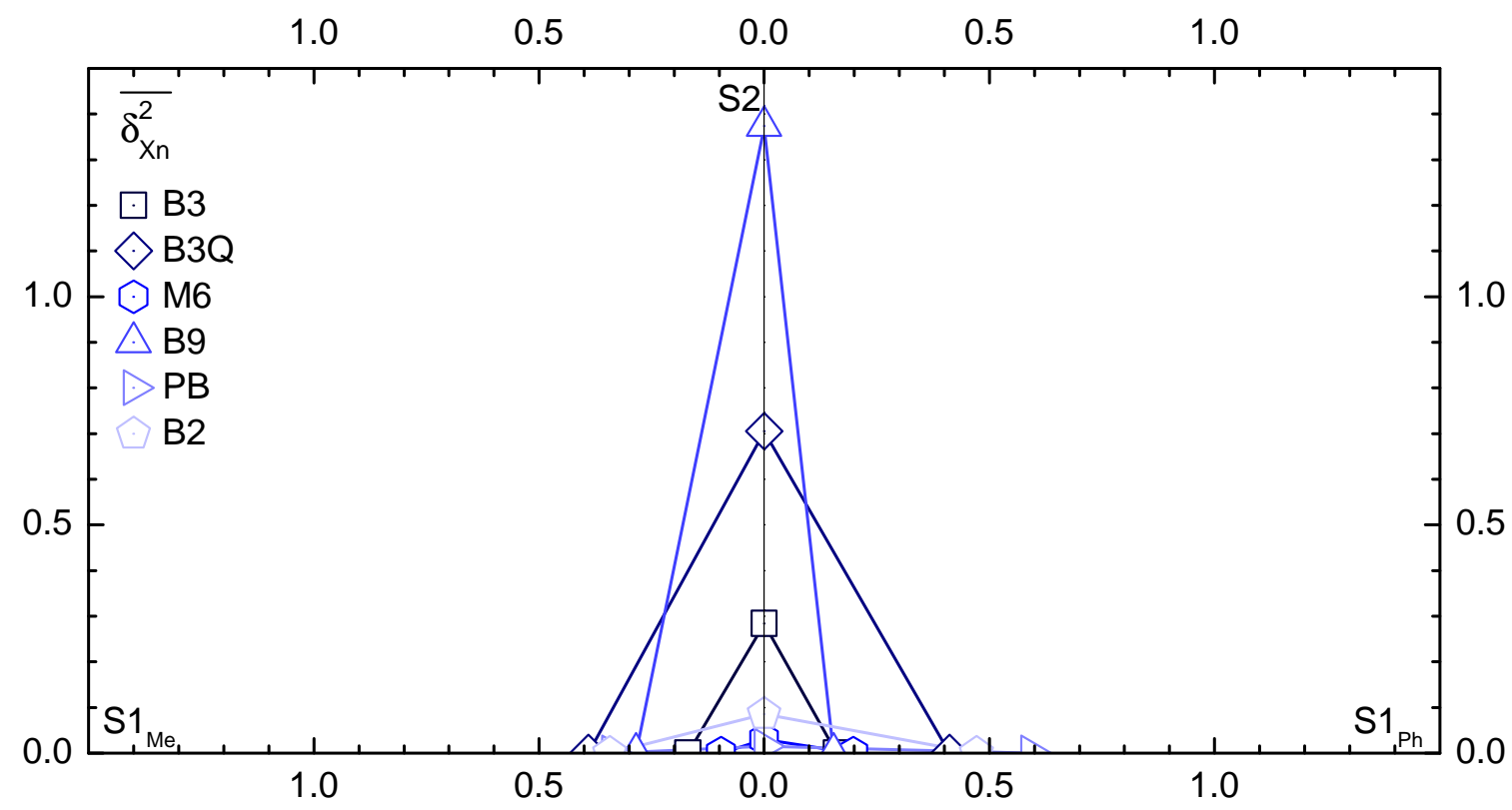

Abbildung 5.14: Über die drei Methanol-Acetophenon(derivat)-Kombinationen $\mathrm{MeOH}+4 \mathrm{FAP}$, $\mathrm{MeOH}+2 \mathrm{FAP}$ und $\mathrm{MeOH}+\mathrm{AP}$ gemittelte $\overline{\delta_{\mathrm{Xn}}^{2}}(\mathrm{Q})-$ Werte ${ }^{[33]}$ für die quantenchemischen Methoden B3 (Quadrate), B3Q (Rauten), M6 (Sechsecke), B9 (aufrechte Dreiecke), PB (seitliche Dreiecke) und B2 (Fünfecke) für die Observablen $\mathrm{OH}$-Streckschwingungsrotverschiebung im $\mathrm{Me}-\left(\mathrm{S} 1_{\mathrm{Me}}\right)$ bzw. Ph-gebundenen ( $\mathrm{S}_{\mathrm{Ph}}$ ) Dimer (Abszisse) bzw. Me/Ph-Dimer-OH-Streckschwingungsbandenaufspaltung (S2, Ordinate).

schneiden, zeigen sich für die Bandenaufspaltungen (S2) größere Unterschiede. PB, M6 und B2 liefern hier die geringsten Abweichungen zum Experiment, wohingegen sich für B3, B3Q und insbesondere B9 größere $\overline{\delta_{\mathrm{Xn}}^{2}}$-Werte ergeben.

Zusammenfassend zeigt somit die M6-Methode die ausgewogenste Leistung hinsichtlich der Beschreibung der untersuchten IR-spektroskopischen Parameter, gefolgt von B2 und PB. Auch die Ergebnisse von B3 sind durchgehend mit den experimentellen Befunden vereinbar, während sich für die Kombination des B3LYP-D3-Funktionals mit dem größeren def2-QZVP-Basissatz (B3Q) Abweichungen ergeben, die sich vermutlich auf Probleme in der Beschreibung der ip/op-Präferenz der Ph-Dimere zurückführen lassen. Die bessere Übereinstimmung mit dem Experiment bei einheitlicher Verwendung der Rotverschiebungen von op- bzw. - nochmals deutlich besser - von ip-Dimeren liefert Hinweise darauf, dass der (ausschließlich) von B3Q vorhergesagte ip/op-Präferenzwechsel bei Substitution am AP im Experiment nicht vorliegt. Erst mikrowellenspektroskopischen Daten würden jedoch eine eindeutige Aussage hierüber und über die tatsächlich vorliegende Präferenz ermöglichen, auch wenn auf Basis des vorliegenden Benchmarks eine durchgängige ip-Präferenz am wahrscheinlichsten erscheint. Die günstige B9-Methode versagt für die Bandenaufspaltungen und sagt hier durchgehend zu große Werte voraus. 
Für die Methoden B3Q und in etwas geringerem Maße M6 (in Ref. [33] wird der größere def2-QZVP-Basissatz verwendet) ist ein Vergleich mit den Ergebnissen für den ENCH51-Benchmarkingdatensatz ${ }^{[33]}$ möglich. Analog zum deutlich umfangreicheren und diverseren ENCH-51-Datensatz ergeben sich im vorliegenden Benchmark in der Observablenklasse S1 (Rotverschiebungen) dabei mit dem M06-2X-Funktional kleinere $\overline{\delta^{2}}$-Werte (ENCH-51: 0.7; ${ }^{[33]}$ hier: 0.1) als für die B3Q-Methode (ENCH-51: 2.0 ; $^{[33]}$ hier: 0.4). Im Gegensatz zum vorliegenden Benchmark erzielt M06-2X für den ENCH-51-Datensatz in der Klasse S2 (Bandenaufspaltungen) jedoch etwas schlechtere Ergebnisse $\left(\overline{\delta^{2}}\right.$-Werte ENCH51: 0.4; ${ }^{[33]}$ hier: 0.03) als B3Q (ENCH-51: 0.3; ${ }^{[33]}$ hier: 0.7). Werden für B3Q konsistent die Daten der ip-Dimere für die Auswertung herangezogen, verbessert sich der $\overline{\delta^{2}}$-Wert für S2 auf 0.02 und liegt damit analog zum ENCH-51-Datensatz unterhalb des entsprechenden Wertes für M06-2X. Zwischen der Größe der Rotverschiebung und dem Diederwinkel $\tau$ zwischen Carbonylebene und Wasserstoffbrückenbindung konnte für die betrachteten gemischten Dimere aus $\mathrm{MeOH}$ und AP, 2FAP und 4FAP kein eindeutiger Zusammenhang gefunden werden. Für den $\mathrm{C}=\mathrm{O} \cdots \mathrm{H}$-Bindungswinkel $\alpha$ ergibt sich hingegen vor allem für die zwischen den Bindungsseiten deutlich unterschiedlichen Werte eine Korrelation mit der berechneten Rotverschiebung. Da die berechneten Rotverschiebungen wiederum zu einem hohen Grad mit den experimentellen Rotverschiebungen korrelieren, ließe sich die Abhängigkeit von $\alpha$ auch auf die experimentellen Werte übertragen. Unklar bleiben jedoch die tatsächlich im Experiment vorliegenden Bindungswinkel $\tau$ und $\alpha$. Die Ermittlung dieser Winkel über mikrowellenspektrokopische Untersuchungen wäre somit wünschenswert und könnte wichtige zusätzliche Daten zu den experimentellen Strukturen der $\mathrm{MeOH}$-Acetophenon(derivat)-Dimere liefern.

\subsection{Analyse des Einflusses von Dispersionswechselwirkungen auf das Konformerengleichgewicht}

Wie schon für die Methanol-Anisol-O/C-Waagensysteme (s. Kap. 4.2.5) soll auch für die Carbonylwaagen zur Analyse des Einflusses von Dispersionswechselwirkungen auf die Regioselektivität der Ausbildung der Wasserstoffbrückenbindung das im Rahmen des DLPNO-CCSD(T)-Verfahrens zur Verfügung stehende LED-Konzept verwendet werden. Da die Ergebnisse keine Observablen darstellen und somit nicht anhand experimenteller Werte verifiziert werden können, bleibt die Zuverlässigkeit der LED-Methode dabei unklar. Dennoch sollten die Ergebnisse zumindest Hinweise darauf geben können, welche Rolle Dispersionswechselwirkungen für das Me/Ph-Konformerengleichgewicht spielen.

Zunächst soll die Größe der Dispersionsbeiträge zur Wechselwirkungsenergie zwischen Methanol und Acetophenon(derivat) für die verschiedenen Strukturtypen $\mathrm{Me}_{\mathrm{ip}}$, $\mathrm{Me}_{\mathrm{op}}, \mathrm{Ph}_{\mathrm{ip}}$ und $\mathrm{Ph}_{\mathrm{op}}$ (s. Abb. 5.3) verglichen werden. Dazu werden mit B3Q+ und B2+ 
Tabelle 5.15: Über die LED-Analyse auf Grundlage des B3Q+- bzw. B2+-Rechenniveaus bestimmte Dispersionsbeiträge $E^{\text {disp }}$ zur Bindungsenergie der Methanol-Acetophenon(derivat)-Dimere. Die schwingungsnullpunktskorrigiert auf den angegebenen Rechenniveaus jeweils stabilste Struktur des Me- bzw. Ph-Dimers ist fett hervorgehoben.

\begin{tabular}{|c|c|c|c|c|c|c|c|c|c|c|c|c|}
\hline \multirow{2}{*}{$\begin{array}{l}E^{\text {disp }} \\
/ \mathrm{kJ} \cdot \mathrm{mol}^{-1}\end{array}$} & \multicolumn{4}{|c|}{$\mathrm{MeOH}+\mathrm{AP}$} & \multicolumn{4}{|c|}{$\mathrm{MeOH}+2 \mathrm{FAP}$} & \multicolumn{4}{|c|}{$\mathrm{MeOH}+4 \mathrm{AP}$} \\
\hline & $\mathrm{Me}_{\mathrm{ip}}$ & $\mathrm{Me}_{\mathrm{op}}$ & $\mathrm{Ph}_{\mathrm{ip}}$ & $\mathrm{Ph}_{\mathrm{op}}$ & $\mathrm{Me}_{\mathrm{ip}}$ & $\mathrm{Me}_{\mathrm{op}}$ & $\mathrm{Ph}_{\mathrm{ip}}$ & $\mathrm{Ph}_{\mathrm{op}}$ & $\mathrm{Me}_{\mathrm{ip}}$ & $\mathrm{Me}_{\mathrm{op}}$ & $\mathrm{Ph}_{\mathrm{ip}}$ & $\mathrm{Ph}_{\mathrm{op}}$ \\
\hline $\begin{array}{l}\text { B3Q+ } \\
\text { B2+ }\end{array}$ & $\begin{array}{l}-11.6 \\
-11.8\end{array}$ & -12.1 & $\begin{array}{r}-12.8 \\
-\mathbf{1 2 . 5}\end{array}$ & $\begin{array}{r}-\mathbf{1 3 . 9} \\
-14.4\end{array}$ & $\begin{array}{l}-11.3 \\
-11.5\end{array}$ & -11.9 & $\begin{array}{l}-12.6 \\
-12.6\end{array}$ & $\begin{array}{l}-14.4 \\
-14.5\end{array}$ & $\begin{array}{l}-11.6 \\
-11.9\end{array}$ & -11.6 & $\begin{array}{l}-12.9 \\
-12.8\end{array}$ & $\begin{array}{l}-14.3 \\
-14.6\end{array}$ \\
\hline
\end{tabular}

die beiden Methoden herangezogen, die auf dem DLPNO-CCSD(T)/aVQZ-Niveau die stabilsten Dimerstrukturen aufweisen (s. Abb. 5.10) und die als einzige Methoden bei Berechnung der elektronischen Energie auf dem DLPNO-CCSD(T)/aVQZ-Niveau in allen Fällen mit dem Experiment kompatible Energiedifferenzen liefern. Die Dispersionsbeiträge der jeweils schwingungsnullpunktskorrigiert stabilsten Dimerstrukturen eines Typs sind in Tabelle 5.15 zusammengefasst. Beide betrachteten Theorieniveaus weisen für die einzelnen Strukturtypen der verschiedenen Methanol-Acetophenon(derivat)Kombination sehr ähnliche Dispersionsbeiträge auf. Die Abweichungen zwischen den Methoden liegen in allen Fällen unterhalb von $0.5 \mathrm{~kJ} \cdot \mathrm{mol}^{-1}$. Die op-Strukturen weisen dabei stets die etwas höheren Dispersionsbeiträge als die ip-Strukturen auf, insbesondere für die Ph-Dimere. Die Unterschiede zwischen ip und op betragen $0.0-0.5 \mathrm{~kJ} \cdot \mathrm{mol}^{-1}$ für die Me- und 1.2-2.0 $\mathrm{kJ} \cdot \mathrm{mol}^{-1}$ für die Ph-Dimere. Dennoch stellen schwingungsnullpunktskorrigiert in nahezu allen Fällen die ip-Strukturen den stabileren Strukturtypen dar. Innerhalb der Bindungsseiten ist die Stärke der Dispersionswechselwirkung somit nicht das entscheidende Kriterium für die Konformationspräferenz.

Die sich zwischen dem schwingungsnullpunktskorrigiert jeweils stabilsten Me- und Ph-Dimer ergebenden Differenzen $\Delta E^{\text {disp }}$ in den Dispersionsbeiträgen zur Wechselwirkungsenergie sind für B3Q+ und B2+ in Tabelle 5.16 aufgelistet. Das Vorzeichen folgt dabei der bisherigen Konvention, sodass negative Werte eine stärkere Dispersionsstabilisierung des Me- und positive Werte des Ph-gebundenen Dimers verdeutlichen. Gemeinsam mit den Dispersionsbeitragsdifferenzen sind noch einmal die schwingungsnullpunktskorrigierten Energiedifferenzen $\Delta E^{0}=E_{\mathrm{Me}}^{0}-E_{\mathrm{Ph}}^{0}$ und die entsprechenden Energiedifferenzen mit subtrahierten Dispersionsbeiträgen, $\Delta E_{\text {nd }}^{0}=\Delta E^{0}-\Delta E^{\text {disp }}$, aufgeführt. Über beide Methoden und alle betrachteten Acetophenonderivate hinweg zeigen sich systematisch stärkere Dispersionswechselwirkungen in den Ph-Dimeren (positive Werte für $\Delta E^{\text {disp }}$ ). Die Unterschiede betragen $0.66(\mathrm{MeOH}+\mathrm{AP}$ auf $\mathbf{B} 2+-\mathrm{Niveau})$ bis $2.32 \mathrm{~kJ} \cdot \mathrm{mol}^{-1}(\mathrm{MeOH}+\mathrm{AP}$ auf B3Q+-Niveau). Die Differenz ist dabei für B3Q+ stets größer als für B2+, wobei sich für $\mathrm{MeOH}+\mathrm{AP}$ besonders große Unterschiede ergeben. Dies kann auf die unterschiedlichen zugrundeliegenden Strukturtypen des Ph-Dimers (s. Tab. 5.15) zurückgeführt werden.

Welchen Einfluss die stärkere Dispersionsstabilisierung des $\mathrm{Ph}$ - gegenüber dem $\mathrm{Me}$ - 
Tabelle 5.16: Ergebnisse der LED-Analyse auf B3Q+- bzw. B2+-Niveau hinsichtlich der Differenzen in den Dispersionsbeiträgen $E^{\text {disp }}$ zwischen dem schwingungsnullpunktskorrigiert jeweils stabilsten Me- und Ph-Dimer für die unterschiedlichen Methanol-Acetophenon(derivat)-Kombinationen. $\Delta E^{0}$ bezeichnet die schwingungsnullpunktskorrigierte Energiedifferenz zwischen diesen beiden Dimeren, $\Delta E_{\text {nd }}^{0}$ die entsprechende Energiedifferenz, wenn die Dispersionsbeiträge subtrahiert werden und $\Delta E^{\text {disp }}=E_{\mathrm{Me}}^{\text {disp }}-E_{\mathrm{Ph}}^{\text {disp }}$ stellt die Differenz in den Dispersionsbeiträgen beider Dimere dar. Negative Vorzeichen entsprechen einer Bevorzugung bzw. stärkeren Dispersionsstabilisierung des Me-Dimers, positive entsprechend des Ph-Dimers.

\begin{tabular}{|c|c|c|c|c|c|c|c|c|c|}
\hline \multirow{2}{*}{$\begin{array}{l}E \\
/ \mathrm{kJ} \cdot \mathrm{mol}^{-1}\end{array}$} & \multicolumn{3}{|c|}{$\mathrm{MeOH}+\mathrm{AP}$} & \multicolumn{3}{|c|}{$\mathrm{MeOH}+2 \mathrm{FAP}$} & \multicolumn{3}{|c|}{$\mathrm{MeOH}+4 \mathrm{AP}$} \\
\hline & $\Delta E^{0}$ & $\Delta E_{\mathrm{nd}}^{0}$ & $\Delta E^{\text {disp }}$ & $\Delta E^{0}$ & $\Delta E_{\mathrm{nd}}^{0}$ & $\Delta E^{\mathrm{disp}}$ & $\Delta E^{0}$ & $\Delta E_{\mathrm{nd}}^{0}$ & $\Delta E^{\text {disp }}$ \\
\hline B3Q+ & -1.13 & -3.44 & 2.32 & -0.27 & -1.58 & 1.31 & -0.53 & -1.76 & 1.23 \\
\hline B2+ & -0.97 & -1.63 & 0.66 & -0.55 & -1.60 & 1.05 & -0.44 & -1.31 & 0.87 \\
\hline
\end{tabular}

gebundenen Dimer auf die Bindungsstellenpräferenz hat, verdeutlicht der Vergleich des schwingungsnullpunktskorrigierten Energieunterschieds $\Delta E^{0}$ mit seinem hypothetischen dispersionsfreien Analogon $\Delta E_{\text {nd }}^{0}$. Dabei liegen in keinem Fall Vorzeichenwechsel vor, d. h. die Dispersionsbeiträge sorgen hier nirgendwo für einen Wechsel der Bindungsstellenpräferenz. Ähnliche Befunde ergeben sich für die Wasser-AP-Dimere aus QTAIM-, NCI-, ELFund NBO-Analysen, gemäß derer die Bindungsstellenpräferenz maßgeblich durch die inäquivalenten freien Elektronenpaare am Carbonyl-Sauerstoffatom bestimmt wird. ${ }^{[51]}$ Für die im Rahmen der vorliegenden Arbeit betrachteten Methanol-Acetophenon(derivat)Waagensysteme spielen die stärkeren Dispersionswechselwirkungen in den Ph-Dimeren dennoch eine wichtige Rolle für die Stabilisierung gegenüber den Me-Dimeren, da die Energiedifferenzen zwischen Me- und Ph-Dimer hierdurch in Bereiche wandern, in denen signifikante Mengen Ph-Dimer im Experiment beobachtet werden können. Basierend auf den Ergebnissen der LED-Analyse scheinen Dispersionswechselwirkungen also einen entscheidenden Beitrag zur Attraktivität der Ph-Andockstelle der betrachteten Acetophenonderivate für den $\mathrm{MeOH}$-Donor zu liefern. Für $\mathrm{MeOH}+\mathrm{AP}$ bestätigt dies die Ergebnisse der Studie von Alkyl-substituierten Acetophenonderivaten mit Methanol und tert-Butylalkohol als Donoren, wobei sich für die weiteren dort betrachteten Systeme je nach Donor und Akzeptor sowohl Alkyl- als auch Phenyl-Seiten-Präferenzen in den Dispersionswechselwirkungen ergeben. ${ }^{[52]}$

\subsection{Zusammenfassung}

Acetophenon bietet mit den beiden freien Elektronenpaaren am Carbonyl-Sauerstoffatom zwei attraktive Andockstellen für Wasserstoffbrückenbindungsdonoren wie Methanol. Durch die unterschiedlichen Substituenten (Me, Ph) auf beiden Seiten der Carbonylgruppe entstehen bei Ausbildung der Wasserstoffbrückenbindung je nach Bindungsseite ver- 
schiedene $\mathrm{OH} \cdots \mathrm{O}$-gebundene Dimere, die sich FTIR-spektroskopisch über die Größe der Rotverschiebungen der OH-Streckschwingungsbanden unterscheiden lassen. Die Unterschiede in den Rotverschiebungen (experimentell $34-38 \mathrm{~cm}^{-1}$ ) können dabei auf verschiedene $\mathrm{C}=\mathrm{O} \cdots \mathrm{H}$-Bindungswinkel $\alpha$, die sich aus den unterschiedlichen sterischen Ansprüchen von $\mathrm{Me}$ - und $\mathrm{Ph}$-Substituent ergeben, zurückgeführt werden. Der Energieunterschied zwischen Me- und Ph-Dimer ist ausreichend klein, sodass sich beide Dimere in einer Überschallexpansion nachweisen lassen. Durch Fluorierung der Phenylgruppe in ortho- (2FAP) bzw. para-Position (4FAP) lässt sich das Me/Ph-Konformerengleichgewicht beeinflussen, wobei in beiden Fällen das Ph-gebundene Dimer relativ zum Me-gebundenen Dimer stabilisiert wird.

Anhand der auf Basis dieses Carbonylwaagensystems gewonnenen experimentellen Daten zu Intensitätsverhältnis, Rotverschiebungen und Aufspaltungen der $\mathrm{OH}$-Streckschwingungsbanden von Me- und Ph-Dimer kann die Leistungsfähigkeit verschiedener quantenchemischer Methoden hinsichtlich der Beschreibung der relativen Energien und der genannten IR-spektroskopischen Parameter untersucht werden. Dazu wurden mehrere DFT-Methoden herangezogen und zusätzlich jeweils mit Einzelpunktrechnungen auf DLPNO-CCSD(T)-Niveau kombiniert. Während für $\mathrm{MeOH}+\mathrm{AP}$ und seine Isotopologe nahezu alle getesteten Methoden dazu in der Lage sind, das Me/Ph-Konformerengleichgewicht treffend zu beschreiben, zeigt sich für die fluorierten Derivate $\mathrm{MeOH}+2 \mathrm{FAP}$ bzw. $\mathrm{MeOH}+4 \mathrm{FAP}$ eine deutlich stärkere Diskrimination der Ergebnisse der quantenchemischen Rechnungen. Die fluorierten Derivate ermöglichen somit einen empfindlicheren Benchmark.

Das gleichmäßige Bandenmuster über die verschiedenen Acetophenonderivate hinweg lässt auf recht konstante anharmonische Beiträge zu Rotverschiebungen bzw. Bandenaufspaltungen schließen. Nach einer Rückkorrektur auf die harmonischen Anteile der im Experiment beobachteten Größen wird deshalb ein Vergleich zu den Ergebnissen der Schwingungsrechnungen in doppelt-harmonischer Näherung möglich. Durch die Betrachtung zweier gleichartiger $\mathrm{OH} \cdots \mathrm{O}$-Wasserstoffbrückenbindungen im vorliegenden $\mathrm{Me} / \mathrm{Ph}$-Konformerengleichgewicht ist die diskriminatorische Leistung des Benchmarks geringer als für die O/C-Waagensysteme (s. Kap. 4), sodass nahezu alle Methoden mit dem Experiment kompatible Ergebnisse erzielen. Die $\overline{\delta_{\mathrm{Xn}}^{2}}$-Werte ermöglichen dennoch eine Abstufung der Qualität der Ergebnisse der quantenchemischen Rechnungen.

Insgesamt zeigen mit B2PLYP-D3(BJ,ABC)/a'VTZ und PBEh-3c nur zwei der sechs betrachteten DFT-Methoden sowohl im energetischen als auch im IR-spektroskopischen Benchmark durchweg gute Ergebnisse. Während das M06-2X-Funktional analog zum ENCH-51-Benchmark ${ }^{[33]}$ in letzterem sehr gut abschneidet, versagt es in ersterem teilweise qualitativ. Die Ergebnisse des dispersionskorrigierten B3LYP-Funktionals sind zwar mit dem kleineren der beiden betrachteten Basissätze (B3LYP-D3(BJ,ABC)/def2TZVP) überall mit den experimentellen Befunden kompatibel, liegen für die Konformationstemperaturen jedoch teilweise außerhalb des Erwartungsbereichs. Für B3LYPD3(BJ,ABC)/def2-QZVP ergibt sich zudem für die Bandenaufspaltungen ein Ergebnis 
außerhalb des Zielbereichs. Dies lässt sich vermutlich auf Probleme in der Bewertung der relativen Stabilität der $\mathrm{Ph}_{\mathrm{ip}}$ - und $\mathrm{Ph}_{\mathrm{op}}$-Dimere zurückführen, wie die Verbesserung des Ergebnisses bei durchgängiger Verwendung der $\mathrm{Ph}_{\mathrm{ip}}$-Rotverschiebungen zeigt. Das bessere Abschneiden der B3LYP-D3- im Vergleich zur M06-2X-Methode im Benchmark relativer Energien deckt sich mit den Befunden für das Methanol-Anisol-Waagensystem (s. Kap. 4.2) und weiteren Untersuchungen. ${ }^{[33,89]}$ Die B97-3c-Methode versagt sowohl für den energetischen als auch den IR-spektroskopischen Benchmark. Für die Methoden B3LYP-D3(BJ,ABC)/def2-TZVP, M06-2X/def2-TZVP und B97-3c bestätigt dies im Wesentlichen die Ergebnisse der bestehenden Studie mehrerer Alkohol-Acetophenon(derivat)Dimere. ${ }^{[52]}$ PBEh-3c zeigt dort jedoch für die meisten Alkohol-Acetophenon(derivat)Kombinationen abseits von $\mathrm{MeOH}+\mathrm{AP}$ eine deutlich schwächere Leistung als für die im Rahmen der vorliegenden Arbeit untersuchten Carbonylwaagen.

Das gute Abschneiden der empirisch parametrisierten PBEh-3c-Methode im vorliegenden Benchmark kann durch die Berechnung der elektronischen Energien auf DLPNO-CCSD(T)/aVQZ-Niveau wie erwartet auf Fehlerkompensation zurückgeführt werden. Zum einen offenbart die Betrachtung der relativen elektronischen DLPNO$\operatorname{CCSD}(\mathrm{T})$-Energien der auf den verschiedenen DFT-Niveaus optimierten Strukturen, dass PBEh-3c die am wenigsten stabilen Strukturen lokalisiert und zum anderen führt die Kombination mit elektronischen Energien auf DLPNO-CCSD $(\mathrm{T}) / \mathrm{aVQZ-Niveau} \mathrm{zu}$ deutlich schlechteren Ergebnissen als für das „reine“ PBEh-3c. Generell ergeben sich durch die Kombination von Geometrieoptimierung und Berechnung der ZPVE auf DFTmit Einzelpunktrechnungen für die elektronische Energie auf Coupled-Cluster-Niveau im energetischen Benchmark der Carbonylwaagensysteme zumindest für die hier verwendeten Kombinationen von DFT- und DLPNO-CCSD(T)-Methoden keine systematischen Verbesserungen gegenüber der vollständigen Berechnung auf DFT-Niveau. Dies gilt auch, wenn bei deutlich gesteigertem Rechenaufwand Kernelektronen mitkorreliert oder eine andere Lokalisierungsmethode (PIPEK-MEzEY) genutzt werden. Nur für die Berechnung von elektronischen Energien auf DLPNO-CCSD(T)/aVQZ-Niveau für die auf diesem Niveau stabilsten Dimerstrukturen (Optimierung und Berechnung der ZPVE auf B3LYP-D3(BJ,ABC)/def2-QZVP- bzw. B2PLYP-D3(BJ,ABC)/a'VTZ-Niveau) zeigen sich durchgehend Konformationstemperaturen im Erwartungsbereich. Die Ergebnisse für die weiteren Methoden verschlechtern sich mit Berücksichtigung der DLPNO$\operatorname{CCSD}(\mathrm{T})$-Einzelpunktrechnungen hingegen. Hier zeigt sich, dass solche Kombinationen von elektronischen Energien auf Basis korrelierter Wellenfunktionsmethoden mit Strukturoptimierung und ZPVE-Berechnung auf DFT-Niveau nur funktionieren, wenn sich die Potentialhyperflächen der kombinierten Niveaus ausreichend stark ähneln. Die Idealvorstellung, auf hohen Theorieniveaus berechnete elektronische Energiedifferenzen möglichst direkt anhand experimenteller Daten zu benchmarken, ist also alles andere als trivial zu erreichen, zumal auch für die Carbonylwaagen noch eine Schätzung des Einflusses der ZPVE nötig ist. Zwar scheint die Berücksichtigung anharmonischer Effekte in den Rechnungen für die untersuchten Systeme nicht nötig zu sein und die Größe der ZPVE-Einflüsse auf die Energiedifferenz bleibt mit maximal $0.6 \mathrm{~kJ} \cdot \mathrm{mol}^{-1}$ geringer 
als für die O/C-Waagensysteme, die Berücksichtigung der harmonischen ZPVE-Effekte sorgt aber teilweise für Wechsel der Bindungsstellenpräferenz und damit für qualitative Unterschiede im Vergleich zur rein elektronischen Energiedifferenz.

Die auf den DLPNO-CCSD(T)/aVQZ-Rechnungen basierende LED-Analyse auf Grundlage der auf B3LYP-D3(BJ,ABC)/def2-QZVP- bzw. B2PLYP-D3(BJ,ABC)/a'VTZ-Niveau optimierten Strukturen zeigt, dass Dispersionswechselwirkungen in den betrachteten Methanol-Acetophenon(derivat)-Dimeren zwar in keinem Fall die Bindungsstellenpräferenz entscheidend beeinflussen, wohl aber wichtige Beiträge zur Stabilisierung der Ph- gegenüber den Me-Dimeren liefern. Für die beiden betrachteten Rechenniveaus ergeben sich dabei zum Teil recht unterschiedlich große relative Stabilisierungen, sodass der Einflussgrad der Dispersion auf das Me/Ph-Gleichgewicht unklar bleibt. Generell kann die Aussagekraft der LED-Ergebnisse nicht abschließend beurteilt werden, da eine Verifizierung der erhaltenen Größen anhand experimenteller Daten nicht möglich ist. Wie auch im Fall der Methanol-Anisol-Waagensysteme wäre somit eine Überprüfung anhand unabhängiger theoretischer Methoden zur Bestimmung der unterschiedlichen Beiträge zur Bindungsenergie ${ }^{[16-19,21]}$ wünschenswert.

Ungeklärt bleibt auf Basis der vorliegenden Daten auch die genaue Struktur der Meund Ph-Dimere, sowohl hinsichtlich der ip/op-Präferenz als auch in Bezug auf die Symmetrie der ip-Dimere. Die betrachteten quantenchemischen Methoden stufen zum Teil ip-, zum Teil aber auch op-Strukturen als stabilste Konformation einer Bindungsseite ein. Werden ip-Strukturen als Minimumsstruktur lokalisiert, weichen diese formal in nahezu allen Fällen leicht von einer $C_{s}$-symmetrischen Anordnung ab. Die Verbesserung des Abschneidens der B3LYP-D3(BJ,ABC)/def2-QZVP-Methode im IR-spektroskopischen Benchmark bei der einheitlichen Verwendung der Daten für ip-Strukturen und die zunehmende relative Stabilität der ip- gegenüber den op-Strukturen bei Berechnung der elektronischen Energien auf DLPNO-CCSD(T)-Niveau deuten auf eine durchgängige ip-Präferenz hin. Hinsichtlich dieser ip-Strukturen legen die Ergebnisse eine tatsächliche oder effektive $C_{s}$-Symmetrie nahe. So liegen die relativen Energien der $C_{s}$-symmetrischen (Übergangs-)Strukturen gegenüber den $C_{1}$-symmetrischen ip-Minima zumindest mit elektronischen DLPNO-CCSD(T)-Energien in allen betrachteten Fällen unterhalb der ZPVE der niederfrequentesten Symmetrisierungsmode. Zudem schneiden die B3LYP-D3(BJ,ABC)/ de2-QZVP- und B2PLYP-D3(BJ,ABC)/a'VTZ-Methoden im energetischen Benchmark besser $a b$, wenn die formale Symmetrie der ip-Minimumsstrukturen durch eine einheitliche Annahme von $C_{s}$-Symmetrie ersetzt wird. Während die quantenchemischen Rechenmethoden somit überwiegend kompaktere und dafür weniger planare Strukturen bevorzugen, sei es in Form $C_{1}$-symmetrischer ip- oder gar noch stärker unplanarer op-Strukturen, deuten die experimentellen Ergebnisse eher auf planarere Anordnungen hin. Für eine eindeutige Identifizierung der im Experiment vorliegenden Strukturen und Symmetrien der Me- und möglicherweise auch der metastabilen Ph-Dimere sind jedoch Jet-mikrowellenspektroskopische Untersuchungen nötig. Dabei ist allerdings zu berücksichtigen, dass auch Mikrowellenspektroskopie nicht ohne Weiteres eindeutig zwischen Planarität und Quasiplanarität des Schweratomgerüsts unterscheiden kann. ${ }^{[80]}$ Dennoch könnte sie 
wie bei der Furan-Mikrosolvatation (s. Kap. 4.1) strukturelle Vermutungen auf der Basis von IR-Korrelationen zumindest am Einzelbeispiel erhärten. Neben wichtigen qualitativen Aufklärungen könnte Jet-Mikrowellenspektroskopie zusätzliche quantitative Daten für den Benchmark der quantenchemischen Methoden liefern, z. B. zu Rotationskonstanten oder auch Bindungswinkeln. Aufseiten der quantenchemischen Rechnungen wären ebenfalls zusätzliche Untersuchungen nötig, um die Gründe für die Bevorzugung kompakterer, unplanarer Strukturen aufzuklären. Da diese vor allem für die Methoden mit kleineren Basissätzen ausgeprägt vorhanden ist, könnten Basissatzüberlagerungsfehler eine wichtige Rolle spielen. Ohne systematische Untersuchungen zur Basissatzkonvergenz für verschiedene Methoden sind hierzu jedoch keine abschließenden Aussagen möglich.

Generell ist eine Erweiterung des kompakten, hier vorgestellten Benchmark-Datensatzes (drei Methanol-Acetophenon(derivat)-Kombinationen sowie drei Isotopologe) auf weitere Carbonylwaagensysteme angezeigt. Für die PBEh-3c-Methode verdeutlicht das schlechte Abschneiden für auf der Alkylseite substituierte Acetophenonderivate und für tert-Butylalkohol als Donor ${ }^{[52]}$ bereits, dass die Erweiterung eine deutlich bessere Unterscheidung der Leistungsfähigkeit und das Aufdecken von zufälliger Fehlerkompensation ermöglichen kann. Eine Untersuchung der Akzeptoren 2FAP und 4FAP mit tert-Butylalkohol als Donor wäre deshalb ebenso wünschenswert wie die Betrachtung weiterer Akzeptoren wie Pinakolon oder den Chlor- oder Brom-Analoga von 2FAP und 4FAP. Auch die in Ref. [52] vorgeschlagenen Erweiterungen auf weitere Donoren wie Phenol ${ }^{[382]}$ oder Akzeptoren wie Cyclohexylphenylketon ${ }^{[383]}$ könnten vielversprechende Ansätze sein. Die konsistente Untersuchung aller Akzeptoren mit den an der Hydroxylgruppe deuterierten Isotopologen der Donoren, also z. B. Methanol-OD oder tert-Butylakohol-OD, würde analog zum Göbench (s. Kap. 4.1) eine genauere Beurteilung der Beschreibung der ZPVE durch die betrachteten quantenchemischen Methoden ermöglichen. Angesichts der am Gratin-Jet im Laufe der Messzeit ansteigenden Wasserkonzentration (s. Kap. 3.6) wäre zudem eine Erweiterung auf den Donor Wasser denkbar. Das Me-Dimer von Wasser mit Acetophenon wurde bereits mittels Jet-Mikrowellenspektroskopie untersucht, wobei das metastabile Ph-gebundene Dimer jedoch bisher nicht nachgewiesen werden konnte. ${ }^{[51]}$ Bei einem berechneten Energieunterschied von $1.3 \mathrm{~kJ} \cdot \mathrm{mol}^{-1}(\mathrm{MP} 2 / 6-311++\mathrm{G}(2 \mathrm{df}, 2 \mathrm{pd})-$ Niveau) ${ }^{[51]}$ sollte Jet-FTIR-Spektroskopie einen experimentellen Nachweis des Ph-Dimers ermöglichen und somit für diese und auch weitere Wasser-Acetophenonderivat-Kombinationen interessante neue, benchmarkrelevante Daten liefern können. 



\section{Kapitel 6}

\section{Zusammenfassung und Ausblick}

In Jet-FTIR-spektroskopischen Messungen konnten im Rahmen der vorliegenden Arbeit experimentelle Daten zu den relativen Energien und zwei Klassen von IR-spektroskopischen Parametern (Rotverschiebungen und Bandenaufspaltungen) in mehreren wasserstoffbrückengebundenen intermolekularen Waagensystemen gewonnen werden. Alle hier betrachteten Waagensysteme lassen sich durch Substitution am organischen Akzeptor hinsichtlich des Gleichgewichts unterschiedlich gebundener Konformere fein austarieren und ermöglichen so die Gewinnung mehrerer Daten- und damit Vergleichspunkte für das Benchmarking quantenchemischer Methoden. Anhand einer in Kooperation mit mehreren Computerchemie-Gruppen durchgeführten (Doppelblind-)Studie (Kap. 4.1) und zwei beispielhaften eigenen Benchmarks (Kap. 4.2 und Kap. 5) konnte gezeigt werden, dass sich diese experimentellen Daten zur Überprüfung der Leistungsfähigkeit quantenchemischer Rechenmethoden eignen. Zudem konnte mit dem Gratin-Jet (Kap. 3) eine neue Messapparatur für Jet-FTIR-Spektroskopie geplant, aufgebaut und in Betrieb genommen werden, die eine vergleichbare Leistungsfähigkeit wie der erfolgreiche Filet-Jet (Kap. 2.2) aufweist, gleichzeitig aber von Verbesserungen wie einem stark reduzierten Substanzverbrauch, einem längeren Absorptionspfad und einer Heizbarkeit der Düse sowie des gesamten Gaskreislaufs profitiert.

Für die Leistungsüberprüfung quantenchemischer Methoden anhand experimenteller Daten müssen sowohl geeignete Bedingungen als auch geeignete „Treffpunkte“, also physikalische Größen bzw. Observablen, zwischen Theorie und Experiment gewählt werden. ${ }^{[7]}$ Im Rahmen der vorliegenden Arbeit wurde für beide Bereiche (Bedingungen und „Treffpunkte“) versucht, der Theorie möglichst weit entgegen zu kommen. Während die für die Messungen verwendete Helium-Jet-FTIR-Spektroskopie den inhärenten Bedingungen der quantenchemischen Rechnungen durch die Betrachtung kalter, isolierter Moleküle bzw. Aggregate recht nahe kommt, müssen bei der „Treffpunkt“-Wahl stets Kompromisse eingegangen werden. So sind die im Rahmen dieser Arbeit untersuchten IR-spektroskopischen Parameter im Experiment direkt zugänglich. Für deren quantenchemische Berechnung sind jedoch mehrere Rechenschritte (Geometrieoptimierung und anschließende Frequenzrechnung) und zahlreiche, teilweise aufeinander aufbauende Näherungen nötig. Durch die Betrachtung von intermolekularen Waagensystemen, also 
Gleichgewichten aus zwei Konformeren desselben Molekülaggregats, und den Rotverschiebungen sowie Aufspaltungen der OH-Streckschwingungsbanden dieser Aggregate, werden jedoch Größen herangezogen, die stark von Kompensation der im Experiment stets vorhandenen anharmonischen Beiträge profitieren. Dies wird auch an den in allen Fällen über die verschieden substituierten Derivate hinweg recht konsistenten Bandenmustern deutlich. Somit können aufseiten der Theorie Schwingungsrechnungen in doppelt-harmonischer Näherung zur Bestimmung der entsprechenden Größen genutzt werden und ein direkter Vergleich zum Experiment wird z. B. durch eine Rückkorrektur der experimentellen Werte auf ihre harmonischen Anteile ${ }^{[33]}$ möglich. Werden wie für einige der im Rahmen des Göbench untersuchten quantenchemischen Methoden anharmonische Korrekturen berücksichtigt, sorgen diese für eine Verschlechterung in der Übereinstimmung mit dem Experiment. Die Gründe hierfür können jedoch ohne zusätzliche Untersuchungen nicht klar benannt werden.

Kompensieren sich Unterschiede in der Schwingungsnullpunktsenergie (ZPVE) zu einem großen Teil, sodass harmonische Schätzungen der ZPVE ausreichen (oder idealerweise keine ZPVE-Berechnung nötig wird), sind Energiedifferenzen zwischen zwei Konformeren in den quantenchemischen Rechnungen vergleichsweise leicht ermittelbar. Somit lag der Fokus der vorliegenden Arbeit auch auf der Bestimmung experimenteller Referenzdaten für relative Energien. Diese sind aus den Schwingungsspektren nicht direkt zugänglich, können aber über die Verwendung einer vereinfachten BoltzmanN-Formel auf Grundlage der experimentellen Daten ermittelt werden (s. Kap. 2.3.2). Dadurch wird eine Überprüfung der berechneten (schwingungsnullpunktskorrigierten) Energieunterschiede möglich. Obwohl hierzu recht grobe Schätzungen für die in der Überschallexpansion erzielte Konformationstemperatur nötig sind - die weiteren unbekannten Größen (IR-Bandenstärken, Entartungsfaktorenverhältnisse) können ebenfalls geschätzt oder aus den quantenchemischen Rechnungen entnommen werden - kann dieser Ansatz erfolgreich für energetische Benchmarks genutzt werden. Problematische Fälle wie das Dimer von Methanol-OD mit Acetophenon, bei denen das zugrundeliegende Intensitätsverhältnis der betrachteten Dimerbanden nur sehr ungenau bestimmt werden kann, verdeutlichen jedoch, dass die experimentelle Unsicherheit des Intensitätsverhältnisses dabei nicht zu groß sein darf. Dies lässt sich abseits solch unglücklicher Fälle, in denen Bandenüberlappung vorliegt, in der Regel durch die Mittelung einer ausreichenden Zahl von Spektrometerscans erreichen. Am bisher genutzten Filet-Jet geht dies jedoch mit einem hohen Substanzverbrauch einher.

Hier verspricht der neu errichtete Gratin-Jet mit seinem Gaskreislaufsystem Besserung, sodass dieser zukünftig womöglich wichtige Beiträge zur Gewinnung experimenteller Referenzdaten liefern kann. Systematische Verfälschungen der ermittelten Bandenintensitäten durch Überlagerung mit durch die ansteigende Wasserkonzentration verursachten Störsignalen müssen dazu jedoch sicher ausgeschlossen werden können, z. B. durch Differenzbildung zu Spektren mit definiertem Wasseranteil, gezielter Messung von WasserAggregaten oder Behebung des Problems der ansteigenden Wasserkonzentration. Ein Ansatz für Letzteres könnte eine Teildeuterierung des Kühlmittels der Schraubenpumpe sein, 
wodurch ein mögliches Leck zwischen Kühlmittelkreislauf und Schöpfraum durch eindringendes deuteriertes Wasser eindeutig nachzuweisen wäre. Ein weiterer Ansatz wäre die Ausdehnung der Kreislaufheizung auf bisher ungeheizte Stellen wie Ventile oder die Verrohrungen des Wälzkolbenpumpstands, um mögliche Adsorption (und darauf im Kreislaufbetrieb folgende kontinuierliche Desorption) größerer Mengen Wasser an den Oberflächen in diesen Bereichen durch einen verbesserten Ausheiz-/Evakuierbetrieb zu unterbinden.

Aufseiten der betrachteten quantenchemischen Methoden erweisen sich Kombinationen von hohen Theorieniveaus (hier insbesondere $\operatorname{CCSD}(\mathrm{T})$-Varianten) für die Berechnung der elektronischen Energie mit niedrigeren Theorieniveaus wie DFT-basierten Methoden zur Geometrieoptimierung und Frequenzrechnung prinzipiell als erfolgreich. Dies gilt allerdings nur, wenn die für die Geometrieoptimierung eingesetzte Methode in der Lage ist, Minimumsstrukturen zu lokalisieren, die auf dem höheren Niveau ebenfalls Punkte vergleichsweise niedriger Energie auf der Potentialhyperfläche darstellen. In allen Benchmarks als erfolgreich erwies sich dabei die Kombination des DLPNO-CCSD(T)-Verfahrens zur Berechnung der elektronischen Energie mit einer Strukturoptimierung und Frequenzrechnung durch das dispersionskorrigierte B3LYP-Funktional. Weitere quantenchemische Methoden wurden jeweils nur auf einen Teil der vorgestellten experimentellen Benchmarks angewandt, wobei die DLPNO-CCSD(T)//B2PLYP-D3-Kombination durch ein gutes Abschneiden sowohl für die Furan-Mikrosolvatation als auch für die Carbonylwaagen positiv und das M06-2X-Funktional durch ein schlechtes Abschneiden (vor allem in den energetischen Benchmarks) für die Anisol- und Carbonylwaagen negativ hervorzuheben sind.

Während das Gleichgewicht zwischen den primären Bindungsstellen für die Wasserstoffbrückenbindungen in allen Fällen mittels Jet-FTIR-Spektroskopie quantifiziert werden konnte, bleiben die für die jeweilige Bindungsstelle vorliegenden experimentellen Strukturtypen mit dieser Technik größtenteils unklar. Für die im Rahmen der vorliegenden Arbeit betrachteten Carbonylwaagen ergeben sich zwar aus den Schwingungsspektren Hinweise auf eine konsistente Bevorzugung planarer Strukturen, definitive Aufklärung können jedoch nur Jet-mikrowellenspektroskopische Daten bringen. Mit diesen konnten die experimentellen Strukturen der O-Dimere von Methanol mit Furan bzw. 2,5Dimethylfuran dem von oberhalb der Ringebene gebundenen Strukturtypen zugeordnet werden. Ein experimenteller Nachweis des metastabilen C-gebundenen Dimers gelang in den Rotationsspektren jedoch nur für Furan, wohingegen mit Jet-FTIR-Spektroskopie benchmarkübergreifend in nahezu allen betrachteten Fällen signifikante Mengen des jeweils metastabilen Dimers beobachtet werden konnten. Jet-mikrowellenspektroskopische Daten zu den weiteren untersuchten Systemen wären für die Strukturaufklärung und zur Gewinnung weiterer benchmarkrelevanter Daten (Rotationskonstanten, Bindungswinkel) von großem Wert. Generell stellen multispektroskopische Ansätze ein wichtiges Werkzeug zur Gewinnung komplementärer experimenteller Daten dar, was sowohl eine wechselseitige Überprüfung der Ergebnisse als auch einen umfassenderen Blick auf die Eigenschaften des untersuchten Systems und damit auch die Gewinnung vielseitigerer 
Referenzdatensätze ermöglicht.

Auch zur genaueren Untersuchung der auf Basis der FTIR-spektroskopischen Daten ungeklärten Symmetrieverhältnisse in den Dimeren könnte Jet-Mikrowellenspektroskopie einen wertvollen Beitrag leisten. Während für das Anisolwaagensystem alle Dimere eindeutig $C_{1}$-Symmetrie aufweisen, können sich im Methanol-Furan- und im MethanolAcetophenon-Waagensystem sowohl $C_{1}$ - als auch $C_{s}$-symmetrische Dimere ergeben. Über die Entartungsfaktorenverhältnisse in der zum energetischen Benchmarking genutzten BolTzMAnN-Verteilung wird die Dimersymmetrie in der Auswertung berücksichtigt. Da für die Entartungsfaktorenverhältnisse nur diskrete Werte möglich sind, ergibt sich im vereinfachten, für die Auswertung genutzten Modell die Notwendigkeit einer strikten Unterscheidung der Punktgruppen. Während aufseiten des Experiments mit Jet-FTIR-Spektroskopie allenfalls Hinweise auf die Dimersymmetrie gewonnen werden können, kann Jet-Mikrowellenspektroskopie die auf der Zeitskala des Experiments vorliegende (effektive) Symmetrie bestimmen. Selbst wenn hierbei für ein gegebenes Dimer $C_{s}$-Symmetrie ermittelt wird, widerspricht dies jedoch nicht zwingend $C_{1}$-symmetrischen Minimumsstrukturen, wie sie in fast allen Fällen von den quantenchemischen Methoden vorhergesagt werden. So könnte beispielsweise ein doppelmuldiges Potential vorliegen, in dem zwei leicht asymmetrische Minima über eine flache Barriere mit einem $C_{s}$-symmetrischen Übergangszustand voneinander getrennt sind, sodass sich auf der Zeitskala des Experiments effektive $C_{s}$-Symmetrie einstellt. Die Beurteilung, ob ein $C_{1}$-symmetrisches Minimum aus einer quantenchemischen Rechnung mit einer im Experiment festgestellten $C_{s^{-}}$ Symmetrie kompatibel ist, kann somit nicht allein auf Basis der Minimumsstruktur erfolgen. Ein mögliches Beurteilungskriterium stellt der Vergleich zwischen Barrierenhöhe und ZPVE der Symmetrisierung (s. Kap. 5.3.2) dar.

Für die Anisol- und Carbonyl-Waagensyteme konnte der Einfluss von Dispersionswechselwirkungen mit dem im Rahmen des DLPNO-CCSD(T)-Verfahrens zur Verfügung stehenden LED-Konzept untersucht werden. Während die Bindungsstellenpräferenz hierbei in keinem Fall maßgeblich durch Dispersionswechselwirkungen bestimmt wird, zeigt sich dennoch bereits für den kompakten Donor Methanol, dass Dispersionswechselwirkungen signifikante Einflüsse auf die relative Stabilität der betrachteten Bindungsstellen untereinander haben können. Für voluminösere Donoren wie tert-Butylalkohol wären hier mutmaßlich noch stärkere Effekte zu erwarten. Interessant wäre zudem eine Bestätigung der mit dem LED-Konzept erhaltenen Befunde durch weitere, unabhängige Rechenverfahren.

Nachdem für die drei hier betrachteten Waagensysteme demonstriert werden konnte, dass sie sich als Grundlage für das Benchmarking quantenchemischer Methoden eignen, wäre eine Erweiterung der hier gezeigten Datenbasis um weitere Systeme und weitere Messgrößen wünschenswert. Erst durch eine Vielzahl möglichst unterschiedlicher Systeme können umfangreiche experimentelle Referenzdatensätze wie beispielsweise ENCH$51^{[33]}$ erhalten werden, die eine breitgefächerte Spanne verschiedener inter- und intramolekularer Wechselwirkungen abdecken. So kann ein Beitrag dazu geleistet werden, die Beurteilung neuentwickelter quantenchemischer Methoden wieder mehr auf experimen- 
telle Daten zu stützen. 



\section{Literatur}

[1] W. Thiel, „Theoretical Chemistry-Quo Vadis?", Angew. Chem. Int. Ed. 2011, 50, 9216-9217, DOI 10.1002/anie.201105305.

[2] G. E. Moore, „Cramming more components onto integrated circuits, Reprinted from Electronics, Volume 38, Number 8, April 19, 1965, pp.114 ff“, IEEE Solid-State Circuits Soc. Newsl. 2006, 11, 33-35, DOI 10.1109/N-SSC.2006.4785860.

[3] M. G. Medvedev, I. S. Bushmarinov, J. Sun, J. P. Perdew, K. A. Lyssenko, „Density functional theory is straying from the path toward the exact functional", Science 2017, 355, 49-52, DOI 10.1126/science. aah5975.

[4] K. P. Kepp, „Comment on "Density functional theory is straying from the path toward the exact functional”“" Science 2017, 356, 496, DOI 10.1126/science. aam9364.

[5] M. G. Medvedev, I. S. Bushmarinov, J. Sun, J. P. Perdew, K. A. Lyssenko, „Response to Comment on "Density functional theory is straying from the path toward the exact functional”", Science 2017, 356, 496, DOI 10.1126/science aam9550.

[6] M. Korth, „Density Functional Theory: Not Quite the Right Answer for the Right Reason Yet“, Angew. Chem. Int. Ed. 2017, 56, 5396-5398, DOI 10 . 1002 / anie . 201701894.

[7] R. A. Mata, M. A. Suhm, „Benchmarking Quantum Chemical Methods: Are We Heading in the Right Direction?", Angew. Chem. Int. Ed. 2017, 56, 11011-11018, DOI 10.1002/anie.201611308.

[8] S. Grimme, P. R. Schreiner, „Computational Chemistry: The Fate of Current Methods and Future Challenges“, Angew. Chem. Int. Ed. 2018, 57, 4170-4176, DOI 10 . 1002/anie. 201709943.

[9] G. Sliwoski, S. Kothiwale, J. Meiler, E. W. Lowe, „Computational Methods in Drug Discovery“, Pharmacol. Rev. 2014, 66, 334-395, DOI 10.1124/pr.112.007336.

[10] T. Weymuth, M. Reiher, „Inverse Quantum Chemistry: Concepts and Strategies for Rational Compound Design“, Int. F. Quantum Chem. 2014, 114, 823-837, DOI $10.1002 /$ qua. 24687.

[11] Y. Cao, J. Romero, A. Aspuru-Guzik, „Potential of quantum computing for drug discovery“, IBM F. Res. \& Dev. 2018, 62, 1-20, DOI 10.1147/JRD. 2018. 2888987. 
[12] K. D. Vogiatzis, M. V. Polynski, J. K. Kirkland, J. Townsend, A. Hashemi, C. Liu, E. A. Pidko, „Computational Approach to Molecular Catalysis by $3 \mathrm{~d}$ Transition Metals: Challenges and Opportunities“, Chem. Rev. 2019, 119, 2453-2523, DOI 10. 1021/acs . chemrev. 8b00361.

[13] (a) L. Himanen, A. Geurts, A. S. Foster, P. Rinke, „Data-Driven Materials Science: Status, Challenges, and Perspectives“, Adv. Sci. 2019, 6, 1900808, DOI 10.1002 / advs . 201900808; (b) L. Himanen, A. Geurts, A. S. Foster, P. Rinke, „Erratum: DataDriven Materials Science: Status, Challenges, and Perspectives“, Adv. Sci. 2020, 7, 1903667, DOI 10.1002/advs. 201903667.

[14] (a) R. Hoffmann, J.-P. Malrieu, „Simulation vs. Understanding: A Tension, in Quantum Chemistry and Beyond. Part A. Stage Setting“, Angew. Chem. Int. Ed. 2020, 59, 12590-12610, DOI 10.1002/anie.201902527; (b) R. Hoffmann, J.-P. Malrieu, „Simulation vs. Understanding: A Tension, in Quantum Chemistry and Beyond. Part B. The March of Simulation, for Better or Worse", Angew. Chem. Int. Ed. 2020, 59, 13156-13178, DOI 10.1002 / anie . 201910283; (c) R. Hoffmann, J.-P. Malrieu, „Simulation vs. Understanding: A Tension, in Quantum Chemistry and Beyond. Part C. Toward Consilience“, Angew. Chem. Int. Ed. 2020, 59, 1369413710, DOI 10.1002/anie.201910285.

[15] F. Neese, „High-Level Spectroscopy, Quantum Chemistry, and Catalysis: Not just a Passing Fad“, Angew. Chem. Int. Ed. 2017, 56, 11003-11010, DOI 10.1002/anie. 201701163.

[16] B. Jeziorski, R. Moszynski, K. Szalewicz, „Perturbation Theory Approach to Intermolecular Potential Energy Surfaces of van der Waals Complexes“, Chem. Rev. 1994, 94, 1887-1930, DOI 10.1021/cr00031a008.

[17] A. Heßelmann, G. Jansen, M. Schütz, „Density-functional theory-symmetryadapted intermolecular perturbation theory with density fitting: A new efficient method to study intermolecular interaction energies“, f. Chem. Phys. 2005, 122, 014103, DOI 10.1063/1.1824898.

[18] K. Szalewicz, „Symmetry-adapted perturbation theory of intermolecular forces“, WIREs Comput. Mol. Sci. 2012, 2, 254-272, DOI 10.1002/wcms . 86.

[19] G. Jansen, „Symmetry-adapted perturbation theory based on density functional theory for noncovalent interactions“, WIREs Comput. Mol. Sci. 2014, 4, 127-144, DOI $10.1002 /$ wcms. 1164 .

[20] W. B. Schneider, G. Bistoni, M. Sparta, M. Saitow, C. Riplinger, A. A. Auer, F. Neese, „Decomposition of Intermolecular Interaction Energies within the Local Pair Natural Orbital Coupled Cluster Framework“, f. Chem. Theory Comput. 2016, 12, 4778-4792, DOI 10.1021/acs.jctc.6b00523.

[21] A. Wuttke, R. A. Mata, „Visualizing Dispersion Interactions Through the Use of Local Orbital Spaces“, f. Comput. Chem. 2017, 38, 15-23, DOI 10.1002/jcc. 24508. 
[22] A. Altun, F. Neese, G. Bistoni, „Local energy decomposition analysis of hydrogenbonded dimers within a domain-based pair natural orbital coupled cluster study", Beilstein f. Org. Chem. 2018, 14, 919-929, DOI 10.3762/bjoc.14.79.

[23] A. Altun, F. Neese, G. Bistoni, „Effect of Electron Correlation on Intermolecular Interactions: A Pair Natural Orbitals Coupled Cluster Based Local Energy Decomposition Study“, f. Chem. Theory Comput. 2019, 15, 215-228, DOI 10.1021 / acs.jctc. 8b00915.

[24] J. F. Stanton, J. Gauss, „A Discussion of Some Problems Associated with the Quantum Mechanical Treatment of Open-Shell Molecules" in Advances in Chemical Physics, (Hrsg.: I. Prigogine, S. A. Rice), John Wiley \& Sons, Inc, Hoboken, USA, 2003, S. 101-146, DOI 10.1002/0471428027.ch2.

[25] M. A. Iron, M. Oren, J. M. L. Martin, „Alkali and alkaline earth metal compounds: core-valence basis sets and importance of subvalence correlation“, Mol. Phys. 2003, 101, 1345-1361, DOI 10.1080/0026897031000094498.

[26] K. Raghavachari, G. W. Trucks, J. A. Pople, M. Head-Gordon, „A fifth-order perturbation comparison of electron correlation theories“, Chem. Phys. Lett. 1989, 157, 479-483, DOI 10.1016/S0009-2614(89)87395-6.

[27] D. E. Taylor, J. G. Ángyán, G. Galli, C. Zhang, F. Gygi, K. Hirao, J. W. Song, K. Rahul, O. Anatole von Lilienfeld, R. Podeszwa, I. W. Bulik, T. M. Henderson, G. E. Scuseria, J. Toulouse, R. Peverati, D. G. Truhlar, K. Szalewicz, „Blind test of densityfunctional-based methods on intermolecular interaction energies“, f. Chem. Phys. 2016, 145, 124105, DOI 10.1063/1.4961095.

[28] P. Morgante, R. Peverati, „ACCDB: A Collection of Chemistry DataBases for Broad Computational Purposes“, f. Comput. Chem. 2019, 40, 839-848, DOI 10.1002/jcc. 25761.

[29] N. O. C. Winter, N. K. Graf, S. Leutwyler, C. Hättig, „Benchmarks for 0-0 transitions of aromatic organic molecules: DFT/B3LYP, ADC(2), CC2, SOS-CC2 and SCS-CC2 compared to high-resolution gas-phase data", Phys. Chem. Chem. Phys. 2013, 15, 6623-6630, DOI 10.1039/C2CP42694C.

[30] A. M. Reilly, R. I. Cooper, C. S. Adjiman, S. Bhattacharya, A. D. Boese, J. G. Brandenburg, P. J. Bygrave, R. Bylsma, J. E. Campbell, R. Car, D. H. Case, R. Chadha, J. C. Cole, K. Cosburn, H. M. Cuppen, F. Curtis, G. M. Day, R. A. DiStasio, A. Dzyabchenko, B. P. van Eijck, D. M. Elking, J. A. van den Ende, J. C. Facelli, M. B. Ferraro, L. Fusti-Molnar, C. A. Gatsiou, T. S. Gee, R. de Gelder, L. M. Ghiringhelli, H. Goto, S. Grimme, R. Guo, D. W. M. Hofmann, J. Hoja, R. K. Hylton, L. Iuzzolino, W. Jankiewicz, D. T. de Jong, J. Kendrick, N. J. J. de Klerk, H. Y. Ko, L. N. Kuleshova, X. Li, S. Lohani, F. J. J. Leusen, A. M. Lund, J. Lv, Y. Ma, N. Marom, A. E. Masunov, P. McCabe, D. P. McMahon, H. Meekes, M. P. Metz, A. J. Misquitta, S. Mohamed, B. Monserrat, R. J. Needs, M. A. Neumann, J. Nyman, S. Obata, H. Oberhofer, A. R. 
Oganov, A. M. Orendt, G. I. Pagola, C. C. Pantelides, C. J. Pickard, R. Podeszwa, L. S. Price, S. L. Price, A. Pulido, M. G. Read, K. Reuter, E. Schneider, C. Schober, G. P. Shields, P. Singh, I. J. Sugden, K. Szalewicz, C. R. Taylor, A. Tkatchenko, M. E. Tuckerman, F. Vacarro, M. Vasileiadis, A. Vazquez-Mayagoitia, L. Vogt, Y. Wang, R. E. Watson, G. A. de Wijs, J. Yang, Q. Zhu, C. R. Groom, „Report on the sixth blind test of organic crystal structure prediction methods“, Acta Cryst. B 2016, 72, 439-459, DOI 10.1107/S2052520616007447.

[31] A. Poblotzki, H. C. Gottschalk, M. A. Suhm, „Tipping the Scales: Spectroscopic Tools for Intermolecular Energy Balances“, f. Phys. Chem. Lett. 2017, 8, 5656-5665, DOI 10.1021/acs.jpclett.7b02337.

[32] D. Bernhard, F. Dietrich, M. Fatima, C. Pérez, H. C. Gottschalk, A. Wuttke, R. A. Mata, M. A. Suhm, M. Schnell, M. Gerhards, „The phenyl vinyl ether-methanol complex: a model system for quantum chemistry benchmarking“, Beilstein f. Org. Chem. 2018, 14, 1642-1654, DOI 10.3762/bjoc.14.140.

[33] S. Oswald, M. A. Suhm, „Soft experimental constraints for soft interactions: a spectroscopic benchmark data set for weak and strong hydrogen bonds", Phys. Chem. Chem. Phys. 2019, 21, 18799-18810, DOI 10.1039/C9CP03651B.

[34] K. A. E. Meyer, M. A. Suhm, „Stretching of cis-formic acid: warm-up and cooldown as molecular work-out", Chem. Sci. 2019, 10, 6285-6294, DOI 10 . 1039 / C9SC $01555 \mathrm{H}$.

[35] R. Knochenmuss, R. K. Sinha, S. Leutwyler, „Benchmark Experimental Gas-Phase Intermolecular Dissociation Energies by the SEP-R2PI Method“, Annu. Rev. Phys. Chem. 2020, 71, 189-211, DOI 10.1146/annurev-physchem-050317-014224.

[36] H. Sasaki, S. Daicho, Y. Yamada, Y. Nibu, „Comparable Strength of OH-O versus $\mathrm{OH}-\pi$ Hydrogen Bonds in Hydrogen-Bonded 2,3-Benzofuran Clusters with Water and Methanol“, f. Phys. Chem. A 2013, 117, 3183-3189, DOI 10.1021/jp400676x.

[37] H. C. Gottschalk, J. Altnöder, M. Heger, M. A. Suhm, „Control over the HydrogenBond Docking Site in Anisole by Ring Methylation", Angew. Chem. Int. Ed. 2016, 55, 1921-1924, DOI 10.1002/anie.201508481.

[38] R. E. Smalley, L. Wharton, D. H. Levy, „Molecular Optical Spectroscopy with Supersonic Beams and Jets“, Acc. Chem. Res. 1977, 10, 139-145, DOI 10.1021/ar50112a006.

[39] A. E. Beylich, „Struktur von Überschall-Freistrahlen aus Schlitzblenden“, Z. Flugwiss. Weltraumforsch. 1979, 3, 48-58.

[40] M. V. Johnston, „Supersonic jet expansions in analytical spectroscopy“, 1984, 3, 58-61, DOI 10.1016/0165-9936(84)87055-7.

[41] J. M. Hayes, „Analytical Spectroscopy in Supersonic Expansions“, Chem. Rev. 1987, 87, 745-760, DOI 10.1021/cr00080a004. 
[42] R. S. Ruoff, T. D. Klots, T. Emilsson, H. S. Gutowsky, „Relaxation of conformers and isomers in seeded supersonic jets of inert gases“, f. Chem. Phys. 1990, 93, 31423150, DOI 10.1063/1.458848.

[43] M. D. Morse, „Supersonic Beam Sources“ in Atomic, Molecular and Optical Physics: Atoms and Molecules, (Hrsg.: F. B. Dunning), Experimental Methods in the Physical Sciences, 29B, Acad. Press, San Diego, CA, 1996, S. 21-47, DOI 10.1016/ S0076695X(08)60784-X.

[44] S. Bocklitz, M. A. Suhm, "Constraining the Conformational Landscape of a Polyether Building Block by Raman Jet Spectroscopy“, Z. Phys. Chem. 2015, 229, 1625-1648, DOI 10.1515/zpch-2015-0633.

[45] K. A. E. Meyer, M. A. Suhm, „Formic acid aggregation in 2D supersonic expansions probed by FTIR imaging“, f. Chem. Phys. 2017, 147, 144305, DOI 10.1063/1. 4989544.

[46] J. M. DeSimone, „Practical Approaches to Green Solvents“, Science 2002, 297, 799803, DOI 10.1126/science.1069622.

[47] S. Leutwyler, J. Boesiger, „Rare-gas Microsolvation of Aromatic Molecules“, Z. Phys. Chem. 1987, 154, 31-57, DOI 10.1524/zpch.1987.154.part_1_2.031.

[48] B. Brutschy, „The Structure of Microsolvated Benzene Derivatives and the Role of Aromatic Substituents“, Chem. Rev. 2000, 100, 3891-3920, DOI 10.1021/cr990055n.

[49] A. Poblotzki, J. Altnöder, M. A. Suhm, „Subtle solvation behaviour of a biofuel additive: the methanol complex with 2,5-dimethylfuran", Phys. Chem. Chem. Phys. 2016, 18, 27265-27271, DOI 10.1039/C6CP05413G.

[50] M. Heger, J. Altnöder, A. Poblotzki, M. A. Suhm, „To $\pi$ or not to $\pi$ - how does methanol dock onto anisole?", Phys. Chem. Chem. Phys. 2015, 17, 13045-13052, DOI $10.1039 / \mathrm{c} 5 \mathrm{cp} 01545 \mathrm{f}$.

[51] J. Lei, J. Zhang, G. Feng, J.-U. Grabow, Q. Gou, „Conformational preference determined by inequivalent n-pairs: rotational studies on acetophenone and its monohydrate“, Phys. Chem. Chem. Phys. 2019, 21, 22888-22894, DOI 10.1039/C9CP03904J.

[52] C. Zimmermann, H. C. Gottschalk, M. A. Suhm, „Three-dimensional docking of alcohols to ketones: an experimental benchmark based on acetophenone solvation energy balances“, Phys. Chem. Chem. Phys. 2020, 22, 2870-2877, DOI 10 . 1039 / C9CP06128B.

[53] C. A. Rice, N. Borho, M. A. Suhm, „Dimerization of Pyrazole in Slit Jet Expansions“, Z. Phys. Chem. 2005, 219, 379-388, DOI 10.1524/zpch.219.3.379.59183.

[54] N. Borho, M. A. Suhm, K. Le Barbu-Debus, A. Zehnacker, „Intra- vs. intermolecular hydrogen bonding: dimers of alpha-hydroxyesters with methanol“, Phys. Chem. Chem. Phys. 2006, 8, 4449-4460, DOI 10.1039/B609725A. 
[55] M. A. Suhm, F. Kollipost, „Femtisecond single-mole infrared spectroscopy of molecular clusters", Phys. Chem. Chem. Phys. 2013, 15, 10702-10721, DOI $10.1039 / \mathrm{C} 3 \mathrm{CP} 51515 \mathrm{~J}$.

[56] M. Herman, R. Georges, M. Hepp, D. Hurtmans, „High resolution Fourier transform spectroscopy of jet-cooled molecules“, Int. Rev. Phys. Chem. 2000, 19, 277325, DOI 10.1080/01442350050020905.

[57] T. Häber, U. Schmitt, M. A. Suhm, „FTIR-spectroscopy of molecular clusters in pulsed supersonic slit-jet expansions", Phys. Chem. Chem. Phys. 1999, 1, 55735582, DOI 10.1039/A907264K.

[58] R. M. Badger, S. H. Bauer, „Spectroscopic Studies of the Hydrogen Bond. II. The Shift of the O-H Vibrational Frequency in the Formation of the Hydrogen Bond", F. Chem. Phys. 1937, 5, 839-851, DOI 10.1063/1.1749952.

[59] E. Arunan, G. R. Desiraju, R. A. Klein, J. Sadlej, S. Scheiner, I. Alkorta, D. C. Clary, R. H. Crabtree, J. J. Dannenberg, P. Hobza, H. G. Kjaergaard, A. C. Legon, B. Mennucci, D. J. Nesbitt, „Definition of the hydrogen bond (IUPAC Recommendations 2011)“, Pure Appl. Chem. 2011, 83, 1637-1641, DOI 10.1351/PAC-REC-10-0102 .

[60] C. Puzzarini, J. F. Stanton, J. Gauss, „Quantum-chemical calculation of spectroscopic parameters for rotational spectroscopy“, Int. Rev. Phys. Chem. 2010, 29, 273367, DOI 10.1080/01442351003643401.

[61] S. Grimme, M. Steinmetz, „Effects of London dispersion correction in density functional theory on the structures of organic molecules in the gas phase", Phys. Chem. Chem. Phys. 2013, 15, 16031-16042, DOI 10.1039/c3cp52293h.

[62] M. K. Kesharwani, B. Brauer, J. M. L. Martin, „Frequency and Zero-Point Vibrational Energy Scale Factors for Double-Hybrid Density Functionals (and Other Selected Methods): Can Anharmonic Force Fields Be Avoided?", f. Phys. Chem. A 2015, 119, 1701-1714, DOI 10.1021/jp508422u.

[63] K. A. Peterson, T. H. Dunning Jr., „Benchmark calculations with correlated molecular wave functions. VII. Binding energy and structure of the HF dimer", f. Chem. Phys. 1995, 102, 2032-2041, DOI 10.1063/1.468725.

[64] J. A. Frey, C. Holzer, W. Klopper, S. Leutwyler, „Experimental and Theoretical Determination of Dissociation Energies of Dispersion-Dominated Aromatic Molecular Complexes“, Chem. Rev. 2016, 116, 5614-5641, DOI 10 . 1021/acs . chemrev . $5 \mathrm{~b} 00652$.

[65] B. Silvi, R. Wieczorek, Z. Latajka, M. E. Alikhani, A. Dkhissi, Y. Bouteiller, „Critical analysis of the calculated frequency shifts of hydrogen-bonded complexes“, $\mathcal{F}$. Chem. Phys. 1999, 111, 6671-6678, DOI 10.1063/1.480038. 
[66] J. Spanget-Larsen, B. K. Hansen, P. E. Hansen, „OH stretching frequencies in systems with intramolecular hydrogen bonds: Harmonic and anharmonic analyses", Chem. Phys. 2011, 389, 107-115, DOI 10.1016/j . chemphys.2011.09.011.

[67] M. Heger, M. A. Suhm, R. A. Mata, „Communication: Towards the binding energy and vibrational red shift of the simplest organic hydrogen bond: Harmonic constraints for methanol dimer", f. Chem. Phys. 2014, 141, 101105, DOI 10.1063/1. 4895728.

[68] F. Jensen, Introduction to Computational Chemistry, Third edition, John Wiley \& Sons, Chichester, UK und Hoboken, NJ, 2017.

[69] J. Tomasi, M. Persico, „Molecular Interactions in Solution: An Overview of Methods Based on Continuous Distributions of the Solvent", Chem. Rev. 1994, 94, 20272094, DOI 10.1021/cr00031a013.

[70] C. J. Cramer, D. G. Truhlar, „Implicit Solvation Models: Equilibria, Structure, Spectra, and Dynamics“, Chem. Rev. 1999, 99, 2161-2200, DOI 10.1021/cr960149m.

[71] M. Orozco, F. J. Luque, „Theoretical Methods for the Description of the Solvent Effect in Biomolecular Systems“, Chem. Rev. 2000, 100, 4187-4226, DOI 10.1021/ cr990052a.

[72] J. Tomasi, B. Mennucci, R. Cammi, „Quantum Mechanical Continuum Solvation Models“, Chem. Rev. 2005, 105, 2999-3093, DOI 10.1021/cr9904009.

[73] B. Tidor, M. Karplus, „The Contribution of Vibrational Entropy to Molecular Association: The Dimerization of Insulin“, f. Mol. Biol. 1994, 238, 405-414, DOI 10. 1006/jmbi.1994.1300.

[74] S. Grimme, „Supramolecular binding thermodynamics by dispersion-corrected density functional theory“, Chem. Eur. f. 2012, 18, 9955-9964, DOI 10.1002/chem. 201200497.

[75] H. C. Gottschalk, A. Poblotzki, M. A. Suhm, M. M. Al-Mogren, J. Antony, A. A. Auer, L. Baptista, D. M. Benoit, G. Bistoni, F. Bohle, R. Dahmani, D. Firaha, S. Grimme, A. Hansen, M. E. Harding, M. Hochlaf, C. Holzer, G. Jansen, W. Klopper, W. A. Kopp, L. C. Kröger, K. Leonhard, H. Mouhib, F. Neese, M. N. Pereira, I. S. Ulusoy, A. Wuttke, R. A. Mata, „The furan microsolvation blind challenge for quantum chemical methods: First steps“, f. Chem. Phys. 2018, 148, 014301, DOI 10.1063/1.5009011.

[76] N. Borho, Chirale Erkennung in Molekülclustern: Maßgeschneiderte Aggregation von $\alpha$-Hydroxyestern, 1. Aufl., Dissertation, Cuvillier Verlag, Göttingen, 2005.

[77] P. Hohenberg, W. Kohn, „Inhomogeneous Electron Gas“, Phys. Rev. 1964, 136, B864-B871, DOI 10.1103/PhysRev.136.B864.

[78] W. Kohn, L. J. Sham, „Self-Consistent Equations Including Exchange and Correlation Effects“, Phys. Rev. 1965, 140, A1133-A1138, DOI 10.1103/PhysRev.140.A1133. 
[79] S. Grimme, J. Antony, T. Schwabe, C. Mück-Lichtenfeld, „Density functional theory with dispersion corrections for supramolecular structures, aggregates, and complexes of (bio)organic molecules“, Org. Biomol. Chem. 2007, 5, 741-758, DOI $10.1039 / \mathrm{b} 615319 \mathrm{~b}$.

[80] H. C. Gottschalk, A. Poblotzki, M. Fatima, D. A. Obenchain, C. Pérez, J. Antony, A. A. Auer, L. Baptista, D. M. Benoit, G. Bistoni, F. Bohle, R. Dahmani, D. Firaha, S. Grimme, A. Hansen, M. E. Harding, M. Hochlaf, C. Holzer, G. Jansen, W. Klopper, W. A. Kopp, M. Krasowska, L. C. Kröger, K. Leonhard, M. Mogren Al-Mogren, H. Mouhib, F. Neese, M. N. Pereira, M. Prakash, I. S. Ulusoy, R. A. Mata, M. A. Suhm, M. Schnell, „The first microsolvation step for furans: New experiments and benchmarking strategies“, f. Chem. Phys. 2020, 152, 164303, DOI 10.1063/5. 0004465 .

[81] S. Grimme, „Density functional theory with London dispersion corrections“, WIREs Comput. Mol. Sci. 2011, 1, 211-228, DOI 10.1002/wcms. 30.

[82] Y. Zhao, D. G. Truhlar, „The M06 suite of density functionals for main group thermochemistry, thermochemical kinetics, noncovalent interactions, excited states, and transition elements: two new functionals and systematic testing of four M06class functionals and 12 other functionals“, Theor. Chem. Account. 2008, 120, 215241, DOI 10.1007/s00214-007-0310-x.

[83] S. Grimme, J. Antony, S. Ehrlich, H. Krieg, „A consistent and accurate ab initio parametrization of density functional dispersion correction (DFT-D) for the 94 elements H-Pu“, f. Chem. Phys. 2010, 132, 154104, DOI 10.1063/1.3382344.

[84] K. S. Thanthiriwatte, E. G. Hohenstein, L. A. Burns, C. D. Sherrill, „Assessment of the Performance of DFT and DFT-D Methods for Describing Distance Dependence of Hydrogen-Bonded Interactions“, f. Chem. Theory Comput. 2011, 7, 88-96, DOI 10.1021/ct100469b.

[85] A. D. Becke, „Density-functional thermochemistry. III. The role of exact exchange", f. Chem. Phys. 1993, 98, 5648-5652, DOI 10.1063/1.464913.

[86] P. J. Stephens, F. J. Devlin, C. F. Chabalowski, M. J. Frisch, „Ab Initio Calculation of Vibrational Absorption and Circular Dichroism Spectra Using Density Functional Force Fields“, F. Phys. Chem. 1994, 98, 11623-11627, DOI 10.1021/j100096a001.

[87] A. D. Becke, „Density-functional exchange-energy approximation with correct asymptotic behavior“, Phys. Rev. A 1988, 38, 3098-3100, DOI 10.1103/PhysRevA. 38.3098.

[88] C. Lee, W. Yang, R. G. Parr, „Development of the Colle-Salvetti correlation-energy formula into a functional of the electron density“, Phys. Rev. B 1988, 37, 785-789, DOI 10.1103/PhysRevB.37.785. 
[89] E. Burevschi, E. R. Alonso, M. E. Sanz, „Binding Site Switch by Dispersion Interactions: Rotational Signatures of Fenchone-Phenol and Fenchone-Benzene Complexes“, Chem. Eur. F. 2020, 26, 11327-11333, DOI 10.1002/chem. 202001713.

[90] A. D. Becke, E. R. Johnson, „A density-functional model of the dispersion interaction“, f. Chem. Phys. 2005, 123, 154101, DOI 10.1063/1.2065267.

[91] E. R. Johnson, A. D. Becke, „A post-Hartree-Fock model of intermolecular interactions“, f. Chem. Phys. 2005, 123, 024101, DOI 10.1063/1.1949201.

[92] E. R. Johnson, A. D. Becke, „A post-Hartree-Fock model of intermolecular interactions: inclusion of higher-order corrections“, f. Chem. Phys. 2006, 124, 174104, DOI 10.1063/1.2190220.

[93] S. Grimme, S. Ehrlich, L. Goerigk, „Effect of the Damping Function in Dispersion Corrected Density Functional Theory“, f. Comput. Chem. 2011, 32, 1456-1465, DOI $10.1002 /$ jcc. 21759 .

[94] E. G. Hohenstein, S. T. Chill, C. D. Sherrill, „Assessment of the Performance of the M05-2X and M06-2X Exchange-Correlation Functionals for Noncovalent Interactions in Biomolecules“, f. Chem. Theory Comput. 2008, 4, 1996-2000, DOI $10.1021 /$ ct $800308 \mathrm{k}$.

[95] I. León, I. Usabiaga, J. Millán, E. J. Cocinero, A. Lesarri, J. A. Fernández, „Mimicking anesthetic-receptor interactions in jets: the propofol-isopropanol cluster", Phys. Chem. Chem. Phys. 2014, 16, 16968-16975, DOI 10.1039/c4cp01702a.

[96] R. Sure, S. Grimme, „Corrected small basis set Hartree-Fock method for large systems“, f. Comput. Chem. 2013, 34, 1672-1685, DOI 10.1002/jcc. 23317.

[97] S. Grimme, J. G. Brandenburg, C. Bannwarth, A. Hansen, „Consistent structures and interactions by density functional theory with small atomic orbital basis sets“, f. Chem. Phys. 2015, 143, 054107, DOI 10.1063/1.4927476.

[98] J. G. Brandenburg, C. Bannwarth, A. Hansen, S. Grimme, „B97-3c: A revised lowcost variant of the B97-D density functional method“, f. Chem. Phys. 2018, 148, 064104, DOI 10.1063/1.5012601.

[99] H. Kruse, S. Grimme, „A geometrical correction for the inter- and intra-molecular basis set superposition error in Hartree-Fock and density functional theory calculations for large systems“, f. Chem. Phys. 2012, 136, 154101, DOI 10 .1063/1. 3700154 .

[100] L. Goerigk, S. Grimme, „Double-hybrid density functionals“, WIREs Comput. Mol. Sci. 2014, 4, 576-600, DOI 10.1002/wcms. 1193.

[101] C. Møller, M. S. Plesset, „Note on an Approximation Treatment for Many-Electron Systems“, Phys. Rev. 1934, 46, 618-622, DOI 10.1103/PhysRev.46.618.

[102] S. Grimme, „Semiempirical hybrid density functional with perturbative secondorder correlation“, f. Chem. Phys. 2006, 124, 034108, DOI 10.1063/1.2148954. 
[103] F. Weigend, R. Ahlrichs, „Balanced basis sets of split valence, triple zeta valence and quadruple zeta valence quality for $\mathrm{H}$ to $\mathrm{Rn}$ : Design and assessment of accuracy“, Phys. Chem. Chem. Phys. 2005, 7, 3297-3305, DOI 10.1039/B508541A.

[104] T. H. Dunning Jr., „Gaussian basis sets for use in correlated molecular calculations. I. The atoms boron through neon and hydrogen“, f. Chem. Phys. 1989, 90, 10071023, DOI 10.1063/1.456153.

[105] R. A. Kendall, T. H. Dunning Jr., R. J. Harrison, „Electron affinities of the first-row atoms revisited. Systematic basis sets and wave functions“, f. Chem. Phys. 1992, 96, 6796-6806, DOI 10.1063/1.462569.

[106] F. Weigend, A. Köhn, C. Hättig, „Efficient use of the correlation consistent basis sets in resolution of the identity MP2 calculations“, f. Chem. Phys. 2002, 116, 31753183, DOI 10.1063/1.1445115.

[107] F. Neese, „The ORCA program system“, WIREs Comput. Mol. Sci. 2012, 2, 73-78, DOI $10.1002 /$ wcms. 81 .

[108] F. Neese, „Software update: the ORCA program system, version 4.0“, WIREs Comput. Mol. Sci. 2018, 8, e1327, DOI 10.1002/wcms. 1327.

[109] F. Neese, F. Wennmohs, U. Becker, C. Riplinger, „The ORCA quantum chemistry program package“, f. Chem. Phys. 2020, 152, 224108, DOI 10.1063/5.0004608.

[110] D. Bykov, T. Petrenko, R. Izsák, S. Kossmann, U. Becker, E. Valeev, F. Neese, „Efficient implementation of the analytic second derivatives of Hartree-Fock and hybrid DFT energies: a detailed analysis of different approximations", Mol. Phys. 2015, 113, 1961-1977, DOI 10.1080/00268976.2015.1025114.

[111] M. J. Frisch, G. W. Trucks, H. B. Schlegel, G. E. Scuseria, M. A. Robb, J. R. Cheeseman, G. Scalmani, V. Barone, G. A. Petersson, H. Nakatsuji, X. Li, M. Caricato, A. V. Marenich, J. Bloino, B. G. Janesko, R. Gomperts, B. Mennucci, H. P. Hratchian, J. V. Ortiz, A. F. Izmaylov, J. L. Sonnenberg, D. Williams-Young, F. Ding, F. Lipparini, F. Egidi, J. Goings, B. Peng, A. Petrone, T. Henderson, D. Ranasinghe, V. G. Zakrzewski, J. Gao, N. Rega, G. Zheng, W. Liang, M. Hada, M. Ehara, K. Toyota, R. Fukuda, J. Hasegawa, M. Ishida, T. Nakajima, Y. Honda, O. Kitao, H. Nakai, T. Vreven, K. Throssell, J. A. Montgomery Jr., J. E. Peralta, F. Ogliaro, M. J. Bearpark, J. J. Heyd, E. N. Brothers, K. N. Kudin, V. N. Staroverov, T. A. Keith, R. Kobayashi, J. Normand, K. Raghavachari, A. P. Rendell, J. C. Burant, S. S. Iyengar, J. Tomasi, M. Cossi, J. M. Millam, M. Klene, C. Adamo, R. Cammi, J. W. Ochterski, R. L. Martin, K. Morokuma, Ö. Farkas, J. B. Foresman, D. J. Fox, Gaussian 16, Revision A.03, Wallingford CT, 2016.

[112] S. E. Wheeler, K. N. Houk, „Integration Grid Errors for Meta-GGA-Predicted Reaction Energies: Origin of Grid Errors for the M06 Suite of Functionals", f. Chem. Theory Comput. 2010, 6, 395-404, DOI 10.1021/ct900639j. 
[113] N. Mardirossian, M. Head-Gordon, „How Accurate Are the Minnesota Density Functionals for Noncovalent Interactions, Isomerization Energies, Thermochemistry, and Barrier Heights Involving Molecules Composed of Main-Group Elements?", f. Chem. Theory Comput. 2016, 12, 4303-4325, DOI 10.1021/acs . jctc . 6b00637.

[114] J. Řezáč, P. Hobza, „Describing Noncovalent Interactions beyond the Common Approximations: How Accurate Is the "Gold Standard," CCSD(T) at the Complete Basis Set Limit?“, f. Chem. Theory Comput. 2013, 9, 2151-2155, DOI 10 . 1021 / ct $400057 \mathrm{w}$.

[115] G. E. Scuseria, T. J. Lee, „Comparison of coupled-cluster methods which include the effects of connected triple excitations“, f. Chem. Phys. 1990, 93, 5851-5855, DOI $10.1063 / 1.459684$.

[116] J. F. Stanton, „Why CCSD(T) works: a different perspective“, Chem. Phys. Lett. 1997, 281, 130-134, DOI 10.1016/S0009-2614(97)01144-5.

[117] L. Šimová, J. Řezáč, P. Hobza, „Convergence of the Interaction Energies in Noncovalent Complexes in the Coupled-Cluster Methods Up to Full Configuration Interaction“, f. Chem. Theory Comput. 2013, 9, 3420-3428, DOI 10.1021/ct4002762.

[118] F. Coester, „Bound states of a many-particle system“, Nucl. Phys. 1958, 7, 421-424, DOI 10.1016/0029-5582(58)90280-3.

[119] F. Coester, H. Kümmel, „Short-range correlations in nuclear wave functions“, Nucl. Phys. 1960, 17, 477-485, DOI 10.1016/0029-5582(60)90140-1.

[120] G. D. Purvis, R. J. Bartlett, „A full coupled-cluster singles and doubles model: The inclusion of disconnected triples“, f. Chem. Phys. 1982, 76, 1910-1918, DOI 10 . $1063 / 1.443164$.

[121] R. J. Bartlett, „Coupled-Cluster Approach to Molecular Structure and Spectra: A Step toward Predictive Quantum Chemistry“, f. Phys. Chem. 1989, 93, 1697-1708, DOI $10.1021 / \mathrm{j} 100342 \mathrm{a} 008$.

[122] F. Neese, A. Hansen, D. G. Liakos, „Efficient and accurate approximations to the local coupled cluster singles doubles method using a truncated pair natural orbital basis“, f. Chem. Phys. 2009, 131, 064103, DOI 10.1063/1.3173827.

[123] C. Riplinger, F. Neese, „An efficient and near linear scaling pair natural orbital based local coupled cluster method“, f. Chem. Phys. 2013, 138, 034106, DOI 10 . 1063/1.4773581.

[124] C. Riplinger, B. Sandhoefer, A. Hansen, F. Neese, „Natural triple excitations in local coupled cluster calculations with pair natural orbitals“, f. Chem. Phys. 2013, 139, 134101, DOI 10.1063/1.4821834. 
[125] C. Riplinger, P. Pinski, U. Becker, E. F. Valeev, F. Neese, „Sparse maps-A systematic infrastructure for reduced-scaling electronic structure methods. II. Linear scaling domain based pair natural orbital coupled cluster theory“, f. Chem. Phys. 2016, 144, 024109, DOI 10.1063/1.4939030.

[126] C. Edmiston, M. Krauss, „Configuration-Interaction Calculation of $\mathrm{H}_{3}$ and $\mathrm{H}_{2}$ “, $\mathcal{F}$. Chem. Phys. 1965, 42,1119-1120, DOI 10.1063/1.1696050.

[127] C. Edmiston, M. Krauss, „Pseudonatural Orbitals as a Basis for the Superposition of Configurations. I. $\mathrm{He}_{2}^{+“}$, f. Chem. Phys. 1966, 45, 1833-1839, DOI 10.1063/1. 1727841.

[128] P. Pulay, „Localizability of dynamic electron correlation“, Chem. Phys. Lett. 1983, 100, 151-154, DOI 10.1016/0009-2614(83)80703-9.

[129] S. Saebø, P. Pulay, „Local Treatment of Electron Correlation“, Annu. Rev. Phys. Chem. 1993, 44, 213-236, DOI 10.1146/annurev.pc.44.100193.001241.

[130] C. Hampel, H.-J. Werner, „Local treatment of electron correlation in coupled cluster theory“, f. Chem. Phys. 1996, 104, 6286-6297, DOI 10.1063/1.471289.

[131] H.-J. Werner, K. Pflüger, „Chapter 4: On the Selection of Domains and Orbital Pairs in Local Correlation Treatments" in Annual Reports in Computational Chemistry, (Hrsg.: D. C. Spellmeyer), Vol. 2, Elsevier, 2006, S. 53-80, DOI 10 . 1016 / S1574 1400(06)02004-4.

[132] S. F. Boys, „Construction of Some Molecular Orbitals to Be Approximately Invariant for Changes from One Molecule to Another", Rev. Mod. Phys. 1960, 32, 296299, DOI 10.1103/RevModPhys.32.296.

[133] J. Pipek, P. G. Mezey, „A fast intrinsic localization procedure applicable for ab initio and semiempirical linear combination of atomic orbital wave functions", f. Chem. Phys. 1989, 90, 4916-4926, DOI 10.1063/1.456588.

[134] F. Neese, F. Wennmohs, D. Aravena, M. Atanasov, U. Becker, G. Bistoni, D. Bykov, V. G. Chilkuri, D. Datta, A. Kumar Dutta, D. Ganyushin, M. Garcia, Y. Guo, A. Hansen, B. Helmich-Paris, L. Huntington, R. Izsák, C. Kollmar, S. Kossmann, Krupička, L. Lang, D. Lenk, D. G. Liakos, D. Manganas, D. A. Pantazis, T. Petrenko, P. Pinski, C. Reimann, M. Retegan, C. Riplinger, T. Risthaus, M. Roemelt, M. Saitow, B. Sandhöfer, I. Schapiro, K. Sivalingam, B. de Souza, G. Stoychev, W. van den Heuvel, B. Wezisla, M. Kállay, S. Grimme, E. Valeev, G. Chan, J. Pittner, M. Brehm, L. Goerigk, V. Åsgeirsson, W. B. Schneider, ORCA - An ab initio, DFT and semiempirical SCF-MO package - Version 4.1.2: Manual, https: //orcaforum. kofo. mpg. de/app.php/dlext/?view=detail\&df_id=53, Mülheim a. d. Ruhr, 2019.

[135] D. G. Liakos, M. Sparta, M. K. Kesharwani, J. M. L. Martin, F. Neese, „Exploring the Accuracy Limits of Local Pair Natural Orbital Coupled-Cluster Theory“, f. Chem. Theory Comput. 2015, 11, 1525-1539, DOI 10.1021/ct501129s. 
[136] L. Goerigk, S. Grimme, „A General Database for Main Group Thermochemistry, Kinetics, and Noncovalent Interactions - Assessment of Common and Reparameterized (meta-)GGA Density Functionals“, f. Chem. Theory Comput. 2010, 6, 107126, DOI $10.1021 /$ ct900489g.

[137] L. Goerigk, S. Grimme, „Efficient and Accurate Double-Hybrid-Meta-GGA Density Functionals-Evaluation with the Extended GMTKN30 Database for General Main Group Thermochemistry, Kinetics, and Noncovalent Interactions“, f. Chem. Theory Comput. 2011, 7, 291-309, DOI 10.1021/ct100466k.

[138] L. Goerigk, A. Hansen, C. Bauer, S. Ehrlich, A. Najibi, S. Grimme, „A look at the density functional theory zoo with the advanced GMTKN55 database for general main group thermochemistry, kinetics and noncovalent interactions", Phys. Chem. Chem. Phys. 2017, 19, 32184-32215, DOI 10.1039/C7CP04913G.

[139] D. G. Liakos, Y. Guo, F. Neese, „Comprehensive Benchmark Results for the Domain Based Local Pair Natural Orbital Coupled Cluster Method (DLPNO-CCSD(T)) for Closed- and Open-Shell Systems“, f. Phys. Chem. A 2020, 124, 90-100, DOI 10 . 1021/acs. jpca.9b05734.

[140] K. A. Peterson, T. H. Dunning Jr., „Accurate correlation consistent basis sets for molecular core-valence correlation effects: The second row atoms $\mathrm{Al}-\mathrm{Ar}$, and the first row atoms B-Ne revisited“, f. Chem. Phys. 2002, 117, 10548-10560, DOI $10.1063 / 1.1520138$.

[141] C. Hättig, „Optimization of auxiliary basis sets for RI-MP2 and RI-CC2 calculations: Core-valence and quintuple- $\zeta$ basis sets for $\mathrm{H}$ to Ar and QZVPP basis sets for Li to Kr“, Phys. Chem. Chem. Phys. 2005, 7, 59-66, DOI 10.1039/B415208E.

[142] D. E. Woon, T. H. Dunning Jr., „Gaussian basis sets for use in correlated molecular calculations. III. The atoms aluminum through argon“, f. Chem. Phys. 1993, 98, 1358-1371, DOI 10.1063/1.464303.

[143] A. K. Wilson, D. E. Woon, K. A. Peterson, T. H. Dunning Jr., „Gaussian basis sets for use in correlated molecular calculations. IX. The atoms gallium through krypton", f. Chem. Phys. 1999, 110, 7667-7676, DOI 10.1063/1.478678.

[144] C. Emmeluth, V. Dyczmons, M. A. Suhm, „Tuning the Hydrogen Bond Donor/Acceptor Isomerism in Jet-Cooled Mixed Dimers of Aliphatic Alcohols“, f. Phys. Chem. A 2006, 110, 2906-2915, DOI 10.1021/jp0540407.

[145] M. Fárník, M. Weimann, C. Steinbach, U. Buck, N. Borho, T. B. Adler, M. A. Suhm, „Size-selected methyl lactate clusters: fragmentation and spectroscopic fingerprints of chiral recognition“, Phys. Chem. Chem. Phys. 2006, 8, 1148-1158, DOI 10.1039/B514117F.

[146] T. B. Adler, N. Borho, M. Reiher, M. A. Suhm, „Chirality-Induced Switch in Hydrogen-Bond Topology: Tetrameric Methyl Lactate Clusters in the Gas Phase", Angew. Chem. Int. Ed. 2006, 45, 3440-3445, DOI 10.1002/anie. 200600380. 
[147] T. N. Wassermann, D. Luckhaus, S. Coussan, M. A. Suhm, „Proton tunneling estimates for malonaldehyde vibrations from supersonic jet and matrix quenching experiments“, Phys. Chem. Chem. Phys. 2006, 8, 2344-2348, DOI 10.1039/b602319n.

[148] P. Zielke, M. A. Suhm, „Concerted proton motion in hydrogen-bonded trimers: A spontaneous Raman scattering perspective“, Phys. Chem. Chem. Phys. 2006, 8, 2826-2830, DOI 10.1039/b605926k.

[149] C. Cézard, C. A. Rice, M. A. Suhm, „OH-stretching Red Shifts in Bulky HydrogenBonded Alcohols: Jet Spectroscopy and Modeling“, f. Phys. Chem. A 2006, 110, 9839-9848, DOI 10.1021/jp0610362.

[150] T. Scharge, T. Häber, M. A. Suhm, „Quantitative chirality synchronization in trifluoroethanol dimers“, Phys. Chem. Chem. Phys. 2006, 8, 4664-4667, DOI 10.1039/ b609868a.

[151] R. Wugt Larsen, M. A. Suhm, „Cooperative organic hydrogen bonds: the librational modes of cyclic methanol clusters“, f. Chem. Phys. 2006, 125, 154314, DOI $10.1063 / 1.2358349$.

[152] C. A. Rice, I. Dauster, M. A. Suhm, „Infrared spectroscopy of pyrrole-2carboxaldehyde and its dimer: A planar $\beta$-sheet peptide model?", f. Chem. Phys. 2007, 126, 134313, DOI 10.1063/1.2715584.

[153] T. N. Wassermann, P. Zielke, J. J. Lee, C. Cézard, M. A. Suhm, „Structural Preferences, Argon Nanocoating, and Dimerization of $n$-Alkanols as Revealed by $\mathrm{OH}$ Stretching Spectroscopy in Supersonic Jets“, f. Phys. Chem. A 2007, 111, 74377448, DOI 10.1021/jp071008z.

[154] R. Wugt Larsen, P. Zielke, M. A. Suhm, „Hydrogen-bonded OH stretching modes of methanol clusters: A combined IR and Raman isotopomer study", f. Chem. Phys. 2007, 126, 194307, DOI 10.1063/1.2732745.

[155] T. Scharge, C. Cézard, P. Zielke, A. Schütz, C. Emmeluth, M. A. Suhm, „A peptide co-solvent under scrutiny: self-aggregation of 2,2,2-trifluoroethanol“, Phys. Chem. Chem. Phys. 2007, 9, 4472-4490, DOI 10.1039/b705498j.

[156] T. Scharge, D. Luckhaus, M. A. Suhm, „Observation and quantification of the hydrogen bond effect on $\mathrm{O}-\mathrm{H}$ overtone intensities in an alcohol dimer", Chem. Phys. 2008, 346, 167-175, DOI 10.1016/j . chemphys. 2008.01.028.

[157] I. Dauster, C. A. Rice, P. Zielke, M. A. Suhm, „N-H $\cdots \pi$ interactions in pyrroles: systematic trends from the vibrational spectroscopy of clusters", Phys. Chem. Chem. Phys. 2008, 10, 2827-2835, DOI 10.1039/b717823a.

[158] M. Albrecht, P. Zielke, C. A. Rice, M. A. Suhm, „Variations of bite angle and coupling patterns in double hydrogen bonds: The case of oxime dimers“', F. Mol. Struct. 2008, 880, 2-13, DOI 10.1016/j .molstruc. 2007.09.027. 
[159] A. Zehnacker, M. A. Suhm, „Chirality Recognition Between Neutral Molecules in the Gas Phase“, Angew. Chem. Int. Ed. 2008, 47, 6970-6992, DOI 10.1002 / anie. 200800957.

[160] M. Albrecht, C. A. Rice, M. A. Suhm, „Elementary Peptide Motifs in the Gas Phase: FTIR Aggregation Study of Formamide, Acetamide, $N$-Methylformamide, and $N$ Methylacetamide“, F. Phys. Chem. A 2008, 112, 7530-7542, DOI 10.1021/jp8039912.

[161] T. Scharge, T. N. Wassermann, M. A. Suhm, „Weak Hydrogen Bonds Make a Difference: Dimers of Jet-Cooled Halogenated Ethanols“, Z. Phys. Chem. 2008, 222, 1407-1452, DOI 10.1524/zpch.2008.5420.

[162] S. Hesse, M. A. Suhm, „Conformation and Aggregation of Proline Esters and Their Aromatic Homologs: Pyramidal vs. Planar RR'N-H in Hydrogen Bonds“, Z. Phys. Chem. 2009, 223, 579-604, DOI 10.1524/zpch.2009.6043.

[163] M. A. Suhm, „Hydrogen Bond Dynamics in Alcohol Clusters“ in Advances in Chemical Physics, (Hrsg.: S. A. Rice), Vol. 74, Wiley-Blackwell, Oxford, 2009, S. 1-57, DOI 10.1002/9780470475935.ch1.

[164] S. Hesse, M. A. Suhm, „On the low volatility of cyclic esters: an infrared spectroscopy comparison between dimers of $\gamma$-butyrolactone and methyl propionate", Phys. Chem. Chem. Phys. 2009, 11, 11157-11170, DOI 10.1039/b915419a.

[165] R. Wugt Larsen, M. A. Suhm, „The benefits of alternation and alkylation: large amplitude hydrogen bond librational modes of alcohol trimers and tetramers", Phys. Chem. Chem. Phys. 2010, 12, 8152-8157, DOI 10.1039/b925578h.

[166] N. O. B. Lüttschwager, T. N. Wassermann, S. Coussan, M. A. Suhm, „Periodic bond breaking and making in the electronic ground state on a sub-picosecond timescale: $\mathrm{OH}$ bending spectroscopy of malonaldehyde in the frequency domain at low temperature“, Phys. Chem. Chem. Phys. 2010, 12, 8201-8207, DOI 10.1039/c002345k.

[167] J. J. Lee, S. Hesse, M. A. Suhm, „Conformational instability upon dimerization: Prolinol“, f. Mol. Struct. 2010, 976, 397-404, DOI 10.1016/j . molstruc. 2010.03.052.

[168] M. Suhm, „Infrared and Raman Detection of Transient Chirality Recognition in the Gas Phase" in Chiral Recognition in the Gas Phase, (Hrsg.: A. Zehnacker), CRC Press, Hoboken, 2010, S. 39-46, DOI 10.1201/9781420082289.

[169] M. Hippler, S. Hesse, M. A. Suhm, „Quantum-chemical study and FTIR jet spectroscopy of $\mathrm{CHCl}_{3}-\mathrm{NH}_{3}$ association in the gas phase", Phys. Chem. Chem. Phys. 2010, 12, 13555-13565, DOI 10.1039/c0cp00530d.

[170] S. Hesse, T. N. Wassermann, M. A. Suhm, „Brightening and Locking a Weak and Floppy N-H Chromophore: The Case of Pyrrolidine“, f. Phys. Chem. A 2010, 114, 10492-10499, DOI 10.1021/jp105517b. 
[171] M. Nedić, T. N. Wassermann, R. Wugt Larsen, M. A. Suhm, „A combined Ramanand infrared jet study of mixed methanol-water and ethanol-water clusters", Phys. Chem. Chem. Phys. 2011, 13, 14050-14063, DOI 10.1039/c1cp20182d.

[172] J. J. J. Dom, B. J. van der Veken, B. Michielsen, S. Jacobs, Z. Xue, S. Hesse, H.-M. Loritz, M. A. Suhm, W. A. Herrebout, „On the weakly C-H $\cdots \pi$ hydrogen bonded complexes of sevoflurane and benzene", Phys. Chem. Chem. Phys. 2011, 13, 1414214152, DOI 10.1039/c1cp20693a.

[173] F. Kollipost, S. Hesse, J. J. Lee, M. A. Suhm, „Dimers of cyclic carbonates: chirality recognition in battery solvents and energy storage“, Phys. Chem. Chem. Phys. 2011, 13, 14176-14182, DOI 10.1039/c1cp21460h.

[174] K. E. Otto, S. Hesse, T. N. Wassermann, C. A. Rice, M. A. Suhm, T. Stafforst, U. Diederichsen, „Temperature-dependent intensity anomalies in amino acid esters: weak hydrogen bonds in protected glycine, alanine and valine“, Phys. Chem. Chem. Phys. 2011, 13, 14119-14130, DOI 10.1039/c1cp20883g.

[175] A. Sharma, I. Reva, R. Fausto, S. Hesse, Z. Xue, M. A. Suhm, S. K. Nayak, R. Sathishkumar, R. Pal, T. N. G. Row, „Conformation-Changing Aggregation in Hydroxyacetone: A Combined Low-Temperature FTIR, Jet, and Crystallographic Study“, $\mathcal{F}$. Am. Chem. Soc. 2011, 133, 20194-20207, DOI 10.1021/ja2030646.

[176] T. N. Wassermann, M. A. Suhm, P. Roubin, S. Coussan, „Isomerization around C-C and $\mathrm{C}-\mathrm{O}$ bonds in 1-propanol: Collisional relaxation in supersonic jets and selective IR photo-isomerization in cryogenic matrices“, f. Mol. Struct. 2012, 1025, 20 32, DOI 10.1016/j.molstruc.2011.12.034.

[177] B. Michielsen, J. J. J. Dom, B. J. van der Veken, S. Hesse, M. A. Suhm, W. A. Herrebout, „Solute-solvent interactions in cryosolutions: a study of halothaneammonia complexes", Phys. Chem. Chem. Phys. 2012, 14, 6469-6478, DOI 10.1039/ c2cp40379j.

[178] F. Kollipost, R. Wugt Larsen, A. V. Domanskaya, M. Nörenberg, M. A. Suhm, „Communication: The highest frequency hydrogen bond vibration and an experimental value for the dissociation energy of formic acid dimer", f. Chem. Phys. 2012, 136, 151101, DOI 10.1063/1.4704827.

[179] N. O. B. Lüttschwager, T. N. Wassermann, S. Coussan, M. A. Suhm, „Vibrational tuning of the Hydrogen transfer in malonaldehyde - a combined FTIR and Raman jet study“, Mol. Phys. 2013, 111, 2211-2227, DOI 10.1080/00268976.2013.798042.

[180] M. Heger, T. Scharge, M. A. Suhm, „From hydrogen bond donor to acceptor: the effect of ethanol fluorination on the first solvating water molecule", Phys. Chem. Chem. Phys. 2013, 15, 16065-16073, DOI 10.1039/c3cp53115e.

[181] F. Kollipost, K. Papendorf, Y.-F. Lee, Y.-P. Lee, M. A. Suhm, „Alcohol dimers - how much diagonal $\mathrm{OH}$ anharmonicity?", Phys. Chem. Chem. Phys. 2014, 16, 1594815956, DOI 10.1039/c4cp01418a. 
[182] F. Kollipost, J. Andersen, D. W. Mahler, J. Heimdal, M. Heger, M. A. Suhm, R. Wugt Larsen, „The effect of hydrogen bonding on torsional dynamics: A combined farinfrared jet and matrix isolation study of methanol dimer", f. Chem. Phys. 2014, 141, 174314, DOI 10.1063/1.4900922.

[183] F. Kollipost, A. V. Domanskaya, M. A. Suhm, „Microscopic Roots of AlcoholKetone Demixing: Infrared Spectroscopy of Methanol-Acetone Clusters“, f. Phys. Chem. A 2015, 119, 2225-2232, DOI 10.1021/jp503999b.

[184] R. Medel, M. Heger, M. A. Suhm, „Molecular Docking via Olefinic OH $\cdots \pi$ Interactions: A Bulky Alkene Model System and Its Cooperativity“, f. Phys. Chem. A 2015, 119, 1723-1730, DOI 10.1021/jp508424p.

[185] M. Heger, R. A. Mata, M. A. Suhm, „Soft hydrogen bonds to alkenes: the methanolethene prototype under experimental and theoretical scrutiny", Chem. Sci. 2015, 6, 3738-3745, DOI 10.1039/c5sc01002k.

[186] F. Kollipost, K. E. Otto, M. A. Suhm, „A Symmetric Recognition Motif between Vicinal Diols: The Fourfold Grip in Ethylene Glycol Dimer", Angew. Chem. Int. Ed. 2016, 55, 4591-4595, DOI 10.1002/anie. 201600603.

[187] C. Medcraft, S. Zinn, M. Schnell, A. Poblotzki, J. Altnöder, M. Heger, M. A. Suhm, D. Bernhard, A. Stamm, F. Dietrich, M. Gerhards, „Aromatic embedding wins over classical hydrogen bonding - a multi-spectroscopic approach for the diphenyl ether-methanol complex“, Phys. Chem. Chem. Phys. 2016, 18, 25975-25983, DOI 10.1039/c6cp03557d.

[188] T. Forsting, H. C. Gottschalk, B. Hartwig, M. Mons, M. A. Suhm, „Correcting the record: the dimers and trimers of trans- $N$-methylacetamide", Phys. Chem. Chem. Phys. 2017, 19, 10727-10737, DOI 10.1039/c6cp07989j.

[189] S. Oswald, M. Wallrabe, M. A. Suhm, „Cooperativity in Alcohol-Nitrogen Complexes: Understanding Cryomatrices through Slit Jet Expansions“, f. Phys. Chem. A 2017, 121, 3411-3422, DOI 10.1021/acs. jpca.7b01265.

[190] D. Bernhard, F. Dietrich, M. Fatima, C. Perez, A. Poblotzki, G. Jansen, M. A. Suhm, M. Schnell, M. Gerhards, „Multi-spectroscopic and theoretical analyses on the diphenyl ether-tert-butyl alcohol complex in the electronic ground and electronically excited state“, Phys. Chem. Chem. Phys. 2017, 19, 18076-18088, DOI 10.1039/ c7cp02967e.

[191] S. Oswald, M. A. Suhm, „Experimental Reference Data for Hexafluorinated Propanol by Exploring an Unusual Intermolecular Torsional Balance“, Angew. Chem. Int. Ed. 2017, 56, 12672-12676, DOI 10.1002/anie. 201705301.

[192] S. Oswald, E. Meyer, M. A. Suhm, „Dinitrogen as a Sensor for Metastable Carboxylic Acid Dimers and a Weak Hydrogen Bond Benchmarking Tool“, f. Phys. Chem. A 2018, 122, 2933-2946, DOI 10.1021/acs. jpca.8b00334. 
[193] K. A. E. Meyer, M. A. Suhm, „Vibrational exciton coupling in homo and hetero dimers of carboxylic acids studied by linear infrared and Raman jet spectroscopy", f. Chem. Phys. 2018, 149, 104307, DOI 10.1063/1.5043400.

[194] S. Oswald, M. A. Suhm, S. Coussan, „Incremental NH stretching downshift through stepwise nitrogen complexation of pyrrole: a combined jet expansion and matrix isolation study“, Phys. Chem. Chem. Phys. 2019, 21, 1277-1284, DOI 10.1039/ c8cp07053a.

[195] S. Oswald, N. A. Seifert, F. Bohle, M. Gawrilow, S. Grimme, W. Jäger, Y. Xu, M. A. Suhm, „The Chiral Trimer and a Metastable Chiral Dimer of Achiral Hexafluoroisopropanol: A Multi-Messenger Study“, Angew. Chem. Int. Ed. 2019, 58, 50805084, DOI 10.1002/anie. 201813881.

[196] G. Karir, N. O. B. Lüttschwager, M. A. Suhm, „Phenylacetylene as a gas phase sliding balance for solvating alcohols“, Phys. Chem. Chem. Phys. 2019, 21, 78317840, DOI 10.1039/c9cp00435a.

[197] R. Medel, C. Stelbrink, M. A. Suhm, „Vibrational Signatures of Chirality Recognition Between $\alpha$-Pinene and Alcohols for Theory Benchmarking“, Angew. Chem. Int. Ed. 2019, 58, 8177-8181, DOI 10.1002/anie. 201901687.

[198] C. Emmeluth, V. Dyczmons, T. Kinzel, P. Botschwina, M. A. Suhm, M. Yáñez, „Combined jet relaxation and quantum chemical study of the pairing preferences of ethanol“, Phys. Chem. Chem. Phys. 2005, 7, 991-997, DOI 10.1039/B417870J.

[199] J. P. Devlin, M. Farník, M. A. Suhm, V. Buch, „Comparative FTIR Spectroscopy of HX Adsorbed on Solid Water: Ragout-Jet Water Clusters vs Ice Nanocrystal Arrays“, F. Phys. Chem. A 2005, 109, 955-958, DOI 10.1021/jp044212k.

[200] T. Scharge, C. Emmeluth, T. Häber, M. A. Suhm, „Competing hydrogen bond topologies in 2-fluoroethanol dimer“, f. Mol. Struct. 2006, 786, 86-95, DOI $10.1016 / j$.molstruc. 2005.09.022.

[201] N. Seurre, K. Le Barbu-Debus, F. Lahmani, A. Zehnacker, N. Borho, M. A. Suhm, „Chiral recognition between lactic acid derivatives and an aromatic alcohol in a supersonic expansion: electronic and vibrational spectroscopy“, Phys. Chem. Chem. Phys. 2006, 8, 1007-1016, DOI 10.1039/B514091A.

[202] M. Weimann, M. Fárník, M. A. Suhm, M. E. Alikhani, J. Sadlej, „Cooperative and anticooperative mixed trimers of $\mathrm{HCl}$ and methanol“, f. Mol. Struct. 2006, 790, 1826, DOI 10.1016/j.molstruc.2005.12.015.

[203] P. Zielke, M. A. Suhm, „Raman jet spectroscopy of formic acid dimers: low frequency vibrational dynamics and beyond“, Phys. Chem. Chem. Phys. 2007, 9, 45284534, DOI 10.1039/b706094g. 
[204] Y. He, H. B. Müller, M. Quack, M. A. Suhm, „High Resolution FTIR and Diode Laser Supersonic Jet Spectroscopy of the $N=2$ HF Stretching Polyad in $(\mathrm{HF})_{2}$ and (HFDF): Hydrogen Bond Switching and Predissociation Dynamics“, Z. Phys. Chem. 2007, 221, 1581-1645, DOI 10.1524/zpch.2007.221.11-12.1581.

[205] T. N. Wassermann, C. A. Rice, M. A. Suhm, D. Luckhaus, „Hydrogen bonding lights up overtones in pyrazoles“, f. Chem. Phys. 2007, 127, 234309, DOI 10.1063/1. 2806181.

[206] I. Dauster, M. A. Suhm, U. Buck, T. Zeuch, „Experimental and theoretical study of the microsolvation of sodium atoms in methanol clusters: differences and similarities to sodium-water and sodium-ammonia“, Phys. Chem. Chem. Phys. 2008, 10, 83-95, DOI 10.1039/b711568g.

[207] M. Nedić, T. N. Wassermann, Z. Xue, P. Zielke, M. A. Suhm, „Raman spectroscopic evidence for the most stable water/ethanol dimer and for the negative mixing energy in cold water/ethanol trimers", Phys. Chem. Chem. Phys. 2008, 10, 59535956, DOI 10.1039/b811154e.

[208] A. Borba, M. Albrecht, A. Gómez-Zavaglia, L. Lapinski, M. J. Nowak, M. A. Suhm, R. Fausto, „Dimer formation in nicotinamide and picolinamide in the gas and condensed phases probed by infrared spectroscopy“, Phys. Chem. Chem. Phys. 2008, 10, 7010-7021, DOI 10.1039/b810002k.

[209] J. L. Wolf, M. A. Suhm, T. Zeuch, „Suppressed Particle Formation by Kinetically Controlled Ozone Removal: Revealing the Role of Transient-Species Chemistry during Alkene Ozonolysis“, Angew. Chem. Int. Ed. 2009, 48, 2231-2235, DOI 10 . 1002/anie. 200805189.

[210] J. J. Lee, S. Höfener, W. Klopper, T. N. Wassermann, M. A. Suhm, „Origin of the Argon Nanocoating Shift in the OH Stretching Fundamental of $n$-Propanol: A Combined Experimental and Quantum Chemical Study“, f. Phys. Chem. C 2009, 113, 10929-10938, DOI 10.1021/jp902194h.

[211] Z. Xue, M. A. Suhm, „Probing the stiffness of the simplest double hydrogen bond: the symmetric hydrogen bond modes of jet-cooled formic acid dimer", $\mathcal{F}$. Chem. Phys. 2009, 131, 054301, DOI 10.1063/1.3191728.

[212] T. N. Wassermann, J. Thelemann, P. Zielke, M. A. Suhm, „The stiffness of a fully stretched polyethylene chain: A Raman jet spectroscopy extrapolation“, f. Chem. Phys. 2009, 131, 161108, DOI 10.1063/1.3256221.

[213] A. Borba, M. Albrecht, A. Gómez-Zavaglia, M. A. Suhm, R. Fausto, „Low Temperature Infrared Spectroscopy Study of Pyrazinamide: From the Isolated Monomer to the Stable Low Temperature Crystalline Phase“, f. Phys. Chem. A 2010, 114, 151161, DOI 10.1021/jp907466h. 
[214] M. Albrecht, V. A. Soloshonok, L. Schrader, M. Yasumoto, M. A. Suhm, „Chiralitydependent sublimation of $\alpha$-(trifluoromethyl)-lactic acid: Relative vapor pressures of racemic, eutectic, and enantiomerically pure forms, and vibrational spectroscopy of isolated $(S, S)$ and $(S, R)$ dimers“, f. Fluorine Chem. 2010, 131, 495-504, DOI 10.1016/j.jfluchem.2009.11.015.

[215] M. Albrecht, A. Borba, K. Le Barbu-Debus, B. Dittrich, R. Fausto, S. Grimme, A. Mahjoub, M. Nedić, U. Schmitt, L. Schrader, M. A. Suhm, A. Zehnacker-Rentien, J. Zischang, „Chirality influence on the aggregation of methyl mandelate“, New $\mathcal{F}$. Chem. 2010, 34, 1266-1285, DOI 10.1039/c0nj00142b.

[216] M. Albrecht, J. Will, M. A. Suhm, „Chirality Recognition in Menthol and Neomenthol: Preference for Homoconfigurational Aggregation“, Angew. Chem. Int. Ed. 2010, 49, 6203-6206, DOI 10.1002/anie.201001565.

[217] Z. Xue, M. A. Suhm, „Adding more weight to a molecular recognition unit: the low-frequency modes of carboxylic acid dimers", Mol. Phys. 2010, 108, 2279-2288, DOI 10.1080/00268976.2010.508600.

[218] T. N. Wassermann, M. A. Suhm, „Ethanol Monomers and Dimers Revisited: A Raman Study of Conformational Preferences and Argon Nanocoating Effects“, $\mathcal{F}$. Phys. Chem. A 2010, 114, 8223-8233, DOI 10.1021/jp104861q.

[219] B. Michielsen, J. J. J. Dom, B. J. van der Veken, S. Hesse, Z. Xue, M. A. Suhm, W. A. Herrebout, „The complexes of halothane with benzene: the temperature dependent direction of the complexation shift of the aliphatic $\mathrm{C}-\mathrm{H}$ stretching", Phys. Chem. Chem. Phys. 2010, 12, 14034-14044, DOI 10.1039/c0cp00771d.

[220] J. Zischang, J. J. Lee, M. A. Suhm, „Communication: Where does the first water molecule go in imidazole?", f. Chem. Phys. 2011, 135, 061102, DOI 10.1063/1. 3624841 .

[221] M. Nedić, M. A. Suhm, „Detailed Assignment of the CH Chromophores in Methyl Mandelate and Mandelic Acid: A Multi-Experimental Approach Using Polarized FTIR Microspectroscopy of Sublimated Crystals“, Cryst. Growth Des. 2012, 12, 1933-1942, DOI 10.1021/cg201673r.

[222] J. Altnöder, J. J. Lee, K. E. Otto, M. A. Suhm, „Molecular Recognition in Glycolaldehyde, the Simplest Sugar: Two Isolated Hydrogen Bonds Win Over One Cooperative Pair“, ChemistryOpen 2012, 1, 269-275, DOI 10.1002/open.201200031.

[223] N. O. B. Lüttschwager, T. N. Wassermann, R. A. Mata, M. A. Suhm, „The Last Globally Stable Extended Alkane“, Angew. Chem. Int. Ed. 2013, 52, 463-466, DOI 10.1002/anie. 201202894.

[224] J. J. Lee, M. Albrecht, C. A. Rice, M. A. Suhm, A. Stamm, M. Zimmer, M. Gerhards, „Adaptive Aggregation of Peptide Model Systems“, f. Phys. Chem. A 2013, 117, 7050-7063, DOI 10.1021/jp400056n. 
[225] J. Altnöder, A. Bouchet, J. J. Lee, K. E. Otto, M. A. Suhm, A. Zehnacker-Rentien, "Chirality-dependent balance between hydrogen bonding and London dispersion in isolated ( \pm )-1-indanol clusters“, Phys. Chem. Chem. Phys. 2013, 15, 10167-10180, DOI $10.1039 /$ c3cp50708d.

[226] J. Zischang, M. A. Suhm, „Infrared absorption imaging of 2D supersonic jet expansions: Free expansion, cluster formation, and shock wave patterns“, $\mathrm{f}$. Chem. Phys. 2013, 139, 024201, DOI 10.1063/1.4812772.

[227] K. E. Otto, Z. Xue, P. Zielke, M. A. Suhm, „The Raman spectrum of isolated water clusters“, Phys. Chem. Chem. Phys. 2014, 16, 9849-9858, DOI 10.1039/c3cp54272f.

[228] J. Zischang, M. A. Suhm, „The OH stretching spectrum of warm water clusters“, f. Chem. Phys. 2014, 140, 064312, DOI 10.1063/1.4865130.

[229] J. Altnöder, S. Oswald, M. A. Suhm, „Phenyl- vs Cyclohexyl-Substitution in Methanol: Implications for the $\mathrm{OH}$ Conformation and for Dispersion-Affected Aggregation from Vibrational Spectra in Supersonic Jets“, f. Phys. Chem. A 2014, 118, 3266-3279, DOI 10.1021/jp501763b.

[230] P. Drawe, N. O. B. Lüttschwager, M. A. Suhm, „The elastic modulus of isolated polytetrafluoroethylene filaments“, ScienceOpen Research - Section: SOR-MATSCI 2014, 0 (ID: bb398bf3-5d4e-4069-b8db-5dcf53481c8a), 1-6, DOI 10 . 14293 / S2199 1006.1.SOR-MATSCI. AKA0J6.v2.

[231] N. O. B. Lüttschwager, M. A. Suhm, „Stretching and folding of 2-nanometer hydrocarbon rods“, Soft Matter 2014, 10, 4885-4901, DOI 10.1039/C4SM00508B.

[232] J. Altnöder, K. Krüger, D. Borodin, L. Reuter, D. Rohleder, F. Hecker, R. A. Schulz, X. T. Nguyen, H. Preiß, M. Eckhoff, M. Levien, M. A. Suhm, „The Guinness Molecules for the Carbohydrate Formula", Chem. Rec. 2014, 14, 1116-1133, DOI $10.1002 /$ tcr. 201402059 .

[233] J. Zischang, D. Skvortsov, M. Y. Choi, R. A. Mata, M. A. Suhm, A. F. Vilesov, „Helium Nanodroplet Study of the Hydrogen-Bonded $\mathrm{OH}$ Vibrations in $\mathrm{HCl}-\mathrm{H}_{2} \mathrm{O}$ Clusters“, f. Phys. Chem. A 2015, 119, 2636-2643, DOI 10.1021/jp509683g.

[234] M. Heger, K. E. Otto, R. A. Mata, M. A. Suhm, „Bracketing subtle conformational energy differences between self-solvated and stretched trifluoropropanol“, Phys. Chem. Chem. Phys. 2015, 17, 9899-9909, DOI 10.1039/c4cp05868b.

[235] M. Heger, J. Andersen, M. A. Suhm, R. Wugt Larsen, „The donor OH stretchinglibration dynamics of hydrogen-bonded methanol dimers in cryogenic matrices", Phys. Chem. Chem. Phys. 2016, 18, 3739-3745, DOI 10.1039/c5cp07387a.

[236] D. M. Hewett, S. Bocklitz, D. P. Tabor, E. L. Sibert III, M. A. Suhm, T. S. Zwier, „Identifying the first folded alkylbenzene via ultraviolet, infrared, and Raman spectroscopy of pentylbenzene through decylbenzene", Chem. Sci. 2017, 8, 5305-5318, DOI $10.1039 / \mathrm{c} 7 \mathrm{sc} 02027 \mathrm{a}$. 
[237] S. Bocklitz, M. A. Suhm, „Polymer Segments at the Folding Limit: Raman Scattering for the Diglyme Benchmark“, ChemPhysChem 2017, 18, 3570-3575, DOI $10.1002 /$ cphc. 201701169.

[238] T. Forsting, J. Zischang, M. A. Suhm, M. Eckhoff, B. Schröder, R. A. Mata, „Strained hydrogen bonding in imidazole trimer: a combined infrared, Raman, and theory study“, Phys. Chem. Chem. Phys. 2019, 21, 5989-5998, DOI 10.1039/c9cp00399a.

[239] D. Bernhard, M. Fatima, A. Poblotzki, A. L. Steber, C. Pérez, M. A. Suhm, M. Schnell, M. Gerhards, „Dispersion-controlled docking preference: multispectroscopic study on complexes of dibenzofuran with alcohols and water", Phys. Chem. Chem. Phys. 2019, 21, 16032-16046, DOI 10.1039/C9CP02635E.

[240] M. A. Suhm, N. O. B. Lüttschwager, Publications of Martin Suhm, https://hbond. uni-goettingen.de/suhm/suhmpubs.html (besucht am 16.05.2020).

[241] M. A. Suhm, M. Albrecht, „Comment on “Theoretical investigations into the enantiomeric and racemic forms of $\alpha$-(trifluoromethyl)lactic acid” by R. Tonner, V. A. Soloshonok and P. Schwerdtfeger, Phys. Chem. Chem. Phys., 2011, 13, 811-817“, Phys. Chem. Chem. Phys. 2011, 13, 4159-4160, DOI 10.1039/c0cp02455d.

[242] M. Heger, M. A. Suhm, „Comment on: “Quantum Confinement in Hydrogen Bond” by Carlos da Silva dos Santos, Elso Drigo Filho, and Regina Maria Ricotta, Int. J. Quantum Chem. 2015, 115, 765-770“, Int. f. Quantum Chem. 2015, 115, 1510-1511, DOI 10.1002 /qua. 24958.

[243] M. Heger, Diagonal and Off-Diagonal Anharmonicity in Hydrogen-Bonded Systems, $\mathrm{PhD}$ thesis, Georg-August-Universität Göttingen, Göttingen, 2015, http : / / hdl . handle. net/11858/00-1735-0000-0028-874B-E.

[244] J. Zischang, Strukturdynamik wasserhaltiger Aggregate in Überschallexpansionen, Dissertation, Georg-August-Universität Göttingen, Göttingen, 2014, http: //hdl. handle. net/11858/00-1735-0000-0022-5F0D-D.

[245] R. Bhargava, D. C. Fernandez, M. D. Schaeberle, I. W. Levin, „Theory and Application of Gain Ranging to Fourier Transform Infrared Spectroscopic Imaging “, Appl. Spectrosc. 2001, 55, 1580-1589, DOI 10.1366/0003702011954143.

[246] P. R. Griffiths, J. A. de Haseth, Fourier Transform Infrared Spectrometry, 2nd ed., Wiley-Interscience, Hoboken, N.J, 2007.

[247] V. Meyer, S. Eisermann, H. C. Gottschalk, R. Hildebrandt, B. Langer, U. Schmitt, M. Zippert, M. A. Suhm, „Gratin Jet Figures: Version 1.0“, 2020, DOI 10. 25625/6V6KSN.

[248] H. C. Gottschalk, Dispersionskontrollierte Regioselektivität in Komplexen zwischen Alkoholen und aromatischen Ethern, Masterarbeit, Georg-August-Universität Göttingen, Göttingen, 2015.

[249] A. Simon, J. Gast, A. Keens, United States Patent No. 5,309,217, 1994. 
[250] L. Mertz, „Auxiliary computation for Fourier spectrometry“, Infrared Phys. 1967, 7, 17-23, DOI 10.1016/0020-0891(67)90026-7.

[251] (a) R. H. Norton, R. Beer, „New apodizing functions for Fourier spectrometry“, $\mathcal{F}$. Opt. Soc. Am. 1976, 66, 259-264, DOI 10.1364/JOSA.66.000259; (b) R. H. Norton, R. Beer, „Errata: New apodizing functions for Fourier spectrometry“, f. Opt. Soc. Am. 1977, 67, 419, DOI 10.1364/JOSA.67.000419.

[252] H. Nyquist, „Certain Topics in Telegraph Transmission Theory“, Trans. Am. Inst. Electr. Eng. 1928, 47, 617-644, DOI 10.1109/T-AIEE.1928.5055024.

[253] C. Camy-Peyret, J. M. Flaud, G. Guelachvili, C. Amiot, „High resolution Fourier

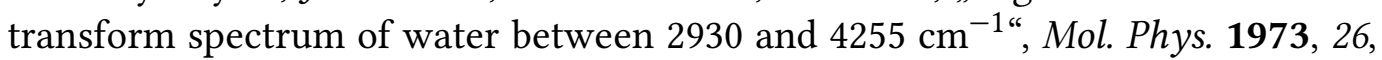
825-855, DOI 10.1080/00268977300102131.

[254] U. Grigull, „Normvolumen und Normkubikmeter“, Brennst.-Wärme-Kraft 1967, 19, 561-563.

[255] DIN 1343:1990-01: Referenzzustand, Normzustand, Normvolumen; Begriffe und Werte, Berlin, 1990, DOI 10.31030/2333155.

[256] D. Sonntag, „Important new Values of the Physical Constants of 1986, Vapour Pressure Formulations based on ITS-90, and Psychrometer Formulae“, Z. Meteorol. 1990, 40, 340-344.

[257] Ethylenglykol: Eintrag in der GESTIS-Stoffdatenbank, http : / gestis . itrust.de / nxt / gateway . dll /gestis_de / 012060 . xml ? f = templates $\$ \mathrm{fn}=$ default . htm $\$ 3.0$ (besucht am 26.11.2019).

[258] Fundamental Physical Constants: molar gas constant: 2018 CODATA recommended values, 2019, https : / / physics . nist . gov / cgi - bin/cuu/value ? eqr (besucht am 26. 11.2019).

[259] P. J. Mohr, D. B. Newell, B. N. Taylor, E. Tiesinga, „Data and analysis for the CODATA 2017 special fundamental constants adjustment“, Metrologia 2018, 55, 125146, DOI 10.1088/1681-7575/aa99bc.

[260] D. B. Newell, F. Cabiati, J. Fischer, K. Fujii, S. G. Karshenboim, H. S. Margolis, E. de Mirandés, P. J. Mohr, F. Nez, K. Pachucki, T. J. Quinn, B. N. Taylor, M. Wang, B. M. Wood, Z. Zhang, „The CODATA 2017 values of $h, e, k$, and $N_{A}$ for the revision of the SI“, Metrologia 2018, 55, L13-L16, DOI 10.1088/1681-7575/aa950a.

[261] T. S. Zwier, „The Spectroscopy of Solvation in Hydrogen-Bonded Aromatic Clusters“, Annu. Rev. Phys. Chem. 1996, 47, 205-241, DOI 10.1146/annurev.physchem. 47.1.205.

[262] F. Lahmani, C. Lardeux-Dedonder, D. Solgadi, A. Zehnacker, „Spectroscopic Study of the Anisole-Benzene Complex Formed in a Supersonic Free Jet“' f. Phys. Chem. 1989, 93, 3984-3989, DOI 10.1021/j100347a024. 
[263] M. Mons, I. Dimicoli, F. Piuzzi, „Gas phase hydrogen-bonded complexes of aromatic molecules: Photoionization and energetics“, Int. Rev. Phys. Chem. 2002, 21, 101-135, DOI 10.1080/01442350110104310.

[264] M. Becucci, F. Mazzoni, G. Pietraperzia, J. Řezáč, D. Natchigallová, P. Hobza, „Noncovalent interactions in anisole- $\left(\mathrm{CO}_{2}\right)_{n}(n=1,2)$ complexes", Phys. Chem. Chem. Phys. 2017, 19, 22749-22758, DOI 10.1039/C7CP03763E.

[265] A. S. Hansen, E. Vogt, H. G. Kjaergaard, „Gibbs energy of complex formation combining infrared spectroscopy and vibrational theory“, Int. Rev. Phys. Chem. 2019, 38, 115-148, DOI 10.1080/0144235X.2019.1608689.

[266] P. Banerjee, T. Chakraborty, „Weak hydrogen bonds: insights from vibrational spectroscopic studies“, Int. Rev. Phys. Chem. 2018, 37, 83-123, DOI 10.1080/0144235X.2018.1419731.

[267] W. Y. Sohn, K.-J. Cho, S. Y. Lee, S. S. Kang, Y. D. Park, H. Kang, „Solvent-assisted conformational isomerization (SACI) of meta-substituted phenols: Tuning relative stability, isomerization barrier, and IVR rate", Chem. Phys. Lett. 2012, 525-526, 3743, DOI 10.1016/j.cplett.2012.01.004.

[268] P. L. Raston, G. E. Douberly, W. Jäger, „Single and double resonance spectroscopy of methanol embedded in superfluid helium nanodroplets“, f. Chem. Phys. 2014, 141, 044301, DOI 10.1063/1.4887348.

[269] A. Metzelthin, O. Birer, E. Sánchez-García, M. Havenith, „High resolution IR spectroscopy of acetylene-furan in ultracold helium nanodroplets", f. Chem. Phys. 2008, 129, 114307, DOI 10.1063/1.2976772.

[270] P. Asselin, B. Madebène, P. Soulard, P. Reinhardt, M. E. Alikhani, „Evidence of an isomeric pair in furan... HCl: Fourier transform infrared spectroscopy and ab initio calculations“, f. Chem. Phys. 2008, 128, 244301, DOI 10.1063/1.2944242.

[271] E. Sánchez-García, G. Jansen, „Competition between $\mathrm{H} \cdots \pi$ and $\mathrm{H} \cdots \mathrm{O}$ interactions in furan heterodimers“, f. Phys. Chem. A 2012, 116, 5689-5697, DOI 10 . 1021/ jp301710y.

[272] M. H. Sirvetz, „The Microwave Spectrum of Furan“, f. Chem. Phys. 1951, 19, 16091610, DOI 10.1063/1.1748135.

[273] A. Mellouki, J. Liévin, M. Herman, „The vibrational spectrum of pyrrole $\left(\mathrm{C}_{4} \mathrm{H}_{5} \mathrm{~N}\right)$ and furan $\left(\mathrm{C}_{4} \mathrm{H}_{4} \mathrm{O}\right)$ in the gas phase“, Chem. Phys. 2001, 271, 239-266, DOI 10 . 1016/S0301-0104(01)00447-5.

[274] I. A. Finneran, S. T. Shipman, S. L. Widicus Weaver, „Rotational spectroscopy of 2-methylfuran from 8.7 to 960GHz“, f. Mol. Spectrosc. 2012, 280, 27-33, DOI 10 . 1016/j.jms.2012.06.005. 
[275] J. Green, D. J. Harrison, „Spectroscopic and thermodynamic properties of furan derivatives-I. 2- and 2,5-substituted compounds“, Spectrochim. Acta A 1977, 33, 843-848, DOI 10.1016/0584-8539(77)80081-0.

[276] V. Van, J. Bruckhuisen, W. Stahl, V. Ilyushin, H. V. L. Nguyen, „The torsional barriers of two equivalent methyl internal rotations in 2,5-dimethylfuran investigated by microwave spectroscopy“, f. Mol. Spectrosc. 2018, 343, 121-125, DOI 10.1016/ j.jms.2017.11.007.

[277] F. Dietrich, D. Bernhard, M. Fatima, C. Pérez, M. Schnell, M. Gerhards, „The Effect of Dispersion on the Structure of Diphenyl Ether Aggregates“, Angew. Chem. Int. Ed. 2018, 57, 9534-9537, DOI 10.1002/anie. 201801842.

[278] J. P. A. Ioannidis, „Author’s Reply“, PLoS Med. 2005, 2, e398, DOI 10.1371/journal. pmed. 0020398.

[279] J. Granatier, M. Pitoňák, P. Hobza, „Accuracy of Several Wave Function and Density Functional Theory Methods for Description of Noncovalent Interaction of Saturated and Unsaturated Hydrocarbon Dimers", f. Chem. Theory Comput. 2012, 8, 2282-2292, DOI 10.1021/ct300215p.

[280] J. C. Howard, G. S. Tschumper, „Wavefunction methods for the accurate characterization of water clusters", WIREs Comput. Mol. Sci. 2014, 4, 199-224, DOI $10.1002 /$ wcms . 1168 .

[281] M. Hapka, Ł. Rajchel, M. Modrzejewski, R. Schäffer, G. Chałasiński, M. M. Szczęśniak, „The nature of three-body interactions in DFT: Exchange and polarization effects“, f. Chem. Phys. 2017, 147, 084106, DOI 10.1063/1.4986291.

[282] W. Jankiewicz, R. Podeszwa, H. A. Witek, „Dispersion-Corrected DFT Struggles with Predicting Three-Body Interaction Energies", f. Chem. Theory Comput. 2018, 14, 5079-5089, DOI 10.1021/acs. jctc.8b00167.

[283] (a) R. Sure, S. Grimme, „Comprehensive Benchmark of Association (Free) Energies of Realistic Host-Guest Complexes“, f. Chem. Theory Comput. 2015, 11, 37853801, DOI 10 . 1021 / acs . jctc . 5b00296; (b) R. Sure, S. Grimme, „Correction to Comprehensive Benchmark of Association (Free) Energies of Realistic Host-Guest Complexes“, f. Chem. Theory Comput. 2015, 11, 5990, DOI 10.1021/acs . jctc . 5b01016.

[284] T. Fuji, Y.-I. Suzuki, T. Horio, T. Suzuki, R. Mitrić, U. Werner, V. Bonačić-Koutecký, „Ultrafast photodynamics of furan“, f. Chem. Phys. 2010, 133, 234303, DOI 10 . 1063/1.3518441.

[285] Equilibrium Molecular Structures: From Spectroscopy to Quantum Chemistry, 1st ed., (Hrsg.: J. Demaison, J. E. Boggs, A. G. Császár), CRC Press, 2010.

[286] S. G. Kukolich, J. A. Shea, „The microwave spectrum and molecular structure of the furan-argon complex“, f. Chem. Phys. 1982, 77, 5242-5243, DOI 10.1063/1. 443664. 
[287] R. M. Spycher, L. Hausherr-Primo, G. Grassi, A. Bauder, „Rotational spectra of isotopic furan-(argon $)_{n}, n=1,2$, complexes and their vibrationally averaged structures“, f. Mol. Struct. 1995, 351, 7-17, DOI 10.1016/0022-2860(94)08494-3.

[288] C. Puzzarini, M. Biczysko, „Microsolvation of 2-Thiouracil: Molecular Structure and Spectroscopic Parameters of the Thiouracil-Water Complex", f. Phys. Chem. A 2015, 119, 5386-5395, DOI 10.1021/jp510511d.

[289] Q. Gou, L. B. Favero, G. Feng, L. Evangelisti, C. Pérez, W. Caminati, „Interactions between Ketones and Alcohols: Rotational Spectrum and Internal Dynamics of the Acetone-Ethanol Complex“, Chem. Eur. F. 2017, 23, 11119-11125, DOI 10 . 1002 / chem. 201702090.

[290] C. Pérez, A. Krin, A. L. Steber, J. C. López, Z. Kisiel, M. Schnell, „Wetting Camphor: Multi-Isotopic Substitution Identifies the Complementary Roles of Hydrogen Bonding and Dispersive Forces“, f. Phys. Chem. Lett. 2016, 7, 154-160, DOI 10.1021/acs. jpclett. 5b02541.

[291] X. Jiang, S. Liu, N. T. Tsona, S. Tang, L. Ding, H. Zhao, L. Du, „Matrix isolation FTIR study of hydrogen-bonded complexes of methanol with heterocyclic organic compounds“, RSC Adv. 2017, 7, 2503-2512, DOI 10.1039/C6RA26076D.

[292] E. Sánchez-García, A. Mardyukov, M. Studentkowski, L. A. Montero, W. Sander, „Furan-Formic Acid Dimers: An ab Initio and Matrix Isolation Study“, f. Phys. Chem. A 2006, 110, 13775-13785, DOI 10.1021/jp065064w.

[293] S. P. Lockwood, T. G. Fuller, J. J. Newby, „Structure and Spectroscopy of Furan: $\mathrm{H}_{2} \mathrm{O}$ Complexes“, f. Phys. Chem. A 2018, 122, 7160-7170, DOI 10.1021/acs . jpca . $8 \mathrm{~b} 06308$.

[294] X. Jiang, N. T. Tsona, S. Tang, L. Du, „Hydrogen bond docking preference in furans: O-H $\cdots \pi$ vs. O-H $\cdots$ O“, Spectrochim. Acta A 2018, 191, 155-164, DOI 10.1016/j . saa.2017.10.006.

[295] R. Knochenmuss, R. K. Sinha, A. Poblotzki, T. Den, S. Leutwyler, „Intermolecular dissociation energies of hydrogen-bonded 1-naphthol complexes“, f. Chem. Phys. 2018, 149, 204311, DOI 10.1063/1.5055720.

[296] A. Poblotzki, Intermolecular energy scales based on aromatic ethers and alcohols, $\mathrm{PhD}$ thesis, Georg-August-Universität Göttingen, Göttingen, 2019, http : / / hdl . handle.net/21.11130/00-1735-0000-0003-C1AD-0.

[297] R. H. Hunt, W. N. Shelton, F. A. Flaherty, W. B. Cook, „Torsion-Rotation Energy Levels and the Hindering Potential Barrier for the Excited Vibrational State of the OH-Stretch Fundamental Band $v_{1}$ of Methanol“, f. Mol. Spectrosc. 1998, 192, 277293, DOI 10.1006/jmsp.1998.7689. 
[298] D. Schmitz, V. Alvin Shubert, T. Betz, M. Schnell, „Multi-resonance effects within a single chirp in broadband rotational spectroscopy: The rapid adiabatic passage regime for benzonitrile“, f. Mol. Spectrosc. 2012, 280, 77-84, DOI 10.1016/j . jms . 2012.08 .001 .

[299] G. G. Brown, B. C. Dian, K. O. Douglass, S. M. Geyer, S. T. Shipman, B. H. Pate, „A broadband Fourier transform microwave spectrometer based on chirped pulse excitation“, Rev. Sci. Instrum. 2008, 79, 053103, DOI 10.1063/1.2919120.

[300] S. Samdal, H. Møllendal, S. Reine, J.-C. Guillemin, „Ring Planarity Problem of 2Oxazoline Revisited Using Microwave Spectroscopy and Quantum Chemical Calculations“, f. Phys. Chem. A 2015, 119, 4875-4884, DOI 10.1021/acs. jpca.5b02528.

[301] R. A. Creswell, I. M. Mills, „Microwave Spectra and Centrifugal Distortion Constants of Oxetane“, f. Mol. Spectrosc. 1974, 52, 392-412, DOI 10.1016/0022-2852(74) 90188-X.

[302] H. A. Harker, M. R. Viant, F. N. Keutsch, E. A. Michael, R. P. McLaughlin, R. J. Saykally, „Water Pentamer: Characterization of the Torsional-Puckering Manifold by Terahertz VRT Spectroscopy“, f. Phys. Chem. A 2005, 109, 6483-6497, DOI 10. 1021/jp051504s.

[303] D. J. Nesbitt, R. Naaman, „On the apparent spectroscopic rigidity of floppy molecular systems“, F. Chem. Phys. 1989, 91, 3801-3809, DOI 10.1063/1.456865.

[304] T. Risthaus, M. Steinmetz, S. Grimme, „Implementation of Nuclear Gradients of Range-Separated Hybrid Density Functionals and Benchmarking on Rotational Constants for Organic Molecules“, f. Comput. Chem. 2014, 35, 1509-1516, DOI $10.1002 /$ jcc. 23649 .

[305] A. Hellweg, S. A. Grün, C. Hättig, „Benchmarking the performance of spincomponent scaled CC2 in ground and electronically excited states", Phys. Chem. Chem. Phys. 2008, 10, 4119-4127, DOI 10.1039/B803727B.

[306] W. Klopper, „Highly accurate coupled-cluster singlet and triplet pair energies from explicitly correlated calculations in comparison with extrapolation techniques", Mol. Phys. 2001, 99, 481-507, DOI 10.1080/00268970010017315.

[307] F. Weigend, „A fully direct RI-HF algorithm: Implementation, optimised auxiliary basis sets, demonstration of accuracy and efficiency“, Phys. Chem. Chem. Phys. 2002, 4, 4285-4291, DOI 10.1039/B204199P.

[308] A. Karton, J. M. L. Martin, „Explicitly correlated Wn theory: W1-F12 and W2-F12“, f. Chem. Phys. 2012, 136, 124114, DOI 10.1063/1.3697678.

[309] E. F. Valeev, „Improving on the resolution of the identity in linear R12 ab initio theories“, Chem. Phys. Lett. 2004, 395, 190-195, DOI 10.1016/j . cplett. 2004.07. 061. 


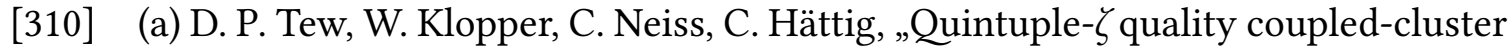
correlation energies with triple- $\zeta$ basis sets", Phys. Chem. Chem. Phys. 2007, 9, 1921-1930, DOI 10 . 1039/B617230J; (b) D. P. Tew, W. Klopper, C. Neiss, C. Hättig, „Comment on Quintuple- $\zeta$ quality coupled-cluster correlation energies with triple- $\zeta$ basis sets by D. P. Tew, W. Klopper, C. Neiss and C. Hättig, Phys. Chem. Chem. Phys., 2007, 9, 1921 [erratum]“, Phys. Chem. Chem. Phys. 2008, 10, 63256327, DOI 10.1039/B811567B.

[311] J. A. Montgomery Jr., M. J. Frisch, J. W. Ochterski, G. A. Petersson, „A complete basis set model chemistry. VI. Use of density functional geometries and frequencies“, F. Chem. Phys. 1999, 110, 2822-2827, DOI 10.1063/1.477924.

[312] J. A. Montgomery Jr., M. J. Frisch, J. W. Ochterski, G. A. Petersson, „A complete basis set model chemistry. VII. Use of the minimum population localization method", f. Chem. Phys. 2000, 112, 6532-6542, DOI 10.1063/1.481224.

[313] J.-D. Chai, M. Head-Gordon, „Long-range corrected hybrid density functionals with damped atom-atom dispersion corrections", Phys. Chem. Chem. Phys. 2008, 10, 6615-6620, DOI 10.1039/B810189B.

[314] V. Barone, „Vibrational zero-point energies and thermodynamic functions beyond the harmonic approximation", f. Chem. Phys. 2004, 120, 3059-3065, DOI 10.1063/ 1.1637580 .

[315] V. Barone, „Anharmonic vibrational properties by a fully automated second-order perturbative approach“, f. Chem. Phys. 2005, 122, 014108, DOI 10.1063/1.1824881.

[316] V. Barone, M. Biczysko, J. Bloino, M. Borkowska-Panek, I. Carnimeo, P. Panek, „Toward Anharmonic Computations of Vibrational Spectra for Large Molecular Systems“, Int. f. Quantum Chem. 2012, 112, 2185-2200, DOI 10.1002/qua. 23224.

[317] D. M. Benoit, B. Madebene, I. Ulusoy, L. Mancera, Y. Scribano, S. Chulkov, „Towards a scalable and accurate quantum approach for describing vibrations of molecule-metal interfaces“, Beilstein f. Nanotechnol. 2011, 2, 427-447, DOI 10.3762/bjnano.2.48.

[318] J. Pfaendtner, X. Yu, L. J. Broadbelt, „The 1-D hindered rotor approximation“, Theor. Chem. Acc. 2007, 118, 881-898, DOI 10.1007/s00214-007-0376-5.

[319] G. M. Chaban, J. O. Jung, R. B. Gerber, „Ab initio calculation of anharmonic vibrational states of polyatomic systems: Electronic structure combined with vibrational self-consistent field“, f. Chem. Phys. 1999, 111, 1823-1829, DOI 10.1063/1. 479452.

[320] C. Qu, J. M. Bowman, "Quantum approaches to vibrational dynamics and spectroscopy: is ease of interpretation sacrificed as rigor increases?", Phys. Chem. Chem. Phys. 2019, 21, 3397-3413, DOI 10.1039/C8CP04990D. 
[321] A. Nejad, M. A. Suhm, „Concerted Pair Motion Due to Double Hydrogen Bonding: The Formic Acid Dimer Case“, f. Indian Inst. Sci. 2020, 100, 5-19, DOI 10.1007 / s41745-019-00137-5.

[322] J. Řezáč, D. Nachtigallová, F. Mazzoni, M. Pasquini, G. Pietraperzia, M. Becucci, K. Müller-Dethlefs, P. Hobza, „Binding Energies of the $\pi$-Stacked Anisole Dimer: New Molecular Beam-Laser Spectroscopy Experiments and CCSD(T) Calculations“, Chem. Eur. F. 2015, 21, 6740-6746, DOI 10.1002/chem. 201406134.

[323] A. Otero-de-la-Roza, E. R. Johnson, „A benchmark for non-covalent interactions in solids“, f. Chem. Phys. 2012, 137, 054103, DOI 10.1063/1.4738961.

[324] L. J. H. Hoffmann, S. Marquardt, A. S. Gemechu, H. Baumgärtel, „The absorption spectra of anisole-h8, anisole-d 3 and anisole-d8. The assignment of fundamental vibrations in the $\mathrm{S}_{0}$ and the $\mathrm{S}_{1}$ states“, Phys. Chem. Chem. Phys. 2006, 8, 2360-2377, DOI $10.1039 /$ B600438P.

[325] H.-D. Barth, K. Buchhold, S. Djafari, B. Reimann, U. Lommatzsch, B. Brutschy, „Hydrogen bonding in (substituted benzene) ( water) $n$ clusters with $n \leq 4$ “, Chem. Phys. 1998, 239, 49-64, DOI 10.1016/S0301-0104(98)00306-1.

[326] B. Reimann, K. Buchhold, H.-D. Barth, B. Brutschy, P. Tarakeshwar, K. S. Kim, „Anisole- $\left(\mathrm{H}_{2} \mathrm{O}\right)_{n}(n=1-3)$ complexes: An experimental and theoretical investigation of the modulation of optimal structures, binding energies, and vibrational spectra in both the ground and first excited states", f. Chem. Phys. 2002, 117, 88058822, DOI 10.1063/1.1510443.

[327] J. B. Nicholas, B. P. Hay, „Anisole as an Ambidentate Ligand: Ab Initio Molecular Orbital Study of Alkali Metal Cations Binding to Anisole“, f. Phys. Chem. A 1999, 103, 9815-9820, DOI 10.1021/jp990570p.

[328] P. J. Breen, E. R. Bernstein, H. V. Secor, J. I. Seeman, „Spectroscopic Observation and Geometry Assignment of the Minimum Energy Conformations of MethoxySubstituted Benzenes“, f. Am. Chem. Soc. 1989, 111, 1958-1968, DOI 10 . 1021 / ja00188a002.

[329] G. Pietraperzia, M. Pasquini, F. Mazzoni, G. Piani, M. Becucci, M. Biczysko, D. Michalski, J. Bloino, V. Barone, „Noncovalent Interactions in the Gas Phase: The Anisole-Phenol Complex“, f. Phys. Chem. A 2011, 115, 9603-9611, DOI 10.1021/ jp200444a.

[330] B. M. Giuliano, W. Caminati, „Isotopomeric Conformational Change in AnisoleWater", Angew. Chem. Int. Ed. 2005, 44, 603-606, DOI 10.1002/anie. 200461860.

[331] B. M. Giuliano, A. Maris, S. Melandri, W. Caminati, „Pure Rotational Spectrum and Model Calculations of Anisole-Ammonia“, f. Phys. Chem. A 2009, 113, 1427714280, DOI 10.1021/jp9026964. 
[332] M. Becucci, S. Melandri, „High-Resolution Spectroscopic Studies of Complexes Formed by Medium-Size Organic Molecules“, Chem. Rev. 2016, 116, 5014-5037, DOI 10.1021/acs.chemrev.5b00512.

[333] L. Ferres, H. Mouhib, W. Stahl, H. V. L. Nguyen, „Methyl Internal Rotation in the Microwave Spectrum of o-Methyl Anisole“, ChemPhysChem 2017, 18, 1855-1859, DOI 10.1002/cphc. 201700276.

[334] F. Lahmani, J. Sepiol, „Spectral hole-burning isolation of fluorescent species in jetcooled 9-methoxyanthracene complexes with water and alcohols“, Chem. Phys. Lett. 1992, 189, 479-485, DOI 10.1016/0009-2614(92)85235-3.

[335] M. Born, R. Oppenheimer, „Zur Quantentheorie der Molekeln“, Ann. Phys. 1927, 389, 457-484, DOI 10.1002/andp.19273892002.

[336] K. A. E. Meyer, J. A. Davies, A. M. Ellis, „Shifting formic acid dimers into perspective: vibrational scrutiny in helium nanodroplets“, Phys. Chem. Chem. Phys. 2020, 22, 9637-9646, DOI 10.1039/D0CP01060J.

[337] S. Liu, „Where does the electron go? The nature of ortho/para and meta group directing in electrophilic aromatic substitution“, f. Chem. Phys. 2014, 141, 194109, DOI $10.1063 / 1.4901898$.

[338] R. Podeszwa, K. Szalewicz, „Communication: Density functional theory overcomes the failure of predicting intermolecular interaction energies“, 7 . Chem. Phys. 2012, 136, 161102, DOI 10.1063/1.4707166.

[339] N. Mardirossian, M. Head-Gordon, „Characterizing and Understanding the Remarkably Slow Basis Set Convergence of Several Minnesota Density Functionals for Intermolecular Interaction Energies“, f. Chem. Theory Comput. 2013, 9, 44534461, DOI 10.1021/ct400660j.

[340] J. P. M. Lommerse, S. L. Price, R. Taylor, „Hydrogen Bonding of Carbonyl, Ether, and Ester Oxygen Atoms with Alkanol Hydroxyl Groups“, f. Comput. Chem. 1997, 18, 757-774, DOI 10 .1002/(SICI) 1096-987X(19970430) 18:6<757: :AID-JCC3>3 . 0 . CO;2-R.

[341] K. Schwing, M. Gerhards, „Investigations on isolated peptides by combined IR/UV spectroscopy in a molecular beam - structure, aggregation, solvation and molecular recognition“, Int. Rev. Phys. Chem. 2016, 35, 569-677, DOI 10.1080/0144235X. 2016.1229331.

[342] P.-S. Huang, S. E. Boyken, D. Baker, „The coming of age of de novo protein design“, Nature 2016, 537, 320-327, DOI 10.1038/nature19946.

[343] E. D. Becker, „Infrared studies of hydrogen bonding in alcohol-base systems“, Spectrochim. Acta 1961, 17, 436-447, DOI 10.1016/0371-1951(61)80095-7. 
[344] C. Dorval, T. Zeegers-Huyskens, „Infrared Study of the Interaction of NMethylacetamide with Phenol Derivatives“, Spectrosc. Lett. 1974, 7, 247-253, DOI $10.1080 / 00387017408067244$.

[345] M. D. G. Faria, J. Teixeira-Dias, R. Fausto, „Hydrogen bonding involving $\alpha, \beta$ unsaturated carboxylic esters and substituted phenols: an infrared spectroscopic study“, f. Mol. Struct. 1991, 263, 87-94, DOI 10.1016/0022-2860(91)80058-C.

[346] M. Ahmed, C. Jelsch, B. Guillot, C. Lecomte, S. Domagała, „Relationship between Stereochemistry and Charge Density in Hydrogen Bonds with Oxygen Acceptors", Cryst. Growth Des. 2013, 13, 315-325, DOI 10.1021/cg3014656.

[347] I. León, A. Lesarri, J. A. Fernández, „Evaluation of the aggregation process in a mixture of propofol and benzocaine", Phys. Chem. Chem. Phys. 2019, 21, 35373544, DOI $10.1039 / \mathrm{C} 8 \mathrm{CP} 04386 \mathrm{H}$.

[348] B. K. Oram, Monu, B. Bandyopadhyay, „Impact of donor acidity and acceptor anharmonicity on $v_{\mathrm{C}=\mathrm{O}}$ spectral shifts in $\mathrm{O}-\mathrm{H} \cdots \mathrm{O}=\mathrm{C} \mathrm{H}$-bonded ketone-alcohol complexes: An IR spectroscopic investigation“, Spectrochim. Acta A 2020, 230, 118070, DOI $10.1016 /$ j.saa.2020.118070.

[349] W. B. Motherwell, J. Moïse, A. E. Aliev, M. Nic, S. J. Coles, P. N. Horton, M. B. Hursthouse, G. Chessari, C. A. Hunter, J. G. Vinter, „Noncovalent Functional-GroupArene Interactions“, Angew. Chem. Int. Ed. 2007, 46, 7823-7826, DOI 10.1002 / anie. 200701463.

[350] A. E. Aliev, J. R. T. Arendorf, I. Pavlakos, R. B. Moreno, M. J. Porter, H. S. Rzepa, W. B. Motherwell, „Surfing $\pi$ Clouds for Noncovalent Interactions: Arenes versus Alkenes“, Angew. Chem. Int. Ed. 2015, 54, 551-555, DOI 10.1002/anie. 201409672.

[351] S. Yamada, N. Yamamoto, E. Takamori, „Synthesis of Molecular Seesaw Balances and the Evaluation of Pyridinium- $\pi$ Interactions", f. Org. Chem. 2016, 81, 1181911830, DOI 10.1021/acs. joc.6b02295.

[352] P. Banerjee, P. Pandey, B. Bandyopadhyay, „Stereo-preference of camphor for Hbonding with phenol, methanol and chloroform: A combined matrix isolation IR spectroscopic and quantum chemical investigation", Spectrochim. Acta A 2019, 209, 186-195, DOI 10.1016/j. saa.2018.10.031.

[353] R. Norman Jones, W. F. Forbes, W. A. Mueller, „The Infrared Carbonyl Stretching Bands of Ring Substituted Acetophenones“, Can. F. Chem. 1957, 35, 504-514, DOI 10.1139/v57-071.

[354] A. Gambi, S. Giorgianni, A. Passerini, R. Visinoni, S. Ghersetti, „Infrared studies of acetophenone and its deuterated derivatives", Spectrochim. Acta A 1980, 36, 871878, DOI 10.1016/0584-8539(80)80036-5. 
[355] D. G. Leopold, R. J. Hemley, V. Vaida, J. L. Roebber, „Direct absorption spectra of higher excited states of jet-cooled monosubstituted benzenes: Phenylacetylene, styrene, benzaldehyde, and acetophenone“, f. Chem. Phys. 1981, 75, 4758-4769, DOI 10.1063/1.441911.

[356] N. Ohmori, T. Suzuki, M. Ito, „Why Does Intersystem Crossing Occur In Isolated Molecules of Benzaldehyde, Acetophenone, and Benzophenone?", f. Phys. Chem. 1988, 92, 1086-1093, DOI 10.1021/j100316a019.

[357] M. Onda, Y. Kohama, K. Suga, I. Yamaguchi, „Microwave spectrum and molecular planarity of acetophenone“, f. Mol. Struct. 1998, 442, 19-22, DOI 10.1016/s0022 2860 (97) 00067-7.

[358] Y. Wang, K. Ushida, Y. Tominaga, A. Kira, „Molecular dynamics of acetophenone and its derivatives investigated by femtosecond optical Kerr effect spectroscopy and depolarized low-frequency Raman scattering“, Chem. Phys. Lett. 1999, 299, 576-582, DOI 10.1016/S0009-2614(98)01306-2.

[359] Y. Yomogida, R. Nozaki, „Dielectric study of acetophenone and its derivatives“, $\mathcal{F}$. Mol. Liq. 2009, 149, 101-104, DOI 10.1016/j .molliq.2009.09.001.

[360] A. Haloui, Y. Arfaoui, „A DFT study of the conformational behavior of parasubstituted acetophenones in vacuum and in various solvents“, F. Mol. Struct. THEOCHEM 2010, 950, 13-19, DOI 10.1016/j.theochem. 2010.03.012.

[361] X. Zhu, V. V. Lozovoy, J. D. Shah, M. Dantus, „Photodissociation Dynamics of Acetophenone and Its Derivatives with Intense Nonresonant Femtosecond Pulses“, $\mathcal{F}$. Phys. Chem. A 2011, 115, 1305-1312, DOI 10.1021/jp1029486.

[362] A. H. Al-Muhtaseb, M. Altarawneh, „Theoretical study on thermochemical parameters of chlorinated isomers of acetophenone", Comput. Theor. Chem. 2011, 966, 38-43, DOI 10.1016/j. comptc.2011.02.009.

[363] F. W. Patureau, T. Besset, F. Glorius, „Rhodium-Catalyzed Oxidative Olefination of $\mathrm{C}-\mathrm{H}$ Bonds in Acetophenones and Benzamides“, Angew. Chem. Int. Ed. 2011, 50, 1064-1067, DOI 10.1002/anie.201006222.

[364] C. E. Garrett, K. Prasad, O. Repič, T. J. Blacklock, „The enantioselective reduction of 2'-fluoroacetophenone utilizing a simplified CBS-reduction procedure", Tetrahedron: Asymmetry 2002, 13, 1347-1349, DOI 10.1016/S0957-4166(02)00373-7.

[365] Z. Weng, S. Muratsugu, N. Ishiguro, S.-i. Ohkoshi, M. Tada, „Preparation of surface molecularly imprinted Ru-complex catalysts for asymmetric transfer hydrogenation in water media“" Dalton Trans. 2011, 40, 2338-2347, DOI 10.1039/C0DT00950D.

[366] H. Gröger, C. Rollmann, F. Chamouleau, I. Sebastien, O. May, W. Wienand, K. Drauz, „Enantioselective Reduction of 4-Fluoroacetophenone at High Substrate Concentration using a Tailor-Made Recombinant Whole-Cell Catalyst", $A d v$. Synth. Catal. 2007, 349, 709-712, DOI 10.1002/adsc. 200600606. 
[367] R. Hess, A. Vargas, T. Mallat, T. Bürgi, A. Baiker, „Inversion of enantioselectivity in the platinum-catalyzed hydrogenation of substituted acetophenones", f. Catal. 2004, 222, 117-128, DOI 10.1016/j. jcat.2003.10.021.

[368] P. A. Dub, N. J. Henson, R. L. Martin, J. C. Gordon, „Unravelling the Mechanism of the Asymmetric Hydrogenation of Acetophenone by $\left[\mathrm{RuX}_{2}\right.$ (diphosphine)(1,2-diamine)] Catalysts“, F. Am. Chem. Soc. 2014, 136, 3505-3521, DOI $10.1021 / j a 411374 j$.

[369] A. A. Koesoema, D. M. Standley, S. Ohshima, M. Tamura, T. Matsuda, „Control of enantioselectivity in the enzymatic reduction of halogenated acetophenone analogs by substituent positions and sizes“, Tetrahedron Lett. 2020, 61, 151820, DOI $10.1016 /$ j.tetlet.2020.151820.

[370] A. Monney, F. Nastri, M. Albrecht, „Peptide-tethered monodentate and chelating histidylidene metal complexes: synthesis and application in catalytic hydrosilylation“, Dalton Trans. 2013, 42, 5655-5660, DOI 10.1039/C3DT50424G.

[371] H. Yoshida, Y. Onodera, S.-i. Fujita, H. Kawamori, M. Arai, „Solvent effects in heterogeneous selective hydrogenation of acetophenone: differences between $\mathrm{Rh} / \mathrm{C}$ and $\mathrm{Rh} / \mathrm{Al}_{2} \mathrm{O}_{3}$ catalysts and the superiority of water as a functional solvent", Green Chem. 2015, 17, 1877-1883, DOI 10.1039/C4GC02211D.

[372] M. Ito, K. Inuzuka, S. Imanishi, „Correlation between Solvent-Induced Frequency Shifts of the $n-\pi^{*}$ Ultraviolet Absorption and the Infrared C=O Band of Ketones", f. Chem. Phys. 1959, 31, 1694, DOI 10.1063/1.1730692.

[373] A. A. Lamola, „Lowest $\pi$, $\pi^{\star}$ Triplet State of Acetophenone“, f. Chem. Phys. 1967, 47, 4810-4816, DOI 10.1063/1.1701703.

[374] M. V. Garcia, M. I. Redondo, „Influence of solvent polarity and acidity properties on the frequency of the carbonyl band of dimethylformamide, acetophenone and benzophenone“, Spectrochim. Acta A 1987, 43, 879-885, DOI 10 .1016/05848539(87)80238-6.

[375] S. K. Ghoshal, S. K. Sarkar, G. S. Kastha, „Effects of Intermolecular Hydrogenbonding on the Luminescence Properties of Acetophenone. Characterization of Emission States“, Bull. Chem. Soc. Jpn. 1981, 54, 3556-3561, DOI 10.1246/bcsj. 54. 3556.

[376] R. Thijs, T. Zeegers-Huyskens, „Infrared and Raman studies of hydrogen bonded complexes involving acetone, acetophenone and benzophenone-I. Thermodynamic constants and frequency shifts of the $v_{\mathrm{OH}}$ and $v_{\mathrm{C}=\mathrm{O}}$ stretching vibrations", Spectrochim. Acta A 1984, 40, 307-313, DOI 10.1016/0584-8539(84)80054-9.

[377] T. Gramstad, „Studies of hydrogen bonding-part VII. Hydrogen-bond association of phenol and pentachlorophenol with carbonyl compounds and ethers", Spectrochim. Acta 1963, 19, 497-508, DOI 10.1016/0371-1951(63)80060-0. 
[378] R. Thijs, T. Zeegers-Huyskens, „Infrared and Raman studies of hydrogen bonded complexes involving acetone, acetophenone and benzophenone-II. Raman intensity of the $v_{\mathrm{C}=\mathrm{O}}$ band“, Spectrochim. Acta A 1984, 40, 1057-1061, DOI 10.1016/ 0584-8539(84)80133-6.

[379] R. Iwamoto, H. Kusanagi, R. Harui, „Novel Hydrogen-Bonding Pattern of Water in Polycarbonate“, Bull. Chem. Soc. Jpn. 2017, 90, 527-536, DOI 10 . 1246 / bcsj . 20160394.

[380] Known Bug: Analytic Hessian with RI, 2020, https : / / orcaforum . kofo . mpg . de / viewtopic. php? $f=26 \& t=5566$ (besucht am 08. 05. 2020).

[381] G. A. Andrienko, ChemCraft - graphical software for visualization of quantum chemistry computations, Version 1.8, build 562b, 2019, https: //chemcraftprog.com/ (besucht am 23.07.2020).

[382] T. Ebata, A. Fujii, N. Mikami, „Vibrational spectroscopy of small-sized hydrogenbonded clusters and their ions", Int. Rev. Phys. Chem. 1998, 17, 331-361, DOI 10 . 1080/014423598230081.

[383] F. D. Lewis, R. W. Johnson, D. E. Johnson, „Conformational Control of Photochemical Behavior. Competitive $\alpha$ Cleavage and $\gamma$-Hydrogen Abstraction of Alkyl Phenyl Ketones“, f. Am. Chem. Soc. 1974, 96, 6090-6099, DOI 10.1021/ja00826a 022. 


\section{Lebenslauf}

\section{Wissenschaftlicher Entwicklungsgang}

2015-2020 Promotion (Promotionsprogramm Chemie), Georg-August-

Universität Göttingen

Dissertation: „IR-Untersuchung von schwach gebundenen

Molekülaggregaten im Überschallstrahl“

2013-2015 Master of Science (Chemie), Georg-August-Universität Göttingen Masterarbeit: „Dispersionskontrollierte Regioselektivität in

Komplexen zwischen Alkoholen und aromatischen Ethern“

2010-2013

Bachelor of Science (Chemie), Georg-August-Universität

Göttingen

Bachelorarbeit: „Theoretische Untersuchung eines

Kupferkatalysators"

2009

Abitur, Kaiserin-Auguste-Viktoria Gymnasium Celle

\section{Publikationen}

[1] H. C. Gottschalk, J. Altnöder, M. Heger, M. A. Suhm, „Control over the HydrogenBond Docking Site in Anisole by Ring Methylation", Angew. Chem. Int. Ed. 2016, 55, 1921-1924, DOI 10.1002/anie. 201508481.

[2] T. Forsting, H. C. Gottschalk, B. Hartwig, M. Mons, M. A. Suhm, „Correcting the record: the dimers and trimers of trans- $N$-methylacetamide“, Phys. Chem. Chem. Phys. 2017, 19, 10727-10737, DOI 10.1039/c6cp07989j.

[3] A. Poblotzki, H. C. Gottschalk, M. A. Suhm, „Tipping the Scales: Spectroscopic Tools for Intermolecular Energy Balances“, f. Phys. Chem. Lett. 2017, 8, 5656-5665, DOI 10.1021/acs.jpclett.7b02337. 
[4] H. C. Gottschalk, A. Poblotzki, M. A. Suhm, M. M. Al-Mogren, J. Antony, A. A. Auer, L. Baptista, D. M. Benoit, G. Bistoni, F. Bohle, R. Dahmani, D. Firaha, S. Grimme, A. Hansen, M. E. Harding, M. Hochlaf, C. Holzer, G. Jansen, W. Klopper, W. A. Kopp, L. C. Kröger, K. Leonhard, H. Mouhib, F. Neese, M. N. Pereira, I. S. Ulusoy, A. Wuttke, R. A. Mata, „The furan microsolvation blind challenge for quantum chemical methods: First steps“, f. Chem. Phys. 2018, 148, 014301, DOI $10.1063 / 1.5009011$.

[5] D. Bernhard, F. Dietrich, M. Fatima, C. Pérez, H. C. Gottschalk, A. Wuttke, R. A. Mata, M. A. Suhm, M. Schnell, M. Gerhards, „The phenyl vinyl ether-methanol complex: a model system for quantum chemistry benchmarking", Beilstein f. Org. Chem. 2018, 14, 1642-1654, DOI 10.3762/bjoc.14.140.

[6] C. Zimmermann, H. C. Gottschalk, M. A. Suhm, „Three-dimensional docking of alcohols to ketones: an experimental benchmark based on acetophenone solvation energy balances“, Phys. Chem. Chem. Phys. 2020, 22, 2870-2877, DOI 10 . 1039 / С9CP06128B.

[7] H. C. Gottschalk, A. Poblotzki, M. Fatima, D. A. Obenchain, C. Pérez, J. Antony, A. A. Auer, L. Baptista, D. M. Benoit, G. Bistoni, F. Bohle, R. Dahmani, D. Firaha, S. Grimme, A. Hansen, M. E. Harding, M. Hochlaf, C. Holzer, G. Jansen, W. Klopper, W. A. Kopp, M. Krasowska, L. C. Kröger, K. Leonhard, M. Mogren Al-Mogren, H. Mouhib, F. Neese, M. N. Pereira, M. Prakash, I. S. Ulusoy, R. A. Mata, M. A. Suhm, M. Schnell, „The first microsolvation step for furans: New experiments and benchmarking strategies“, f. Chem. Phys. 2020, 152, 164303, DOI 10.1063/5. 0004465 . 
